\title{
Connecting and Analyzing Enantioselective Bifunctional Hydrogen Bond Donor Catalysis Using Data Science Tools
}

\author{
Jacob Werth and Matthew S. Sigman* \\ Department of Chemistry, University of Utah, 315 South 1400 East, Salt Lake City, UT 84112, \\ United States
}

\section{Table of Contents}

Computational Methods

S2

Cartesian Coordinates for Catalysts

S3

Cartesian Coordinates for Electrophiles

S60

Cartesian Coordinates for Nucleophiles

S158

Cartesian Coordinates for Solvents

S228

Collected Parameters

S233

Data Curation

S239

Model Development

S262

References

S289 


\section{Computational Methods}

All reaction components (catalyst, electrophile, nucleophile and solvent) were optimized in the gas-phase with B3LYPD3BJ/6-31(d,p) $)^{1-4}$ as implemented in Gaussian 16 (revision A.03). ${ }^{5}$ Single point calculations were run with M06-2X/def2-TZVP. ${ }^{6-7}$ The optimized geometries were verified by frequency computations as minima (zero imaginary frequencies). Parameters were acquired from these ground state structures. NBO charges were calculated using NBO 3.1 at the M062X/def2-TZVP level. ${ }^{8}$ NMR computations were calculated at the M06-2X/def2-TZVP level using the GIAO method and referenced to an optimized structure of tetramethylsilane. ${ }^{9}$ Sterimol values were calculated using a modified version of Paton's Python script to accommodate nonterminal reference atoms. ${ }^{10}$

Conformational searches were performed using Macromodel version 11.8 and the OPLS3 force field. ${ }^{11-12}$ The hydrogen bond donor dihedral angle of the catalysts was constrained to an antianti conformation during searches to obtain structures relevant to catalysis. ${ }^{13-14}$

Multidimensional regression analyses were performed using MATLAB ${ }^{\circledR 15}$ 


\section{Cartesian Coordinates - Catalysts}<smiles>CN(C)C1CCCC[C@@H]1NC(=S)Nc1cc(C(F)(F)F)cc(C(F)(F)F)c1</smiles>

Thiourea-1

$\begin{array}{lrrr}\mathrm{C} & -1.79446900 & 0.92398600 & 0.24653800 \\ \mathrm{C} & -1.28613000 & -0.34702800 & -0.05068100 \\ \mathrm{C} & -2.17480000 & -1.40588900 & -0.27155000 \\ \mathrm{C} & -3.54984400 & -1.19728000 & -0.20361600 \\ \mathrm{C} & -4.06439900 & 0.06738200 & 0.07005100 \\ \mathrm{C} & -3.17302200 & 1.11613300 & 0.29179800 \\ \mathrm{C} & -4.48171700 & -2.36566700 & -0.37016300 \\ \mathrm{C} & -3.70964800 & 2.50079800 & 0.54112000 \\ \mathrm{~F} & -3.97094300 & -3.29956000 & -1.20418900 \\ \mathrm{~F} & -4.71593400 & -2.98294900 & 0.81147800 \\ \mathrm{~F} & -5.67915600 & -1.98749800 & -0.86270700 \\ \mathrm{~F} & -3.87047400 & 3.18370200 & -0.61328700 \\ \mathrm{~F} & -4.91637200 & 2.46437100 & 1.14987100 \\ \mathrm{~F} & -2.88166300 & 3.22739900 & 1.32123400 \\ \mathrm{~N} & 0.08626500 & -0.63756000 & -0.07523100 \\ \mathrm{C} & 1.15639700 & 0.15041700 & -0.46511800 \\ \mathrm{H} & 0.30062700 & -1.62378400 & -0.00570500 \\ \mathrm{~N} & 2.33417100 & -0.50369900 & -0.27209200 \\ \mathrm{~S} & 1.03121300 & 1.68892400 & -1.11530200 \\ \mathrm{H} & 2.37263400 & -1.14635100 & 0.52149200 \\ \mathrm{C} & 3.63889000 & 0.11224200 & -0.48436600 \\ \mathrm{C} & 4.66274600 & -0.68103700 & 0.35116000 \\ \mathrm{C} & 6.03466700 & 0.00812800 & 0.26299900 \\ \mathrm{C} & 6.49933200 & 0.09044400 & -1.19903700 \\ \mathrm{C} & 5.45643200 & 0.78552900 & -2.08470500 \\ \mathrm{C} & 4.07437800 & 0.13280200 & -1.95434600 \\ \mathrm{~N} & 4.13201800 & -0.95307200 & 1.69748800 \\ \mathrm{C} & 3.97209200 & 0.22820900 & 2.54252500 \\ \mathrm{C} & 4.85102700 & -2.01327400 & 2.39234900 \\ \mathrm{H} & -1.12161500 & 1.74674800 & 0.43318700 \\ \mathrm{H} & -1.79486100 & -2.39391600 & -0.51023600 \\ \mathrm{H} & -5.13310700 & 0.23127300 & 0.11379000 \\ \mathrm{H} & 3.59448600 & 1.15270100 & -0.13519700 \\ \mathrm{H} & 4.75834100 & -1.66985600 & -0.12054800 \\ \mathrm{H} & 6.76921600 & -0.53871300 & 0.86408000 \\ \mathrm{H} & 5.96596000 & 1.01892400 & 0.68364600 \\ \mathrm{H} & 6.67172200 & -0.92735400 & -1.57528000 \\ \mathrm{H} & 7.46093000 & 0.61190400 & -1.25872000 \\ \mathrm{H} & 5.77901800 & 0.77359800 & -3.13118800 \\ \mathrm{H} & 5.37949400 & 1.84184000 & -1.79427500\end{array}$




$\begin{array}{lrrr}\mathrm{H} & 4.10161700 & -0.89856400 & -2.32955700 \\ \mathrm{H} & 3.33152200 & 0.67805400 & -2.54022700 \\ \mathrm{H} & 3.43894300 & -0.05745100 & 3.45406600 \\ \mathrm{H} & 4.92901700 & 0.68621700 & 2.84134100 \\ \mathrm{H} & 3.37430500 & 0.98458700 & 2.02897400 \\ \mathrm{H} & 4.30448200 & -2.28734300 & 3.30006200 \\ \mathrm{H} & 4.91476100 & -2.89769900 & 1.75151700 \\ \mathrm{H} & 5.87521000 & -1.73183400 & 2.69110800\end{array}$<smiles>FC(F)(F)c1cc(NC(=S)N[C@H]2CCCCC2N2CCCC2)cc(C(F)(F)F)c1</smiles>

Thiourea-2

$\begin{array}{lrrc}\text { C } & -2.39799600 & -1.23053300 & -0.74026200 \\ \text { C } & -1.51671800 & -0.23893400 & -0.29302000 \\ \text { C } & -2.02794300 & 0.88282700 & 0.37236400 \\ \text { C } & -3.40340500 & 1.00269100 & 0.55427500 \\ \text { C } & -4.28752000 & 0.02087600 & 0.10930400 \\ \text { C } & -3.76867800 & -1.10078100 & -0.53238700 \\ \text { C } & -3.94810500 & 2.24574000 & 1.20662700 \\ \text { C } & -4.68675400 & -2.21436400 & -0.95463700 \\ \text { F } & -4.20415600 & 3.21012000 & 0.29600300 \\ \text { F } & -5.10745400 & 1.99892500 & 1.85708800 \\ \text { F } & -3.08120000 & 2.76414800 & 2.10213300 \\ \text { F } & -4.82525400 & -3.13844300 & 0.02474900 \\ \text { F } & -5.92307800 & -1.76251300 & -1.24925900 \\ \text { F } & -4.21755800 & -2.86594300 & -2.04272700 \\ \text { N } & -0.14427800 & -0.45999300 & -0.48065500 \\ \text { C } & 0.88267500 & 0.44396400 & -0.69919300 \\ \text { N } & 2.08219300 & -0.19563100 & -0.76345000 \\ \text { S } & 0.68555500 & 2.09610000 & -0.88986100 \\ \text { C } & 3.35942500 & 0.50251900 & -0.86324900 \\ \text { C } & 4.44432100 & -0.45139100 & -0.32452500 \\ \text { C } & 5.79436600 & 0.28483300 & -0.28806200 \\ \text { C } & 6.17226100 & 0.77627100 & -1.69372600 \\ \text { C } & 5.06825400 & 1.65191900 & -2.30199200 \\ \text { C } & 3.70784000 & 0.94296000 & -2.28885500 \\ \text { N } & 3.98374400 & -1.07370300 & 0.91736800 \\ \text { C } & 4.74471600 & -2.23130400 & 1.38895800 \\ \text { C } & 4.10318100 & -2.55288400 & 2.75256900 \\ \text { C } & 3.44620000 & -1.21580300 & 3.20007800 \\ \text { C } & 3.81878600 & -0.21404600 & 2.09441100 \\ \text { H } & -2.01654600 & -2.10107200 & -1.26390700 \\ \text { H } & -1.35995800 & 1.64886000 & 0.73498900\end{array}$




$\begin{array}{rrrr}\mathrm{H} & -5.35352100 & 0.12573000 & 0.26278300 \\ \mathrm{H} & 0.09292100 & -1.41785100 & -0.70355000 \\ \mathrm{H} & 2.18426400 & -1.02574500 & -0.17518400 \\ \mathrm{H} & 3.31472300 & 1.40101000 & -0.23241400 \\ \mathrm{H} & 4.53642100 & -1.27745800 & -1.04560700 \\ \mathrm{H} & 6.57385000 & -0.37673200 & 0.10471700 \\ \mathrm{H} & 5.72788600 & 1.14116100 & 0.39418100 \\ \mathrm{H} & 6.34068900 & -0.09469900 & -2.34201900 \\ \mathrm{H} & 7.11944600 & 1.32555300 & -1.65739300 \\ \mathrm{H} & 5.33181800 & 1.93767600 & -3.32583300 \\ \mathrm{H} & 4.98837200 & 2.58513400 & -1.72857300 \\ \mathrm{H} & 2.92281500 & 1.60348200 & -2.66304500 \\ \mathrm{H} & 3.73268400 & 0.05652400 & -2.93608300 \\ \mathrm{H} & 4.67518800 & -3.05200400 & 0.66766300 \\ \mathrm{H} & 5.81497200 & -1.99533200 & 1.52118200 \\ \mathrm{H} & 4.84880800 & -2.91090600 & 3.46657500 \\ \mathrm{H} & 3.34812500 & -3.33634400 & 2.64928500 \\ \mathrm{H} & 3.79547500 & -0.88156400 & 4.17983900 \\ \mathrm{H} & 2.36015300 & -1.32519700 & 3.25920300 \\ \mathrm{H} & 4.75260600 & 0.31259600 & 2.35401300 \\ \mathrm{H} & 3.04717900 & 0.54085700 & 1.92019300\end{array}$<smiles>FC(F)(F)c1cc(NC(=S)N[C@H]2CCCCC2N2CCCCC2)cc(C(F)(F)F)c1</smiles>

Thiourea-3

$\begin{array}{lrrr}\mathrm{C} & 2.14769900 & -0.82169700 & 0.51928700 \\ \mathrm{C} & 1.62669700 & 0.10001200 & -0.39798900 \\ \mathrm{C} & 2.49448400 & 0.98832600 & -1.04435500 \\ \mathrm{C} & 3.86184900 & 0.95218600 & -0.78408800 \\ \mathrm{C} & 4.39118000 & 0.02526200 & 0.11022000 \\ \mathrm{C} & 3.52032100 & -0.85401000 & 0.75215000 \\ \mathrm{C} & 4.76321400 & 1.96574500 & -1.43328100 \\ \mathrm{C} & 4.07869800 & -1.89526500 & 1.68565600 \\ \mathrm{~F} & 6.01555200 & 1.49235500 & -1.59790800 \\ \mathrm{~F} & 4.30310800 & 2.34119900 & -2.64815400 \\ \mathrm{~F} & 4.85964800 & 3.09080800 & -0.68682300 \\ \mathrm{~F} & 3.19908100 & -2.22443000 & 2.65515900 \\ \mathrm{~F} & 4.39023900 & -3.03303300 & 1.02738800 \\ \mathrm{~F} & 5.20980500 & -1.46624500 & 2.28963500 \\ \mathrm{~N} & 0.25465000 & 0.22973900 & -0.65961500 \\ \mathrm{C} & -0.74321300 & -0.73103500 & -0.68416200 \\ \mathrm{~N} & -1.95546200 & -0.16126500 & -0.92358000\end{array}$




$\begin{array}{rrrr}\mathrm{S} & -0.50178100 & -2.37527900 & -0.47682400 \\ \mathrm{C} & -3.21224000 & -0.89829400 & -0.86106600 \\ \mathrm{C} & -4.32746200 & 0.12581600 & -0.56802500 \\ \mathrm{C} & -5.65538700 & -0.61710100 & -0.34845600 \\ \mathrm{C} & -6.01314400 & -1.44750100 & -1.59081100 \\ \mathrm{C} & -4.87878800 & -2.40633000 & -1.97869800 \\ \mathrm{C} & -3.54148000 & -1.67187900 & -2.14223800 \\ \mathrm{~N} & -3.89947100 & 1.06561000 & 0.47702800 \\ \mathrm{C} & -3.73305600 & 0.48185500 & 1.81371500 \\ \mathrm{C} & -2.99632500 & 1.46105600 & 2.72931300 \\ \mathrm{C} & -3.73651100 & 2.80073300 & 2.80237400 \\ \mathrm{C} & -3.98987200 & 3.34669700 & 1.39280100 \\ \mathrm{C} & -4.68687600 & 2.29913900 & 0.52252900 \\ \mathrm{H} & 1.48951600 & -1.50346100 & 1.03550500 \\ \mathrm{H} & 2.10555200 & 1.70255000 & -1.76276900 \\ \mathrm{H} & 5.45497400 & -0.00865300 & 0.30559100 \\ \mathrm{H} & -0.00014400 & 1.10330600 & -1.10167200 \\ \mathrm{H} & -2.09012800 & 0.78345600 & -0.55542000 \\ \mathrm{H} & -3.14658900 & -1.62728600 & -0.04238100 \\ \mathrm{H} & -4.43905100 & 0.74002500 & -1.47353200 \\ \mathrm{H} & -6.45354700 & 0.09995000 & -0.12750300 \\ \mathrm{H} & -5.56752800 & -1.27668800 & 0.52371800 \\ \mathrm{H} & -6.21052700 & -0.76493800 & -2.42889400 \\ \mathrm{H} & -6.94079500 & -2.00375600 & -1.41694400 \\ \mathrm{H} & -5.13077700 & -2.93650900 & -2.90323000 \\ \mathrm{H} & -4.76943300 & -3.17278400 & -1.19982300 \\ \mathrm{H} & -2.73486500 & -2.37761000 & -2.35232200 \\ \mathrm{H} & -3.59226400 & -0.96552400 & -2.98111600 \\ \mathrm{H} & -4.70906700 & 0.22464000 & 2.26322600 \\ \mathrm{H} & -3.16278100 & -0.44641300 & 1.72613000 \\ \mathrm{H} & -1.98482000 & 1.61661800 & 2.33345500 \\ \mathrm{H} & -2.89052600 & 1.01952300 & 3.72612000 \\ \mathrm{H} & -3.17256700 & 3.52362700 & 3.40145000 \\ \mathrm{H} & -4.69991800 & 2.65212300 & 3.30930600 \\ \mathrm{H} & -3.03427200 & 3.61181600 & 0.92325600 \\ \mathrm{H} & -4.59922900 & 4.25644000 & 1.43058900 \\ \mathrm{H} & -4.80280500 & 2.66938300 & -0.50180700 \\ \mathrm{H} & -5.70356800 & 2.11788100 & 0.91847800\end{array}$<smiles>CCN(CC)C1CCCC[C@H]1NC(=S)Nc1cc(C(F)(F)F)cc(C(F)(F)F)c1</smiles>

Thiourea-4

\begin{tabular}{|c|c|c|}
\hline \multirow[t]{3}{*}{$\mathrm{C}$} & 2.0149680 & -0.90145400 \\
\hline & 1.5043800 & 0.27911800 \\
\hline & 2.3862790 & 1.30332900 \\
\hline
\end{tabular}




$\begin{array}{lrrr}\mathrm{C} & 3.75826700 & 1.14828200 & -0.45941600 \\ \mathrm{C} & 4.27650100 & -0.03023800 & 0.07111700 \\ \mathrm{C} & 3.39125500 & -1.04466400 & 0.43333400 \\ \mathrm{C} & 4.67903900 & 2.29111300 & -0.78716000 \\ \mathrm{C} & 3.93496400 & -2.34467100 & 0.96409700 \\ \mathrm{~F} & 4.83558100 & 3.11864200 & 0.27247100 \\ \mathrm{~F} & 5.90855600 & 1.86140400 & -1.13806300 \\ \mathrm{~F} & 4.20149500 & 3.04411100 & -1.80375100 \\ \mathrm{~F} & 3.07347400 & -2.93816300 & 1.81723200 \\ \mathrm{~F} & 4.17656300 & -3.22319200 & -0.03320400 \\ \mathrm{~F} & 5.10184500 & -2.16389300 & 1.62261600 \\ \mathrm{~N} & 0.13046900 & 0.52105400 & -0.42780300 \\ \mathrm{C} & -0.89762300 & -0.35508500 & -0.73407500 \\ \mathrm{~N} & -2.09947000 & 0.28353100 & -0.70773100 \\ \mathrm{~S} & -0.69979000 & -1.97570100 & -1.10333800 \\ \mathrm{C} & -3.37705700 & -0.40867100 & -0.84775100 \\ \mathrm{C} & -4.44667900 & 0.48090900 & -0.18466400 \\ \mathrm{C} & -5.81956500 & -0.21055400 & -0.24132100 \\ \mathrm{C} & -6.20707000 & -0.53856300 & -1.69062200 \\ \mathrm{C} & -5.12252200 & -1.37173800 & -2.38537000 \\ \mathrm{C} & -3.75227300 & -0.68804300 & -2.30760900 \\ \mathrm{~N} & -3.98518600 & 0.93973700 & 1.13562200 \\ \mathrm{C} & -4.70883100 & 2.11648000 & 1.62209300 \\ \mathrm{C} & -3.83871300 & -0.12161600 & 2.15323100 \\ \mathrm{C} & -3.95282500 & 2.87062900 & 2.71318100 \\ \mathrm{C} & -2.41918900 & -0.22778600 & 2.71311500 \\ \mathrm{H} & 1.34579800 & -1.69367600 & 0.57591500 \\ \mathrm{H} & 2.00443600 & 2.21974800 & -1.07874000 \\ \mathrm{H} & 5.34333600 & -0.15404200 & 0.20343000 \\ \mathrm{H} & -0.10881100 & 1.49787800 & -0.53686100 \\ \mathrm{H} & -2.18649300 & 1.03935100 & -0.02573100 \\ \mathrm{H} & -3.31058200 & -1.37196300 & -0.32397000 \\ \mathrm{H} & -4.51545800 & 1.39128700 & -0.79741200 \\ \mathrm{H} & -6.57497300 & 0.43553600 & 0.21905200 \\ \mathrm{H} & -5.79278100 & -1.13639400 & 0.34650700 \\ \mathrm{H} & -2.08515700 & 0.72458000 & 3.13278900 \\ \mathrm{H} & -6.35021700 & 0.39983500 & -2.24397300 \\ \mathrm{H} & -7.16851400 & -1.06332900 & -1.71252200 \\ \mathrm{H} & -5.39214500 & -1.55128900 & -3.43151100 \\ \mathrm{H} & -5.05831500 & -2.35794200 & -1.90616900 \\ \mathrm{H} & -2.97925900 & -1.31180800 & -2.76145000 \\ \mathrm{H} & -3.77172300 & 0.26449300 & -2.85323700 \\ \mathrm{H} & -5.71414000 & 1.85148500 & 1.99627200 \\ \mathrm{H} & -4.86052100 & 2.78458600 & 0.76699100 \\ \mathrm{H} & -4.11643800 & -1.08636100 & 1.71942800 \\ \mathrm{H} & -4.55185400 & 0.05202100 & 2.97100400 \\ -4.49812000 & 3.77767300 & 2.99158300 \\ \mathrm{H} & -3.83387000 & 2.26739300 & 3.61751300 \\ \mathrm{H} & -2.95644600 & 3.15705700 & 2.36285900 \\ \mathrm{H} & -1.77703300 & -0.98347300 & 3.50461600 \\ \mathrm{H} & & -0.5254100 & 1.93359700 \\ \mathrm{H} & & & \\ \mathrm{H} & & & \end{array}$


<smiles>O=[N+]([O-])c1ccc(NC(=S)N[C@H]2CCCCC2N2CCCC2)cc1</smiles>

Thiourea-5

$\begin{array}{lrrr}\mathrm{C} & 3.13126100 & 0.47161500 & -1.42245800 \\ \mathrm{C} & 2.33774200 & -0.13224100 & -0.42620900 \\ \mathrm{C} & 2.94889400 & -0.59737500 & 0.75118200 \\ \mathrm{C} & 4.32161400 & -0.46650000 & 0.91206800 \\ \mathrm{C} & 5.08592400 & 0.12929300 & -0.08857400 \\ \mathrm{C} & 4.49914400 & 0.60520900 & -1.26031200 \\ \mathrm{~N} & 0.96434400 & -0.17798500 & -0.67536800 \\ \mathrm{C} & -0.06922500 & -0.90018800 & -0.10100400 \\ \mathrm{~N} & -1.26953100 & -0.48527300 & -0.59470400 \\ \mathrm{~S} & 0.10279300 & -2.13823100 & 1.01306800 \\ \mathrm{C} & -2.55245900 & -0.98205700 & -0.10931000 \\ \mathrm{C} & -3.61100800 & 0.08692500 & -0.44797800 \\ \mathrm{C} & -4.96328700 & -0.32870700 & 0.15565600 \\ \mathrm{C} & -5.39609300 & -1.69548600 & -0.39623500 \\ \mathrm{C} & -4.32101000 & -2.76532600 & -0.16186600 \\ \mathrm{C} & -2.95678200 & -2.33119300 & -0.71307900 \\ \mathrm{~N} & -3.10008200 & 1.41467300 & -0.10427300 \\ \mathrm{C} & -3.83226500 & 2.55731700 & -0.65259700 \\ \mathrm{C} & -3.14376300 & 3.77307000 & -0.00235500 \\ \mathrm{C} & -2.48217200 & 3.20494600 & 1.28570900 \\ \mathrm{C} & -2.90439700 & 1.72677500 & 1.31580100 \\ \mathrm{H} & 2.66298600 & 0.83292900 & -2.33413100 \\ \mathrm{H} & 2.34878400 & -1.06398200 & 1.51699100 \\ \mathrm{H} & 4.81082000 & -0.81783700 & 1.81125200 \\ \mathrm{H} & 5.11920200 & 1.06348000 & -2.01936900 \\ \mathrm{H} & 0.68926600 & 0.32812600 & -1.50735700 \\ \mathrm{H} & -1.34883600 & 0.51001800 & -0.81778800 \\ \mathrm{H} & -2.48947600 & -1.10327400 & 0.98094900 \\ \mathrm{H} & -3.72441600 & 0.09477300 & -1.54243700 \\ \mathrm{H} & -5.72339800 & 0.42806400 & -0.06584900 \\ \mathrm{H} & -4.87669100 & -0.38515700 & 1.24766500 \\ \mathrm{H} & -5.58272600 & -1.60118400 & -1.47486400 \\ \mathrm{H} & -6.34474600 & -2.00069600 & 0.05874700 \\ \mathrm{H} & -4.62420000 & -3.71384700 & -0.61759900 \\ \mathrm{H} & -4.22549500 & -2.95361800 & 0.91585100 \\ \mathrm{H} & -2.19090300 & -3.07417700 & -0.48089300 \\ \mathrm{H} & -3.00071600 & -2.22982800 & -1.80531100 \\ \mathrm{H} & -4.90016900 & 2.53417900 & -0.37436800 \\ \mathrm{H} & -3.77682900 & 2.55542100 & -1.74599000 \\ \mathrm{H} & -3.86322100 & 4.56653600 & 0.21366300 \\ \mathrm{H} & -2.38714700 & 4.19240200 & -0.67039600 \\ \mathrm{H} & -2.79732700 & 3.73189400 & 2.18935500 \\ \mathrm{H} & -1.39372800 & 3.28464000 & 1.22277800\end{array}$




$\begin{array}{lrrr}\mathrm{H} & -2.14830200 & 1.07213700 & 1.75788000 \\ \mathrm{H} & -3.83495100 & 1.60623100 & 1.89557700 \\ \mathrm{~N} & 6.52851900 & 0.26455800 & 0.09133400 \\ \mathrm{O} & 7.17118600 & 0.80665200 & -0.81111300 \\ \mathrm{O} & 7.02157300 & -0.16837800 & 1.13448900\end{array}$<smiles>CN(C)C1CCCC[C@H]1NC(=O)Nc1cc(C(F)(F)F)cc(C(F)(F)F)c1</smiles>

Urea-1

$\begin{array}{lrrr}\mathrm{C} & 2.17391700 & 1.35991100 & -0.32210900 \\ \mathrm{C} & 1.26314100 & 0.29882600 & -0.20873200 \\ \mathrm{C} & 1.74837400 & -0.99324300 & 0.04492700 \\ \mathrm{C} & 3.12072200 & -1.19188900 & 0.18191800 \\ \mathrm{C} & 4.03146800 & -0.14360200 & 0.07023800 \\ \mathrm{C} & 3.53949400 & 1.13488800 & -0.18558100 \\ \mathrm{C} & 3.61970800 & -2.57040600 & 0.52588300 \\ \mathrm{C} & 4.49835700 & 2.27622800 & -0.38388500 \\ \mathrm{~F} & 4.89065600 & -2.76228500 & 0.10749200 \\ \mathrm{~F} & 3.60973100 & -2.77734500 & 1.86323400 \\ \mathrm{~F} & 2.85784300 & -3.53573900 & -0.02841800 \\ \mathrm{~F} & 4.88086300 & 2.38390600 & -1.67720100 \\ \mathrm{~F} & 3.94673300 & 3.46251300 & -0.03893200 \\ \mathrm{~F} & 5.62168600 & 2.12108300 & 0.34789600 \\ \mathrm{~N} & -0.09463500 & 0.59657000 & -0.34692000 \\ \mathrm{C} & -1.17435000 & -0.28696400 & -0.30948500 \\ \mathrm{~N} & -2.38036900 & 0.35385100 & -0.46667400 \\ \mathrm{O} & -1.05758700 & -1.49997500 & -0.17706800 \\ \mathrm{C} & -3.61425800 & -0.39681500 & -0.25450200 \\ \mathrm{C} & -4.74846600 & 0.62550200 & -0.05454100 \\ \mathrm{C} & -6.06505300 & -0.11064000 & 0.24551400 \\ \mathrm{C} & -6.41650800 & -1.07666200 & -0.89549500 \\ \mathrm{C} & -5.27254400 & -2.06274400 & -1.16606200 \\ \mathrm{C} & -3.94788700 & -1.33366200 & -1.42163700 \\ \mathrm{~N} & -4.34105700 & 1.67228100 & 0.90003100 \\ \mathrm{C} & -5.14931800 & 2.88090800 & 0.80114400 \\ \mathrm{C} & -4.22289200 & 1.22439500 & 2.28506100 \\ \mathrm{H} & 1.81605800 & 2.36696300 & -0.51080000 \\ \mathrm{H} & 1.05356600 & -1.81620700 & 0.11907300 \\ \mathrm{H} & 5.09386900 & -0.31699700 & 0.17832700 \\ \mathrm{H} & -0.30622700 & 1.55135200 & -0.59688100 \\ \mathrm{H} & -2.46852900 & 1.29656900 & -0.08932300 \\ \mathrm{H} & -3.51332600 & -1.01725900 & 0.64796200 \\ \mathrm{H} & -4.86892100 & 1.14713600 & -1.01449600 \\ \mathrm{H} & -6.87379300 & 0.61329400 & 0.39388900\end{array}$




$\begin{array}{lrrr}\mathrm{H} & -5.96620600 & -0.67415400 & 1.18166500 \\ \mathrm{H} & -6.61647100 & -0.49618200 & -1.80665000 \\ \mathrm{H} & -7.34058600 & -1.61602800 & -0.65988000 \\ \mathrm{H} & -5.51751500 & -2.70489900 & -2.01879700 \\ \mathrm{H} & -5.15572900 & -2.72744800 & -0.29938200 \\ \mathrm{H} & -3.12822100 & -2.04416800 & -1.54795800 \\ \mathrm{H} & -4.01332500 & -0.73704900 & -2.34096100 \\ \mathrm{H} & -5.17704800 & 3.22267300 & -0.23765600 \\ \mathrm{H} & -6.18907700 & 2.74872300 & 1.14697200 \\ \mathrm{H} & -4.69668900 & 3.67095100 & 1.40863500 \\ \mathrm{H} & -3.57714800 & 0.34545500 & 2.34583600 \\ \mathrm{H} & -3.76217500 & 2.01990500 & 2.87847200 \\ \mathrm{H} & -5.19042300 & 0.97487400 & 2.75087200\end{array}$<smiles>CN(C)C1CCCC[C@@H]1Nc1c(Nc2cc(C(F)(F)F)cc(C(F)(F)F)c2)c(=O)c1=O</smiles>

Squaramide-1

$\begin{array}{lrrr}\text { C } & 2.53993900 & -0.98640300 & 0.09185100 \\ \text { C } & 1.75030500 & 0.14099100 & -0.16947400 \\ \text { C } & 2.35979200 & 1.38908000 & -0.35580100 \\ \text { C } & 3.74334100 & 1.50839300 & -0.28000800 \\ \text { C } & 4.54228900 & 0.39632800 & -0.02187400 \\ \text { C } & 3.92377400 & -0.83957200 & 0.15978400 \\ \text { C } & 4.37461800 & 2.86758400 & -0.40996800 \\ \text { C } & 4.77389400 & -2.06649300 & 0.37016700 \\ \text { F } & 5.61329100 & 2.79666000 & -0.93853900 \\ \text { F } & 3.63986400 & 3.68858900 & -1.19503800 \\ \text { F } & 4.49041600 & 3.47555700 & 0.79289400 \\ \text { F } & 4.16154600 & -2.97262300 & 1.15583600 \\ \text { F } & 5.05179700 & -2.67527100 & -0.80420800 \\ \text { F } & 5.95798200 & -1.75727500 & 0.94534400 \\ \text { N } & 0.35325200 & 0.08451800 & -0.24909600 \\ \text { C } & -0.48005300 & -0.98652300 & -0.13864800 \\ \text { H } & -0.09262800 & 0.96739100 & -0.46024100 \\ \text { C } & -1.88121200 & -1.01093800 & -0.15607500 \\ \text { C } & -1.92011600 & -2.49692100 & -0.02178800 \\ \text { C } & -0.37741300 & -2.46798300 & 0.01402000 \\ \text { N } & -2.80757800 & -0.04785600 & -0.25678800 \\ \text { O } & -2.79039200 & -3.33907700 & 0.03056100 \\ \text { O } & 0.50873500 & -3.28704700 & 0.14140100 \\ \text { C } & -4.24388400 & -0.28531200 & -0.08051500 \\ \text { H } & -2.56897700 & 0.90320300 & 0.02926600 \\ \text { C } & -4.91945800 & 1.09860500 & -0.03835500 \\ \text { C } & -6.42370800 & 0.93444000 & 0.23102100 \\ & & & \end{array}$




$\begin{array}{lrrr}\mathrm{C} & -7.07079800 & 0.06275800 & -0.85536300 \\ \mathrm{C} & -6.36710700 & -1.29506900 & -0.97601600 \\ \mathrm{C} & -4.85668800 & -1.13908100 & -1.19653400 \\ \mathrm{~N} & -4.17535300 & 2.00979700 & 0.85048500 \\ \mathrm{C} & -4.45735500 & 3.41675100 & 0.58841700 \\ \mathrm{C} & -4.29109200 & 1.70267100 & 2.27471500 \\ \mathrm{H} & 2.08545600 & -1.96269800 & 0.23166400 \\ \mathrm{H} & 1.75824600 & 2.26772400 & -0.56549800 \\ \mathrm{H} & 5.61851200 & 0.49009400 & 0.03607000 \\ \mathrm{H} & -4.41480400 & -0.80378400 & 0.87398100 \\ \mathrm{H} & -4.80445500 & 1.52573800 & -1.04448900 \\ \mathrm{H} & -6.90600100 & 1.91710500 & 0.26959400 \\ \mathrm{H} & -6.57390700 & 0.46670200 & 1.21193400 \\ \mathrm{H} & -7.01150200 & 0.58873200 & -1.81801700 \\ \mathrm{H} & -8.13585200 & -0.07575100 & -0.64027700 \\ \mathrm{H} & -6.80185300 & -1.87815100 & -1.79430700 \\ \mathrm{H} & -6.53542800 & -1.87464700 & -0.05856200 \\ \mathrm{H} & -4.65908000 & -0.65048200 & -2.15948400 \\ \mathrm{H} & -4.37311700 & -2.11807900 & -1.20818800 \\ \mathrm{H} & -3.76197100 & 4.03843000 & 1.16072900 \\ \mathrm{H} & -4.31256400 & 3.63098900 & -0.47440700 \\ \mathrm{H} & -5.48139000 & 3.72043500 & 0.86396900 \\ \mathrm{H} & -3.58292200 & 2.32335700 & 2.83143900 \\ \mathrm{H} & -5.29722200 & 1.89016000 & 2.68280500 \\ \mathrm{H} & -4.03600500 & 0.65673000 & 2.46018100\end{array}$<smiles>O=c1c(Nc2cc(C(F)(F)F)cc(C(F)(F)F)c2)c(N[C@H]2CCCCC2N2CCCC2)c1=O</smiles>

Squaramide-2

$\begin{array}{lrrr}\text { C } & 2.49093800 & 1.26062600 & -0.57523300 \\ \text { C } & 1.94577100 & 0.00698900 & -0.26666800 \\ \text { C } & 2.78769700 & -1.03623100 & 0.14047200 \\ \text { C } & 4.15827800 & -0.80159400 & 0.22835300 \\ \text { C } & 4.71294900 & 0.44089800 & -0.07413600 \\ \text { C } & 3.86243500 & 1.46878600 & -0.47639100 \\ \text { C } & 5.06708500 & -1.94607000 & 0.59795700 \\ \text { C } & 4.42077900 & 2.84028800 & -0.74084400 \\ \text { F } & 6.21846100 & -1.50626400 & 1.15417600 \\ \text { F } & 4.48424200 & -2.78641800 & 1.47411200 \\ \text { F } & 5.40567300 & -2.67210900 & -0.49061000 \\ \text { F } & 4.45919200 & 3.58424200 & 0.38841100 \\ \text { F } & 5.67944100 & 2.78539100 & -1.22172700 \\ \text { F } & 3.66943000 & 3.52354600 & -1.63471200\end{array}$




$\begin{array}{lrrr}\mathrm{N} & 0.55765300 & -0.14009900 & -0.37636500 \\ \mathrm{C} & -0.21784200 & -1.24023500 & -0.16961000 \\ \mathrm{C} & -0.03918300 & -2.68727000 & 0.14897400 \\ \mathrm{C} & -1.57638200 & -2.80938800 & 0.07617000 \\ \mathrm{C} & -1.61429600 & -1.34660500 & -0.21957700 \\ \mathrm{~N} & -2.58553800 & -0.45289500 & -0.44959800 \\ \mathrm{O} & 0.88603000 & -3.43461300 & 0.38987100 \\ \mathrm{O} & -2.40019300 & -3.69002900 & 0.19788500 \\ \mathrm{C} & -4.01259200 & -0.74259100 & -0.27225400 \\ \mathrm{C} & -4.75301000 & 0.60351200 & -0.39271400 \\ \mathrm{C} & -6.25282600 & 0.39340800 & -0.12848200 \\ \mathrm{C} & -6.83294100 & -0.62250700 & -1.12361300 \\ \mathrm{C} & -6.06467900 & -1.94993200 & -1.08067200 \\ \mathrm{C} & -4.55835000 & -1.74709500 & -1.29205300 \\ \mathrm{~N} & -4.06771500 & 1.62119700 & 0.40868800 \\ \mathrm{C} & -4.39952200 & 3.01510000 & 0.10257600 \\ \mathrm{C} & -3.65898800 & 3.81338600 & 1.19360700 \\ \mathrm{C} & -3.43981700 & 2.79173900 & 2.34564300 \\ \mathrm{C} & -4.15756800 & 1.51871500 & 1.86961000 \\ \mathrm{H} & 1.84875200 & 2.07372900 & -0.89826800 \\ \mathrm{H} & 2.38357500 & -2.01623900 & 0.37642700 \\ \mathrm{H} & 5.77982100 & 0.60343300 & 0.00143600 \\ \mathrm{H} & 0.07052700 & 0.68509900 & -0.69985500 \\ \mathrm{H} & -2.40471800 & 0.53564000 & -0.26246000 \\ \mathrm{H} & -4.17921000 & -1.15150000 & 0.73518900 \\ \mathrm{H} & -4.63865100 & 0.93108300 & -1.43631000 \\ \mathrm{H} & -6.78438700 & 1.34800500 & -0.20325400 \\ \mathrm{H} & -6.39908600 & 0.02605900 & 0.89475900 \\ \mathrm{H} & -6.77566300 & -0.20296000 & -2.13731800 \\ \mathrm{H} & --7.89514800 & -0.78858900 & -0.91393200 \\ \mathrm{H} & -6.45435100 & -2.64010100 & -1.83595900 \\ \mathrm{H} & -6.22641500 & -2.43175200 & -0.10718600 \\ \mathrm{H} & -4.36249900 & -1.36172900 & -2.30112400 \\ \mathrm{H} & -4.02947400 & -2.69640200 & -1.18376200 \\ \mathrm{H} & -4.08269800 & 3.26604200 & -0.91470000 \\ \mathrm{H} & -5.48509200 & 3.20232300 & 0.16781800 \\ \mathrm{H} & -4.24198100 & 4.68062200 & 1.51234800 \\ \mathrm{H} & -2.70121200 & 4.18509800 & 0.82070000 \\ \mathrm{H} & -3.83344300 & 3.14276700 & 3.30230700 \\ \mathrm{H} & -2.37332700 & 2.59414500 & 2.48301600 \\ \mathrm{H} & -5.20353500 & 1.51646400 & 2.21879900 \\ \mathrm{H} & -3.68484300 & 0.59939600 & 2.22666400\end{array}$<smiles>O=c1c(Nc2cc(C(F)(F)F)cc(C(F)(F)F)c2)c(N[C@@H]2CCCCC2N2CCCCC2)c1=O</smiles> 
Squaramide-3

$\begin{array}{lrrr}\mathrm{C} & 2.53296700 & 1.20380200 & -0.58326300 \\ \mathrm{C} & 2.02888700 & -0.07368800 & -0.30400100 \\ \mathrm{C} & 2.90384800 & -1.09775100 & 0.08160700 \\ \mathrm{C} & 4.26517000 & -0.81862400 & 0.18328400 \\ \mathrm{C} & 4.77915900 & 0.44782200 & -0.09050300 \\ \mathrm{C} & 3.89670200 & 1.45461200 & -0.47699500 \\ \mathrm{C} & 5.19370600 & -1.90284300 & 0.66764000 \\ \mathrm{C} & 4.42812500 & 2.81200300 & -0.84823100 \\ \mathrm{~F} & 5.25037400 & -1.92802900 & 2.01856100 \\ \mathrm{~F} & 4.79657300 & -3.12316400 & 0.26132600 \\ \mathrm{~F} & 6.45603200 & -1.71137900 & 0.22279000 \\ \mathrm{~F} & 4.75964800 & 2.87237900 & -2.15790400 \\ \mathrm{~F} & 3.51342100 & 3.78502700 & -0.62990700 \\ \mathrm{~F} & 5.53490700 & 3.12660500 & -0.14452900 \\ \mathrm{~N} & 0.64669800 & -0.26351600 & -0.42225600 \\ \mathrm{C} & -0.09916500 & -1.38125400 & -0.20140600 \\ \mathrm{C} & -1.49163200 & -1.52670900 & -0.25957200 \\ \mathrm{C} & -1.41758700 & -2.97920400 & 0.07522400 \\ \mathrm{C} & 0.11554800 & -2.81491200 & 0.15358500 \\ \mathrm{O} & -2.21954400 & -3.87667000 & 0.21891700 \\ \mathrm{O} & 1.05879300 & -3.53179800 & 0.41619600 \\ \mathrm{~N} & -2.48354100 & -0.66514900 & -0.52088200 \\ \mathrm{C} & -3.90169700 & -0.97332200 & -0.31179700 \\ \mathrm{C} & -4.66696500 & 0.35522200 & -0.47316200 \\ \mathrm{C} & -6.15736200 & 0.13682100 & -0.16966000 \\ \mathrm{C} & -6.74211800 & -0.93068000 & -1.10671000 \\ \mathrm{C} & -5.94794100 & -2.24161600 & -1.03109200 \\ \mathrm{C} & -4.45054300 & -2.02130500 & -1.28557300 \\ \mathrm{~N} & -3.98350300 & 1.43297200 & 0.25878000 \\ \mathrm{C} & -4.31740000 & 2.77679600 & -0.22116200 \\ \mathrm{C} & -3.34598000 & 3.80741100 & 0.35717300 \\ \mathrm{C} & -3.34251200 & 3.75384000 & 1.88890200 \\ \mathrm{C} & -3.09002300 & 2.32153300 & 2.37136400 \\ \mathrm{C} & -4.07595000 & 1.34896000 & 1.72172800 \\ \mathrm{H} & 1.86317500 & 2.00576100 & -0.87672200 \\ \mathrm{H} & 2.53246700 & -2.09747200 & 0.28762700 \\ \mathrm{H} & -4.83962200 & 0.64442000 & -0.00590200 \\ \mathrm{H} & -3.90205900 & -2.95683300 & -1.15619100 \\ \mathrm{H} & & & \\ \mathrm{H} & -13653800 & 0.54755400 & -0.74584800 \\ \mathrm{H} & -2.32217400 & 0.33389400 & -0.37476300 \\ \mathrm{H} & -4.04521000 & -1.35521300 & 0.70926400 \\ \mathrm{H} & -4.57943000 & 0.63060300 & -1.53362600 \\ \mathrm{H} & -6.70160100 & 1.08126900 & -0.27802400 \\ \mathrm{H} & -6.27864900 & -0.18337400 & 0.87258100 \\ \mathrm{H} & -6.71787900 & -0.55191900 & -2.13765600 \\ \mathrm{H} & -6.79548700 & -1.10740200 & -0.86378000 \\ \mathrm{H} & -6.07799400 & -2.68835400 & -0.03633000 \\ \mathrm{H} & -28420100 & -1.66848800 & -2.31165700 \\ \mathrm{H} & & & \end{array}$




$\begin{array}{lrrr}\mathrm{H} & -4.26299800 & 2.77303200 & -1.31487000 \\ \mathrm{H} & -5.35113300 & 3.05924300 & 0.05149600 \\ \mathrm{H} & -3.61815600 & 4.80743500 & 0.00250600 \\ \mathrm{H} & -2.33896600 & 3.58998600 & -0.02129000 \\ \mathrm{H} & -4.31847900 & 4.09208300 & 2.26299000 \\ \mathrm{H} & -2.59268800 & 4.43891100 & 2.29826000 \\ \mathrm{H} & -3.17697200 & 2.25664100 & 3.46122900 \\ \mathrm{H} & -2.06935900 & 2.01569600 & 2.10821500 \\ \mathrm{H} & -5.09806400 & 1.57789000 & 2.07223900 \\ \mathrm{H} & -3.85138300 & 0.32496900 & 2.03229300\end{array}$<smiles>O=c1c(Nc2ccc(C(F)(F)F)cc2)c(N[C@@H]2CCCCC2N2CCCCC2)c1=O</smiles>

Squaramide-4

$\begin{array}{lrrr}\mathrm{C} & -1.03975900 & -1.50073400 & 0.00831300 \\ \mathrm{~N} & -2.00749500 & -0.67932400 & -0.42438200 \\ \mathrm{C} & -3.43282200 & -0.90771800 & -0.16876300 \\ \mathrm{C} & -4.16614700 & 0.38474800 & -0.57827300 \\ \mathrm{C} & -5.66223000 & 0.26052200 & -0.24982300 \\ \mathrm{C} & -6.27045300 & -0.94810200 & -0.97678000 \\ \mathrm{C} & -5.50846900 & -2.24011800 & -0.65320100 \\ \mathrm{C} & -4.00563900 & -2.10564100 & -0.93364600 \\ \mathrm{~N} & -3.46068300 & 1.56438000 & -0.05363600 \\ \mathrm{C} & -3.76580400 & 2.80301400 & -0.77448600 \\ \mathrm{C} & -2.77351500 & 3.90262700 & -0.39169500 \\ \mathrm{C} & -2.77272400 & 4.13235900 & 1.12364100 \\ \mathrm{C} & -2.55070000 & 2.80865900 & 1.86301500 \\ \mathrm{C} & -3.55640900 & 1.75347500 & 1.39910500 \\ \mathrm{C} & -1.00787200 & -2.86110800 & 0.61546700 \\ \mathrm{C} & 0.52811300 & -2.72583500 & 0.67796400 \\ \mathrm{C} & 0.35632000 & -1.38043800 & 0.04996600 \\ \mathrm{O} & -1.83518700 & -3.69452700 & 0.91975600 \\ \mathrm{O} & 1.44673000 & -3.40566700 & 1.08618000 \\ \mathrm{~N} & 1.12814100 & -0.34529400 & -0.37711000 \\ \mathrm{C} & 2.51561600 & -0.16417300 & -0.29382100 \\ \mathrm{C} & 3.04836400 & 1.01738200 & -0.83325500 \\ \mathrm{C} & 4.41342300 & 1.26042600 & -0.77647000 \\ \mathrm{C} & 5.26433600 & 0.32905800 & -0.17812700 \\ \mathrm{C} & 4.73148000 & -0.84610700 & 0.35700700 \\ \mathrm{C} & 3.36624200 & -1.10274700 & 0.30742300 \\ \mathrm{C} & 6.74500000 & 0.56053200 & -0.16918300 \\ \mathrm{~F} & 7.33896000 & -0.00935900 & 0.90312300 \\ \mathrm{~F} & 7.34589300 & 0.04174600 & -1.26788400 \\ \mathrm{~F} & 7.04836000 & 1.87979700 & -0.14964600 \\ & & & \end{array}$




$\begin{array}{rrrr}\mathrm{H} & -1.81907900 & 0.32450600 & -0.46309200 \\ \mathrm{H} & -3.58711400 & -1.09006900 & 0.90459600 \\ \mathrm{H} & -4.06932100 & 0.45718600 & -1.67076000 \\ \mathrm{H} & -6.18363700 & 1.18088500 & -0.53451200 \\ \mathrm{H} & -5.79361200 & 0.14147000 & 0.83282500 \\ \mathrm{H} & -6.23411300 & -0.76805700 & -2.05994000 \\ \mathrm{H} & -7.32855300 & -1.05177900 & -0.71291800 \\ \mathrm{H} & -5.91865200 & -3.07742800 & -1.22710900 \\ \mathrm{H} & -5.65187300 & -2.49073900 & 0.40637100 \\ \mathrm{H} & -3.47999700 & -3.01281900 & -0.62786600 \\ \mathrm{H} & -3.82814700 & -1.95454400 & -2.00636300 \\ \mathrm{H} & -4.79355300 & 3.15249000 & -0.56350200 \\ \mathrm{H} & -3.71066600 & 2.59654000 & -1.84851600 \\ \mathrm{H} & -3.02449500 & 4.82547500 & -0.92595700 \\ \mathrm{H} & -1.77101600 & 3.59828600 & -0.71867500 \\ \mathrm{H} & -3.74185800 & 4.55355300 & 1.42412300 \\ \mathrm{H} & -2.00922600 & 4.86585400 & 1.40351200 \\ \mathrm{H} & -2.63999500 & 2.94775900 & 2.94575300 \\ \mathrm{H} & -1.53656400 & 2.43866300 & 1.66547300 \\ \mathrm{H} & -3.35378100 & 0.80003400 & 1.89429400 \\ \mathrm{H} & -4.57372500 & 2.06441100 & 1.69671000 \\ \mathrm{H} & 0.63665300 & 0.39567200 & -0.85939900 \\ \mathrm{H} & 2.38843800 & 1.74780000 & -1.29430900 \\ \mathrm{H} & 4.81901900 & 2.17731100 & -1.18837000 \\ \mathrm{H} & 5.39021600 & -1.56825000 & 0.82606900 \\ \mathrm{H} & 2.96555600 & -2.02112200 & 0.72338000\end{array}$<smiles>C=CC1CN2CCC1CC2[C@H](Nc1c(Nc2cc(C(F)(F)F)cc(C(F)(F)F)c2)c(=O)c1=O)c1ccnc2ccc(OC)cc12</smiles>

Squaramide-5

$\begin{array}{lrrr}\mathrm{N} & -1.30664500 & -0.07067600 & -0.58552200 \\ \mathrm{C} & -0.42018400 & -0.27384700 & 0.39181100 \\ \mathrm{C} & 0.98102300 & -0.25531600 & 0.48692300 \\ \mathrm{C} & 0.95879700 & -0.68831400 & 1.91841100 \\ \mathrm{C} & -0.57094900 & -0.72633300 & 1.79557900 \\ \mathrm{O} & 1.77044800 & -0.92716200 & 2.78801300 \\ \mathrm{O} & -1.51777700 & -1.01796100 & 2.50561600 \\ \mathrm{~N} & 1.90438100 & 0.06893800 & -0.45523300 \\ \mathrm{C} & 3.30514900 & 0.07474200 & -0.37422100 \\ \mathrm{C} & 4.01453900 & 0.44689400 & -1.52366600 \\ \mathrm{C} & 5.40478500 & 0.47149900 & -1.50737000 \\ \mathrm{C} & 6.11036600 & 0.12536200 & -0.35660800 \\ \mathrm{C} & 5.39236900 & -0.24232200 & 0.77971100\end{array}$




\begin{tabular}{|c|c|c|c|}
\hline $\mathrm{C}$ & 3.99915300 & -0.27201200 & 0.79125100 \\
\hline $\mathrm{C}$ & 6.13616400 & -0.68425800 & 2.01444700 \\
\hline $\mathrm{C}$ & 6.15026000 & 0.94173200 & -2.72696900 \\
\hline $\mathrm{F}$ & 7.36838300 & -0.13247500 & 2.07607700 \\
\hline $\mathrm{F}$ & 5.47956300 & -0.34913600 & 3.14123300 \\
\hline $\mathrm{F}$ & 6.30097600 & -2.02579800 & 2.02946900 \\
\hline $\mathrm{F}$ & 6.37843300 & 2.27371300 & -2.68184500 \\
\hline $\mathrm{F}$ & 7.34993100 & 0.33732800 & -2.84314800 \\
\hline $\mathrm{F}$ & 5.45608400 & 0.69948500 & -3.86217400 \\
\hline $\mathrm{C}$ & -2.74604900 & 0.11361500 & -0.33319700 \\
\hline $\mathrm{C}$ & -3.52200300 & -0.73145300 & -1.32831400 \\
\hline $\mathrm{C}$ & -3.11054500 & 1.60724400 & -0.33907400 \\
\hline $\mathrm{C}$ & -3.73689900 & -2.12188600 & -1.07331200 \\
\hline $\mathrm{C}$ & -4.43265400 & -2.87015900 & -2.07796700 \\
\hline $\mathrm{N}$ & -4.87764300 & -2.32670200 & -3.24447600 \\
\hline $\mathrm{C}$ & -4.65009300 & -1.04513700 & -3.44597300 \\
\hline $\mathrm{C}$ & -3.97955800 & -0.21235500 & -2.52230200 \\
\hline $\mathrm{C}$ & -3.29876900 & -2.78053200 & 0.10679100 \\
\hline $\mathrm{C}$ & -3.55328500 & -4.12826500 & 0.28177700 \\
\hline $\mathrm{C}$ & -4.25042700 & -4.86933100 & -0.71223000 \\
\hline $\mathrm{C}$ & -4.67583900 & -4.25391000 & -1.85776500 \\
\hline $\mathrm{O}$ & -3.18221100 & -4.84972900 & 1.36792600 \\
\hline $\mathrm{C}$ & -2.45586600 & -4.18009700 & 2.39563700 \\
\hline $\mathrm{C}$ & -4.62186000 & 1.85864900 & -0.06931000 \\
\hline $\mathrm{C}$ & -4.73937300 & 3.08542600 & 0.85372300 \\
\hline $\mathrm{C}$ & -3.90397600 & 4.23932200 & 0.23404600 \\
\hline $\mathrm{C}$ & -2.40367000 & 3.78780800 & 0.29855500 \\
\hline $\mathrm{N}$ & -2.27388500 & 2.35810300 & 0.61580800 \\
\hline $\mathrm{C}$ & -4.12045100 & 5.56510000 & 0.89980000 \\
\hline $\mathrm{C}$ & -4.60363700 & 6.65149200 & 0.29778600 \\
\hline $\mathrm{C}$ & -4.16754200 & 2.70089300 & 2.22818500 \\
\hline $\mathrm{C}$ & -2.72904200 & 2.15109300 & 2.00769100 \\
\hline $\mathrm{H}$ & -0.98460800 & 0.23193600 & -1.49575100 \\
\hline $\mathrm{H}$ & 1.53967800 & 0.36537400 & -1.35038900 \\
\hline $\mathrm{H}$ & 3.48664400 & 0.71140500 & -2.43427700 \\
\hline $\mathrm{H}$ & 7.19208300 & 0.14252300 & -0.34547500 \\
\hline $\mathrm{H}$ & 3.46570000 & -0.55336200 & 1.69419500 \\
\hline $\mathrm{H}$ & -2.92420900 & -0.26928200 & 0.67144100 \\
\hline $\mathrm{H}$ & -2.84802600 & 2.00926500 & -1.32608400 \\
\hline $\mathrm{H}$ & -5.01095900 & -0.62565900 & -4.38355100 \\
\hline $\mathrm{H}$ & -3.84392300 & 0.83489200 & -2.76885800 \\
\hline $\mathrm{H}$ & -2.76954900 & -2.23090300 & 0.87177300 \\
\hline $\mathrm{H}$ & -4.42760800 & -5.92358300 & -0.52977500 \\
\hline $\mathrm{H}$ & -5.20765700 & -4.79606300 & -2.63164000 \\
\hline $\mathrm{H}$ & -2.27341700 & -4.92780700 & 3.16723000 \\
\hline $\mathrm{H}$ & -1.50126400 & -3.79531900 & 2.02175400 \\
\hline $\mathrm{H}$ & -3.02587600 & -3.34451200 & 2.81425100 \\
\hline $\mathrm{H}$ & -5.07916500 & 0.98625900 & 0.41312500 \\
\hline $\mathrm{H}$ & -5.16234900 & 2.01791500 & -1.00676000 \\
\hline $\mathrm{H}$ & -5.78461300 & 3.39498100 & 0.94253900 \\
\hline $\mathrm{H}$ & -4.20532800 & 4.33814200 & -0.81667800 \\
\hline $\mathrm{H}$ & -1.86857900 & 4.34907200 & 1.07141500 \\
\hline $\mathrm{H}$ & -1.90255400 & 3.99325300 & -0.65351300 \\
\hline
\end{tabular}




$\begin{array}{lrrr}\mathrm{H} & -3.84215600 & 5.62448100 & 1.95248500 \\ \mathrm{H} & -4.73444100 & 7.58977600 & 0.82771300 \\ \mathrm{H} & -4.88752300 & 6.63999100 & -0.75189300 \\ \mathrm{H} & -4.80488100 & 1.94240200 & 2.69469100 \\ \mathrm{H} & -4.16416800 & 3.56491200 & 2.89968200 \\ \mathrm{H} & -2.67061100 & 1.08858200 & 2.24648400 \\ \mathrm{H} & -2.00771200 & 2.65240600 & 2.65980800\end{array}$<smiles>C=CC1CN2CCC1CN2[C@H](NC(=S)Nc1cc(C(F)(F)F)cc(C(F)(F)F)c1)c1ccnc2ccc(OC)cc12</smiles>

Thiourea-6

$\begin{array}{lcrr}\mathrm{C} & 3.07676900 & -0.49935600 & 0.13524600 \\ \mathrm{C} & 3.49457100 & 0.43502200 & -0.82064000 \\ \mathrm{C} & 4.85412600 & 0.69541800 & -0.97384500 \\ \mathrm{C} & 5.81533900 & 0.03365200 & -0.21157300 \\ \mathrm{C} & 5.39136100 & -0.90775300 & 0.72304100 \\ \mathrm{C} & 4.03620900 & -1.17053700 & 0.90294000 \\ \mathrm{C} & 5.28828700 & 1.75285200 & -1.95430600 \\ \mathrm{C} & 6.40551600 & -1.69506100 & 1.50650200 \\ \mathrm{~F} & 6.52026900 & 1.49709800 & -2.44830000 \\ \mathrm{~F} & 4.44052300 & 1.84477900 & -3.00102800 \\ \mathrm{~F} & 5.33971900 & 2.97198900 & -1.37532300 \\ \mathrm{~F} & 6.74957400 & -2.83456100 & 0.86243800 \\ \mathrm{~F} & 7.54072300 & -0.99444400 & 1.70483600 \\ \mathrm{~F} & 5.92975200 & -2.05906300 & 2.71858700 \\ \mathrm{~N} & 1.73314100 & -0.85465800 & 0.32926800 \\ \mathrm{C} & 0.58110800 & -0.10701800 & 0.17781400 \\ \mathrm{~N} & -0.52698800 & -0.87396700 & 0.39545700 \\ \mathrm{~S} & 0.51362000 & 1.52332800 & -0.18370100 \\ \mathrm{C} & -1.87404900 & -0.34364300 & 0.23030000 \\ \mathrm{C} & -2.81406600 & -1.55879800 & 0.07885800 \\ \mathrm{C} & -2.31525100 & 0.50946800 & 1.40671400 \\ \mathrm{C} & -2.08982700 & 0.05822300 & 2.69064700 \\ \mathrm{C} & -2.58557400 & 0.78410100 & 3.79295400 \\ \mathrm{~N} & -3.28565400 & 1.89635900 & 3.68280300 \\ \mathrm{C} & -3.50692600 & 2.37513100 & 2.42836100 \\ \mathrm{C} & -3.03043800 & 1.73125100 & 1.24014300 \\ \mathrm{C} & -4.24877700 & 3.58301100 & 2.30417200 \\ \mathrm{C} & -4.49332800 & 4.13956600 & 1.07842800 \\ \mathrm{C} & -3.99357900 & 3.51732800 & -0.09757300 \\ \mathrm{C} & -3.28215100 & 2.33786800 & -0.02077500\end{array}$




$\begin{array}{lrrr}\mathrm{O} & -4.27149800 & 4.18044700 & -1.25369400 \\ \mathrm{C} & -3.76041100 & 3.63989600 & -2.46209000 \\ \mathrm{~N} & -2.21824500 & -2.55009900 & -0.84667100 \\ \mathrm{C} & -3.02242200 & -3.77978400 & -0.78656800 \\ \mathrm{C} & -4.50368000 & -3.53752100 & -1.24353200 \\ \mathrm{C} & -4.25241800 & -1.16654400 & -0.35763500 \\ \mathrm{C} & -4.63343400 & -2.02636100 & -1.57475500 \\ \mathrm{C} & -2.21521400 & -2.04411300 & -2.23438700 \\ \mathrm{C} & -3.65429300 & -1.69753600 & -2.71497600 \\ \mathrm{C} & -5.49247300 & -4.00469300 & -0.21546600 \\ \mathrm{C} & -6.46599100 & -4.88632300 & -0.43982600 \\ \mathrm{H} & 2.76756600 & 0.95283000 & -1.42702300 \\ \mathrm{H} & 6.86838100 & 0.24397200 & -0.34501000 \\ \mathrm{H} & 3.72644800 & -1.89154200 & 1.65245300 \\ \mathrm{H} & 1.60387800 & -1.71800800 & 0.84054800 \\ \mathrm{H} & -0.48721400 & -1.85387900 & 0.10841700 \\ \mathrm{H} & -1.89817400 & 0.25601000 & -0.68268900 \\ \mathrm{H} & -2.85067100 & -2.05814800 & 1.05340100 \\ \mathrm{H} & -1.52537700 & -0.85420500 & 2.85361100 \\ \mathrm{H} & -2.40353800 & 0.42096000 & 4.80312800 \\ \mathrm{H} & -4.60354800 & 4.04442300 & 3.21899000 \\ \mathrm{H} & -5.05086400 & 5.06336800 & 0.96962600 \\ \mathrm{H} & -2.87488700 & 1.89164700 & -0.91519600 \\ \mathrm{H} & -4.07110900 & 4.32177800 & -3.25403700 \\ \mathrm{H} & -2.66559800 & 3.57647800 & -2.44057100 \\ \mathrm{H} & -4.17287300 & 2.64157400 & -2.65972200 \\ \mathrm{H} & -2.99520600 & -4.15036000 & 0.24329500 \\ \mathrm{H} & -2.54282900 & -4.53536000 & -1.41588300 \\ \mathrm{H} & -4.69055300 & -4.09351000 & -2.17001900 \\ \mathrm{H} & -4.30721400 & -0.10876800 & -0.63097600 \\ \mathrm{H} & -4.95617500 & -1.30745700 & 0.46633500 \\ \mathrm{H} & -5.66121500 & -1.81062900 & -1.88019900 \\ \mathrm{H} & -1.76416700 & -2.82086200 & -2.85868100 \\ \mathrm{H} & -1.55403300 & -1.17528500 & -2.28732400 \\ \mathrm{H} & -3.91355600 & -2.26732700 & -3.61346500 \\ \mathrm{H} & -3.73304600 & -0.63573400 & -2.97364500 \\ \mathrm{H} & -5.38138800 & -3.58378100 & 0.78450100 \\ \mathrm{H} & -7.15482600 & -5.19021300 & 0.34207900 \\ \mathrm{H} & -6.60888100 & -5.33687700 & -1.41911800 \\ & & & \\ & & & \\ & & & \end{array}$<smiles>CCC1CN2CCC1CC2[C@H](Nc1c(Nc2cc(C(F)(F)F)cc(C(F)(F)F)c2)c(=O)c1=O)c1ccnc2ccc(OC)cc12</smiles>

Squaramide-6 


\begin{tabular}{|c|c|c|c|}
\hline $\mathrm{N}$ & 0.90208600 & 0.36650000 & 0.59045800 \\
\hline $\mathrm{C}$ & 0.10207900 & -0.45203100 & -0.12256000 \\
\hline $\mathrm{C}$ & -1.28817400 & -0.57178200 & -0.23425900 \\
\hline $\mathrm{C}$ & -1.18423300 & -1.67549100 & -1.24354400 \\
\hline $\mathrm{C}$ & 0.34765500 & -1.52008500 & -1.12716700 \\
\hline $\mathrm{O}$ & -1.94781400 & -2.34398000 & -1.90534800 \\
\hline ) & 1.32816400 & -1.99695000 & -1.66333500 \\
\hline $\mathrm{N}$ & -2.25447500 & 0.12546400 & 0.41409100 \\
\hline $\mathrm{C}$ & 2.36430200 & 0.21451900 & 0.48455200 \\
\hline $\mathrm{C}$ & 2.93143100 & -0.65449400 & 1.59585400 \\
\hline $\mathrm{C}$ & 3.00006400 & 1.61648800 & 0.52929500 \\
\hline $\mathrm{C}$ & 3.78146100 & -1.76871200 & 1.33339300 \\
\hline $\mathrm{C}$ & 4.35948800 & -2.42866400 & 2.46941900 \\
\hline $\mathrm{N}$ & 4.10460000 & -2.06893100 & 3.75586300 \\
\hline $\mathrm{C}$ & 3.27034200 & -1.06613000 & 3.95442800 \\
\hline $\mathrm{C}$ & 2.66903100 & -0.33190300 & 2.91234400 \\
\hline $\mathrm{C}$ & 4.06694500 & -2.25765200 & 0.02966900 \\
\hline $\mathrm{C}$ & 4.89938300 & -3.34583600 & -0.13735500 \\
\hline $\mathrm{C}$ & 5.50930800 & -3.97125200 & 0.98482300 \\
\hline $\mathrm{C}$ & 5.23890200 & -3.52543300 & 2.24993600 \\
\hline $\mathrm{O}$ & 5.19518700 & -3.91053200 & -1.33642300 \\
\hline $\mathrm{C}$ & 4.54694400 & -3.38280400 & -2.48990400 \\
\hline $\mathrm{N}$ & 2.21580000 & 2.55888100 & -0.29600800 \\
\hline $\mathrm{C}$ & 2.75959400 & 3.91245800 & -0.09352100 \\
\hline $\mathrm{C}$ & 4.26344800 & 4.01355400 & -0.50793300 \\
\hline $\mathrm{C}$ & 4.68849500 & 2.61889000 & -1.02418400 \\
\hline $\mathrm{C}$ & 4.49751400 & 1.59575900 & 0.10913800 \\
\hline $\mathrm{C}$ & 4.49480100 & 5.16290000 & -1.49908200 \\
\hline $\mathrm{C}$ & 5.95432500 & 5.35942200 & -1.91276500 \\
\hline $\mathrm{C}$ & -3.64772100 & 0.08041400 & 0.26033200 \\
\hline $\mathrm{C}$ & -4.41511800 & 0.92067100 & 1.07725800 \\
\hline $\mathrm{C}$ & -5.80122700 & 0.92613100 & 0.96391500 \\
\hline $\mathrm{C}$ & -6.44429400 & 0.10461400 & 0.04018100 \\
\hline $\mathrm{C}$ & -5.66892600 & -0.72769100 & -0.76504900 \\
\hline $\mathrm{C}$ & -4.27901700 & -0.75504500 & -0.66930400 \\
\hline $\mathrm{C}$ & -6.34051600 & -1.57459600 & -1.81599100 \\
\hline $\mathrm{C}$ & -6.61546600 & 1.78249700 & 1.89556700 \\
\hline $\mathrm{F}$ & -5.93275400 & 2.88165200 & 2.28968700 \\
\hline $\mathrm{F}$ & -7.76189900 & 2.19742900 & 1.31910000 \\
\hline $\mathrm{F}$ & -6.95520900 & 1.10724700 & 3.01626600 \\
\hline $\mathrm{F}$ & -6.39355900 & -0.92650500 & -3.00167800 \\
\hline $\mathrm{F}$ & -5.68445700 & -2.73191100 & -2.02200800 \\
\hline $\mathrm{F}$ & -7.61217400 & -1.87662700 & -1.47258400 \\
\hline $\mathrm{C}$ & 3.80004700 & 2.19221600 & -2.20621400 \\
\hline $\mathrm{C}$ & 2.31974000 & 2.21582500 & -1.73020500 \\
\hline $\mathrm{H}$ & 0.63071500 & 1.34927100 & 0.63024100 \\
\hline $\mathrm{H}$ & -1.92679000 & 0.73799200 & 1.15008100 \\
\hline $\mathrm{H}$ & 2.58576200 & -0.23840900 & -0.48029600 \\
\hline $\mathrm{H}$ & 2.91224900 & 1.98606600 & 1.55705400 \\
\hline $\mathrm{H}$ & 3.06234500 & -0.80253000 & 4.99007400 \\
\hline $\mathrm{H}$ & 2.00081600 & 0.48940800 & 3.15233900 \\
\hline $\mathrm{H}$ & 3.56317700 & -1.83129400 & -0.82335300 \\
\hline
\end{tabular}




$\begin{array}{rrrr}\mathrm{H} & 6.16590100 & -4.81535200 & 0.80419200 \\ \mathrm{H} & 5.67225200 & -3.99802800 & 3.12432800 \\ \mathrm{H} & 4.87223400 & -4.00316000 & -3.32521400 \\ \mathrm{H} & 3.45702900 & -3.42552400 & -2.39252400 \\ \mathrm{H} & 4.84775200 & -2.34271900 & -2.67082400 \\ \mathrm{H} & 2.62150900 & 4.18008000 & 0.95916500 \\ \mathrm{H} & 2.15112300 & 4.60438000 & -0.68635200 \\ \mathrm{H} & 4.86533200 & 4.22451100 & 0.38646800 \\ \mathrm{H} & 5.73884600 & 2.63217800 & -1.32750000 \\ \mathrm{H} & 4.79467600 & 0.59906300 & -0.23369500 \\ \mathrm{H} & 5.13777800 & 1.84023000 & 0.96198100 \\ \mathrm{H} & 4.12233700 & 6.08701700 & -1.03773300 \\ \mathrm{H} & 3.87517300 & 5.00784700 & -2.39209500 \\ \mathrm{H} & 6.06825900 & 6.24653500 & -2.54295000 \\ \mathrm{H} & 6.59838200 & 5.48828500 & -1.03567000 \\ \mathrm{H} & 6.33439800 & 4.50379000 & -2.47923900 \\ \mathrm{H} & -3.93465700 & 1.57559500 & 1.79687100 \\ \mathrm{H} & -7.52249200 & 0.11172600 & -0.04906200 \\ \mathrm{H} & -3.70134700 & -1.42671900 & -1.29686600 \\ \mathrm{H} & 4.08750000 & 1.18674000 & -2.53246800 \\ \mathrm{H} & 3.94488000 & 2.85493800 & -3.06426200 \\ \mathrm{H} & 1.83283700 & 1.25179800 & -1.89901800 \\ \mathrm{H} & 1.73847400 & 2.96249000 & -2.27912700\end{array}$<smiles>C=CC1CN2CCC1CN2[C@H](NC(=S)Nc1cc(C(F)(F)F)cc(C(F)(F)F)c1)c1ccnc2ccccc12</smiles>

Thiourea-7

$\begin{array}{lccc}\mathrm{C} & 2.83753500 & -0.44714200 & 0.14917300 \\ \mathrm{C} & 3.35889400 & 0.34400800 & -0.88220600 \\ \mathrm{C} & 4.73870900 & 0.49611500 & -0.99637600 \\ \mathrm{C} & 5.61892200 & -0.13630500 & -0.12009000 \\ \mathrm{C} & 5.09176000 & -0.93664800 & 0.89051900 \\ \mathrm{C} & 3.71539900 & -1.08859100 & 1.03171400 \\ \mathrm{~N} & 1.46581200 & -0.69002000 & 0.31758200 \\ \mathrm{C} & 0.37403500 & 0.10415500 & 0.02269200 \\ \mathrm{~N} & -0.79108200 & -0.55667100 & 0.28600200 \\ \mathrm{~S} & 0.42968900 & 1.67552200 & -0.54150500 \\ \mathrm{C} & -2.09295300 & 0.02307200 & -0.01378800 \\ \mathrm{C} & -3.10848200 & -1.14188600 & 0.01246700 \\ \mathrm{C} & -2.50361700 & 1.08410700 & 0.99254100 \\ \mathrm{C} & -2.30663800 & 0.85013200 & 2.33604400 \\ \mathrm{C} & -2.77616400 & 1.77834000 & 3.29043000\end{array}$




\begin{tabular}{|c|c|c|c|}
\hline $\mathrm{N}$ & -3.42238900 & 2.88487700 & 2.98741000 \\
\hline $\mathrm{C}$ & -3.61776000 & 3.15332700 & 1.66380900 \\
\hline $\mathrm{C}$ & -3.16525000 & 2.28920700 & 0.61131400 \\
\hline $\mathrm{C}$ & -4.30146500 & 4.35276000 & 1.33472200 \\
\hline $\mathrm{C}$ & -4.51922200 & 4.70025200 & 0.02299600 \\
\hline $\mathrm{C}$ & -4.05062600 & 3.86515300 & -1.01843700 \\
\hline $\mathrm{C}$ & -3.39026700 & 2.69249400 & -0.73280500 \\
\hline $\mathrm{N}$ & -2.55536900 & -2.31918400 & -0.69618400 \\
\hline $\mathrm{C}$ & -3.40829700 & -3.47983000 & -0.39771600 \\
\hline $\mathrm{C}$ & -4.89416200 & -3.24919200 & -0.84698100 \\
\hline $\mathrm{C}$ & -4.50562900 & -0.75407100 & -0.54066000 \\
\hline $\mathrm{C}$ & -4.94978200 & -1.85244300 & -1.52227800 \\
\hline $\mathrm{C}$ & -2.53733500 & -2.09561500 & -2.15616900 \\
\hline $\mathrm{C}$ & -3.97161200 & -1.85332600 & -2.70977200 \\
\hline $\mathrm{C}$ & -5.85714800 & -3.39003000 & 0.29626400 \\
\hline $\mathrm{C}$ & -6.90231000 & -4.21619500 & 0.31426600 \\
\hline $\mathrm{C}$ & 5.28634300 & 1.40576700 & -2.06447700 \\
\hline $\mathrm{C}$ & 6.01576900 & -1.69436100 & 1.80378100 \\
\hline $\mathrm{F}$ & 6.51702200 & 1.01619700 & -2.46599900 \\
\hline $\mathrm{F}$ & 4.49172100 & 1.43421200 & -3.15523200 \\
\hline $\mathrm{F}$ & 5.39927400 & 2.67634100 & -1.62040700 \\
\hline $\mathrm{F}$ & 5.46121400 & -1.90081900 & 3.01955400 \\
\hline $\mathrm{F}$ & 6.31811200 & -2.91311600 & 1.29900000 \\
\hline $\mathrm{F}$ & 7.18208700 & -1.04318500 & 1.99030400 \\
\hline $\mathrm{H}$ & 2.69561400 & 0.83633300 & -1.57651300 \\
\hline $\mathrm{H}$ & 6.68871800 & -0.01121600 & -0.22432600 \\
\hline $\mathrm{H}$ & 3.32537600 & -1.69898800 & 1.83971500 \\
\hline $\mathrm{H}$ & 1.25771400 & -1.47695700 & 0.91832300 \\
\hline $\mathrm{H}$ & -0.80955500 & -1.56875200 & 0.14278800 \\
\hline $\mathrm{H}$ & -2.05764200 & 0.46344400 & -1.01344400 \\
\hline $\mathrm{H}$ & -3.20385700 & -1.45413700 & 1.05837100 \\
\hline $\mathrm{H}$ & -1.78415400 & -0.04319300 & 2.66168300 \\
\hline $\mathrm{H}$ & -2.61555200 & 1.58422300 & 4.35001900 \\
\hline $\mathrm{H}$ & -4.63249900 & 4.97561400 & 2.15839800 \\
\hline $\mathrm{H}$ & -5.03962100 & 5.62155600 & -0.21935600 \\
\hline $\mathrm{H}$ & -4.20341400 & 4.15867700 & -2.05225000 \\
\hline $\mathrm{H}$ & -3.01379000 & 2.08637000 & -1.54742300 \\
\hline $\mathrm{H}$ & -3.35844900 & -3.66954900 & 0.67931900 \\
\hline $\mathrm{H}$ & -2.98335100 & -4.35318100 & -0.90124100 \\
\hline $\mathrm{H}$ & -5.16039000 & -3.99268700 & -1.60754300 \\
\hline $\mathrm{H}$ & -4.47041100 & 0.20450800 & -1.06655000 \\
\hline $\mathrm{H}$ & -5.22183200 & -0.63115000 & 0.27537900 \\
\hline $\mathrm{H}$ & -5.96935200 & -1.65867500 & -1.86712200 \\
\hline $\mathrm{H}$ & -2.07640000 & -2.97565400 & -2.61393800 \\
\hline $\mathrm{H}$ & -1.87832000 & -1.24952800 & -2.36756600 \\
\hline $\mathrm{H}$ & -4.02766000 & -0.89568700 & -3.23803600 \\
\hline $\mathrm{H}$ & -4.25047200 & -2.63297900 & -3.42653600 \\
\hline $\mathrm{H}$ & -5.66332700 & -2.76527500 & 1.16891400 \\
\hline $\mathrm{H}$ & -7.56864400 & -4.27836000 & 1.16886900 \\
\hline $\mathrm{H}$ & -7.12930000 & -4.86234300 & -0.53036500 \\
\hline
\end{tabular}


<smiles>CCC1CN2CCC1C2[C@H](NC(=S)Nc1cc(C(F)(F)F)cc(C(F)(F)F)c1)c1ccnc2ccccc12</smiles>

Thiourea-8

$\begin{array}{lrrr}\text { C } & 2.89583900 & -0.44663300 & 0.13970700 \\ \text { C } & 3.42008100 & 0.36302100 & -0.87583600 \\ \text { C } & 4.80021900 & 0.51681700 & -0.98368700 \\ \text { C } & 5.67817300 & -0.13145000 & -0.11674900 \\ \text { C } & 5.14824900 & -0.94981300 & 0.87786900 \\ \text { C } & 3.77152300 & -1.10407300 & 1.01271100 \\ \text { N } & 1.52393000 & -0.69235000 & 0.30049300 \\ \text { C } & 0.43199800 & 0.10547500 & 0.01527400 \\ \text { N } & -0.73273300 & -0.56010300 & 0.26654300 \\ \text { S } & 0.48807800 & 1.68527600 & -0.52522100 \\ \text { C } & -2.03453100 & 0.02084400 & -0.03036000 \\ \text { C } & -3.04878800 & -1.14597900 & -0.01271500 \\ \text { C } & -2.44887600 & 1.07329400 & 0.98308600 \\ \text { C } & -2.24940000 & 0.83195800 & 2.32492600 \\ \text { C } & -2.72434600 & 1.75080400 & 3.28560800 \\ \text { N } & -3.37836300 & 2.85493500 & 2.99049100 \\ \text { C } & -3.57651700 & 3.13063000 & 1.66873300 \\ \text { C } & -3.11899900 & 2.27643200 & 0.61035500 \\ \text { C } & -4.26862000 & 4.32746300 & 1.34784800 \\ \text { C } & -4.48968000 & 4.68198800 & 0.03853000 \\ \text { C } & -4.01617900 & 3.85686900 & -1.00860000 \\ \text { C } & -3.34772400 & 2.68688100 & -0.73091400 \\ \text { N } & -2.49233200 & -2.31979800 & -0.72476100 \\ \text { C } & -3.33001500 & -3.49088900 & -0.41212200 \\ \text { C } & -4.82963800 & -3.25516200 & -0.78605000 \\ \text { C } & -4.44412300 & -0.75807500 & -0.56691900 \\ \text { C } & -4.90789900 & -1.88760200 & -1.50383600 \\ \text { C } & -2.50513800 & -2.09996000 & -2.18520900 \\ \text { C } & -3.95711200 & -1.92001000 & -2.71411300 \\ \text { C } & -5.74295700 & -3.38133500 & 0.44229000 \\ \text { C } & -7.23077400 & -3.18413000 & 0.14677300 \\ \text { C } & 5.35075500 & 1.44544200 & -2.03379100 \\ \text { C } & 6.06971700 & -1.72405900 & 1.77968000 \\ \text { F } & 4.55907000 & 1.49383400 & -3.12593600 \\ \text { F } & 5.46294200 & 2.70782400 & -1.56664400 \\ \text { F } & 6.58246400 & 1.06287800 & -2.43912800 \\ \text { F } & 7.23587200 & -1.07686800 & 1.98073900 \\ \text { F } & 5.51217900 & -1.95204000 & 2.99028300 \\ \text { F } & 6.37296800 & -2.93382100 & 1.25409000 \\ \text { H } & 2.75850000 & 0.86801800 & -1.56262500 \\ \text { H } & 6.74824100 & -0.00468600 & -0.21597400\end{array}$




$\begin{array}{rrrr}\mathrm{H} & 3.37934000 & -1.72898200 & 1.80848200 \\ \mathrm{H} & 1.31434500 & -1.48946900 & 0.88710100 \\ \mathrm{H} & -0.75158600 & -1.57010300 & 0.10601900 \\ \mathrm{H} & -1.99860500 & 0.46840100 & -1.02685700 \\ \mathrm{H} & -3.14715800 & -1.46276100 & 1.03143000 \\ \mathrm{H} & -1.72112700 & -0.06025300 & 2.64414500 \\ \mathrm{H} & -2.56153200 & 1.55085300 & 4.34380200 \\ \mathrm{H} & -4.60318600 & 4.94280100 & 2.17575200 \\ \mathrm{H} & -5.01639500 & 5.60137900 & -0.19745300 \\ \mathrm{H} & -4.17162200 & 4.15602000 & -2.04042000 \\ \mathrm{H} & -2.96795100 & 2.08833400 & -1.54956000 \\ \mathrm{H} & -3.23055400 & -3.69997400 & 0.65891100 \\ \mathrm{H} & -2.91552300 & -4.34912700 & -0.95004000 \\ \mathrm{H} & -5.14106400 & -4.02082000 & -1.50917400 \\ \mathrm{H} & -4.40052400 & 0.18074300 & -1.12726100 \\ \mathrm{H} & -5.14922300 & -0.59466400 & 0.25198800 \\ \mathrm{H} & -5.92959000 & -1.69749700 & -1.84336900 \\ \mathrm{H} & -2.01354900 & -2.95928800 & -2.65053500 \\ \mathrm{H} & -1.88732000 & -1.22577400 & -2.40695600 \\ \mathrm{H} & -4.04884100 & -0.99203900 & -3.28839000 \\ \mathrm{H} & -4.23291500 & -2.74140500 & -3.38416500 \\ \mathrm{H} & -5.42361800 & -2.66871400 & 1.21395500 \\ \mathrm{H} & -5.58873300 & -4.37749500 & 0.87731000 \\ \mathrm{H} & -7.83729600 & -3.37861000 & 1.03631000 \\ \mathrm{H} & -7.44716200 & -2.16267400 & -0.18066600 \\ \mathrm{H} & -7.56900900 & -3.86418100 & -0.64314000\end{array}$<smiles>CCN(CC)C(=O)[C@H](NC(=S)N[C@H]1CCCCC1n1c(C)ccc1C)C(C)(C)C</smiles>

Thiourea-9

$\begin{array}{lrrr}\mathrm{C} & 2.08641600 & 1.28540500 & 2.75735400 \\ \mathrm{C} & 3.26426000 & 0.78929600 & 1.91895800 \\ \mathrm{~N} & 3.12444400 & 1.10270100 & 0.49405600 \\ \mathrm{C} & 3.58005400 & 2.42649600 & 0.05529400 \\ \mathrm{C} & 4.98422400 & 2.37928900 & -0.54483300 \\ \mathrm{C} & 2.53100700 & 0.31010200 & -0.43616900 \\ \mathrm{C} & 1.99280400 & -1.06653300 & -0.02363200 \\ \mathrm{O} & 2.39812900 & 0.67364900 & -1.61103100 \\ \mathrm{~N} & 0.63367000 & -1.12508200 & -0.54600400 \\ \mathrm{C} & 2.87720300 & -2.24434800 & -0.54664200 \\ \mathrm{C} & 2.29498400 & -3.55331500 & 0.01154100 \\ \mathrm{C} & 4.31413400 & -2.06496600 & -0.02880700 \\ \mathrm{C} & 2.88940000 & -2.30683800 & -2.08213600 \\ \mathrm{C} & -0.46930000 & -1.32342600 & 0.22181900 \\ \mathrm{~N} & -1.63637900 & -1.29442500 & -0.49105600 \\ \mathrm{~S} & -0.42971800 & -1.65696600 & 1.87927400\end{array}$




\begin{tabular}{|c|c|c|c|}
\hline $\mathrm{C}$ & -2.90934200 & -0.88700900 & 0.08454200 \\
\hline $\mathrm{C}$ & -3.32503800 & 0.49984400 & -0.46556700 \\
\hline $\mathrm{C}$ & -4.59473500 & 0.99290700 & 0.23856600 \\
\hline $\mathrm{C}$ & -5.73097200 & -0.01709600 & 0.01822900 \\
\hline $\mathrm{C}$ & -5.33616200 & -1.42341600 & 0.49211400 \\
\hline $\mathrm{C}$ & -4.02999200 & -1.90187200 & -0.15811100 \\
\hline $\mathrm{N}$ & -2.20675200 & 1.43746200 & -0.48318500 \\
\hline $\mathrm{C}$ & -1.48769100 & 1.91035300 & 0.61308500 \\
\hline $\mathrm{C}$ & -0.32119800 & 2.46729200 & 0.13284000 \\
\hline $\mathrm{C}$ & -0.30557900 & 2.31928200 & -1.28059900 \\
\hline $\mathrm{C}$ & -1.46552800 & 1.67369000 & -1.64403200 \\
\hline $\mathrm{C}$ & -1.94497800 & 1.83348600 & 2.03355600 \\
\hline $\mathrm{C}$ & -1.94219500 & 1.31886400 & -3.01676600 \\
\hline $\mathrm{H}$ & 2.25935400 & 1.06902900 & 3.81644100 \\
\hline $\mathrm{H}$ & 1.16223900 & 0.78889500 & 2.45104300 \\
\hline $\mathrm{H}$ & 1.95646100 & 2.36689200 & 2.64894700 \\
\hline $\mathrm{H}$ & 4.20022000 & 1.24779300 & 2.25571100 \\
\hline $\mathrm{H}$ & 3.39370400 & -0.28481100 & 2.04719700 \\
\hline $\mathrm{H}$ & 3.54830300 & 3.09119700 & 0.92362100 \\
\hline $\mathrm{H}$ & 2.86855100 & 2.79889100 & -0.68327800 \\
\hline $\mathrm{H}$ & 5.31160300 & 3.38113900 & -0.84065500 \\
\hline $\mathrm{H}$ & 5.70856900 & 1.98198000 & 0.17378900 \\
\hline $\mathrm{H}$ & 4.98426600 & 1.74012800 & -1.43089700 \\
\hline $\mathrm{H}$ & 1.90237100 & -1.16488700 & 1.05629700 \\
\hline $\mathrm{H}$ & 0.51988800 & -0.57687000 & -1.39463300 \\
\hline $\mathrm{H}$ & 2.28914000 & -3.55378200 & 1.10543600 \\
\hline $\mathrm{H}$ & 2.88940900 & -4.40658700 & -0.33202100 \\
\hline $\mathrm{H}$ & 1.26494400 & -3.69912900 & -0.32380000 \\
\hline $\mathrm{H}$ & 4.76595700 & -1.13672600 & -0.39200500 \\
\hline $\mathrm{H}$ & 4.34824500 & -2.05791200 & 1.06601400 \\
\hline $\mathrm{H}$ & 4.94069900 & -2.89530000 & -0.36911400 \\
\hline $\mathrm{H}$ & 3.49928700 & -3.15469900 & -2.41146900 \\
\hline $\mathrm{H}$ & 1.87790900 & -2.45377000 & -2.47257100 \\
\hline $\mathrm{H}$ & 3.29046500 & -1.39155400 & -2.52053300 \\
\hline $\mathrm{H}$ & -1.53269300 & -1.10983300 & -1.48128200 \\
\hline $\mathrm{H}$ & -2.73670800 & -0.81792900 & 1.15989000 \\
\hline $\mathrm{H}$ & -3.59608700 & 0.35728800 & -1.51685900 \\
\hline $\mathrm{H}$ & -4.86390800 & 1.98082400 & -0.14901100 \\
\hline $\mathrm{H}$ & -4.41175900 & 1.10438300 & 1.31128500 \\
\hline $\mathrm{H}$ & -5.98016800 & -0.05109500 & -1.05148200 \\
\hline $\mathrm{H}$ & -6.63445900 & 0.31855600 & 0.53841100 \\
\hline $\mathrm{H}$ & -6.14288800 & -2.13413400 & 0.28326500 \\
\hline $\mathrm{H}$ & -5.20411500 & -1.41106700 & 1.58224400 \\
\hline $\mathrm{H}$ & -4.17615900 & -2.03453800 & -1.23977000 \\
\hline $\mathrm{H}$ & -3.72300900 & -2.87092700 & 0.24603000 \\
\hline $\mathrm{H}$ & 0.42923400 & 2.94678100 & 0.74428500 \\
\hline $\mathrm{H}$ & 0.47002100 & 2.63669400 & -1.96139500 \\
\hline $\mathrm{H}$ & -2.08485700 & 0.80418900 & 2.37896700 \\
\hline $\mathrm{H}$ & -1.17192000 & 2.27547500 & 2.66644000 \\
\hline $\mathrm{H}$ & -2.87252200 & 2.38950000 & 2.20940600 \\
\hline $\mathrm{H}$ & -2.05777100 & 0.23901000 & -3.18981000 \\
\hline $\mathrm{H}$ & -2.90668700 & 1.78087200 & -3.26147300 \\
\hline $\mathrm{H}$ & -1.21022100 & 1.67876800 & -3.74308800 \\
\hline
\end{tabular}


<smiles>CCCc1cc(C(C)C)c(S(=O)NC(=O)N[C@H]2CCCCC2N(C)C)c(C(C)C)c1</smiles>

Urea-2

$\begin{array}{lrrr}\mathrm{C} & -2.21419700 & 0.46168700 & 1.73385900 \\ \mathrm{C} & -2.75997400 & 1.28679100 & 0.75490100 \\ \mathrm{C} & -2.65464000 & 0.88539400 & -0.58024000 \\ \mathrm{C} & -2.04505600 & -0.31220600 & -0.95210100 \\ \mathrm{C} & -1.51910100 & -1.13149100 & 0.07097000 \\ \mathrm{C} & -1.58219800 & -0.75014400 & 1.42472200 \\ \mathrm{C} & -1.02438000 & -1.58934000 & 2.57023100 \\ \mathrm{C} & -1.92196000 & -0.63355800 & -2.43796600 \\ \mathrm{C} & -3.43479100 & 2.59456500 & 1.12784600 \\ \mathrm{C} & -4.90258700 & 2.62301900 & 0.67171000 \\ \mathrm{C} & -2.65949700 & 3.80289300 & 0.57649600 \\ \mathrm{C} & -2.16951200 & -2.19459200 & 3.39923600 \\ \mathrm{C} & -0.05459500 & -0.78026900 & 3.44493900 \\ \mathrm{C} & -0.88994100 & 0.30309400 & -3.09334100 \\ \mathrm{C} & -3.27699500 & -0.57584900 & -3.15877900 \\ \mathrm{~S} & -0.82129400 & -2.78581500 & -0.28087100 \\ \mathrm{~N} & 0.61784400 & -2.31895700 & -1.18077800 \\ \mathrm{O} & -1.69211500 & -3.45036600 & -1.31095500 \\ \mathrm{C} & 1.62443000 & -1.67252600 & -0.47395000 \\ \mathrm{~N} & 2.61769200 & -1.13023800 & -1.24974700 \\ \mathrm{O} & 1.61739800 & -1.65901400 & 0.75659900 \\ \mathrm{C} & 3.52727800 & -0.11522800 & -0.70137200 \\ \mathrm{C} & 2.76646800 & 1.07092800 & -0.06562600 \\ \mathrm{C} & 3.76464700 & 2.12465100 & 0.44822000 \\ \mathrm{C} & 4.75161300 & 1.51131800 & 1.44880900 \\ \mathrm{C} & 5.50341000 & 0.33282400 & 0.81901600 \\ \mathrm{C} & 4.53023400 & -0.72585900 & 0.28862700 \\ \mathrm{~N} & 1.71955300 & 1.57114400 & -0.96833700 \\ \mathrm{C} & 2.19194200 & 2.39435700 & -2.07194900 \\ \mathrm{C} & 0.62238200 & 2.21171600 & -0.25826300 \\ \mathrm{H} & -2.28276200 & 0.76769400 & 2.77338700 \\ \mathrm{H} & -3.05422700 & 1.52836300 & -1.35799500 \\ \mathrm{H} & -0.43977300 & -2.40747800 & 2.15169800 \\ \mathrm{H} & -1.58592200 & -1.66385000 & -2.54117600 \\ \mathrm{H} & -3.42361800 & 2.66366800 & 2.22288000 \\ \mathrm{H} & -5.45858200 & 1.77416000 & 1.07991600 \\ \mathrm{H} & -5.39116400 & 3.54573600 & 1.00119600 \\ \mathrm{H} & -4.97807400 & 2.57761000 & -0.41972800 \\ \mathrm{H} & -1.62590600 & 3.80588000 & 0.93414800 \\ \mathrm{H} & -2.63502500 & 3.78783600 & -0.51833200 \\ \mathrm{H} & -3.13291900 & 4.74025000 & 0.88643600 \\ \mathrm{H} & -2.82684200 & -2.80922800 & 2.77628200 \\ \mathrm{H} & -1.77022100 & -2.82515600 & 4.20024200\end{array}$




$\begin{array}{lrrr}\mathrm{H} & -2.78284300 & -1.41279600 & 3.86020300 \\ \mathrm{H} & 0.76903400 & -0.39696800 & 2.83919400 \\ \mathrm{H} & -0.54960900 & 0.05916400 & 3.94409700 \\ \mathrm{H} & 0.36790200 & -1.42403500 & 4.22306900 \\ \mathrm{H} & 0.05597100 & 0.30740800 & -2.54087200 \\ \mathrm{H} & -0.70285700 & 0.00490800 & -4.13118400 \\ \mathrm{H} & -1.25533500 & 1.33593500 & -3.10889200 \\ \mathrm{H} & -3.98517300 & -1.26979100 & -2.69860200 \\ \mathrm{H} & -3.71494900 & 0.42722100 & -3.13663900 \\ \mathrm{H} & -3.15548400 & -0.85941000 & -4.20918700 \\ \mathrm{H} & 0.43828900 & -2.08696500 & -2.15155200 \\ \mathrm{H} & 2.37003200 & -0.98276900 & -2.21717800 \\ \mathrm{H} & 4.09378300 & 0.25369100 & -1.56559300 \\ \mathrm{H} & 2.23813400 & 0.65848400 & 0.79840600 \\ \mathrm{H} & 3.21790700 & 2.95577900 & 0.90789900 \\ \mathrm{H} & 4.32750500 & 2.54619100 & -0.39518200 \\ \mathrm{H} & 4.20095200 & 1.15901900 & 2.33132600 \\ \mathrm{H} & 5.45725500 & 2.27267100 & 1.80014600 \\ \mathrm{H} & 6.18486000 & -0.12084700 & 1.54699800 \\ \mathrm{H} & 6.12882500 & 0.70515800 & -0.00505700 \\ \mathrm{H} & 3.96816700 & -1.16982600 & 1.11488200 \\ \mathrm{H} & 5.06850900 & -1.53858700 & -0.21019400 \\ \mathrm{H} & 1.37662900 & 2.53251200 & -2.78954800 \\ \mathrm{H} & 2.54249100 & 3.39584700 & -1.76689900 \\ \mathrm{H} & 3.01449000 & 1.89845000 & -2.59595100 \\ \mathrm{H} & -0.18336500 & 2.43648300 & -0.96117700 \\ \mathrm{H} & 0.22172300 & 1.53051700 & 0.49552100 \\ \mathrm{H} & 0.90713100 & 3.15844600 & 0.23786400 \\ & & & \end{array}$

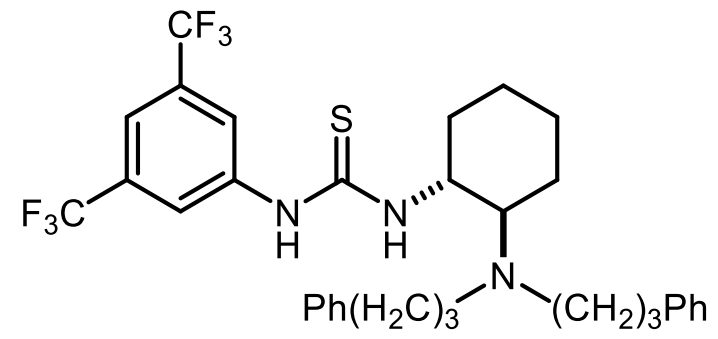

Thiourea-10

$\begin{array}{lrrr}\text { C } & -2.46270500 & 1.71616700 & -0.63413600 \\ \text { C } & -1.63431300 & 0.60479700 & -0.81927400 \\ \text { C } & -2.21249400 & -0.67141300 & -0.88421700 \\ \text { C } & -3.58105500 & -0.83708200 & -0.75088100 \\ \text { C } & -4.41592200 & 0.26095700 & -0.54325900 \\ \text { C } & -3.83687900 & 1.52363000 & -0.48265200 \\ \text { C } & -4.17858600 & -2.21374000 & -0.80046500 \\ \text { C } & -4.68803700 & 2.70971300 & -0.12442800 \\ \text { F } & -5.03214700 & -2.42333600 & 0.22835500 \\ \text { F } & -4.88458200 & -2.41405200 & -1.93647200 \\ \text { F } & -3.23771000 & -3.18742900 & -0.74310300 \\ \text { F } & -5.98070400 & 2.53227200 & -0.47650700 \\ \text { F } & -4.67237800 & 2.92733300 & 1.21528100\end{array}$




\begin{tabular}{|c|c|c|c|}
\hline $\mathrm{F}$ & -4.25674100 & 3.84445400 & -0.71109100 \\
\hline $\mathrm{N}$ & -0.23944700 & 0.64557200 & -0.95450400 \\
\hline $\mathrm{C}$ & 0.69170500 & 1.58751300 & -0.58920700 \\
\hline $\mathrm{N}$ & 1.96285500 & 1.13134200 & -0.73989600 \\
\hline S & 0.34857800 & 3.12728800 & -0.00041400 \\
\hline $\mathrm{C}$ & 3.14513400 & 1.88180300 & -0.33387200 \\
\hline $\mathrm{C}$ & 4.30194700 & 0.90278600 & -0.07753200 \\
\hline $\mathrm{C}$ & 5.54737100 & 1.69277300 & 0.37681500 \\
\hline $\mathrm{C}$ & 5.95563400 & 2.74772200 & -0.65933400 \\
\hline $\mathrm{C}$ & 4.79234400 & 3.69506700 & -0.96934700 \\
\hline $\mathrm{C}$ & 3.54890000 & 2.91268600 & -1.39980600 \\
\hline $\mathrm{N}$ & 3.90937700 & -0.22932500 & 0.78506300 \\
\hline $\mathrm{C}$ & 3.48244200 & 0.13475100 & 2.15421700 \\
\hline $\mathrm{C}$ & 1.96883100 & 0.27657300 & 2.35847400 \\
\hline $\mathrm{C}$ & 1.20001800 & -0.98807600 & 1.98174000 \\
\hline $\mathrm{C}$ & -0.30290500 & -0.95080300 & 2.17043100 \\
\hline $\mathrm{C}$ & -1.01884100 & -2.15680700 & 2.12374700 \\
\hline $\mathrm{C}$ & -2.40323800 & -2.18089900 & 2.26826700 \\
\hline $\mathrm{C}$ & -3.10475700 & -0.98900100 & 2.46149800 \\
\hline $\mathrm{C}$ & -2.40912000 & 0.21674900 & 2.50380700 \\
\hline $\mathrm{C}$ & -1.01940600 & 0.23522800 & 2.36236400 \\
\hline $\mathrm{C}$ & 4.95259500 & -1.26450400 & 0.80569700 \\
\hline $\mathrm{C}$ & 4.42913600 & -2.68299000 & 1.07990900 \\
\hline $\mathrm{C}$ & 4.05617100 & -3.49215900 & -0.17829800 \\
\hline $\mathrm{C}$ & 2.85911300 & -3.00326100 & -0.95881100 \\
\hline $\mathrm{C}$ & 1.57498500 & -3.47349000 & -0.65482800 \\
\hline $\mathrm{C}$ & 0.46738800 & -3.08395300 & -1.40634400 \\
\hline $\mathrm{C}$ & 0.62662000 & -2.20083200 & -2.47835800 \\
\hline $\mathrm{C}$ & 1.89820200 & -1.70415500 & -2.77938700 \\
\hline $\mathrm{C}$ & 3.00192600 & -2.10839700 & -2.02725300 \\
\hline $\mathrm{H}$ & -2.03512800 & 2.70510400 & -0.57618800 \\
\hline $\mathrm{H}$ & -1.57826700 & -1.53936500 & -0.97760100 \\
\hline $\mathrm{H}$ & -5.48270100 & 0.13321900 & -0.41068500 \\
\hline $\mathrm{H}$ & 0.15093700 & -0.24271000 & -1.25054900 \\
\hline $\mathrm{H}$ & 2.11132000 & 0.12925300 & -0.80402600 \\
\hline $\mathrm{H}$ & 2.90597900 & 2.42351200 & 0.58910700 \\
\hline $\mathrm{H}$ & 4.54467700 & 0.44711300 & -1.04907700 \\
\hline $\mathrm{H}$ & 6.37571800 & 0.99959200 & 0.55505600 \\
\hline $\mathrm{H}$ & 5.34025700 & 2.18959000 & 1.33234800 \\
\hline $\mathrm{H}$ & 6.26638400 & 2.24226200 & -1.58439600 \\
\hline $\mathrm{H}$ & 6.82710000 & 3.30700100 & -0.30125200 \\
\hline $\mathrm{H}$ & 5.07543200 & 4.40990700 & -1.74940300 \\
\hline $\mathrm{H}$ & 4.55378800 & 4.28595400 & -0.07483100 \\
\hline $\mathrm{H}$ & 3.74476600 & 2.37979400 & -2.33996100 \\
\hline $\mathrm{H}$ & 2.70482700 & 3.58371300 & -1.57208300 \\
\hline $\mathrm{H}$ & 3.97651000 & 1.05783100 & 2.48790500 \\
\hline $\mathrm{H}$ & 3.83981100 & -0.65013600 & 2.82883900 \\
\hline $\mathrm{H}$ & 1.59021800 & 1.13518900 & 1.80192400 \\
\hline $\mathrm{H}$ & 1.80030000 & 0.50848500 & 3.41779200 \\
\hline $\mathrm{H}$ & 1.59666800 & -1.83181900 & 2.56345200 \\
\hline $\mathrm{H}$ & 1.42667700 & -1.23761900 & 0.93907200 \\
\hline $\mathrm{H}$ & -0.47652400 & -3.08766200 & 1.97393300 \\
\hline $\mathrm{H}$ & -2.93852400 & -3.12351500 & 2.21374300 \\
\hline
\end{tabular}




$\begin{array}{lrrr}\mathrm{H} & -4.18557100 & -1.00282100 & 2.55251100 \\ \mathrm{H} & -2.94909600 & 1.15195400 & 2.61272600 \\ \mathrm{H} & -0.50394000 & 1.18868600 & 2.37458600 \\ \mathrm{H} & 5.73224400 & -1.02397500 & 1.54767500 \\ \mathrm{H} & 5.45613100 & -1.25844500 & -0.16756100 \\ \mathrm{H} & 3.57388800 & -2.64089400 & 1.76084000 \\ \mathrm{H} & 5.21260400 & -3.24384700 & 1.60301700 \\ \mathrm{H} & 3.86969800 & -4.52802800 & 0.12666900 \\ \mathrm{H} & 4.92871700 & -3.51981600 & -0.84225700 \\ \mathrm{H} & 1.44607100 & -4.16900100 & 0.16973100 \\ \mathrm{H} & -0.51525500 & -3.47684400 & -1.16416000 \\ \mathrm{H} & -0.22886100 & -1.91292500 & -3.08196900 \\ \mathrm{H} & 2.03061200 & -1.01490200 & -3.60749700 \\ \mathrm{H} & 3.99091700 & -1.74085500 & -2.28527700\end{array}$<smiles>CN(C)C1CCCC[C@H]1NC(N)=S</smiles>

Thiourea-11

$\begin{array}{lrrr}\mathrm{C} & 1.34736400 & -0.23776900 & -0.30679800 \\ \mathrm{C} & -0.01682300 & 0.44145100 & -0.08077200 \\ \mathrm{C} & 0.00390600 & 1.85588600 & -0.67260200 \\ \mathrm{C} & 1.10515800 & 2.69111200 & -0.00812000 \\ \mathrm{C} & 2.47762200 & 2.01453800 & -0.12444500 \\ \mathrm{C} & 2.44332000 & 0.57336400 & 0.40620200 \\ \mathrm{~N} & -1.04275800 & -0.41350800 & -0.66714100 \\ \mathrm{C} & -2.34312200 & -0.38891200 & -0.27719900 \\ \mathrm{~N} & -3.09595300 & -1.40941200 & -0.79807100 \\ \mathrm{~S} & -3.01433700 & 0.73636500 & 0.77845600 \\ \mathrm{~N} & 1.27226900 & -1.67521600 & 0.00155900 \\ \mathrm{C} & 1.03884300 & -1.98913800 & 1.40860800 \\ \mathrm{C} & 2.39145900 & -2.43502300 & -0.53781300 \\ \mathrm{H} & 1.55092500 & -0.19010300 & -1.38675000 \\ \mathrm{H} & -0.21514400 & 0.53556900 & 0.99535900 \\ \mathrm{H} & -0.97553100 & 2.31556900 & -0.52437600 \\ \mathrm{H} & 0.17849800 & 1.78442300 & -1.75431300 \\ \mathrm{H} & 0.85645100 & 2.82756900 & 1.05284500 \\ \mathrm{H} & 1.13685100 & 3.69225300 & -0.45136700 \\ \mathrm{H} & 3.23787900 & 2.59420000 & 0.41093300 \\ \mathrm{H} & 2.78318300 & 1.99772000 & -1.17982300 \\ \mathrm{H} & 3.41813100 & 0.09421800 & 0.26392900 \\ \mathrm{H} & 2.24757200 & 0.58492200 & 1.48558700 \\ \mathrm{H} & -0.66161400 & -1.32874200 & -0.90633800 \\ \mathrm{H} & -4.09032500 & -1.32007700 & -0.65615800 \\ \mathrm{H} & -2.80473400 & -1.82864800 & -1.67103900 \\ \mathrm{H} & 0.15128100 & -1.46666500 & 1.77203500 \\ \mathrm{H} & 0.85704100 & -3.06358500 & 1.50631600\end{array}$




$$
\begin{array}{lrrr}
\mathrm{H} & 1.88751200 & -1.73045000 & 2.06335800 \\
\mathrm{H} & 2.18622400 & -3.50538200 & -0.43567700 \\
\mathrm{H} & 2.51154700 & -2.21039900 & -1.60199700 \\
\mathrm{H} & 3.35238200 & -2.23032900 & -0.03462000
\end{array}
$$<smiles>CNC(=S)N[C@H]1CCCCC1N(C)C</smiles>

Thiourea-12

$\begin{array}{lrrr}\mathrm{C} & -1.61221200 & 0.38934200 & -0.32251600 \\ \mathrm{C} & -0.37675500 & -0.49634900 & -0.07179000 \\ \mathrm{C} & -0.59693200 & -1.88124700 & -0.69215300 \\ \mathrm{C} & -1.83567100 & -2.54680400 & -0.08056200 \\ \mathrm{C} & -3.08216900 & -1.66383200 & -0.22641800 \\ \mathrm{C} & -2.84404600 & -0.25482200 & 0.33702500 \\ \mathrm{~N} & 0.78562900 & 0.19680800 & -0.61437500 \\ \mathrm{C} & 2.05676100 & -0.02408100 & -0.16307800 \\ \mathrm{~N} & 2.96633400 & 0.85782300 & -0.66541000 \\ \mathrm{~S} & 2.48527800 & -1.24509000 & 0.91497300 \\ \mathrm{~N} & -1.32741900 & 1.79180700 & 0.02501500 \\ \mathrm{C} & -1.09527000 & 2.03626700 & 1.44589000 \\ \mathrm{C} & -2.29758200 & 2.72665700 & -0.52738100 \\ \mathrm{C} & 4.39266200 & 0.76484800 & -0.41068000 \\ \mathrm{H} & -1.78398300 & 0.39612400 & -1.40896200 \\ \mathrm{H} & -0.23176300 & -0.63766200 & 1.00777900 \\ \mathrm{H} & 0.29455200 & -2.48901000 & -0.52299900 \\ \mathrm{H} & -0.72083000 & -1.76689800 & -1.77718200 \\ \mathrm{H} & -1.64818500 & -2.73644300 & 0.98485700 \\ \mathrm{H} & -2.00639900 & -3.52401300 & -0.54513400 \\ \mathrm{H} & -3.94161900 & -2.12717100 & 0.27101800 \\ \mathrm{H} & -3.34373700 & -1.58192300 & -1.29056900 \\ \mathrm{H} & -3.72807700 & 0.37181800 & 0.17558700 \\ \mathrm{H} & -2.68952900 & -0.31580900 & 1.42143500 \\ \mathrm{H} & 0.55398300 & 1.16849500 & -0.82116300 \\ \mathrm{H} & 2.67902800 & 1.39436400 & -1.47206200 \\ \mathrm{H} & -0.31215500 & 1.37420500 & 1.82175000 \\ \mathrm{H} & -0.75301300 & 3.06732200 & 1.57675900 \\ \mathrm{H} & -1.99492700 & 1.89934300 & 2.06866600 \\ \mathrm{H} & -3.29481100 & 2.66294700 & -0.05826300 \\ \mathrm{H} & -1.93356300 & 3.75016700 & -0.39192400 \\ \mathrm{H} & -2.41554100 & 2.54537200 & -1.60002500 \\ \mathrm{H} & 4.81997300 & -0.16254500 & -0.80881700 \\ \mathrm{H} & 4.87800600 & 1.62326900 & -0.88080200 \\ \mathrm{H} & 4.58361800 & 0.78456000 & 0.66384500\end{array}$


<smiles>CCNC(=S)N[C@H]1CCCCC1N(C)C</smiles>

Thiourea-13

$\begin{array}{lrrr}\mathrm{C} & -1.92244400 & 0.36900200 & -0.37561800 \\ \mathrm{C} & -0.69589900 & -0.49234700 & -0.01880600 \\ \mathrm{C} & -0.84041900 & -1.88432600 & -0.64587900 \\ \mathrm{C} & -2.11218800 & -2.56913500 & -0.13109700 \\ \mathrm{C} & -3.35861700 & -1.71027600 & -0.38297000 \\ \mathrm{C} & -3.19186700 & -0.29371700 & 0.18723800 \\ \mathrm{~N} & 0.49399700 & 0.21974700 & -0.46979900 \\ \mathrm{C} & 1.73038700 & 0.02762000 & 0.07770400 \\ \mathrm{~N} & 2.65263800 & 0.93462900 & -0.35634000 \\ \mathrm{~S} & 2.08496600 & -1.18658000 & 1.19366700 \\ \mathrm{~N} & -1.69194300 & 1.77869700 & -0.01776800 \\ \mathrm{C} & -1.57757700 & 2.03679600 & 1.41500700 \\ \mathrm{C} & -2.63222900 & 2.69139600 & -0.65268300 \\ \mathrm{C} & 4.07533600 & 0.87334100 & -0.04403300 \\ \mathrm{C} & 4.84916400 & -0.08112000 & -0.95376700 \\ \mathrm{H} & -2.00598700 & 0.36547100 & -1.47242100 \\ \mathrm{H} & -0.63689300 & -0.62483100 & 1.06969600 \\ \mathrm{H} & 0.04535800 & -2.47461300 & -0.40176700 \\ \mathrm{H} & -0.87892800 & -1.77772400 & -1.73806600 \\ \mathrm{H} & -2.00756000 & -2.74927300 & 0.94723400 \\ \mathrm{H} & -2.22734900 & -3.55194500 & -0.60092500 \\ \mathrm{H} & -4.24677500 & -2.18647800 & 0.04732700 \\ \mathrm{H} & -3.53523200 & -1.63956700 & -1.46525300 \\ \mathrm{H} & -4.07097400 & 0.31549900 & -0.04992200 \\ \mathrm{H} & -3.12462900 & -0.34518000 & 1.28102800 \\ \mathrm{H} & 0.26149000 & 1.18363300 & -0.70960400 \\ \mathrm{H} & 2.40152300 & 1.46123900 & -1.18301700 \\ \mathrm{H} & -0.81293300 & 1.39409500 & 1.85678100 \\ \mathrm{H} & -1.26805200 & 3.07565100 & 1.56417100 \\ \mathrm{H} & -2.52057600 & 1.88472700 & 1.96597500 \\ \mathrm{H} & -2.29851500 & 3.72241900 & -0.49767000 \\ \mathrm{H} & -2.66199500 & 2.50042400 & -1.72971300 \\ \mathrm{H} & -3.66202800 & 2.61243100 & -0.26300700 \\ \mathrm{H} & 4.16298600 & 0.55693700 & 0.99665200 \\ \mathrm{H} & 4.46787100 & 1.89287300 & -0.12375200 \\ \mathrm{H} & 4.76276300 & 0.21505700 & -2.00492600 \\ \mathrm{H} & 5.91134300 & -0.08431600 & -0.68838800 \\ \mathrm{H} & 4.46091200 & -1.09616100 & -0.84233300\end{array}$


<smiles>CC(C)NC(=S)N[C@H]1CCCCC1N(C)C</smiles>

Thiourea-14

$\begin{array}{lrrr}\mathrm{C} & -2.15467000 & 0.49704500 & -0.37536900 \\ \mathrm{C} & -1.04934700 & -0.51620900 & -0.02141600 \\ \mathrm{C} & -1.36806500 & -1.87401900 & -0.65859400 \\ \mathrm{C} & -2.71874200 & -2.39442700 & -0.15215900 \\ \mathrm{C} & -3.84453000 & -1.38160800 & -0.40053800 \\ \mathrm{C} & -3.49999500 & -0.00169400 & 0.17997100 \\ \mathrm{~N} & 0.22335800 & 0.04108800 & -0.46421600 \\ \mathrm{C} & 1.42312400 & -0.30644400 & 0.08962100 \\ \mathrm{~N} & 2.45227200 & 0.48204200 & -0.33017400 \\ \mathrm{~S} & 1.61412500 & -1.56597900 & 1.19642000 \\ \mathrm{~N} & -1.74714900 & 1.86333400 & -0.00679600 \\ \mathrm{C} & -1.60167200 & 2.09381700 & 1.42780300 \\ \mathrm{C} & -2.56297200 & 2.89302100 & -0.63471000 \\ \mathrm{C} & 3.86349700 & 0.26031700 & -0.01188100 \\ \mathrm{C} & 4.59627900 & 1.60052500 & -0.06842700 \\ \mathrm{C} & 4.48453300 & -0.78698200 & -0.94124000 \\ \mathrm{H} & -2.23440100 & 0.51166700 & -1.47238000 \\ \mathrm{H} & -1.01171900 & -0.66229500 & 1.06632900 \\ \mathrm{H} & -0.56567200 & -2.57398800 & -0.41606400 \\ \mathrm{H} & -1.38858800 & -1.75626200 & -1.75012800 \\ \mathrm{H} & -2.64166400 & -2.59417400 & 0.92505900 \\ \mathrm{H} & -2.95697500 & -3.35109400 & -0.62962100 \\ \mathrm{H} & -4.78757500 & -1.74334600 & 0.02440300 \\ \mathrm{H} & -4.00734300 & -1.28154200 & -1.48268100 \\ \mathrm{H} & -4.29340400 & 0.71636400 & -0.05479900 \\ \mathrm{H} & -3.44307600 & -0.06858600 & 1.27351300 \\ \mathrm{H} & 0.11610000 & 1.02758100 & -0.70044700 \\ \mathrm{H} & 2.27342700 & 1.05314800 & -1.14719800 \\ \mathrm{H} & -2.55656300 & 2.05962400 & 1.97829400 \\ \mathrm{H} & -0.92591000 & 1.35494000 & 1.86401400 \\ \mathrm{H} & -1.16173000 & 3.08333800 & 1.58478200 \\ \mathrm{H} & -2.61558900 & 2.71557800 & -1.71318600 \\ \mathrm{H} & -3.59489500 & 2.94284600 & -0.24580900 \\ \mathrm{H} & -2.10093700 & 3.87200200 & -0.47175400 \\ \mathrm{H} & 3.87915400 & -0.12563800 & 1.01016000 \\ \mathrm{H} & 4.14966200 & 2.32193600 & 0.62141400 \\ \mathrm{H} & 5.64824900 & 1.46865900 & 0.19927500 \\ \mathrm{H} & 4.56614100 & 2.02616500 & -1.07956300 \\ \mathrm{H} & 5.52826600 & -0.97350300 & -0.66852300 \\ \mathrm{H} & 4.46143700 & -0.44610800 & -1.98305700 \\ \mathrm{H} & 3.93359500 & -1.72695400 & -0.86480100\end{array}$


<smiles>CN(C)C1CCCC[C@H]1NC(=S)NCc1ccccc1</smiles>

Thiourea-15

$\begin{array}{lrrr}\mathrm{C} & -3.06289200 & 0.29351900 & -0.65100300 \\ \mathrm{C} & -1.95414200 & -0.46993000 & 0.09872100 \\ \mathrm{C} & -1.90701200 & -1.92323500 & -0.38843400 \\ \mathrm{C} & -3.25593900 & -2.60970700 & -0.14162200 \\ \mathrm{C} & -4.41147300 & -1.83818100 & -0.79295200 \\ \mathrm{C} & -4.42217100 & -0.36502200 & -0.35788300 \\ \mathrm{~N} & -0.70223200 & 0.24762000 & -0.11170000 \\ \mathrm{C} & 0.36409200 & 0.16237400 & 0.73288200 \\ \mathrm{~N} & 1.34893100 & 1.06006600 & 0.43435900 \\ \mathrm{~S} & 0.45100900 & -0.92418900 & 2.02227000 \\ \mathrm{~N} & -2.95592400 & 1.74146100 & -0.40634200 \\ \mathrm{C} & -3.71572500 & 2.54044000 & -1.35770900 \\ \mathrm{C} & -3.21933600 & 2.15154100 & 0.97025000 \\ \mathrm{C} & 2.65800700 & 1.10665400 & 1.07422200 \\ \mathrm{C} & 3.74247800 & 0.47232800 & 0.23031200 \\ \mathrm{C} & 3.65047300 & -0.87856900 & -0.13363300 \\ \mathrm{C} & 4.64224900 & -1.46385600 & -0.91654500 \\ \mathrm{C} & 5.73843800 & -0.71040300 & -1.34541500 \\ \mathrm{C} & 5.83677600 & 0.63267600 & -0.98643200 \\ \mathrm{C} & 4.84008500 & 1.21961600 & -0.20409100 \\ \mathrm{H} & -2.86158900 & 0.17227800 & -1.72554200 \\ \mathrm{H} & -2.17428000 & -0.48747100 & 1.17432800 \\ \mathrm{H} & -1.10352000 & -2.44550100 & 0.13516000 \\ \mathrm{H} & -1.66520900 & -1.92905300 & -1.45946900 \\ \mathrm{H} & -3.42858100 & -2.67596600 & 0.94097600 \\ \mathrm{H} & -3.22836400 & -3.63884700 & -0.51570500 \\ \mathrm{H} & -5.37136100 & -2.30839600 & -0.55175400 \\ \mathrm{H} & -4.30604400 & -1.88538700 & -1.88570400 \\ \mathrm{H} & -5.22161200 & 0.17670600 & -0.87514700 \\ \mathrm{H} & -4.63648900 & -0.30170200 & 0.71619300 \\ \mathrm{H} & -0.87967700 & 1.16683100 & -0.51667500 \\ \mathrm{H} & 1.30045000 & 1.48072900 & -0.48492900 \\ \mathrm{H} & -3.45003800 & 3.59575500 & -1.23925700 \\ \mathrm{H} & -3.46373100 & 2.23742900 & -2.37846600 \\ \mathrm{H} & -4.80963800 & 2.45652600 & -1.23620500 \\ \mathrm{H} & -2.97491900 & 3.21273000 & 1.07676100 \\ \mathrm{H} & -4.27002700 & 2.01625100 & 1.27615400 \\ \mathrm{H} & -2.58466200 & 1.59195100 & 1.66085400 \\ \mathrm{H} & 2.54871400 & 0.58461500 & 2.02786800 \\ \mathrm{H} & 2.90773800 & 2.15241600 & 1.28369000 \\ \mathrm{H} & 2.80193600 & -1.46214200 & 0.21044100 \\ \mathrm{H} & 4.56399500 & -2.51197100 & -1.18978200 \\ \mathrm{H} & 6.51072300 & -1.16983300 & -1.95494200\end{array}$


$\begin{array}{llll}\mathrm{H} & 6.68473400 & 1.22538000 & -1.31649600 \\ \mathrm{H} & 4.91643900 & 2.26819000 & 0.07310000\end{array}$<smiles>CN(C)C1CCCC[C@H]1NC(=S)NC1CCCCC1</smiles>

Thiourea-16

$\begin{array}{lrrr}\mathrm{C} & -3.04397100 & 0.51959000 & -0.41435700 \\ \mathrm{C} & -1.97098900 & -0.51001200 & -0.01142300 \\ \mathrm{C} & -2.30989000 & -1.87656800 & -0.61898200 \\ \mathrm{C} & -3.68262700 & -2.35070000 & -0.12687500 \\ \mathrm{C} & -4.77813600 & -1.31847900 & -0.42537000 \\ \mathrm{C} & -4.41197000 & 0.06782100 & 0.12578100 \\ \mathrm{~N} & -0.67645500 & 0.00490700 & -0.44187200 \\ \mathrm{C} & 0.50263800 & -0.35397200 & 0.14763700 \\ \mathrm{~N} & 1.55844400 & 0.39999800 & -0.26871400 \\ \mathrm{~S} & 0.64042700 & -1.58770300 & 1.29139000 \\ \mathrm{~N} & -2.61085700 & 1.88497500 & -0.07291900 \\ \mathrm{C} & -3.38975900 & 2.91703400 & -0.74231300 \\ \mathrm{C} & -2.48588600 & 2.14928700 & 1.35775400 \\ \mathrm{C} & 2.95125200 & 0.16021100 & 0.09332000 \\ \mathrm{C} & 3.59844100 & -0.91200800 & -0.79596200 \\ \mathrm{C} & 5.06932200 & -1.12715900 & -0.41878600 \\ \mathrm{C} & 5.85794900 & 0.18763600 & -0.47319700 \\ \mathrm{C} & 5.20571100 & 1.26221900 & 0.40670300 \\ \mathrm{C} & 3.73225900 & 1.47754500 & 0.03594500 \\ \mathrm{H} & -3.10139200 & 0.50726100 & -1.51281200 \\ \mathrm{H} & -1.95892600 & -0.62784800 & 1.08033500 \\ \mathrm{H} & -1.52967300 & -2.58850800 & -0.34140300 \\ \mathrm{H} & -2.30568000 & -1.78788500 & -1.71344900 \\ \mathrm{H} & -3.63200100 & -2.52287500 & 0.95664700 \\ \mathrm{H} & -3.93451300 & -3.31403900 & -0.58348800 \\ \mathrm{H} & -5.73815200 & -1.64617900 & -0.01092400 \\ \mathrm{H} & -4.91642400 & -1.24358200 & -1.51294600 \\ \mathrm{H} & -5.18287900 & 0.79792200 & -0.14416400 \\ \mathrm{H} & -4.37866400 & 0.02883700 & 1.22164900 \\ \mathrm{H} & -0.75516900 & 0.98655100 & -0.70749400 \\ \mathrm{H} & 1.41172000 & 0.95733600 & -1.10122300 \\ \mathrm{H} & -4.42684500 & 3.00222000 & -0.37375500 \\ \mathrm{H} & -2.90657600 & 3.88851800 & -0.59667200 \\ \mathrm{H} & -3.42776600 & 2.71226900 & -1.81655600 \\ \mathrm{H} & -2.02550800 & 3.13209300 & 1.49742400 \\ \mathrm{H} & -3.45108000 & 2.15177300 & 1.89110600 \\ \mathrm{H} & -1.83575000 & 1.40664400 & 1.82538900 \\ \mathrm{H} & 2.93676000 & -0.20796100 & 1.12331200 \\ \mathrm{H} & 3.02801000 & -1.84014900 & -0.69955500 \\ \mathrm{H} & 3.52934900 & -0.58912200 & -1.84508600\end{array}$




$\begin{array}{lrrr}\mathrm{H} & 5.52236200 & -1.87358100 & -1.08067100 \\ \mathrm{H} & 5.12068000 & -1.53897500 & 0.59820700 \\ \mathrm{H} & 5.89308600 & 0.54503300 & -1.51206400 \\ \mathrm{H} & 6.89619000 & 0.02444900 & -0.16259400 \\ \mathrm{H} & 5.75279200 & 2.20828400 & 0.32713400 \\ \mathrm{H} & 5.26639700 & 0.95241000 & 1.45871000 \\ \mathrm{H} & 3.67372700 & 1.88759600 & -0.98426700 \\ \mathrm{H} & 3.26510700 & 2.21093500 & 0.70224800\end{array}$<smiles>CN(C)C1CCCC[C@H]1NC(=S)Nc1ccccc1</smiles>

Thiourea-17

$\begin{array}{lrrr}\mathrm{C} & -2.84453600 & 0.59907500 & -0.29742900 \\ \mathrm{C} & -1.78999800 & -0.50703100 & -0.09994300 \\ \mathrm{C} & -2.20702700 & -1.76485000 & -0.87152500 \\ \mathrm{C} & -3.56962000 & -2.26301200 & -0.37425300 \\ \mathrm{C} & -4.64122800 & -1.16834500 & -0.46528100 \\ \mathrm{C} & -4.19813900 & 0.11639700 & 0.25110500 \\ \mathrm{~N} & -0.50341400 & 0.03068300 & -0.52346100 \\ \mathrm{C} & 0.69343600 & -0.44974700 & -0.06974200 \\ \mathrm{~N} & 1.73814800 & 0.31834900 & -0.53129800 \\ \mathrm{~S} & 0.83923100 & -1.81338300 & 0.89765100 \\ \mathrm{~N} & -2.34244900 & 1.89001400 & 0.20228000 \\ \mathrm{C} & -3.10672100 & 3.02961100 & -0.28620600 \\ \mathrm{C} & -2.14892900 & 1.95395400 & 1.64862000 \\ \mathrm{C} & 3.12574800 & 0.23231300 & -0.29350200 \\ \mathrm{C} & 3.97555000 & 0.66843900 & -1.31989500 \\ \mathrm{C} & 5.35571800 & 0.67059300 & -1.14132000 \\ \mathrm{C} & 5.90753600 & 0.22956100 & 0.06142900 \\ \mathrm{C} & 5.05973800 & -0.20005800 & 1.08270800 \\ \mathrm{C} & 3.67581600 & -0.19551900 & 0.92123600 \\ \mathrm{H} & -2.95851300 & 0.74024600 & -1.38223800 \\ \mathrm{H} & -1.72492400 & -0.77707900 & 0.96274900 \\ \mathrm{H} & -1.44113700 & -2.53202500 & -0.73952200 \\ \mathrm{H} & -2.25517900 & -1.52376200 & -1.94160400 \\ \mathrm{H} & -3.47002600 & -2.58725600 & 0.67032200 \\ \mathrm{H} & -3.87885300 & -3.14493300 & -0.94549600 \\ \mathrm{H} & -5.58944700 & -1.52388800 & -0.04690700 \\ \mathrm{H} & -4.83286000 & -0.93807100 & -1.52248400 \\ \mathrm{H} & -4.95470400 & 0.89901300 & 0.12761800 \\ \mathrm{H} & -4.11043600 & -0.07631900 & 1.32751600 \\ \mathrm{H} & -0.56376600 & 1.04376300 & -0.63357200 \\ \mathrm{H} & 1.49667400 & 0.91770600 & -1.30977500 \\ \mathrm{H} & -4.12389100 & 3.09700200 & 0.13689500 \\ \mathrm{H} & -2.58062900 & 3.95521100 & -0.03198100 \\ \mathrm{H} & -3.19528500 & 2.97514800 & -1.37542000 \\ \mathrm{H} & -1.64163000 & 2.89092300 & 1.89715500\end{array}$




$\begin{array}{rrrr}\mathrm{H} & -3.09130400 & 1.91959600 & 2.21987800 \\ \mathrm{H} & -1.51328000 & 1.13116300 & 1.98318700 \\ \mathrm{H} & 3.54862500 & 0.99939300 & -2.26364400 \\ \mathrm{H} & 5.99806200 & 1.01060200 & -1.94779200 \\ \mathrm{H} & 6.98366300 & 0.22274900 & 0.20129400 \\ \mathrm{H} & 5.47621800 & -0.53879600 & 2.02643900 \\ \mathrm{H} & 3.02484700 & -0.52788600 & 1.71631800\end{array}$<smiles>CN(C)C1CCCC[C@H]1NC(=S)NC(C)(C)C</smiles>

Thiourea-18

$\begin{array}{lrrr}\mathrm{C} & -2.38095700 & 0.53042900 & -0.34950100 \\ \mathrm{C} & -1.29642000 & -0.52728400 & -0.06859900 \\ \mathrm{C} & -1.67997000 & -1.85327700 & -0.73634700 \\ \mathrm{C} & -3.02759300 & -2.34857900 & -0.19788400 \\ \mathrm{C} & -4.12876400 & -1.29438900 & -0.37341500 \\ \mathrm{C} & -3.72096800 & 0.05456100 & 0.23799900 \\ \mathrm{~N} & -0.02360200 & 0.00594100 & -0.53787000 \\ \mathrm{C} & 1.18658900 & -0.38378600 & -0.02519800 \\ \mathrm{~N} & 2.20987200 & 0.37926700 & -0.49671200 \\ \mathrm{~S} & 1.34170500 & -1.66386700 & 1.06536100 \\ \mathrm{~N} & -1.91713300 & 1.87027600 & 0.04859700 \\ \mathrm{C} & -1.70733400 & 2.04572200 & 1.48308600 \\ \mathrm{C} & -2.72460200 & 2.94522500 & -0.51038000 \\ \mathrm{C} & 3.66514400 & 0.29730100 & -0.23312500 \\ \mathrm{C} & 3.96078400 & 0.53503800 & 1.25508800 \\ \mathrm{C} & 4.28562700 & 1.43475600 & -1.05868700 \\ \mathrm{C} & 4.23520300 & -1.04924600 & -0.70596600 \\ \mathrm{H} & -2.49934900 & 0.58491600 & -1.44180700 \\ \mathrm{H} & -1.22592400 & -0.71063200 & 1.01182900 \\ \mathrm{H} & -0.89191000 & -2.58472900 & -0.54482900 \\ \mathrm{H} & -1.73490600 & -1.69994200 & -1.82226900 \\ \mathrm{H} & -2.91882000 & -2.58522200 & 0.86907400 \\ \mathrm{H} & -3.31282700 & -3.28157500 & -0.69603400 \\ \mathrm{H} & -5.06741600 & -1.64106900 & 0.07327700 \\ \mathrm{H} & -4.32587800 & -1.15382600 & -1.44530400 \\ \mathrm{H} & -4.49942900 & 0.80340200 & 0.05496400 \\ \mathrm{H} & -3.62785600 & -0.05010100 & 1.32608400 \\ \mathrm{H} & -0.11329100 & 1.00210700 & -0.73788700 \\ \mathrm{H} & 1.96260000 & 0.98325000 & -1.26938100 \\ \mathrm{H} & -1.23358100 & 3.01695800 & 1.65535400 \\ \mathrm{H} & -2.63981700 & 2.01810900 & 2.07105200 \\ \mathrm{H} & -1.03574300 & 1.27395100 & 1.86559000 \\ \mathrm{H} & -2.22544500 & 3.90359800 & -0.33473500 \\ \mathrm{H} & -2.82671600 & 2.80638700 & -1.59096800 \\ \mathrm{H} & -3.73773100 & 3.01303400 & -0.07733100\end{array}$




$\begin{array}{rrrr}\mathrm{H} & 3.52191800 & -0.25183600 & 1.86806800 \\ \mathrm{H} & 5.04349400 & 0.54881200 & 1.41578300 \\ \mathrm{H} & 3.55086600 & 1.49805400 & 1.57477700 \\ \mathrm{H} & 5.36890900 & 1.44961300 & -0.91800600 \\ \mathrm{H} & 4.08928300 & 1.29921400 & -2.12874300 \\ \mathrm{H} & 3.88858000 & 2.40745000 & -0.74908300 \\ \mathrm{H} & 5.31868200 & -1.06863200 & -0.55026000 \\ \mathrm{H} & 3.78300300 & -1.87260800 & -0.15264800 \\ \mathrm{H} & 4.03763300 & -1.19403700 & -1.77288200\end{array}$<smiles>CCC(CC)(CC)NC(=S)N[C@H]1CCCCC1N(C)C</smiles>

Thiourea-19

$\begin{array}{lrrr}\mathrm{C} & -2.94881000 & 0.52539500 & -0.52393200 \\ \mathrm{C} & -1.96912600 & -0.55255300 & -0.02307100 \\ \mathrm{C} & -2.45808700 & -1.94178900 & -0.44721900 \\ \mathrm{C} & -3.85238800 & -2.21143500 & 0.13174100 \\ \mathrm{C} & -4.85146600 & -1.11613000 & -0.26519800 \\ \mathrm{C} & -4.33315800 & 0.28288100 & 0.10190100 \\ \mathrm{~N} & -0.64528900 & -0.22447100 & -0.53726300 \\ \mathrm{C} & 0.51286000 & -0.57837800 & 0.10678000 \\ \mathrm{~N} & 1.60664300 & -0.00675100 & -0.46259800 \\ \mathrm{~S} & 0.52806000 & -1.61260200 & 1.44157300 \\ \mathrm{~N} & -2.36776900 & 1.86902800 & -0.36187500 \\ \mathrm{C} & -2.13640000 & 2.27721700 & 1.02114300 \\ \mathrm{C} & -3.07711200 & 2.89465700 & -1.11320900 \\ \mathrm{C} & 3.02392000 & 0.01928900 & -0.03255500 \\ \mathrm{C} & 3.14641300 & 0.61691900 & 1.38850100 \\ \mathrm{C} & 3.62646800 & -1.40486600 & -0.03446200 \\ \mathrm{C} & 3.70749500 & 0.92496300 & -1.08671700 \\ \mathrm{C} & 3.53891300 & -2.14650300 & -1.36769600 \\ \mathrm{C} & 5.20871500 & 1.14064000 & -0.90251200 \\ \mathrm{C} & 2.53870700 & 2.00818300 & 1.56738000 \\ \mathrm{H} & -3.04917100 & 0.38979100 & -1.61112200 \\ \mathrm{H} & -1.93244500 & -0.53854600 & 1.07415300 \\ \mathrm{H} & -1.74084900 & -2.68839800 & -0.09868700 \\ \mathrm{H} & -2.48264000 & -1.99071100 & -1.54389100 \\ \mathrm{H} & -3.77975600 & -2.25622100 & 1.22668500 \\ \mathrm{H} & -4.21450100 & -3.19177100 & -0.19658500 \\ \mathrm{H} & -5.82338400 & -1.29304000 & 0.20896100 \\ \mathrm{H} & -5.02080700 & -1.15862900 & -1.35014400 \\ \mathrm{H} & -5.03964700 & 1.04837300 & -0.23735400 \\ \mathrm{H} & -4.26330900 & 0.37329600 & 1.19303200 \\ \mathrm{H} & -0.64894400 & 0.71519600 & -0.93374500 \\ \mathrm{H} & 1.42260400 & 0.48493000 & -1.32671900 \\ \mathrm{H} & -1.52994300 & 1.53224400 & 1.54125500 \\ \mathrm{H} & -1.58168400 & 3.22058500 & 1.02310300\end{array}$




$\begin{array}{rrrr}\mathrm{H} & -3.06430400 & 2.43267300 & 1.59637700 \\ \mathrm{H} & -2.49261700 & 3.82030200 & -1.10564700 \\ \mathrm{H} & -3.19602400 & 2.57429500 & -2.15275700 \\ \mathrm{H} & -4.07811200 & 3.12939200 & -0.71151100 \\ \mathrm{H} & 4.20930600 & 0.63532100 & 1.65320700 \\ \mathrm{H} & 2.66404100 & -0.07758700 & 2.07930200 \\ \mathrm{H} & 3.10366100 & -1.98087200 & 0.73161800 \\ \mathrm{H} & 4.67157800 & -1.32720200 & 0.28578500 \\ \mathrm{H} & 3.20175000 & 1.89798700 & -1.08306400 \\ \mathrm{H} & 3.52864800 & 0.49115200 & -2.07823300 \\ \mathrm{H} & 4.13341700 & -1.66928500 & -2.15407400 \\ \mathrm{H} & 3.90677300 & -3.17076300 & -1.25695500 \\ \mathrm{H} & 2.50228200 & -2.20351200 & -1.71310900 \\ \mathrm{H} & 5.76288000 & 0.19918300 & -0.94919900 \\ \mathrm{H} & 5.59527700 & 1.79037500 & -1.69329800 \\ \mathrm{H} & 5.43610600 & 1.61681400 & 0.05529300 \\ \mathrm{H} & 3.05540600 & 2.77081100 & 0.97524300 \\ \mathrm{H} & 1.48327100 & 2.01485100 & 1.27790500 \\ \mathrm{H} & 2.59541700 & 2.31501700 & 2.61591300\end{array}$<smiles>CN(C)C1CCCC[C@H]1NC(=S)NC(c1ccccc1)c1ccccc1</smiles>

Thiourea-20

$\begin{array}{lccc}\mathrm{C} & 3.61247600 & -0.12210300 & 0.90539900 \\ \mathrm{C} & 2.81452600 & -0.66487700 & -0.29475100 \\ \mathrm{C} & 3.49660100 & -1.91183200 & -0.86601100 \\ \mathrm{C} & 4.92473700 & -1.57452900 & -1.31224900 \\ \mathrm{C} & 5.74132300 & -0.95162700 & -0.17160900 \\ \mathrm{C} & 5.02688300 & 0.26388700 & 0.43891400 \\ \mathrm{~N} & 1.44637100 & -0.90550800 & 0.14982300 \\ \mathrm{C} & 0.37203900 & -0.76598300 & -0.68003300 \\ \mathrm{~N} & -0.82099900 & -0.79934700 & -0.01959700 \\ \mathrm{~S} & 0.48841400 & -0.57834000 & -2.34882200 \\ \mathrm{~N} & 2.83699100 & 0.90776700 & 1.61878100 \\ \mathrm{C} & 2.56534700 & 2.11855300 & 0.84763200 \\ \mathrm{C} & 3.37627000 & 1.21957100 & 2.93496100 \\ \mathrm{C} & -2.08875000 & -0.36607800 & -0.59205800 \\ \mathrm{C} & -2.24335100 & 1.15061500 & -0.60871200 \\ \mathrm{C} & -3.21025000 & -1.08152200 & 0.14949800 \\ \mathrm{C} & -3.29162300 & 1.71494400 & -1.34420000 \\ \mathrm{C} & -3.47672600 & 3.09450100 & -1.37363000 \\ \mathrm{C} & -2.61292300 & 3.93275400 & -0.66460600 \\ \mathrm{C} & -1.56612900 & 3.37797400 & 0.06887600 \\ \mathrm{C} & -1.38336400 & 1.99410700 & 0.09602400 \\ \mathrm{C} & -3.51329100 & -2.40529900 & -0.19131500 \\ \mathrm{C} & -4.49800800 & -3.10723100 & 0.49977500 \\ \mathrm{C} & -5.18938800 & -2.49338700 & 1.54680100\end{array}$




$\begin{array}{lrrr}\mathrm{C} & -4.88947600 & -1.17717200 & 1.89532700 \\ \mathrm{C} & -3.90471700 & -0.47377800 & 1.19941200 \\ \mathrm{H} & 3.71648300 & -0.94785400 & 1.62517600 \\ \mathrm{H} & 2.78821100 & 0.08866700 & -1.09238600 \\ \mathrm{H} & 2.90281700 & -2.28242100 & -1.70518800 \\ \mathrm{H} & 3.51037200 & -2.69555300 & -0.09716600 \\ \mathrm{H} & 4.87715900 & -0.86774400 & -2.15147700 \\ \mathrm{H} & 5.42465800 & -2.47311100 & -1.68967300 \\ \mathrm{H} & 6.73685300 & -0.66260800 & -0.52657100 \\ \mathrm{H} & 5.89610400 & -1.70429200 & 0.61394300 \\ \mathrm{H} & 5.60657200 & 0.65944400 & 1.28007600 \\ \mathrm{H} & 4.96090400 & 1.06412900 & -0.30861500 \\ \mathrm{H} & 1.31240900 & -0.54070300 & 1.09201800 \\ \mathrm{H} & -0.80975200 & -0.95644300 & 0.97936600 \\ \mathrm{H} & 1.88526400 & 2.75634600 & 1.42110400 \\ \mathrm{H} & 3.46829500 & 2.71005900 & 0.62281300 \\ \mathrm{H} & 2.07177400 & 1.87009800 & -0.09454200 \\ \mathrm{H} & 2.65943100 & 1.83870700 & 3.48383100 \\ \mathrm{H} & 3.53031200 & 0.29460100 & 3.49897200 \\ \mathrm{H} & 4.33496000 & 1.76579000 & 2.90608800 \\ \mathrm{H} & -2.07794500 & -0.70700900 & -1.63182500 \\ \mathrm{H} & -3.96551100 & 1.06420600 & -1.89470300 \\ \mathrm{H} & -4.29202400 & 3.51743000 & -1.95282300 \\ \mathrm{H} & -2.75392300 & 5.00891900 & -0.68934000 \\ \mathrm{H} & -0.88481500 & 4.02099300 & 0.61847300 \\ \mathrm{H} & -0.55876100 & 1.57042700 & 0.65858300 \\ \mathrm{H} & -2.96828000 & -2.88121000 & -1.00172300 \\ \mathrm{H} & -4.72887000 & -4.13084100 & 0.22072900 \\ \mathrm{H} & -5.95946800 & -3.03816000 & 2.08442300 \\ \mathrm{H} & -5.42464500 & -0.69331600 & 2.70687300 \\ \mathrm{H} & -3.68297900 & 0.55558500 & 1.46100300\end{array}$<smiles>C=CC1CN2CCC1CC2[C@H](NC(=S)NC)c1ccnc2ccc(OC)cc12</smiles>

Thiourea-21

$\begin{array}{lrrr}\mathrm{C} & 1.51892800 & 0.20878200 & 0.50063200 \\ \mathrm{C} & 0.10349900 & 0.76673600 & 0.24118400 \\ \mathrm{~N} & 0.15867800 & 2.20388700 & 0.47287900 \\ \mathrm{C} & -0.76375000 & 3.04957500 & -0.08789200 \\ \mathrm{~N} & -0.48227900 & 4.36380200 & 0.12093400 \\ \mathrm{~S} & -2.10401100 & 2.52712100 & -0.95652800 \\ \mathrm{C} & -1.35427400 & 5.44266400 & -0.30877500 \\ \mathrm{~N} & 2.53305300 & 1.07064200 & -0.14989800 \\ \mathrm{C} & 3.86529200 & 0.61938500 & 0.27515000\end{array}$




$\begin{array}{lrrr}\mathrm{C} & 4.16807300 & -0.84781000 & -0.19093700 \\ \mathrm{C} & 2.88173300 & -1.37520800 & -0.88133400 \\ \mathrm{C} & 1.67466800 & -1.27591800 & 0.06646700 \\ \mathrm{C} & -0.89827900 & 0.06938500 & 1.14597500 \\ \mathrm{C} & -1.77520800 & -0.95183500 & 0.67647600 \\ \mathrm{C} & -2.57240400 & -1.63054000 & 1.65470100 \\ \mathrm{~N} & -2.55342500 & -1.32690200 & 2.98138100 \\ \mathrm{C} & -1.76040000 & -0.34548000 & 3.36609000 \\ \mathrm{C} & -0.91727300 & 0.37057900 & 2.49204800 \\ \mathrm{C} & -1.90369600 & -1.31698000 & -0.69144200 \\ \mathrm{C} & -2.76635400 & -2.32583000 & -1.06574300 \\ \mathrm{C} & -3.53130300 & -3.02318000 & -0.09202000 \\ \mathrm{C} & -3.43781100 & -2.67658800 & 1.22852600 \\ \mathrm{O} & -2.97096600 & -2.73286400 & -2.34974200 \\ \mathrm{C} & -2.27743000 & -2.03982800 & -3.37561700 \\ \mathrm{C} & 2.44045000 & 0.97905100 & -1.62077000 \\ \mathrm{C} & 2.59741900 & -0.49076200 & -2.10813300 \\ \mathrm{C} & 4.62946600 & -1.71355000 & 0.94440700 \\ \mathrm{C} & 5.78308200 & -2.37977200 & 0.97602900 \\ \mathrm{H} & 1.70891400 & 0.29813400 & 1.57604700 \\ \mathrm{H} & -0.17071600 & 0.59310100 & -0.80168500 \\ \mathrm{H} & 1.12494000 & 2.53094800 & 0.48507400 \\ \mathrm{H} & 0.18705300 & 4.57404400 & 0.84821500 \\ \mathrm{H} & -1.52753300 & 5.37731500 & -1.38456500 \\ \mathrm{H} & -2.32893000 & 5.40190900 & 0.19027800 \\ \mathrm{H} & -0.86281100 & 6.39020400 & -0.07727100 \\ \mathrm{H} & 3.91207100 & 0.69079500 & 1.36681700 \\ \mathrm{H} & 4.61039900 & 1.31242300 & -0.12724500 \\ \mathrm{H} & 4.96260900 & -0.82951500 & -0.94675700 \\ \mathrm{H} & 3.03511200 & -2.41414800 & -1.18726700 \\ \mathrm{H} & 0.77921000 & -1.63134000 & -0.45129700 \\ \mathrm{H} & 1.80073800 & -1.92129700 & 0.93947900 \\ \mathrm{H} & -1.76705100 & -0.10225300 & 4.42755200 \\ \mathrm{H} & -0.28526600 & 1.16409900 & 2.87729000 \\ \mathrm{H} & -1.35857200 & -0.76236500 & -1.43967800 \\ \mathrm{H} & -4.19270300 & -3.81284000 & -0.43150900 \\ \mathrm{H} & -4.02284900 & -3.17910300 & 1.99078000 \\ \mathrm{H} & -2.59422200 & -2.49355600 & -4.31517200 \\ \mathrm{H} & -2.52950400 & -0.97240100 & -3.37879200 \\ \mathrm{H} & -1.19005600 & -2.14929700 & -3.26786900 \\ \mathrm{H} & 3.22746800 & 1.61845600 & -2.03157500 \\ \mathrm{H} & 1.48739600 & 1.40878400 & -1.93923500 \\ \mathrm{H} & 1.68385600 & -0.83562100 & -2.60548100 \\ \mathrm{H} & 3.41165300 & -0.57644900 & -2.83557200 \\ & 3.95775500 & -1.78004300 & 1.80101800 \\ \mathrm{H} & 6.48609200 & -2.99179900 & 1.82605800 \\ \mathrm{H} & & -2.33646500 & 0.14754400\end{array}$


<smiles>C=CC1CN2CCC1CC2[C@H](NC(=S)NCC)c1ccnc2ccc(OC)cc12</smiles>

Thiourea-22

$\begin{array}{lrrr}\mathrm{C} & 1.48338900 & 0.61607400 & 0.46540900 \\ \mathrm{C} & -0.02973100 & 0.59878100 & 0.16064300 \\ \mathrm{~N} & -0.51331000 & 1.96444900 & 0.31187200 \\ \mathrm{C} & -1.67191300 & 2.38411900 & -0.28732500 \\ \mathrm{~N} & -1.88521100 & 3.72234600 & -0.15368400 \\ \mathrm{~S} & -2.70705600 & 1.34913000 & -1.11739400 \\ \mathrm{C} & -3.10057300 & 4.40501900 & -0.58280800 \\ \mathrm{~N} & 2.12862200 & 1.76028300 & -0.21921200 \\ \mathrm{C} & 3.51658600 & 1.85956800 & 0.25294100 \\ \mathrm{C} & 4.35637800 & 0.58497300 & -0.11034700 \\ \mathrm{C} & 3.38599300 & -0.41272300 & -0.79771400 \\ \mathrm{C} & 2.18815400 & -0.72454100 & 0.11507500 \\ \mathrm{C} & -0.73062300 & -0.37294200 & 1.09509900 \\ \mathrm{C} & -1.15194500 & -1.66965100 & 0.67883900 \\ \mathrm{C} & -1.67153200 & -2.54409400 & 1.68798200 \\ \mathrm{~N} & -1.80797000 & -2.18681100 & 2.99429100 \\ \mathrm{C} & -1.44701300 & -0.96259300 & 3.32742900 \\ \mathrm{C} & -0.90176800 & -0.03105500 & 2.42054000 \\ \mathrm{C} & -1.09367500 & -2.12662200 & -0.66607300 \\ \mathrm{C} & -1.50926300 & -3.40172700 & -0.98785000 \\ \mathrm{C} & -1.99182600 & -4.28173500 & 0.01827200 \\ \mathrm{C} & -2.07456900 & -3.85722700 & 1.31659800 \\ \mathrm{O} & -1.50811800 & -3.92196300 & -2.24704400 \\ \mathrm{C} & -1.08690600 & -3.07621800 & -3.30572800 \\ \mathrm{C} & 2.13050400 & 1.56828700 & -1.68318700 \\ \mathrm{C} & 2.84813800 & 0.24547200 & -2.08041700 \\ \mathrm{C} & 5.04832300 & 0.00748800 & 1.08940000 \\ \mathrm{C} & 6.36218900 & -0.19034700 & 1.19071800 \\ \mathrm{C} & -4.23606600 & 4.30126200 & 0.43539500 \\ \mathrm{H} & 1.59412200 & 0.81924600 & 1.53640400 \\ \mathrm{H} & -0.18979900 & 0.28501000 & -0.87309600 \\ \mathrm{H} & 0.26354000 & 2.62569900 & 0.31651600 \\ \mathrm{H} & -1.33740700 & 4.19022800 & 0.55683400 \\ \mathrm{H} & -3.40193500 & 3.95837600 & -1.53189000 \\ \mathrm{H} & -2.83531700 & 5.45139500 & -0.76798300 \\ \mathrm{H} & 3.49339900 & 2.00561700 & 1.33790800 \\ \mathrm{H} & 3.96734700 & 2.75527900 & -0.18512500 \\ \mathrm{H} & 5.12384900 & 0.85592900 & -0.84549000 \\ \mathrm{H} & 3.92492000 & -1.33363300 & -1.03857400 \\ \mathrm{H} & 1.50654300 & -1.40440700 & -0.40405300\end{array}$




$\begin{array}{rrrr}\mathrm{H} & 2.50472000 & -1.24240100 & 1.02399400 \\ \mathrm{H} & -1.57647300 & -0.68448100 & 4.37232500 \\ \mathrm{H} & -0.62088200 & 0.95929200 & 2.76380500 \\ \mathrm{H} & -0.76906000 & -1.44887900 & -1.44066300 \\ \mathrm{H} & -2.30293800 & -5.27701000 & -0.27986900 \\ \mathrm{H} & -2.45560100 & -4.50081000 & 2.10173900 \\ \mathrm{H} & -1.18198600 & -3.66415000 & -4.21911800 \\ \mathrm{H} & -1.71601800 & -2.18064800 & -3.37737600 \\ \mathrm{H} & -0.04047700 & -2.76795200 & -3.17949600 \\ \mathrm{H} & 2.63147600 & 2.43732300 & -2.11995300 \\ \mathrm{H} & 1.09762000 & 1.58833100 & -2.03977600 \\ \mathrm{H} & 2.15389600 & -0.43885100 & -2.58093300 \\ \mathrm{H} & 3.66807800 & 0.44032700 & -2.77987000 \\ \mathrm{H} & 4.40842400 & -0.25419200 & 1.93288100 \\ \mathrm{H} & 6.81089800 & -0.61130700 & 2.08503500 \\ \mathrm{H} & 7.03835300 & 0.06267200 & 0.37751600 \\ \mathrm{H} & -5.12121100 & 4.83474800 & 0.07439700 \\ \mathrm{H} & -4.50212500 & 3.25314000 & 0.59031900 \\ \mathrm{H} & -3.94654100 & 4.73647800 & 1.39802300\end{array}$<smiles>C=CC1CN2CCC1CC2[C@H](NC(=S)NC(C)C)c1ccnc2ccc(OC)cc12</smiles>

Thiourea-23

$\begin{array}{lrrr}\mathrm{C} & -1.09973300 & -1.19404300 & 0.52898700 \\ \mathrm{C} & 0.15570800 & -0.34867400 & 0.22609500 \\ \mathrm{~N} & 1.31242100 & -1.20901900 & 0.43512900 \\ \mathrm{C} & 2.52129100 & -0.93955400 & -0.15240900 \\ \mathrm{~N} & 3.43624400 & -1.92764000 & 0.03702800 \\ \mathrm{~S} & 2.82330800 & 0.46465200 & -1.03081600 \\ \mathrm{C} & 4.84078400 & -1.85768700 & -0.37163700 \\ \mathrm{~N} & -0.98322100 & -2.52447400 & -0.11237200 \\ \mathrm{C} & -2.10697800 & -3.35627400 & 0.33982900 \\ \mathrm{C} & -3.49097000 & -2.77822900 & -0.11978000 \\ \mathrm{C} & -3.20281200 & -1.42016000 & -0.81430400 \\ \mathrm{C} & -2.42227200 & -0.48386800 & 0.12356900 \\ \mathrm{C} & 0.17755500 & 0.88040700 & 1.11915600 \\ \mathrm{C} & -0.17997200 & 2.17764700 & 0.64844800 \\ \mathrm{C} & -0.25477300 & 3.22855300 & 1.61947600 \\ \mathrm{~N} & 0.02626400 & 3.05314800 & 2.93969400 \\ \mathrm{C} & 0.39563000 & 1.84665300 & 3.32436800 \\ \mathrm{C} & 0.47862000 & 0.73773800 & 2.45777800 \\ \mathrm{C} & -0.45074900 & 2.47770600 & -0.71452700 \\ \mathrm{C} & -0.80292000 & 3.75715900 & -1.09014200 \\ \mathrm{C} & -0.91124200 & 4.79219000 & -0.12240100\end{array}$




$\begin{array}{rrrr}\mathrm{C} & -0.63739700 & 4.53093500 & 1.19269500 \\ \mathrm{O} & -1.06274200 & 4.14445300 & -2.37028100 \\ \mathrm{C} & -0.92006300 & 3.16997600 & -3.39184400 \\ \mathrm{C} & -1.03204500 & -2.40872700 & -1.58370600 \\ \mathrm{C} & -2.32995300 & -1.68946200 & -2.05260400 \\ \mathrm{C} & -4.45999500 & -2.66879400 & 1.02044200 \\ \mathrm{C} & -5.65935100 & -3.24812700 & 1.06088500 \\ \mathrm{C} & 5.35867700 & -3.27815600 & -0.59493500 \\ \mathrm{C} & 5.67614900 & -1.08201800 & 0.65119700 \\ \mathrm{H} & -1.10433100 & -1.39045300 & 1.60692000 \\ \mathrm{H} & 0.13952400 & -0.03455500 & -0.81968400 \\ \mathrm{H} & 1.03382400 & -2.18951900 & 0.47353500 \\ \mathrm{H} & 3.22338500 & -2.60158800 & 0.76242100 \\ \mathrm{H} & 4.84678800 & -1.30965800 & -1.31700600 \\ \mathrm{H} & -2.06185300 & -3.41661300 & 1.43227000 \\ \mathrm{H} & -1.96600700 & -4.37020100 & -0.04684000 \\ \mathrm{H} & -3.92846300 & -3.44555200 & -0.87227600 \\ \mathrm{H} & -4.14859900 & -0.95541500 & -1.10761400 \\ \mathrm{H} & -2.22158800 & 0.45924000 & -0.39247000 \\ \mathrm{H} & -3.01006600 & -0.22988800 & 1.00938500 \\ \mathrm{H} & 0.63310900 & 1.72398700 & 4.38005200 \\ \mathrm{H} & 0.78563500 & -0.22932200 & 2.84251700 \\ \mathrm{H} & -0.32862000 & 1.70655900 & -1.45958000 \\ \mathrm{H} & -1.19613200 & 5.78184100 & -0.46252900 \\ \mathrm{H} & -0.69448500 & 5.30515600 & 1.94976400 \\ \mathrm{H} & -1.14442000 & 3.67918800 & -4.32958600 \\ \mathrm{H} & 0.10103700 & 2.77092600 & -3.42478300 \\ \mathrm{H} & -1.62374100 & 2.33842900 & -3.25271800 \\ \mathrm{H} & -0.97517700 & -3.42448800 & -1.98622500 \\ \mathrm{H} & -0.13584100 & -1.88427600 & -1.92456400 \\ \mathrm{H} & -2.09234200 & -0.74079900 & -2.54716500 \\ \mathrm{H} & -2.87604300 & -2.30221600 & -2.77769000 \\ \mathrm{H} & -4.13249600 & -2.07290400 & 1.8731100 \\ \mathrm{H} & -6.32092500 & -3.13671100 & 1.91426900 \\ \mathrm{H} & -6.02349400 & -3.85680400 & 0.23661500 \\ \mathrm{H} & 6.39768100 & -3.25279000 & -0.93449600 \\ \mathrm{H} & 5.33181300 & -3.85999400 & 0.33510500 \\ \mathrm{H} & 4.76035500 & -3.80196100 & -1.34558800 \\ \mathrm{H} & 6.71487900 & -0.99961300 & 0.31571900 \\ \mathrm{H} & 5.27230700 & -0.07517000 & 0.77766300 \\ \mathrm{H} & 5.67219200 & -1.59017300 & 1.62260200\end{array}$<smiles>C=CC1CN2CCC1CN2[C@H](NC(=S)NCc1ccccc1)c1ccnc2ccc(OC)cc12</smiles>

Thiourea-24 


$\begin{array}{lrrr}\mathrm{C} & -2.31623900 & -0.32963200 & -0.09896300 \\ \mathrm{C} & -1.23236200 & -0.36328000 & -1.20628900 \\ \mathrm{~N} & -0.24210100 & -1.33361900 & -0.77960900 \\ \mathrm{C} & 0.89873300 & -1.64655700 & -1.44486200 \\ \mathrm{~N} & 1.68793800 & -2.53208000 & -0.75599300 \\ \mathrm{~S} & 1.28242300 & -1.01694700 & -2.95517100 \\ \mathrm{C} & 3.12189800 & -2.68401400 & -1.01221000 \\ \mathrm{~N} & -2.61840000 & -1.69653600 & 0.38497300 \\ \mathrm{C} & -3.37496800 & -1.58186100 & 1.64067400 \\ \mathrm{C} & -4.68974500 & -0.74391100 & 1.46002900 \\ \mathrm{C} & -4.81241800 & -0.42174700 & -0.05399300 \\ \mathrm{C} & -3.60828200 & 0.40926000 & -0.53138700 \\ \mathrm{C} & -0.70278700 & 1.04461500 & -1.42885900 \\ \mathrm{C} & 0.16505700 & 1.72159500 & -0.51587700 \\ \mathrm{C} & 0.48896500 & 3.08552500 & -0.81627100 \\ \mathrm{~N} & 0.00993300 & 3.75559400 & -1.90042000 \\ \mathrm{C} & -0.78392600 & 3.09274100 & -2.71737200 \\ \mathrm{C} & -1.15937400 & 1.74788100 & -2.52325300 \\ \mathrm{C} & 0.72866900 & 1.12609100 & 0.64703100 \\ \mathrm{C} & 1.57710900 & 1.84590400 & 1.46338200 \\ \mathrm{C} & 1.89174300 & 3.19847900 & 1.16797900 \\ \mathrm{C} & 1.35901600 & 3.79531400 & 0.05717700 \\ \mathrm{O} & 2.18250300 & 1.35153100 & 2.57793800 \\ \mathrm{C} & 1.99617700 & -0.02436200 & 2.87384100 \\ \mathrm{C} & -3.42871500 & -2.43495700 & -0.60426100 \\ \mathrm{C} & -4.80861100 & -1.75114200 & -0.82830100 \\ \mathrm{C} & -4.70123200 & 0.48346200 & 2.32524300 \\ \mathrm{C} & -5.66339800 & 0.79390400 & 3.19310800 \\ \mathrm{H} & 3.93360500 & -1.76382900 & -0.12434100 \\ \mathrm{H} & 2.56157500 & 3.72688100 & 1.83773100 \\ \mathrm{H} & 1.58447000 & 4.82469700 & -0.19849100 \\ \mathrm{H} & 2.59340100 & -0.22494000 & 3.76315000 \\ \mathrm{C} & 0.94138900 & -0.24658600 & 3.08359400 \\ \mathrm{C} & & -2.27349700 & 0.94652100 \\ \mathrm{C} & 5.36408000 & -1.41656800 & 1.80766900 \\ \mathrm{H} & 5.31372800 & -0.03816800 & 1.60571900 \\ \mathrm{H} & 4.57673500 & 0.47964200 & 0.53766000 \\ \mathrm{H} & 3.89158900 & -0.37451600 & -0.32245000 \\ \mathrm{H} & -1.87442900 & 0.18705800 & 0.75902100 \\ \mathrm{H} & -1.68471200 & -0.68790500 & -2.15239500 \\ \mathrm{H} & -0.60247400 & -1.94853400 & -0.05024100 \\ \mathrm{H} & 1.44470000 & -2.63839700 & 0.22198400 \\ \mathrm{H} & 3.27234100 & -2.45943400 & -2.06988100 \\ \mathrm{H} & 3.39727400 & -3.72834400 & -0.83583200 \\ \mathrm{H} & -2.72482900 & -1.11820400 & 2.38971400 \\ \mathrm{H} & -3.60612800 & -2.59224400 & 1.99134400 \\ \mathrm{H} & -5.55228700 & -1.36181200 & 1.73641100 \\ \mathrm{H} & -3.74339800 & 0.12372800 & -0.23246900 \\ \mathrm{H} & -1.154115000 & 0.51301600 & -1.62091000 \\ \mathrm{H} & 0.51951000 & 0.63486200 & -3.58588900 \\ \mathrm{H} & & & \end{array}$




$\begin{array}{lrrr}\mathrm{H} & 2.35124700 & -0.65506400 & 2.05210500 \\ \mathrm{H} & -3.54517000 & -3.45872500 & -0.23660000 \\ \mathrm{H} & -2.85755200 & -2.49606900 & -1.53411200 \\ \mathrm{H} & -4.98079800 & -1.56440300 & -1.89346100 \\ \mathrm{H} & -5.62485000 & -2.39190100 & -0.47743000 \\ \mathrm{H} & -3.84589400 & 1.15243900 & 2.22477600 \\ \mathrm{H} & -5.62209400 & 1.69601100 & 3.79541100 \\ \mathrm{H} & -6.53101800 & 0.15307800 & 3.33147600 \\ \mathrm{H} & 4.71155400 & -3.34825400 & 1.10760000 \\ \mathrm{H} & 5.93575000 & -1.82665500 & 2.63495400 \\ \mathrm{H} & 5.84084400 & 0.63156100 & 2.27801400 \\ \mathrm{H} & 4.52290700 & 1.55202800 & 0.38151900 \\ \mathrm{H} & 3.31054100 & 0.02719300 & -1.14582300\end{array}$<smiles>C=CC1CN2CCC1CC2[C@H](NC(=S)NC1CCCCC1)c1ccnc2ccc(OC)cc12</smiles>

Thiourea-25

$\begin{array}{lrrr}\mathrm{C} & -1.23677600 & 1.60459200 & -1.28753700 \\ \mathrm{C} & -0.51504200 & 0.38002800 & -0.67540900 \\ \mathrm{~N} & 0.91370400 & 0.53852300 & -0.91836800 \\ \mathrm{C} & 1.84670300 & 0.00435400 & -0.07087600 \\ \mathrm{~N} & 3.11778700 & 0.38308600 & -0.36989800 \\ \mathrm{~S} & 1.44907700 & -1.00013000 & 1.22393000 \\ \mathrm{C} & 4.31730300 & -0.12272600 & 0.29081900 \\ \mathrm{~N} & -0.44085600 & 2.83538900 & -1.07108400 \\ \mathrm{C} & -1.07222300 & 3.94035100 & -1.81054500 \\ \mathrm{C} & -2.52375800 & 4.20194300 & -1.30538500 \\ \mathrm{C} & -2.82769100 & 3.19240000 & -0.18493500 \\ \mathrm{C} & -2.68500200 & 1.76713100 & -0.74293900 \\ \mathrm{C} & -1.05699300 & -0.90175600 & -1.28922100 \\ \mathrm{C} & -1.99786900 & -1.74282600 & -0.62595600 \\ \mathrm{C} & -2.50789900 & -2.86048700 & -1.36636800 \\ \mathrm{~N} & -2.12447700 & -3.16109400 & -2.63702400 \\ \mathrm{C} & -1.22542500 & -2.37647400 & -3.19913100 \\ \mathrm{C} & -0.67236800 & -1.24376000 & -2.56975600 \\ \mathrm{C} & -2.44010900 & -1.54038700 & 0.71102000 \\ \mathrm{C} & -3.36158700 & -2.39969000 & 1.27430200 \\ \mathrm{C} & -3.88691800 & -3.49133300 & 0.53150300 \\ \mathrm{C} & -3.46500900 & -3.71376900 & -0.75046700 \\ \mathrm{O} & -3.83991600 & -2.30215300 & 2.54597200 \\ \mathrm{C} & -3.33600900 & -1.25085200 & 3.35454900 \\ \mathrm{C} & -0.38536300 & 3.17941100 & 0.36176600 \\ \mathrm{C} & -1.80281200 & 3.40896300 & 0.96178100 \\ \mathrm{C} & 4.76645200 & -1.46936300 & -0.29551200\end{array}$




$\begin{array}{rrrr}\mathrm{C} & 6.04428000 & -1.96936800 & 0.38943500 \\ \mathrm{C} & 7.16638100 & -0.92648600 & 0.30750800 \\ \mathrm{C} & 6.71390000 & 0.42172300 & 0.88363200 \\ \mathrm{C} & 5.43282900 & 0.92426700 & 0.20437300 \\ \mathrm{C} & -2.14095500 & 2.59654300 & 2.18696500 \\ \mathrm{C} & -1.36948400 & 1.73507400 & 2.85243200 \\ \mathrm{H} & -1.26269800 & 1.46461000 & -2.37359200 \\ \mathrm{H} & -0.67836200 & 0.36415000 & 0.40245800 \\ \mathrm{H} & 1.12116600 & 1.46113400 & -1.30127000 \\ \mathrm{H} & 3.25990800 & 0.79429200 & -1.28426500 \\ \mathrm{H} & 4.04378300 & -0.27566000 & 1.33890900 \\ \mathrm{H} & -1.06372200 & 3.68198500 & -2.87398900 \\ \mathrm{H} & -0.44303300 & 4.82718600 & -1.68578500 \\ \mathrm{H} & -3.24757200 & 4.08296700 & -2.11850000 \\ \mathrm{H} & -2.62802700 & 5.22535800 & -0.92894000 \\ \mathrm{H} & -3.84261700 & 3.34499800 & 0.19720500 \\ \mathrm{H} & -2.89665900 & 1.03517600 & 0.03928100 \\ \mathrm{H} & -3.41658100 & 1.59942400 & -1.53902000 \\ \mathrm{H} & -0.91668100 & -2.63604400 & -4.21072500 \\ \mathrm{H} & 0.06542900 & -0.64187400 & -3.09012400 \\ \mathrm{H} & -2.02223800 & -0.73146600 & 1.29196900 \\ \mathrm{H} & -4.61100300 & -4.13674900 & 1.01673800 \\ \mathrm{H} & -3.83785800 & -4.54664100 & -1.33622800 \\ \mathrm{H} & -3.82750700 & -1.35111900 & 4.32303200 \\ \mathrm{H} & -2.24952000 & -1.33097300 & 3.48249200 \\ \mathrm{H} & -3.56627000 & -0.26926700 & 2.92353600 \\ \mathrm{H} & 0.22626000 & 4.08096100 & 0.45865000 \\ \mathrm{H} & 0.14967400 & 2.38283500 & 0.87921700 \\ \mathrm{H} & -1.89333200 & 4.46367000 & 1.26102000 \\ \mathrm{H} & 4.95025300 & -1.34130800 & -1.37220100 \\ \mathrm{H} & 3.95243300 & -2.19130700 & -0.18509500 \\ \mathrm{H} & 6.36597200 & -2.91510300 & -0.06028500 \\ \mathrm{H} & 5.82420000 & -2.18374700 & 1.44399800 \\ \mathrm{H} & 7.45649300 & -0.78995400 & -0.74381100 \\ \mathrm{H} & 8.05856600 & -1.28191500 & 0.83528600 \\ \mathrm{H} & 7.50807100 & 1.16963100 & 0.78156300 \\ \mathrm{H} & 6.52552000 & 0.30912600 & 1.95985200 \\ \mathrm{H} & 5.09303100 & 1.86234800 & 0.65684000 \\ \mathrm{H} & 5.64617200 & 1.13730400 & -0.85452800 \\ \mathrm{H} & -3.14817600 & 2.77836200 & 2.56633400 \\ \mathrm{H} & -1.73939600 & 1.24288900 & 3.74599400 \\ \mathrm{H} & -0.35602200 & 1.47767900 & 2.56165900\end{array}$<smiles>C=CC1CN2CCC1CN2[C@H](NC(=S)Nc1ccccc1)c1ccnc2ccc(OC)cc12</smiles>

Thiourea-26 


\begin{tabular}{|c|c|c|c|}
\hline $\mathrm{C}$ & -1.26972500 & -1.42707800 & 0.47229900 \\
\hline $\mathrm{C}$ & -0.28861100 & -0.25103400 & 0.27784900 \\
\hline $\mathrm{N}$ & 1.04491000 & -0.75948100 & 0.56621300 \\
\hline $\mathrm{C}$ & 2.17074200 & -0.12965500 & 0.09934300 \\
\hline $\mathrm{N}$ & 3.29996000 & -0.83592300 & 0.43223400 \\
\hline $\mathrm{S}$ & 2.11889400 & 1.32394100 & -0.73106500 \\
\hline $\mathrm{C}$ & 4.65933300 & -0.62041800 & 0.12318900 \\
\hline $\mathrm{N}$ & -0.72877500 & -2.65038700 & -0.16449600 \\
\hline $\mathrm{C}$ & -1.58342400 & -3.78513700 & 0.21246900 \\
\hline $\mathrm{C}$ & -3.05256400 & -3.61601000 & -0.31154700 \\
\hline $\mathrm{C}$ & -3.12758200 & -2.22837500 & -1.00349500 \\
\hline $\mathrm{C}$ & -2.70517000 & -1.11205700 & -0.03354100 \\
\hline $\mathrm{C}$ & -0.68139800 & 0.90408600 & 1.18285200 \\
\hline $\mathrm{C}$ & -1.36404600 & 2.05863900 & 0.69979700 \\
\hline $\mathrm{C}$ & -1.80239700 & 3.01392800 & 1.67356800 \\
\hline $\mathrm{N}$ & -1.57279200 & 2.88521800 & 3.00892900 \\
\hline $\mathrm{C}$ & -0.90041100 & 1.82297400 & 3.40800300 \\
\hline $\mathrm{C}$ & -0.44443600 & 0.81110100 & 2.53858900 \\
\hline $\mathrm{C}$ & -1.61847100 & 2.31052200 & -0.67608100 \\
\hline $\mathrm{C}$ & -2.29780900 & 3.44685600 & -1.06297400 \\
\hline $\mathrm{C}$ & -2.76210400 & 4.37801500 & -0.09505900 \\
\hline $\mathrm{C}$ & -2.51299300 & 4.16553200 & 1.23359800 \\
\hline $\mathrm{O}$ & -2.57387600 & 3.78216800 & -2.35414800 \\
\hline $\mathrm{C}$ & -2.09427900 & 2.91939400 & -3.37336900 \\
\hline $\mathrm{C}$ & -0.71981800 & -2.52220800 & -1.63561800 \\
\hline $\mathrm{C}$ & -2.14485800 & -2.22728100 & -2.18746200 \\
\hline $\mathrm{C}$ & -4.06286000 & -3.78691100 & 0.78482200 \\
\hline $\mathrm{C}$ & -5.05905200 & -4.67179900 & 0.77290300 \\
\hline $\mathrm{C}$ & 5.60114800 & -1.13525200 & 1.02572800 \\
\hline $\mathrm{C}$ & 6.96348300 & -1.01354500 & 0.77090000 \\
\hline $\mathrm{C}$ & 7.40573700 & -0.37054400 & -0.38535100 \\
\hline $\mathrm{C}$ & 6.46679600 & 0.13683800 & -1.28343300 \\
\hline $\mathrm{C}$ & 5.09934500 & 0.01294000 & -1.04558500 \\
\hline $\mathrm{H}$ & -1.29932000 & -1.64752400 & 1.54513200 \\
\hline $\mathrm{H}$ & -0.31568000 & 0.07987800 & -0.76293700 \\
\hline $\mathrm{H}$ & 1.05026400 & -1.78035900 & 0.55587500 \\
\hline $\mathrm{H}$ & 3.15393900 & -1.52953800 & 1.15424000 \\
\hline $\mathrm{H}$ & -1.57369000 & -3.86549700 & 1.30441900 \\
\hline $\mathrm{H}$ & -1.13585300 & -4.70146200 & -0.18406700 \\
\hline $\mathrm{H}$ & -3.25232100 & -4.37564800 & -1.07692700 \\
\hline $\mathrm{H}$ & -4.14868300 & -2.05438700 & -1.35492700 \\
\hline $\mathrm{H}$ & -2.73825500 & -0.15039300 & -0.55349600 \\
\hline $\mathrm{H}$ & -3.40004500 & -1.03087600 & 0.80610200 \\
\hline $\mathrm{H}$ & -0.70989100 & 1.74069600 & 4.47695200 \\
\hline $\mathrm{H}$ & 0.09908700 & -0.04005800 & 2.93561300 \\
\hline $\mathrm{H}$ & -1.23371000 & 1.62852600 & -1.41880500 \\
\hline $\mathrm{H}$ & -3.29573300 & 5.25503600 & -0.44473600 \\
\hline $\mathrm{H}$ & -2.84006200 & 4.86753400 & 1.99253500 \\
\hline $\mathrm{H}$ & -1.00162600 & 2.82925800 & -3.34035200 \\
\hline $\mathrm{H}$ & -2.53767000 & 1.91806000 & -3.29151600 \\
\hline $\mathrm{H}$ & -2.39543800 & 3.37098300 & -4.31903600 \\
\hline $\mathrm{H}$ & -0.32572200 & -3.46062900 & -2.03663800 \\
\hline $\mathrm{H}$ & -0.01056200 & -1.73869600 & -1.91422200 \\
\hline
\end{tabular}




$\begin{array}{lrrr}\mathrm{H} & -2.17433600 & -1.25309000 & -2.68835900 \\ \mathrm{H} & -2.44045200 & -2.97848500 & -2.92750900 \\ \mathrm{H} & -3.94788300 & -3.13284100 & 1.64991400 \\ \mathrm{H} & -5.76240000 & -4.75366900 & 1.59564800 \\ \mathrm{H} & -5.20681200 & -5.34847400 & -0.06540600 \\ \mathrm{H} & 5.25988500 & -1.62635200 & 1.93396900 \\ \mathrm{H} & 7.67771200 & -1.41660600 & 1.48214600 \\ \mathrm{H} & 8.46760800 & -0.26750700 & -0.58365900 \\ \mathrm{H} & 6.79753700 & 0.63436200 & -2.18998600 \\ \mathrm{H} & 4.37823800 & 0.41022400 & -1.74427300\end{array}$<smiles>C=CC1CN2CCC1CC2[C@H](NC(=S)NC(C)(C)C)c1ccnc2ccc(OC)cc12</smiles>

Thiourea-27

$\begin{array}{lrrr}\mathrm{C} & -1.04535100 & -1.34185400 & 0.54132600 \\ \mathrm{C} & 0.03246400 & -0.26673600 & 0.28556200 \\ \mathrm{~N} & 1.32370600 & -0.88396100 & 0.55349700 \\ \mathrm{C} & 2.48613000 & -0.38983800 & 0.00900200 \\ \mathrm{~N} & 3.55471300 & -1.17786300 & 0.29489300 \\ \mathrm{~S} & 2.51422500 & 1.03057100 & -0.89676900 \\ \mathrm{C} & 4.98099400 & -1.02225800 & -0.07614300 \\ \mathrm{~N} & -0.64135300 & -2.62374800 & -0.08227900 \\ \mathrm{C} & -1.59899100 & -3.66009900 & 0.32744900 \\ \mathrm{C} & -3.04774600 & -3.36490000 & -0.19682600 \\ \mathrm{C} & -3.00067400 & -1.97723600 & -0.89100100 \\ \mathrm{C} & -2.46176400 & -0.90528800 & 0.07127200 \\ \mathrm{C} & -0.22733900 & 0.94402400 & 1.16591900 \\ \mathrm{C} & -0.81062300 & 2.14526200 & 0.66670300 \\ \mathrm{C} & -1.13280900 & 3.16296000 & 1.62236400 \\ \mathrm{~N} & -0.88195600 & 3.04852700 & 2.95531800 \\ \mathrm{C} & -0.30122800 & 1.93842300 & 3.36825300 \\ \mathrm{C} & 0.03583400 & 0.86569700 & 2.51779300 \\ \mathrm{C} & -1.07431800 & 2.38371400 & -0.70978600 \\ \mathrm{C} & -1.65357400 & 3.56833700 & -1.11375600 \\ \mathrm{C} & -2.00520200 & 4.56399000 & -0.16268600 \\ \mathrm{C} & -1.74379400 & 4.36417600 & 1.16567300 \\ \mathrm{O} & -1.92751900 & 3.89433300 & -2.40805400 \\ \mathrm{C} & -1.55335100 & 2.96359900 & -3.41165300 \\ \mathrm{C} & -0.64564700 & -2.52003000 & -1.55535700 \\ \mathrm{C} & -2.03732700 & -2.07064700 & -2.08729800 \\ \mathrm{C} & -4.06871800 & -3.44652600 & 0.89956900 \\ \mathrm{C} & -5.13151200 & -4.25037100 & 0.89430100 \\ \mathrm{C} & 5.56548700 & 0.26471300 & 0.52674900 \\ \mathrm{C} & 5.14848600 & -1.04875200 & -1.60230300 \\ \mathrm{C} & 5.68827700 & -2.24278000 & 0.53183000 \\ & & & \end{array}$




$\begin{array}{lrrr}\mathrm{H} & -1.06010700 & -1.53528500 & 1.61970600 \\ \mathrm{H} & 0.00182100 & 0.03684800 & -0.76307000 \\ \mathrm{H} & 1.23634500 & -1.89979300 & 0.58416800 \\ \mathrm{H} & 3.38348800 & -1.87606000 & 1.00648200 \\ \mathrm{H} & -1.59139900 & -3.71088400 & 1.42126900 \\ \mathrm{H} & -1.24484900 & -4.62644400 & -0.04433800 \\ \mathrm{H} & -3.31251300 & -4.10570300 & -0.96108300 \\ \mathrm{H} & -4.00513000 & -1.70747900 & -1.22988500 \\ \mathrm{H} & -2.42656700 & 0.05835000 & -0.44458900 \\ \mathrm{H} & -3.12727200 & -0.77076200 & 0.92780900 \\ \mathrm{H} & -0.09169800 & 1.86656900 & 4.43449500 \\ \mathrm{H} & 0.50866200 & -0.02180200 & 2.92554100 \\ \mathrm{H} & -0.77008600 & 1.65013200 & -1.44048000 \\ \mathrm{H} & -2.46326300 & 5.47778900 & -0.52520300 \\ \mathrm{H} & -1.98494700 & 5.11363000 & 1.91133300 \\ \mathrm{H} & -1.83203200 & 3.41634600 & -4.36368400 \\ \mathrm{H} & -0.47355000 & 2.77164700 & -3.39738300 \\ \mathrm{H} & -2.08645200 & 2.01091300 & -3.29257600 \\ \mathrm{H} & -0.37217700 & -3.50481000 & -1.94585900 \\ \mathrm{H} & 0.14424700 & -1.82929300 & -1.86092800 \\ \mathrm{H} & -1.96882700 & -1.09419900 & -2.58006200 \\ \mathrm{H} & -2.41941000 & -2.77947800 & -2.82960400 \\ \mathrm{H} & -3.90255100 & -2.79669000 & 1.75951800 \\ \mathrm{H} & -5.83901500 & -4.27008000 & 1.71735300 \\ \mathrm{H} & -5.33234400 & -4.91989100 & 0.06128400 \\ \mathrm{H} & 5.45939700 & 0.25895400 & 1.61613900 \\ \mathrm{H} & 6.63056100 & 0.33703000 & 0.28446600 \\ \mathrm{H} & 5.05239300 & 1.14179400 & 0.13168800 \\ \mathrm{H} & 4.72731700 & -1.97046900 & -2.01543900 \\ \mathrm{H} & 4.64707600 & -0.19789300 & -2.06299600 \\ \mathrm{H} & 6.21304500 & -1.01140100 & -1.85376100 \\ \mathrm{H} & 5.28232500 & -3.17562900 & 0.12626400 \\ \mathrm{H} & 6.75557700 & -2.20792300 & 0.30150200 \\ \mathrm{H} & 5.58248800 & -2.25801000 & 1.62289800\end{array}$<smiles>CNc1c(N[C@@H]2CCCCC2N(C)C)c(=O)c1=O</smiles>

Squaramide-7

$\begin{array}{lrrr}\mathrm{C} & 1.38383800 & -0.19587400 & -0.11551300 \\ \mathrm{C} & 1.91432300 & -1.46931300 & 0.44871400 \\ \mathrm{C} & 3.31977400 & -0.83798800 & 0.36098700 \\ \mathrm{C} & 2.66337600 & 0.36703000 & -0.21156200 \\ \mathrm{O} & 1.42850600 & -2.52381300 & 0.81062000 \\ \mathrm{O} & 4.46489500 & -1.13485500 & 0.64336700 \\ \mathrm{~N} & 3.14561400 & 1.55297900 & -0.62271500 \\ \mathrm{~N} & 0.15205100 & 0.25460500 & -0.43266000 \\ \mathrm{C} & 4.57711400 & 1.82634900 & -0.68969600\end{array}$




$\begin{array}{lrrr}\mathrm{C} & -1.06605600 & -0.45337100 & -0.03130500 \\ \mathrm{C} & -2.24838600 & 0.50296200 & -0.27407500 \\ \mathrm{C} & -3.55649500 & -0.15535800 & 0.19484700 \\ \mathrm{C} & -3.78846000 & -1.48166800 & -0.54341500 \\ \mathrm{C} & -2.59199600 & -2.42742900 & -0.37983100 \\ \mathrm{C} & -1.27731400 & -1.76130200 & -0.80406900 \\ \mathrm{~N} & -1.95231100 & 1.84404100 & 0.26248000 \\ \mathrm{C} & -2.79762100 & 2.88527600 & -0.30757300 \\ \mathrm{C} & -1.91954200 & 1.92601300 & 1.72024500 \\ \mathrm{H} & 2.52213600 & 2.18659700 & -1.10249200 \\ \mathrm{H} & -0.00340100 & 1.26192700 & -0.43258300 \\ \mathrm{H} & 4.93611600 & 1.84165400 & -1.72575300 \\ \mathrm{H} & 4.80560500 & 2.78994500 & -0.22357000 \\ \mathrm{H} & 5.09996600 & 1.03489600 & -0.15147500 \\ \mathrm{H} & -1.01506800 & -0.70303100 & 1.03885500 \\ \mathrm{H} & -2.31350800 & 0.64028300 & -1.36273900 \\ \mathrm{H} & -4.39848500 & 0.52568200 & 0.02939000 \\ \mathrm{H} & -3.50669000 & -0.34390500 & 1.27450900 \\ \mathrm{H} & -3.94289000 & -1.27404900 & -1.61125400 \\ \mathrm{H} & -4.70681500 & -1.95721200 & -0.18163000 \\ \mathrm{H} & -2.74834100 & -3.34341200 & -0.95922100 \\ \mathrm{H} & -2.51275600 & -2.73504700 & 0.67144100 \\ \mathrm{H} & -1.29274800 & -1.53350300 & -1.87798200 \\ \mathrm{H} & -0.43491200 & -2.42855600 & -0.61036000 \\ \mathrm{H} & -2.76301400 & 2.83233900 & -1.39971500 \\ \mathrm{H} & -3.85393800 & 2.82036600 & 0.00563000 \\ \mathrm{H} & -2.42163300 & 3.86648800 & -0.00080700 \\ \mathrm{H} & -2.90823900 & 1.79758300 & 2.19062700 \\ \mathrm{H} & -1.24581500 & 1.16902800 & 2.12793100 \\ \mathrm{H} & -1.53558900 & 2.90792500 & 2.01303800\end{array}$<smiles>CCNc1c(N[C@@H]2CCCCC2N(C)C)c(=O)c1=O</smiles>

Squaramide-8

$\begin{array}{lrrr}\mathrm{C} & 1.07472000 & -0.30134900 & 0.09423000 \\ \mathrm{C} & 1.50492200 & -1.62985700 & 0.62061400 \\ \mathrm{C} & 2.94237700 & -1.06411100 & 0.66190600 \\ \mathrm{C} & 2.38198800 & 0.20000900 & 0.11849700 \\ \mathrm{O} & 0.94749300 & -2.67823200 & 0.88106200 \\ \mathrm{O} & 4.05196600 & -1.43762400 & 0.98971800 \\ \mathrm{~N} & 2.94717100 & 1.38763300 & -0.18505400 \\ \mathrm{~N} & -0.10647400 & 0.22552900 & -0.28619200 \\ \mathrm{C} & 4.40799000 & 1.52529800 & -0.22197100 \\ \mathrm{C} & -1.38347000 & -0.44439300 & -0.02853600 \\ \mathrm{C} & -2.49881200 & 0.57951500 & -0.30931300 \\ \mathrm{C} & -3.86873200 & -0.04038700 & 0.01229200 \\ \mathrm{C} & -4.09515700 & -1.31086400 & -0.82005200\end{array}$




$\begin{array}{lrrr}\mathrm{C} & -2.95832700 & -2.32055900 & -0.61728600 \\ \mathrm{C} & -1.58509900 & -1.69530300 & -0.89259400 \\ \mathrm{~N} & -2.19050100 & 1.87282700 & 0.32751800 \\ \mathrm{C} & -2.93855600 & 2.98384600 & -0.24655900 \\ \mathrm{C} & -2.27552800 & 1.87244700 & 1.78544200 \\ \mathrm{C} & 5.04042100 & 0.95215600 & -1.48968300 \\ \mathrm{H} & 2.42298200 & 2.00563000 & -0.79160300 \\ \mathrm{H} & -0.21517000 & 1.23801400 & -0.24079800 \\ \mathrm{H} & 4.80079800 & 1.00385100 & 0.65306300 \\ \mathrm{H} & 4.63956600 & 2.58942900 & -0.11298100 \\ \mathrm{H} & -1.43321400 & -0.75543500 & 1.02541800 \\ \mathrm{H} & -2.46693700 & 0.77948600 & -1.38974000 \\ \mathrm{H} & -4.66362000 & 0.68848700 & -0.18014400 \\ \mathrm{H} & -3.91777600 & -0.29091400 & 1.07931000 \\ \mathrm{H} & -4.15107400 & -1.03676500 & -1.88259500 \\ \mathrm{H} & -5.06001400 & -1.76160700 & -0.56259400 \\ \mathrm{H} & -3.10487700 & -3.19381800 & -1.26157000 \\ \mathrm{H} & -2.98075800 & -2.69056100 & 0.41649100 \\ \mathrm{H} & -0.79163000 & -2.41216700 & -0.67152600 \\ \mathrm{H} & -1.50059000 & -1.40729000 & -1.94870200 \\ \mathrm{H} & -4.01915900 & 2.95345100 & -0.02410700 \\ \mathrm{H} & -2.54643400 & 3.92703400 & 0.14689500 \\ \mathrm{H} & -2.81523600 & 2.98970100 & -1.33355200 \\ \mathrm{H} & -1.87601400 & 2.81750100 & 2.16546500 \\ \mathrm{H} & -3.30449400 & 1.76446800 & 2.16608400 \\ \mathrm{H} & -1.67034200 & 1.06352000 & 2.20081500 \\ \mathrm{H} & 4.86169300 & -0.12494800 & -1.54328000 \\ \mathrm{H} & 4.63016800 & 1.42300600 & -2.38966100 \\ \mathrm{H} & 6.12266000 & 1.11616600 & -1.48275800\end{array}$<smiles>CC(C)Nc1c(N[C@@H]2CCCCC2N(C)C)c(=O)c1=O</smiles>

Squaramide-9

$\begin{array}{lrrr}\mathrm{C} & 0.76546100 & -0.56278000 & 0.09660500 \\ \mathrm{C} & 1.04105900 & -1.93337100 & 0.61746300 \\ \mathrm{C} & 2.53272500 & -1.53329000 & 0.66649100 \\ \mathrm{C} & 2.12110200 & -0.21187600 & 0.12585500 \\ \mathrm{O} & 0.36808200 & -2.91328300 & 0.87230100 \\ \mathrm{O} & 3.59172300 & -2.02994100 & 0.99867900 \\ \mathrm{~N} & 2.81657300 & 0.90547000 & -0.16774000 \\ \mathrm{~N} & -0.34730100 & 0.09682700 & -0.28366400 \\ \mathrm{C} & 4.29013700 & 0.88719200 & -0.21670500 \\ \mathrm{C} & -1.69283600 & -0.42279300 & -0.02867400 \\ \mathrm{C} & -2.68332300 & 0.72291100 & -0.30729600 \\ \mathrm{C} & -4.11556200 & 0.26253700 & 0.01160000 \\ \mathrm{C} & -4.48482100 & -0.97109100 & -0.82487600 \\ \mathrm{C} & -3.47149300 & -2.10496800 & -0.62389800\end{array}$




$\begin{array}{lrrr}\mathrm{C} & -2.03546300 & -1.64022000 & -0.89624300 \\ \mathrm{~N} & -2.22963400 & 1.97080900 & 0.33295900 \\ \mathrm{C} & -2.84489100 & 3.16161900 & -0.23865600 \\ \mathrm{C} & -2.31426500 & 1.97692300 & 1.79081000 \\ \mathrm{C} & 4.80416100 & 2.31389500 & -0.04294100 \\ \mathrm{C} & 4.80112000 & 0.23067100 & -1.50260100 \\ \mathrm{H} & 2.36568100 & 1.59173100 & -0.76184000 \\ \mathrm{H} & -0.33895800 & 1.11492600 & -0.23581600 \\ \mathrm{H} & 4.60656000 & 0.27312300 & 0.63080200 \\ \mathrm{H} & -1.77922700 & -0.72890700 & 1.02438000 \\ \mathrm{H} & -2.62781700 & 0.92082000 & -1.38714500 \\ \mathrm{H} & -4.82178900 & 1.07804800 & -0.17899900 \\ \mathrm{H} & -4.19392500 & 0.01605300 & 1.07782700 \\ \mathrm{H} & -4.50737700 & -0.68925500 & -1.88661700 \\ \mathrm{H} & -5.49537300 & -1.30902600 & -0.56991200 \\ \mathrm{H} & -3.71632000 & -2.95401500 & -1.27068500 \\ \mathrm{H} & -3.53740900 & -2.47253900 & 0.40888100 \\ \mathrm{H} & -1.32936200 & -2.44366800 & -0.67634300 \\ \mathrm{H} & -1.91727400 & -1.36105900 & -1.95148300 \\ \mathrm{H} & -2.34720300 & 4.05266900 & 0.15698500 \\ \mathrm{H} & -2.72123500 & 3.15572500 & -1.32561000 \\ \mathrm{H} & -3.92196500 & 3.25507700 & -0.01647300 \\ \mathrm{H} & -1.80789000 & 2.86843700 & 2.17293400 \\ \mathrm{H} & -3.34877000 & 1.98828100 & 2.17158300 \\ \mathrm{H} & -1.80696400 & 1.10236500 & 2.20424500 \\ \mathrm{H} & 5.89722800 & 2.32764900 & -0.03778000 \\ \mathrm{H} & 4.47049700 & 2.95238300 & -0.87089500 \\ \mathrm{H} & 4.44547100 & 2.74966000 & 0.89333100 \\ \mathrm{H} & 5.89537900 & 0.21133600 & -1.51542800 \\ \mathrm{H} & 4.44402400 & -0.79980600 & -1.56978100 \\ \mathrm{H} & 4.45944300 & 0.78189400 & -2.38648800 \\ & & & \end{array}$<smiles>CN(C)C1CCCC[C@H]1Nc1c(NCc2ccccc2)c(=O)c1=O</smiles>

Squaramide-10

$\begin{array}{lrrr}\mathrm{C} & 0.14280500 & -0.61106500 & -0.57685100 \\ \mathrm{C} & -0.05111700 & -2.07160800 & -0.82119800 \\ \mathrm{C} & -1.45792300 & -1.71950600 & -1.34690400 \\ \mathrm{C} & -1.14329600 & -0.30251300 & -1.04093800 \\ \mathrm{O} & 0.61956700 & -3.07216200 & -0.66026900 \\ \mathrm{O} & -2.42018400 & -2.30569200 & -1.81019400 \\ \mathrm{~N} & -1.82914200 & 0.84830400 & -1.21262300 \\ \mathrm{~N} & 1.14402200 & 0.13818400 & -0.08145900 \\ \mathrm{C} & -3.27395100 & 0.81434700 & -1.50515300 \\ \mathrm{C} & 2.48760200 & -0.39131600 & 0.16294500 \\ \mathrm{C} & 3.40448300 & 0.81366200 & 0.44486300 \\ \mathrm{C} & 4.85257000 & 0.33290900 & 0.63507800\end{array}$




$\begin{array}{lrrr}\mathrm{C} & 4.93786300 & -0.67694800 & 1.78863400 \\ \mathrm{C} & 3.97733900 & -1.85331200 & 1.57251200 \\ \mathrm{C} & 2.53809700 & -1.37856900 & 1.33558400 \\ \mathrm{~N} & 3.18361700 & 1.87973200 & -0.54875900 \\ \mathrm{C} & 3.66046000 & 1.57066000 & -1.89411500 \\ \mathrm{C} & 3.66121300 & 3.18247700 & -0.10316800 \\ \mathrm{C} & -4.12073300 & 0.71098800 & -0.25590600 \\ \mathrm{C} & -4.59308700 & 1.86385300 & 0.37989000 \\ \mathrm{C} & -5.32305100 & 1.77229900 & 1.56510500 \\ \mathrm{C} & -5.58792900 & 0.52149900 & 2.12340800 \\ \mathrm{C} & -5.12485600 & -0.63373800 & 1.49040400 \\ \mathrm{C} & -4.39470100 & -0.54207900 & 0.30645600 \\ \mathrm{H} & -1.52187800 & 1.64290800 & -0.66406100 \\ \mathrm{H} & 1.18331500 & 1.12349500 & -0.33952100 \\ \mathrm{H} & -3.44105900 & -0.04564400 & -2.15558200 \\ \mathrm{H} & -3.51909000 & 1.72317300 & -2.06269500 \\ \mathrm{H} & 2.84564000 & -0.91852800 & -0.73358500 \\ \mathrm{H} & 3.06532700 & 1.24003200 & 1.39956600 \\ \mathrm{H} & 5.50841200 & 1.18888000 & 0.82805000 \\ \mathrm{H} & 5.20830200 & -0.13891200 & -0.28935200 \\ \mathrm{H} & 4.68213500 & -0.16841900 & 2.72839400 \\ \mathrm{H} & 5.96700200 & -1.03646600 & 1.89800300 \\ \mathrm{H} & 4.00747300 & -2.53258700 & 2.43085200 \\ \mathrm{H} & 4.30786600 & -2.43723300 & 0.70307300 \\ \mathrm{H} & 1.89035900 & -2.22921700 & 1.11378600 \\ \mathrm{H} & 2.14812300 & -0.87728700 & 2.23108900 \\ \mathrm{H} & 4.75916100 & 1.52538500 & -1.97188400 \\ \mathrm{H} & 3.25376900 & 0.61434600 & -2.23066300 \\ \mathrm{H} & 3.30822200 & 2.34428100 & -2.58298600 \\ \mathrm{H} & 3.31991000 & 3.95242800 & -0.80228200 \\ \mathrm{H} & 3.24755000 & 3.40971700 & 0.88373800 \\ \mathrm{H} & 4.76067900 & 3.25381200 & -0.03917000 \\ \mathrm{H} & -4.39474400 & 2.83885300 & -0.05996200 \\ \mathrm{H} & -5.68863300 & 2.67367600 & 2.04787800 \\ \mathrm{H} & -6.15804800 & 0.44679200 & 3.04444300 \\ \mathrm{H} & -5.33699700 & -1.60929700 & 1.91732600 \\ \mathrm{H} & -4.03807600 & -1.43713700 & -0.19473200\end{array}$<smiles>CN(C)C1CCCC[C@H]1Nc1c(Nc2ccccc2)c(=O)c1=O</smiles>

Squaramide-11

$\begin{array}{lrrr}\mathrm{C} & -0.01645600 & 0.87060700 & -0.02246100 \\ \mathrm{C} & -0.09015000 & 2.30164600 & 0.37334800 \\ \mathrm{C} & -1.62385500 & 2.14827900 & 0.40311000 \\ \mathrm{C} & -1.41197700 & 0.72629200 & -0.01441200 \\ \mathrm{O} & 0.71373600 & 3.18940700 & 0.57661800 \\ \mathrm{O} & -2.56547300 & 2.86350900 & 0.67643400\end{array}$




$\begin{array}{lrrr}\mathrm{N} & -2.15114700 & -0.36979300 & -0.31509000 \\ \mathrm{~N} & 0.98361600 & 0.01470500 & -0.29499600 \\ \mathrm{C} & -3.54443700 & -0.56600800 & -0.26168100 \\ \mathrm{C} & 2.39287100 & 0.33699000 & -0.05330100 \\ \mathrm{C} & 3.18790800 & -0.97106700 & -0.22274100 \\ \mathrm{C} & 4.67150800 & -0.72308900 & 0.09474200 \\ \mathrm{C} & 5.24308200 & 0.36694000 & -0.82379300 \\ \mathrm{C} & 4.42304700 & 1.66034600 & -0.73366200 \\ \mathrm{C} & 2.93353600 & 1.41186200 & -1.00350600 \\ \mathrm{~N} & 2.52775700 & -2.07564000 & 0.49707200 \\ \mathrm{C} & 2.94098200 & -3.39127700 & 0.02398900 \\ \mathrm{C} & 2.60332300 & -1.98772500 & 1.95346300 \\ \mathrm{C} & -4.03489300 & -1.81790800 & -0.65972400 \\ \mathrm{C} & -5.40149300 & -2.07700600 & -0.62428000 \\ \mathrm{C} & -6.29265400 & -1.09423700 & -0.19271700 \\ \mathrm{C} & -5.79621800 & 0.14930100 & 0.20070700 \\ \mathrm{C} & -4.43112400 & 0.42725500 & 0.17255200 \\ \mathrm{H} & -1.63202100 & -1.16130100 & -0.67178900 \\ \mathrm{H} & 0.82068400 & -0.98441900 & -0.16450600 \\ \mathrm{H} & 2.51523800 & 0.70640200 & 0.97539100 \\ \mathrm{H} & 3.11322800 & -1.23953000 & -1.28596900 \\ \mathrm{H} & 5.23875000 & -1.65340100 & -0.01810900 \\ \mathrm{H} & 4.77700000 & -0.41037000 & 1.14102100 \\ \mathrm{H} & 5.23242000 & 0.00260800 & -1.86022900 \\ \mathrm{H} & 6.29157300 & 0.55901300 & -0.57100300 \\ \mathrm{H} & 4.80807900 & 2.40534500 & -1.43765300 \\ \mathrm{H} & 4.53628100 & 2.09344300 & 0.26915100 \\ \mathrm{H} & 2.36445400 & 2.33316900 & -0.86251900 \\ \mathrm{H} & 2.78306500 & 1.07279000 & -2.03672800 \\ \mathrm{H} & 2.30176400 & -4.15630300 & 0.47569600 \\ \mathrm{H} & 2.82480700 & -3.44653000 & -1.06232900 \\ \mathrm{H} & 3.98681900 & -3.64195100 & 0.27071000 \\ \mathrm{H} & 1.95387800 & -2.75211900 & 2.39033300 \\ \mathrm{H} & 3.61980100 & -2.14205700 & 2.35045500 \\ \mathrm{H} & 2.24711900 & -1.01290000 & 2.29421900 \\ \mathrm{H} & -3.34354200 & -2.58704300 & -0.99653400 \\ \mathrm{H} & -5.76737100 & -3.05058700 & -0.93555700 \\ \mathrm{H} & -7.35855200 & -1.29478700 & -0.16450700 \\ \mathrm{H} & -6.47898500 & 0.92379900 & 0.53644000 \\ \mathrm{H} & -4.06018100 & 1.40129500 & 0.47289700 \\ & & & \\ & & & \end{array}$<smiles>CN(C)C1CCCC[C@H]1Nc1c(NC(C)(C)C)c(=O)c1=O</smiles>

Squaramide-12

\begin{tabular}{|c|c|c|c|}
\hline $\mathrm{C}$ & 0.52443300 & -0.66422800 & 0.02591100 \\
\hline C & 0.76769800 & -2.04806300 & 0.5099330 \\
\hline $\mathrm{C}$ & 2.26970000 & -1.70487800 & 0.52103700 \\
\hline
\end{tabular}




$\begin{array}{rrrr}\mathrm{C} & 1.89306200 & -0.35099700 & 0.01949200 \\ \mathrm{O} & 0.07405200 & -3.01398600 & 0.76624800 \\ \mathrm{O} & 3.30705200 & -2.26811300 & 0.81700100 \\ \mathrm{~N} & -0.57075300 & 0.04777700 & -0.30502800 \\ \mathrm{C} & -1.92918700 & -0.42610100 & -0.03006100 \\ \mathrm{C} & -2.88069400 & 0.76052500 & -0.27004000 \\ \mathrm{C} & -4.32296900 & 0.34966500 & 0.06964100 \\ \mathrm{C} & -4.75438000 & -0.85530700 & -0.77886000 \\ \mathrm{C} & -3.78139700 & -2.02986700 & -0.61512500 \\ \mathrm{C} & -2.33392000 & -1.61609200 & -0.90848600 \\ \mathrm{~N} & -2.36669100 & 1.97892600 & 0.38201300 \\ \mathrm{C} & -2.42735400 & 1.96430600 & 1.84109900 \\ \mathrm{C} & -2.94377900 & 3.20242900 & -0.15988600 \\ \mathrm{~N} & 2.54637200 & 0.77921200 & -0.31261800 \\ \mathrm{C} & 4.02058100 & 0.94235700 & -0.34891000 \\ \mathrm{C} & 4.27521800 & 2.37948700 & -0.81850900 \\ \mathrm{C} & 4.64598900 & -0.06215000 & -1.33065800 \\ \mathrm{C} & 4.58634600 & 0.73622000 & 1.06375500 \\ \mathrm{H} & -0.52255800 & 1.06360100 & -0.22946500 \\ \mathrm{H} & -2.00507100 & -0.74543800 & 1.01996500 \\ \mathrm{H} & -2.83807800 & 0.97485700 & -1.34728800 \\ \mathrm{H} & -5.00113700 & 1.19428000 & -0.09422900 \\ \mathrm{H} & -4.39044500 & 0.08869900 & 1.13315500 \\ \mathrm{H} & -4.78599000 & -0.55547400 & -1.83542200 \\ \mathrm{H} & -5.77209600 & -1.15902900 & -0.50980400 \\ \mathrm{H} & -4.07075100 & -2.85853100 & -1.26987300 \\ \mathrm{H} & -3.84188400 & -2.41116300 & 0.41300100 \\ \mathrm{H} & -1.65488800 & -2.44902100 & -0.71392300 \\ \mathrm{H} & -2.22502700 & -1.32543000 & -1.96161300 \\ \mathrm{H} & -1.87873400 & 2.82798300 & 2.22879000 \\ \mathrm{H} & -3.45422900 & 2.01147600 & 2.23926200 \\ \mathrm{H} & -1.94982300 & 1.06300300 & 2.23195300 \\ \mathrm{H} & -2.40516000 & 4.06657400 & 0.24176900 \\ \mathrm{H} & -2.83807900 & 3.20999000 & -1.24870300 \\ \mathrm{H} & -4.01255100 & 3.33392200 & 0.08199500 \\ \mathrm{H} & 2.01858300 & 1.46474200 & -0.83617400 \\ \mathrm{H} & 3.81674200 & 3.10211300 & -0.13554100 \\ \mathrm{H} & 5.34906100 & 2.57714100 & -0.85214700 \\ \mathrm{H} & 3.87433100 & 2.54415800 & -1.82559100 \\ \mathrm{H} & 4.48078200 & -1.08453500 & -0.98471000 \\ \mathrm{H} & 4.21419100 & 0.05325100 & -2.32999600 \\ \mathrm{H} & 5.72558700 & 0.10381300 & -1.40207900 \\ \mathrm{H} & 4.41098900 & -0.28724800 & 1.40096500 \\ \mathrm{H} & 5.66618000 & 0.91414500 & 1.05943100 \\ \mathrm{H} & 4.12265600 & 1.43368300 & 1.76770700\end{array}$<smiles>CN(Cc1ccccc1)C(=O)C(NC(=S)Nc1cc(C(F)(F)F)cc(C(F)(F)F)c1)C(C)(C)C</smiles> 
Thiourea-28

$\begin{array}{lrrc}\mathrm{N} & 2.26596700 & -0.50083800 & -0.61743000 \\ \mathrm{C} & 1.05738700 & -0.85174400 & -0.10623000 \\ \mathrm{~N} & 0.02151400 & -0.30808300 & -0.84230400 \\ \mathrm{~S} & 0.86616700 & -1.77634000 & 1.27985900 \\ \mathrm{C} & -1.35988100 & -0.40531400 & -0.59652100 \\ \mathrm{C} & -2.13544300 & 0.72335300 & -0.86332300 \\ \mathrm{C} & -3.50762900 & 0.70919600 & -0.63046400 \\ \mathrm{C} & -4.13639300 & -0.43840700 & -0.15858700 \\ \mathrm{C} & -3.36100400 & -1.57861900 & 0.05930500 \\ \mathrm{C} & -1.98497400 & -1.57897800 & -0.15748500 \\ \mathrm{C} & -4.01689800 & -2.82259000 & 0.59699000 \\ \mathrm{C} & -4.26294400 & 1.99282300 & -0.82288200 \\ \mathrm{~F} & -3.95587100 & 2.89071200 & 0.15155300 \\ \mathrm{~F} & -3.94664200 & 2.58722000 & -1.99624900 \\ \mathrm{~F} & -5.59693100 & 1.81686100 & -0.79610800 \\ \mathrm{~F} & -3.36858600 & -3.94081300 & 0.20905800 \\ \mathrm{~F} & -4.03972600 & -2.82392700 & 1.94830300 \\ \mathrm{~F} & -5.29877100 & -2.92760700 & 0.18104400 \\ \mathrm{C} & 3.57604700 & -0.87046400 & -0.09032400 \\ \mathrm{C} & 4.42479600 & 0.41969000 & 0.07072200 \\ \mathrm{C} & 4.25331800 & -1.99245700 & -0.93782800 \\ \mathrm{C} & 3.33620200 & -3.22970600 & -0.92200300 \\ \mathrm{C} & 5.59395100 & -2.37578900 & -0.28555300 \\ \mathrm{C} & 4.47157500 & -1.56107200 & -2.39908800 \\ \mathrm{~N} & 3.98761000 & 1.35028300 & 0.98064200 \\ \mathrm{O} & 5.45163000 & 0.60290500 & -0.57908700 \\ \mathrm{C} & 4.79048100 & 2.55227400 & 1.19031600 \\ \mathrm{C} & 2.74318900 & 1.28984500 & 1.72881600 \\ \mathrm{C} & 1.59527400 & 2.07270700 & 1.10749200 \\ \mathrm{C} & 1.71714700 & 2.73507700 & -0.11756800 \\ \mathrm{C} & 0.64198000 & 3.45712700 & -0.64557400 \\ \mathrm{C} & -0.56941800 & 3.51617900 & 0.04347400 \\ \mathrm{C} & -0.70633200 & 2.83244600 & 1.25403200 \\ \mathrm{C} & 0.36833200 & 2.11984900 & 1.78079900 \\ \mathrm{H} & 2.27509400 & -0.01993000 & -1.50688600 \\ \mathrm{H} & 5.27229800 & 0.53910300 & -1.33933800 \\ \mathrm{H} & -1.66164300 & 1.62850800 & -1.22005700 \\ \mathrm{H} & -5.20316600 & -0.45162900 & 0.02141000 \\ \mathrm{H} & -1.40727600 & -2.47363800 & 0.01730300 \\ \mathrm{H} & 3.38564900 & -1.28875900 & 0.90172600 \\ \mathrm{H} & 2.36591200 & -3.02417500 & -1.38188900 \\ \mathrm{H} & 3.80922900 & -4.03796700 & -1.48865300 \\ \mathrm{H} & 3.15217300 & -3.58378800 & 0.09541400 \\ \mathrm{H} & 6.31013800 & -1.55600200 & -0.33074100 \\ \mathrm{H} & 5.44859100 & -2.65989000 & 0.76334900 \\ \mathrm{H} & 3.51847100 & -3.24123700 & -0.80302700 \\ \mathrm{H} & & -1.34032400 & -2.89566600 \\ \mathrm{H} & -11903000 & -0.68711800 & -2.46261700 \\ \mathrm{H} & -2.38152400 & -2.95823500 \\ \mathrm{H} & 2.55691000 & 0.46994400\end{array}$




$\begin{array}{lrrr}\mathrm{H} & 4.17410900 & 3.44701300 & 1.06027400 \\ \mathrm{H} & 5.20155100 & 2.55754100 & 2.20643800 \\ \mathrm{H} & 2.94168000 & 1.69159200 & 2.73002600 \\ \mathrm{H} & 2.43120900 & 0.25492100 & 1.88225000 \\ \mathrm{H} & 2.65863700 & 2.69543600 & -0.65573700 \\ \mathrm{H} & 0.75814200 & 3.98090800 & -1.58997700 \\ \mathrm{H} & -1.41178100 & 4.06600100 & -0.36411600 \\ \mathrm{H} & -1.65972500 & 2.84180200 & 1.77140100 \\ \mathrm{H} & 0.25291200 & 1.58034600 & 2.71649200\end{array}$<smiles>CN(C)C(c1ccccc1)[C@H](NC(=S)Nc1cc(C(F)(F)F)cc(C(F)(F)F)c1)c1ccccc1</smiles>

Thiourea-29

$\begin{array}{lrrr}\mathrm{C} & 3.37445100 & -1.34610300 & -0.26363900 \\ \mathrm{C} & 2.46737200 & -0.28019000 & -0.22078200 \\ \mathrm{C} & 2.95407400 & 1.02973700 & -0.12239800 \\ \mathrm{C} & 4.32784300 & 1.24525400 & -0.04588900 \\ \mathrm{C} & 5.23694200 & 0.18920000 & -0.08502400 \\ \mathrm{C} & 4.74478400 & -1.10822600 & -0.20273100 \\ \mathrm{C} & 4.83688300 & 2.65132300 & 0.13135900 \\ \mathrm{C} & 5.69963500 & -2.26335700 & -0.32597100 \\ \mathrm{~F} & 4.02465200 & 3.55700000 & -0.45362100 \\ \mathrm{~F} & 4.92766300 & 2.98303800 & 1.43769100 \\ \mathrm{~F} & 6.06989600 & 2.80483900 & -0.40180800 \\ \mathrm{~F} & 6.86225400 & -2.02512500 & 0.31513900 \\ \mathrm{~F} & 5.17690000 & -3.40269000 & 0.18135300 \\ \mathrm{~F} & 6.00506200 & -2.51578700 & -1.62027700 \\ \mathrm{~N} & 1.10478100 & -0.58761600 & -0.34454300 \\ \mathrm{C} & -0.00735300 & 0.06142600 & 0.16417000 \\ \mathrm{~N} & -1.15283600 & -0.53743700 & -0.26779600 \\ \mathrm{~S} & 0.01989000 & 1.37554000 & 1.19544000 \\ \mathrm{C} & -2.47602300 & -0.03146800 & 0.06630000 \\ \mathrm{C} & -2.92511700 & -0.42204200 & 1.46172600 \\ \mathrm{C} & -3.65996200 & 0.47336900 & 2.24093200 \\ \mathrm{C} & -4.13557900 & 0.09391800 & 3.49488100 \\ \mathrm{C} & -3.87486200 & -1.18608400 & 3.98459900 \\ \mathrm{C} & -3.13474500 & -2.08330100 & 3.21342900 \\ \mathrm{C} & -2.66546700 & -1.70245900 & 1.95761400 \\ \mathrm{C} & -3.45326400 & -0.62063100 & -0.98712300 \\ \mathrm{C} & -4.83273500 & 0.01096900 & -0.91568500 \\ \mathrm{~N} & -2.82784200 & -0.61758400 & -2.32938700 \\ \mathrm{C} & -5.96269500 & -0.81402000 & -0.90557300 \\ \mathrm{C} & -7.24812000 & -0.27440700 & -0.88156400 \\ \mathrm{C} & -7.42284800 & 1.10889000 & -0.86863700 \\ \mathrm{C} & -6.30526500 & 1.94412700 & -0.87696500 \\ & & & \end{array}$




$\begin{array}{lrrr}\mathrm{C} & -5.02211900 & 1.39984400 & -0.89989500 \\ \mathrm{C} & -3.52469100 & -1.49306200 & -3.26653500 \\ \mathrm{C} & -2.60424300 & 0.70705300 & -2.90615800 \\ \mathrm{H} & 3.01161600 & -2.36653800 & -0.33197900 \\ \mathrm{H} & 2.26785700 & 1.86219700 & -0.09886400 \\ \mathrm{H} & 6.30175400 & 0.37329800 & -0.02877600 \\ \mathrm{H} & 0.92186600 & -1.52639600 & -0.67402900 \\ \mathrm{H} & -1.15025700 & -0.95951100 & -1.19917000 \\ \mathrm{H} & -2.45851500 & 1.06171400 & 0.00815000 \\ \mathrm{H} & -3.85605500 & 1.47179000 & 1.86403300 \\ \mathrm{H} & -4.70273000 & 0.80094400 & 4.09246200 \\ \mathrm{H} & -4.23984600 & -1.48050400 & 4.96377300 \\ \mathrm{H} & -2.92017100 & -3.07837800 & 3.59158700 \\ \mathrm{H} & -2.07699900 & -2.39291900 & 1.36080800 \\ \mathrm{H} & -3.56818700 & -1.68147800 & -0.73727600 \\ \mathrm{H} & -5.83062300 & -1.89250000 & -0.90388700 \\ \mathrm{H} & -8.11050200 & -0.93375700 & -0.86675400 \\ \mathrm{H} & -8.42153700 & 1.53371300 & -0.84668100 \\ \mathrm{H} & -6.43120500 & 3.02235200 & -0.86064400 \\ \mathrm{H} & -4.16645200 & 2.06620900 & -0.89287500 \\ \mathrm{H} & -2.94466400 & -1.56319700 & -4.19147700 \\ \mathrm{H} & -3.61125100 & -2.49627500 & -2.83864500 \\ \mathrm{H} & -4.53683500 & -1.13913300 & -3.51962900 \\ \mathrm{H} & -2.01204500 & 0.59569000 & -3.81906000 \\ \mathrm{H} & -3.53664700 & 1.23202600 & -3.16286000 \\ \mathrm{H} & -2.03476300 & 1.33045600 & -2.21209300\end{array}$<smiles>OC1Cc2ccccc2[C@H]1NC(=S)Nc1cc(C(F)(F)F)cc(C(F)(F)F)c1</smiles>

Thiourea-30

$\begin{array}{lrrr}\text { C } & 2.09016600 & 1.50227500 & 0.07104900 \\ \mathrm{C} & 1.30490100 & 0.34694400 & 0.09558700 \\ \mathrm{C} & 1.92197400 & -0.90811400 & 0.14925400 \\ \mathrm{C} & 3.31141200 & -0.98624100 & 0.15020600 \\ \mathrm{C} & 4.10419600 & 0.16144800 & 0.12458600 \\ \mathrm{C} & 3.48044600 & 1.40502000 & 0.09435000 \\ \mathrm{C} & 3.97198800 & -2.33966900 & 0.13458100 \\ \mathrm{C} & 4.30557100 & 2.66175200 & 0.14408200 \\ \mathrm{~N} & -0.09410100 & 0.50308900 & 0.13752000 \\ \mathrm{C} & -1.06801300 & -0.25934200 & -0.47052000 \\ \mathrm{~N} & -2.31696600 & 0.13489500 & -0.12705100 \\ \mathrm{~S} & -0.75460300 & -1.49855900 & -1.56763800 \\ \mathrm{~F} & 4.20387800 & -2.75953400 & -1.12792800 \\ \mathrm{~F} & 5.16501800 & -2.31597300 & 0.76912500 \\ \mathrm{~F} & 3.20855000 & -3.27726500 & 0.73360500 \\ \mathrm{~F} & 4.49070500 & 3.07898700 & 1.41784000\end{array}$




$\begin{array}{lrrr}\text { F } & 5.52768300 & 2.48207500 & -0.39645500 \\ \text { F } & 3.70776200 & 3.67793500 & -0.51767000 \\ \text { C } & -3.54434600 & -0.47979300 & -0.62840300 \\ \text { C } & -3.94196600 & -1.77991000 & 0.16102900 \\ \text { C } & -4.92183500 & -1.30813800 & 1.25305200 \\ \text { C } & -5.49726500 & -0.03282700 & 0.68697300 \\ \mathrm{C} & -4.71921700 & 0.43630900 & -0.37949800 \\ \mathrm{C} & -6.62616200 & 0.67819100 & 1.08987200 \\ \mathrm{C} & -6.95990100 & 1.85885000 & 0.42209100 \\ \mathrm{C} & -6.17651700 & 2.32686300 & -0.63807700 \\ \mathrm{C} & -5.04645600 & 1.61529500 & -1.04549600 \\ \mathrm{O} & -2.86569700 & -2.46440900 & 0.74957100 \\ \mathrm{H} & 1.62134000 & 2.47934300 & 0.01927700 \\ \mathrm{H} & 1.32513700 & -1.80691500 & 0.18761100 \\ \mathrm{H} & 5.18377400 & 0.08635300 & 0.13288700 \\ \mathrm{H} & -0.40065800 & 1.39871700 & 0.49466200 \\ \mathrm{H} & -2.42432900 & 0.70980600 & 0.69892800 \\ \mathrm{H} & -3.37266200 & -0.69748100 & -1.68402500 \\ \mathrm{H} & -4.48640000 & -2.41660100 & -0.55344900 \\ \mathrm{H} & -4.35729300 & -1.11762100 & 2.17504200 \\ \mathrm{H} & -5.67825900 & -2.06166900 & 1.48897900 \\ \mathrm{H} & -7.24089500 & 0.32002900 & 1.91058700 \\ \mathrm{H} & -7.83970800 & 2.41780500 & 0.72619600 \\ \mathrm{H} & -6.45323700 & 3.24333400 & -1.14978800 \\ \mathrm{H} & -4.43610100 & 1.96977800 & -1.87117100 \\ \mathrm{H} & -2.23022900 & -2.66805400 & 0.04165300\end{array}$<smiles>CCC1CN2CCC1CN2[C@H](NC(=S)Nc1ccc([N+](=O)[O-])cc1)c1ccnc2ccc(OC)cc12</smiles>

Thiourea-31

$\begin{array}{llcc}\mathrm{C} & 3.96267400 & -0.45414600 & 0.29064900 \\ \mathrm{C} & 4.85771300 & -1.29130900 & 0.98784100 \\ \mathrm{C} & 6.22509700 & -1.17790400 & 0.80991200 \\ \mathrm{C} & 6.70926500 & -0.21643100 & -0.07623400 \\ \mathrm{C} & 5.84456100 & 0.61976700 & -0.77782400 \\ \mathrm{C} & 4.47196700 & 0.50599000 & -0.60049900 \\ \mathrm{~N} & 2.60436300 & -0.67906800 & 0.52781200 \\ \mathrm{C} & 1.46007300 & 0.01257300 & 0.17899200 \\ \mathrm{~N} & 0.34695600 & -0.70132100 & 0.52391400 \\ \mathrm{~S} & 1.38545600 & 1.52601100 & -0.52620500 \\ \mathrm{C} & -0.99662400 & -0.23348600 & 0.21128300 \\ \mathrm{C} & -1.92826900 & -1.45974600 & 0.32995800 \\ \mathrm{C} & -1.46905200 & 0.86434600 & 1.14795200 \\ \mathrm{C} & -1.26089300 & 0.73018200 & 2.50497700\end{array}$




\begin{tabular}{|c|c|c|c|}
\hline $\mathrm{C}$ & -1.78754400 & 1.68454200 & 3.39919900 \\
\hline $\mathrm{N}$ & -2.50186300 & 2.72684100 & 3.02154400 \\
\hline $\mathrm{C}$ & -2.70710800 & 2.89443600 & 1.68670900 \\
\hline $\mathrm{C}$ & -2.19961600 & 1.99930600 & 0.68941000 \\
\hline $\mathrm{C}$ & -3.46413100 & 4.02575400 & 1.27239000 \\
\hline $\mathrm{C}$ & -3.69439200 & 4.27548000 & -0.05302300 \\
\hline $\mathrm{C}$ & -3.16446400 & 3.40455200 & -1.04315400 \\
\hline $\mathrm{C}$ & -2.43711300 & 2.28933200 & -0.68194700 \\
\hline $\mathrm{N}$ & -1.30617900 & -2.63876000 & -0.31647600 \\
\hline $\mathrm{C}$ & -2.07054000 & -3.83728000 & 0.07023300 \\
\hline $\mathrm{C}$ & -3.57970300 & -3.72207300 & -0.32134700 \\
\hline $\mathrm{C}$ & -3.35523800 & -1.19836700 & -0.21984100 \\
\hline $\mathrm{C}$ & -3.74689200 & -2.39714900 & -1.10114200 \\
\hline $\mathrm{C}$ & -1.33762500 & -2.50756100 & -1.78722900 \\
\hline $\mathrm{C}$ & -2.79927900 & -2.42382500 & -2.31405800 \\
\hline $\mathrm{C}$ & -4.49053000 & -3.85533200 & 0.90800100 \\
\hline $\mathrm{C}$ & -5.98652900 & -3.77618800 & 0.59906200 \\
\hline $\mathrm{O}$ & -3.43188500 & 3.77316300 & -2.32640500 \\
\hline $\mathrm{C}$ & -2.89379700 & 2.97294900 & -3.36718500 \\
\hline $\mathrm{H}$ & 4.46873800 & -2.03579100 & 1.67745900 \\
\hline $\mathrm{H}$ & 6.92165700 & -1.81296500 & 1.34108700 \\
\hline $\mathrm{H}$ & 6.25636600 & 1.35281500 & -1.45928900 \\
\hline $\mathrm{H}$ & 3.79324800 & 1.15652100 & -1.13015400 \\
\hline $\mathrm{H}$ & 2.43051600 & -1.46195800 & 1.14508500 \\
\hline $\mathrm{H}$ & 0.39052900 & -1.72036800 & 0.44426500 \\
\hline $\mathrm{H}$ & -1.00390700 & 0.13658100 & -0.81691100 \\
\hline $\mathrm{H}$ & -1.98841500 & -1.71233400 & 1.39433200 \\
\hline $\mathrm{H}$ & -0.68619600 & -0.10825200 & 2.88487300 \\
\hline $\mathrm{H}$ & -1.61869000 & 1.57134100 & 4.46884000 \\
\hline $\mathrm{H}$ & -3.84214100 & 4.68184100 & 2.04848200 \\
\hline $\mathrm{H}$ & -4.26332000 & 5.13769900 & -0.38317100 \\
\hline $\mathrm{H}$ & -2.00840900 & 1.65282800 & -1.44094900 \\
\hline $\mathrm{H}$ & -1.96515100 & -3.96811400 & 1.15303200 \\
\hline $\mathrm{H}$ & -1.59976800 & -4.70169600 & -0.40826200 \\
\hline $\mathrm{H}$ & -3.83257400 & -4.53929600 & -1.01010900 \\
\hline $\mathrm{H}$ & -3.38649600 & -0.28253300 & -0.81794400 \\
\hline $\mathrm{H}$ & -4.06096100 & -1.05240500 & 0.60170600 \\
\hline $\mathrm{H}$ & -4.77942900 & -2.28904400 & -1.44394300 \\
\hline $\mathrm{H}$ & -0.81221900 & -3.37203900 & -2.20345800 \\
\hline $\mathrm{H}$ & -0.75913900 & -1.62328000 & -2.06743800 \\
\hline $\mathrm{H}$ & -2.94268700 & -1.52246700 & -2.91998700 \\
\hline $\mathrm{H}$ & -3.03318400 & -3.28152900 & -2.95376700 \\
\hline $\mathrm{H}$ & -4.22605000 & -3.08754300 & 1.64682100 \\
\hline $\mathrm{H}$ & -4.27018600 & -4.81733100 & 1.38900900 \\
\hline $\mathrm{H}$ & -6.58350400 & -3.97287800 & 1.49452400 \\
\hline $\mathrm{H}$ & -6.27182900 & -2.78797400 & 0.22565200 \\
\hline $\mathrm{H}$ & -6.27151500 & -4.51279800 & -0.16055600 \\
\hline $\mathrm{H}$ & -3.20170900 & 3.44366100 & -4.30116700 \\
\hline $\mathrm{H}$ & -1.79858700 & 2.93614800 & -3.31827100 \\
\hline $\mathrm{H}$ & -3.28803100 & 1.94880000 & -3.32920600 \\
\hline $\mathrm{N}$ & 8.15092300 & -0.08946600 & -0.27157500 \\
\hline $\mathrm{O}$ & 8.55129900 & 0.76956300 & -1.05905300 \\
\hline $\mathrm{O}$ & 8.88487100 & -0.85145800 & 0.36216400 \\
\hline
\end{tabular}




\section{Cartesian Coordinates - Electrophiles}<smiles>O=[N+]([O-])C=Cc1ccccc1</smiles>

Nitroalkene-1

$\begin{array}{lrrr}\mathrm{C} & -3.08182300 & 1.06707700 & 0.00032100 \\ \mathrm{C} & -1.70406100 & 1.26621100 & 0.00014000 \\ \mathrm{C} & -0.81826900 & 0.17485700 & -0.00010500 \\ \mathrm{C} & -1.35311500 & -1.12746700 & -0.00049700 \\ \mathrm{C} & -2.72789100 & -1.32474200 & -0.00029900 \\ \mathrm{C} & -3.59696200 & -0.22890600 & 0.00013200 \\ \mathrm{C} & 0.61358000 & 0.44624100 & 0.00004600 \\ \mathrm{C} & 1.60147400 & -0.45849800 & 0.00060900 \\ \mathrm{H} & -3.75155300 & 1.92086900 & 0.00062900 \\ \mathrm{H} & -1.30061100 & 2.27447200 & 0.00023700 \\ \mathrm{H} & -0.69044200 & -1.98633400 & -0.00108700 \\ \mathrm{H} & -3.12717300 & -2.33384500 & -0.00061400 \\ \mathrm{H} & -4.67058100 & -0.38806000 & 0.00021300 \\ \mathrm{H} & 0.92028900 & 1.48891900 & -0.00079300 \\ \mathrm{H} & 1.51186600 & -1.53461300 & 0.00143200 \\ \mathrm{~N} & 2.98673800 & -0.03179700 & 0.00014200 \\ \mathrm{O} & 3.24889200 & 1.17340600 & -0.00069800 \\ \mathrm{O} & 3.82653700 & -0.93684000 & 0.00031000\end{array}$<smiles>O=[N+]([O-])C=Cc1ccc(F)cc1</smiles>

Nitroalkene-2

$\begin{array}{lrrr}\mathrm{C} & -2.59746300 & 1.17784200 & -0.00011300 \\ \mathrm{C} & -1.21581300 & 1.33434000 & -0.00011800 \\ \mathrm{C} & -0.35432900 & 0.22245500 & 0.00002700 \\ \mathrm{C} & -0.92287500 & -1.06673800 & -0.00002100 \\ \mathrm{C} & -2.29927400 & -1.23938600 & 0.00003800 \\ \mathrm{C} & -3.11713800 & -0.11089500 & -0.00003100 \\ \mathrm{C} & 1.08206800 & 0.45800400 & 0.00012600 \\ \mathrm{C} & 2.04915200 & -0.46945400 & 0.00020400 \\ \mathrm{~N} & 3.44303200 & -0.07351000 & 0.00008800 \\ \mathrm{O} & 4.26266400 & -0.99676600 & -0.00030800 \\ \mathrm{O} & 3.73120100 & 1.12588600 & 0.00010700 \\ \mathrm{~F} & -4.45044500 & -0.27766200 & 0.00006100 \\ \mathrm{H} & -3.26917000 & 2.02822000 & -0.00015900 \\ \mathrm{H} & -0.79124000 & 2.33341000 & -0.00025300 \\ \mathrm{H} & -0.28347100 & -1.94258900 & -0.00017600 \\ \mathrm{H} & -2.75017800 & -2.22492600 & -0.00001700 \\ \mathrm{H} & 1.41428100 & 1.49282400 & -0.00001000 \\ \mathrm{H} & 1.93567700 & -1.54337900 & 0.00039200\end{array}$


<smiles>O=[N+]([O-])C=Cc1cccc2ccccc12</smiles>

Nitroalkene-3

$\begin{array}{lrrr}\mathrm{C} & -1.22355200 & 2.62787000 & -0.08851800 \\ \mathrm{C} & 0.00814100 & 1.94970000 & -0.13473600 \\ \mathrm{C} & 0.07634300 & 0.56227700 & -0.10551100 \\ \mathrm{C} & -1.14030700 & -0.20153800 & -0.03073700 \\ \mathrm{C} & -2.39095400 & 0.49866100 & 0.00564800 \\ \mathrm{C} & -2.39990500 & 1.91617500 & -0.02276800 \\ \mathrm{C} & 1.36587200 & -0.11570900 & -0.18450500 \\ \mathrm{C} & 2.53755900 & 0.39981300 & 0.21054800 \\ \mathrm{~N} & 3.76536400 & -0.35146500 & 0.03957000 \\ \mathrm{O} & 4.79516100 & 0.20591000 & 0.43061900 \\ \mathrm{O} & 3.72080100 & -1.47566500 & -0.46712100 \\ \mathrm{C} & -3.60136500 & -0.23988600 & 0.07637300 \\ \mathrm{C} & -3.58902400 & -1.61365000 & 0.11740200 \\ \mathrm{C} & -2.35728400 & -2.30668300 & 0.09700300 \\ \mathrm{C} & -1.16575900 & -1.61969800 & 0.03096200 \\ \mathrm{H} & -1.23863000 & 3.71225400 & -0.12072900 \\ \mathrm{H} & 0.92339200 & 2.52387100 & -0.23230100 \\ \mathrm{H} & -3.35566900 & 2.43150200 & 0.00320000 \\ \mathrm{H} & 1.40748200 & -1.11845300 & -0.59705500 \\ \mathrm{H} & 2.70679300 & 1.35618800 & 0.68312600 \\ \mathrm{H} & -4.54024600 & 0.30568400 & 0.10076300 \\ \mathrm{H} & -4.51997100 & -2.16879200 & 0.17121000 \\ \mathrm{H} & -2.35056300 & -3.39118000 & 0.13972100 \\ \mathrm{H} & -0.23642500 & -2.17678700 & 0.04012400 \\ & & & \\ \mathrm{Me} & & & \\ & & & \end{array}$

Nitroalkene-4

$\begin{array}{lrrr}\mathrm{C} & 0.19948700 & 1.26816900 & 0.00001600 \\ \mathrm{C} & -1.27007000 & 0.98793700 & 0.00006000 \\ \mathrm{C} & -1.82368800 & -0.22362200 & 0.00007200 \\ \mathrm{~N} & -3.27276200 & -0.37870900 & 0.00006500 \\ \mathrm{O} & -3.98647500 & 0.62523400 & -0.00012400 \\ \mathrm{O} & -3.68624400 & -1.54030100 & -0.00002000 \\ \mathrm{C} & 1.12373900 & 0.04976600 & 0.00001100 \\ \mathrm{C} & 2.60506300 & 0.43529000 & -0.00000900 \\ \mathrm{C} & 3.54300800 & -0.77494000 & -0.00001000 \\ \mathrm{C} & 5.02176200 & -0.38320600 & -0.00002800 \\ \mathrm{H} & 0.41861700 & 1.90481500 & 0.87013000 \\ \mathrm{H} & 0.41858600 & 1.90479600 & -0.87012200 \\ \mathrm{H} & -1.94794400 & 1.83812600 & 0.00005700 \\ \mathrm{H} & -1.31639100 & -1.17679900 & 0.00006400\end{array}$




$\begin{array}{lrrr}\mathrm{H} & 0.90929500 & -0.57164900 & -0.87908400 \\ \mathrm{H} & 0.90931500 & -0.57163900 & 0.87911700 \\ \mathrm{H} & 2.81899600 & 1.06073400 & 0.87790800 \\ \mathrm{H} & 2.81897600 & 1.06072100 & -0.87794000 \\ \mathrm{H} & 3.32662500 & -1.39872500 & -0.87734500 \\ \mathrm{H} & 3.32664300 & -1.39871200 & 0.87734000 \\ \mathrm{H} & 5.27155400 & 0.21428500 & -0.88377200 \\ \mathrm{H} & 5.66943700 & -1.26511600 & -0.00002900 \\ \mathrm{H} & 5.27157300 & 0.21429800 & 0.88370200\end{array}$

Nitroalkene-5

\begin{tabular}{|c|c|c|c|}
\hline $\mathrm{C}$ & -0.83830000 & -1.20231400 & 0.00002300 \\
\hline $\mathrm{C}$ & -1.71932700 & 0.04764300 & 0.00000500 \\
\hline $\mathrm{C}$ & 0.64018500 & -0.97368600 & 0.00009000 \\
\hline $\mathrm{C}$ & 1.23586700 & 0.21773400 & 0.00009500 \\
\hline $\mathrm{N}$ & 2.68951100 & 0.32187100 & 0.00007500 \\
\hline $\mathrm{O}$ & 3.36746900 & -0.70654400 & -0.00016300 \\
\hline $\mathrm{O}$ & 3.14343900 & 1.46825200 & -0.00001100 \\
\hline $\mathrm{C}$ & -3.21402800 & -0.28546100 & -0.00002800 \\
\hline $\mathrm{C}$ & -4.09881900 & 0.96220900 & -0.00004100 \\
\hline $\mathrm{H}$ & -1.07949000 & -1.83086800 & -0.87017000 \\
\hline $\mathrm{H}$ & -1.07953800 & -1.83088000 & 0.87019000 \\
\hline $\mathrm{H}$ & -1.48395800 & 0.66157200 & -0.87911200 \\
\hline $\mathrm{H}$ & -1.48399400 & 0.66157100 & 0.87913300 \\
\hline $\mathrm{H}$ & 1.28783600 & -1.84710800 & 0.00008800 \\
\hline $\mathrm{H}$ & 0.76231700 & 1.18812200 & 0.00007500 \\
\hline $\mathrm{H}$ & -3.44660600 & -0.90298000 & 0.87757200 \\
\hline H & -3.44657000 & -0.90297400 & -0.87764000 \\
\hline H & -5.16055700 & 0.69839800 & -0.00006200 \\
\hline $\mathrm{H}$ & -3.90839400 & 1.58081600 & 0.88377900 \\
\hline & -3.90836000 & 1.58082100 & -0.88385000 \\
\hline
\end{tabular}

Nitroalkene-6

$\begin{array}{lrrr}\mathrm{C} & 0.86712600 & 0.38757500 & 0.00001900 \\ \mathrm{C} & -0.55511500 & 0.58421600 & 0.00006500 \\ \mathrm{C} & -1.49625800 & -0.37389900 & 0.00007200 \\ \mathrm{~N} & -2.89669800 & -0.01934400 & 0.00009500 \\ \mathrm{O} & -3.21682700 & 1.17322200 & -0.00006100 \\ \mathrm{O} & -3.69205100 & -0.96395900 & -0.00004200 \\ \mathrm{~S} & 1.63322300 & -1.19019000 & -0.00008200 \\ \mathrm{C} & 3.20724800 & -0.47201300 & 0.00012700 \\ \mathrm{C} & 3.15300600 & 0.89810100 & -0.00001500 \\ \mathrm{C} & 1.82147400 & 1.38766400 & -0.00006000 \\ \mathrm{H} & -0.90369000 & 1.61370000 & 0.00008000\end{array}$




$$
\begin{array}{rrrr}
\mathrm{H} & -1.33895300 & -1.44317300 & 0.00004700 \\
\mathrm{H} & 4.08408000 & -1.10420700 & 0.00022500 \\
\mathrm{H} & 4.03281700 & 1.52939100 & -0.00002200 \\
\mathrm{H} & 1.55719900 & 2.43877600 & -0.00010600
\end{array}
$$<smiles>CC(C)CC=C[N+](=O)[O-]</smiles>

Nitroalkene-7

$$
\begin{aligned}
& \begin{array}{llll}
\text { C } & -0.20464500 & -0.62053500 & 0.13100100
\end{array} \\
& \begin{array}{llll}
\mathrm{C} & -1.22447900 & -0.14971500 & -0.58424600
\end{array} \\
& \begin{array}{llll}
\mathrm{N} & -2.54114600 & 0.03153600 & 0.01273700
\end{array} \\
& \begin{array}{llll}
\mathrm{O} & -3.41510700 & 0.46900100 & -0.73870000
\end{array} \\
& \begin{array}{llll}
\mathrm{O} & -2.70615200 & -0.25184400 & 1.20018400
\end{array} \\
& \begin{array}{llll}
\mathrm{C} & 1.17556300 & -0.82569600 & -0.40213600
\end{array} \\
& \begin{array}{llll}
\mathrm{C} & 2.24155000 & 0.00469100 & 0.35170600
\end{array} \\
& \begin{array}{llll}
\mathrm{C} & 1.99162000 & 1.50808400 & 0.19306100
\end{array} \\
& \begin{array}{llll}
\mathrm{C} & 3.64405600 & -0.37933100 & -0.13044600
\end{array} \\
& \mathrm{H} \quad-0.39317400 \quad-0.85623500 \quad 1.17613700 \\
& \mathrm{H} \quad \begin{array}{llll}
\mathrm{H} & -1.20561600 & 0.13557400 & -1.62700100
\end{array} \\
& \mathrm{H} \quad \begin{array}{llll}
1.21321900 & -0.58612300 & -1.47161600
\end{array} \\
& \mathrm{H} \quad \begin{array}{llll}
1.42895900 & -1.89006300 & -0.30202500
\end{array} \\
& \begin{array}{llll}
\mathrm{H} & 2.16352000 & -0.24765400 & 1.41877400
\end{array} \\
& \mathrm{H} \quad 2.05011200 \quad 1.79975800 \quad-0.86243700 \\
& \begin{array}{llll}
\mathrm{H} & 1.00384200 & 1.79659500 & 0.56373400
\end{array} \\
& \begin{array}{llll}
\mathrm{H} & 2.74103800 & 2.08755500 & 0.74064900
\end{array} \\
& \mathrm{H} \quad 3.76343600 \quad-0.15520800 \quad-1.19690900 \\
& \begin{array}{lllll}
\mathrm{H} & 4.41111600 & 0.17996100 & 0.41387800
\end{array} \\
& \mathrm{H} \quad 3.83964400 \quad-1.44716000 \quad 0.01215100
\end{aligned}
$$

Nitroalkene-8

$\begin{array}{lrrr}\mathrm{C} & -1.11963600 & 0.29918800 & -0.00004100 \\ \mathrm{C} & 0.28728700 & 0.53972100 & -0.00009800 \\ \mathrm{C} & 1.23426100 & -0.41319900 & -0.00011500 \\ \mathrm{~N} & 2.63095700 & -0.04769400 & -0.00017200 \\ \mathrm{O} & 3.43504800 & -0.98505100 & 0.00014300 \\ \mathrm{O} & 2.94108700 & 1.14808200 & 0.00012800 \\ \mathrm{O} & -1.57377300 & -0.99794800 & 0.00001600 \\ \mathrm{C} & -2.92999500 & -0.94685700 & -0.00000900 \\ \mathrm{C} & -3.36708800 & 0.34758400 & 0.00011400 \\ \mathrm{C} & -2.19557500 & 1.15730400 & -0.00002300 \\ \mathrm{H} & 0.60981200 & 1.57605200 & -0.00012900 \\ \mathrm{H} & 1.07203000 & -1.48044300 & -0.00008500 \\ \mathrm{H} & -3.43677700 & -1.89910500 & -0.00000800 \\ \mathrm{H} & -4.39481200 & 0.67809700 & 0.00020900 \\ \mathrm{H} & -2.14136200 & 2.23615300 & -0.00003700\end{array}$


<smiles>O=[N+]([O-])C=Cc1c[nH]c2ccccc12</smiles>

Nitroalkene-9

$\begin{array}{lrrr}\mathrm{C} & 0.00678300 & 0.90927800 & 0.00005100 \\ \mathrm{C} & -1.41873500 & 0.75842000 & 0.00003400 \\ \mathrm{C} & -2.12081900 & -0.38920300 & -0.00013500 \\ \mathrm{~N} & -3.56230000 & -0.35662700 & -0.00005200 \\ \mathrm{O} & -4.12891400 & -1.45596000 & -0.00028300 \\ \mathrm{O} & -4.14801600 & 0.73220500 & 0.00030300 \\ \mathrm{C} & 0.62257100 & 2.15282400 & 0.00001900 \\ \mathrm{~N} & 1.97912500 & 2.00228800 & -0.00020600 \\ \mathrm{C} & 2.29842200 & 0.65094800 & -0.00007300 \\ \mathrm{C} & 1.07752600 & -0.07398600 & 0.00007400 \\ \mathrm{C} & 3.54844100 & 0.03214800 & -0.00013000 \\ \mathrm{C} & 3.57082100 & -1.35765200 & -0.00001000 \\ \mathrm{C} & 2.37678000 & -2.10140000 & 0.00015000 \\ \mathrm{C} & 1.13545300 & -1.47605900 & 0.00019400 \\ \mathrm{H} & -2.01064200 & 1.67048700 & 0.00025900 \\ \mathrm{H} & -1.73965800 & -1.39716700 & -0.00040300 \\ \mathrm{H} & 0.16764100 & 3.13292900 & -0.00004700 \\ \mathrm{H} & 2.64707800 & 2.75617700 & 0.00047100 \\ \mathrm{H} & 4.46596500 & 0.61223500 & -0.00023800 \\ \mathrm{H} & 4.52400400 & -1.87614000 & -0.00004300 \\ \mathrm{H} & 2.42699600 & -3.18533600 & 0.00024000 \\ \mathrm{H} & 0.23281800 & -2.07468700 & 0.00035700\end{array}$<smiles>O=[N+]([O-])C=CC1CCCCC1</smiles>

Nitroalkene-10

$\begin{array}{lrrr}\mathrm{C} & -2.64971900 & 1.31827600 & -0.34369500 \\ \mathrm{C} & -1.13064000 & 1.17141000 & -0.18990900 \\ \mathrm{C} & -0.66814400 & -0.25098900 & -0.53542100 \\ \mathrm{C} & -1.42732500 & -1.29562600 & 0.31826700 \\ \mathrm{C} & -2.94561700 & -1.14245700 & 0.16763300 \\ \mathrm{C} & -3.40212000 & 0.28301000 & 0.50135500 \\ \mathrm{C} & 0.80729900 & -0.47880900 & -0.41906700 \\ \mathrm{C} & 1.70099300 & 0.40771400 & 0.01647600 \\ \mathrm{~N} & 3.11354800 & 0.05904500 & 0.10376400 \\ \mathrm{O} & 3.86061200 & 0.95070200 & 0.51290500 \\ \mathrm{O} & 3.47742600 & -1.07110300 & -0.22452500 \\ \mathrm{H} & -2.91911000 & 1.18800900 & -1.40089100 \\ \mathrm{H} & -2.95518500 & 2.33308800 & -0.06676000 \\ \mathrm{H} & -0.84980300 & 1.39495600 & 0.84844700 \\ \mathrm{H} & -0.61216300 & 1.89974000 & -0.82378100\end{array}$




$$
\begin{array}{lrrr}
\mathrm{H} & -0.93191900 & -0.45053300 & -1.58721300 \\
\mathrm{H} & -1.14348200 & -1.15765900 & 1.36988000 \\
\mathrm{H} & -1.11302300 & -2.30703600 & 0.03655700 \\
\mathrm{H} & -3.45659000 & -1.86872900 & 0.80923600 \\
\mathrm{H} & -3.22996800 & -1.38153200 & -0.86637500 \\
\mathrm{H} & -4.48259900 & 0.38098400 & 0.34905500 \\
\mathrm{H} & -3.21505900 & 0.48170400 & 1.56567600 \\
\mathrm{H} & 1.18386400 & -1.46137600 & -0.69523000 \\
\mathrm{H} & 1.51752700 & 1.42310400 & 0.33418400
\end{array}
$$<smiles>O=[N+]([O-])C1=CCCCC1</smiles>

Nitroalkene-11

$\begin{array}{lrrr}\mathrm{C} & 0.35711700 & 0.03461300 & -0.01420600 \\ \mathrm{~N} & 1.82897400 & 0.02774200 & 0.01562700 \\ \mathrm{O} & 2.37034900 & -1.07385500 & 0.13550200 \\ \mathrm{O} & 2.43607500 & 1.09581100 & -0.07034300 \\ \mathrm{C} & -0.26725800 & 1.21511300 & -0.06560200 \\ \mathrm{C} & -1.76330800 & 1.32861800 & -0.04905200 \\ \mathrm{C} & -2.44684400 & 0.01636200 & 0.35920000 \\ \mathrm{C} & -1.79570700 & -1.17766500 & -0.34686900 \\ \mathrm{C} & -0.31219700 & -1.30747600 & 0.03009300 \\ \mathrm{H} & 0.33451200 & 2.11631800 & -0.11766100 \\ \mathrm{H} & -2.09616700 & 1.64017800 & -1.05057600 \\ \mathrm{H} & -2.05389000 & 2.14669600 & 0.62121900 \\ \mathrm{H} & -3.51627200 & 0.06570800 & 0.13195100 \\ \mathrm{H} & -2.35735700 & -0.11348200 & 1.44504900 \\ \mathrm{H} & -1.88106400 & -1.04450900 & -1.43284700 \\ \mathrm{H} & -2.31897300 & -2.10615000 & -0.09917700 \\ \mathrm{H} & 0.20614800 & -1.99469600 & -0.64385800 \\ \mathrm{H} & -0.20196700 & -1.73730200 & 1.03385200\end{array}$<smiles>O=[N+]([O-])C=Cc1ccc2ccccc2c1</smiles>

Nitroalkene-12 


$\begin{array}{lrrr}\mathrm{C} & -3.62259300 & 1.12221000 & -0.00000700 \\ \mathrm{C} & -4.58701200 & 0.13829000 & -0.00001500 \\ \mathrm{C} & -4.21761200 & -1.22787200 & -0.00001600 \\ \mathrm{C} & -2.88930300 & -1.58737200 & -0.00000700 \\ \mathrm{H} & -0.21451100 & -1.97990400 & 0.00001100 \\ \mathrm{H} & 0.86345800 & 2.18990900 & 0.00003500 \\ \mathrm{H} & -1.50587100 & 2.81839900 & 0.00001700 \\ \mathrm{H} & 2.07536100 & -1.44999000 & 0.00004300 \\ \mathrm{H} & 2.98382900 & 1.49560000 & -0.00003500 \\ \mathrm{H} & -3.90492400 & 2.17102900 & -0.00000600 \\ \mathrm{H} & -5.63816700 & 0.40851100 & -0.00002200 \\ \mathrm{H} & -4.98842000 & -1.99173200 & -0.00002300 \\ \mathrm{H} & -2.59981000 & -2.63425500 & -0.00000800\end{array}$<smiles>O=[N+]([O-])C/C=C/c1ccccc1[N+](=O)[O-]</smiles>

Nitroalkene-13

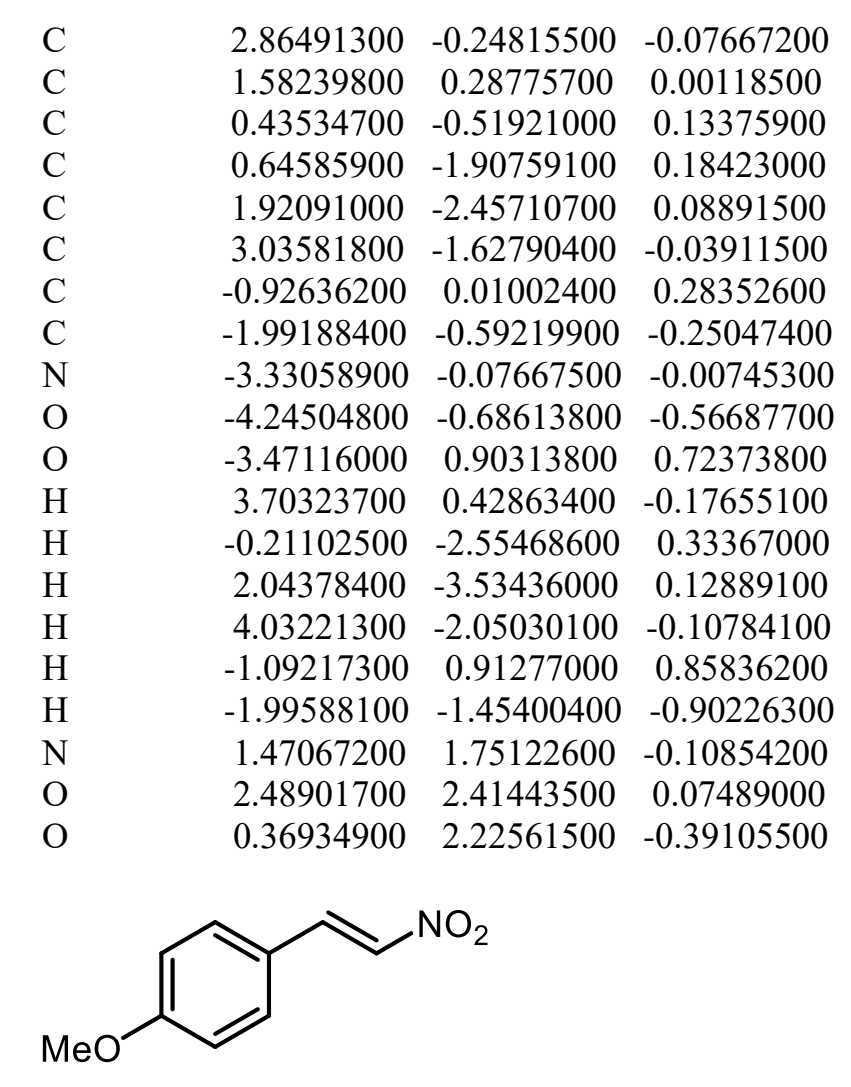

Nitroalkene-14

$\begin{array}{lrrr}\mathrm{C} & -2.15964800 & 1.02582600 & 0.00003600 \\ \mathrm{C} & -0.78304900 & 1.22911800 & -0.00000100 \\ \mathrm{C} & 0.12379500 & 0.15780400 & -0.00004100\end{array}$




\begin{tabular}{lrrr}
$\mathrm{C}$ & -0.40409300 & -1.15228600 & -0.00006100 \\
$\mathrm{C}$ & -1.76711500 & -1.37060200 & -0.00002800 \\
$\mathrm{C}$ & -2.65938700 & -0.28230500 & 0.00002500 \\
$\mathrm{C}$ & 1.54577900 & 0.44596300 & -0.00005800 \\
$\mathrm{C}$ & 2.55024900 & -0.44424100 & 0.00003200 \\
$\mathrm{O}$ & -3.97549400 & -0.60527900 & 0.00005700 \\
$\mathrm{C}$ & -4.92841400 & 0.45053700 & 0.00009500 \\
$\mathrm{H}$ & -2.82582100 & 1.87886700 & 0.00007100 \\
$\mathrm{H}$ & -0.39879300 & 2.24494600 & 0.00000700 \\
$\mathrm{H}$ & 0.26507600 & -2.00610800 & -0.00011600 \\
$\mathrm{H}$ & -2.17923700 & -2.37338000 & -0.00004800 \\
$\mathrm{H}$ & 1.83930900 & 1.49253400 & -0.00014800 \\
$\mathrm{H}$ & 2.47703400 & -1.52155500 & 0.00014900 \\
$\mathrm{H}$ & -4.83019900 & 1.07722600 & -0.89419800 \\
$\mathrm{H}$ & -5.90667400 & -0.02979900 & 0.00010800 \\
$\mathrm{H}$ & -4.83016100 & 1.07719600 & 0.89440400 \\
$\mathrm{~N}$ & 3.92483500 & 0.00357400 & -0.00000500 \\
$\mathrm{O}$ & 4.16947300 & 1.21396100 & -0.00015800 \\
$\mathrm{O}$ & 4.78188600 & -0.88666000 & 0.00007900 \\
& & & \\
$i-\mathrm{Pr}$ & $\mathrm{NO}_{2}$ & & \\
\hline
\end{tabular}

Nitroalkene-15

$\begin{array}{lrrr}\mathrm{C} & 0.35985400 & -0.41680200 & -0.33325400 \\ \mathrm{C} & -0.61151200 & 0.43199400 & -0.00266700 \\ \mathrm{~N} & -2.00101200 & -0.00768200 & 0.04152400 \\ \mathrm{O} & -2.27187000 & -1.17835300 & -0.22916700 \\ \mathrm{O} & -2.82472800 & 0.85376700 & 0.35689400 \\ \mathrm{C} & 1.82485900 & -0.09459100 & -0.39724100 \\ \mathrm{C} & 2.59565000 & -1.03898900 & 0.54721900 \\ \mathrm{C} & 2.15783100 & 1.37281100 & -0.11687600 \\ \mathrm{H} & 0.06362100 & -1.43867600 & -0.55950200 \\ \mathrm{H} & -0.51224800 & 1.47604100 & 0.25408900 \\ \mathrm{H} & 2.14342200 & -0.33301300 & -1.42378000 \\ \mathrm{H} & 3.67319200 & -0.88183400 & 0.44393200 \\ \mathrm{H} & 2.32065400 & -0.84797100 & 1.58934900 \\ \mathrm{H} & 2.38159800 & -2.08924500 & 0.32766200 \\ \mathrm{H} & 1.87463700 & 1.65165200 & 0.90379600 \\ \mathrm{H} & 3.23284900 & 1.54327600 & -0.22085500 \\ \mathrm{H} & 1.64205600 & 2.04369400 & -0.81025900\end{array}$<smiles>O=[N+]([O-])/C=C/c1ccc(Cl)cc1Cl</smiles>

Nitroalkene-16
C
$-2.13695100$
$0.77690100-0.02991700$
C
$-0.75630600$
$0.95602200-0.00103000$ 


$\begin{array}{lrrc}\mathrm{C} & 0.13723600 & -0.13349200 & 0.05985700 \\ \mathrm{C} & -0.43208600 & -1.42150600 & 0.10298500 \\ \mathrm{C} & -1.80231000 & -1.62606700 & 0.07533500 \\ \mathrm{C} & -2.64794200 & -0.51696400 & 0.00715300 \\ \mathrm{C} & 1.57962000 & 0.07229100 & 0.09175200 \\ \mathrm{C} & 2.50552700 & -0.87607200 & -0.09359300 \\ \mathrm{Cl} & -0.16193500 & 2.60315400 & -0.06138000 \\ \mathrm{Cl} & -4.37888400 & -0.75140600 & -0.02526100 \\ \mathrm{H} & -2.79806800 & 1.63209400 & -0.08207100 \\ \mathrm{H} & 0.22213200 & -2.28267400 & 0.17854500 \\ \mathrm{H} & -2.21684800 & -2.62592900 & 0.11404100 \\ \mathrm{H} & 1.94611500 & 1.07706000 & 0.27163900 \\ \mathrm{H} & 2.35177000 & -1.92117800 & -0.31862700 \\ \mathrm{~N} & 3.91770200 & -0.54447100 & -0.01660100 \\ \mathrm{O} & 4.25209700 & 0.61614400 & 0.22549000 \\ \mathrm{O} & 4.69592700 & -1.48295100 & -0.20670100\end{array}$<smiles>Cc1cc(C)c(/C=C/[N+](=O)[O-])c(C)c1</smiles>

Nitroalkene-17

$\begin{array}{lrrr}\mathrm{C} & -2.28623400 & -1.25922100 & -0.04554100 \\ \mathrm{C} & -0.89404300 & -1.17352600 & -0.07119300 \\ \mathrm{C} & -0.27893300 & 0.10642400 & -0.03899200 \\ \mathrm{C} & -1.10362900 & 1.26638700 & 0.00942200 \\ \mathrm{C} & -2.48779800 & 1.12447500 & 0.04553500 \\ \mathrm{C} & -3.10332300 & -0.12983200 & 0.02196400 \\ \mathrm{C} & 1.15970600 & 0.32370200 & -0.08074900 \\ \mathrm{C} & 2.18632100 & -0.51732500 & 0.12856100 \\ \mathrm{~N} & 3.54630800 & -0.01535300 & 0.03584600 \\ \mathrm{O} & 3.74697000 & 1.16333000 & -0.27040000 \\ \mathrm{O} & 4.43545900 & -0.83734300 & 0.28020500 \\ \mathrm{C} & -0.52139800 & 2.66108400 & 0.03652800 \\ \mathrm{C} & -0.11102100 & -2.45803400 & -0.15699900 \\ \mathrm{C} & -4.60264100 & -0.25758900 & 0.08839000 \\ \mathrm{H} & -2.74712600 & -2.24268500 & -0.08249700 \\ \mathrm{H} & -3.10601900 & 2.01710200 & 0.08924100 \\ \mathrm{H} & 1.48419400 & 1.33600000 & -0.29757400 \\ \mathrm{H} & 2.17709200 & -1.55793500 & 0.39925200 \\ \mathrm{H} & -1.31949300 & 3.40318300 & 0.10400800 \\ \mathrm{H} & 0.14606200 & 2.81133300 & 0.89092600 \\ \mathrm{H} & 0.05591500 & 2.88188600 & -0.86819800 \\ \mathrm{H} & 0.64267500 & -2.42412000 & -0.94971800 \\ \mathrm{H} & 0.41028900 & -2.67822600 & 0.78184800 \\ \mathrm{H} & -0.77934400 & -3.29722200 & -0.36110700 \\ \mathrm{H} & -5.09677000 & 0.56913300 & -0.42995400 \\ \mathrm{H} & -4.94423700 & -1.19608200 & -0.35624400 \\ \mathrm{H} & -4.94887300 & -0.24207200 & 1.12910300\end{array}$


<smiles>O=[N+]([O-])C=Cc1ccccc1F</smiles>

Nitroalkene-18

$\begin{array}{lrrr}\mathrm{C} & 2.98545200 & -1.35984200 & 0.00002200 \\ \mathrm{C} & 1.60254200 & -1.49812300 & -0.00000900 \\ \mathrm{C} & 0.74628500 & -0.37927800 & -0.00002600 \\ \mathrm{C} & 1.36319900 & 0.88307000 & -0.00000700 \\ \mathrm{C} & 2.73876100 & 1.04687600 & 0.00002400 \\ \mathrm{C} & 3.55475900 & -0.08466500 & 0.00004000 \\ \mathrm{C} & -0.69128700 & -0.59668600 & -0.00005800 \\ \mathrm{C} & -1.66892800 & 0.32026100 & -0.00006700 \\ \mathrm{~N} & -3.05597100 & -0.11330100 & -0.00007700 \\ \mathrm{O} & -3.89714200 & 0.78913400 & 0.00010200 \\ \mathrm{O} & -3.31601300 & -1.31927900 & 0.00005100 \\ \mathrm{~F} & 0.59408100 & 1.99215000 & -0.00002000 \\ \mathrm{H} & 3.61781100 & -2.24076800 & 0.00003600 \\ \mathrm{H} & 1.15514400 & -2.48733900 & -0.00001900 \\ \mathrm{H} & 3.14778700 & 2.05052100 & 0.00003700 \\ \mathrm{H} & 4.63322600 & 0.03383200 & 0.00006500 \\ \mathrm{H} & -1.01668100 & -1.63369500 & -0.00007000 \\ \mathrm{H} & -1.57168300 & 1.39268500 & -0.00007500\end{array}$<smiles>Cc1ccccc1/C=C/[N+](=O)[O-]</smiles>

Nitroalkene-19

$\begin{array}{lrrr}\mathrm{C} & 2.48292900 & -1.77139900 & -0.05418200 \\ \mathrm{C} & 1.13459700 & -1.44459700 & -0.08704300 \\ \mathrm{C} & 0.70692900 & -0.10425600 & -0.04406500 \\ \mathrm{C} & 1.67381300 & 0.92994400 & 0.02275000 \\ \mathrm{C} & 3.02621900 & 0.57839800 & 0.05608600 \\ \mathrm{C} & 3.43504500 & -0.75303200 & 0.01971700 \\ \mathrm{C} & -0.71047100 & 0.23705700 & -0.07348900 \\ \mathrm{C} & -1.73923800 & -0.60867900 & 0.07710400 \\ \mathrm{C} & 1.28127300 & 2.38691900 & 0.07067600 \\ \mathrm{H} & 2.79127300 & -2.81100000 & -0.09213600 \\ \mathrm{H} & 0.39772900 & -2.23703000 & -0.16397900 \\ \mathrm{H} & 3.77124800 & 1.36655000 & 0.11166200 \\ \mathrm{H} & 4.49304600 & -0.99464100 & 0.04395900 \\ \mathrm{H} & -0.98388500 & 1.27611200 & -0.22275000 \\ \mathrm{H} & -1.70294800 & -1.67176900 & 0.26264400 \\ \mathrm{H} & 2.16889300 & 3.02043600 & 0.12752300 \\ \mathrm{H} & 0.71855900 & 2.68671800 & -0.82024100 \\ \mathrm{H} & 0.65539200 & 2.61210100 & 0.94037000\end{array}$




$\begin{array}{lrrr}\mathrm{N} & -3.10231800 & -0.11988900 & 0.01070900 \\ \mathrm{O} & -3.98513200 & -0.96981700 & 0.16176800 \\ \mathrm{O} & -3.30732500 & 1.08101900 & -0.18518400\end{array}$<smiles>O=[N+]([O-])/C=C/c1cccc(Cl)c1</smiles>

Nitroalkene-20

$\begin{array}{lrrr}\mathrm{C} & 2.05071800 & 2.13170200 & 0.00007400 \\ \mathrm{C} & 0.66512700 & 1.99614300 & 0.00001700 \\ \mathrm{C} & 0.07038600 & 0.72372000 & -0.00007600 \\ \mathrm{C} & 0.89670900 & -0.41486100 & -0.00006800 \\ \mathrm{C} & 2.27552400 & -0.26035500 & 0.00000000 \\ \mathrm{C} & 2.86937000 & 1.00439300 & 0.00006900 \\ \mathrm{C} & -1.38594300 & 0.63727700 & -0.00011400 \\ \mathrm{C} & -2.12102100 & -0.48109100 & 0.00004900 \\ \mathrm{~N} & -3.57084100 & -0.40435000 & 0.00004100 \\ \mathrm{O} & -4.11628700 & 0.70118900 & -0.00006000 \\ \mathrm{O} & -4.16336100 & -1.48642200 & 0.00008500 \\ \mathrm{Cl} & 3.29781300 & -1.68588400 & -0.00001900 \\ \mathrm{H} & 2.49948900 & 3.11940500 & 0.00013200 \\ \mathrm{H} & 0.03200600 & 2.87763600 & 0.00002600 \\ \mathrm{H} & 0.47375700 & -1.41193600 & -0.00015000 \\ \mathrm{H} & 3.94914200 & 1.09513700 & 0.00012700 \\ \mathrm{H} & -1.93677600 & 1.57392600 & -0.00024900 \\ \mathrm{H} & -1.77260000 & -1.50339200 & 0.00023300\end{array}$<smiles>COc1cccc(/C=C/[N+](=O)[O-])c1</smiles>

Nitroalkene-21

$\begin{array}{lrrr}\mathrm{C} & 2.10723700 & -2.22076900 & 0.00002900 \\ \mathrm{C} & 0.71867900 & -2.07824400 & -0.00047900 \\ \mathrm{C} & 0.14366200 & -0.80024700 & -0.00023900 \\ \mathrm{C} & 0.97717000 & 0.33706400 & 0.00029500 \\ \mathrm{C} & 2.36130800 & 0.18497700 & 0.00066300 \\ \mathrm{C} & 2.92779600 & -1.10236700 & 0.00060900 \\ \mathrm{C} & -1.30990800 & -0.70032600 & -0.00043400 \\ \mathrm{C} & -2.03724200 & 0.42498800 & 0.00067700 \\ \mathrm{~N} & -3.48478300 & 0.36540700 & 0.00020000 \\ \mathrm{O} & -4.04606800 & -0.73290300 & -0.00105100 \\ \mathrm{O} & -4.06651900 & 1.45489300 & 0.00094000 \\ \mathrm{O} & 3.25136600 & 1.21374200 & 0.00130200 \\ \mathrm{C} & 2.74058000 & 2.53911500 & -0.00177400 \\ \mathrm{H} & 2.55005800 & -3.21135500 & -0.00007600 \\ \mathrm{H} & 0.07726800 & -2.95342200 & -0.00102700 \\ \mathrm{H} & 0.53494500 & 1.32415300 & 0.00037000 \\ \mathrm{H} & 4.00867100 & -1.18889800 & 0.00091400\end{array}$




$$
\begin{array}{lrrr}
\mathrm{H} & -1.86905300 & -1.63199600 & -0.00149100 \\
\mathrm{H} & -1.67816700 & 1.44333800 & 0.00201400 \\
\mathrm{H} & 3.61039700 & 3.19604700 & -0.00328400 \\
\mathrm{H} & 2.13669700 & 2.73453100 & -0.89650000 \\
\mathrm{H} & 2.13673100 & 2.73874700 & 0.89206600
\end{array}
$$<smiles>O=[N+]([O-])C=Cc1ccc(Br)cc1</smiles>

Nitroalkene-22

$\begin{array}{lrrr}\mathrm{C} & 1.34596200 & 1.30638900 & 0.00014000 \\ \mathrm{C} & -0.04022600 & 1.42042200 & 0.00003900 \\ \mathrm{C} & -0.86607400 & 0.28324400 & -0.00026800 \\ \mathrm{C} & -0.25656500 & -0.98586000 & -0.00067900 \\ \mathrm{C} & 1.12505600 & -1.11386300 & -0.00053800 \\ \mathrm{C} & 1.91790400 & 0.03637700 & -0.00016000 \\ \mathrm{C} & -2.31012300 & 0.47336500 & -0.00010400 \\ \mathrm{C} & -3.24393600 & -0.48677600 & 0.00053900 \\ \mathrm{~N} & -4.65189100 & -0.13931400 & 0.00022200 \\ \mathrm{O} & -5.43803900 & -1.09078500 & 0.00046800 \\ \mathrm{O} & -4.98060300 & 1.04918200 & -0.00047300 \\ \mathrm{Br} & 3.81265300 & -0.13861300 & 0.00015800 \\ \mathrm{H} & 1.97560800 & 2.18787100 & 0.00048000 \\ \mathrm{H} & -0.49383100 & 2.40685400 & 0.00021800 \\ \mathrm{H} & -0.86542300 & -1.88341600 & -0.00125200 \\ \mathrm{H} & 1.59036800 & -2.09219200 & -0.00084600 \\ \mathrm{H} & -2.67584100 & 1.49673900 & -0.00082500 \\ \mathrm{H} & -3.09333200 & -1.55616700 & 0.00135400\end{array}$<smiles>CCCc1ccc(/C=C/[N+](=O)[O-])cc1</smiles>

Nitroalkene-23

$\begin{array}{lrrr}\mathrm{C} & -1.52742900 & -0.92995700 & -0.00008400 \\ \mathrm{C} & -0.14263400 & -0.87498100 & -0.00012000 \\ \mathrm{C} & 0.52771500 & 0.36399400 & 0.00001200 \\ \mathrm{C} & -0.24801500 & 1.53455900 & 0.00014700 \\ \mathrm{C} & -1.63787300 & 1.47119400 & 0.00020300 \\ \mathrm{C} & -2.30390400 & 0.24172800 & 0.00011900 \\ \mathrm{C} & 1.97747800 & 0.48797300 & 0.00000600 \\ \mathrm{C} & 2.86903200 & -0.51296800 & -0.00004200 \\ \mathrm{~N} & 4.28938100 & -0.23050600 & -0.00002800 \\ \mathrm{O} & 4.67445100 & 0.94179800 & 0.00004900 \\ \mathrm{O} & 5.03332700 & -1.21641800 & -0.00005900 \\ \mathrm{C} & -3.81947100 & 0.17630400 & 0.00011200 \\ \mathrm{C} & -4.35236800 & -0.51130400 & 1.26857500 \\ \mathrm{C} & -4.35233400 & -0.51054000 & -1.26879600\end{array}$




$\begin{array}{lrrr}\mathrm{H} & -2.01803200 & -1.89836700 & -0.00019200 \\ \mathrm{H} & 0.42572800 & -1.79925300 & -0.00024600 \\ \mathrm{H} & 0.24974000 & 2.49997300 & 0.00023100 \\ \mathrm{H} & -2.21695900 & 2.39020000 & 0.00032300 \\ \mathrm{H} & 2.38903800 & 1.49392500 & 0.00005200 \\ \mathrm{H} & 2.66960700 & -1.57421700 & -0.00008100 \\ \mathrm{H} & -4.18786000 & 1.20971500 & 0.00040900 \\ \mathrm{H} & -3.99334600 & -0.00896000 & 2.17138900 \\ \mathrm{H} & -5.44684900 & -0.49948700 & 1.28015600 \\ \mathrm{H} & -4.03005600 & -1.55672300 & 1.31598300 \\ \mathrm{H} & -3.99322900 & -0.00768400 & -2.17129300 \\ \mathrm{H} & -4.03003400 & -1.55593400 & -1.31678300 \\ \mathrm{H} & -5.44681500 & -0.49870100 & -1.28046500\end{array}$<smiles>O=[N+]([O-])C=Cc1ccc(Cl)c(Cl)c1</smiles>

Nitroalkene-24

$\begin{array}{lrrr}\mathrm{C} & -1.20163300 & -1.71756800 & 0.00012200 \\ \mathrm{C} & 0.14801700 & -1.40582800 & 0.00018700 \\ \mathrm{C} & 0.57262800 & -0.06437700 & 0.00011500 \\ \mathrm{C} & -0.40001100 & 0.94567900 & 0.00017400 \\ \mathrm{C} & -1.75817300 & 0.63734700 & 0.00001700 \\ \mathrm{C} & -2.16535900 & -0.70256300 & 0.00005800 \\ \mathrm{C} & 1.97541900 & 0.33172900 & 0.00012600 \\ \mathrm{C} & 3.03341500 & -0.48830300 & -0.00024000 \\ \mathrm{~N} & 4.38010100 & 0.05389400 & -0.00012700 \\ \mathrm{O} & 4.53759600 & 1.27628200 & 0.00027600 \\ \mathrm{O} & 5.29061000 & -0.77854900 & -0.00024200 \\ \mathrm{Cl} & -3.84761900 & -1.14070400 & -0.00004900 \\ \mathrm{Cl} & -2.91601300 & 1.93891400 & -0.00014200 \\ \mathrm{H} & -1.53026400 & -2.75006400 & 0.00022600 \\ \mathrm{H} & 0.87357500 & -2.21110400 & 0.00046100 \\ \mathrm{H} & -0.10034900 & 1.98761600 & 0.00024800 \\ \mathrm{H} & 2.19229500 & 1.39645400 & 0.00035800 \\ \mathrm{H} & 3.03430400 & -1.56828800 & -0.00078000\end{array}$<smiles>COc1ccc(/C=C/[N+](=O)[O-])cc1OC</smiles>

Nitroalkene-25

$\begin{array}{lrrr}\mathrm{C} & 2.13680500 & -1.12107500 & 0.00000000 \\ \mathrm{C} & 0.82441500 & -1.59437500 & 0.00000000 \\ \mathrm{C} & -0.25924500 & -0.71088700 & 0.00000000 \\ \mathrm{C} & 0.00000000 & 0.68001200 & 0.00000000 \\ \mathrm{C} & 1.29703400 & 1.16323200 & 0.00000000 \\ \mathrm{C} & 2.38968200 & 0.24908700 & 0.00000000\end{array}$




\begin{tabular}{|c|c|c|c|}
\hline $\mathrm{C}$ & -1.60231100 & -1.25703400 & 0.00000000 \\
\hline $\mathrm{C}$ & -2.75491400 & -0.56800900 & 0.00000000 \\
\hline $\mathrm{N}$ & -4.02290900 & -1.25917100 & 0.00000000 \\
\hline $\mathrm{O}$ & -5.02927800 & -0.54089800 & 0.00000000 \\
\hline $\mathrm{O}$ & -4.04235300 & -2.49408100 & 0.00000000 \\
\hline $\mathrm{O}$ & 3.61747200 & 0.81527900 & 0.00000000 \\
\hline $\mathrm{C}$ & 4.74833400 & -0.04533300 & 0.00000000 \\
\hline $\mathrm{O}$ & 1.64440800 & 2.47535500 & 0.00000000 \\
\hline $\mathrm{C}$ & 0.59776000 & 3.43401700 & 0.00000000 \\
\hline $\mathrm{H}$ & 2.95781100 & -1.82628200 & 0.00000000 \\
\hline $\mathrm{H}$ & 0.64216100 & -2.66416000 & 0.00000000 \\
\hline $\mathrm{H}$ & -0.82862300 & 1.37521500 & 0.00000000 \\
\hline $\mathrm{H}$ & -1.69520600 & -2.33977300 & 0.00000000 \\
\hline $\mathrm{H}$ & -2.88116400 & 0.50432000 & 0.00000000 \\
\hline $\mathrm{H}$ & 5.61988600 & 0.60899100 & 0.00000000 \\
\hline $\mathrm{H}$ & 4.76783600 & -0.67970000 & 0.89431400 \\
\hline $\mathrm{H}$ & 4.76783600 & -0.67970000 & -0.89431400 \\
\hline $\mathrm{H}$ & 1.08538200 & 4.40884300 & 0.00000000 \\
\hline $\mathrm{H}$ & -0.03145300 & 3.34169700 & -0.89411200 \\
\hline & -0.03145300 & 3.34169700 & 0.89411200 \\
\hline & & & \\
\hline
\end{tabular}

Nitroalkene-26

$\begin{array}{lrrc}\mathrm{C} & -1.68973500 & 1.83968700 & -0.43019800 \\ \mathrm{C} & -0.32512300 & 1.79397000 & -0.29299500 \\ \mathrm{C} & 0.33465700 & 0.57923900 & 0.05667400 \\ \mathrm{C} & -0.42915800 & -0.55358500 & 0.28413200 \\ \mathrm{C} & -1.83979800 & -0.53334900 & 0.16471000 \\ \mathrm{C} & -2.48891400 & 0.68766100 & -0.20588600 \\ \mathrm{C} & 1.81084100 & 0.55002200 & 0.17380100 \\ \mathrm{C} & 2.44365400 & -0.54729800 & -0.29180900 \\ \mathrm{C} & 2.49262900 & 1.74462500 & 0.79011300 \\ \mathrm{~N} & 3.86340500 & -0.78501600 & -0.22372200 \\ \mathrm{O} & 4.62511500 & 0.11829700 & 0.14193900 \\ \mathrm{O} & 4.23393300 & -1.91586400 & -0.55674100 \\ \mathrm{C} & -3.90151800 & 0.70557400 & -0.32808200 \\ \mathrm{C} & -4.64069200 & -0.43145400 & -0.09305900 \\ \mathrm{C} & -3.99987800 & -1.63869300 & 0.27759000 \\ \mathrm{C} & -2.63126300 & -1.68775800 & 0.40556600 \\ \mathrm{H} & -2.17793400 & 2.76715900 & -0.71526900 \\ \mathrm{H} & 0.26461200 & 2.68491800 & -0.47945400 \\ \mathrm{H} & 0.05186200 & -1.47571200 & 0.59538100 \\ \mathrm{H} & 1.93184900 & -1.37613100 & -0.75772900 \\ \mathrm{H} & 1.75977400 & 2.40760600 & 1.25123200 \\ \mathrm{H} & 3.22039400 & 1.42751100 & 1.53802600 \\ \mathrm{H} & 3.06225000 & 2.30084700 & 0.03929900 \\ \mathrm{H} & -4.39058100 & 1.63341700 & -0.61064900\end{array}$




$$
\begin{array}{lrrr}
H & -5.72152600 & -0.40693400 & -0.19000700 \\
\mathrm{H} & -4.59563700 & -2.52724900 & 0.46082300 \\
\mathrm{H} & -2.13550200 & -2.61162300 & 0.68946700
\end{array}
$$<smiles>CC(=C[N+](=O)[O-])c1cccc(Cl)c1</smiles>

Nitroalkene-27

$\begin{array}{lrrr}\mathrm{C} & -1.67184600 & 2.15744100 & -0.36825600 \\ \mathrm{C} & -0.38637700 & 1.62646400 & -0.42630700 \\ \mathrm{C} & -0.17273000 & 0.25356500 & -0.22276600 \\ \mathrm{C} & -1.27817300 & -0.57593700 & 0.02467200 \\ \mathrm{C} & -2.55335100 & -0.02487900 & 0.08978100 \\ \mathrm{C} & -2.76904000 & 1.33833900 & -0.10495900 \\ \mathrm{C} & 1.19002100 & -0.33295100 & -0.25870200 \\ \mathrm{C} & 2.18622200 & 0.40526900 & 0.27081400 \\ \mathrm{C} & 1.35327300 & -1.70424500 & -0.86169100 \\ \mathrm{~N} & 3.57980900 & 0.03017900 & 0.30257800 \\ \mathrm{O} & 3.91756100 & -1.11792900 & -0.00583800 \\ \mathrm{O} & 4.36226000 & 0.91691400 & 0.65803300 \\ \mathrm{Cl} & -3.92063600 & -1.07390500 & 0.42254700 \\ \mathrm{H} & -1.82568300 & 3.21783000 & -0.53934500 \\ \mathrm{H} & 0.45314400 & 2.26998400 & -0.66380500 \\ \mathrm{H} & -1.14736900 & -1.63820000 & 0.19052100 \\ \mathrm{H} & -3.77344400 & 1.74217800 & -0.05920200 \\ \mathrm{H} & 2.03720100 & 1.37628400 & 0.71910100 \\ \mathrm{H} & 1.52916800 & -2.45270500 & -0.08234500 \\ \mathrm{H} & 0.46398000 & -1.98077400 & -1.42907200 \\ \mathrm{H} & 2.22859600 & -1.73975400 & -1.51026400\end{array}$<smiles>CC(=C[N+](=O)[O-])c1ccc(Cl)cc1</smiles>

Nitroalkene-28

$\begin{array}{lrrr}\mathrm{C} & -1.98659300 & -1.14472200 & 0.43666300 \\ \mathrm{C} & -0.61197800 & -0.94158700 & 0.49484800 \\ \mathrm{C} & -0.03986700 & 0.27980000 & 0.10165800 \\ \mathrm{C} & -0.89512700 & 1.30300600 & -0.34015800 \\ \mathrm{C} & -2.27042200 & 1.10944200 & -0.41541200 \\ \mathrm{C} & -2.80721200 & -0.11636600 & -0.02450400 \\ \mathrm{C} & 1.42574700 & 0.49565500 & 0.13891500 \\ \mathrm{C} & 2.20989900 & -0.55159200 & -0.19030700 \\ \mathrm{C} & 1.92903500 & 1.86365600 & 0.52243800 \\ \mathrm{~N} & 3.65241400 & -0.55311300 & -0.17984400\end{array}$




$\begin{array}{lrrr}\mathrm{O} & 4.27068700 & 0.50626800 & -0.02568300 \\ \mathrm{O} & 4.18597500 & -1.65535600 & -0.34088100 \\ \mathrm{Cl} & -4.53987000 & -0.36315000 & -0.10101300 \\ \mathrm{H} & -2.42108100 & -2.08488100 & 0.75531500 \\ \mathrm{H} & 0.02219900 & -1.73107300 & 0.88303100 \\ \mathrm{H} & -0.48182800 & 2.25492100 & -0.65496400 \\ \mathrm{H} & -2.92184600 & 1.89810800 & -0.77298900 \\ \mathrm{H} & 1.82535700 & -1.51566900 & -0.48819400 \\ \mathrm{H} & 1.12588900 & 2.45881500 & 0.95814900 \\ \mathrm{H} & 2.75309700 & 1.78615500 & 1.23194100 \\ \mathrm{H} & 2.33491500 & 2.38790500 & -0.34849300\end{array}$<smiles>CC(=C[N+](=O)[O-])c1ccc(C#N)cc1</smiles>

Nitroalkene-29

$\begin{array}{lrrr}\mathrm{C} & -2.12489800 & -1.15626300 & 0.44174200 \\ \mathrm{C} & -0.75298900 & -0.95325800 & 0.50499300 \\ \mathrm{C} & -0.18399600 & 0.26759500 & 0.10386900 \\ \mathrm{C} & -1.03544000 & 1.28999300 & -0.34913600 \\ \mathrm{C} & -2.40750300 & 1.09368800 & -0.42689900 \\ \mathrm{C} & -2.96217600 & -0.13323500 & -0.03001500 \\ \mathrm{C} & 1.28246500 & 0.48737600 & 0.14696400 \\ \mathrm{C} & 2.06559900 & -0.55541300 & -0.19350400 \\ \mathrm{C} & 1.77848200 & 1.85413800 & 0.54334200 \\ \mathrm{~N} & 3.51052600 & -0.55235800 & -0.18883000 \\ \mathrm{O} & 4.04475700 & -1.65511800 & -0.33605600 \\ \mathrm{O} & 4.12345400 & 0.51143700 & -0.05190800 \\ \mathrm{C} & -4.37815700 & -0.33641100 & -0.09673400 \\ \mathrm{~N} & -5.52805900 & -0.50103600 & -0.15171600 \\ \mathrm{H} & -2.55677800 & -2.09680400 & 0.76416200 \\ \mathrm{H} & -0.11635400 & -1.73709600 & 0.89978200 \\ \mathrm{H} & -0.61782400 & 2.23901200 & -0.66604600 \\ \mathrm{H} & -3.05593500 & 1.88181000 & -0.79227000 \\ \mathrm{H} & 1.68256700 & -1.51904800 & -0.49537700 \\ \mathrm{H} & 0.97816700 & 2.43146100 & 1.00775600 \\ \mathrm{H} & 2.62072000 & 1.77580000 & 1.23063200 \\ \mathrm{H} & 2.15417800 & 2.39881000 & -0.32883500\end{array}$<smiles>CC(=C[N+](=O)[O-])c1ccc(F)cc1</smiles>

Nitroalkene-30

$\mathrm{C}$

$\begin{array}{llll}-2.72667300 & 0.99631300 & -0.43730100\end{array}$ 


$\begin{array}{lrrr}\mathrm{C} & -1.35834800 & 1.23071700 & -0.35190900 \\ \mathrm{C} & -0.47468000 & 0.22951000 & 0.08794600 \\ \mathrm{C} & -1.01092400 & -1.01325700 & 0.46887600 \\ \mathrm{C} & -2.37806300 & -1.25874500 & 0.40054400 \\ \mathrm{C} & -3.21579200 & -0.24843200 & -0.05793000 \\ \mathrm{C} & 0.98298500 & 0.48874400 & 0.13477800 \\ \mathrm{C} & 1.80228700 & -0.53308400 & -0.19116300 \\ \mathrm{C} & 1.44413100 & 1.86962900 & 0.52563200 \\ \mathrm{~N} & 3.24304800 & -0.49178300 & -0.16711500 \\ \mathrm{O} & 3.81157400 & -1.57562300 & -0.33606500 \\ \mathrm{O} & 3.82893100 & 0.58353000 & 0.00511300 \\ \mathrm{~F} & -4.53951600 & -0.47824700 & -0.12622600 \\ \mathrm{H} & -3.41291000 & 1.75718600 & -0.79065200 \\ \mathrm{H} & -0.97065200 & 2.19638400 & -0.65690100 \\ \mathrm{H} & -0.35334600 & -1.78434000 & 0.85478500 \\ \mathrm{H} & -2.80090700 & -2.20847800 & 0.70700200 \\ \mathrm{H} & 1.44963800 & -1.50676000 & -0.49691300 \\ \mathrm{H} & 1.85262800 & 2.40388200 & -0.33787500 \\ \mathrm{H} & 0.61898100 & 2.44420300 & 0.94748200 \\ \mathrm{H} & 2.25730200 & 1.81300900 & 1.24968500\end{array}$<smiles>CC(=C[N+](=O)[O-])c1ccc(C)cc1</smiles>

Nitroalkene-31

$\begin{array}{rrrr}\mathrm{C} & 2.35334900 & -1.22595700 & 0.39421800 \\ \mathrm{C} & 0.98382600 & -0.99870600 & 0.46728700 \\ \mathrm{C} & 0.43732000 & 0.23875400 & 0.08775500 \\ \mathrm{C} & 1.31805100 & 1.23988700 & -0.35431700 \\ \mathrm{C} & 2.68541500 & 1.00069300 & -0.43756400 \\ \mathrm{C} & 3.23016100 & -0.23314500 & -0.06099900 \\ \mathrm{C} & -1.02129100 & 0.48928300 & 0.13281400 \\ \mathrm{C} & -1.83522900 & -0.53949300 & -0.18700000 \\ \mathrm{C} & -1.49229600 & 1.86899400 & 0.51583700 \\ \mathrm{~N} & -3.27514500 & -0.50642300 & -0.16486800 \\ \mathrm{O} & -3.83866000 & -1.59215000 & -0.34218500 \\ \mathrm{O} & -3.86836900 & 0.56422100 & 0.01438900 \\ \mathrm{C} & 4.71715700 & -0.47318500 & -0.10908800 \\ \mathrm{H} & 2.75138100 & -2.18811400 & 0.70427300 \\ \mathrm{H} & 0.33225400 & -1.77620900 & 0.85190700 \\ \mathrm{H} & 0.92749400 & 2.20275800 & -0.66617300 \\ \mathrm{H} & 3.34268900 & 1.78511800 & -0.80219400 \\ \mathrm{H} & -1.47587500 & -1.51233200 & -0.48711200 \\ \mathrm{H} & -0.66654600 & 2.45824300 & 0.91533500 \\ \mathrm{H} & -1.92480000 & 2.38784200 & -0.34515500 \\ \mathrm{H} & -2.29067500 & 1.81011600 & 1.25636800 \\ \mathrm{H} & 5.19209800 & 0.12085500 & -0.89475700 \\ \mathrm{H} & 5.18964700 & -0.19565100 & 0.84108000 \\ \mathrm{H} & 4.94579500 & -1.52698500 & -0.29079800 \\ & & & \end{array}$


<smiles>CC/C(C)=C/[N+](=O)[O-]</smiles>

Nitroalkene-32

$\begin{array}{lrrr}\mathrm{C} & -2.15098500 & 0.22409100 & 0.00002000 \\ \mathrm{C} & -0.66804200 & 0.51326300 & -0.00005500 \\ \mathrm{C} & 0.20524700 & -0.50731300 & -0.00008700 \\ \mathrm{C} & -0.30321200 & 1.97374500 & 0.00010300 \\ \mathrm{~N} & 1.64388200 & -0.36942800 & -0.00002000 \\ \mathrm{O} & 2.28367700 & -1.42566800 & 0.00019200 \\ \mathrm{O} & 2.15673700 & 0.75510300 & -0.00018700 \\ \mathrm{C} & -2.59308600 & -1.23706500 & -0.00003200 \\ \mathrm{H} & -2.57708500 & 0.74442700 & -0.86984500 \\ \mathrm{H} & -2.57689100 & 0.74431500 & 0.87005200 \\ \mathrm{H} & -0.07716000 & -1.54842800 & -0.00023100 \\ \mathrm{H} & -1.20599400 & 2.58960300 & -0.00035700 \\ \mathrm{H} & 0.30552000 & 2.22624500 & 0.87155800 \\ \mathrm{H} & 0.30652300 & 2.22617300 & -0.87066000 \\ \mathrm{H} & -3.68449000 & -1.29789800 & 0.00034800 \\ \mathrm{H} & -2.23053100 & -1.76700500 & -0.88603500 \\ \mathrm{H} & -2.22991300 & -1.76724200 & 0.88557800\end{array}$<smiles>CC(=C[N+](=O)[O-])c1ccco1</smiles>

Nitroalkene-33

$\begin{array}{lrrr}\mathrm{C} & 1.18894400 & 0.19343700 & -0.00000700 \\ \mathrm{C} & -0.22427100 & 0.50386600 & -0.00000400 \\ \mathrm{C} & -1.09882500 & -0.53174800 & 0.00005900 \\ \mathrm{C} & -0.60570800 & 1.96189500 & -0.00003400 \\ \mathrm{~N} & -2.52680300 & -0.40110200 & 0.00002900 \\ \mathrm{O} & -3.16437700 & -1.46111600 & -0.00013000 \\ \mathrm{O} & -3.04796500 & 0.72287300 & 0.00009200 \\ \mathrm{O} & 1.56205800 & -1.13094700 & 0.00003600 \\ \mathrm{C} & 2.91794600 & -1.17377400 & 0.00002700 \\ \mathrm{C} & 3.43903700 & 0.08718900 & -0.00001900 \\ \mathrm{C} & 2.32284800 & 0.97409300 & -0.00004100 \\ \mathrm{H} & -0.79064900 & -1.56557900 & 0.00007200 \\ \mathrm{H} & 0.27620800 & 2.60164600 & -0.00028900 \\ \mathrm{H} & -1.21990100 & 2.19521100 & -0.87165300 \\ \mathrm{H} & -1.21948200 & 2.19534600 & 0.87184800 \\ \mathrm{H} & 3.35758600 & -2.15885400 & 0.00005700 \\ \mathrm{H} & 4.48573300 & 0.35142700 & -0.00003400 \\ \mathrm{H} & 2.36056900 & 2.05229800 & -0.00007300\end{array}$


<smiles>CC/C(=C\[N+](=O)[O-])c1ccccc1</smiles>

Nitroalkene-34

$\begin{array}{lrrr}\mathrm{C} & -3.27621400 & 0.41280600 & -0.86406700 \\ \mathrm{C} & -1.91107500 & 0.67344900 & -0.94861200 \\ \mathrm{C} & -1.00036600 & -0.00384000 & -0.12142100 \\ \mathrm{C} & -1.49964900 & -0.93435500 & 0.80557600 \\ \mathrm{C} & -2.86628300 & -1.18495200 & 0.89703200 \\ \mathrm{C} & -3.75898600 & -0.51472200 & 0.06013300 \\ \mathrm{C} & 0.45893400 & 0.25406900 & -0.21589500 \\ \mathrm{C} & 1.26672600 & -0.82537100 & -0.17025500 \\ \mathrm{C} & 0.91057500 & 1.69091700 & -0.29558500 \\ \mathrm{~N} & 2.71105400 & -0.80745400 & -0.17992200 \\ \mathrm{O} & 3.32461400 & 0.25366000 & -0.01534700 \\ \mathrm{O} & 3.25522900 & -1.90352100 & -0.34647000 \\ \mathrm{C} & 1.08695600 & 2.28096200 & 1.11559300 \\ \mathrm{H} & -3.96401800 & 0.93431900 & -1.52214500 \\ \mathrm{H} & -1.54910000 & 1.38477600 & -1.68307500 \\ \mathrm{H} & -0.81008400 & -1.43739200 & 1.47523700 \\ \mathrm{H} & -3.23421400 & -1.89775500 & 1.62822800 \\ \mathrm{H} & -4.82438900 & -0.70957100 & 0.13100800 \\ \mathrm{H} & 0.89729700 & -1.84035400 & -0.16979400 \\ \mathrm{H} & 0.15069900 & 2.26767500 & -0.82991200 \\ \mathrm{H} & 1.85061700 & 1.76624100 & -0.83982400 \\ \mathrm{H} & 1.35097100 & 3.34013500 & 1.05078700 \\ \mathrm{H} & 0.16443300 & 2.19181100 & 1.69728300 \\ \mathrm{H} & 1.88796300 & 1.75741100 & 1.64121400\end{array}$<smiles>CC(=C[N+](=O)[O-])c1ccccc1</smiles>

Nitroalkene-35

$\begin{array}{lrrr}\mathrm{C} & 3.18056200 & 0.80146000 & -0.48615500 \\ \mathrm{C} & 1.82545000 & 1.10478200 & -0.38653400 \\ \mathrm{C} & 0.90737300 & 0.14405700 & 0.07005800 \\ \mathrm{C} & 1.39141700 & -1.11938500 & 0.44938600 \\ \mathrm{C} & 2.74855100 & -1.41638200 & 0.36100300 \\ \mathrm{C} & 3.64732700 & -0.45914700 & -0.11156600 \\ \mathrm{C} & -0.53800800 & 0.46743800 & 0.13606500 \\ \mathrm{C} & -1.40332000 & -0.51425200 & -0.19298500 \\ \mathrm{C} & -0.93378900 & 1.86243400 & 0.54711700 \\ \mathrm{H} & 3.87289300 & 1.55102700 & -0.85617800 \\ \mathrm{H} & 1.47298300 & 2.08472900 & -0.69024700 \\ \mathrm{H} & 0.70307500 & -1.85754000 & 0.84699500\end{array}$




$\begin{array}{rrrr}\mathrm{H} & 3.10570400 & -2.39325900 & 0.67117400 \\ \mathrm{H} & 4.70541800 & -0.69115700 & -0.17961500 \\ \mathrm{H} & -1.09509400 & -1.49901500 & -0.51089700 \\ \mathrm{H} & -1.34189200 & 2.41905400 & -0.30219600 \\ \mathrm{H} & -1.73064000 & 1.83251400 & 1.29096900 \\ \mathrm{H} & -0.07565900 & 2.39973900 & 0.95174600 \\ \mathrm{~N} & -2.84075600 & -0.40937400 & -0.15814200 \\ \mathrm{O} & -3.45867000 & -1.46343800 & -0.34199200 \\ \mathrm{O} & -3.37693900 & 0.68762500 & 0.03785500\end{array}$<smiles>CCCC(=C[N+](=O)[O-])c1ccccc1</smiles>

Nitroalkene-36

$\begin{array}{lrrr}\mathrm{C} & 3.37746600 & 0.47722500 & -0.91140200 \\ \mathrm{C} & 1.99233100 & 0.51822700 & -1.04720700 \\ \mathrm{C} & 1.16387100 & -0.16967400 & -0.14566800 \\ \mathrm{C} & 1.76341000 & -0.88247600 & 0.90650700 \\ \mathrm{C} & 3.14826600 & -0.91253200 & 1.04775800 \\ \mathrm{C} & 3.96028400 & -0.23558600 & 0.13723800 \\ \mathrm{C} & -0.31339000 & -0.14547800 & -0.29220200 \\ \mathrm{C} & -0.95920900 & -1.31467700 & -0.10062200 \\ \mathrm{C} & -0.96536600 & 1.18141400 & -0.57916600 \\ \mathrm{~N} & -2.38969100 & -1.51043400 & -0.13015100 \\ \mathrm{O} & -2.76520700 & -2.68707100 & -0.13587200 \\ \mathrm{O} & -3.15514300 & -0.53875900 & -0.13554800 \\ \mathrm{C} & -1.28560000 & 1.93032100 & 0.73138800 \\ \mathrm{C} & -1.89869800 & 3.30478100 & 0.46560600 \\ \mathrm{H} & 4.00267700 & 1.00218200 & -1.62670300 \\ \mathrm{H} & 1.55414500 & 1.06279000 & -1.87655200 \\ \mathrm{H} & 1.13343600 & -1.38743000 & 1.63115800 \\ \mathrm{H} & 3.59245000 & -1.45783000 & 1.87447700 \\ \mathrm{H} & 5.03986600 & -0.25852900 & 0.24699800 \\ \mathrm{H} & -0.44852000 & -2.25433700 & 0.05038600 \\ \mathrm{H} & -1.88601000 & 1.04395300 & -1.14526400 \\ \mathrm{H} & -0.27959500 & 1.79429100 & -1.17186000 \\ \mathrm{H} & -0.36621400 & 2.03318400 & 1.32046300 \\ \mathrm{H} & -1.97803300 & 1.31645100 & 1.31479400 \\ \mathrm{H} & -2.12264400 & 3.82332700 & 1.40272200 \\ \mathrm{H} & -1.21821200 & 3.93909900 & -0.11348600 \\ \mathrm{H} & -2.83290100 & 3.21325800 & -0.09809300\end{array}$<smiles>O=[N+]([O-])/C=C/c1ccccc1Br</smiles>

Nitroalkene-37 


$\begin{array}{lrrr}\mathrm{C} & -2.67527300 & 0.69404700 & -0.03163900 \\ \mathrm{C} & -1.42677800 & 0.07831400 & 0.00554200 \\ \mathrm{C} & -0.23297000 & 0.82567700 & 0.05776000 \\ \mathrm{C} & -0.35691200 & 2.22944400 & 0.08316100 \\ \mathrm{C} & -1.59534300 & 2.85354900 & 0.04583800 \\ \mathrm{C} & -2.75950000 & 2.08370100 & -0.01294500 \\ \mathrm{C} & 1.06997600 & 0.17192000 & 0.10018200 \\ \mathrm{C} & 2.25053300 & 0.76952000 & -0.09875500 \\ \mathrm{Br} & -1.39126100 & -1.83144800 & -0.03545600 \\ \mathrm{~N} & 3.48104800 & 0.00352300 & -0.00898600 \\ \mathrm{O} & 3.42588500 & -1.19866300 & 0.25511000 \\ \mathrm{O} & 4.52018200 & 0.63793200 & -0.21170100 \\ \mathrm{H} & -3.56954300 & 0.08434000 & -0.07572000 \\ \mathrm{H} & 0.54127300 & 2.83323500 & 0.15201100 \\ \mathrm{H} & -1.65582000 & 3.93627200 & 0.07029200 \\ \mathrm{H} & -3.73347000 & 2.56132500 & -0.03904800 \\ \mathrm{H} & 1.09371100 & -0.89456000 & 0.29786200 \\ \mathrm{H} & 2.43972700 & 1.80424700 & -0.34367600\end{array}$<smiles>COc1cccc(OC)c1/C=C/[N+](=O)[O-]</smiles>

Nitroalkene-38

$\begin{array}{lrrr}\mathrm{C} & 2.16188300 & 1.76191900 & 0.00000600 \\ \mathrm{C} & 0.82415000 & 1.35629100 & 0.00000900 \\ \mathrm{C} & 0.47175500 & -0.01750200 & 0.00000700 \\ \mathrm{C} & 1.53165000 & -0.96588300 & 0.00000900 \\ \mathrm{C} & 2.86896500 & -0.56232700 & 0.00000600 \\ \mathrm{C} & 3.16538700 & 0.79768800 & 0.00000400 \\ \mathrm{C} & -0.88788600 & -0.51853600 & -0.00000700 \\ \mathrm{C} & -2.04696300 & 0.16153000 & -0.00000400 \\ \mathrm{O} & -0.21161500 & 2.23267500 & 0.00001400 \\ \mathrm{~N} & -3.29785100 & -0.57079000 & -0.00000900 \\ \mathrm{O} & -4.32279600 & 0.12138100 & -0.00000300 \\ \mathrm{O} & -3.29104700 & -1.80578100 & 0.00001600 \\ \mathrm{O} & 1.15068200 & -2.26876600 & 0.00001500 \\ \mathrm{C} & 0.06616800 & 3.62748100 & -0.00002000 \\ \mathrm{C} & 2.15161600 & -3.27683100 & -0.00002500 \\ \mathrm{H} & 2.42367600 & 2.81098400 & 0.00000700 \\ \mathrm{H} & 3.66973200 & -1.28893500 & 0.00000800 \\ \mathrm{H} & 4.20375000 & 1.11399900 & 0.00000400 \\ \mathrm{H} & -0.98520600 & -1.59835000 & -0.00002200 \\ \mathrm{H} & -2.19560200 & 1.22601000 & 0.00001400 \\ \mathrm{H} & -0.90495300 & 4.12224600 & -0.00003500 \\ \mathrm{H} & 0.62603400 & 3.92188900 & 0.89483100 \\ \mathrm{H} & 0.62603900 & 3.92184400 & -0.89488400 \\ \mathrm{H} & 1.61536300 & -4.22561500 & -0.00004200 \\ \mathrm{H} & 2.78197200 & -3.21377600 & -0.89469100 \\ & & & \end{array}$


<smiles>O=[N+]([O-])C=Cc1ccc2c(c1)OCO2</smiles>

Nitroalkene-39

$\begin{array}{lrrr}\mathrm{C} & -1.84963300 & -0.61194200 & -0.00017500 \\ \mathrm{C} & -0.48041700 & -0.69873700 & -0.00039600 \\ \mathrm{C} & 0.24868700 & 0.52106700 & -0.00019400 \\ \mathrm{C} & -0.44002600 & 1.74582600 & 0.00011300 \\ \mathrm{C} & -1.83980800 & 1.81745300 & 0.00031700 \\ \mathrm{C} & -2.52007400 & 0.61627100 & 0.00018200 \\ \mathrm{C} & 1.70010300 & 0.55581100 & -0.00026600 \\ \mathrm{C} & 2.53133200 & -0.49756000 & 0.00024900 \\ \mathrm{~N} & 3.96374700 & -0.29929200 & 0.00008300 \\ \mathrm{O} & 4.41722200 & 0.84903800 & -0.00057300 \\ \mathrm{O} & 4.64986700 & -1.32681900 & 0.00063400 \\ \mathrm{O} & -2.76390300 & -1.63318800 & -0.00033100 \\ \mathrm{O} & -3.86815700 & 0.40737100 & 0.00026300 \\ \mathrm{C} & -4.05717200 & -1.01737400 & 0.00003200 \\ \mathrm{H} & 0.01322100 & -1.66228200 & -0.00078100 \\ \mathrm{H} & 0.13419400 & 2.66635400 & 0.00022300 \\ \mathrm{H} & -2.36560000 & 2.76428500 & 0.00056300 \\ \mathrm{H} & 2.17179100 & 1.53464500 & -0.00074000 \\ \mathrm{H} & 2.26915200 & -1.54505900 & 0.00088000 \\ \mathrm{H} & -4.60375800 & -1.30933300 & -0.90325800 \\ \mathrm{H} & -4.60341200 & -1.30968000 & 0.90342200\end{array}$<smiles>O=[N+]([O-])C=Cc1cn([As])c2ccccc12</smiles>

Nitroalkene-40

$\begin{array}{lrrr}\mathrm{C} & 1.07173700 & -0.06234400 & 1.06537300 \\ \mathrm{~N} & -0.05485300 & 0.74062900 & 1.15202800 \\ \mathrm{C} & 0.01018800 & 1.73954200 & 0.16069700 \\ \mathrm{C} & 1.23388700 & 1.55591100 & -0.52432900 \\ \mathrm{C} & 1.90169600 & 0.40902100 & 0.07176200 \\ \mathrm{C} & -0.88118100 & 2.76489600 & -0.15364900 \\ \mathrm{C} & -0.52438300 & 3.61145200 & -1.19921000 \\ \mathrm{C} & 0.68652100 & 3.44820400 & -1.89517400 \\ \mathrm{C} & 1.57483100 & 2.43234400 & -1.56183500 \\ \mathrm{~S} & -1.50157600 & 0.19886300 & 1.92896600 \\ \mathrm{C} & 3.18543200 & -0.10720600 & -0.32959100\end{array}$




$\begin{array}{rrrr}\mathrm{C} & 3.87695200 & -1.08352300 & 0.27977700 \\ \mathrm{O} & -1.04207500 & -0.77724800 & 2.91116500 \\ \mathrm{O} & -2.23753000 & 1.40440900 & 2.29018500 \\ \mathrm{C} & -2.35017900 & -0.63710200 & 0.61112100 \\ \mathrm{C} & -3.29608700 & 0.05512900 & -0.14384600 \\ \mathrm{C} & -3.91359100 & -0.60012900 & -1.20550400 \\ \mathrm{C} & -3.59536700 & -1.92694200 & -1.52240500 \\ \mathrm{C} & -2.64569900 & -2.59762800 & -0.73683700 \\ \mathrm{C} & -2.01737800 & -1.96334000 & 0.32823600 \\ \mathrm{C} & -4.24313700 & -2.61741500 & -2.69433700 \\ \mathrm{H} & 1.17736100 & -0.89867700 & 1.73851400 \\ \mathrm{H} & -1.79388300 & 2.90697000 & 0.40989600 \\ \mathrm{H} & -1.19127200 & 4.42239400 & -1.47315300 \\ \mathrm{H} & 0.93423400 & 4.13346200 & -2.69923500 \\ \mathrm{H} & 2.51351900 & 2.32174400 & -2.09532900 \\ \mathrm{H} & 3.64666600 & 0.32641500 & -1.21252000 \\ \mathrm{H} & 3.59877300 & -1.62108000 & 1.17477300 \\ \mathrm{H} & -3.55146000 & 1.07637000 & 0.11001000 \\ \mathrm{H} & -4.65744300 & -0.07356700 & -1.79552900 \\ \mathrm{H} & -2.40110800 & -3.63153900 & -0.96098700 \\ \mathrm{H} & -1.29548100 & -2.48642800 & 0.94460000 \\ \mathrm{H} & -4.41802100 & -3.67650700 & -2.48591100 \\ \mathrm{H} & -5.19884300 & -2.15500300 & -2.95297200 \\ \mathrm{H} & -3.59859900 & -2.56138200 & -3.57962200 \\ \mathrm{~N} & 5.14630500 & -1.52240900 & -0.25183800 \\ \mathrm{O} & 5.59526100 & -0.98486800 & -1.26918400 \\ \mathrm{O} & 5.70624000 & -2.43201100 & 0.36923500\end{array}$<smiles>Cc1ccc(/C=C/[N+](=O)[O-])cc1</smiles>

Nitroalkene-41

$\begin{array}{lrrr}\mathrm{C} & -2.55473000 & 1.17977100 & 0.00057300 \\ \mathrm{C} & -1.17275500 & 1.34278800 & 0.00027700 \\ \mathrm{C} & -0.31382300 & 0.23211400 & 0.00014800 \\ \mathrm{C} & -0.89152900 & -1.05299900 & 0.00039600 \\ \mathrm{C} & -2.26835900 & -1.20830100 & 0.00068800 \\ \mathrm{C} & -3.12747100 & -0.09578800 & 0.00058700 \\ \mathrm{C} & 1.12294600 & 0.46188300 & 0.00002500 \\ \mathrm{C} & 2.08552700 & -0.47093600 & -0.00000100 \\ \mathrm{~N} & 3.48128500 & -0.08481300 & -0.00019800 \\ \mathrm{O} & 3.77899000 & 1.11264800 & -0.00034600 \\ \mathrm{O} & 4.29564500 & -1.01337800 & -0.00020500 \\ \mathrm{C} & -4.62186700 & -0.28527700 & -0.00115700 \\ \mathrm{H} & -3.19836100 & 2.05447600 & 0.00087400 \\ \mathrm{H} & -0.74664300 & 2.34183900 & 0.00037900 \\ \mathrm{H} & -0.25728600 & -1.93329300 & 0.00061300 \\ \mathrm{H} & -2.69371300 & -2.20808100 & 0.00111400 \\ \mathrm{H} & 1.45969900 & 1.49528700 & -0.00010300 \\ \mathrm{H} & 1.96469900 & -1.54398900 & 0.00010900\end{array}$




$$
\begin{array}{lrrr}
\mathrm{H} & -5.14694200 & 0.67256100 & 0.01876300 \\
\mathrm{H} & -4.94857700 & -0.83115200 & -0.89329300 \\
\mathrm{H} & -4.94658700 & -0.86764800 & 0.86811800
\end{array}
$$<smiles>[C-][N+](=O)[O-]</smiles>

Nitroalkene-42

$\begin{array}{lrrr}\mathrm{C} & -2.89325000 & 1.18979700 & -0.36598000 \\ \mathrm{C} & -1.51839600 & 0.97846800 & -0.42115600 \\ \mathrm{C} & -0.97330300 & -0.27470100 & -0.08628800 \\ \mathrm{C} & -1.85357200 & -1.31362200 & 0.26951900 \\ \mathrm{C} & -3.22561000 & -1.09620400 & 0.33755200 \\ \mathrm{C} & -3.75004600 & 0.15900900 & 0.02337800 \\ \mathrm{C} & 0.45724100 & -0.57314500 & -0.12569500 \\ \mathrm{C} & 1.48824100 & 0.26255000 & 0.08967900 \\ \mathrm{~N} & 2.83909400 & -0.32235900 & -0.07193400 \\ \mathrm{O} & 3.79226000 & 0.44610100 & 0.07520200 \\ \mathrm{O} & 2.95248300 & -1.51758400 & -0.34939100 \\ \mathrm{C} & 1.48426200 & 1.69651700 & 0.50821000 \\ \mathrm{H} & -3.29799900 & 2.15977600 & -0.63714500 \\ \mathrm{H} & -0.86968500 & 1.77549900 & -0.76343700 \\ \mathrm{H} & -1.44637600 & -2.29230200 & 0.50582000 \\ \mathrm{H} & -3.88690500 & -1.90660800 & 0.62726500 \\ \mathrm{H} & -4.82122700 & 0.32861300 & 0.06735000 \\ \mathrm{H} & 0.72770500 & -1.60470100 & -0.33038500 \\ \mathrm{H} & 0.51480100 & 1.95681300 & 0.93425900 \\ \mathrm{H} & 2.26450400 & 1.87112700 & 1.25049100 \\ \mathrm{H} & 1.69017600 & 2.36815600 & -0.33249100\end{array}$<smiles>C/C(=C\c1ccc(F)cc1)[N+](=O)[O-]</smiles>

Nitroalkene-43

$\begin{array}{lrrc}\mathrm{C} & -2.48316800 & 1.11976600 & -0.36830100 \\ \mathrm{C} & -1.10602800 & 0.93098900 & -0.41031500 \\ \mathrm{C} & -0.53404700 & -0.31418100 & -0.08609800 \\ \mathrm{C} & -1.39729400 & -1.37604900 & 0.24731900 \\ \mathrm{C} & -2.77409700 & -1.19893800 & 0.30674100 \\ \mathrm{C} & -3.29581100 & 0.05353400 & 0.00106200 \\ \mathrm{C} & 0.90079200 & -0.58317000 & -0.11597200 \\ \mathrm{C} & 1.91657100 & 0.27413700 & 0.08940200 \\ \mathrm{~N} & 3.27690300 & -0.28904300 & -0.06478200 \\ \mathrm{O} & 4.21690500 & 0.49756800 & 0.07037400 \\ \mathrm{O} & 3.41043200 & -1.48622000 & -0.32488000 \\ \mathrm{C} & 1.88566400 & 1.71355900 & 0.48778100 \\ \mathrm{~F} & -4.62789200 & 0.23463500 & 0.04696800\end{array}$




$$
\begin{array}{lrrr}
\mathrm{H} & -2.93389400 & 2.07086500 & -0.62728500 \\
\mathrm{H} & -0.47528600 & 1.74842200 & -0.73595100 \\
\mathrm{H} & -0.97405200 & -2.34944600 & 0.47511600 \\
\mathrm{H} & -3.44297400 & -2.00837900 & 0.57520600 \\
\mathrm{H} & 1.19189000 & -1.61174200 & -0.30656700 \\
\mathrm{H} & 0.92114200 & 1.95563900 & 0.93592700 \\
\mathrm{H} & 2.67926600 & 1.91865500 & 1.20737900 \\
\mathrm{H} & 2.05242000 & 2.37891100 & -0.36672400
\end{array}
$$

Nitroalkene-44

$\begin{array}{lrrr}\mathrm{C} & 1.34291300 & -0.99910900 & -0.40694700 \\ \mathrm{C} & -0.03964200 & -0.85064000 & -0.44193700 \\ \mathrm{C} & -0.64611700 & 0.37275100 & -0.10283200 \\ \mathrm{C} & 0.18717500 & 1.45424500 & 0.23866600 \\ \mathrm{C} & 1.56889200 & 1.31495400 & 0.29091900 \\ \mathrm{C} & 2.13662100 & 0.08251900 & -0.02908900 \\ \mathrm{C} & -2.08932200 & 0.59854400 & -0.12488200 \\ \mathrm{C} & -3.07386500 & -0.29080700 & 0.09231700 \\ \mathrm{~N} & -4.45459000 & 0.22726600 & -0.05283800 \\ \mathrm{O} & -5.36597400 & -0.59008800 & 0.09170600 \\ \mathrm{O} & -4.62864900 & 1.41828600 & -0.31519400 \\ \mathrm{C} & -2.99360600 & -1.72653300 & 0.49618400 \\ \mathrm{Br} & 4.02963900 & -0.12050300 & 0.02859200 \\ \mathrm{H} & 1.80331500 & -1.94133400 & -0.67920300 \\ \mathrm{H} & -0.64624200 & -1.68385200 & -0.77422700 \\ \mathrm{H} & -0.26075700 & 2.41356200 & 0.47937900 \\ \mathrm{H} & 2.20064600 & 2.15005900 & 0.56878300 \\ \mathrm{H} & -2.41365500 & 1.61669000 & -0.31768300 \\ \mathrm{H} & -2.01361500 & -1.93960500 & 0.92471300 \\ \mathrm{H} & -3.76605900 & -1.95045700 & 1.23313600 \\ \mathrm{H} & -3.16018400 & -2.39944600 & -0.35225400\end{array}$<smiles>CC(=C1COC1)[N+](=O)[O-]</smiles>

Nitroalkene-45
$\begin{array}{llll}\text { C } & -0.73605400 & 0.18632300 & 0.00015200\end{array}$
$\begin{array}{llll}\mathrm{C} & 0.54750700 & 0.54425300 & 0.00002800\end{array}$
$\begin{array}{llll}\mathrm{N} & 1.54331800 & -0.52939900 & 0.00003500\end{array}$
$\begin{array}{llll}\mathrm{O} & 2.72775500 & -0.19123000 & -0.00015800\end{array}$
$\begin{array}{llll}\mathrm{O} & 1.14750500 & -1.69984500 & 0.00010400\end{array}$
$\begin{array}{llll}\mathrm{C} & 1.08795500 & 1.93673600 & 0.00001000\end{array}$
C $\quad-1.52390400 \quad-1.08937100 \quad-0.00002500$
$\begin{array}{llll}\mathrm{O} & -2.76743600 & -0.33362200 & -0.00012500\end{array}$ 


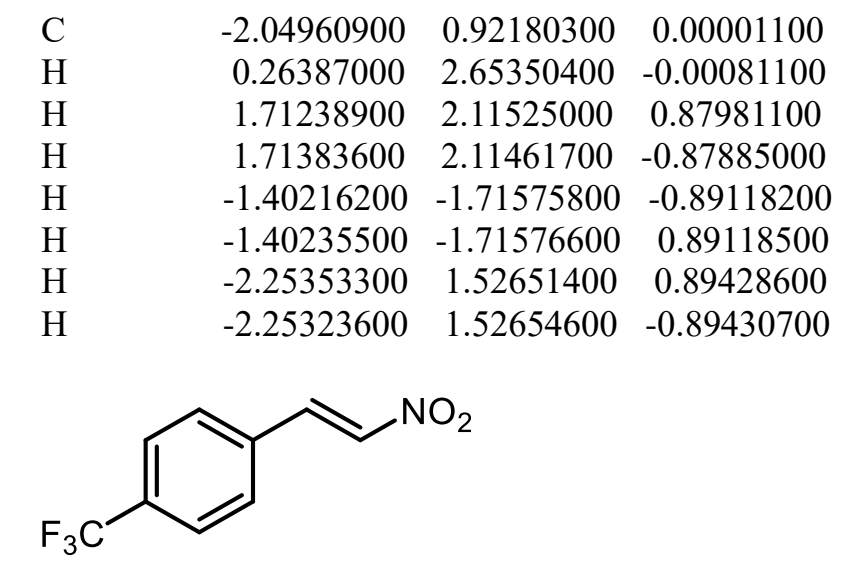

Nitroalkene-46

$\begin{array}{lrrr}\mathrm{C} & -2.24571200 & 0.47922800 & -0.00406000 \\ \mathrm{C} & -3.17841300 & -0.48031900 & -0.00121700 \\ \mathrm{~N} & -4.58838300 & -0.13231400 & 0.01118600 \\ \mathrm{O} & -5.37307600 & -1.08421500 & 0.01318300 \\ \mathrm{O} & -4.91585200 & 1.05581400 & 0.01878300 \\ \mathrm{C} & -0.79965700 & 0.28547000 & -0.01536200 \\ \mathrm{C} & -0.19494100 & -0.98572300 & -0.02638600 \\ \mathrm{C} & 1.18609600 & -1.11058300 & -0.03814100 \\ \mathrm{C} & 1.99171400 & 0.03341000 & -0.03979500 \\ \mathrm{C} & 1.41075300 & 1.30099800 & -0.03045400 \\ \mathrm{C} & 0.02547600 & 1.42244300 & -0.01894000 \\ \mathrm{C} & 3.48863500 & -0.11259400 & 0.00763500 \\ \mathrm{~F} & 4.11460400 & 0.96228600 & -0.51566900 \\ \mathrm{~F} & 3.92807500 & -0.25030400 & 1.27900800 \\ \mathrm{~F} & 3.90343900 & -1.20232500 & -0.67356900 \\ \mathrm{H} & -2.60972100 & 1.50309500 & 0.00325800 \\ \mathrm{H} & -3.02868600 & -1.54985900 & -0.00714100 \\ \mathrm{H} & -0.80737700 & -1.88052400 & -0.02874400 \\ \mathrm{H} & 1.64647400 & -2.09175500 & -0.05446300 \\ \mathrm{H} & 2.03967600 & 2.18334700 & -0.03943000 \\ \mathrm{H} & -0.42903100 & 2.40819800 & -0.01511800 \\ & & & \\ \mathrm{Me} & \mathrm{NO} & & \\ \mathrm{NO} & & & \end{array}$

Nitroalkene-47

$\begin{array}{lrrr}\mathrm{C} & 2.67402900 & -0.12977700 & 0.00004400 \\ \mathrm{C} & 1.24936600 & 0.31603000 & -0.00002500 \\ \mathrm{C} & 0.20775200 & -0.51366100 & -0.00005700 \\ \mathrm{H} & 3.19640300 & 0.26595600 & -0.87871600 \\ \mathrm{H} & 3.19632400 & 0.26598500 & 0.87883800 \\ \mathrm{H} & 2.76702100 & -1.21822900 & 0.00006700 \\ \mathrm{H} & 1.03812400 & 1.38238200 & -0.00006000 \\ \mathrm{H} & 0.24007200 & -1.59447200 & -0.00003000 \\ \mathrm{~N} & -1.15715400 & -0.00266900 & -0.00015400 \\ \mathrm{O} & -2.04774200 & -0.85466100 & 0.00009100\end{array}$


<smiles>O=[N+]([O-])C=C[Pb]</smiles>

Nitroalkene-48

$\begin{array}{lrrr}\mathrm{C} & -1.51544300 & 0.85334300 & 0.00000100 \\ \mathrm{C} & -0.01948800 & 0.83259600 & 0.00000600 \\ \mathrm{C} & 0.73656800 & -0.26411600 & 0.00001400 \\ \mathrm{~N} & 2.19067800 & -0.16436400 & 0.00001500 \\ \mathrm{O} & 2.71853200 & 0.94853000 & -0.00001400 \\ \mathrm{O} & 2.80017000 & -1.23610100 & -0.00001000 \\ \mathrm{C} & -2.21519800 & -0.50731900 & 0.00000100 \\ \mathrm{C} & -3.73898300 & -0.37370400 & -0.00000800 \\ \mathrm{H} & -1.84242500 & 1.44201700 & -0.87017900 \\ \mathrm{H} & -1.84243100 & 1.44202000 & 0.87017800 \\ \mathrm{H} & 0.50041000 & 1.78759600 & 0.00000300 \\ \mathrm{H} & 0.40344900 & -1.29121500 & 0.00001700 \\ \mathrm{H} & -1.89478200 & -1.08019500 & -0.87880600 \\ \mathrm{H} & -1.89479200 & -1.08019200 & 0.87881400 \\ \mathrm{H} & -4.08819400 & 0.17054400 & -0.88417000 \\ \mathrm{H} & -4.08820400 & 0.17054900 & 0.88414800 \\ \mathrm{H} & -4.22213100 & -1.35481200 & -0.00000700\end{array}$<smiles>O=[N+]([O-])C=Cc1cccc(Br)c1</smiles>

Nitroalkene-49

$\begin{array}{lrrr}\mathrm{C} & 2.05795400 & 0.66467500 & 0.00007800 \\ \mathrm{C} & 2.62722200 & -0.54662200 & 0.00012300 \\ \mathrm{~N} & 4.07291800 & -0.67616700 & 0.00002800 \\ \mathrm{O} & 4.50608800 & -1.83140500 & -0.00011500 \\ \mathrm{O} & 4.76968500 & 0.34085500 & 0.00000100 \\ \mathrm{C} & 0.62867600 & 0.95576500 & 0.00000500 \\ \mathrm{C} & -0.35007100 & -0.05517400 & -0.00003100 \\ \mathrm{C} & -1.69276700 & 0.29141300 & -0.00000200 \\ \mathrm{C} & -2.10351500 & 1.62622400 & 0.00001000 \\ \mathrm{C} & -1.13499300 & 2.62803500 & -0.00002600 \\ \mathrm{C} & 0.21793000 & 2.29899300 & -0.00004800 \\ \mathrm{Br} & -3.01112300 & -1.08706200 & 0.00000100 \\ \mathrm{H} & 2.73572300 & 1.51405100 & 0.00003700 \\ \mathrm{H} & 2.13670200 & -1.50881200 & 0.00020600 \\ \mathrm{H} & -0.07058500 & -1.10139000 & -0.00012000 \\ \mathrm{H} & -3.15932800 & 1.86909800 & 0.00001600 \\ \mathrm{H} & -1.44088100 & 3.66892900 & -0.00002500 \\ \mathrm{H} & 0.96847300 & 3.08301200 & -0.00009300\end{array}$




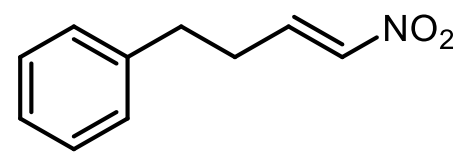

Nitroalkene-50

\begin{tabular}{|c|c|c|c|}
\hline $\mathrm{C}$ & -1.43048300 & 1.29100100 & 0.02080400 \\
\hline $\mathrm{C}$ & -1.78242000 & 0.20960700 & -0.67058600 \\
\hline $\mathrm{N}$ & -2.80961400 & -0.69529900 & -0.17327700 \\
\hline $\mathrm{O}$ & -3.07047200 & -1.66023700 & -0.89436400 \\
\hline $\mathrm{O}$ & -3.34538500 & -0.45745100 & 0.91068300 \\
\hline $\mathrm{C}$ & -0.34447800 & 2.22972200 & -0.39271700 \\
\hline $\mathrm{C}$ & 0.91558300 & 2.07367000 & 0.50314100 \\
\hline $\mathrm{C}$ & 1.57061900 & 0.72430400 & 0.33354700 \\
\hline $\mathrm{C}$ & 1.23547700 & -0.34919700 & 1.16661300 \\
\hline $\mathrm{C}$ & 1.79626600 & -1.61018000 & 0.96649800 \\
\hline $\mathrm{C}$ & 2.70195600 & -1.81579000 & -0.07443100 \\
\hline $\mathrm{C}$ & 3.04409900 & -0.75310900 & -0.91162700 \\
\hline $\mathrm{C}$ & 2.48082100 & 0.50585600 & -0.70741100 \\
\hline $\mathrm{H}$ & -1.93451000 & 1.46685400 & 0.96812900 \\
\hline $\mathrm{H}$ & -1.36913700 & -0.11638100 & -1.61469400 \\
\hline $\mathrm{H}$ & -0.70892900 & 3.26097900 & -0.31134100 \\
\hline $\mathrm{H}$ & -0.06472100 & 2.05717300 & -1.43668600 \\
\hline $\mathrm{H}$ & 0.63390800 & 2.23039900 & 1.55025400 \\
\hline $\mathrm{H}$ & 1.61799000 & 2.87086500 & 0.23835000 \\
\hline $\mathrm{H}$ & 0.52706400 & -0.19649000 & 1.97615300 \\
\hline $\mathrm{H}$ & 1.52452400 & -2.43129900 & 1.62263100 \\
\hline $\mathrm{H}$ & 3.13951200 & -2.79675800 & -0.23118500 \\
\hline $\mathrm{H}$ & 3.75180500 & -0.90358400 & -1.72126900 \\
\hline $\mathrm{H}$ & 2.75200700 & 1.33152900 & -1.36092500 \\
\hline
\end{tabular}

Nitroalkene-51

$\begin{array}{lrrr}\mathrm{C} & -0.28165700 & 0.31842500 & -0.59219900 \\ \mathrm{C} & -1.15033600 & -0.46227400 & 0.03934200 \\ \mathrm{~N} & -2.57196200 & -0.12805600 & 0.05870300 \\ \mathrm{O} & -3.29700100 & -0.91414800 & 0.66999900 \\ \mathrm{O} & -2.95544200 & 0.88989900 & -0.51783500 \\ \mathrm{C} & 1.19515900 & 0.03609100 & -0.66498700 \\ \mathrm{O} & 1.48240400 & -1.06039700 & 0.15882900 \\ \mathrm{O} & 1.96088100 & 1.17983200 & -0.35776700 \\ \mathrm{C} & 2.80549400 & -1.55925700 & -0.00814800 \\ \mathrm{C} & 1.75996500 & 1.68388300 & 0.96370200 \\ \mathrm{H} & -0.63409100 & 1.21090700 & -1.09944100 \\ \mathrm{H} & -0.92013400 & -1.37117000 & 0.57395400 \\ \mathrm{H} & 1.46708400 & -0.18571500 & -1.70983800 \\ \mathrm{H} & 2.91163800 & -2.39953200 & 0.67910700 \\ \mathrm{H} & 2.96633000 & -1.90993500 & -1.03707900\end{array}$




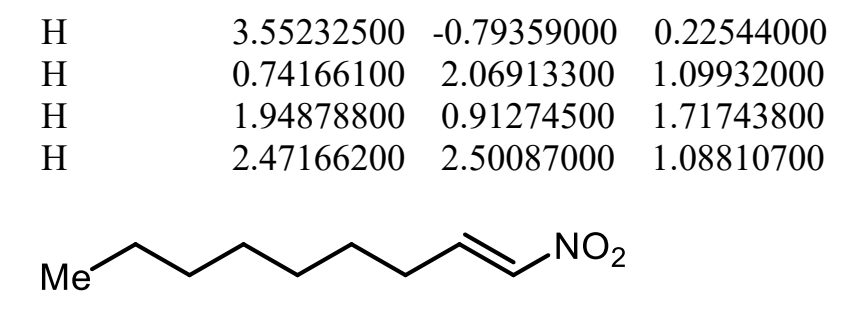

Nitroalkene-52

$\begin{array}{lrrr}\mathrm{C} & 2.54748900 & -0.99066600 & -0.00002200 \\ \mathrm{C} & 2.97822300 & 0.26994400 & -0.00005500 \\ \mathrm{~N} & 4.40468700 & 0.56830100 & -0.00007700 \\ \mathrm{O} & 4.70070300 & 1.76524200 & 0.00004700 \\ \mathrm{O} & 5.21483500 & -0.35957300 & 0.00008500 \\ \mathrm{C} & 1.11290300 & -1.41528900 & -0.00000100 \\ \mathrm{C} & 0.07251300 & -0.29436100 & -0.00002300 \\ \mathrm{C} & -1.36354200 & -0.82436600 & 0.00000400 \\ \mathrm{C} & -2.41540400 & 0.28756000 & -0.00001700 \\ \mathrm{C} & -3.85303300 & -0.23786800 & 0.00001400 \\ \mathrm{C} & -4.90657800 & 0.87340900 & -0.00000600 \\ \mathrm{C} & -6.34003100 & 0.33882800 & 0.00002600 \\ \mathrm{H} & 3.30645800 & -1.76930400 & -0.00001000 \\ \mathrm{H} & 2.37872900 & 1.16798100 & -0.00006900 \\ \mathrm{H} & 0.95787000 & -2.07045600 & 0.87013200 \\ \mathrm{H} & 0.95785900 & -2.07050200 & -0.87009600 \\ \mathrm{H} & 0.22443100 & 0.34518700 & -0.87909400 \\ \mathrm{H} & 0.22444600 & 0.34523400 & 0.87901100 \\ \mathrm{H} & -1.51395400 & -1.46780200 & 0.87789800 \\ \mathrm{H} & -1.51397000 & -1.46785100 & -0.87785200 \\ \mathrm{H} & -2.26317300 & 0.93096600 & -0.87769000 \\ \mathrm{H} & -2.26315500 & 0.93101700 & 0.87761600 \\ \mathrm{H} & -4.00512400 & -0.88176400 & 0.87766700 \\ \mathrm{H} & -4.00514400 & -0.88181500 & -0.87759800 \\ \mathrm{H} & -4.75298800 & 1.51591200 & -0.87722100 \\ \mathrm{H} & -4.75296800 & 1.51596400 & 0.87716700 \\ \mathrm{H} & -6.52972600 & -0.28081500 & -0.88351600 \\ \mathrm{H} & -6.52970500 & -0.28076500 & 0.88360800 \\ \mathrm{H} & -7.07223300 & 1.15220100 & 0.00001200 \\ & & & \end{array}$<smiles>O=[N+]([O-])C=Cc1ccc(O)cc1</smiles>

Nitroalkene-53
$\begin{array}{llll}\text { C } & 0.42408200 & 2.80620700 & 0.00000000\end{array}$
$\begin{array}{llll}\text { C } & -0.45945300 & 1.73457200 & 0.00000000\end{array}$
$\begin{array}{llll}\mathrm{C} & 0.00000000 & 0.40617200 & 0.00000000\end{array}$
$\begin{array}{llll}\mathrm{C} & 1.39427700 & 0.18958600 & 0.00000000\end{array}$
$\begin{array}{llll}\mathrm{C} & 2.28453000 & 1.24791200 & 0.00000000\end{array}$
$\begin{array}{llll}\mathrm{C} & 1.80247200 & 2.56626300 & 0.00000000\end{array}$ 


$\begin{array}{lrrr}\mathrm{C} & -0.97513400 & -0.66888300 & 0.00000000 \\ \mathrm{C} & -0.72463200 & -1.98716100 & 0.00000000 \\ \mathrm{O} & 2.72452900 & 3.56294800 & 0.00000000 \\ \mathrm{H} & 0.04814400 & 3.82611200 & 0.00000000 \\ \mathrm{H} & -1.52849400 & 1.92491100 & 0.00000000 \\ \mathrm{H} & 1.78382500 & -0.82280700 & 0.00000000 \\ \mathrm{H} & 3.35625100 & 1.08483200 & 0.00000000 \\ \mathrm{H} & -2.02459500 & -0.38585900 & 0.00000000 \\ \mathrm{H} & 0.23816600 & -2.47604700 & 0.00000000 \\ \mathrm{H} & 2.27554800 & 4.41900800 & 0.00000000 \\ \mathrm{~N} & -1.81355500 & -2.93859500 & 0.00000000 \\ \mathrm{O} & -2.97819400 & -2.52852600 & 0.00000000 \\ \mathrm{O} & -1.48768700 & -4.13042200 & 0.00000000\end{array}$<smiles>O=[N+]([O-])/C=C/c1ccccc1Cl</smiles>

Nitroalkene-54

$\begin{array}{lrrr}\mathrm{C} & -2.22885400 & -2.14954000 & 0.06110100 \\ \mathrm{C} & -0.90787900 & -1.72773500 & 0.10094800 \\ \mathrm{C} & -0.56482700 & -0.36155700 & 0.05980500 \\ \mathrm{C} & -1.62555300 & 0.56463200 & -0.01035000 \\ \mathrm{C} & -2.95552400 & 0.15197500 & -0.05036400 \\ \mathrm{C} & -3.25676600 & -1.20670700 & -0.01608200 \\ \mathrm{C} & 0.82380300 & 0.08245000 & 0.10446700 \\ \mathrm{C} & 1.89364800 & -0.69346500 & -0.10585000 \\ \mathrm{~N} & 3.23206600 & -0.13794800 & -0.01149700 \\ \mathrm{O} & 4.15410400 & -0.92918100 & -0.22851500 \\ \mathrm{O} & 3.37291200 & 1.05319700 & 0.26993500 \\ \mathrm{Cl} & -1.30758400 & 2.28991200 & -0.07022800 \\ \mathrm{H} & -2.45936800 & -3.20875300 & 0.09856600 \\ \mathrm{H} & -0.11572400 & -2.46333100 & 0.18647100 \\ \mathrm{H} & -3.74133300 & 0.89560800 & -0.10929400 \\ \mathrm{H} & -4.29341900 & -1.52584600 & -0.04438300 \\ \mathrm{H} & 1.01758700 & 1.12862500 & 0.31509100 \\ \mathrm{H} & 1.91229400 & -1.74162800 & -0.36551000\end{array}$<smiles>O=[N+]([O-])/C=C/c1c(F)cccc1Cl</smiles>

Nitroalkene-55
$\begin{array}{lllll}\text { C } & -2.94882000 & 0.43397900 & -0.00022100\end{array}$
$\begin{array}{lllll}\mathrm{C} & & -1.60377000 & 0.79040200 & -0.00004200\end{array}$ 


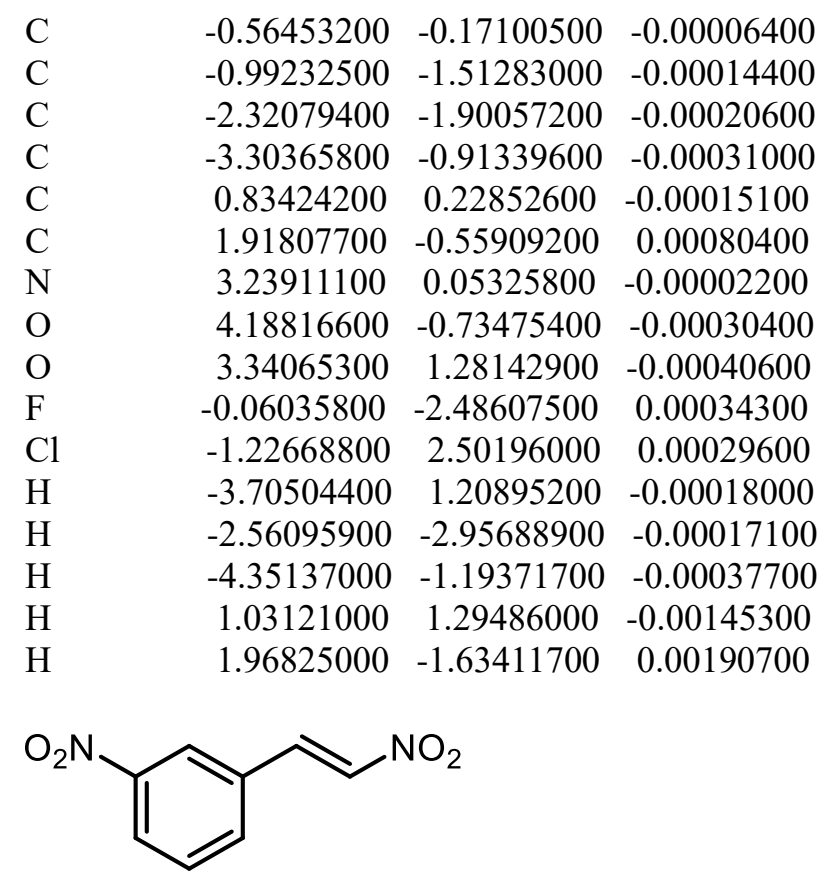

Nitroalkene-56

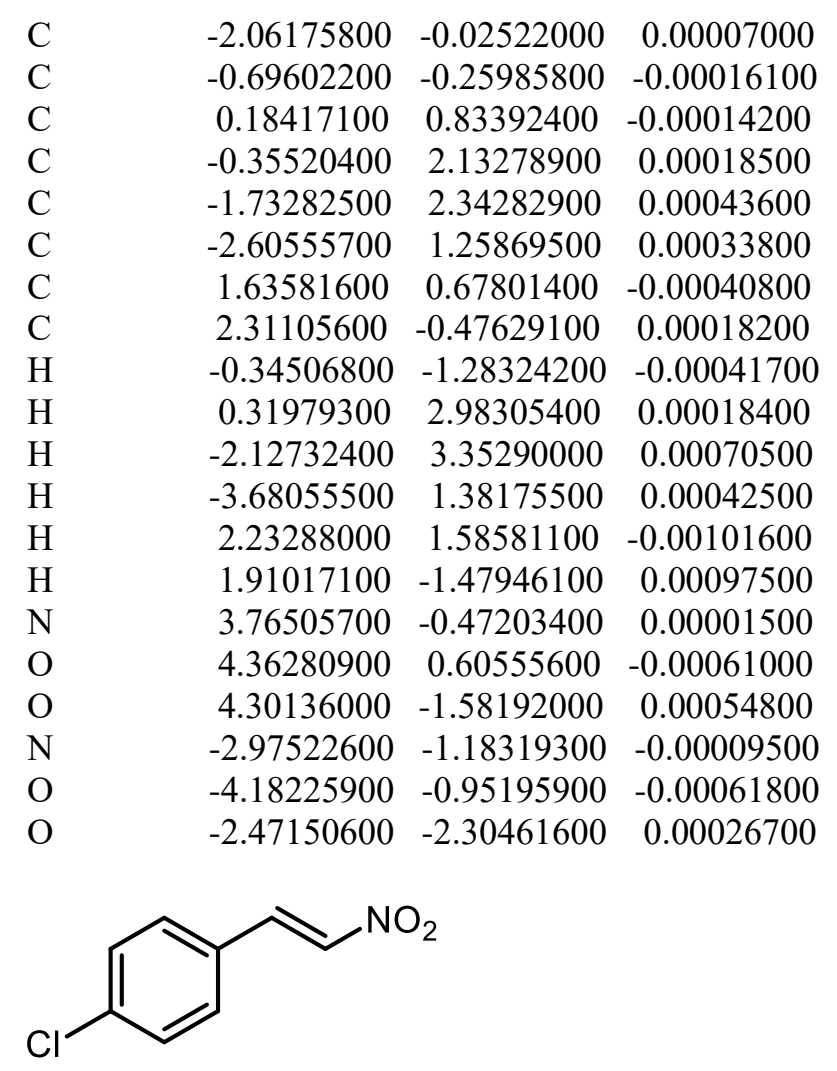

Nitroalkene-57

$\begin{array}{llll}\text { C } & -2.12245600 & 1.24553100 & 0.00024200\end{array}$ 


$\begin{array}{lrrr}\mathrm{C} & -0.73829500 & 1.37959900 & 0.00007500 \\ \mathrm{C} & 0.10475400 & 0.25520200 & -0.00029100 \\ \mathrm{C} & -0.48595700 & -1.02261300 & -0.00057900 \\ \mathrm{C} & -1.86526300 & -1.17178900 & -0.00042300 \\ \mathrm{C} & -2.67599800 & -0.03331400 & 0.00002000 \\ \mathrm{C} & 1.54577400 & 0.46719300 & -0.00032800 \\ \mathrm{C} & 2.49430800 & -0.47829900 & 0.00024400 \\ \mathrm{~N} & 3.89690200 & -0.10904800 & 0.00014300 \\ \mathrm{O} & 4.69765000 & -1.04820800 & 0.00062200 \\ \mathrm{O} & 4.20725900 & 1.08437500 & -0.00038100 \\ \mathrm{Cl} & -4.41585100 & -0.22018500 & 0.00024100 \\ \mathrm{H} & -2.76623500 & 2.11700000 & 0.00052900 \\ \mathrm{H} & -0.29930700 & 2.37258100 & 0.00024700 \\ \mathrm{H} & 0.13648000 & -1.91075700 & -0.00104200 \\ \mathrm{H} & -2.31681900 & -2.15677100 & -0.00067200 \\ \mathrm{H} & 1.89591300 & 1.49599400 & -0.00073900 \\ \mathrm{H} & 2.36065400 & -1.54996200 & 0.00089100\end{array}$<smiles>O=[N+]([O-])C=Cc1ccc(-c2ccccc2)cc1</smiles>

Nitroalkene-58

$\begin{array}{lrrr}\mathrm{C} & -0.57147300 & -1.02411100 & 0.24528100 \\ \mathrm{C} & 0.81047700 & -0.93051800 & 0.22253400 \\ \mathrm{C} & 1.44507500 & 0.29160000 & -0.07173900 \\ \mathrm{C} & 0.63468000 & 1.40824900 & -0.34118900 \\ \mathrm{C} & -0.75024000 & 1.31199500 & -0.31932100 \\ \mathrm{C} & -1.38330400 & 0.09397900 & -0.02525300 \\ \mathrm{C} & 2.89000700 & 0.45321600 & -0.10959300 \\ \mathrm{C} & 3.80966500 & -0.49332300 & 0.12610400 \\ \mathrm{~N} & 5.22136700 & -0.17932800 & 0.05099700 \\ \mathrm{O} & 5.99296100 & -1.11527100 & 0.28350300 \\ \mathrm{O} & 5.57235500 & 0.96920800 & -0.23215900 \\ \mathrm{C} & -2.85808600 & -0.01345000 & 0.00036200 \\ \mathrm{C} & -3.64758000 & 1.04629900 & 0.47541100 \\ \mathrm{C} & -5.03619000 & 0.94514900 & 0.49950000 \\ \mathrm{C} & -5.66356400 & -0.21665100 & 0.04769700 \\ \mathrm{C} & -4.89093800 & -1.27704800 & -0.42767100 \\ \mathrm{C} & -3.50216300 & -1.17712300 & -0.45025100 \\ \mathrm{H} & -1.03812100 & -1.96907400 & 0.50224200 \\ \mathrm{H} & 1.40420600 & -1.81086600 & 0.44501000 \\ \mathrm{H} & 1.10501300 & 2.35770000 & -0.57972400 \\ \mathrm{H} & -1.35172300 & 2.18227900 & -0.55895400 \\ \mathrm{H} & 3.27259100 & 1.44100400 & -0.35283100 \\ \mathrm{H} & 3.64036100 & -1.52868100 & 0.38199300 \\ \mathrm{H} & -3.16646700 & 1.94207000 & 0.85479400 \\ \mathrm{H} & -5.62892900 & 1.77138500 & 0.87969000 \\ \mathrm{H} & -6.74599200 & -0.29509200 & 0.06609300 \\ \mathrm{H} & -5.37087000 & -2.18104400 & -0.78961500 \\ \mathrm{H} & -2.91036000 & -1.99545000 & -0.84765400\end{array}$


<smiles>O=[N+]([O-])/C=C/c1c(Cl)cccc1Cl</smiles>

Nitroalkene-59

$\begin{array}{lrrr}\mathrm{C} & -2.91193700 & -0.83751400 & 0.11746900 \\ \mathrm{C} & -1.54473000 & -1.06932400 & 0.02262800 \\ \mathrm{C} & -0.59141500 & -0.02534200 & -0.05287700 \\ \mathrm{C} & -1.11992900 & 1.28562800 & -0.04096700 \\ \mathrm{C} & -2.48669700 & 1.53567500 & 0.04513100 \\ \mathrm{C} & -3.38035200 & 0.47270100 & 0.12840700 \\ \mathrm{C} & 0.82409500 & -0.37170700 & -0.18081400 \\ \mathrm{C} & 1.88187600 & 0.30734400 & 0.27135700 \\ \mathrm{~N} & 3.22045700 & -0.23048100 & 0.05770600 \\ \mathrm{O} & 4.13842800 & 0.41991500 & 0.56183700 \\ \mathrm{O} & 3.35945900 & -1.26847300 & -0.59029800 \\ \mathrm{Cl} & -1.01363700 & -2.74154700 & 0.03463000 \\ \mathrm{Cl} & -0.08194800 & 2.69161400 & -0.18866100 \\ \mathrm{H} & -3.59259600 & -1.67724300 & 0.18363200 \\ \mathrm{H} & -2.83795100 & 2.56013700 & 0.04158800 \\ \mathrm{H} & -4.44522900 & 0.66651200 & 0.19917400 \\ \mathrm{H} & 1.04650500 & -1.31643000 & -0.66623300 \\ \mathrm{H} & 1.90245200 & 1.23294400 & 0.82211900\end{array}$<smiles>Cc1ccccc1/C(=C\[N+](=O)[O-])C(F)(F)F</smiles>

Nitroalkene-60

$\begin{array}{lrrr}\mathrm{C} & -0.70375500 & -0.09540800 & -0.19263300 \\ \mathrm{C} & 0.77738000 & -0.17977000 & -0.07612500 \\ \mathrm{C} & 1.67066800 & 0.81125400 & -0.08623300 \\ \mathrm{C} & 1.32778900 & -1.58995600 & 0.03380500 \\ \mathrm{C} & -1.28759600 & -0.36442300 & -1.43460300 \\ \mathrm{C} & -2.67281900 & -0.33914200 & -1.58024600 \\ \mathrm{C} & -3.47361300 & -0.06479600 & -0.47423100 \\ \mathrm{C} & -2.88799000 & 0.18484100 & 0.76663400 \\ \mathrm{C} & -1.50152500 & 0.17546600 & 0.93538200 \\ \mathrm{~F} & 1.01851800 & -2.30782600 & -1.06408500 \\ \mathrm{~F} & 2.66729200 & -1.62688000 & 0.17844000 \\ \mathrm{~F} & 0.78664800 & -2.21727300 & 1.09794000 \\ \mathrm{~N} & 1.32854500 & 2.21829200 & -0.25145800 \\ \mathrm{O} & 2.23744000 & 2.92272200 & -0.69335000 \\ \mathrm{O} & 0.20228400 & 2.61006600 & 0.04441000 \\ \mathrm{C} & -0.88250600 & 0.46896700 & 2.27625100\end{array}$




$\begin{array}{crrc}\mathrm{H} & 2.73931500 & 0.66143300 & -0.04748700 \\ \mathrm{H} & -0.65287200 & -0.58725900 & -2.28536700 \\ \mathrm{H} & -3.11848800 & -0.53668400 & -2.54954000 \\ \mathrm{H} & -4.55441300 & -0.04698800 & -0.57385600 \\ \mathrm{H} & -3.51717100 & 0.39543800 & 1.62644600 \\ \mathrm{H} & -1.64689300 & 0.53277100 & 3.05371600 \\ \mathrm{H} & -0.16765200 & -0.30712400 & 2.56491700 \\ \mathrm{H} & -0.34774900 & 1.42368000 & 2.24423300\end{array}$<smiles>O=[N+]([O-])/C=C(/c1ccc2ccccc2c1)C(F)(F)F</smiles>

Nitroalkene-61

\begin{tabular}{|c|c|c|c|}
\hline $\mathrm{C}$ & 0.27599700 & -0.31784800 & 0.15153500 \\
\hline $\mathrm{C}$ & 1.71760700 & -0.03480500 & -0.00124500 \\
\hline $\mathrm{C}$ & 2.31163700 & 1.15945800 & 0.09813400 \\
\hline $\mathrm{C}$ & 2.61184500 & -1.20135100 & -0.37848700 \\
\hline $\mathrm{C}$ & -0.17528400 & -1.44470600 & 0.89546100 \\
\hline $\mathrm{C}$ & -1.51801700 & -1.70102700 & 1.01736500 \\
\hline $\mathrm{C}$ & -2.48627300 & -0.86710800 & 0.39971500 \\
\hline $\mathrm{C}$ & -2.03666700 & 0.25737200 & -0.36382600 \\
\hline $\mathrm{C}$ & -0.64810200 & 0.50216400 & -0.47013200 \\
\hline $\mathrm{F}$ & 2.07635600 & -1.91805300 & -1.38120700 \\
\hline $\mathrm{F}$ & 3.84252400 & -0.80942500 & -0.76463000 \\
\hline $\mathrm{F}$ & 2.77571900 & -2.03621500 & 0.67551700 \\
\hline $\mathrm{N}$ & 1.62328300 & 2.38194400 & 0.50762100 \\
\hline $\mathrm{O}$ & 1.90349400 & 3.38079900 & -0.15694200 \\
\hline $\mathrm{O}$ & 0.86537200 & 2.35056600 & 1.47039300 \\
\hline $\mathrm{C}$ & -3.87932100 & -1.11028000 & 0.50675700 \\
\hline $\mathrm{C}$ & -4.78573900 & -0.28024400 & -0.11235200 \\
\hline $\mathrm{C}$ & -4.34092100 & 0.83153300 & -0.86882600 \\
\hline $\mathrm{C}$ & -2.99672900 & 1.09344400 & -0.99319100 \\
\hline $\mathrm{H}$ & 3.34297500 & 1.34502400 & -0.16296100 \\
\hline $\mathrm{H}$ & 0.54786800 & -2.09103300 & 1.37603200 \\
\hline $\mathrm{H}$ & -1.85659000 & -2.55524300 & 1.59624100 \\
\hline $\mathrm{H}$ & -0.31369000 & 1.34534800 & -1.06692400 \\
\hline $\mathrm{H}$ & -4.21839400 & -1.96352300 & 1.08706700 \\
\hline $\mathrm{H}$ & -5.84982900 & -0.47509900 & -0.02300000 \\
\hline $\mathrm{H}$ & -5.06802300 & 1.47761100 & -1.35027100 \\
\hline $\mathrm{H}$ & -2.64982200 & 1.94602300 & -1.56968700 \\
\hline & & & \\
\hline
\end{tabular}

Nitroalkene-62 


$\begin{array}{lrrr}\text { C } & -0.37911500 & -0.42050700 & 0.16220000 \\ \mathrm{C} & 1.02041700 & 0.03630400 & 0.02092800 \\ \mathrm{C} & 1.46679500 & 1.29569200 & 0.08506600 \\ \mathrm{C} & 2.05509300 & -1.02460000 & -0.30858400 \\ \mathrm{C} & -0.70595500 & -1.52720900 & 0.95631300 \\ \mathrm{C} & -2.03436600 & -1.93477900 & 1.05207500 \\ \mathrm{C} & -3.03215400 & -1.25933900 & 0.35406500 \\ \mathrm{C} & -2.72335100 & -0.15875100 & -0.45694400 \\ \mathrm{C} & -1.39162100 & 0.24428300 & -0.54475800 \\ \mathrm{~F} & 1.63897700 & -1.80765600 & -1.31832900 \\ \mathrm{~F} & 3.24620600 & -0.50022000 & -0.66057800 \\ \mathrm{~F} & 2.27623400 & -1.82300400 & 0.76224700 \\ \mathrm{~N} & 0.63792900 & 2.44516700 & 0.43738200 \\ \mathrm{O} & 0.90488800 & 3.48146200 & -0.17372900 \\ \mathrm{O} & -0.21900800 & 2.32806900 & 1.30696800 \\ \mathrm{C} & -3.80630700 & 0.58309800 & -1.19919000 \\ \mathrm{H} & 2.47597900 & 1.59023800 & -0.16092100 \\ \mathrm{H} & 0.06718800 & -2.05467900 & 1.50009600 \\ \mathrm{H} & -2.28979900 & -2.78725200 & 1.67332200 \\ \mathrm{H} & -4.06391000 & -1.59106200 & 0.43122500 \\ \mathrm{H} & -1.13213800 & 1.08183900 & -1.18510600 \\ \mathrm{H} & -3.39319400 & 1.18438900 & -2.01302000 \\ \mathrm{H} & -4.54295500 & -0.10584600 & -1.62313300 \\ \mathrm{H} & -4.34307400 & 1.26274000 & -0.52714200\end{array}$<smiles>O=[N+]([O-])/C=C(/c1cn([As])c2ccccc12)C(F)(F)F</smiles>

Nitroalkene-63

$\begin{array}{lrrr}\mathrm{C} & -1.50846300 & -0.16123600 & -0.28727100 \\ \mathrm{C} & -2.68889100 & 0.67215900 & -0.12298400 \\ \mathrm{C} & -2.81359500 & 2.01608700 & -0.16893200 \\ \mathrm{C} & -4.00183700 & -0.04162000 & 0.19985500 \\ \mathrm{~F} & -4.04632200 & -1.26495900 & -0.34552400 \\ \mathrm{~F} & -4.15859000 & -0.17952700 & 1.53917800 \\ \mathrm{~F} & -5.07341000 & 0.64440000 & -0.24617900 \\ \mathrm{~N} & -1.74398900 & 2.98217600 & -0.23660400 \\ \mathrm{O} & -1.99724000 & 4.07469600 & 0.27811400 \\ \mathrm{O} & -0.66803500 & 2.69361800 & -0.77025700 \\ \mathrm{C} & -0.56027600 & 0.00732700 & -1.27087400 \\ \mathrm{~N} & 0.37566100 & -1.00462700 & -1.20348900 \\ \mathrm{C} & 0.07613900 & -1.83480200 & -0.10438000 \\ \mathrm{C} & -1.12349500 & -1.35042000 & 0.47181700 \\ \mathrm{C} & 0.76264800 & -2.94222000 & 0.38895000 \\ \mathrm{C} & 0.22203700 & -3.57597500 & 1.50349700 \\ \mathrm{C} & -0.96525900 & -3.11592000 & 2.09557900 \\ \mathrm{C} & -1.64505100 & -2.01240900 & 1.59243800\end{array}$




$\begin{array}{lrrr}\mathrm{S} & 1.96036000 & -0.77398200 & -1.87436300 \\ \mathrm{O} & 2.54233200 & -2.10483300 & -2.00582200 \\ \mathrm{O} & 1.74546000 & 0.10932500 & -3.01417200 \\ \mathrm{C} & 2.77318700 & 0.10507300 & -0.56255000 \\ \mathrm{C} & 2.44890100 & 1.44739100 & -0.34670400 \\ \mathrm{C} & 3.03926100 & 2.10874700 & 0.72275900 \\ \mathrm{C} & 3.94690000 & 1.45514200 & 1.57081500 \\ \mathrm{C} & 4.24994200 & 0.11053300 & 1.32430100 \\ \mathrm{C} & 3.66531600 & -0.57617600 & 0.26302000 \\ \mathrm{C} & 4.59455000 & 2.19818500 & 2.71040000 \\ \mathrm{H} & -3.76349900 & 2.50524900 & -0.01644200 \\ \mathrm{H} & -0.48731200 & 0.75490000 & -2.03994300 \\ \mathrm{H} & 1.66661400 & -3.29505700 & -0.08970600 \\ \mathrm{H} & 0.72579400 & -4.44375200 & 1.91650500 \\ \mathrm{H} & -1.36297000 & -3.63325000 & 2.96270000 \\ \mathrm{H} & -2.55626100 & -1.67143600 & 2.06433800 \\ \mathrm{H} & 1.74723000 & 1.96170600 & -0.99190300 \\ \mathrm{H} & 2.78849900 & 3.14943100 & 0.90434500 \\ \mathrm{H} & 4.94950000 & -0.40765100 & 1.97309400 \\ \mathrm{H} & 3.89583400 & -1.61652800 & 0.06835000 \\ \mathrm{H} & 5.40132800 & 2.84357000 & 2.34354200 \\ \mathrm{H} & 3.87419700 & 2.84124300 & 3.22439500 \\ \mathrm{H} & 5.02624600 & 1.51157900 & 3.44259400\end{array}$<smiles>O=[N+]([O-])C=C(c1ccsc1)C(F)(F)F</smiles>

Nitroalkene-64

$\begin{array}{lrrr}\mathrm{C} & -0.70699300 & 0.12700000 & 0.04461400 \\ \mathrm{C} & 0.70353100 & -0.27177200 & 0.00694700 \\ \mathrm{C} & 1.80893100 & 0.49170400 & -0.03051200 \\ \mathrm{C} & 0.96460600 & -1.77148800 & 0.08305600 \\ \mathrm{~F} & 0.53376700 & -2.38229000 & -1.04721000 \\ \mathrm{~F} & 2.26949600 & -2.07360300 & 0.21860300 \\ \mathrm{~F} & 0.31027500 & -2.32591400 & 1.11722000 \\ \mathrm{~N} & 1.85347000 & 1.93415800 & -0.19243800 \\ \mathrm{O} & 2.91382800 & 2.46203600 & 0.15109100 \\ \mathrm{O} & 0.89100000 & 2.52874300 & -0.67594700 \\ \mathrm{C} & -1.71802600 & -0.61928300 & -0.52739500 \\ \mathrm{~S} & -3.26930400 & 0.07552000 & -0.26244300 \\ \mathrm{C} & -2.57741100 & 1.37732600 & 0.66072600 \\ \mathrm{C} & -1.22252300 & 1.27997300 & 0.73403100 \\ \mathrm{H} & 2.80271700 & 0.08313900 & 0.06367000 \\ \mathrm{H} & -1.62095800 & -1.52948000 & -1.09761000 \\ \mathrm{H} & -3.21512800 & 2.13567400 & 1.09094500 \\ \mathrm{H} & -0.60522300 & 1.99252400 & 1.26168800\end{array}$


<smiles>O=[N+]([O-])/C=C(/c1ccc(Br)cc1)C(F)(F)F</smiles>

Nitroalkene-65

$\begin{array}{lrrr}\mathrm{C} & -0.53390300 & -0.11543700 & 0.02203400 \\ \mathrm{C} & -2.01201400 & -0.15316300 & 0.00951500 \\ \mathrm{C} & -2.85199200 & 0.88707900 & 0.06042700 \\ \mathrm{C} & -2.65191100 & -1.52198900 & -0.14965600 \\ \mathrm{C} & 0.20758200 & -0.98061600 & 0.83911800 \\ \mathrm{C} & 1.59877300 & -0.94585000 & 0.82750100 \\ \mathrm{C} & 2.25088600 & -0.05298500 & -0.01906600 \\ \mathrm{C} & 1.53507000 & 0.80399500 & -0.85380200 \\ \mathrm{C} & 0.14657700 & 0.76877600 & -0.82790700 \\ \mathrm{~F} & -3.97365400 & -1.45408000 & -0.39914500 \\ \mathrm{~F} & -2.07893900 & -2.20662900 & -1.15361400 \\ \mathrm{~F} & -2.49850300 & -2.25063200 & 0.98130000 \\ \mathrm{~N} & -2.44455500 & 2.27353900 & 0.26873200 \\ \mathrm{O} & -3.16593500 & 3.11009800 & -0.27664300 \\ \mathrm{O} & -1.46733800 & 2.51910500 & 0.96914300 \\ \mathrm{Br} & 4.15450600 & -0.00905500 & -0.04594400 \\ \mathrm{H} & -3.91868700 & 0.80293600 & -0.08307400 \\ \mathrm{H} & -0.30067500 & -1.67495500 & 1.49597500 \\ \mathrm{H} & 2.17074500 & -1.60587400 & 1.46827100 \\ \mathrm{H} & 2.05757500 & 1.48488100 & -1.51455100 \\ \mathrm{H} & -0.41313800 & 1.42476700 & -1.48556000\end{array}$<smiles>O=[N+]([O-])C=C(c1ccc(C(F)(F)F)cc1)C(F)(F)F</smiles>

Nitroalkene-66

$\begin{array}{lrrr}\mathrm{C} & -0.48089000 & -0.10375600 & 0.03610800 \\ \mathrm{C} & -1.96183100 & -0.15728100 & 0.01689500 \\ \mathrm{C} & -2.80835600 & 0.87545600 & 0.06714200 \\ \mathrm{C} & -2.58051700 & -1.53374400 & -0.15191400 \\ \mathrm{C} & 0.25778800 & -0.88004900 & 0.93858600 \\ \mathrm{C} & 1.64800600 & -0.82912000 & 0.92830200 \\ \mathrm{C} & 2.30917000 & -0.01621700 & 0.00790300 \\ \mathrm{C} & 1.58070300 & 0.74995300 & -0.90410400 \\ \mathrm{C} & 0.19277500 & 0.70634400 & -0.88848200 \\ \mathrm{~F} & -2.38169400 & -2.28115200 & 0.95801700 \\ \mathrm{~F} & -3.90902600 & -1.48499800 & -0.36572000 \\ \mathrm{~F} & -2.02011000 & -2.18366000 & -1.18569000 \\ \mathrm{~N} & -2.40231500 & 2.26511700 & 0.26270400 \\ \mathrm{O} & -3.14821900 & 3.09686600 & -0.25530600\end{array}$




$\begin{array}{lrrr}\mathrm{O} & -1.39892600 & 2.51431300 & 0.92341000 \\ \mathrm{C} & 3.81279500 & -0.01435000 & -0.04656200 \\ \mathrm{~F} & 4.29596000 & 1.16966100 & -0.47889800 \\ \mathrm{~F} & 4.35768700 & -0.26392000 & 1.16315600 \\ \mathrm{~F} & 4.27487000 & -0.96338400 & -0.89271400 \\ \mathrm{H} & -3.87573700 & 0.78525300 & -0.06833500 \\ \mathrm{H} & -0.25304700 & -1.51020700 & 1.65521700 \\ \mathrm{H} & 2.21924600 & -1.41495400 & 1.63880100 \\ \mathrm{H} & 2.09936800 & 1.38090700 & -1.61625100 \\ \mathrm{H} & -0.37349500 & 1.29741700 & -1.59978400\end{array}$<smiles>O=[N+]([O-])C=C(c1ccc(F)cc1)C(F)(F)F</smiles>

Nitroalkene-67

$\begin{array}{lrrr}\mathrm{C} & -0.42853200 & -0.11724000 & 0.00392100 \\ \mathrm{C} & 1.04835600 & -0.15403600 & 0.00926400 \\ \mathrm{C} & 1.89066400 & 0.88503400 & 0.06694700 \\ \mathrm{C} & 1.69204200 & -1.52286700 & -0.13834700 \\ \mathrm{C} & -1.18007600 & -1.00042400 & 0.79438700 \\ \mathrm{C} & -2.57057400 & -0.96620400 & 0.76797800 \\ \mathrm{C} & -3.20170200 & -0.05376700 & -0.06835000 \\ \mathrm{C} & -2.48685400 & 0.82178100 & -0.87907600 \\ \mathrm{C} & -1.09938200 & 0.78508600 & -0.83667300 \\ \mathrm{~F} & 1.12602300 & -2.21642900 & -1.14028100 \\ \mathrm{~F} & 3.01510300 & -1.45496800 & -0.38177300 \\ \mathrm{~F} & 1.53519700 & -2.24489900 & 0.99694400 \\ \mathrm{~N} & 1.48971700 & 2.27284600 & 0.27470500 \\ \mathrm{O} & 2.22608700 & 3.10711600 & -0.25465300 \\ \mathrm{O} & 0.50418000 & 2.52422200 & 0.96172100 \\ \mathrm{~F} & -4.54530000 & -0.02191000 & -0.10300500 \\ \mathrm{H} & 2.95796900 & 0.79729900 & -0.06883100 \\ \mathrm{H} & -0.67778300 & -1.70755200 & 1.44182900 \\ \mathrm{H} & -3.16521900 & -1.63264600 & 1.38160300 \\ \mathrm{H} & -3.01734200 & 1.50926700 & -1.52719700 \\ \mathrm{H} & -0.53064300 & 1.45268100 & -1.47416300\end{array}$<smiles>Cc1ccc(/C(=C\[N+](=O)[O-])C(F)(F)F)cc1</smiles>

Nitroalkene-68
C
$\begin{array}{lll}-0.39832400 & -0.12386600 & 0.00499700\end{array}$
$\begin{array}{llll}\mathrm{C} & 1.07818600 & -0.14962300 & 0.01031300\end{array}$
$\begin{array}{llll}\mathrm{C} & 1.91146400 & 0.89703600 & 0.06632800\end{array}$ 


\begin{tabular}{|c|c|c|c|}
\hline $\mathrm{C}$ & 1.73459600 & -1.51199300 & -0.13671000 \\
\hline $\mathrm{C}$ & -1.14760000 & -1.01884400 & 0.78110900 \\
\hline $\mathrm{C}$ & -2.53825400 & -0.98538900 & 0.74378300 \\
\hline $\mathrm{C}$ & -3.22159800 & -0.07861500 & -0.07321800 \\
\hline $\mathrm{C}$ & -2.46475500 & 0.79683500 & -0.86449400 \\
\hline $\mathrm{C}$ & -1.07768600 & 0.77941500 & -0.82677600 \\
\hline $\mathrm{F}$ & 1.17382900 & -2.21420700 & -1.13580200 \\
\hline $\mathrm{F}$ & 3.05705200 & -1.43168600 & -0.38495400 \\
\hline $\mathrm{F}$ & 1.58999700 & -2.23397100 & 1.00006500 \\
\hline $\mathrm{N}$ & 1.49634400 & 2.28030600 & 0.27429800 \\
\hline $\mathrm{O}$ & 2.20292300 & 3.12132500 & -0.28511000 \\
\hline $\mathrm{O}$ & 0.53054400 & 2.52324000 & 0.99128900 \\
\hline $\mathrm{C}$ & -4.72736400 & -0.02823200 & -0.09291400 \\
\hline $\mathrm{H}$ & 2.97911300 & 0.82040200 & -0.07341400 \\
\hline $\mathrm{H}$ & -0.64449300 & -1.73241200 & 1.42144800 \\
\hline $\mathrm{H}$ & -3.10258800 & -1.67913000 & 1.36011100 \\
\hline $\mathrm{H}$ & -2.97117700 & 1.49798300 & -1.52150400 \\
\hline $\mathrm{H}$ & -0.51419900 & 1.45568000 & -1.46072400 \\
\hline $\mathrm{H}$ & -5.09761900 & 0.78453500 & 0.54344000 \\
\hline $\mathrm{H}$ & -5.10714900 & 0.15277200 & -1.10278600 \\
\hline $\mathrm{H}$ & -5.16393100 & -0.96006800 & 0.27561500 \\
\hline
\end{tabular}<smiles>COc1ccc(/C(=C\[N+](=O)[O-])C(F)(F)F)cc1</smiles>

Nitroalkene-69

$\begin{array}{rrrr}\mathrm{C} & 0.08792600 & -0.03434800 & -0.04818800 \\ \mathrm{C} & -1.37166800 & -0.21460600 & -0.00779400 \\ \mathrm{C} & -2.33428600 & 0.71961200 & 0.06548300 \\ \mathrm{C} & -1.87549000 & -1.64719600 & -0.12277400 \\ \mathrm{C} & 0.94759300 & -0.90163300 & 0.64004300 \\ \mathrm{C} & 2.32969700 & -0.74610900 & 0.58169100 \\ \mathrm{C} & 2.87909900 & 0.27914400 & -0.19701200 \\ \mathrm{C} & 2.02961300 & 1.13943200 & -0.91240800 \\ \mathrm{C} & 0.65949500 & 0.98561100 & -0.83341600 \\ \mathrm{~F} & -1.23304400 & -2.31362900 & -1.09696200 \\ \mathrm{~F} & -1.66407600 & -2.31817100 & 1.03624700 \\ \mathrm{~F} & -3.19581600 & -1.72230300 & -0.38142100 \\ \mathrm{~N} & -2.13212200 & 2.14128200 & 0.29869200 \\ \mathrm{O} & -3.07135400 & 2.86058200 & -0.05243900 \\ \mathrm{O} & -1.10490500 & 2.53729600 & 0.84538500 \\ \mathrm{O} & 4.20694900 & 0.51650500 & -0.33359700 \\ \mathrm{C} & 5.11590300 & -0.32398600 & 0.36563400 \\ \mathrm{H} & -3.38299000 & 0.49335500 & -0.04826500 \\ \mathrm{H} & 0.53737200 & -1.70008100 & 1.24516100 \\ \mathrm{H} & 2.96157800 & -1.42448500 & 1.14013800 \\ \mathrm{H} & 2.47419600 & 1.91652900 & -1.52348300 \\ \mathrm{H} & 0.01992500 & 1.65077500 & -1.40121000 \\ \mathrm{H} & 6.11228700 & 0.04259000 & 0.11899800\end{array}$


$\begin{array}{llll}\mathrm{H} & 4.96356800 & -0.26289500 & 1.44974700 \\ \mathrm{H} & 5.02253400 & -1.36842400 & 0.04494400\end{array}$<smiles>O=[N+]([O-])C=C(CBr)C(F)(F)F</smiles>

Nitroalkene-70

$\begin{array}{lrrr}\mathrm{C} & -0.11112000 & -0.35401900 & 1.21941400 \\ \mathrm{C} & -1.18511400 & 0.11533000 & 0.26236100 \\ \mathrm{C} & -1.27600200 & 1.28357600 & -0.37815200 \\ \mathrm{C} & -2.29218500 & -0.88137100 & 0.00103900 \\ \mathrm{~F} & -1.79169000 & -2.04780100 & -0.46712900 \\ \mathrm{~F} & -2.93487300 & -1.16080800 & 1.15447000 \\ \mathrm{~F} & -3.20821400 & -0.45176600 & -0.88491500 \\ \mathrm{~N} & -0.32564800 & 2.37424700 & -0.23578900 \\ \mathrm{O} & 0.40633800 & 2.41327800 & 0.75327200 \\ \mathrm{O} & -0.34329100 & 3.20945400 & -1.13973600 \\ \mathrm{C} & 1.24502200 & -0.54094600 & 0.55480900 \\ \mathrm{C} & 2.37161400 & 0.11660300 & 1.05671500 \\ \mathrm{C} & 3.62247500 & -0.07299200 & 0.46969700 \\ \mathrm{C} & 3.75893200 & -0.91771100 & -0.63098200 \\ \mathrm{C} & 2.63754900 & -1.57461100 & -1.13978200 \\ \mathrm{C} & 1.38878000 & -1.38909300 & -0.55016100 \\ \mathrm{H} & -0.43979100 & -1.29711700 & 1.66506100 \\ \mathrm{H} & -0.02242000 & 0.37711300 & 2.02399000 \\ \mathrm{H} & -2.04991400 & 1.51996000 & -1.09269400 \\ \mathrm{H} & 2.26477500 & 0.78766400 & 1.90224200 \\ \mathrm{H} & 4.48759600 & 0.44677600 & 0.86951400 \\ \mathrm{H} & 4.73141500 & -1.06219900 & -1.09119700 \\ \mathrm{H} & 2.73463600 & -2.23485000 & -1.99620800 \\ \mathrm{H} & 0.52215600 & -1.90415700 & -0.95004000\end{array}$<smiles>CCCCCCCCC/C(=C\[N+](=O)[O-])C(F)(F)F</smiles>

Nitroalkene-71

$\begin{array}{lrrr}\text { C } & -1.75346900 & -0.17287600 & 0.89384400 \\ \text { C } & -3.07009300 & -0.14959400 & 0.16915000 \\ \text { C } & -3.76894600 & 0.90133100 & -0.27286400 \\ \text { C } & -3.66340000 & -1.51421500 & -0.11325600 \\ \text { F } & -2.78506700 & -2.27220300 & -0.80525200 \\ \text { F } & -3.92437800 & -2.15739400 & 1.04422300 \\ \text { F } & -4.80731300 & -1.47107800 & -0.82078600 \\ \text { N } & -3.38772500 & 2.29036400 & -0.07833500 \\ \text { O } & -4.25548800 & 3.11722700 & -0.36066600\end{array}$




$\begin{array}{lrrr}\mathrm{O} & -2.26062500 & 2.56596000 & 0.33967600 \\ \mathrm{C} & -0.55323700 & -0.10183600 & -0.07405100 \\ \mathrm{C} & 0.78099900 & -0.13108600 & 0.67314100 \\ \mathrm{C} & 1.98815600 & -0.06348000 & -0.26571800 \\ \mathrm{C} & 3.32933500 & -0.08628200 & 0.47198500 \\ \mathrm{C} & 4.53816900 & -0.01815700 & -0.46469600 \\ \mathrm{C} & 5.87996800 & -0.03993800 & 0.27159200 \\ \mathrm{C} & 7.09002300 & 0.02805000 & -0.66437000 \\ \mathrm{C} & 8.42603300 & 0.00603300 & 0.08089200 \\ \mathrm{H} & -1.69885200 & -1.09458800 & 1.48092700 \\ \mathrm{H} & -1.70555000 & 0.67279100 & 1.58088700 \\ \mathrm{H} & -4.71618800 & 0.82149700 & -0.78402900 \\ \mathrm{H} & -0.63218400 & 0.82132900 & -0.65624600 \\ \mathrm{H} & -0.60731500 & -0.93881000 & -0.77847900 \\ \mathrm{H} & 0.81854800 & 0.71070400 & 1.37747400 \\ \mathrm{H} & 0.84169900 & -1.04463200 & 1.28080400 \\ \mathrm{H} & 1.92277100 & 0.84892100 & -0.87405500 \\ \mathrm{H} & 1.94674300 & -0.90412900 & -0.97214400 \\ \mathrm{H} & 3.39311900 & -0.99818500 & 1.08203000 \\ \mathrm{H} & 3.36901800 & 0.75444600 & 1.17841000 \\ \mathrm{H} & 4.47332000 & 0.89351000 & -1.07490400 \\ \mathrm{H} & 4.49776700 & -0.85888900 & -1.17139900 \\ \mathrm{H} & 5.92071400 & 0.80093300 & 0.97825600 \\ \mathrm{H} & 5.94507500 & -0.95142800 & 0.88247700 \\ \mathrm{H} & 7.04802400 & -0.81257300 & -1.36985400 \\ \mathrm{H} & 7.02354000 & 0.93902100 & -1.27408000 \\ \mathrm{H} & 8.50710400 & 0.85450400 & 0.76951900 \\ \mathrm{H} & 8.53182100 & -0.90971000 & 0.67331200 \\ \mathrm{H} & 9.27338100 & 0.05561500 & -0.61018800 \\ & & & \end{array}$<smiles>O=[N+]([O-])C=C(c1ccccc1)C(F)(F)F</smiles>

Nitroalkene-72

$\begin{array}{lrrr}\text { C } & -0.75139700 & -0.14720600 & -0.00963400 \\ \text { C } & 0.72779200 & -0.13186200 & 0.01751400 \\ \text { C } & 1.52464400 & 0.94000900 & 0.09075900 \\ \text { C } & 1.42324400 & -1.47380500 & -0.12990100 \\ \text { C } & -1.48281300 & -1.02891100 & 0.79892000 \\ \text { C } & -2.87445000 & -1.03529000 & 0.74324900 \\ \text { C } & -3.54781100 & -0.17893700 & -0.12739400 \\ \text { C } & -2.82372100 & 0.68848800 & -0.94706600 \\ \text { C } & -1.43460900 & 0.70565000 & -0.88904400 \\ \text { F } & 1.26571000 & -2.21635800 & 0.99114200 \\ \text { F } & 0.90790500 & -2.17336300 & -1.15490000 \\ \text { F } & 2.74921900 & -1.35463200 & -0.33994500 \\ \text { H } & 2.59703400 & 0.90100100 & -0.02787700 \\ \text { H } & -0.96479400 & -1.69603300 & 1.47629800 \\ \text { H } & -3.43210300 & -1.71259400 & 1.38193100\end{array}$




$$
\begin{array}{lrrr}
\mathrm{H} & -4.63222000 & -0.19016700 & -0.17146800 \\
\mathrm{H} & -3.34060400 & 1.35055700 & -1.63400200 \\
\mathrm{H} & -0.87332300 & 1.37102700 & -1.53648900 \\
\mathrm{~N} & 1.05291800 & 2.30792900 & 0.28674900 \\
\mathrm{O} & 0.07317800 & 2.51444200 & 0.99543100 \\
\mathrm{O} & 1.72742200 & 3.17194100 & -0.27627200
\end{array}
$$<smiles>C/C(=C\[N+](=O)[O-])C(C)(C)C</smiles>

Nitroalkene-73

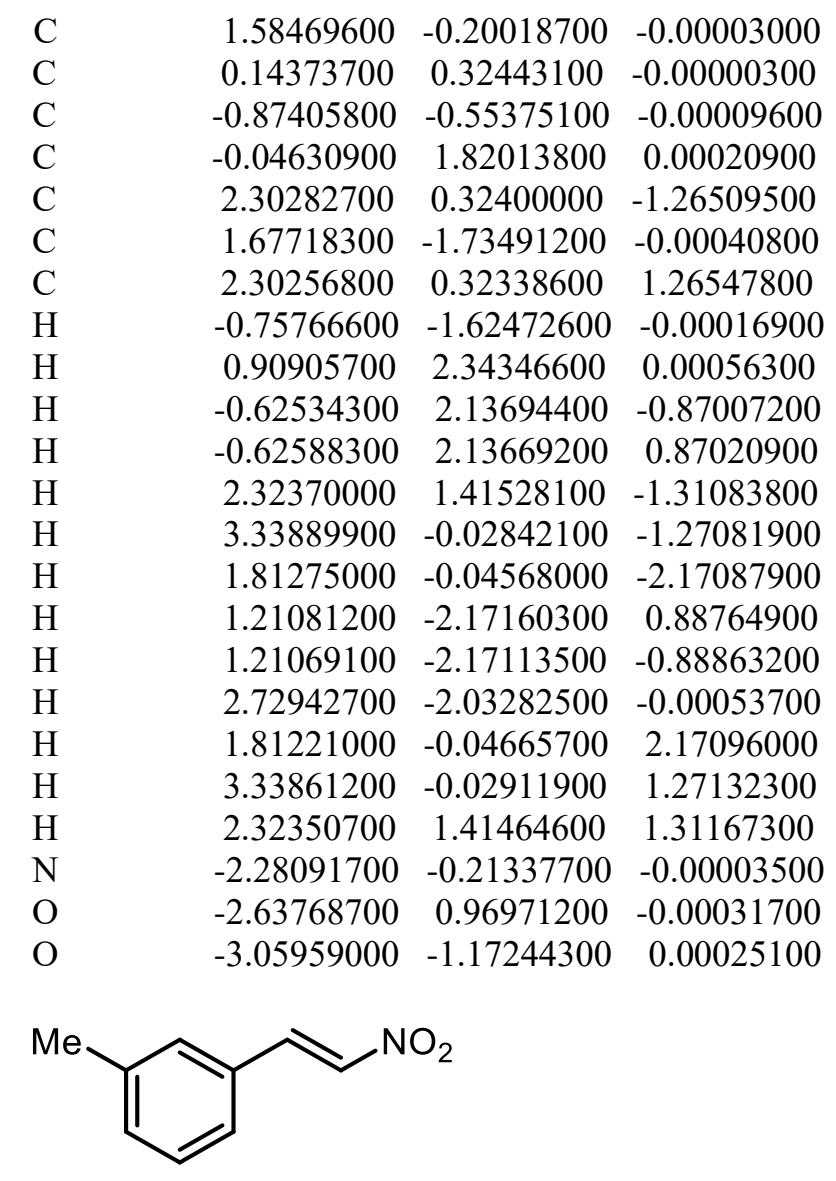

Nitroalkene-74

$\begin{array}{lrrr}\mathrm{C} & 2.54252900 & 0.71650100 & 0.00005100 \\ \mathrm{C} & 1.15093200 & 0.69116400 & 0.00010100 \\ \mathrm{C} & 0.43667300 & -0.52243500 & 0.00003800 \\ \mathrm{C} & 1.15463000 & -1.72840900 & -0.00005200 \\ \mathrm{C} & 2.54733600 & -1.71705000 & -0.00008100 \\ \mathrm{C} & 3.23436900 & -0.50663400 & -0.00002900 \\ \mathrm{C} & -1.01905500 & -0.58415900 & 0.00007600\end{array}$




$\begin{array}{lrrr}\mathrm{C} & -1.86644900 & 0.45360200 & -0.00005000 \\ \mathrm{~N} & -3.29850800 & 0.23199100 & -0.00001600 \\ \mathrm{O} & -3.73295200 & -0.92256400 & 0.00010500 \\ \mathrm{O} & -3.99887600 & 1.24904600 & -0.00011700 \\ \mathrm{C} & 3.29990000 & 2.02091900 & 0.00000600 \\ \mathrm{H} & 0.61083100 & 1.63281900 & 0.00022300 \\ \mathrm{H} & 0.61462900 & -2.67036800 & -0.00010100 \\ \mathrm{H} & 3.09659400 & -2.65309700 & -0.00013600 \\ \mathrm{H} & 4.32098500 & -0.50267800 & -0.00002200 \\ \mathrm{H} & -1.47292400 & -1.57166200 & 0.00016500 \\ \mathrm{H} & -1.62251400 & 1.50548100 & -0.00020100 \\ \mathrm{H} & 3.94481000 & 2.10289600 & -0.88158600 \\ \mathrm{H} & 3.94543200 & 2.10265600 & 0.88115600 \\ \mathrm{H} & 2.62113500 & 2.87717100 & 0.00034500\end{array}$<smiles>O=[N+]([O-])/C=C/c1ccc([N+](=O)[O-])cc1</smiles>

Nitroalkene-75

$\begin{array}{rrrr}\mathrm{C} & 1.57926000 & -1.15134400 & 0.00020900 \\ \mathrm{C} & 0.20035300 & -1.00833900 & 0.00029300 \\ \mathrm{C} & -0.39108200 & 0.27027100 & 0.00017900 \\ \mathrm{C} & 0.44276600 & 1.40291300 & 0.00002600 \\ \mathrm{C} & 1.82638500 & 1.27472100 & -0.00008000 \\ \mathrm{C} & 2.37267000 & -0.00439300 & 0.00000100 \\ \mathrm{C} & -1.83608600 & 0.47725500 & 0.00018600 \\ \mathrm{C} & -2.77463900 & -0.47579700 & -0.00013700 \\ \mathrm{~N} & -4.18407500 & -0.11633200 & -0.00008300 \\ \mathrm{~N} & 3.83687000 & -0.15345700 & -0.00011100 \\ \mathrm{O} & -4.97422000 & -1.06265800 & -0.00049300 \\ \mathrm{O} & -4.50032700 & 1.07410800 & 0.00032800 \\ \mathrm{O} & 4.51219800 & 0.87416400 & -0.00038100 \\ \mathrm{O} & 4.29083500 & -1.29644900 & 0.00014400 \\ \mathrm{H} & 2.05289200 & -2.12422100 & 0.00031600 \\ \mathrm{H} & -0.42069100 & -1.89680800 & 0.00050400 \\ \mathrm{H} & -0.00403500 & 2.39183100 & -0.00001700 \\ \mathrm{H} & 2.48213500 & 2.13540900 & -0.00022600 \\ \mathrm{H} & -2.19184300 & 1.50392900 & 0.00046000 \\ \mathrm{H} & -2.63366100 & -1.54665100 & -0.00051900\end{array}$<smiles>CC/C(=C\c1ccccc1)[N+](=O)[O-]</smiles>

Nitroalkene-76

$\mathrm{C}$

$\begin{array}{lll}-3.48692200 & -1.08569700 & 0.19784000\end{array}$ 


$\begin{array}{lrrr}\mathrm{C} & -2.13388700 & -1.39713300 & 0.11817400 \\ \mathrm{C} & -1.16820600 & -0.39215800 & -0.08179200 \\ \mathrm{C} & -1.61062000 & 0.93365900 & -0.24753200 \\ \mathrm{C} & -2.96638800 & 1.24126800 & -0.18011500 \\ \mathrm{C} & -3.90787700 & 0.23735600 & 0.05260100 \\ \mathrm{C} & 0.23295800 & -0.80435700 & -0.13560000 \\ \mathrm{C} & 1.33461300 & -0.09336900 & 0.16634700 \\ \mathrm{~N} & 2.62361500 & -0.80199300 & -0.01389100 \\ \mathrm{O} & 2.62607800 & -1.96003600 & -0.43419700 \\ \mathrm{O} & 3.64615500 & -0.17186000 & 0.27002600 \\ \mathrm{C} & 1.45944000 & 1.30349900 & 0.69271000 \\ \mathrm{C} & 1.85202400 & 2.33529100 & -0.37766800 \\ \mathrm{H} & -4.21355100 & -1.87434200 & 0.36527100 \\ \mathrm{H} & -1.80746200 & -2.42752000 & 0.22398100 \\ \mathrm{H} & -0.89924900 & 1.71894400 & -0.46812700 \\ \mathrm{H} & -3.29002000 & 2.26795200 & -0.31945400 \\ \mathrm{H} & -4.96389200 & 0.48256700 & 0.10594400 \\ \mathrm{H} & 0.41184900 & -1.83493200 & -0.42737500 \\ \mathrm{H} & 2.21702700 & 1.30522900 & 1.48023600 \\ \mathrm{H} & 0.50687800 & 1.57528700 & 1.15453700 \\ \mathrm{H} & 1.91673400 & 3.33470000 & 0.06205200 \\ \mathrm{H} & 2.82582000 & 2.08467800 & -0.80355000 \\ \mathrm{H} & 1.12188200 & 2.36639800 & -1.19270200\end{array}$<smiles>CC(=Cc1ccccc1Br)[N+](=O)[O-]</smiles>

Nitroalkene-77

$\begin{array}{lrrr}\mathrm{C} & -1.73552000 & 2.79987700 & -0.31354300 \\ \mathrm{C} & -0.50514500 & 2.15541700 & -0.38740400 \\ \mathrm{C} & -0.39611400 & 0.76059100 & -0.23765900 \\ \mathrm{C} & -1.58830200 & 0.04217300 & -0.03084300 \\ \mathrm{C} & -2.82456500 & 0.67469500 & 0.04942100 \\ \mathrm{C} & -2.89679500 & 2.05986700 & -0.09006900 \\ \mathrm{C} & 0.88711000 & 0.06630400 & -0.35648000 \\ \mathrm{C} & 2.06887600 & 0.48546600 & 0.12135100 \\ \mathrm{~N} & 3.23679400 & -0.36987700 & -0.19828700 \\ \mathrm{O} & 3.07163800 & -1.38953800 & -0.86757400 \\ \mathrm{O} & 4.32750200 & 0.01112100 & 0.23136100 \\ \mathrm{C} & 2.38878800 & 1.66940100 & 0.97346100 \\ \mathrm{Br} & -1.53096600 & -1.85519300 & 0.17839600 \\ \mathrm{H} & -1.78863500 & 3.87597600 & -0.44050400 \\ \mathrm{H} & 0.39021600 & 2.72835100 & -0.59887600 \\ \mathrm{H} & -3.71738900 & 0.08535900 & 0.22072500 \\ \mathrm{H} & -3.86099700 & 2.55413900 & -0.03112400 \\ \mathrm{H} & 0.88484000 & -0.88977300 & -0.86927700 \\ \mathrm{H} & 2.93752600 & 2.43448000 & 0.41438900 \\ \mathrm{H} & 1.46976800 & 2.10789300 & 1.36292200\end{array}$


<smiles>CC(=Cc1ccccc1Cl)[N+](=O)[O-]</smiles>

Nitroalkene-78

\begin{tabular}{|c|c|c|c|}
\hline $\mathrm{C}$ & -2.39010400 & -2.05699600 & -0.36235700 \\
\hline $\mathrm{C}$ & -1.06995700 & -1.62452000 & -0.42683900 \\
\hline $\mathrm{C}$ & -0.72621700 & -0.27701900 & -0.21325800 \\
\hline $\mathrm{C}$ & -1.77846100 & 0.62132400 & 0.04788000 \\
\hline $\mathrm{C}$ & -3.10291900 & 0.19988000 & 0.12020100 \\
\hline $\mathrm{C}$ & -3.40793000 & -1.14469500 & -0.08352500 \\
\hline $\mathrm{C}$ & 0.65492900 & 0.19669000 & -0.32206600 \\
\hline $\mathrm{C}$ & 1.75051500 & -0.43942000 & 0.12071100 \\
\hline $\mathrm{N}$ & 3.04688900 & 0.21426800 & -0.18024500 \\
\hline $\mathrm{O}$ & 4.05765300 & -0.36520700 & 0.22214300 \\
\hline $\mathrm{O}$ & 3.05678300 & 1.27201200 & -0.80946400 \\
\hline $\mathrm{C}$ & 1.86725800 & -1.69594000 & 0.91928500 \\
\hline $\mathrm{Cl}$ & -1.43092000 & 2.32010300 & 0.32317800 \\
\hline $\mathrm{H}$ & -2.62453700 & -3.10127100 & -0.53951200 \\
\hline $\mathrm{H}$ & -0.28505500 & -2.32712600 & -0.68150000 \\
\hline $\mathrm{H}$ & -3.88130800 & 0.92230600 & 0.33587600 \\
\hline $\mathrm{H}$ & -4.44079200 & -1.47297300 & -0.03060300 \\
\hline $\mathrm{H}$ & 0.81538600 & 1.15838000 & -0.79763400 \\
\hline $\mathrm{H}$ & 0.88884000 & -1.98701200 & 1.30202300 \\
\hline $\mathrm{H}$ & 2.27363400 & -2.51891000 & 0.32214400 \\
\hline $\mathrm{H}$ & 2.55308900 & -1.54526900 & 1.75525700 \\
\hline
\end{tabular}

Nitroalkene-79

$\begin{array}{lrrr}\mathrm{C} & -1.86803700 & 0.34365100 & -0.00144500 \\ \mathrm{C} & -0.52508500 & 0.01468300 & -0.14251800 \\ \mathrm{C} & 0.45166100 & 1.02079500 & -0.03980300 \\ \mathrm{C} & 0.03521000 & 2.34806500 & 0.16907000 \\ \mathrm{C} & -1.31308500 & 2.65514700 & 0.31837100 \\ \mathrm{C} & -2.28045600 & 1.65276600 & 0.23808500 \\ \mathrm{C} & 1.88435500 & 0.75957100 & -0.17774200 \\ \mathrm{C} & 2.55700000 & -0.35815300 & 0.14415600 \\ \mathrm{~N} & 4.00917700 & -0.34591500 & -0.15890900 \\ \mathrm{O} & 4.62789200 & -1.38484900 & 0.07819100 \\ \mathrm{O} & 4.51841600 & 0.67100300 & -0.63165300 \\ \mathrm{C} & 2.07416100 & -1.61229300 & 0.79577800 \\ \mathrm{Br} & -3.17899000 & -1.03617400 & -0.15168500\end{array}$




$$
\begin{array}{lrrr}
\mathrm{H} & -0.24850800 & -1.00622700 & -0.36692900 \\
\mathrm{H} & 0.78154800 & 3.13402600 & 0.22602600 \\
\mathrm{H} & -1.61978800 & 3.68136000 & 0.49233700 \\
\mathrm{H} & -3.33389400 & 1.88151100 & 0.34572400 \\
\mathrm{H} & 2.48673700 & 1.57562700 & -0.56495400 \\
\mathrm{H} & 1.12497800 & -1.43027600 & 1.30094400 \\
\mathrm{H} & 1.93560400 & -2.42111400 & 0.06995600 \\
\mathrm{H} & 2.80891500 & -1.96204600 & 1.52221600
\end{array}
$$

Nitroalkene-80

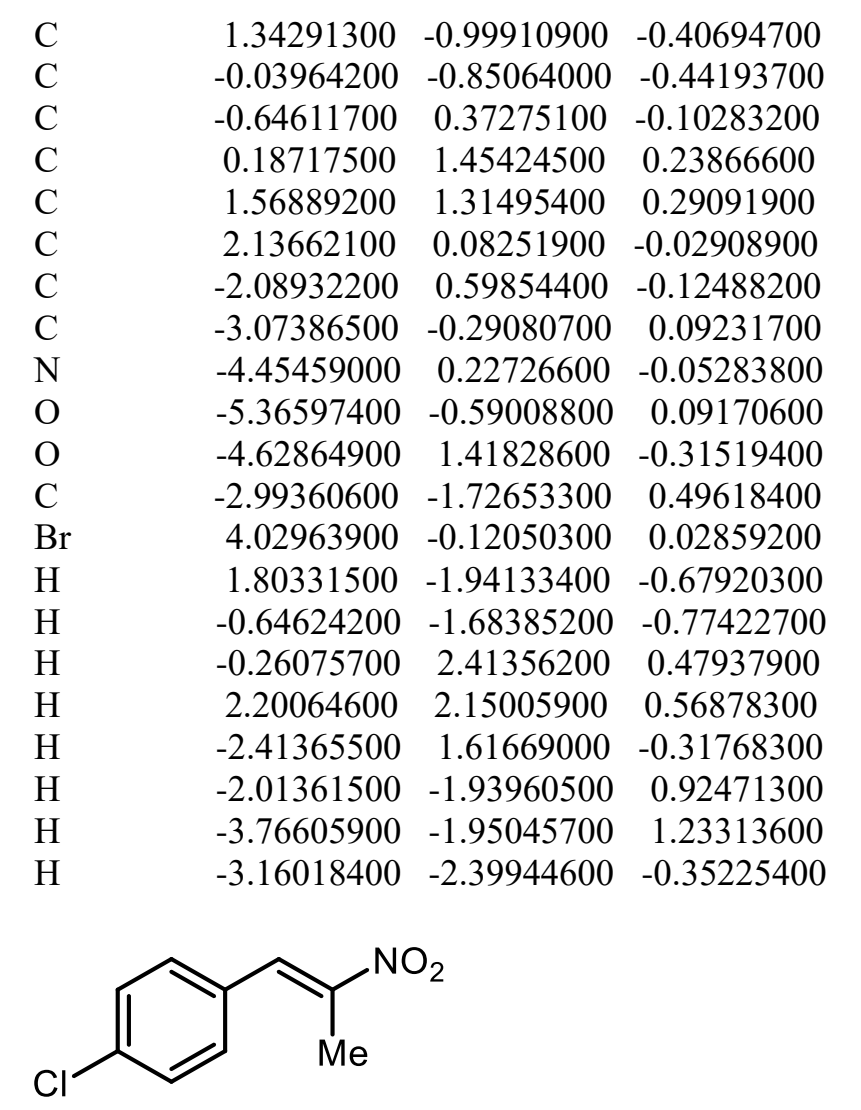

Nitroalkene-81

$\begin{array}{lrrr}C & -2.06596700 & -1.05215000 & -0.39418100 \\ \mathrm{C} & -0.68596900 & -0.88411500 & -0.43367600 \\ \mathrm{C} & -0.09516500 & 0.34756300 & -0.09745900 \\ \mathrm{C} & -0.94241200 & 1.41752800 & 0.24559400 \\ \mathrm{C} & -2.32173300 & 1.25974200 & 0.30252700 \\ \mathrm{C} & -2.87417000 & 0.01889800 & -0.01457100\end{array}$




$\begin{array}{lrrr}\mathrm{C} & 1.34473400 & 0.59300400 & -0.12421400 \\ \mathrm{C} & 2.34196600 & -0.28252500 & 0.09147600 \\ \mathrm{~N} & 3.71500200 & 0.25411800 & -0.05839400 \\ \mathrm{O} & 4.63784900 & -0.55045100 & 0.08535800 \\ \mathrm{O} & 3.87207800 & 1.44687300 & -0.32361500 \\ \mathrm{C} & 2.28248500 & -1.71855600 & 0.49780600 \\ \mathrm{Cl} & -4.61225300 & -0.19148900 & 0.04403600 \\ \mathrm{H} & -2.51527600 & -2.00050000 & -0.66448700 \\ \mathrm{H} & -0.06880800 & -1.70898700 & -0.76716600 \\ \mathrm{H} & -0.50690800 & 2.38304700 & 0.48401000 \\ \mathrm{H} & -2.96511000 & 2.08582600 & 0.58146200 \\ \mathrm{H} & 1.65477900 & 1.61507600 & -0.31971900 \\ \mathrm{H} & 1.30678700 & -1.94424700 & 0.92966700 \\ \mathrm{H} & 2.45560400 & -2.39059200 & -0.35004600 \\ \mathrm{H} & 3.06019500 & -1.93084000 & 1.23266800\end{array}$<smiles>C/C(=C\c1ccc(F)cc1)[N+](=O)[O-]</smiles>

Nitroalkene-82

$\begin{array}{lrrr}\mathrm{C} & -2.48316800 & 1.11976600 & -0.36830100 \\ \mathrm{C} & -1.10602800 & 0.93098900 & -0.41031500 \\ \mathrm{C} & -0.53404700 & -0.31418100 & -0.08609800 \\ \mathrm{C} & -1.39729400 & -1.37604900 & 0.24731900 \\ \mathrm{C} & -2.77409700 & -1.19893800 & 0.30674100 \\ \mathrm{C} & -3.29581100 & 0.05353400 & 0.00106200 \\ \mathrm{C} & 0.90079200 & -0.58317000 & -0.11597200 \\ \mathrm{C} & 1.91657100 & 0.27413700 & 0.08940200 \\ \mathrm{~N} & 3.27690300 & -0.28904300 & -0.06478200 \\ \mathrm{O} & 4.21690500 & 0.49756800 & 0.07037400 \\ \mathrm{O} & 3.41043200 & -1.48622000 & -0.32488000 \\ \mathrm{C} & 1.88566400 & 1.71355900 & 0.48778100 \\ \mathrm{~F} & -4.62789200 & 0.23463500 & 0.04696800 \\ \mathrm{H} & -2.93389400 & 2.07086500 & -0.62728500 \\ \mathrm{H} & -0.47528600 & 1.74842200 & -0.73595100 \\ \mathrm{H} & -0.97405200 & -2.34944600 & 0.47511600 \\ \mathrm{H} & -3.44297400 & -2.00837900 & 0.57520600 \\ \mathrm{H} & 1.19189000 & -1.61174200 & -0.30656700 \\ \mathrm{H} & 0.92114200 & 1.95563900 & 0.93592700 \\ \mathrm{H} & 2.67926600 & 1.91865500 & 1.20737900 \\ \mathrm{H} & 2.05242000 & 2.37891100 & -0.36672400\end{array}$


<smiles>C/C(=C\c1ccc([N+](=O)[O-])cc1)[N+](=O)[O-]</smiles>

Nitroalkene-83

$\begin{array}{lrrr}\mathrm{C} & -1.78733700 & 1.04141400 & -0.38756000 \\ \mathrm{C} & -0.40874000 & 0.87805600 & -0.43962600 \\ \mathrm{C} & 0.18192100 & -0.36254600 & -0.13337500 \\ \mathrm{C} & -0.65510800 & -1.44762200 & 0.19103900 \\ \mathrm{C} & -2.03314300 & -1.29521700 & 0.25816800 \\ \mathrm{C} & -2.57862600 & -0.04627200 & -0.02856700 \\ \mathrm{C} & 1.62589700 & -0.59666000 & -0.17612600 \\ \mathrm{C} & 2.60727000 & 0.27321600 & 0.11276600 \\ \mathrm{~N} & 3.99247500 & -0.23563500 & -0.06561900 \\ \mathrm{O} & 4.90033400 & 0.57054700 & 0.13929800 \\ \mathrm{O} & 4.16361900 & -1.40408400 & -0.41181100 \\ \mathrm{C} & 2.52963500 & 1.67383200 & 0.62330700 \\ \mathrm{~N} & -4.03792700 & 0.12501500 & 0.03306600 \\ \mathrm{O} & -4.49137800 & 1.23906600 & -0.22521500 \\ \mathrm{O} & -4.71317400 & -0.85606300 & 0.34058000 \\ \mathrm{H} & -2.25897100 & 1.98551200 & -0.62677900 \\ \mathrm{H} & 0.20959100 & 1.70875300 & -0.75546800 \\ \mathrm{H} & -0.21136400 & -2.41460200 & 0.40467500 \\ \mathrm{H} & -2.68827700 & -2.11573800 & 0.51974700 \\ \mathrm{H} & 1.94679300 & -1.59833600 & -0.44560200 \\ \mathrm{H} & 1.53660000 & 1.87207300 & 1.02738800 \\ \mathrm{H} & 3.27488200 & 1.82742400 & 1.40536000 \\ \mathrm{H} & 2.74308800 & 2.40432900 & -0.16442400\end{array}$<smiles>COc1ccc(C=C(C)[N+](=O)[O-])cc1</smiles>

Nitroalkene-84

$\begin{array}{lrrr}\mathrm{C} & -1.97707200 & 1.27276000 & -0.36551200 \\ \mathrm{C} & -0.61249700 & 1.04448400 & -0.38356400 \\ \mathrm{C} & -0.08289600 & -0.23501900 & -0.10719200 \\ \mathrm{C} & -0.99561100 & -1.27277000 & 0.14968900 \\ \mathrm{C} & -2.36870300 & -1.05529400 & 0.18532900 \\ \mathrm{C} & -2.86795400 & 0.22898000 & -0.06841200 \\ \mathrm{C} & 1.33599900 & -0.55951100 & -0.10971600 \\ \mathrm{C} & 2.39204800 & 0.25203400 & 0.09431300 \\ \mathrm{~N} & 3.72139500 & -0.37620700 & -0.03668200 \\ \mathrm{O} & 4.69991500 & 0.36420100 & 0.09832200 \\ \mathrm{O} & 3.80204700 & -1.58311100 & -0.27964300 \\ \mathrm{C} & 2.42318800 & 1.69790000 & 0.47101100\end{array}$




$\begin{array}{lrrr}\mathrm{O} & -4.18423300 & 0.55957000 & -0.06765900 \\ \mathrm{C} & -5.13305000 & -0.46103400 & 0.21294600 \\ \mathrm{H} & -2.38662300 & 2.25132700 & -0.59000900 \\ \mathrm{H} & 0.04857500 & 1.85790000 & -0.65408400 \\ \mathrm{H} & -0.61526000 & -2.27214600 & 0.33970700 \\ \mathrm{H} & -3.03419500 & -1.88150500 & 0.40055700 \\ \mathrm{H} & 1.58454700 & -1.60263900 & -0.28060100 \\ \mathrm{H} & 1.48197500 & 1.97940900 & 0.94536400 \\ \mathrm{H} & 3.24516600 & 1.88542900 & 1.16254000 \\ \mathrm{H} & 2.58376200 & 2.34667500 & -0.39777000 \\ \mathrm{H} & -6.11098300 & 0.01797400 & 0.16484400 \\ \mathrm{H} & -5.08588300 & -1.26677000 & -0.52943000 \\ \mathrm{H} & -4.98339400 & -0.88267300 & 1.21413800\end{array}$<smiles>CC(=CCCc1ccccc1)[N+](=O)[O-]</smiles>

Nitroalkene-85

$\begin{array}{lrrr}\mathrm{C} & -1.35548200 & 1.37569000 & 0.03734800 \\ \mathrm{C} & -1.57424500 & 0.14667100 & 0.51769900 \\ \mathrm{~N} & -2.69288400 & -0.61165100 & -0.09203600 \\ \mathrm{O} & -3.36897500 & -0.08610900 & -0.97761200 \\ \mathrm{O} & -2.87585100 & -1.75375100 & 0.33285600 \\ \mathrm{C} & -0.82699700 & -0.60545600 & 1.56861700 \\ \mathrm{C} & -0.23869500 & 2.28756200 & 0.43685100 \\ \mathrm{C} & 0.98020600 & 2.15376800 & -0.52257200 \\ \mathrm{C} & 1.61821700 & 0.78979600 & -0.43256100 \\ \mathrm{C} & 2.55213400 & 0.51054900 & 0.57266500 \\ \mathrm{C} & 3.07365800 & -0.77285700 & 0.72792600 \\ \mathrm{C} & 2.66594300 & -1.80035300 & -0.12424000 \\ \mathrm{C} & 1.74397700 & -1.53204500 & -1.13690500 \\ \mathrm{C} & 1.22677900 & -0.24564400 & -1.28899900 \\ \mathrm{H} & -2.01382600 & 1.70885600 & -0.75921700 \\ \mathrm{H} & -1.50393200 & -0.97739200 & 2.34117100 \\ \mathrm{H} & -0.07022400 & 0.03303400 & 2.02297800 \\ \mathrm{H} & -0.31804900 & -1.46907300 & 1.13155700 \\ \mathrm{H} & -0.59388700 & 3.32351000 & 0.40889400 \\ \mathrm{H} & 0.09591400 & 2.08471200 & 1.45811100 \\ \mathrm{H} & 1.70534800 & 2.93089200 & -0.25857800 \\ \mathrm{H} & 0.65279400 & 2.35380900 & -1.54837300 \\ \mathrm{H} & 2.86813900 & 1.30677000 & 1.24236500 \\ \mathrm{H} & 3.79738500 & -0.97127700 & 1.51266300 \\ \mathrm{H} & 3.06816500 & -2.80136200 & -0.00374000 \\ \mathrm{H} & 1.42558700 & -2.32405300 & -1.80773500 \\ \mathrm{H} & 0.50242500 & -0.04407200 & -2.07276300\end{array}$




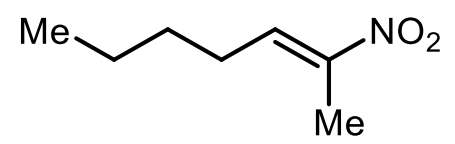

Nitroalkene-86

$\begin{array}{lrrr}\mathrm{C} & -0.23101200 & -0.29859200 & 0.93850400 \\ \mathrm{C} & -1.16032700 & 0.35615300 & 0.23388600 \\ \mathrm{~N} & -2.30121400 & -0.44653300 & -0.26811500 \\ \mathrm{O} & -2.34818600 & -1.65197700 & -0.02201400 \\ \mathrm{O} & -3.15267200 & 0.16248700 & -0.91891800 \\ \mathrm{C} & -1.22402800 & 1.80152000 & -0.13984900 \\ \mathrm{C} & 1.01741800 & 0.27465900 & 1.53059500 \\ \mathrm{C} & 2.28632500 & -0.26549400 & 0.83703900 \\ \mathrm{C} & 2.41522300 & 0.17700900 & -0.62310000 \\ \mathrm{C} & 3.68599500 & -0.35134100 & -1.29041600 \\ \mathrm{H} & -0.39032700 & -1.36538400 & 1.07037900 \\ \mathrm{H} & -2.13388000 & 2.26782300 & 0.24724200 \\ \mathrm{H} & -0.35793500 & 2.33275700 & 0.25540600 \\ \mathrm{H} & -1.24317700 & 1.92097000 & -1.22644700 \\ \mathrm{H} & 1.05011800 & -0.00254300 & 2.59211100 \\ \mathrm{H} & 1.01588100 & 1.36793200 & 1.48803200 \\ \mathrm{H} & 2.29062200 & -1.36213600 & 0.89040000 \\ \mathrm{H} & 3.16200400 & 0.07491700 & 1.40317300 \\ \mathrm{H} & 2.40256900 & 1.27446900 & -0.66762100 \\ \mathrm{H} & 1.53546100 & -0.16077900 & -1.18419100 \\ \mathrm{H} & 3.70603500 & -1.44666000 & -1.28669800 \\ \mathrm{H} & 3.75756900 & -0.02063700 & -2.33094100 \\ \mathrm{H} & 4.58285900 & -0.00256000 & -0.76653000\end{array}$<smiles>C/C(=C\c1ccccc1)[N+](=O)[O-]</smiles>

Nitroalkene-87

$\begin{array}{lrrr}\mathrm{C} & -2.89325000 & 1.18979700 & -0.36598000 \\ \mathrm{C} & -1.51839600 & 0.97846800 & -0.42115600 \\ \mathrm{C} & -0.97330300 & -0.27470100 & -0.08628800 \\ \mathrm{C} & -1.85357200 & -1.31362200 & 0.26951900 \\ \mathrm{C} & -3.22561000 & -1.09620400 & 0.33755200 \\ \mathrm{C} & -3.75004600 & 0.15900900 & 0.02337800 \\ \mathrm{C} & 0.45724100 & -0.57314500 & -0.12569500 \\ \mathrm{C} & 1.48824100 & 0.26255000 & 0.08967900 \\ \mathrm{~N} & 2.83909400 & -0.32235900 & -0.07193400 \\ \mathrm{O} & 3.79226000 & 0.44610100 & 0.07520200 \\ \mathrm{O} & 2.95248300 & -1.51758400 & -0.34939100 \\ \mathrm{C} & 1.48426200 & 1.69651700 & 0.50821000 \\ \mathrm{H} & -3.29799900 & 2.15977600 & -0.63714500 \\ \mathrm{H} & -0.86968500 & 1.77549900 & -0.76343700\end{array}$




$\begin{array}{rrrr}\mathrm{H} & -1.44637600 & -2.29230200 & 0.50582000 \\ \mathrm{H} & -3.88690500 & -1.90660800 & 0.62726500 \\ \mathrm{H} & -4.82122700 & 0.32861300 & 0.06735000 \\ \mathrm{H} & 0.72770500 & -1.60470100 & -0.33038500 \\ \mathrm{H} & 0.51480100 & 1.95681300 & 0.93425900 \\ \mathrm{H} & 2.26450400 & 1.87112700 & 1.25049100 \\ \mathrm{H} & 1.69017600 & 2.36815600 & -0.33249100\end{array}$<smiles>CCOC(=O)NC(Cl)C(=O)OCC</smiles>

Chloroglycine-1

\begin{tabular}{|c|c|c|c|}
\hline $\mathrm{N}$ & 1.44709500 & -0.46163000 & -0.24918700 \\
\hline $\mathrm{C}$ & 0.36089800 & 0.22617200 & 0.30587300 \\
\hline $\mathrm{C}$ & -0.93076000 & -0.52881500 & 0.02351500 \\
\hline $\mathrm{O}$ & -1.88294000 & -0.19623400 & 0.89320800 \\
\hline $\mathrm{O}$ & -1.03912000 & -1.32027200 & -0.88992200 \\
\hline $\mathrm{C}$ & -3.20237900 & -0.75928300 & 0.65186800 \\
\hline $\mathrm{C}$ & -3.94306100 & 0.04351300 & -0.40237900 \\
\hline $\mathrm{C}$ & 2.73208400 & -0.31487300 & 0.24426500 \\
\hline $\mathrm{O}$ & 2.96241200 & 0.34948000 & 1.23921400 \\
\hline $\mathrm{C}$ & 3.79755400 & -1.03040400 & -0.55922800 \\
\hline $\mathrm{Cl}$ & 0.10847400 & 1.92169100 & -0.45532900 \\
\hline $\mathrm{H}$ & 1.25995300 & -0.98329900 & -1.09596200 \\
\hline $\mathrm{H}$ & 0.52966700 & 0.43609700 & 1.35859800 \\
\hline $\mathrm{H}$ & -3.69249900 & -0.70738600 & 1.62517900 \\
\hline $\mathrm{H}$ & -3.08488000 & -1.80374300 & 0.35526200 \\
\hline $\mathrm{H}$ & -4.95908500 & -0.34715800 & -0.51599800 \\
\hline $\mathrm{H}$ & -4.00537600 & 1.09582000 & -0.11362700 \\
\hline $\mathrm{H}$ & -3.43660300 & -0.02963200 & -1.36733000 \\
\hline $\mathrm{H}$ & 3.44869200 & -1.99402100 & -0.94158400 \\
\hline $\mathrm{H}$ & 4.08363700 & -0.40857200 & -1.41450100 \\
\hline $\mathrm{H}$ & 4.67393900 & -1.17710100 & 0.07138800 \\
\hline
\end{tabular}

Chloroglycine-2
$\begin{array}{llll}\mathrm{N} & -0.06503100 & 0.52307900 & 1.43627600\end{array}$
$\begin{array}{llll}\text { C } & 0.58520400 & 0.24478400 & 0.25886900\end{array}$
$\begin{array}{llll}\mathrm{C} & 1.97298200 & -0.30750600 & 0.51913900\end{array}$
$\begin{array}{lllll}\mathrm{Cl} & 0.96900700 & 1.83971700 & -0.84817600\end{array}$
$\begin{array}{llll}\mathrm{O} & 2.38107800 & -1.07974000 & -0.49029800\end{array}$ 


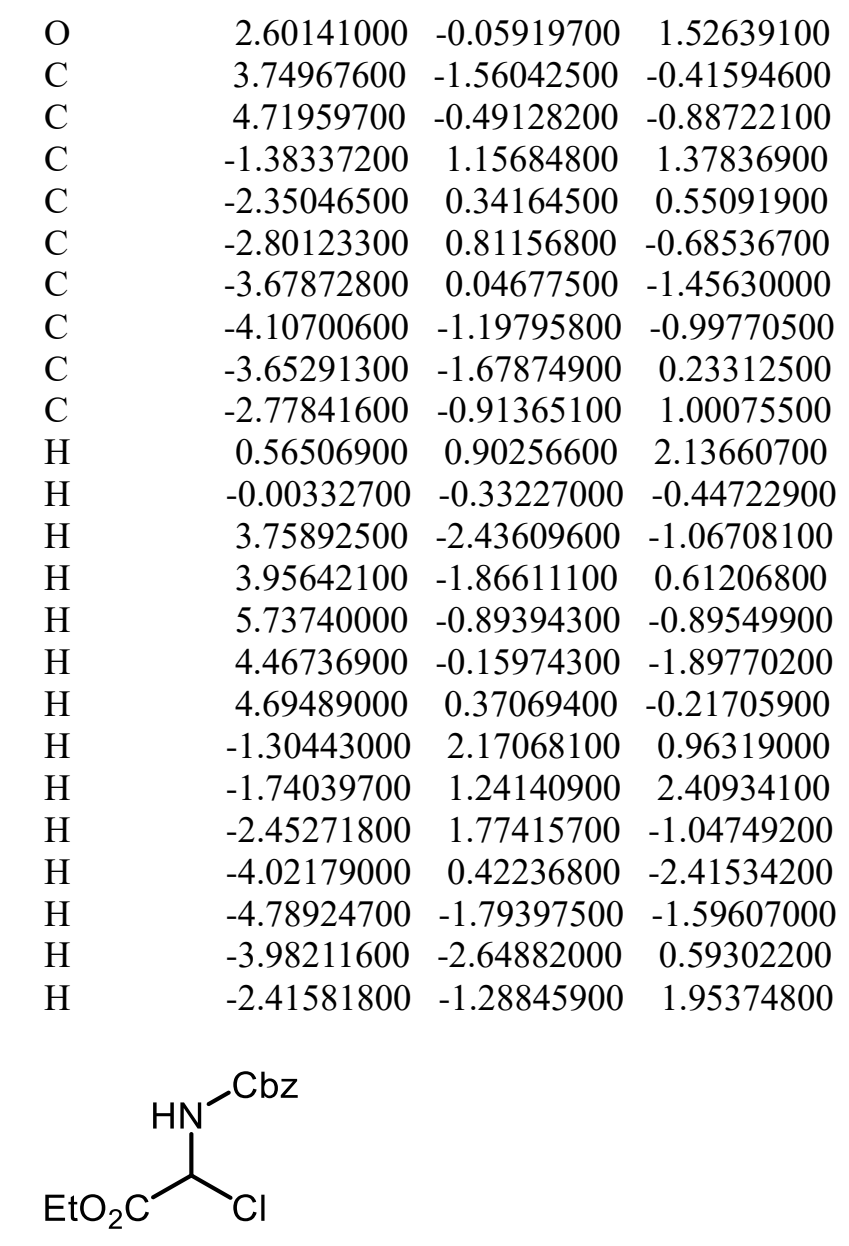

Chloroglycine-3

$\begin{array}{lrrr}\mathrm{N} & -0.67076500 & 0.48186200 & -0.47413200 \\ \mathrm{C} & -1.80975000 & 0.41281200 & 0.33214900 \\ \mathrm{C} & -2.76902000 & -0.63275200 & -0.22059500 \\ \mathrm{Cl} & -2.79993700 & 2.01124300 & 0.35199100 \\ \mathrm{O} & -3.58834200 & -1.08003000 & 0.73032100 \\ \mathrm{O} & -2.76006100 & -0.98056800 & -1.38204300 \\ \mathrm{C} & -4.63617100 & -1.99227400 & 0.29963100 \\ \mathrm{C} & -5.80545600 & -1.22529900 & -0.29131800 \\ \mathrm{C} & 0.51116600 & 1.00376100 & 0.00065800 \\ \mathrm{O} & 1.39388900 & 1.11105400 & -1.01192100 \\ \mathrm{O} & 0.70733500 & 1.30307500 & 1.16234400 \\ \mathrm{C} & 2.73159100 & 1.54408500 & -0.63388200 \\ \mathrm{C} & 3.59295900 & 0.37876600 & -0.22829700 \\ \mathrm{C} & 3.62828800 & -0.04973300 & 1.10460000 \\ \mathrm{C} & 4.41069500 & -1.14427600 & 1.46920000 \\ \mathrm{C} & 5.16359900 & -1.82012100 & 0.50754000 \\ \mathrm{C} & 5.13218000 & -1.39902800 & -0.82250300 \\ \mathrm{C} & 4.34941800 & -0.30446300 & -1.18651600 \\ \mathrm{H} & -0.78687500 & 0.31467700 & -1.46549100 \\ \mathrm{H} & -1.54800300 & 0.26075500 & 1.37539300 \\ \mathrm{H} & -4.91385000 & -2.52339300 & 1.21129000\end{array}$




$$
\begin{array}{lrrr}
\mathrm{H} & -4.21012300 & -2.69528900 & -0.41936000 \\
\mathrm{H} & -6.61356200 & -1.92035700 & -0.53975300 \\
\mathrm{H} & -6.18600900 & -0.49034400 & 0.42259500 \\
\mathrm{H} & -5.50349700 & -0.70834300 & -1.20482400 \\
\mathrm{H} & 2.64118400 & 2.27659900 & 0.16937500 \\
\mathrm{H} & 3.11479400 & 2.02679100 & -1.53394300 \\
\mathrm{H} & 3.02861000 & 0.47206600 & 1.84250100 \\
\mathrm{H} & 4.43447900 & -1.46893300 & 2.50498600 \\
\mathrm{H} & 5.77467200 & -2.67073400 & 0.79392000 \\
\mathrm{H} & 5.71801000 & -1.92013900 & -1.57347200 \\
\mathrm{H} & 4.32487500 & 0.02538300 & -2.22175700 \\
& & & \\
& & & \\
& & &
\end{array}
$$

Chloroglycine-4

$$
\begin{aligned}
& \begin{array}{lrrr}
\mathrm{N} & 1.06664400 & -0.39771100 & -0.22643600
\end{array}
\end{aligned}
$$

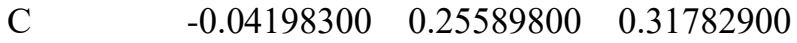

$$
\begin{aligned}
& \begin{array}{llll}
\mathrm{C} & -1.30680400 & -0.53688400 & 0.01702200
\end{array} \\
& \mathrm{Cl} \quad-0.34620200 \quad 1.95042900 \quad-0.43891100 \\
& \begin{array}{lllll}
\mathrm{O} & -2.26950200 & -0.25198300 & 0.89287800
\end{array} \\
& \begin{array}{lllll}
\mathrm{O} & & -1.38823900 & -1.31146400 & -0.91230700
\end{array} \\
& \begin{array}{llll}
\mathrm{C} & -3.57018900 & -0.85103200 & 0.63673400
\end{array} \\
& \begin{array}{llll}
\text { C } & -4.33379200 & -0.05021300 & -0.40254900
\end{array} \\
& \begin{array}{llll}
\mathrm{C} & 2.33989200 & -0.14358900 & 0.23361900
\end{array} \\
& \begin{array}{llll}
\mathrm{O} & 3.23938900 & -0.76342400 & -0.55851500
\end{array} \\
& \begin{array}{llll}
\mathrm{O} & 2.60037000 & 0.51936200 & 1.21586500
\end{array} \\
& \text { C } \quad 4.60650700 \quad-0.59415600 \quad-0.15122800 \\
& \mathrm{H} \quad 0.93349200 \quad-0.89863400 \quad-1.09569100 \\
& \begin{array}{llll}
\mathrm{H} & 0.10261500 & 0.46897700 & 1.37319200
\end{array} \\
& \begin{array}{llll}
\mathrm{H} & -4.06339800 & -0.83479800 & 1.60974900
\end{array} \\
& \mathrm{H} \quad-3.41926900 \quad-1.88490500 \quad 0.31892800 \\
& \mathrm{H} \quad-5.33665900 \quad-0.47041900 \quad-0.52714600 \\
& \mathrm{H} \quad-4.43009800 \quad 0.99334100 \quad-0.09211300 \\
& \mathrm{H} \quad-3.82320800 \quad-0.08705300 \quad-1.36737300 \\
& \mathrm{H} \quad \begin{array}{llll}
5.19756300 & -1.14071500 & -0.88497900
\end{array} \\
& \mathrm{H} \quad 4.87734100 \quad 0.46388000 \quad-0.14956400 \\
& \mathrm{H} \quad 4.76261500 \quad-1.00305300 \quad 0.84960100
\end{aligned}
$$

Chloroglycine-5
$\begin{array}{llll}\mathrm{N} & -0.43884600 & -0.26056200 & -0.17614700\end{array}$
$\begin{array}{llll}\mathrm{C} & -1.59755600 & 0.35446100 & 0.31055600\end{array}$
$\begin{array}{llll}\mathrm{C} & -2.79631900 & -0.55612300 & 0.07682000\end{array}$
$\begin{array}{llll}\mathrm{Cl} & -2.01339500 & 1.94865400 & -0.58677200\end{array}$ 


\begin{tabular}{|c|c|c|c|}
\hline $\mathrm{O}$ & -3.78925200 & -0.26309100 & 0.91439100 \\
\hline $\mathrm{O}$ & -2.80545300 & -1.41827100 & -0.77570700 \\
\hline $\mathrm{C}$ & -5.03834500 & -0.98065900 & 0.70901400 \\
\hline $\mathrm{C}$ & -5.84592700 & -0.34297700 & -0.40703600 \\
\hline $\mathrm{C}$ & 0.80653000 & 0.11932200 & 0.26409800 \\
\hline $\mathrm{O}$ & 1.74132000 & -0.52830100 & -0.48736200 \\
\hline $\mathrm{O}$ & 1.01373800 & 0.88340500 & 1.17823800 \\
\hline $\mathrm{C}$ & 3.10007600 & -0.33300500 & -0.22416400 \\
\hline $\mathrm{C}$ & 3.88888900 & -1.47907100 & -0.21972600 \\
\hline $\mathrm{C}$ & 5.26608700 & -1.35475700 & -0.04149800 \\
\hline $\mathrm{C}$ & 5.83929500 & -0.09501800 & 0.13460400 \\
\hline $\mathrm{C}$ & 5.03018100 & 1.04214100 & 0.12612200 \\
\hline $\mathrm{C}$ & 3.65205400 & 0.93507200 & -0.05842800 \\
\hline $\mathrm{H}$ & -0.53115900 & -0.85754400 & -0.98832100 \\
\hline $\mathrm{H}$ & -1.47800700 & 0.66327100 & 1.34529600 \\
\hline $\mathrm{H}$ & -5.54401700 & -0.90891800 & 1.67302200 \\
\hline $\mathrm{H}$ & -4.80614200 & -2.02570400 & 0.49313200 \\
\hline $\mathrm{H}$ & -6.81278000 & -0.84805100 & -0.49583300 \\
\hline $\mathrm{H}$ & -6.02428700 & 0.71516100 & -0.19930300 \\
\hline $\mathrm{H}$ & -5.32197600 & -0.43356900 & -1.36108600 \\
\hline $\mathrm{H}$ & 3.41797200 & -2.44595700 & -0.35829100 \\
\hline $\mathrm{H}$ & 5.88794300 & -2.24429500 & -0.03873700 \\
\hline $\mathrm{H}$ & 6.91085300 & 0.00073400 & 0.27676500 \\
\hline $\mathrm{H}$ & 5.47132700 & 2.02452000 & 0.26165000 \\
\hline $\mathrm{H}$ & 3.01729800 & 1.81090800 & -0.05879100 \\
\hline & & & \\
\hline
\end{tabular}

Chloroglycine-6

$\begin{array}{lrrr}\mathrm{N} & -2.09400200 & -0.51673800 & -0.65134600 \\ \mathrm{C} & -3.16419300 & -0.68115200 & 0.23322100 \\ \mathrm{C} & -4.46572600 & -0.29460100 & -0.45630400 \\ \mathrm{Cl} & -3.05302100 & 0.45299000 & 1.72843800 \\ \mathrm{O} & -5.50888700 & -0.87995900 & 0.12948800 \\ \mathrm{O} & -4.50314800 & 0.45714600 & -1.40679500 \\ \mathrm{C} & -6.82327800 & -0.49082400 & -0.35833600 \\ \mathrm{C} & -7.26024500 & 0.82167900 & 0.26688200 \\ \mathrm{C} & -0.88312000 & -1.13200600 & -0.42364900 \\ \mathrm{O} & 0.03852100 & -0.63578500 & -1.27418500 \\ \mathrm{O} & -0.69543800 & -1.99431400 & 0.41061200 \\ \mathrm{C} & 1.37819300 & -1.14683600 & -1.10648800 \\ \mathrm{C} & 2.07952500 & -0.53421000 & 0.11325300 \\ \mathrm{C} & 3.51715500 & -1.01710900 & 0.18629200 \\ \mathrm{C} & 4.40248800 & 0.07628200 & 0.09218200 \\ \mathrm{C} & 3.61312400 & 1.30991300 & -0.00612700 \\ \mathrm{C} & 2.24352500 & 0.97251500 & 0.02143700 \\ \mathrm{C} & 4.01366100 & 2.64262200 & -0.09881900 \\ \mathrm{C} & 3.03429800 & 3.63545900 & -0.16099300 \\ \mathrm{C} & 1.67692200 & 3.30138600 & -0.12603100\end{array}$




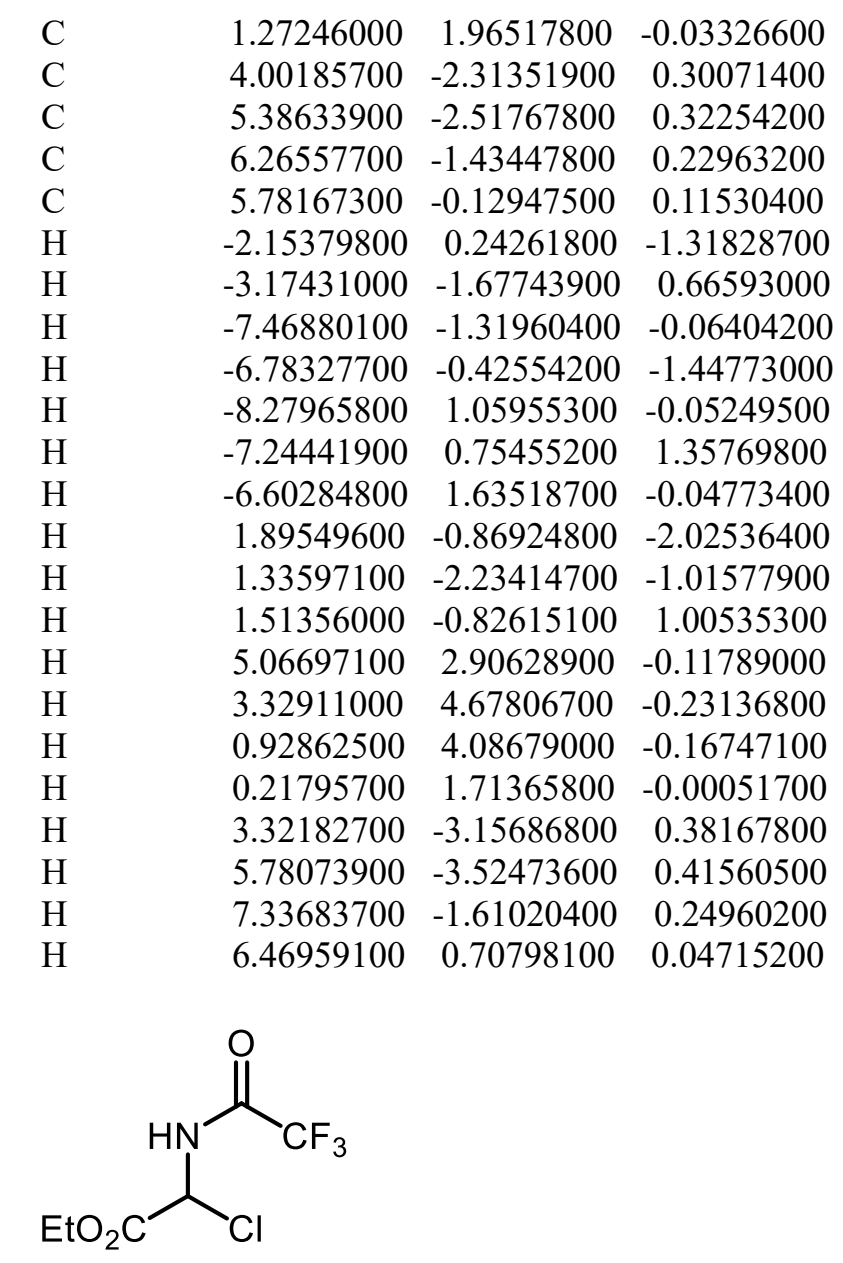

Chloroglycine-7

$\begin{array}{lrrr}\mathrm{N} & 0.62605900 & -0.08141300 & -0.04247700 \\ \mathrm{C} & -0.58273700 & 0.51185000 & 0.37789100 \\ \mathrm{C} & -1.72707100 & -0.46805200 & 0.13748000 \\ \mathrm{Cl} & -0.99468900 & 2.03585900 & -0.59160400 \\ \mathrm{O} & -2.78381500 & -0.16175500 & 0.88282400 \\ \mathrm{O} & -1.63746000 & -1.38849200 & -0.64730600 \\ \mathrm{C} & -3.98689100 & -0.95202000 & 0.65647600 \\ \mathrm{C} & -4.74603200 & -0.43735200 & -0.55272900 \\ \mathrm{C} & 1.82657600 & 0.30425800 & 0.47527600 \\ \mathrm{C} & 3.03927800 & -0.44255400 & -0.12472200 \\ \mathrm{O} & 1.98283500 & 1.13697000 & 1.34391500 \\ \mathrm{~F} & 3.90635100 & 0.43342600 & -0.64397000 \\ \mathrm{~F} & 3.65993200 & -1.14760900 & 0.83025000 \\ \mathrm{~F} & 2.66877100 & -1.30128400 & -1.10602900 \\ \mathrm{H} & 0.56835600 & -0.76990700 & -0.78353100 \\ \mathrm{H} & -0.51217000 & 0.85216300 & 1.40838900 \\ \mathrm{H} & -4.55538200 & -0.83209600 & 1.57959800 \\ \mathrm{H} & -3.69558400 & -1.99761900 & 0.53716100 \\ \mathrm{H} & -5.68292200 & -0.99250400 & -0.66052300 \\ \mathrm{H} & -4.98240800 & 0.62361000 & -0.43853600\end{array}$


<smiles>CCOC(=O)O[Te]NC(Cl)C(=O)OCC</smiles>

Chloroglycine-8

$\begin{array}{lrrr}\mathrm{N} & -0.92430400 & -0.32398500 & 0.35556400 \\ \mathrm{C} & -2.15350900 & -0.61685200 & -0.25259100 \\ \mathrm{C} & -3.12300500 & 0.53429100 & -0.00995600 \\ \mathrm{Cl} & -2.98250100 & -2.12609700 & 0.47283100 \\ \mathrm{O} & -4.09419300 & 0.54016700 & -0.91952500 \\ \mathrm{O} & -2.99050700 & 1.31438200 & 0.90869200 \\ \mathrm{C} & -5.15388400 & 1.51955200 & -0.72543100 \\ \mathrm{C} & -6.16500400 & 1.02087400 & 0.29087900 \\ \mathrm{C} & 0.23282500 & -0.92823000 & -0.04980700 \\ \mathrm{O} & 1.23889000 & -0.57220500 & 0.80562800 \\ \mathrm{O} & 0.34773900 & -1.65748900 & -1.00911300 \\ \mathrm{C} & 2.54278400 & -0.99260200 & 0.44231400 \\ \mathrm{C} & 3.39326300 & 0.21226500 & 0.01922200 \\ \mathrm{Cl} & 3.50957000 & 1.39877500 & 1.36242700 \\ \mathrm{Cl} & 5.03290500 & -0.41343800 & -0.37915000 \\ \mathrm{Cl} & 2.67712700 & 1.00785200 & -1.42002600 \\ \mathrm{H} & -0.93831900 & 0.24437400 & 1.19346800 \\ \mathrm{H} & -2.02644600 & -0.86429300 & -1.30305300 \\ \mathrm{H} & -5.59168200 & 1.63189800 & -1.71825100 \\ \mathrm{H} & -4.70011100 & 2.46310800 & -0.41510700 \\ \mathrm{H} & -6.99163900 & 1.73406900 & 0.36652700 \\ \mathrm{H} & -6.56937100 & 0.05093900 & -0.00967400 \\ \mathrm{H} & -5.70435400 & 0.92335200 & 1.27640500 \\ \mathrm{H} & 2.49680600 & -1.70335800 & -0.38423600 \\ \mathrm{H} & 3.00826600 & -1.44738900 & 1.31833400\end{array}$<smiles>[As]/N=C/c1ccccc1</smiles>

Imine-1

$\begin{array}{lrrr}\mathrm{C} & 1.67684300 & 0.72731900 & 0.28178300 \\ \mathrm{~N} & 0.69324000 & 0.61717200 & -0.53791600 \\ \mathrm{H} & 1.63516000 & 1.42881100 & 1.12321600 \\ \mathrm{~S} & -0.62720900 & 1.67852100 & -0.24051900 \\ \mathrm{O} & -0.46035400 & 2.38709700 & 1.03965600 \\ \mathrm{O} & -0.82798400 & 2.42632800 & -1.48357500 \\ \mathrm{C} & -1.93920200 & 0.48790400 & -0.05641900 \\ \mathrm{C} & -2.44670200 & -0.14468100 & -1.19264900 \\ \mathrm{C} & -3.46702300 & -1.07652700 & -1.04236400 \\ \mathrm{C} & -3.98812900 & -1.38273000 & 0.22413800 \\ \mathrm{C} & -3.46450700 & -0.72413700 & 1.34307700\end{array}$




$$
\begin{aligned}
& \begin{array}{lrrr}
\mathrm{C} & -2.43909800 & 0.21024600 & 1.21331100 \\
\mathrm{C} & -5.07892400 & -2.41275700 & 0.37168500 \\
\mathrm{C} & 2.89014400 & -0.07579300 & 0.14587500 \\
\mathrm{C} & 3.91431500 & 0.07785500 & 1.09173600 \\
\mathrm{C} & 5.08112200 & -0.67602300 & 0.99242100 \\
\mathrm{C} & 5.23030200 & -1.58685800 & -0.05392300 \\
\mathrm{C} & 4.21305900 & -1.74401900 & -1.00213800 \\
\mathrm{C} & 3.04826900 & -0.99384200 & -0.90616000 \\
\mathrm{H} & -2.05344300 & 0.10608600 & -2.17107600 \\
\mathrm{H} & -3.87309000 & -1.57124500 & -1.92009500 \\
\mathrm{H} & -3.86783200 & -0.94188600 & 2.32768700 \\
\mathrm{H} & -2.03522600 & 0.73167600 & 2.07334300 \\
\mathrm{H} & -5.57987600 & -2.32934300 & 1.33934500 \\
\mathrm{H} & -5.83300800 & -2.30885800 & -0.41423300 \\
\mathrm{H} & -4.67003900 & -3.42740800 & 0.29629200 \\
\mathrm{H} & 3.78947200 & 0.79042400 & 1.90225500 \\
\mathrm{H} & 5.87098000 & -0.55371700 & 1.72640500 \\
\mathrm{H} & 6.13950800 & -2.17477900 & -0.13388600 \\
\mathrm{H} & 4.33535200 & -2.45240000 & -1.81531200 \\
\mathrm{H} & 2.24858900 & -1.09704100 & -1.63111300
\end{array} \\
& \prod^{-\mathrm{P}(\mathrm{O}) \mathrm{Ph}_{2}}
\end{aligned}
$$

Imine-2

$\begin{array}{lrrr}\mathrm{C} & 1.78664700 & -0.00021600 & -0.85567100 \\ \mathrm{~N} & 0.74466300 & -0.00012700 & -0.10717500 \\ \mathrm{H} & 1.69885400 & -0.00012300 & -1.95092100 \\ \mathrm{P} & -0.78450100 & -0.00001800 & -0.87566800 \\ \mathrm{O} & -0.82765200 & -0.00003900 & -2.37477500 \\ \mathrm{C} & 3.14993700 & -0.00016200 & -0.31679100 \\ \mathrm{C} & 3.38762600 & -0.00015200 & 1.06728600 \\ \mathrm{C} & 4.69004300 & -0.00011600 & 1.55004000 \\ \mathrm{C} & 5.76886600 & -0.00008600 & 0.65857400 \\ \mathrm{C} & 5.54142400 & -0.00009300 & -0.71791000 \\ \mathrm{C} & 4.23564500 & -0.00012600 & -1.20360700 \\ \mathrm{C} & -1.57172300 & -1.46370500 & -0.14588800 \\ \mathrm{C} & -1.57151900 & 1.46379400 & -0.14592000 \\ \mathrm{C} & -2.42770000 & -2.21278200 & -0.96223600 \\ \mathrm{C} & -3.07953200 & -3.33223100 & -0.44620900 \\ \mathrm{C} & -2.87700500 & -3.70711500 & 0.88254800 \\ \mathrm{C} & -2.01774200 & -2.96582000 & 1.69700500 \\ \mathrm{C} & -1.36526700 & -1.84578000 & 1.18647500 \\ \mathrm{C} & -2.42748100 & 2.21290800 & -0.96225000 \\ \mathrm{C} & -3.07916400 & 3.33245400 & -0.44624300 \\ \mathrm{C} & -2.87650100 & 3.70739900 & 0.88247500 \\ \mathrm{C} & -2.01725000 & 2.96606900 & 1.69691300 \\ \mathrm{C} & -1.36492500 & 1.84593300 & 1.18640400 \\ \mathrm{H} & 2.53546900 & -0.00018000 & 1.73797600 \\ \mathrm{H} & 4.87216500 & -0.00011300 & 2.62021500 \\ & & & \end{array}$




$\begin{array}{lrrr}\mathrm{H} & 6.78559600 & -0.00005800 & 1.03950300 \\ \mathrm{H} & 6.37825800 & -0.00007100 & -1.40896100 \\ \mathrm{H} & 4.04862300 & -0.00013200 & -2.27394500 \\ \mathrm{H} & -2.56072500 & -1.91390500 & -1.99676100 \\ \mathrm{H} & -3.74166200 & -3.91318500 & -1.08085700 \\ \mathrm{H} & -3.38427000 & -4.57950000 & 1.28349500 \\ \mathrm{H} & -1.85404500 & -3.26414000 & 2.72807500 \\ \mathrm{H} & -0.68489900 & -1.27762100 & 1.81102100 \\ \mathrm{H} & -2.56061200 & 1.91398500 & -1.99674800 \\ \mathrm{H} & -3.74128200 & 3.91343500 & -1.08087900 \\ \mathrm{H} & -3.38365000 & 4.57985900 & 1.28340700 \\ \mathrm{H} & -1.85344500 & 3.26443800 & 2.72795100 \\ \mathrm{H} & -0.68456200 & 1.27774800 & 1.81093100\end{array}$<smiles>Cc1ccc(/C=N/[PH+]=C/c2ccccc2)cc1</smiles>

Imine-3

$\begin{array}{lrrr}\mathrm{C} & -1.39238600 & -0.00012100 & 0.98211000 \\ \mathrm{~N} & -0.38449100 & 0.00021200 & 0.18660900 \\ \mathrm{H} & -1.25401800 & -0.00052800 & 2.07220000 \\ \mathrm{P} & 1.17671700 & -0.00010000 & 0.88382200 \\ \mathrm{O} & 1.29128800 & -0.00054900 & 2.37935400 \\ \mathrm{C} & -2.77671500 & 0.00000500 & 0.50805700 \\ \mathrm{C} & -3.82342500 & -0.00020300 & 1.43820400 \\ \mathrm{C} & -5.14886400 & -0.00008300 & 1.01064300 \\ \mathrm{C} & -5.46124500 & 0.00023800 & -0.35270500 \\ \mathrm{C} & -4.40550700 & 0.00042600 & -1.28176500 \\ \mathrm{C} & -3.08451000 & 0.00031600 & -0.86327200 \\ \mathrm{C} & 1.93180200 & -1.46298600 & 0.11829000 \\ \mathrm{C} & 1.93209900 & 1.46296400 & 0.11895300 \\ \mathrm{C} & 2.82663500 & -2.21040200 & 0.89334100 \\ \mathrm{C} & 3.45539500 & -3.32895000 & 0.34748400 \\ \mathrm{C} & 3.19124900 & -3.70458700 & -0.97018500 \\ \mathrm{C} & 2.29323900 & -2.96492200 & -1.74331200 \\ \mathrm{C} & 1.66355300 & -1.84590400 & -1.20276800 \\ \mathrm{C} & 2.82971700 & 2.20786100 & 0.89323200 \\ \mathrm{C} & 3.45896300 & 3.32631500 & 0.34776900 \\ \mathrm{C} & 3.19251400 & 3.70442000 & -0.96873800 \\ \mathrm{C} & 2.29168800 & 2.96734400 & -1.74104300 \\ \mathrm{C} & 1.66150500 & 1.84840300 & -1.20089500 \\ \mathrm{C} & -6.89186200 & 0.00041700 & -0.82641000 \\ \mathrm{H} & -3.59342800 & -0.00046000 & 2.50023700 \\ \mathrm{H} & -5.95164800 & -0.00024600 & 1.74218800 \\ \mathrm{H} & -4.63431500 & 0.00065100 & -2.34422700 \\ \mathrm{H} & -2.26773800 & 0.00043600 & -1.57678900 \\ \mathrm{H} & 3.00768600 & -1.91094700 & 1.92040400 \\ \mathrm{H} & 4.14768000 & -3.90859900 & 0.95042200 \\ & & & \end{array}$




$\begin{array}{lrrr}\mathrm{H} & 3.68065200 & -4.57617900 & -1.39445600 \\ \mathrm{H} & 2.08168100 & -3.26374500 & -2.76553500 \\ \mathrm{H} & 0.95332000 & -1.27903000 & -1.79438900 \\ \mathrm{H} & 3.01251300 & 1.90662800 & 1.91946700 \\ \mathrm{H} & 4.15339900 & 3.90399700 & 0.95011800 \\ \mathrm{H} & 3.68229200 & 4.57595400 & -1.39269800 \\ \mathrm{H} & 2.07829400 & 3.26813400 & -2.76230800 \\ \mathrm{H} & 0.94900100 & 1.28360000 & -1.79176100 \\ \mathrm{H} & -7.10320400 & 0.88179900 & -1.44210100 \\ \mathrm{H} & -7.10293600 & -0.87995200 & -1.44363000 \\ \mathrm{H} & -7.59192700 & -0.00040600 & 0.01249100\end{array}$<smiles>Clc1ccc(/C=N/[PH](c2ccccc2)c2ccccc2)cc1</smiles>

Imine-4

$\begin{array}{lrrr}\mathrm{C} & 1.03564600 & 0.00013000 & 1.05005900 \\ \mathrm{~N} & 0.05366500 & 0.00002700 & 0.22522100 \\ \mathrm{H} & 0.86738500 & 0.00014900 & 2.13574200 \\ \mathrm{P} & -1.52899200 & 0.00002600 & 0.88307100 \\ \mathrm{O} & -1.67163200 & 0.00011200 & 2.37578400 \\ \mathrm{C} & 2.43412300 & 0.00008000 & 0.61127100 \\ \mathrm{C} & 2.77091400 & 0.00002500 & -0.75180500 \\ \mathrm{C} & 4.09995400 & -0.00001200 & -1.15047800 \\ \mathrm{C} & 5.10151000 & 0.00000300 & -0.17484500 \\ \mathrm{C} & 4.79442500 & 0.00005700 & 1.18466000 \\ \mathrm{C} & 3.45669800 & 0.00009400 & 1.56939000 \\ \mathrm{C} & -2.25992400 & 1.46500000 & 0.10079700 \\ \mathrm{C} & -2.25987900 & -1.46504000 & 0.10093100 \\ \mathrm{C} & -3.16141400 & 2.22099800 & 0.85980100 \\ \mathrm{C} & -3.77286600 & 3.34258100 & 0.30076700 \\ \mathrm{C} & -3.48471300 & 3.71246900 & -1.01347100 \\ \mathrm{C} & -2.58018600 & 2.96404000 & -1.77040500 \\ \mathrm{C} & -1.96766100 & 1.84190800 & -1.21696000 \\ \mathrm{C} & -3.16184200 & -2.22062600 & 0.85978900 \\ \mathrm{C} & -3.77330800 & -3.34223900 & 0.30083500 \\ \mathrm{C} & -3.48469700 & -3.71257700 & -1.01317600 \\ \mathrm{C} & -2.57969000 & -2.96457300 & -1.76995500 \\ \mathrm{C} & -1.96714800 & -1.84240900 & -1.21659000 \\ \mathrm{Cl} & 6.78161700 & -0.00004400 & -0.67496600 \\ \mathrm{H} & 1.97065200 & 0.00002100 & -1.48340400 \\ \mathrm{H} & 4.36929900 & -0.00005100 & -2.20018400 \\ \mathrm{H} & 5.58882500 & 0.00006900 & 1.92142000 \\ \mathrm{H} & 3.20077800 & 0.00013700 & 2.62504000 \\ \mathrm{H} & -3.36157500 & 1.92570200 & 1.88447800 \\ \mathrm{H} & -4.47043100 & 3.92894500 & 0.89084400 \\ \mathrm{H} & -3.96062100 & 4.58648900 & -1.44781000 \\ \mathrm{H} & -2.35029000 & 3.25837500 & -2.78987800 \\ & & & \end{array}$




$$
\begin{array}{lrrr}
\mathrm{H} & -1.25307600 & 1.26825700 & -1.79666400 \\
\mathrm{H} & -3.36235200 & -1.92500400 & 1.88430300 \\
\mathrm{H} & -4.47123900 & -3.92827900 & 0.89080100 \\
\mathrm{H} & -3.96061700 & -4.58662300 & -1.44745100 \\
\mathrm{H} & -2.34942800 & -3.25926800 & -2.78924100 \\
\mathrm{H} & -1.25217200 & -1.26910200 & -1.79615200
\end{array}
$$<smiles>O=P(N=Cc1ccc2ccccc2c1)(c1ccccc1)c1ccccc1</smiles>

Imine-5

$\begin{array}{lrrr}\mathrm{C} & 0.73982700 & 0.00003200 & -0.80403700 \\ \mathrm{~N} & -0.31712000 & -0.00002400 & -0.07532600 \\ \mathrm{H} & 0.67497800 & 0.00009800 & -1.90075300 \\ \mathrm{P} & -1.83103700 & 0.00001900 & -0.87225800 \\ \mathrm{O} & -1.84730500 & 0.00009500 & -2.37198100 \\ \mathrm{C} & 2.08824300 & 0.00001000 & -0.23626700 \\ \mathrm{C} & 2.29142900 & -0.00004000 & 1.17352700 \\ \mathrm{C} & 3.56036600 & -0.00006400 & 1.68873600 \\ \mathrm{C} & 4.70031700 & -0.00004000 & 0.83528700 \\ \mathrm{C} & 4.50283700 & 0.00001200 & -0.58364800 \\ \mathrm{C} & 3.17917900 & 0.00003700 & -1.08678600 \\ \mathrm{C} & -2.63226800 & -1.46351200 & -0.15712500 \\ \mathrm{C} & -2.63224400 & 1.46351000 & -0.15700900 \\ \mathrm{C} & -3.47574700 & -2.21074700 & -0.98799800 \\ \mathrm{C} & -4.13739800 & -3.32984400 & -0.48379800 \\ \mathrm{C} & -3.95718300 & -3.70622300 & 0.84774700 \\ \mathrm{C} & -3.11036900 & -2.96677100 & 1.67680200 \\ \mathrm{C} & -2.44816000 & -1.84712600 & 1.17807500 \\ \mathrm{C} & -3.47526700 & 2.21115000 & -0.98797700 \\ \mathrm{C} & -4.13685700 & 3.33025200 & -0.48370300 \\ \mathrm{C} & -3.95703500 & 3.70622500 & 0.84800800 \\ \mathrm{C} & -3.11068300 & 2.96635700 & 1.67716500 \\ \mathrm{C} & -2.44853700 & 1.84670900 & 1.17836500 \\ \mathrm{C} & 6.02408600 & -0.00006500 & 1.34053000 \\ \mathrm{C} & 7.10404400 & -0.00004000 & 0.48548100 \\ \mathrm{C} & 6.90916100 & 0.00001100 & -0.91617700 \\ \mathrm{C} & 5.63606000 & 0.00003700 & -1.43842000 \\ \mathrm{H} & 1.41754600 & -0.00005500 & 1.81488600 \\ \mathrm{H} & 3.71722200 & -0.00010000 & 2.76375600 \\ \mathrm{H} & 3.02371000 & 0.00007800 & -2.16292000 \\ \mathrm{H} & -3.59136200 & -1.91083700 & -2.02433200 \\ \mathrm{H} & -4.78975300 & -3.90938800 & -1.12979700 \\ \mathrm{H} & -4.47206400 & -4.57834000 & 1.23951500 \\ \mathrm{H} & -2.96385200 & -3.26626600 & 2.71013700 \\ \mathrm{H} & -1.77716900 & -1.28051400 & 1.81406700 \\ \mathrm{H} & -3.59058600 & 1.91153100 & -2.02442800 \\ \mathrm{H} & -4.78886100 & 3.91011200 & -1.12977400 \\ & & & \\ & & & \\ & & & \end{array}$




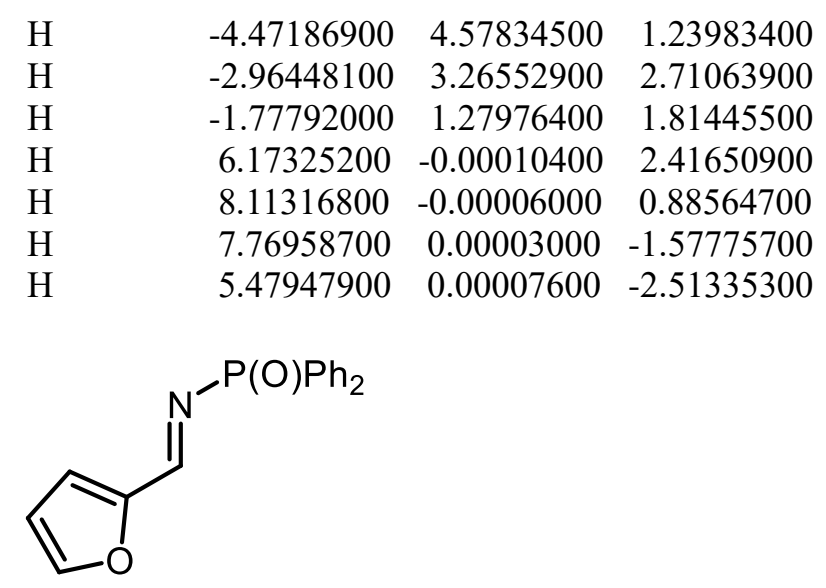

Imine-6

$\begin{array}{lrrr}\mathrm{C} & 2.03298100 & -0.00001300 & -0.84229800 \\ \mathrm{~N} & 0.98996000 & -0.00001100 & -0.09136000 \\ \mathrm{H} & 1.96213000 & -0.00000300 & -1.93807900 \\ \mathrm{P} & -0.52999500 & 0.00000000 & -0.87304800 \\ \mathrm{O} & -0.55914400 & 0.00000200 & -2.37310200 \\ \mathrm{C} & 3.37550400 & -0.00001200 & -0.32580900 \\ \mathrm{C} & -1.32513900 & -1.46229800 & -0.14919800 \\ \mathrm{C} & -1.32512300 & 1.46230500 & -0.14919500 \\ \mathrm{C} & -2.19678400 & -2.19675500 & -0.96224200 \\ \mathrm{C} & -2.85546100 & -3.31260400 & -0.44721500 \\ \mathrm{C} & -2.64386400 & -3.69826300 & 0.87712900 \\ \mathrm{C} & -1.76849700 & -2.97173200 & 1.68772500 \\ \mathrm{C} & -1.10895100 & -1.85506700 & 1.17856800 \\ \mathrm{C} & -2.19674300 & 2.19678600 & -0.96224400 \\ \mathrm{C} & -2.85540600 & 3.31264300 & -0.44721600 \\ \mathrm{C} & -2.64382000 & 3.69828500 & 0.87713600 \\ \mathrm{C} & -1.76847800 & 2.97172900 & 1.68773700 \\ \mathrm{C} & -1.10894600 & 1.85505700 & 1.17857800 \\ \mathrm{O} & 3.58369800 & -0.00002900 & 1.02941700 \\ \mathrm{C} & 4.92352900 & -0.00000200 & 1.22422500 \\ \mathrm{C} & 5.59280500 & -0.00000500 & 0.03180300 \\ \mathrm{C} & 4.58746000 & -0.00001700 & -0.97636900 \\ \mathrm{H} & -2.33657100 & -1.89029300 & -1.99369900 \\ \mathrm{H} & -3.52989200 & -3.88244400 & -1.07906600 \\ \mathrm{H} & -3.15654800 & -4.56787500 & 1.27734900 \\ \mathrm{H} & -1.59749400 & -3.27893300 & 2.71502800 \\ \mathrm{H} & -0.41469200 & -1.29856400 & 1.79843000 \\ \mathrm{H} & -2.33652200 & 1.89033600 & -1.99370500 \\ \mathrm{H} & -3.52981700 & 3.88250200 & -1.07907100 \\ \mathrm{H} & -3.15649300 & 4.56790300 & 1.27735600 \\ \mathrm{H} & -1.59748400 & 3.27891700 & 2.71504500 \\ \mathrm{H} & -0.41470700 & 1.29853500 & 1.79844400 \\ \mathrm{H} & 5.24951500 & 0.00000200 & 2.25278000 \\ \mathrm{H} & 6.66373700 & 0.00000100 & -0.10520300 \\ \mathrm{H} & 4.72821200 & -0.00002100 & -2.04757400\end{array}$


<smiles>CCCCOPN=Cc1ccccn1</smiles>

Imine-7

$\begin{array}{llll}\mathrm{C} & 1.79226400 & 0.00018700 & -0.86999000\end{array}$

$\begin{array}{llll}\mathrm{N} & 0.75243000 & 0.00006200 & -0.12275700\end{array}$

$\mathrm{H} \quad 1.74209600 \quad 0.00018700 \quad-1.96519100$

$\begin{array}{lllll}\mathrm{P} & -0.78471300 & 0.00003300 & -0.88500600\end{array}$

$\begin{array}{lllll}\mathrm{O} & -0.84069000 & 0.00012400 & -2.38224500\end{array}$

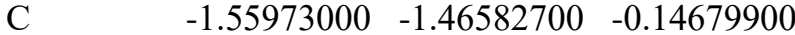

$\begin{array}{llll}\text { C } & -1.55983000 & 1.46577300 & -0.14666100\end{array}$

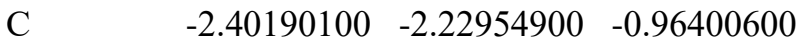

$\begin{array}{llll}\text { C } & -3.04537100 & -3.35231700 & -0.44468700\end{array}$

$\begin{array}{llll}\text { C } & -2.84829100 & -3.71590400 & 0.88795400\end{array}$

$\begin{array}{llll}\text { C } & -2.00279200 & -2.95991800 & 1.70334800\end{array}$

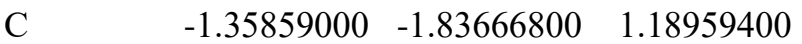

$\begin{array}{lllll}\text { C } & -2.40152000 & 2.22989800 & -0.96398200\end{array}$

$\begin{array}{llll}\text { C } & -3.04501400 & 3.35261400 & -0.44457600\end{array}$

$\begin{array}{llll}\text { C } & -2.84843900 & 3.71573800 & 0.88826400\end{array}$

$\begin{array}{llll}\mathrm{C} & -2.00342900 & 2.95933500 & 1.70378200\end{array}$

$\begin{array}{llll}\mathrm{C} & -1.35920500 & 1.83613900 & 1.18994100\end{array}$

$\begin{array}{llll}\mathrm{C} & 3.15628000 & 0.00009500 & -0.30784400\end{array}$

$\begin{array}{llll}\mathrm{C} & 3.37866500 & 0.00001800 & 1.07535900\end{array}$

$\begin{array}{llll}\mathrm{C} & 4.69006700 & -0.00003900 & 1.53550800\end{array}$

$\begin{array}{llll}\mathrm{C} & 5.72709800 & -0.00001300 & 0.60207300\end{array}$

$\begin{array}{llll}\mathrm{C} & 5.40300000 & 0.00007500 & -0.75653800\end{array}$

$\mathrm{N} \quad 4.14904800 \quad 0.00012200 \quad-1.21532400$

$\mathrm{H} \quad-2.53087000 \quad-1.93875300 \quad-2.00131200$

$\mathrm{H} \quad-3.69677900 \quad-3.94453100 \quad-1.07995500$

$\mathrm{H} \quad-3.34911500 \quad-4.59082900 \quad 1.29140100$

$\mathrm{H} \quad-1.84351800 \quad-3.24926000 \quad 2.73766000$

$\mathrm{H} \quad-\quad-6.68942200 \quad-1.25728700 \quad 1.81588700$

$\mathrm{H} \quad-2.53010900 \quad 1.93943700 \quad-2.00142900$

$\mathrm{H} \quad-3.69605100 \quad 3.94514300 \quad-1.07993000$

$\mathrm{H} \quad-3.34928200 \quad 4.59062100 \quad 1.29178000$

$\mathrm{H} \quad-1.84456100 \quad 3.24830700 \quad 2.73825900$

$\mathrm{H} \quad \begin{array}{llll}\mathrm{H} & -0.69044100 & 1.25641900 & 1.81635100\end{array}$

$\begin{array}{llll}\mathrm{H} & 2.52690300 & 0.00001500 & 1.74528600\end{array}$

$\mathrm{H} \quad 4.90304300 \quad-0.00009900 \quad 2.59997900$

$\mathrm{H} \quad 6.76582900 \quad-0.00005600 \quad 0.91530900$

$\begin{array}{lllll}\mathrm{H} & 6.18857400 & 0.00009600 & -1.50892900\end{array}$<smiles>CCCCOPN=Cc1cccs1</smiles> 
Imine-8

$\begin{array}{lccc}\mathrm{C} & -1.78853100 & 0.00003100 & 0.99911500 \\ \mathrm{~N} & -0.77241300 & -0.00000500 & 0.21154200 \\ \mathrm{H} & -1.67435300 & 0.00005900 & 2.09151700 \\ \mathrm{P} & 0.78346100 & 0.00001000 & 0.92064800 \\ \mathrm{O} & 0.87798400 & 0.00004400 & 2.41760400 \\ \mathrm{C} & -3.14494000 & 0.00001800 & 0.50787600 \\ \mathrm{C} & 1.54295500 & -1.46311800 & 0.16171500 \\ \mathrm{C} & 1.54295200 & 1.46311300 & 0.16166300 \\ \mathrm{C} & 2.44024600 & -2.20510600 & 0.93921200 \\ \mathrm{C} & 3.07234400 & -3.32326500 & 0.39657600 \\ \mathrm{C} & 2.80874200 & -3.70374300 & -0.91986900 \\ \mathrm{C} & 1.90803900 & -2.96954000 & -1.69502600 \\ \mathrm{C} & 1.27493700 & -1.85065000 & -1.15815400 \\ \mathrm{C} & 2.44005300 & 2.20527400 & 0.93921300 \\ \mathrm{C} & 3.07213000 & 3.32342900 & 0.39654600 \\ \mathrm{C} & 2.80869600 & 3.70373100 & -0.91998400 \\ \mathrm{C} & 1.90818500 & 2.96935100 & -1.69519700 \\ \mathrm{C} & 1.27510500 & 1.85046500 & -1.15829400 \\ \mathrm{~S} & -3.49867000 & -0.00002200 & -1.20474700 \\ \mathrm{C} & -5.19779100 & -0.00004500 & -0.88259300 \\ \mathrm{C} & -5.47971800 & -0.00001600 & 0.46080000 \\ \mathrm{C} & -4.30424800 & 0.00005700 & 1.25646900 \\ \mathrm{H} & 2.62065500 & -1.90253000 & 1.96548500 \\ \mathrm{H} & 3.76661400 & -3.89893000 & 1.00102400 \\ \mathrm{H} & 3.30065500 & -4.57520200 & -1.34149800 \\ \mathrm{H} & 1.69667600 & -3.27247300 & -2.71603300 \\ \mathrm{H} & 0.56222100 & -1.28832100 & -1.75117200 \\ \mathrm{H} & 2.62033300 & 1.90282600 & 1.96554700 \\ \mathrm{H} & 3.76625300 & 3.89922900 & 1.00103400 \\ \mathrm{H} & 3.30059400 & 4.57518600 & -1.34163800 \\ \mathrm{H} & 1.69695600 & 3.27214300 & -2.71627400 \\ \mathrm{H} & 0.56254300 & 1.28799500 & -1.75136300 \\ \mathrm{H} & -5.90062300 & -0.00008500 & -1.70431500 \\ \mathrm{H} & -6.48598800 & -0.00002500 & 0.86142300 \\ \mathrm{H} & -4.29765800 & 0.00009900 & 2.34046200\end{array}$

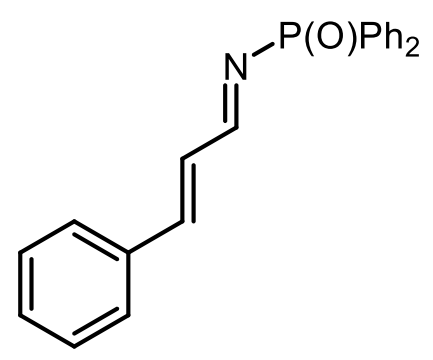

Imine-9

$\begin{array}{lrrr}\mathrm{C} & 0.94127300 & -0.00015600 & -0.61353700 \\ \mathrm{~N} & -0.14792800 & -0.00011800 & 0.07384600 \\ \mathrm{H} & 0.92260300 & -0.00015600 & -1.71261700 \\ \mathrm{P} & -1.62261800 & -0.00001500 & -0.79049400\end{array}$




$\begin{array}{lrrr}\mathrm{O} & -1.57550400 & -0.00000700 & -2.29013900 \\ \mathrm{C} & -2.45969200 & -1.46197100 & -0.11245500 \\ \mathrm{C} & -2.45948400 & 1.46205100 & -0.11243700 \\ \mathrm{C} & -3.27166500 & -2.20377100 & -0.97876400 \\ \mathrm{C} & -3.95940900 & -3.32019700 & -0.50439600 \\ \mathrm{C} & -3.83669100 & -3.69934400 & 0.83292700 \\ \mathrm{C} & -3.02117100 & -2.96541200 & 1.69749900 \\ \mathrm{C} & -2.33298400 & -1.84845400 & 1.22854700 \\ \mathrm{C} & -3.27137100 & 2.20396000 & -0.97873300 \\ \mathrm{C} & -3.95895900 & 3.32047800 & -0.50435400 \\ \mathrm{C} & -3.83616700 & 3.69960900 & 0.83296700 \\ \mathrm{C} & -3.02073100 & 2.96556700 & 1.69752600 \\ \mathrm{C} & -2.33270200 & 1.84851700 & 1.22856300 \\ \mathrm{C} & 2.24278000 & -0.00021600 & 0.01877100 \\ \mathrm{C} & 3.37709900 & -0.00012400 & -0.71415000 \\ \mathrm{C} & 4.75235400 & -0.00011600 & -0.22734800 \\ \mathrm{C} & 5.80142200 & 0.00005400 & -1.16359200 \\ \mathrm{C} & 7.13102200 & 0.00010400 & -0.75126400 \\ \mathrm{C} & 7.43806700 & -0.00000900 & 0.60926500 \\ \mathrm{C} & 6.40639600 & -0.00018500 & 1.55313500 \\ \mathrm{C} & 5.07929000 & -0.00024100 & 1.14214800 \\ \mathrm{H} & -3.34258000 & -1.90161200 & -2.01848500 \\ \mathrm{H} & -4.58738400 & -3.89544900 & -1.17787500 \\ \mathrm{H} & -4.37200700 & -4.56925400 & 1.20162100 \\ \mathrm{H} & -2.91936600 & -3.26703700 & 2.73562000 \\ \mathrm{H} & -1.68531600 & -1.28616400 & 1.89204400 \\ \mathrm{H} & -3.34234400 & 1.90181200 & -2.01845300 \\ \mathrm{H} & -4.58686700 & 3.89581300 & -1.17782300 \\ \mathrm{H} & -4.37136000 & 4.56959200 & 1.20167000 \\ \mathrm{H} & -2.91886800 & 3.26717900 & 2.73564500 \\ \mathrm{H} & -1.68510000 & 1.28613800 & 1.89205000 \\ \mathrm{H} & 2.25160300 & -0.00030200 & 1.10475900 \\ \mathrm{H} & 3.27040900 & -0.00006100 & -1.79875800 \\ \mathrm{H} & 5.56277100 & 0.00014900 & -2.22346100 \\ \mathrm{H} & 7.92609900 & 0.00022900 & -1.49018100 \\ \mathrm{H} & 8.47349200 & 0.00004500 & 0.93500800 \\ \mathrm{H} & 6.64118900 & -0.00027500 & 2.61298900 \\ \mathrm{H} & 4.28976100 & -0.00038300 & 1.88592700\end{array}$<smiles>CC(C)(C)OC(=O)N=Cc1ccccc1</smiles>

Imine-10
$\begin{array}{llll}\text { C } & -4.81138600 & -0.80383000 & 0.05431300\end{array}$
$\begin{array}{llll}\text { C } & -3.47279900 & -1.18825200 & 0.05087600\end{array}$
$\begin{array}{llll}\text { C } & -2.45767600 & -0.22093000 & 0.00499100\end{array}$ 


$\begin{array}{lrrr}\mathrm{C} & -2.80024400 & 1.14185100 & -0.03662700 \\ \mathrm{C} & -4.13596000 & 1.52209200 & -0.03290800 \\ \mathrm{C} & -5.14298200 & 0.55104400 & 0.01245300 \\ \mathrm{C} & -1.06294600 & -0.65181200 & 0.00083900 \\ \mathrm{~N} & -0.08749900 & 0.18407900 & -0.04238400 \\ \mathrm{C} & 1.20271700 & -0.41127000 & -0.05080100 \\ \mathrm{O} & 1.44504600 & -1.60413800 & -0.11350700 \\ \mathrm{O} & 2.10835300 & 0.57086600 & 0.01493200 \\ \mathrm{C} & 3.54839000 & 0.26885300 & 0.02462100 \\ \mathrm{C} & 3.94330600 & -0.42842100 & -1.27845200 \\ \mathrm{C} & 3.89898500 & -0.56293400 & 1.25969600 \\ \mathrm{C} & 4.17581800 & 1.65936400 & 0.10939500 \\ \mathrm{H} & -0.87401100 & -1.73201800 & 0.03449700 \\ \mathrm{H} & -5.59311800 & -1.55582400 & 0.08947900 \\ \mathrm{H} & -3.20426500 & -2.24059800 & 0.08304100 \\ \mathrm{H} & -2.00038900 & 1.87309100 & -0.07059300 \\ \mathrm{H} & -4.40002600 & 2.57450100 & -0.06500400 \\ \mathrm{H} & -6.18591800 & 0.85307500 & 0.01534200 \\ \mathrm{H} & 3.62740200 & 0.17099400 & -2.13738800 \\ \mathrm{H} & 5.03174500 & -0.53442600 & -1.31788400 \\ \mathrm{H} & 3.48967000 & -1.41668200 & -1.34790000 \\ \mathrm{H} & 3.55278200 & -0.05697100 & 2.16583200 \\ \mathrm{H} & 3.44501700 & -1.55218900 & 1.20858000 \\ \mathrm{H} & 4.98553300 & -0.67492100 & 1.32541000 \\ \mathrm{H} & 3.85071800 & 2.17194900 & 1.01865000 \\ \mathrm{H} & 5.26627300 & 1.57769100 & 0.12474600 \\ \mathrm{H} & 3.88256700 & 2.26542000 & -0.75190300\end{array}$<smiles>CC(C)(C)OC(=O)N=Cc1ccccc1</smiles>

Imine-11

$\begin{array}{lrrr}\mathrm{C} & -3.15161900 & 0.17726000 & -0.13023400 \\ \mathrm{C} & -1.79064600 & 0.05633400 & -0.64228200 \\ \mathrm{~N} & -0.96923100 & -0.83103000 & -0.20536400 \\ \mathrm{C} & -3.99500000 & 1.16759600 & -0.65603900 \\ \mathrm{C} & -5.29950800 & 1.30168100 & -0.18745500 \\ \mathrm{C} & -5.76801400 & 0.44667100 & 0.81102500 \\ \mathrm{C} & -4.93219000 & -0.54279400 & 1.34153300 \\ \mathrm{C} & -3.63088000 & -0.67931800 & 0.87622800 \\ \mathrm{C} & 0.29805100 & -0.82359200 & -0.83184000 \\ \mathrm{O} & 1.13419800 & -1.58954400 & -0.11274400 \\ \mathrm{O} & 0.60108900 & -0.24918800 & -1.86263600 \\ \mathrm{C} & 2.50271500 & -1.65408600 & -0.59395600 \\ \mathrm{C} & 3.32388200 & -0.50166700 & -0.07817500 \\ \mathrm{C} & 3.47190000 & 0.66591300 & -0.83619800 \\ \mathrm{C} & 4.21685900 & 1.73551900 & -0.34125300\end{array}$




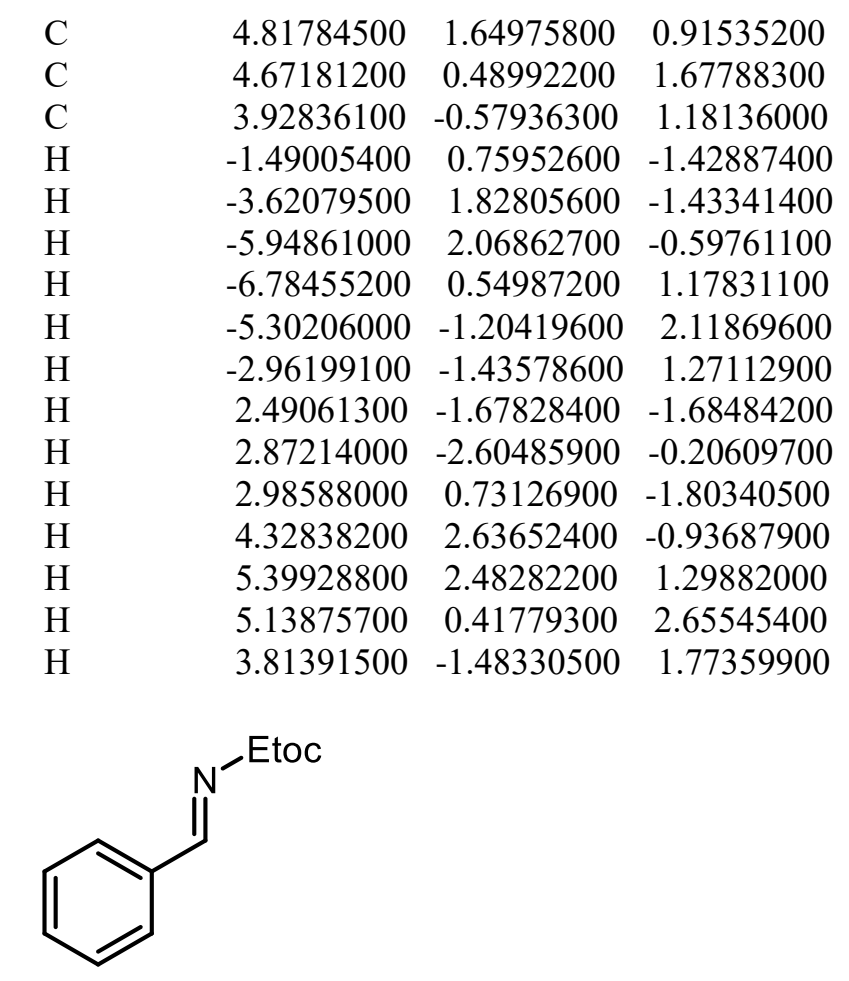

Imine-12

$\begin{array}{lrrr}\mathrm{C} & 1.80556900 & -0.22142300 & -0.00002600 \\ \mathrm{C} & 0.42473500 & -0.69311400 & -0.00004900 \\ \mathrm{~N} & -0.57617500 & 0.11424700 & 0.00001100 \\ \mathrm{C} & 2.10758600 & 1.15166300 & 0.00002600 \\ \mathrm{C} & 3.43155900 & 1.57076800 & 0.00005000 \\ \mathrm{C} & 4.46683400 & 0.62877300 & 0.00002400 \\ \mathrm{C} & 4.17556100 & -0.73601300 & -0.00002800 \\ \mathrm{C} & 2.84891500 & -1.15954000 & -0.00005200 \\ \mathrm{C} & -1.84334200 & -0.51909300 & 0.00004100 \\ \mathrm{O} & -2.79537000 & 0.42666300 & -0.00020200 \\ \mathrm{O} & -2.07279200 & -1.71461800 & 0.00023200 \\ \mathrm{C} & -4.15212100 & -0.06566200 & -0.00017500 \\ \mathrm{C} & -5.06893500 & 1.14046600 & 0.00013500 \\ \mathrm{H} & 0.26801400 & -1.77895000 & -0.00008100 \\ \mathrm{H} & 1.28659200 & 1.85988600 & 0.00004500 \\ \mathrm{H} & 3.66452300 & 2.63095100 & 0.00008800 \\ \mathrm{H} & 5.50046700 & 0.96121200 & 0.00004200 \\ \mathrm{H} & 4.97930400 & -1.46524000 & -0.00004800 \\ \mathrm{H} & 2.61158300 & -2.21982600 & -0.00009200 \\ \mathrm{H} & -4.29905600 & -0.69721600 & 0.88165300 \\ \mathrm{H} & -4.29921000 & -0.69685000 & -0.88224600 \\ \mathrm{H} & -6.11310400 & 0.81363400 & 0.00013200 \\ \mathrm{H} & -4.89932300 & 1.75749200 & 0.88670900 \\ \mathrm{H} & -4.89943900 & 1.75787100 & -0.88619700\end{array}$


<smiles>Cc1ccccc1/C=N/C(=O)OCc1ccccc1</smiles>

Imine-13

$\begin{array}{lrrr}\mathrm{C} & -2.31936100 & -0.36827500 & 0.00650300 \\ \mathrm{C} & -0.90763400 & -0.73031500 & -0.02326400 \\ \mathrm{~N} & 0.06543500 & 0.11050500 & -0.08202400 \\ \mathrm{C} & -3.22472100 & -1.44346200 & 0.08350400 \\ \mathrm{C} & -4.59734300 & -1.23729700 & 0.11928400 \\ \mathrm{C} & -5.08393200 & 0.06890200 & 0.07861100 \\ \mathrm{C} & -4.19818900 & 1.14381000 & 0.00267000 \\ \mathrm{C} & -2.81233400 & 0.96341500 & -0.03566800 \\ \mathrm{C} & 1.35422900 & -0.48324300 & -0.12270400 \\ \mathrm{O} & 2.25888900 & 0.48642100 & 0.06014400 \\ \mathrm{O} & 1.59919700 & -1.66308700 & -0.30604900 \\ \mathrm{C} & 3.69795100 & 0.18166700 & 0.07062600 \\ \mathrm{C} & 4.02175400 & -0.77495800 & 1.21968800 \\ \mathrm{C} & 4.32355200 & 1.55428800 & 0.31345900 \\ \mathrm{C} & 4.12191600 & -0.37705100 & -1.28893500 \\ \mathrm{C} & -1.91574400 & 2.16847200 & -0.11949500 \\ \mathrm{H} & -0.69458000 & -1.80676500 & 0.00620200 \\ \mathrm{H} & -2.82770500 & -2.45432500 & 0.11448200 \\ \mathrm{H} & -5.27803400 & -2.08012500 & 0.17811900 \\ \mathrm{H} & -6.15351800 & 0.25428200 & 0.10596200 \\ \mathrm{H} & -4.59043500 & 2.15615900 & -0.02849400 \\ \mathrm{H} & 3.57007700 & -1.75286200 & 1.05532500 \\ \mathrm{H} & 5.10665400 & -0.89455300 & 1.29797200 \\ \mathrm{H} & 3.65460200 & -0.36591000 & 2.16568700 \\ \mathrm{H} & 4.04837600 & 2.24764400 & -0.48573200 \\ \mathrm{H} & 3.97955000 & 1.96980400 & 1.26438600 \\ \mathrm{H} & 5.41344700 & 1.46923200 & 0.34284900 \\ \mathrm{H} & 3.82592400 & 0.30969800 & -2.08746300 \\ \mathrm{H} & 5.21089400 & -0.48196400 & -1.31501200 \\ \mathrm{H} & 3.66900600 & -1.35115400 & -1.47129400 \\ \mathrm{H} & -2.51329600 & 3.08392200 & -0.13005900 \\ \mathrm{H} & -1.21459400 & 2.20338200 & 0.71811800 \\ \mathrm{H} & -1.28995300 & 2.13761300 & -1.01532000\end{array}$


<smiles>CC(C)(C)OC(=O)N=Cc1ccc(F)cc1</smiles>

Imine-14

$\begin{array}{lrrr}\mathrm{C} & 2.01122300 & -0.35139900 & -0.00002300 \\ \mathrm{C} & 0.60606600 & -0.73910100 & -0.00002600 \\ \mathrm{~N} & -0.34260200 & 0.12854900 & 0.00013200 \\ \mathrm{C} & 3.00118900 & -1.34651500 & -0.00010900 \\ \mathrm{C} & 4.35063000 & -1.00847700 & -0.00009800 \\ \mathrm{C} & 4.69195600 & 0.33945700 & -0.00000400 \\ \mathrm{C} & 3.73530700 & 1.35422900 & 0.00007100 \\ \mathrm{C} & 2.39369200 & 1.00275500 & 0.00006300 \\ \mathrm{C} & -1.65117300 & -0.42544000 & 0.00014300 \\ \mathrm{O} & -2.52521000 & 0.58659700 & 0.00008200 \\ \mathrm{O} & -1.93038000 & -1.61194200 & 0.00022300 \\ \mathrm{C} & -3.97464800 & 0.33107900 & -0.00005400 \\ \mathrm{C} & -4.37120900 & -0.42193200 & 1.27108000 \\ \mathrm{C} & -4.37096800 & -0.42226900 & -1.27105800 \\ \mathrm{C} & -4.55776100 & 1.74322900 & -0.00031300 \\ \mathrm{~F} & 5.99346100 & 0.67875000 & -0.00001600 \\ \mathrm{H} & 0.38347300 & -1.81333100 & -0.00016200 \\ \mathrm{H} & 2.70686000 & -2.39197800 & -0.00018200 \\ \mathrm{H} & 5.13184700 & -1.75954400 & -0.00016200 \\ \mathrm{H} & 4.05732600 & 2.38930100 & 0.00013200 \\ \mathrm{H} & 1.61528000 & 1.75728500 & 0.00012000 \\ \mathrm{H} & -4.02290100 & 0.12061400 & 2.15493900 \\ \mathrm{H} & -5.46171100 & -0.49571500 & 1.32375100 \\ \mathrm{H} & -3.94892100 & -1.42636400 & 1.28037100 \\ \mathrm{H} & -4.02253200 & 0.12005400 & -2.15500400 \\ \mathrm{H} & -3.94869700 & -1.42671000 & -1.28003600 \\ \mathrm{H} & -5.46146300 & -0.49606400 & -1.32387700 \\ \mathrm{H} & -4.23099000 & 2.29287100 & -0.88702300 \\ \mathrm{H} & -5.65037600 & 1.69696000 & -0.00045200 \\ \mathrm{H} & -4.23122600 & 2.29310300 & 0.88634000\end{array}$<smiles>COc1ccc(/C=N/C(=O)OCc2ccccc2)cc1</smiles>

Imine-15 


$\begin{array}{lrrr}\mathrm{C} & 3.84460500 & -1.37490600 & -0.00020200 \\ \mathrm{C} & 2.48466400 & -1.63191600 & -0.00010600 \\ \mathrm{C} & 1.55010400 & -0.57981500 & 0.00011000 \\ \mathrm{C} & 2.02309000 & 0.74231600 & 0.00023000 \\ \mathrm{C} & 3.38396600 & 1.01479000 & 0.00013600 \\ \mathrm{C} & 4.30353800 & -0.04790900 & -0.00008200 \\ \mathrm{C} & 0.12839000 & -0.87774700 & 0.00020200 \\ \mathrm{~N} & -0.77065100 & 0.04519700 & 0.00042500 \\ \mathrm{C} & -2.10496900 & -0.43051300 & 0.00052900 \\ \mathrm{O} & -2.45839100 & -1.59804300 & 0.00003200 \\ \mathrm{O} & -2.92185100 & 0.63181900 & 0.00028700 \\ \mathrm{C} & -4.38112500 & 0.45840500 & -0.00013200 \\ \mathrm{C} & -4.82194600 & -0.27069000 & 1.27067100 \\ \mathrm{C} & -4.82115700 & -0.27095500 & -1.27105500 \\ \mathrm{C} & -4.88492300 & 1.90108400 & -0.00043500 \\ \mathrm{O} & 5.65141100 & 0.10620600 & -0.00019000 \\ \mathrm{C} & 6.18066100 & 1.42582700 & -0.00007500 \\ \mathrm{H} & -0.15944200 & -1.93651600 & 0.00010600 \\ \mathrm{H} & 4.57635100 & -2.17484000 & -0.00037000 \\ \mathrm{H} & 2.12991100 & -2.65882700 & -0.00019900 \\ \mathrm{H} & 1.29619100 & 1.54705200 & 0.00039600 \\ \mathrm{H} & 3.72373900 & 2.04275500 & 0.00023000 \\ \mathrm{H} & -4.44354200 & 0.25112800 & 2.15471500 \\ \mathrm{H} & -5.91501300 & -0.28233700 & 1.32279300 \\ \mathrm{H} & -4.45676000 & -1.29723300 & 1.28000200 \\ \mathrm{H} & -4.44224500 & 0.25070300 & -2.15497500 \\ \mathrm{H} & -4.45592100 & -1.29748600 & -1.27996200 \\ \mathrm{H} & -5.91419300 & -0.28266200 & -1.32382900 \\ \mathrm{H} & -4.52739000 & 2.43159900 & -0.88700400 \\ \mathrm{H} & -5.97849400 & 1.91647900 & -0.00076700 \\ \mathrm{H} & -4.52792700 & 2.43178300 & 0.88624000 \\ \mathrm{H} & 7.26486000 & 1.31269300 & -0.00019000 \\ \mathrm{H} & 5.87276400 & 1.98093600 & -0.89407800 \\ \mathrm{H} & 5.87293100 & 1.98071300 & 0.89412500\end{array}$<smiles>CC(C)(C)OC(=O)N=Cc1cccc2ccccc12</smiles>

Imine-16

\begin{tabular}{|c|c|c|c|}
\hline $\mathrm{C}$ & 3.73568500 & -2.32284300 & 4700 \\
\hline $\mathrm{C}$ & 2.33046100 & -2.27247300 & 0.00023400 \\
\hline $\mathrm{C}$ & 1.63396300 & -1.06785700 & 0.0000500 \\
\hline $\mathrm{C}$ & 2.36692300 & 0.17417100 & -0.0000190 \\
\hline $\mathrm{C}$ & 3.80065100 & 0.11025200 & 0.00009100 \\
\hline $\mathrm{C}$ & 4.45391500 & -1.14978400 & 0.0002680 \\
\hline$C$ & 0.18212100 & -1.19716300 & -0.000063 \\
\hline
\end{tabular}




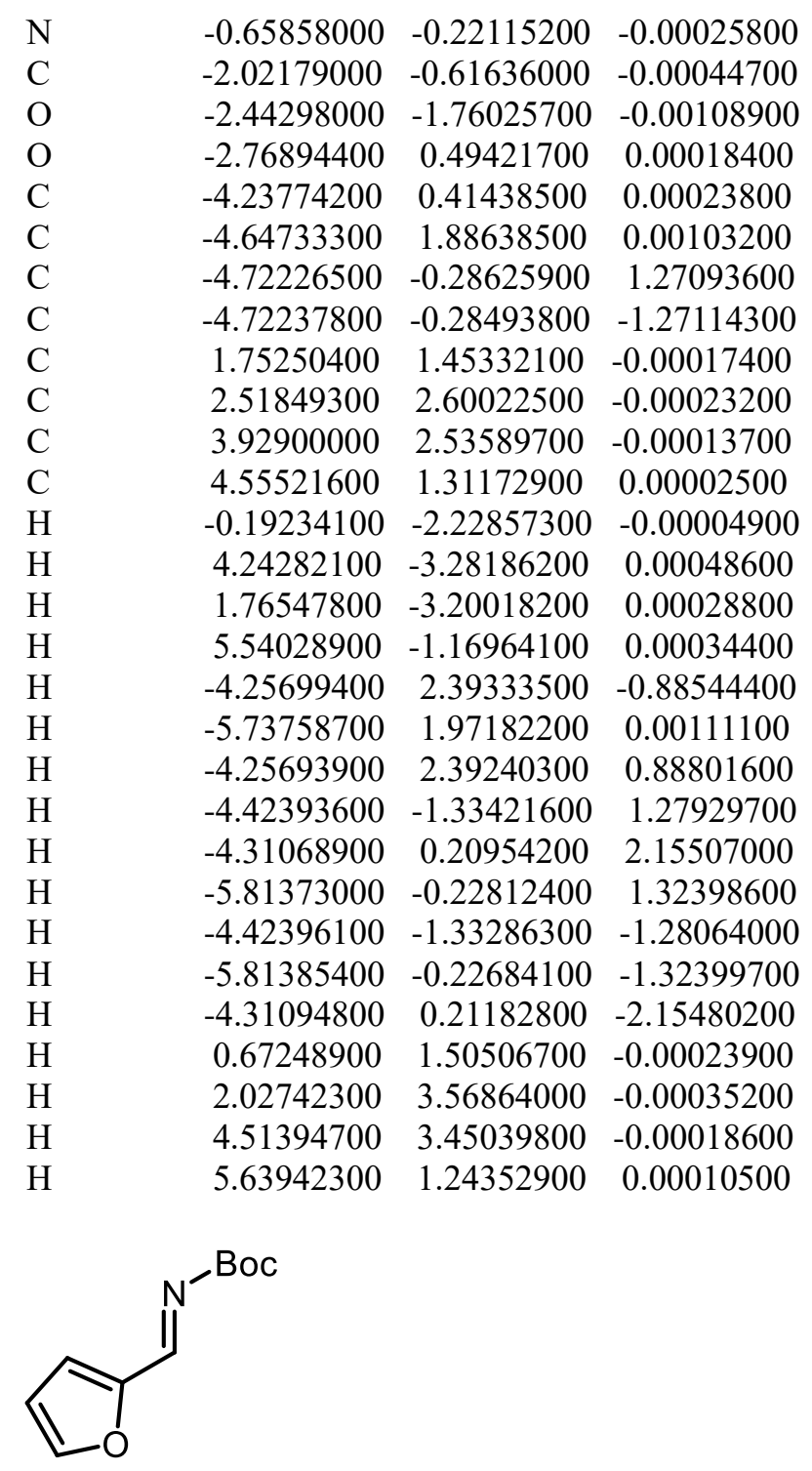

Imine-17

$\begin{array}{lrrr}\mathrm{C} & 2.72558300 & -0.03820100 & 0.00002000 \\ \mathrm{C} & 1.37968100 & -0.53013300 & 0.00003000 \\ \mathrm{~N} & 0.37774700 & 0.28170300 & -0.00042400 \\ \mathrm{C} & -0.88732300 & -0.35929900 & -0.00045600 \\ \mathrm{O} & -1.08600000 & -1.56253400 & -0.00089900 \\ \mathrm{O} & -1.83017700 & 0.59070000 & 0.00003400 \\ \mathrm{C} & -3.25713400 & 0.23468700 & 0.00020200 \\ \mathrm{C} & -3.60133800 & -0.54379700 & -1.27104000 \\ \mathrm{C} & -3.93795100 & 1.60258200 & 0.00084900 \\ \mathrm{C} & -3.60084800 & -0.54474400 & 1.27099400 \\ \mathrm{O} & 3.73168700 & -0.97796800 & 0.00056500 \\ \mathrm{C} & 4.90359100 & -0.30294100 & 0.00045200 \\ \mathrm{C} & 4.68694100 & 1.04943500 & -0.00015600 \\ \mathrm{C} & 3.27641500 & 1.22244600 & -0.00043000\end{array}$




$\begin{array}{lrrr}\mathrm{H} & 1.24708000 & -1.61763700 & 0.00041900 \\ \mathrm{H} & -4.68410300 & -0.69350900 & -1.32319000 \\ \mathrm{H} & -3.10974500 & -1.51615800 & -1.28090000 \\ \mathrm{H} & -3.29213700 & 0.02209800 & -2.15483200 \\ \mathrm{H} & -3.65024800 & 2.17351600 & 0.88766000 \\ \mathrm{H} & -3.65055200 & 2.17419500 & -0.88562300 \\ \mathrm{H} & -5.02467600 & 1.47998100 & 0.00098800 \\ \mathrm{H} & -4.68360200 & -0.69441100 & 1.32348500 \\ \mathrm{H} & -3.10932300 & -1.51714700 & 1.27991800 \\ \mathrm{H} & -3.29123800 & 0.02045900 & 2.15508700 \\ \mathrm{H} & 5.79732300 & -0.90731300 & 0.00083500 \\ \mathrm{H} & 5.44422500 & 1.81922500 & -0.00037700 \\ \mathrm{H} & 2.71298100 & 2.14299900 & -0.00089100\end{array}$<smiles>CC(C)(C)OC(=O)N=Cc1cccs1</smiles>

Imine-18

$\begin{array}{lrrr}\mathrm{C} & 2.47480800 & -0.41628600 & 0.00003700 \\ \mathrm{C} & 1.09161700 & -0.80118800 & 0.00018300 \\ \mathrm{~N} & 0.14305900 & 0.07151400 & 0.00020000 \\ \mathrm{C} & -1.16360800 & -0.47974600 & 0.00026400 \\ \mathrm{O} & -1.44276200 & -1.66711200 & 0.00071100 \\ \mathrm{O} & -2.03838200 & 0.53186100 & -0.00047500 \\ \mathrm{C} & -3.48676000 & 0.27596700 & -0.00013500 \\ \mathrm{C} & -3.88342700 & -0.47811300 & -1.27072900 \\ \mathrm{C} & -4.07078500 & 1.68783400 & -0.00078900 \\ \mathrm{C} & -3.88334400 & -0.47677400 & 1.27126700 \\ \mathrm{~S} & 2.96179200 & 1.26342400 & 0.00008800 \\ \mathrm{C} & 4.62825100 & 0.80784400 & 0.00027300 \\ \mathrm{C} & 4.80605900 & -0.55472500 & -0.00036200 \\ \mathrm{C} & 3.57355200 & -1.25499500 & -0.00054700 \\ \mathrm{H} & 0.87726600 & -1.87662500 & 0.00000900 \\ \mathrm{H} & -3.46051100 & -1.48231300 & -1.27925100 \\ \mathrm{H} & -4.97392700 & -0.55235800 & -1.32339200 \\ \mathrm{H} & -3.53513200 & 0.06395600 & -2.15490500 \\ \mathrm{H} & -3.74390800 & 2.23823800 & 0.88540000 \\ \mathrm{H} & -3.74433500 & 2.23725200 & -0.88774800 \\ \mathrm{H} & -5.16341800 & 1.64102600 & -0.00050300 \\ \mathrm{H} & -3.53500900 & 0.06617600 & 2.15488600 \\ \mathrm{H} & -4.97385600 & -0.55080500 & 1.32400300 \\ \mathrm{H} & -3.46062700 & -1.48103800 & 1.28085700 \\ \mathrm{H} & 5.39417500 & 1.57128100 & 0.00064600 \\ \mathrm{H} & 5.77827300 & -1.03196600 & -0.00055700 \\ \mathrm{H} & 3.48188500 & -2.33511600 & -0.00091500\end{array}$


<smiles>O=C(NC([12F])c1ccccc1)c1ccccc1</smiles>

Amino Sulfone-1

$\begin{array}{lrrr}\mathrm{C} & -2.95689700 & 0.37735600 & -0.27323800 \\ \mathrm{C} & -1.48996800 & 0.05114700 & -0.36322700 \\ \mathrm{~S} & -1.19624900 & -1.59893000 & 0.48598700 \\ \mathrm{~N} & -0.60321400 & 0.99852000 & 0.23484900 \\ \mathrm{C} & -3.86098200 & -0.33698300 & -1.06983300 \\ \mathrm{C} & -5.22677500 & -0.08068300 & -0.98354100 \\ \mathrm{C} & -5.70353700 & 0.89078700 & -0.10222600 \\ \mathrm{C} & -4.80690900 & 1.60397100 & 0.69272500 \\ \mathrm{C} & -3.43957300 & 1.34561200 & 0.61124200 \\ \mathrm{O} & -1.43216400 & -1.36777900 & 1.92474900 \\ \mathrm{O} & -1.92136600 & -2.64505500 & -0.25485200 \\ \mathrm{C} & 0.56249800 & -1.84629900 & 0.25842600 \\ \mathrm{C} & 1.42676800 & -1.61918900 & 1.32741700 \\ \mathrm{C} & 2.79494100 & -1.79071400 & 1.13560900 \\ \mathrm{C} & 3.30811600 & -2.16496300 & -0.11324000 \\ \mathrm{C} & 2.41341600 & -2.39033700 & -1.16839100 \\ \mathrm{C} & 1.04257600 & -2.23653100 & -0.99113600 \\ \mathrm{C} & 4.79468500 & -2.28284200 & -0.32832200 \\ \mathrm{C} & 0.57267400 & 1.34432400 & -0.39596100 \\ \mathrm{O} & 1.34971900 & 2.02252900 & 0.46840100 \\ \mathrm{O} & 0.82283800 & 1.07833500 & -1.55837500 \\ \mathrm{C} & 2.63781600 & 2.58747600 & 0.02890600 \\ \mathrm{C} & 2.40253400 & 3.60451900 & -1.08814000 \\ \mathrm{C} & 3.58076800 & 1.46128200 & -0.39565300 \\ \mathrm{C} & 3.14508700 & 3.27323100 & 1.29577400 \\ \mathrm{H} & -1.16783600 & -0.10019700 & -1.39484800 \\ \mathrm{H} & -0.63816200 & 1.11576900 & 1.23836900 \\ \mathrm{H} & -3.49087900 & -1.10289200 & -1.74219100 \\ \mathrm{H} & -5.91852300 & -0.63936700 & -1.60594300 \\ \mathrm{H} & -6.76829500 & 1.09218400 & -0.03728000 \\ \mathrm{H} & -5.17023700 & 2.36271000 & 1.37873500 \\ \mathrm{H} & -2.74916600 & 1.91550400 & 1.22233100 \\ \mathrm{H} & 1.01953000 & -1.32966900 & 2.28919500 \\ \mathrm{H} & 3.47647100 & -1.62736100 & 1.96564600 \\ \mathrm{H} & 2.79702100 & -2.69063100 & -2.13887400 \\ \mathrm{H} & 0.35195300 & -2.42566700 & -1.80517800 \\ \mathrm{H} & 5.03085800 & -3.01708100 & -1.10309000 \\ \mathrm{H} & 5.21268900 & -1.32092600 & -0.64955500 \\ \mathrm{H} & 5.31079600 & -2.57375800 & 0.59059700 \\ \mathrm{H} & 1.68015300 & 4.36013600 & -0.76554100 \\ \mathrm{H} & 3.34371100 & 4.11053400 & -1.32349200 \\ \mathrm{H} & 2.02900800 & 3.11687600 & -1.98821000 \\ \mathrm{H} & 3.69496600 & 0.74087700 & 0.41770900 \\ \mathrm{H} & 3.19716500 & 0.94005700 & -1.27226700\end{array}$




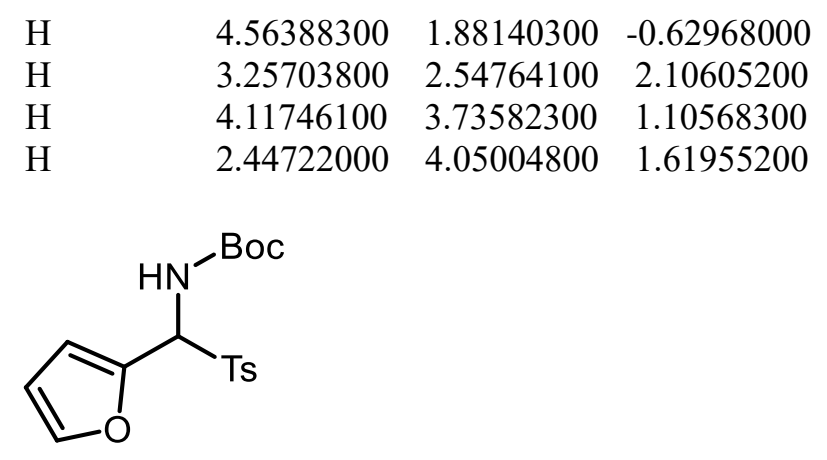

Amino Sulfone-2

$\begin{array}{lrrr}\mathrm{C} & -3.08772000 & 0.53886900 & -0.36798700 \\ \mathrm{C} & -1.66769400 & 0.14193700 & -0.49717400 \\ \mathrm{~S} & -1.49399100 & -1.58571900 & 0.25664500 \\ \mathrm{~N} & -0.75880200 & 1.03542300 & 0.13490000 \\ \mathrm{O} & -1.87159700 & -1.47260500 & 1.67733400 \\ \mathrm{O} & -2.18120400 & -2.51966500 & -0.65260900 \\ \mathrm{C} & 0.26716900 & -1.88552600 & 0.18078500 \\ \mathrm{C} & 1.04338900 & -1.66403200 & 1.31733500 \\ \mathrm{C} & 2.41974200 & -1.85503500 & 1.23970000 \\ \mathrm{C} & 3.02827500 & -2.24662300 & 0.03954300 \\ \mathrm{C} & 2.22049000 & -2.46885100 & -1.08373800 \\ \mathrm{C} & 0.84232300 & -2.29082800 & -1.02251500 \\ \mathrm{C} & 4.52575900 & -2.38700500 & -0.05012600 \\ \mathrm{C} & 0.46327800 & 1.31634100 & -0.42703200 \\ \mathrm{O} & 1.17915500 & 2.06939900 & 0.42790700 \\ \mathrm{O} & 0.80130100 & 0.93287400 & -1.53393200 \\ \mathrm{C} & 2.51947600 & 2.55225600 & 0.05217700 \\ \mathrm{C} & 2.42045100 & 3.45075400 & -1.18103600 \\ \mathrm{C} & 3.45605200 & 1.36341000 & -0.16566900 \\ \mathrm{C} & 2.93623900 & 3.35598600 & 1.28222000 \\ \mathrm{O} & -3.41505900 & 1.30438800 & 0.71869500 \\ \mathrm{C} & -4.76961000 & 1.46613400 & 0.69770700 \\ \mathrm{C} & -5.30864300 & 0.81365200 & -0.36890900 \\ \mathrm{C} & -4.21283800 & 0.20458000 & -1.06225100 \\ \mathrm{H} & -1.37152500 & 0.01021900 & -1.53848500 \\ \mathrm{H} & -0.96222700 & 1.36145400 & 1.06813800 \\ \mathrm{H} & 0.56271900 & -1.36375000 & 2.24106400 \\ \mathrm{H} & 3.03318900 & -1.69542100 & 2.12197400 \\ \mathrm{H} & 2.67841700 & -2.78356100 & -2.01674400 \\ \mathrm{H} & 0.21663800 & -2.47078900 & -1.88911500 \\ \mathrm{H} & 4.95646200 & -2.70406800 & 0.90362200 \\ \mathrm{H} & 4.98531500 & -1.42675100 & -0.31447900 \\ \mathrm{H} & 4.81571800 & -3.11078600 & -0.81636300 \\ \mathrm{H} & 1.69941500 & 4.25469800 & -1.00588800 \\ \mathrm{H} & 3.39603100 & 3.90471800 & -1.37976400 \\ \mathrm{H} & 2.11115600 & 2.88000900 & -2.05620000 \\ \mathrm{H} & 3.46963600 & 0.72741800 & 0.72289200 \\ \mathrm{H} & 3.13541700 & 0.76459500 & -1.01765100 \\ \mathrm{H} & 4.47110800 & 1.73067800 & -0.34617100 \\ \mathrm{H} & 2.95266800 & 2.71812900 & 2.17011200\end{array}$




$\begin{array}{rrrr}\mathrm{H} & 3.93624700 & 3.77280400 & 1.13413400 \\ \mathrm{H} & 2.23864300 & 4.17900000 & 1.45919200 \\ \mathrm{H} & -5.18183900 & 2.06909000 & 1.49063800 \\ \mathrm{H} & -6.35441000 & 0.77041000 & -0.63421400 \\ \mathrm{H} & -4.25090400 & -0.41581500 & -1.94465900\end{array}$<smiles>CC(C)(C)OC(=O)NC([12S])c1ccc2c(c1)OCO2</smiles>

Amino Sulfone-3

$\begin{array}{lrrr}\mathrm{C} & 2.24716000 & 0.00004600 & 0.54390500 \\ \mathrm{C} & 0.75711700 & -0.18342800 & 0.51791300 \\ \mathrm{~S} & 0.36313100 & -1.78815500 & -0.36592900 \\ \mathrm{~N} & 0.01486000 & 0.86598200 & -0.11209900 \\ \mathrm{O} & 0.70158400 & -1.57811900 & -1.78982600 \\ \mathrm{O} & 0.95250700 & -2.89251900 & 0.40756900 \\ \mathrm{C} & -1.42112900 & -1.87428300 & -0.25134300 \\ \mathrm{C} & -2.19260600 & -1.52621700 & -1.35846600 \\ \mathrm{C} & -3.57979400 & -1.55448700 & -1.24835600 \\ \mathrm{C} & -4.20192600 & -1.90551500 & -0.04294400 \\ \mathrm{C} & -3.39962900 & -2.25848000 & 1.05056900 \\ \mathrm{C} & -2.01207000 & -2.24763600 & 0.95483900 \\ \mathrm{C} & -5.70261000 & -1.86433700 & 0.08508000 \\ \mathrm{C} & -1.16947900 & 1.30781400 & 0.43090700 \\ \mathrm{O} & -1.78017000 & 2.12396200 & -0.44910500 \\ \mathrm{O} & -1.56177400 & 1.00144100 & 1.54390100 \\ \mathrm{C} & -3.03868200 & 2.79942100 & -0.08879500 \\ \mathrm{C} & -2.81063600 & 3.71129200 & 1.11723600 \\ \mathrm{C} & -4.13219600 & 1.76065900 & 0.16382100 \\ \mathrm{C} & -3.34397600 & 3.61692600 & -1.34228900 \\ \mathrm{C} & 2.94834000 & 0.30858000 & -0.64347300 \\ \mathrm{C} & 4.31462900 & 0.43810500 & -0.54236800 \\ \mathrm{C} & 4.99056900 & 0.26742900 & 0.66616200 \\ \mathrm{C} & 4.32547500 & -0.04338900 & 1.83375400 \\ \mathrm{C} & 2.92979500 & -0.17883100 & 1.74797900 \\ \mathrm{O} & 6.33338700 & 0.47518000 & 0.47723500 \\ \mathrm{C} & 6.50830700 & 0.59925900 & -0.94095200 \\ \mathrm{O} & 5.21332700 & 0.75782100 & -1.53115400 \\ \mathrm{H} & 0.35414600 & -0.30825800 & 1.52312300 \\ \mathrm{H} & 0.19755000 & 1.08562900 & -1.08047900 \\ \mathrm{H} & -1.70128700 & -1.25405900 & -2.28524000 \\ \mathrm{H} & -4.19064700 & -1.29504700 & -2.10846500 \\ \mathrm{H} & -3.86925600 & -2.54396200 & 1.98713300 \\ \mathrm{H} & -1.39334300 & -2.53179000 & 1.79860900 \\ \mathrm{H} & -6.03433700 & -0.86071100 & 0.37817800 \\ \mathrm{H} & -6.19244500 & -2.10683500 & -0.86201500 \\ \mathrm{H} & -6.05996200 & -2.56287000 & 0.84622100 \\ \mathrm{H} & -2.58050400 & 3.12883700 & 2.00895600 \\ & & & \\ & & & \end{array}$




$\begin{array}{lrrr}\mathrm{H} & -1.98510200 & 4.40113400 & 0.91851500 \\ \mathrm{H} & -3.71258900 & 4.30254100 & 1.30228200 \\ \mathrm{H} & -5.08418300 & 2.27212000 & 0.33696600 \\ \mathrm{H} & -4.24108700 & 1.10970600 & -0.70698500 \\ \mathrm{H} & -3.89350300 & 1.14549500 & 1.03091200 \\ \mathrm{H} & -3.45115400 & 2.96167200 & -2.21102800 \\ \mathrm{H} & -4.27632500 & 4.17218500 & -1.20774800 \\ \mathrm{H} & -2.53976300 & 4.32994700 & -1.54267100 \\ \mathrm{H} & 2.44611600 & 0.40280500 & -1.59759100 \\ \mathrm{H} & 4.85601300 & -0.17641200 & 2.76868200 \\ \mathrm{H} & 2.36853600 & -0.43206800 & 2.64092300 \\ \mathrm{H} & 6.98099300 & -0.31258200 & -1.33128700 \\ \mathrm{H} & 7.11719400 & 1.48143500 & -1.15548000\end{array}$<smiles>COc1ccc(C([18F])NC(=O)OC(C)(C)C)c(OC)c1OC</smiles>

Amino Sulfone-4

$\begin{array}{lrrr}\mathrm{C} & 1.82580500 & 0.19624400 & -0.27301900 \\ \mathrm{C} & 0.36170400 & -0.04007400 & -0.05841600 \\ \mathrm{~S} & -0.14082800 & -1.53423900 & -1.06770400 \\ \mathrm{~N} & -0.50331800 & 1.04048700 & -0.41747000 \\ \mathrm{O} & -0.07554400 & -1.12643600 & -2.48709400 \\ \mathrm{O} & 0.61394900 & -2.70332600 & -0.58003100 \\ \mathrm{C} & -1.86774700 & -1.73988100 & -0.64654000 \\ \mathrm{C} & -2.84340400 & -1.34304400 & -1.55855100 \\ \mathrm{C} & -4.18445300 & -1.46556600 & -1.20586500 \\ \mathrm{C} & -4.55860900 & -1.95774000 & 0.05138700 \\ \mathrm{C} & -3.55443200 & -2.35459600 & 0.94493800 \\ \mathrm{C} & -2.21004200 & -2.25131700 & 0.60440200 \\ \mathrm{C} & -6.01053500 & -2.01831500 & 0.44952300 \\ \mathrm{C} & -1.59456800 & 1.35052900 & 0.36154700 \\ \mathrm{O} & -2.37002500 & 2.23178500 & -0.30160200 \\ \mathrm{O} & -1.78804700 & 0.89701000 & 1.47566900 \\ \mathrm{C} & -3.57478500 & 2.78781200 & 0.33577500 \\ \mathrm{C} & -3.18479400 & 3.56367400 & 1.59448500 \\ \mathrm{C} & -4.57352700 & 1.66708100 & 0.62806200 \\ \mathrm{C} & -4.11299400 & 3.72831600 & -0.74080500 \\ \mathrm{C} & 2.32500500 & 0.77676900 & -1.43909100 \\ \mathrm{C} & 3.69191000 & 0.96620500 & -1.62745400 \\ \mathrm{C} & 4.59236000 & 0.56894200 & -0.63245100 \\ \mathrm{C} & 4.10753900 & -0.03792200 & 0.54191700 \\ \mathrm{C} & 2.73321600 & -0.22724300 & 0.71360800 \\ \mathrm{O} & 4.99727200 & -0.45637300 & 1.50052200 \\ \mathrm{C} & 5.11278900 & 0.44694400 & 2.60433600 \\ \mathrm{O} & 2.25159300 & -0.77091000 & 1.87670700 \\ & & & \end{array}$




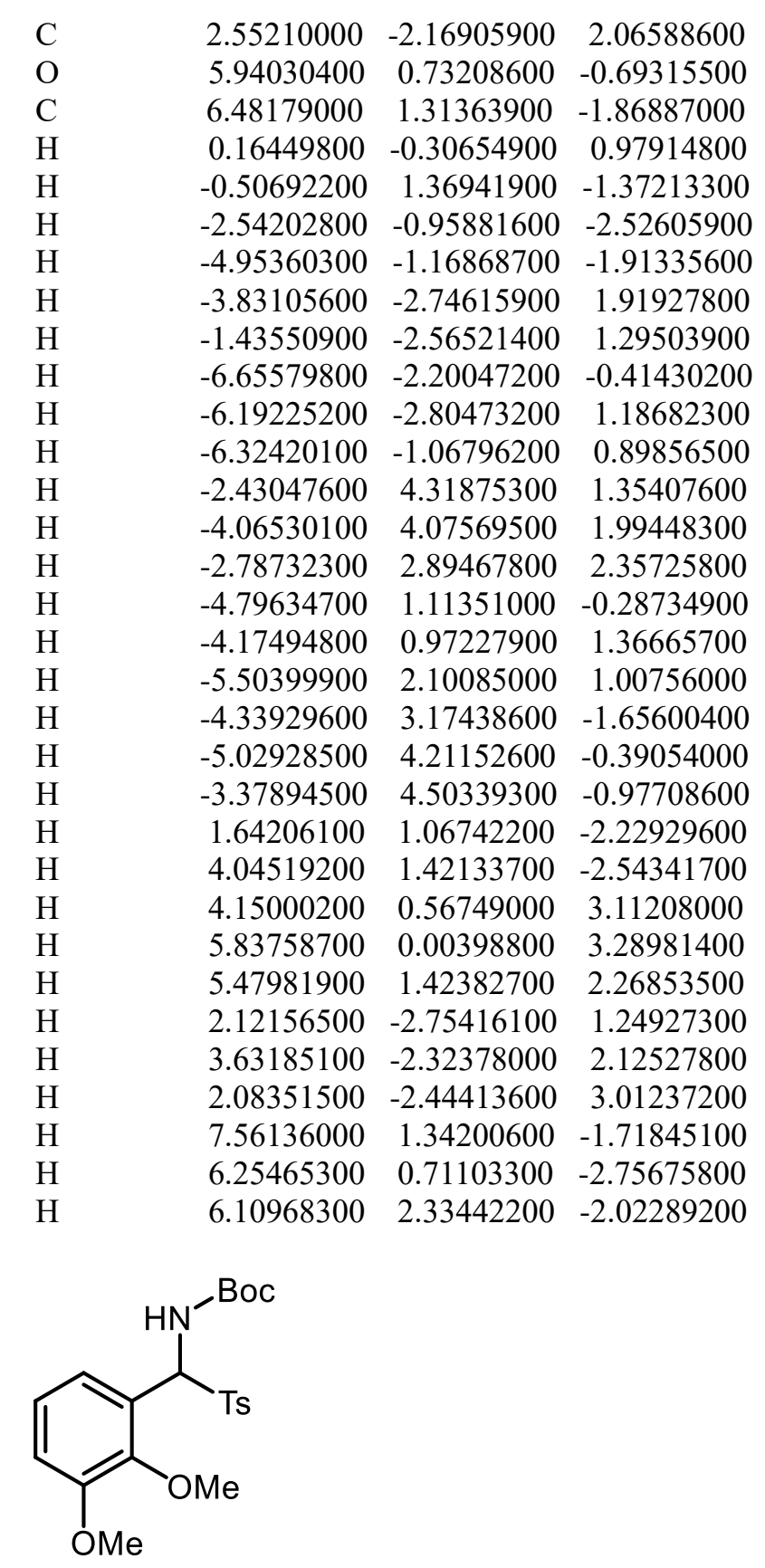

Amino Sulfone-5

$\begin{array}{llrc}\mathrm{C} & -2.20796300 & -0.25297000 & -0.86705900 \\ \mathrm{C} & -0.71018400 & -0.36980400 & -0.79902600 \\ \mathrm{~S} & -0.30506200 & -1.99112700 & 0.09136200 \\ \mathrm{~N} & -0.01958400 & 0.71669100 & -0.19843500 \\ \mathrm{O} & -0.90076200 & -1.94868900 & 1.44257900 \\ \mathrm{O} & -0.63444200 & -3.08328100 & -0.84302400 \\ \mathrm{C} & 1.47045300 & -1.91018700 & 0.29024700 \\ \mathrm{C} & 2.00161400 & -1.40314900 & 1.47513600 \\ \mathrm{C} & 3.38330100 & -1.29889100 & 1.60294100\end{array}$




$\begin{array}{lrrr}\mathrm{C} & 4.23620200 & -1.68422200 & 0.55953800 \\ \mathrm{C} & 3.67273600 & -2.19925700 & -0.61513400 \\ \mathrm{C} & 2.29363800 & -2.31177600 & -0.75965500 \\ \mathrm{C} & 5.72761100 & -1.50975700 & 0.68486500 \\ \mathrm{C} & 1.16695700 & 1.16644700 & -0.71340400 \\ \mathrm{O} & 1.62032100 & 2.18411700 & 0.04678800 \\ \mathrm{O} & 1.70315400 & 0.69875100 & -1.70540500 \\ \mathrm{C} & 2.88297700 & 2.85723600 & -0.28957800 \\ \mathrm{C} & 4.04096200 & 1.86157100 & -0.20276600 \\ \mathrm{C} & 2.99413600 & 3.91648300 & 0.80566900 \\ \mathrm{C} & 2.77732500 & 3.50858300 & -1.66937600 \\ \mathrm{C} & -2.86250300 & -0.86531600 & -1.94627200 \\ \mathrm{C} & -4.24507100 & -0.82422400 & -2.04475700 \\ \mathrm{C} & -5.00157800 & -0.15416500 & -1.08115000 \\ \mathrm{C} & -4.36593700 & 0.46695800 & -0.00531200 \\ \mathrm{C} & -2.96154600 & 0.38987000 & 0.12314100 \\ \mathrm{O} & -5.01088300 & 1.17385900 & 0.96776800 \\ \mathrm{C} & -6.42454900 & 1.26837300 & 0.89217300 \\ \mathrm{O} & -2.33048100 & 1.01625500 & 1.17406900 \\ \mathrm{C} & -2.60089000 & 0.48358700 & 2.48844300 \\ \mathrm{H} & -0.28548600 & -0.54067600 & -1.78936600 \\ \mathrm{H} & -0.46417700 & 1.18724800 & 0.57897400 \\ \mathrm{H} & 1.33446500 & -1.11286000 & 2.27807300 \\ \mathrm{H} & 3.80851100 & -0.91143600 & 2.52453600 \\ \mathrm{H} & 4.32306900 & -2.51114800 & -1.42701100 \\ \mathrm{H} & 1.85552300 & -2.71102600 & -1.66688800 \\ \mathrm{H} & 6.26925000 & -2.23759800 & 0.07490000 \\ \mathrm{H} & 6.02617800 & -0.51016300 & 0.34607100 \\ \mathrm{H} & 6.05817200 & -1.61581400 & 1.72182700 \\ \mathrm{H} & 3.94485600 & 1.08500700 & -0.96097000 \\ \mathrm{H} & 4.98769200 & 2.39138900 & -0.34778300 \\ \mathrm{H} & 4.05509600 & 1.38773400 & 0.78216600 \\ \mathrm{H} & 2.14045800 & 4.59857700 & 0.76881400 \\ \mathrm{H} & 3.01937600 & 3.44636000 & 1.79256400 \\ \mathrm{H} & 3.91093000 & 4.49756500 & 0.67254400 \\ \mathrm{H} & 1.90663600 & 4.16994400 & -1.70829600 \\ \mathrm{H} & 3.67179800 & 4.11005600 & -1.85854400 \\ \mathrm{H} & 2.68547800 & 2.75401600 & -2.44995400 \\ \mathrm{H} & -2.27201900 & -1.38722500 & -2.69056100 \\ \mathrm{H} & -4.74593900 & -1.30555100 & -2.87794600 \\ \mathrm{H} & -6.07885400 & -0.11119300 & -1.17951200 \\ \mathrm{H} & -6.73075600 & 1.86537000 & 1.75161600 \\ \mathrm{H} & -6.74452100 & 1.76933900 & -0.02972400 \\ \mathrm{H} & -6.89852400 & 0.28058700 & 0.94798200 \\ \mathrm{H} & -1.94308100 & 1.03263000 & 3.16437200 \\ \mathrm{H} & & & \end{array}$


<smiles>CC(C)(C)OC(=O)NC([12SH])c1ccccc1F</smiles>

Amino Sulfone-6

$\begin{array}{lrrr}\mathrm{C} & -2.83008200 & 0.30498600 & -0.39455400 \\ \mathrm{C} & -1.36350700 & 0.00609200 & -0.50188000 \\ \mathrm{~S} & -1.10257700 & -1.70741500 & 0.24305700 \\ \mathrm{~N} & -0.48385900 & 0.94839600 & 0.10325100 \\ \mathrm{C} & -3.40688800 & 0.87939300 & 0.73976200 \\ \mathrm{C} & -4.76997600 & 1.10532300 & 0.85463500 \\ \mathrm{C} & -5.60519400 & 0.72102200 & -0.19336000 \\ \mathrm{C} & -5.06750800 & 0.12308700 & -1.33368300 \\ \mathrm{C} & -3.69351300 & -0.08292300 & -1.42659700 \\ \mathrm{O} & -1.51249600 & -1.63842200 & 1.65757800 \\ \mathrm{O} & -1.71566200 & -2.67231700 & -0.68729900 \\ \mathrm{C} & 0.67480600 & -1.90216000 & 0.19796700 \\ \mathrm{C} & 1.41836100 & -1.63141000 & 1.34539100 \\ \mathrm{C} & 2.80406600 & -1.75224100 & 1.29312100 \\ \mathrm{C} & 3.45238200 & -2.12528700 & 0.10808100 \\ \mathrm{C} & 2.67686600 & -2.39716000 & -1.02709800 \\ \mathrm{C} & 1.29082000 & -2.28710900 & -0.99172200 \\ \mathrm{C} & 4.95623000 & -2.19372200 & 0.04490000 \\ \mathrm{C} & 0.70763800 & 1.27900300 & -0.49413500 \\ \mathrm{O} & 1.40884400 & 2.07483400 & 0.33523700 \\ \mathrm{O} & 1.03756400 & 0.90256000 & -1.60651800 \\ \mathrm{C} & 2.71775000 & 2.60977700 & -0.07527400 \\ \mathrm{C} & 3.69998600 & 1.45894500 & -0.29617300 \\ \mathrm{C} & 3.12488800 & 3.44841800 & 1.13463200 \\ \mathrm{C} & 2.55549100 & 3.48582900 & -1.31805000 \\ \mathrm{~F} & -2.61409800 & 1.25108500 & 1.77705700 \\ \mathrm{H} & -1.05928100 & -0.11449300 & -1.54216000 \\ \mathrm{H} & -0.67207200 & 1.26621800 & 1.04159200 \\ \mathrm{H} & -5.15297700 & 1.56364200 & 1.75900000 \\ \mathrm{H} & -6.67456100 & 0.88689400 & -0.11396100 \\ \mathrm{H} & -5.71538400 & -0.18385100 & -2.14745200 \\ \mathrm{H} & -3.27094500 & -0.56119300 & -2.30368300 \\ \mathrm{H} & 0.90707600 & -1.34929500 & 2.25838800 \\ \mathrm{H} & 3.39297100 & -1.55283700 & 2.18392300 \\ \mathrm{H} & 3.16646200 & -2.69715600 & -1.94882100 \\ \mathrm{H} & 0.69001700 & -2.50463200 & -1.86716500 \\ \mathrm{H} & 5.29358600 & -2.93226700 & -0.68721600 \\ \mathrm{H} & 5.37176000 & -1.22322000 & -0.25277400 \\ \mathrm{H} & 5.38711700 & -2.45038000 & 1.01645600 \\ \mathrm{H} & 3.38577000 & 0.83218800 & -1.13029800 \\ \mathrm{H} & 4.69398800 & 1.86572600 & -0.50668400 \\ \mathrm{H} & 3.76064500 & 0.83986900 & 0.60229400 \\ \mathrm{H} & 2.39657600 & 4.24368100 & 1.31473000 \\ \mathrm{H} & 3.18596800 & 2.82480700 & 2.03065400\end{array}$




$$
\begin{array}{lrrr}
\mathrm{H} & 4.10316100 & 3.90482100 & 0.96023700 \\
\mathrm{H} & 1.80274200 & 4.25929400 & -1.13896900 \\
\mathrm{H} & 3.50584300 & 3.97999000 & -1.54176100 \\
\mathrm{H} & 2.25496100 & 2.88987300 & -2.17930400
\end{array}
$$<smiles>COc1cc(OC)cc(C([18F])NC(=O)OCc2ccccc2)c1</smiles>

Amino Sulfone-7

$\begin{array}{lrrr}\mathrm{C} & -2.19691700 & 0.04511500 & -0.18521800 \\ \mathrm{C} & -0.71130900 & -0.17392100 & -0.29803900 \\ \mathrm{~S} & -0.26354900 & -1.84317100 & 0.42311200 \\ \mathrm{~N} & 0.09356300 & 0.82354500 & 0.33755500 \\ \mathrm{O} & -0.45993000 & -1.75445500 & 1.88646500 \\ \mathrm{O} & -0.93268500 & -2.88031100 & -0.37768000 \\ \mathrm{C} & 1.50155700 & -1.92134300 & 0.13551300 \\ \mathrm{C} & 1.97445500 & -2.21982000 & -1.14113700 \\ \mathrm{C} & 3.34666900 & -2.21676400 & -1.36932300 \\ \mathrm{C} & 4.24949700 & -1.92274700 & -0.33883200 \\ \mathrm{C} & 3.74535600 & -1.64829200 & 0.93951500 \\ \mathrm{C} & 2.37530500 & -1.63579100 & 1.18307600 \\ \mathrm{C} & 5.73121900 & -1.86341400 & -0.60573900 \\ \mathrm{C} & 1.22983100 & 1.30533100 & -0.26703000 \\ \mathrm{O} & 1.89631300 & 2.08680800 & 0.60440700 \\ \mathrm{O} & 1.54368900 & 1.05501100 & -1.41853800 \\ \mathrm{C} & 3.12100000 & 2.79030800 & 0.18642500 \\ \mathrm{C} & 2.80028300 & 3.75542000 & -0.95548200 \\ \mathrm{C} & 4.20003000 & 1.77547000 & -0.19377100 \\ \mathrm{C} & 3.51151600 & 3.55157600 & 1.45166000 \\ \mathrm{C} & -2.80370100 & 0.11663000 & 1.07184700 \\ \mathrm{C} & -4.18563300 & 0.32924400 & 1.14396600 \\ \mathrm{C} & -4.94120500 & 0.47839200 & -0.02017100 \\ \mathrm{C} & -4.32205700 & 0.39861100 & -1.26721700 \\ \mathrm{C} & -2.94096700 & 0.17681900 & -1.35830100 \\ \mathrm{O} & -5.14415800 & 0.54903600 & -2.34394900 \\ \mathrm{O} & -4.88211100 & 0.39955000 & 2.31228200 \\ \mathrm{C} & -4.57252300 & 0.43885400 & -3.63794400 \\ \mathrm{C} & -4.17022300 & 0.17630900 & 3.52108100 \\ \mathrm{H} & -0.39481400 & -0.23007600 & -1.33972800 \\ \mathrm{H} & -0.05705000 & 1.03394100 & 1.31294600 \\ \mathrm{H} & 1.27742500 & -2.45686000 & -1.93707900 \\ \mathrm{H} & 3.72482400 & -2.44388600 & -2.36158200 \\ \mathrm{H} & 4.43560500 & -1.43653800 & 1.75129100 \\ \mathrm{H} & 1.97477900 & -1.42157700 & 2.16688100 \\ \mathrm{H} & 6.30840100 & -2.18761800 & 0.26473000 \\ \mathrm{H} & 6.01141600 & -2.48931700 & -1.45694300 \\ & & & \\ & & & \end{array}$




\begin{tabular}{|c|c|c|c|}
\hline $\mathrm{H}$ & 6.03750800 & -0.83594700 & -0.83735100 \\
\hline $\mathrm{H}$ & 1.98701300 & 4.42713600 & -0.66516400 \\
\hline $\mathrm{H}$ & 3.68255600 & 4.36352000 & -1.17720900 \\
\hline $\mathrm{H}$ & 2.50963100 & 3.21296400 & -1.85473900 \\
\hline $\mathrm{H}$ & 4.37455800 & 1.08355800 & 0.63383800 \\
\hline $\mathrm{H}$ & 3.90398900 & 1.20131700 & -1.07122100 \\
\hline $\mathrm{H}$ & 5.13433500 & 2.30372700 & -0.40788800 \\
\hline $\mathrm{H}$ & 3.68679400 & 2.85774700 & 2.27837400 \\
\hline $\mathrm{H}$ & 4.42748100 & 4.12240700 & 1.27604700 \\
\hline $\mathrm{H}$ & 2.71907800 & 4.24597800 & 1.74354500 \\
\hline $\mathrm{H}$ & -2.21276300 & -0.04325100 & 1.96212700 \\
\hline $\mathrm{H}$ & -6.00981900 & 0.64275800 & 0.04433000 \\
\hline $\mathrm{H}$ & -2.44315100 & 0.10872200 & -2.31632700 \\
\hline $\mathrm{H}$ & -5.39472600 & 0.57242800 & -4.34172800 \\
\hline $\mathrm{H}$ & -3.81706200 & 1.21512800 & -3.81342200 \\
\hline $\mathrm{H}$ & -4.11743100 & -0.54688800 & -3.79479400 \\
\hline $\mathrm{H}$ & -4.91140300 & 0.22840800 & 4.31919800 \\
\hline $\mathrm{H}$ & -3.69087500 & -0.80969100 & 3.52926100 \\
\hline $\mathrm{H}$ & -3.40650400 & 0.94678800 & 3.68675900 \\
\hline
\end{tabular}

Amino Sulfone-8

$\begin{array}{lrrr}\mathrm{C} & -1.82551100 & -0.01661400 & -0.27376400 \\ \mathrm{C} & -0.33179800 & -0.18413600 & -0.36290800 \\ \mathrm{~S} & 0.14079800 & -1.79377500 & 0.48325800 \\ \mathrm{~N} & 0.44291500 & 0.85480200 & 0.23806300 \\ \mathrm{C} & -2.65516700 & -0.86004200 & -1.02421300 \\ \mathrm{C} & -4.03981400 & -0.74836300 & -0.94431700 \\ \mathrm{C} & -4.59477300 & 0.21665000 & -0.10665700 \\ \mathrm{C} & -3.79064200 & 1.06577500 & 0.64787300 \\ \mathrm{C} & -2.40571300 & 0.94059800 & 0.56219300 \\ \mathrm{O} & -0.12269600 & -1.59185200 & 1.92122900 \\ \mathrm{O} & -0.46671100 & -2.90957600 & -0.26201600 \\ \mathrm{C} & 1.91508000 & -1.84505300 & 0.25615000 \\ \mathrm{C} & 2.74847200 & -1.53270300 & 1.32831400 \\ \mathrm{C} & 4.12724200 & -1.55222900 & 1.13695100 \\ \mathrm{C} & 4.67891300 & -1.85868300 & -0.11405200 \\ \mathrm{C} & 3.81481600 & -2.17220400 & -1.17228000 \\ \mathrm{C} & 2.43537600 & -2.17096100 & -0.99588700 \\ \mathrm{C} & 6.16952800 & -1.81240500 & -0.32779700 \\ \mathrm{C} & 1.57457000 & 1.32851000 & -0.39405300 \\ \mathrm{O} & 2.27569500 & 2.08137800 & 0.47146800 \\ \mathrm{O} & 1.84684400 & 1.09542500 & -1.55829600 \\ \mathrm{C} & 3.49380000 & 2.78683300 & 0.03169900 \\ \mathrm{C} & 3.14476200 & 3.77626400 & -1.08024500 \\ \mathrm{C} & 4.55287800 & 1.77222100 & -0.39968700\end{array}$




$\begin{array}{lrrr}\mathrm{C} & 3.92543400 & 3.51855900 & 1.30071000 \\ \mathrm{Br} & -6.49347900 & 0.38305700 & 0.00234400 \\ \mathrm{H} & 0.00557300 & -0.29588200 & -1.39494200 \\ \mathrm{H} & 0.40365100 & 0.95462500 & 1.24367800 \\ \mathrm{H} & -2.21511400 & -1.61963500 & -1.65995200 \\ \mathrm{H} & -4.68090100 & -1.40038400 & -1.52514200 \\ \mathrm{H} & -4.23939800 & 1.81290100 & 1.29135900 \\ \mathrm{H} & -1.77933700 & 1.61317000 & 1.13619300 \\ \mathrm{H} & 2.31180300 & -1.29689000 & 2.29183800 \\ \mathrm{H} & 4.78626800 & -1.32189600 & 1.96919800 \\ \mathrm{H} & 4.22943800 & -2.42142400 & -2.14440600 \\ \mathrm{H} & 1.77042000 & -2.42866200 & -1.81246400 \\ \mathrm{H} & 6.48463800 & -2.51039400 & -1.10789800 \\ \mathrm{H} & 6.48027500 & -0.80815300 & -0.64095000 \\ \mathrm{H} & 6.71359600 & -2.05255000 & 0.58960700 \\ \mathrm{H} & 2.82552800 & 3.25469000 & -1.98221000 \\ \mathrm{H} & 2.34428300 & 4.44622400 & -0.75284500 \\ \mathrm{H} & 4.02375800 & 4.38414700 & -1.31476100 \\ \mathrm{H} & 4.74936800 & 1.06685800 & 0.41110000 \\ \mathrm{H} & 4.22577300 & 1.21421500 & -1.27657400 \\ \mathrm{H} & 5.48264500 & 2.29874400 & -0.63624300 \\ \mathrm{H} & 4.11805800 & 2.80630800 & 2.10763000 \\ \mathrm{H} & 4.84088700 & 4.08553500 & 1.11086400 \\ \mathrm{H} & 3.14761700 & 4.21313500 & 1.62904700\end{array}$<smiles>Cc1ccc(C(NC(=O)OC(C)(C)C)C(=S)OC(C)(C)C)cc1</smiles>

Amino Sulfone-9

$\begin{array}{lrrr}\mathrm{C} & -2.67189000 & 0.19781500 & -0.27463400 \\ \mathrm{C} & -1.19207700 & -0.05431500 & -0.36690400 \\ \mathrm{~S} & -0.81654500 & -1.68855800 & 0.48170600 \\ \mathrm{~N} & -0.35293400 & 0.93573700 & 0.23113900 \\ \mathrm{C} & -3.54360200 & -0.54974000 & -1.07706500 \\ \mathrm{C} & -4.91816500 & -0.36587700 & -0.98313400 \\ \mathrm{C} & -5.46467800 & 0.56646800 & -0.09125100 \\ \mathrm{C} & -4.58677600 & 1.30570000 & 0.70744600 \\ \mathrm{C} & -3.20810900 & 1.12323100 & 0.62260500 \\ \mathrm{O} & -1.06504300 & -1.47173800 & 1.92076000 \\ \mathrm{O} & -1.48822200 & -2.76869700 & -0.26102600 \\ \mathrm{C} & 0.95284500 & -1.84950800 & 0.25697600 \\ \mathrm{C} & 1.45385300 & -2.21253600 & -0.99254300 \\ \mathrm{C} & 2.83095100 & -2.29746300 & -1.16791700 \\ \mathrm{C} & 3.71169800 & -2.03006300 & -0.11089900 \\ \mathrm{C} & 3.17855900 & -1.68461400 & 1.13789000 \\ \mathrm{C} & 1.80322600 & -1.58195000 & 1.32777700 \\ \mathrm{C} & 5.20266100 & -2.07275700 & -0.32377700 \\ \mathrm{C} & 0.80546000 & 1.33811300 & -0.39741700\end{array}$




\begin{tabular}{|c|c|c|c|}
\hline $\mathrm{O}$ & 1.54625200 & 2.05574100 & 0.46755700 \\
\hline $\mathrm{O}$ & 1.07185200 & 1.08319700 & -1.55878800 \\
\hline $\mathrm{C}$ & 2.80546400 & 2.68260100 & 0.02941400 \\
\hline $\mathrm{C}$ & 2.52291400 & 3.68365400 & -1.09125100 \\
\hline $\mathrm{C}$ & 3.80436300 & 1.60347800 & -0.38953100 \\
\hline $\mathrm{C}$ & 3.27511500 & 3.39659000 & 1.29523000 \\
\hline $\mathrm{C}$ & -6.95561500 & 0.77938800 & -0.01616800 \\
\hline $\mathrm{H}$ & -0.86308700 & -0.19005900 & -1.39851900 \\
\hline $\mathrm{H}$ & -0.39746600 & 1.05514800 & 1.23393200 \\
\hline $\mathrm{H}$ & -3.14011100 & -1.28950800 & -1.75967300 \\
\hline $\mathrm{H}$ & -5.57959000 & -0.95587800 & -1.61156800 \\
\hline $\mathrm{H}$ & -4.98648600 & 2.03295300 & 1.40859900 \\
\hline $\mathrm{H}$ & -2.55288300 & 1.71975500 & 1.24717200 \\
\hline $\mathrm{H}$ & 0.77473800 & -2.43390000 & -1.80813700 \\
\hline $\mathrm{H}$ & 3.23054100 & -2.57604600 & -2.13844100 \\
\hline $\mathrm{H}$ & 3.84983100 & -1.48921500 & 1.96938800 \\
\hline $\mathrm{H}$ & 1.38047700 & -1.31505200 & 2.28940900 \\
\hline $\mathrm{H}$ & 5.73115600 & -2.34199900 & 0.59473600 \\
\hline $\mathrm{H}$ & 5.47641800 & -2.79062500 & -1.10151900 \\
\hline $\mathrm{H}$ & 5.57280900 & -1.08968800 & -0.63966200 \\
\hline $\mathrm{H}$ & 2.17688100 & 3.17547100 & -1.99093600 \\
\hline $\mathrm{H}$ & 1.76249300 & 4.40281100 & -0.77272400 \\
\hline $\mathrm{H}$ & 3.43815000 & 4.23578800 & -1.32532600 \\
\hline $\mathrm{H}$ & 3.95435600 & 0.89345600 & 0.42715800 \\
\hline $\mathrm{H}$ & 3.44786600 & 1.05935900 & -1.26367000 \\
\hline $\mathrm{H}$ & 4.76515900 & 2.07156200 & -0.62527100 \\
\hline $\mathrm{H}$ & 3.42059900 & 2.67994600 & 2.10813400 \\
\hline $\mathrm{H}$ & 4.22398200 & 3.90601800 & 1.10600800 \\
\hline $\mathrm{H}$ & 2.53892300 & 4.13903100 & 1.61480000 \\
\hline $\mathrm{H}$ & -7.31021300 & 1.38910100 & -0.85604100 \\
\hline $\mathrm{H}$ & -7.49562600 & -0.17157100 & -0.05662200 \\
\hline $\mathrm{H}$ & -7.23955300 & 1.29257500 & 0.90640400 \\
\hline
\end{tabular}

Amino Sulfone-10

$\begin{array}{lrrr}\mathrm{C} & -2.19494700 & 0.03558500 & -0.26743600 \\ \mathrm{C} & -0.70292700 & -0.15545900 & -0.35254100 \\ \mathrm{~S} & -0.25787900 & -1.76292700 & 0.51456400 \\ \mathrm{~N} & 0.08609900 & 0.87992600 & 0.23379200 \\ \mathrm{O} & -0.51971400 & -1.53205300 & 1.94795600 \\ \mathrm{O} & -0.88760200 & -2.87581200 & -0.21546500 \\ \mathrm{C} & 1.51336900 & -1.84417300 & 0.28490700 \\ \mathrm{C} & 2.35319700 & -1.52971900 & 1.35154900 \\ \mathrm{C} & 3.73099800 & -1.57523200 & 1.15804100 \\ \mathrm{C} & 4.27511200 & -1.90970000 & -0.08916300 \\ \mathrm{C} & 3.40400500 & -2.22433700 & -1.14155800\end{array}$




\begin{tabular}{|c|c|c|c|}
\hline $\mathrm{C}$ & 2.02523800 & -2.19739100 & -0.96337800 \\
\hline $\mathrm{C}$ & 5.76574400 & -1.89267000 & -0.30598600 \\
\hline $\mathrm{C}$ & 1.22319100 & 1.32740100 & -0.41200800 \\
\hline $\mathrm{O}$ & 1.94220400 & 2.07649400 & 0.43924900 \\
\hline $\mathrm{O}$ & 1.48039700 & 1.07492100 & -1.57537800 \\
\hline $\mathrm{C}$ & 3.17072200 & 2.75679700 & -0.01602000 \\
\hline $\mathrm{C}$ & 2.83054700 & 3.73958700 & -1.13626500 \\
\hline $\mathrm{C}$ & 4.20822100 & 1.71859500 & -0.44295500 \\
\hline $\mathrm{C}$ & 3.62252200 & 3.49400600 & 1.24251100 \\
\hline $\mathrm{C}$ & -2.75509800 & 1.03140300 & 0.53962600 \\
\hline $\mathrm{C}$ & -4.13521900 & 1.18133600 & 0.62214800 \\
\hline $\mathrm{C}$ & -4.94452000 & 0.31753600 & -0.10866400 \\
\hline $\mathrm{C}$ & -4.41583300 & -0.68504200 & -0.91762200 \\
\hline $\mathrm{C}$ & -3.03542000 & -0.82102200 & -0.99396700 \\
\hline $\mathrm{H}$ & -0.37006400 & -0.28420700 & -1.38427500 \\
\hline $\mathrm{H}$ & 0.06023000 & 0.98421100 & 1.23965700 \\
\hline $\mathrm{H}$ & 1.92278900 & -1.27284200 & 2.31248700 \\
\hline $\mathrm{H}$ & 4.39520500 & -1.34393200 & 1.98576900 \\
\hline $\mathrm{H}$ & 3.81265400 & -2.49525400 & -2.11032500 \\
\hline $\mathrm{H}$ & 1.35484800 & -2.45641600 & -1.77508100 \\
\hline $\mathrm{H}$ & 6.09150000 & -0.90168400 & -0.64498500 \\
\hline $\mathrm{H}$ & 6.30750700 & -2.11820900 & 0.61636000 \\
\hline $\mathrm{H}$ & 6.06817800 & -2.61490800 & -1.06879200 \\
\hline $\mathrm{H}$ & 2.04387000 & 4.42669200 & -0.81107300 \\
\hline $\mathrm{H}$ & 3.71806800 & 4.32988900 & -1.38295200 \\
\hline $\mathrm{H}$ & 2.49699500 & 3.21414800 & -2.03081200 \\
\hline $\mathrm{H}$ & 4.39768000 & 1.01878500 & 0.37430300 \\
\hline $\mathrm{H}$ & 3.86608300 & 1.15723600 & -1.31198200 \\
\hline $\mathrm{H}$ & 5.14551300 & 2.22596200 & -0.69103100 \\
\hline $\mathrm{H}$ & 3.80836500 & 2.78718500 & 2.05575000 \\
\hline $\mathrm{H}$ & 4.54621800 & 4.04311900 & 1.04078900 \\
\hline $\mathrm{H}$ & 2.85898800 & 4.20539200 & 1.56823900 \\
\hline $\mathrm{H}$ & -2.11283100 & 1.70886300 & 1.08847400 \\
\hline $\mathrm{H}$ & -4.59051500 & 1.94766500 & 1.23536600 \\
\hline $\mathrm{H}$ & -5.08364100 & -1.33337400 & -1.46914900 \\
\hline $\mathrm{H}$ & -2.60404700 & -1.60532500 & -1.60404300 \\
\hline $\mathrm{N}$ & -6.40425900 & 0.47356900 & -0.02907900 \\
\hline $\mathrm{O}$ & -7.09768100 & -0.29718400 & -0.69156000 \\
\hline $\mathrm{O}$ & -6.84433300 & 1.36668200 & 0.69444400 \\
\hline
\end{tabular}

Amino Sulfone-11
$\begin{array}{llll}\text { C } & -1.90898600 & 0.42805600 & -0.10462600\end{array}$
$\begin{array}{lllll}\text { C } & & -0.47570800 & 0.02879000 & -0.25773200\end{array}$
$\begin{array}{llll}\mathrm{S} & -0.19780800 & -1.61813900 & 0.60109300\end{array}$
$\begin{array}{llll}\mathrm{N} & 0.44281100 & 0.97543500 & 0.29168100\end{array}$
$\begin{array}{llll}\mathrm{O} & -0.35968600 & -1.36825500 & 2.04503000\end{array}$ 


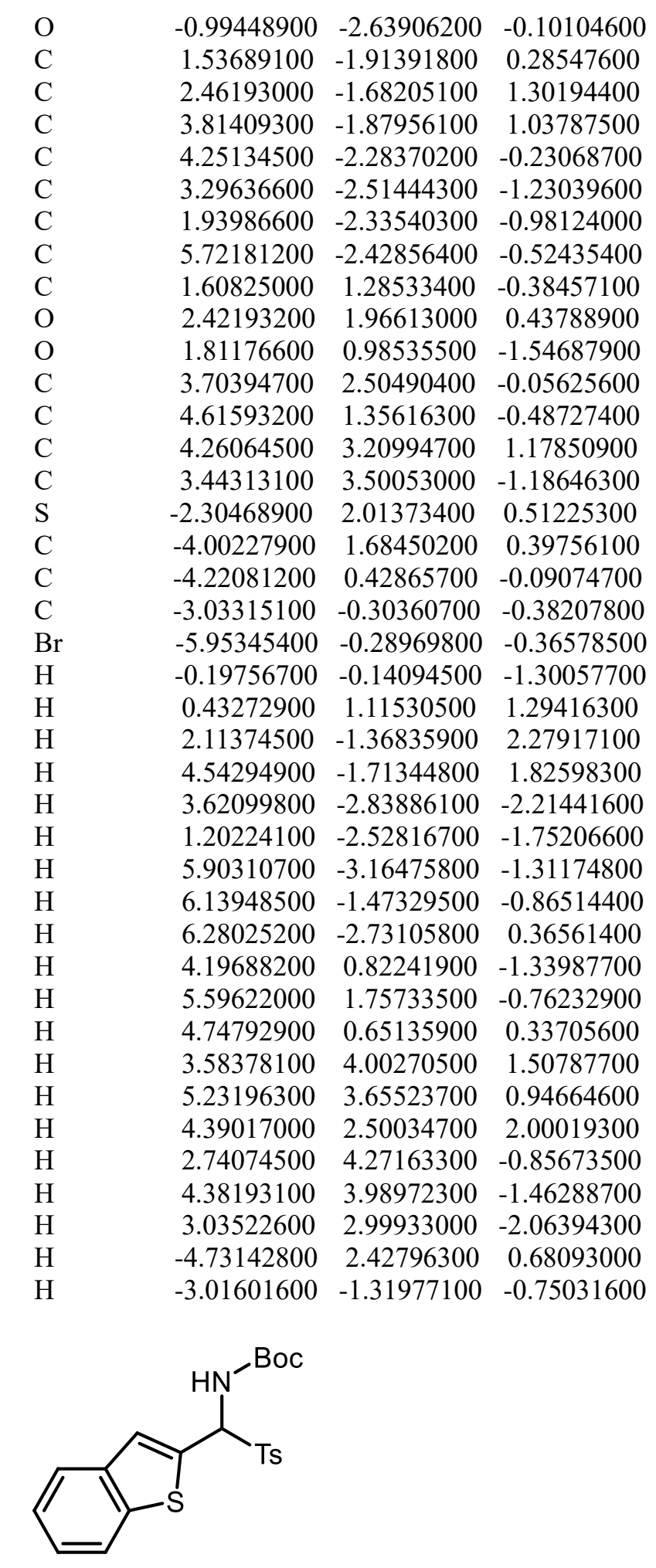

Amino Sulfone-12

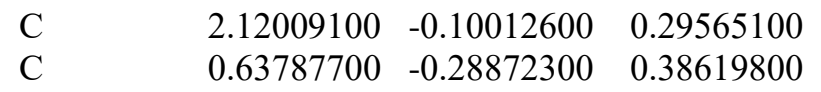




\begin{tabular}{|c|c|c|c|}
\hline $\mathrm{S}$ & 0.15979800 & -1.89119600 & -0.47185400 \\
\hline $\mathrm{N}$ & -0.11358400 & 0.77080400 & -0.20789600 \\
\hline $\mathrm{O}$ & 0.43783900 & -1.70145000 & -1.90702900 \\
\hline $\mathrm{O}$ & 0.74475200 & -3.01189800 & 0.28562500 \\
\hline $\mathrm{C}$ & -1.61563400 & -1.91276000 & -0.25575800 \\
\hline $\mathrm{C}$ & -2.43502000 & -1.55223100 & -1.32398500 \\
\hline $\mathrm{C}$ & -3.81426900 & -1.53661900 & -1.13800600 \\
\hline $\mathrm{C}$ & -4.37973500 & -1.85591200 & 0.10379100 \\
\hline $\mathrm{C}$ & -3.52984900 & -2.22026200 & 1.15701100 \\
\hline $\mathrm{C}$ & -2.15000100 & -2.25380600 & 0.98612800 \\
\hline $\mathrm{C}$ & -5.86924900 & -1.76999400 & 0.31265300 \\
\hline $\mathrm{C}$ & -1.25066500 & 1.25348400 & 0.40856400 \\
\hline $\mathrm{O}$ & -1.90689900 & 2.05251500 & -0.44894600 \\
\hline $\mathrm{O}$ & -1.56088200 & 0.98571000 & 1.55542500 \\
\hline $\mathrm{C}$ & -3.11171000 & 2.78427900 & -0.01504900 \\
\hline $\mathrm{C}$ & -4.21370200 & 1.79264300 & 0.35863600 \\
\hline $\mathrm{C}$ & -3.48752100 & 3.57005500 & -1.26936400 \\
\hline $\mathrm{C}$ & -2.75700200 & 3.72535000 & 1.13633100 \\
\hline $\mathrm{S}$ & 2.74860800 & 1.44948000 & -0.26624100 \\
\hline $\mathrm{C}$ & 4.39510400 & 0.86699300 & -0.09593100 \\
\hline $\mathrm{C}$ & 4.42377600 & -0.46712800 & 0.38206600 \\
\hline $\mathrm{C}$ & 3.10312900 & -0.99221500 & 0.59702900 \\
\hline $\mathrm{C}$ & 5.56910100 & 1.57405500 & -0.36753200 \\
\hline $\mathrm{C}$ & 6.78585100 & 0.93412700 & -0.16264200 \\
\hline $\mathrm{C}$ & 6.83465300 & -0.39017300 & 0.30835700 \\
\hline $\mathrm{C}$ & 5.66859500 & -1.09007000 & 0.58056600 \\
\hline $\mathrm{H}$ & 0.29200800 & -0.40447700 & 1.41625700 \\
\hline $\mathrm{H}$ & -0.01122100 & 0.92928500 & -1.20185900 \\
\hline $\mathrm{H}$ & -1.98776700 & -1.30578600 & -2.27992200 \\
\hline $\mathrm{H}$ & -4.46296800 & -1.26808500 & -1.96691200 \\
\hline $\mathrm{H}$ & -3.95576700 & -2.48056400 & 2.12129800 \\
\hline $\mathrm{H}$ & -1.49538000 & -2.54827100 & 1.79856900 \\
\hline $\mathrm{H}$ & -6.20603200 & -2.46137700 & 1.08949400 \\
\hline $\mathrm{H}$ & -6.15353300 & -0.75841600 & 0.62734100 \\
\hline $\mathrm{H}$ & -6.41646100 & -1.99238400 & -0.60744100 \\
\hline $\mathrm{H}$ & -3.92740700 & 1.19835900 & 1.22594300 \\
\hline $\mathrm{H}$ & -5.13176900 & 2.34242700 & 0.58785300 \\
\hline $\mathrm{H}$ & -4.41217500 & 1.11859500 & -0.47802800 \\
\hline $\mathrm{H}$ & -2.67878500 & 4.24715400 & -1.55675400 \\
\hline $\mathrm{H}$ & -3.68430000 & 2.89079500 & -2.10326400 \\
\hline $\mathrm{H}$ & -4.38770400 & 4.16191100 & -1.08240100 \\
\hline $\mathrm{H}$ & -1.92659100 & 4.37719800 & 0.84967800 \\
\hline $\mathrm{H}$ & -3.62067400 & 4.35520200 & 1.36981800 \\
\hline $\mathrm{H}$ & -2.47770200 & 3.16491900 & 2.02821300 \\
\hline $\mathrm{H}$ & 2.89717600 & -1.99872900 & 0.93798900 \\
\hline $\mathrm{H}$ & 5.53266000 & 2.59554100 & -0.73181900 \\
\hline $\mathrm{H}$ & 7.70975900 & 1.46452400 & -0.37049800 \\
\hline $\mathrm{H}$ & 7.79735000 & -0.86812400 & 0.45928400 \\
\hline $\mathrm{H}$ & 5.70650000 & -2.11269300 & 0.94308100 \\
\hline
\end{tabular}


<smiles>CC(C)(C)OC(=O)NC([15F])c1cccs1</smiles>

Amino Sulfone-13

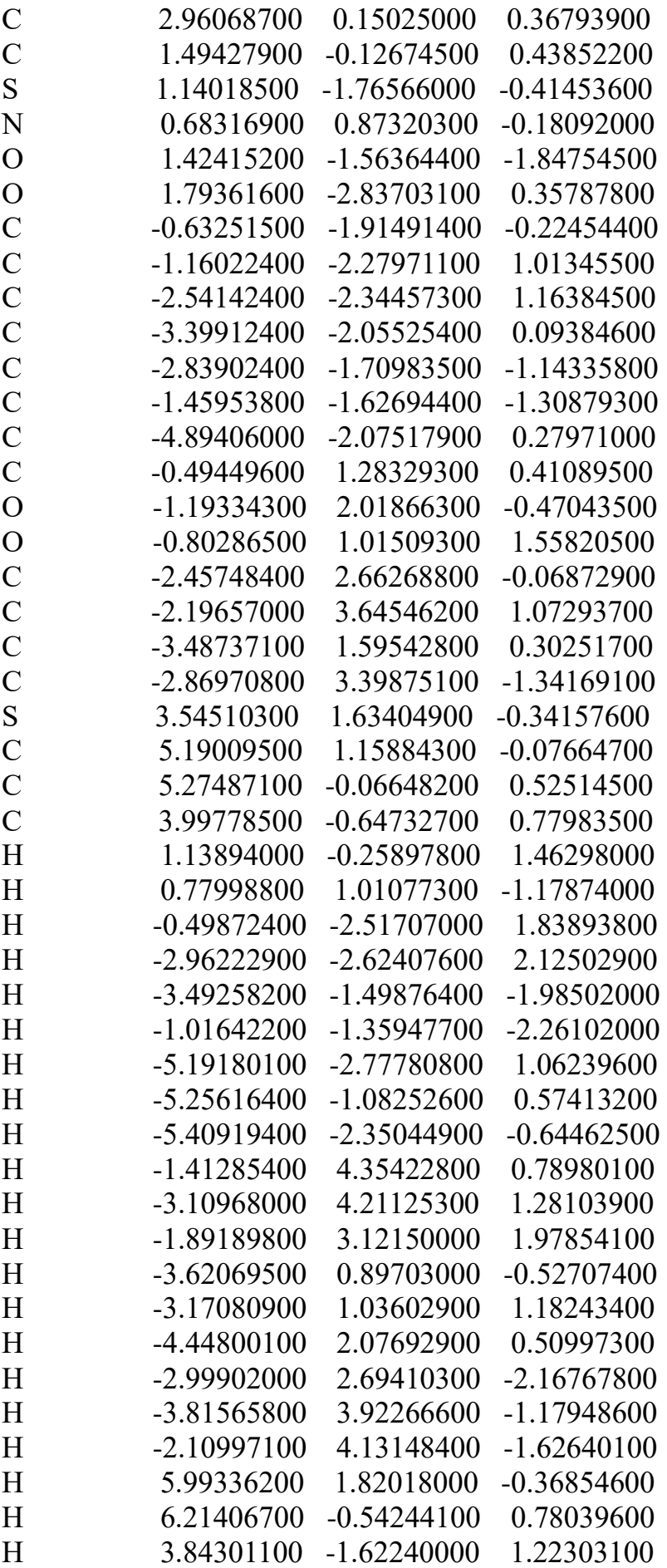


<smiles>C=CC(=O)c1ccccc1</smiles>

Enone-1

$\begin{array}{lrrr}\mathrm{C} & -2.09675500 & -1.37491400 & -0.07702800 \\ \mathrm{C} & -0.71484200 & -1.19134200 & -0.08059900 \\ \mathrm{C} & -0.17072700 & 0.09894000 & -0.00388300 \\ \mathrm{C} & -1.03780700 & 1.20029200 & 0.07187900 \\ \mathrm{C} & -2.41515500 & 1.01623400 & 0.08435900 \\ \mathrm{C} & -2.94826200 & -0.27378100 & 0.01039100 \\ \mathrm{C} & 1.30022100 & 0.37705300 & -0.01943300 \\ \mathrm{C} & 2.25759200 & -0.75824300 & 0.13525000 \\ \mathrm{O} & 1.71050000 & 1.52616600 & -0.14682800 \\ \mathrm{C} & 3.57281200 & -0.55353000 & 0.02562300 \\ \mathrm{H} & -2.50781500 & -2.37736600 & -0.14340200 \\ \mathrm{H} & -0.07071300 & -2.05896400 & -0.16372000 \\ \mathrm{H} & -0.59861700 & 2.19028600 & 0.11990000 \\ \mathrm{H} & -3.07651000 & 1.87461800 & 0.14987800 \\ \mathrm{H} & -4.02425900 & -0.41907300 & 0.01844900 \\ \mathrm{H} & 1.87306100 & -1.74776400 & 0.35626400 \\ \mathrm{H} & 4.29059000 & -1.35839100 & 0.14416500 \\ \mathrm{H} & 3.94779500 & 0.44307300 & -0.18627000\end{array}$<smiles>C=CC(=O)c1cccs1</smiles>

Enone-2

$\begin{array}{lrrr}\mathrm{C} & 0.00000000 & 0.23096700 & 0.00000000 \\ \mathrm{C} & 0.40949500 & -1.18041900 & 0.00000000 \\ \mathrm{C} & 1.87031600 & -1.47835100 & 0.00000000 \\ \mathrm{O} & -0.43352700 & -2.07469400 & 0.00000000 \\ \mathrm{C} & 2.30290900 & -2.74141900 & 0.00000000 \\ \mathrm{~S} & -1.71033400 & 0.58718200 & 0.00000000 \\ \mathrm{C} & -1.39193400 & 2.28332700 & 0.00000000 \\ \mathrm{C} & -0.04809900 & 2.56634700 & 0.00000000 \\ \mathrm{C} & 0.74825800 & 1.39126900 & 0.00000000 \\ \mathrm{H} & 2.57102900 & -0.64968300 & 0.00000000 \\ \mathrm{H} & 3.35975200 & -2.98603500 & 0.00000000 \\ \mathrm{H} & 1.58559600 & -3.55632000 & 0.00000000 \\ \mathrm{H} & -2.21300500 & 2.98708300 & 0.00000000 \\ \mathrm{H} & 0.35356900 & 3.57241800 & 0.00000000 \\ \mathrm{H} & 1.83095800 & 1.40485000 & 0.00000000\end{array}$


<smiles>C=CC(=O)c1ccc(C)cc1</smiles>

Enone-3

$\begin{array}{rrrr}\mathrm{C} & 1.88934200 & 1.14025900 & 0.05867700 \\ \mathrm{C} & 0.50866400 & 1.26937900 & 0.05484200 \\ \mathrm{C} & -0.31848700 & 0.13577500 & -0.00520900 \\ \mathrm{C} & 0.28368300 & -1.12769700 & -0.07394300 \\ \mathrm{C} & 1.67143300 & -1.25084900 & -0.07732700 \\ \mathrm{C} & 2.49671300 & -0.12396400 & -0.00454700 \\ \mathrm{C} & -1.79660800 & 0.35456100 & -0.01354100 \\ \mathrm{C} & -2.70811600 & -0.82144000 & 0.11707000 \\ \mathrm{O} & -2.25490300 & 1.48862500 & -0.11637500 \\ \mathrm{C} & -4.03125000 & -0.66509700 & 0.02566100 \\ \mathrm{C} & 3.99755300 & -0.25685100 & 0.02275800 \\ \mathrm{H} & 2.51344000 & 2.02858800 & 0.10862100 \\ \mathrm{H} & 0.03373000 & 2.24315100 & 0.09551800 \\ \mathrm{H} & -0.31852500 & -2.02594100 & -0.14627800 \\ \mathrm{H} & 2.12126200 & -2.23777900 & -0.13924700 \\ \mathrm{H} & -2.28404300 & -1.80186800 & 0.30359500 \\ \mathrm{H} & -4.71620200 & -1.50049400 & 0.12609500 \\ \mathrm{H} & -4.44630500 & 0.32218400 & -0.15247100 \\ \mathrm{H} & 4.31779100 & -1.25361700 & -0.29107900 \\ \mathrm{H} & 4.47346700 & 0.47815400 & -0.63391900 \\ \mathrm{H} & 4.38704600 & -0.08584500 & 1.03351700\end{array}$<smiles>C=CC(=O)c1ccco1</smiles>

Enone-4

\begin{tabular}{|c|c|c|c|}
\hline $\mathrm{C}$ & -0.44964300 & 0.20581900 & 0.00008200 \\
\hline $\mathrm{C}$ & 0.99803100 & 0.43599300 & 0.00020000 \\
\hline $\mathrm{C}$ & 1.88420200 & -0.76098500 & 0.00003800 \\
\hline $\mathrm{O}$ & 1.42989500 & 1.58606300 & -0.00004400 \\
\hline $\mathrm{C}$ & 3.21174900 & -0.61666200 & -0.00010500 \\
\hline $\mathrm{O}$ & -0.93226100 & -1.08368800 & 0.00005900 \\
\hline $\mathrm{C}$ & -2.28511700 & -1.00104500 & -0.00006600 \\
\hline $\mathrm{C}$ & -2.69049900 & 0.30501800 & -0.00004700 \\
\hline $\mathrm{C}$ & -1.50075200 & 1.08705400 & -0.00003900 \\
\hline $\mathrm{H}$ & 1.41506900 & -1.73936200 & 0.00005500 \\
\hline $\mathrm{H}$ & 3.88198700 & -1.46965700 & -0.00019900 \\
\hline
\end{tabular}




$\begin{array}{lrrr}\mathrm{H} & 3.64654300 & 0.37836400 & -0.00011500 \\ \mathrm{H} & -2.81583100 & -1.94042100 & -0.00009800 \\ \mathrm{H} & -3.71079700 & 0.65882300 & -0.00008500 \\ \mathrm{H} & -1.40586400 & 2.16209500 & -0.00005500\end{array}$<smiles>C=CC(=O)C1CCCCC1</smiles>

Enone-5

$\begin{array}{rrrr}\mathrm{C} & 0.00452200 & -0.08093300 & -0.35746900 \\ \mathrm{C} & -1.43689700 & -0.36622300 & 0.03785800 \\ \mathrm{C} & -2.46306300 & 0.60413400 & -0.45195500 \\ \mathrm{O} & -1.74065300 & -1.32005100 & 0.73867600 \\ \mathrm{C} & -3.75060100 & 0.47400600 & -0.12356900 \\ \mathrm{C} & 0.52684300 & 1.13308800 & 0.44947900 \\ \mathrm{C} & 1.98323300 & 1.45326300 & 0.09053200 \\ \mathrm{C} & 2.88907900 & 0.23035200 & 0.28022100 \\ \mathrm{C} & 2.37013000 & -0.97362000 & -0.51618400 \\ \mathrm{C} & 0.91444900 & -1.29745900 & -0.16042300 \\ \mathrm{H} & 0.00582500 & 0.20836900 & -1.42025100 \\ \mathrm{H} & -2.12431000 & 1.41623100 & -1.09097500 \\ \mathrm{H} & -4.50996500 & 1.16437800 & -0.47579600 \\ \mathrm{H} & -4.06065500 & -0.34795300 & 0.51509600 \\ \mathrm{H} & -0.10960800 & 2.00912600 & 0.28090400 \\ \mathrm{H} & 0.45550900 & 0.89218600 & 1.51836300 \\ \mathrm{H} & 2.33954500 & 2.29258500 & 0.69876900 \\ \mathrm{H} & 2.03097100 & 1.78117200 & -0.95725700 \\ \mathrm{H} & 2.92091100 & -0.03039500 & 1.34709100 \\ \mathrm{H} & 3.91664800 & 0.47064800 & -0.01553400 \\ \mathrm{H} & 3.00296200 & -1.84982700 & -0.33567600 \\ \mathrm{H} & 2.44262300 & -0.75253600 & -1.59055500 \\ \mathrm{H} & 0.84302900 & -1.61688900 & 0.88542500 \\ \mathrm{H} & 0.54557400 & -2.13633500 & -0.75995400\end{array}$<smiles>C=CC(=O)c1cc(OC)c(OC)c(OC)c1</smiles>

Enone-6

C

$\begin{array}{lll}-0.66478100 & 1.30924700 & -0.12118600\end{array}$ 


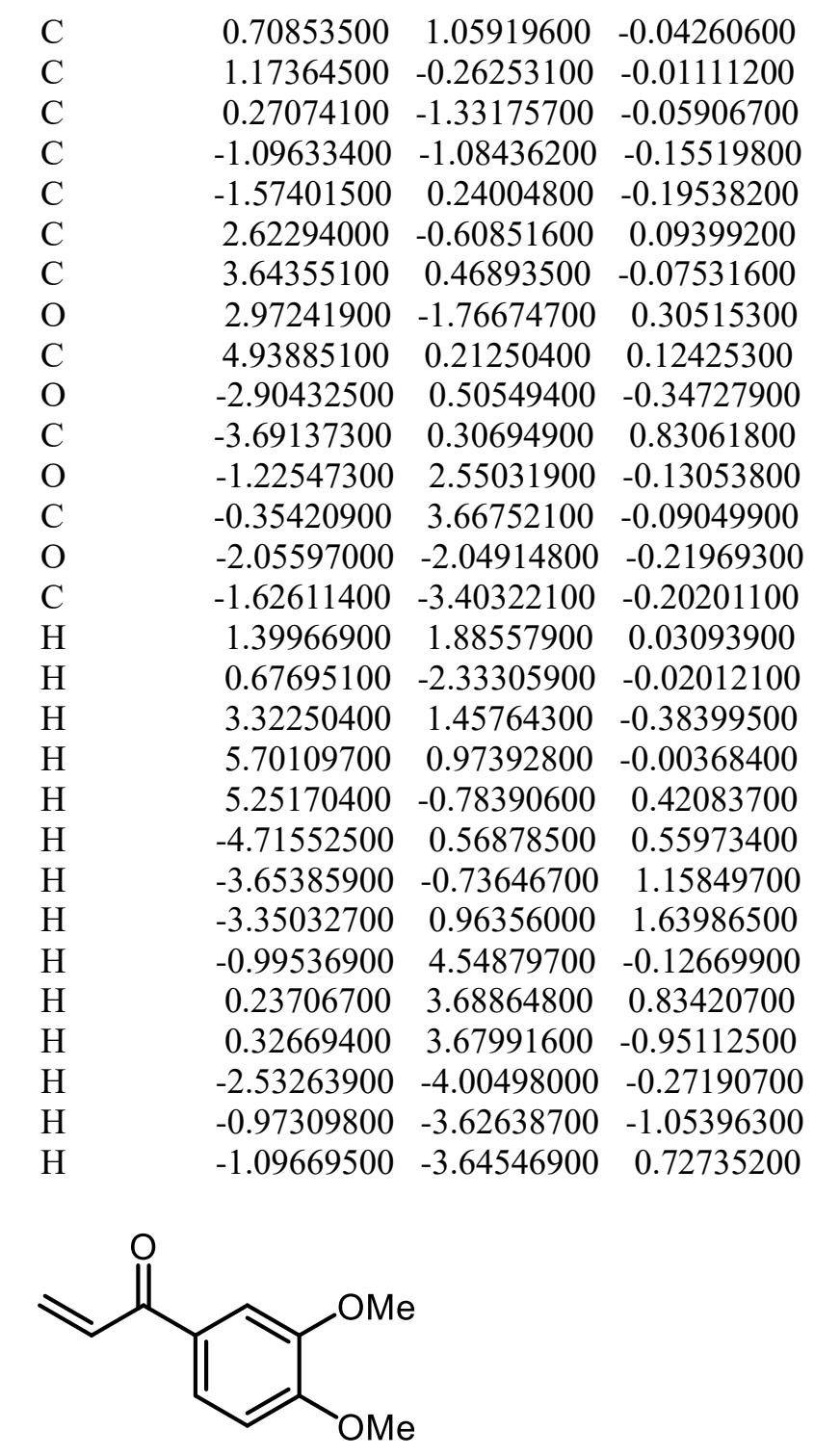

Enone-7

$\begin{array}{lrrr}\mathrm{C} & 1.22000800 & 0.72173000 & -0.00000500 \\ \mathrm{C} & -0.14818600 & 0.92774300 & 0.00001400 \\ \mathrm{C} & -1.05045700 & -0.15583900 & 0.00003700 \\ \mathrm{C} & -0.54412000 & -1.45632300 & 0.00005800 \\ \mathrm{C} & 0.83522600 & -1.67933300 & 0.00003100 \\ \mathrm{C} & 1.72571600 & -0.60729300 & -0.00000500 \\ \mathrm{C} & -2.50165300 & 0.17808900 & 0.00003900 \\ \mathrm{C} & -3.50742700 & -0.92612900 & -0.00026300 \\ \mathrm{O} & -2.87062500 & 1.35098500 & 0.00030400 \\ \mathrm{C} & -4.81408400 & -0.65038300 & -0.00005100 \\ \mathrm{O} & 3.07602800 & -0.71713100 & -0.00007200 \\ \mathrm{C} & 3.63674600 & -2.02104200 & 0.00008600 \\ \mathrm{O} & 2.16146400 & 1.70248900 & -0.00000500\end{array}$




$\begin{array}{rrrr}\mathrm{C} & 1.70016900 & 3.04508400 & -0.00014200 \\ \mathrm{H} & -0.57409400 & 1.92217200 & 0.00001500 \\ \mathrm{H} & -1.20171200 & -2.31641300 & 0.00014500 \\ \mathrm{H} & 1.20875600 & -2.69539300 & 0.00002700 \\ \mathrm{H} & -3.16794000 & -1.95570000 & -0.00069300 \\ \mathrm{H} & -5.56570700 & -1.43284800 & -0.00028400 \\ \mathrm{H} & -5.14629500 & 0.38316300 & 0.00034000 \\ \mathrm{H} & 3.34238400 & -2.58462100 & 0.89402900 \\ \mathrm{H} & 3.34249200 & -2.58480200 & -0.89378000 \\ \mathrm{H} & 4.71787500 & -1.88107800 & 0.00013500 \\ \mathrm{H} & 2.59479200 & 3.66837300 & -0.00022600 \\ \mathrm{H} & 1.10142500 & 3.26418400 & -0.89278700 \\ \mathrm{H} & 1.10145900 & 3.26438400 & 0.89247600\end{array}$<smiles>C=CC(=O)c1ccc(OC)cc1</smiles>

Enone-8

$\begin{array}{rrrr}\mathrm{C} & -1.35505400 & 1.42880500 & -0.03524700 \\ \mathrm{C} & 0.02592200 & 1.41602500 & -0.02949400 \\ \mathrm{C} & 0.74456800 & 0.20462400 & -0.00064100 \\ \mathrm{C} & 0.01960900 & -0.99286300 & 0.02950400 \\ \mathrm{C} & -1.37433600 & -0.99767800 & 0.02848500 \\ \mathrm{C} & -2.06831000 & 0.21778700 & -0.00656400 \\ \mathrm{C} & 2.23207000 & 0.28326000 & 0.00773700 \\ \mathrm{C} & 3.03327500 & -0.97625300 & -0.06149900 \\ \mathrm{O} & 2.79645600 & 1.37308300 & 0.06558400 \\ \mathrm{C} & 4.36674000 & -0.93254200 & -0.00511800 \\ \mathrm{O} & -3.42128100 & 0.33165700 & -0.01448900 \\ \mathrm{C} & -4.19611700 & -0.85872600 & 0.01396700 \\ \mathrm{H} & -1.91549600 & 2.35691000 & -0.06089100 \\ \mathrm{H} & 0.59271300 & 2.33988400 & -0.04737400 \\ \mathrm{H} & 0.52957100 & -1.94830400 & 0.06494800 \\ \mathrm{H} & -1.90343900 & -1.94172400 & 0.05584200 \\ \mathrm{H} & 2.52332200 & -1.92723500 & -0.16829100 \\ \mathrm{H} & 4.97338100 & -1.83048500 & -0.05874900 \\ \mathrm{H} & 4.87006300 & 0.02404400 & 0.09615000 \\ \mathrm{H} & -5.23817300 & -0.53875100 & 0.00036300 \\ \mathrm{H} & -3.99745900 & -1.48758400 & -0.86235500 \\ \mathrm{H} & -4.00608600 & -1.43930700 & 0.92483100\end{array}$<smiles>C=CC(=O)c1ccc([N+](=O)[O-])cc1</smiles> 
Enone-9

$\begin{array}{lrrr}\mathrm{C} & 1.02369400 & -1.19628900 & -0.09259700 \\ \mathrm{C} & -0.36412100 & -1.09398000 & -0.09428600 \\ \mathrm{C} & -0.98651000 & 0.16024000 & -0.01280700 \\ \mathrm{C} & -0.19618700 & 1.31775500 & 0.06451100 \\ \mathrm{C} & 1.18890500 & 1.23179600 & 0.07756400 \\ \mathrm{C} & 1.77609800 & -0.02989800 & -0.00047200 \\ \mathrm{C} & -2.47865600 & 0.34219200 & -0.03105000 \\ \mathrm{C} & -3.35585500 & -0.84264400 & 0.18183500 \\ \mathrm{O} & -2.95034200 & 1.45884500 & -0.20794300 \\ \mathrm{C} & -4.68210800 & -0.73104700 & 0.06397400 \\ \mathrm{H} & 1.52778200 & -2.15121300 & -0.16112200 \\ \mathrm{H} & -0.95203400 & -1.99957600 & -0.18069500 \\ \mathrm{H} & -0.69771500 & 2.27710600 & 0.11240200 \\ \mathrm{H} & 1.81864700 & 2.10925200 & 0.14440800 \\ \mathrm{H} & -2.90533700 & -1.79079100 & 0.45396900 \\ \mathrm{H} & -5.34423000 & -1.57493900 & 0.22551200 \\ \mathrm{H} & -5.12360200 & 0.22535500 & -0.19876700 \\ \mathrm{~N} & 3.24544500 & -0.13360200 & 0.01104100 \\ \mathrm{O} & 3.88863500 & 0.91129200 & 0.09251300 \\ \mathrm{O} & 3.73755900 & -1.25872700 & -0.06119900\end{array}$<smiles>C=CC(=O)c1cccc(Br)c1</smiles>

Enone-10

$\begin{array}{lrrc}\mathrm{C} & 0.20066900 & 2.52505900 & 0.06589600 \\ \mathrm{C} & -1.07010400 & 1.96289500 & 0.04036500 \\ \mathrm{C} & -1.23033100 & 0.56966200 & 0.00726800 \\ \mathrm{C} & -0.09550600 & -0.25239600 & -0.01058600 \\ \mathrm{C} & 1.16859600 & 0.32870700 & 0.00840400 \\ \mathrm{C} & 1.33510300 & 1.71021500 & 0.05031700 \\ \mathrm{C} & -2.63091800 & 0.03198600 & -0.02494400 \\ \mathrm{C} & -2.85396900 & -1.43413400 & 0.13077900 \\ \mathrm{O} & -3.57545800 & 0.79988400 & -0.16955300 \\ \mathrm{C} & -4.08397900 & -1.94430400 & 0.02505600 \\ \mathrm{Br} & 2.71038800 & -0.79995600 & -0.02458400 \\ \mathrm{H} & 0.31934100 & 3.60343100 & 0.09687800 \\ \mathrm{H} & -1.96236200 & 2.57770700 & 0.04485000 \\ \mathrm{H} & -0.17442000 & -1.33027300 & -0.05411000 \\ \mathrm{H} & 2.33078600 & 2.13745500 & 0.06842400 \\ \mathrm{H} & -2.01014100 & -2.07999800 & 0.34569300 \\ \mathrm{H} & -4.27580500 & -3.00557000 & 0.14227900 \\ \mathrm{H} & -4.92471000 & -1.28952300 & -0.18249600\end{array}$


<smiles>C=CC(=O)c1ccc(C(F)(F)F)cc1</smiles>

Enone-11

$\begin{array}{lrrr}\text { C } & -0.65427700 & -1.16485700 & -0.13730500 \\ \mathrm{C} & 0.73549500 & -1.07882200 & -0.12302400 \\ \mathrm{C} & 1.36911500 & 0.16692800 & -0.02077100 \\ \mathrm{C} & 0.58421300 & 1.32713100 & 0.05917200 \\ \mathrm{C} & -0.80128300 & 1.24574000 & 0.05456000 \\ \mathrm{C} & -1.42136400 & -0.00384000 & -0.04279500 \\ \mathrm{C} & 2.85995200 & 0.33812300 & -0.01933500 \\ \mathrm{C} & 3.72799200 & -0.86065600 & 0.16053300 \\ \mathrm{O} & 3.34664000 & 1.45507700 & -0.15316900 \\ \mathrm{C} & 5.05599700 & -0.75453200 & 0.06200100 \\ \mathrm{C} & -2.92186400 & -0.09735000 & 0.01123100 \\ \mathrm{~F} & -3.51085500 & 0.97794300 & -0.55525600 \\ \mathrm{~F} & -3.38152500 & -1.19723900 & -0.62405100 \\ \mathrm{~F} & -3.36541100 & -0.16311400 & 1.28773800 \\ \mathrm{H} & -1.14333400 & -2.12752300 & -0.22976900 \\ \mathrm{H} & 1.31534600 & -1.98930100 & -0.21547200 \\ \mathrm{H} & 1.09115000 & 2.28305600 & 0.12096200 \\ \mathrm{H} & -1.40601200 & 2.14335200 & 0.11631600 \\ \mathrm{H} & 3.26874200 & -1.81538000 & 0.39203300 \\ \mathrm{H} & 5.71086100 & -1.60834400 & 0.19982800 \\ \mathrm{H} & 5.50639500 & 0.20802600 & -0.16003200\end{array}$<smiles>C=CC(=O)/C(C)=C/c1ccccc1</smiles>

Enone-12

$\begin{array}{lrrr}\mathrm{C} & 1.10638300 & 0.60858000 & -0.06274300 \\ \mathrm{C} & 2.55944400 & 0.24795200 & -0.07900100 \\ \mathrm{C} & 3.00084500 & -1.08331300 & 0.44145500 \\ \mathrm{O} & 3.38935800 & 1.04988200 & -0.49990800 \\ \mathrm{C} & 4.26754500 & -1.47999200 & 0.29627400 \\ \mathrm{C} & 0.17370000 & -0.36824800 & 0.03752200 \\ \mathrm{C} & -1.28697300 & -0.25846200 & 0.04012900 \\ \mathrm{C} & -2.03539700 & -1.35832700 & -0.42114800 \\ \mathrm{C} & -3.42537600 & -1.32327200 & -0.44966900 \\ \mathrm{C} & -4.10445200 & -0.19211000 & 0.00708400 \\ \mathrm{C} & -3.38004400 & 0.89530900 & 0.49701900 \\ \mathrm{C} & -1.98791700 & 0.86609200 & 0.51431900\end{array}$




$\begin{array}{lrrr}\mathrm{C} & 0.81663800 & 2.06969600 & -0.27612900 \\ \mathrm{H} & 2.28814000 & -1.70727100 & 0.96926900 \\ \mathrm{H} & 4.61742200 & -2.43195200 & 0.68179800 \\ \mathrm{H} & 4.97952200 & -0.84021600 & -0.21597600 \\ \mathrm{H} & 0.52608900 & -1.39548100 & 0.06603900 \\ \mathrm{H} & -1.50992000 & -2.24237700 & -0.77174400 \\ \mathrm{H} & -3.97990300 & -2.17921600 & -0.82171100 \\ \mathrm{H} & -5.18947700 & -0.16334100 & -0.00775000 \\ \mathrm{H} & -3.90155800 & 1.76925300 & 0.87513200 \\ \mathrm{H} & -1.44388300 & 1.70477000 & 0.92887800 \\ \mathrm{H} & 0.75844300 & 2.61051400 & 0.67670000 \\ \mathrm{H} & 1.63794700 & 2.51027400 & -0.84346800 \\ \mathrm{H} & -0.12406500 & 2.22254900 & -0.80857200\end{array}$<smiles>C=CC(=O)c1ccc2ccccc2c1</smiles>

Enone-13

$\begin{array}{lrrr}\mathrm{C} & -0.10339700 & 2.52705300 & -0.34500200 \\ \mathrm{C} & -1.09968100 & 1.52940700 & -0.39274800 \\ \mathrm{C} & -0.78573700 & 0.18569200 & -0.26673900 \\ \mathrm{C} & 0.58188000 & -0.21203800 & -0.07801600 \\ \mathrm{C} & 1.58321200 & 0.81228400 & 0.00034800 \\ \mathrm{C} & 1.20904200 & 2.17353700 & -0.14487600 \\ \mathrm{C} & -1.88094600 & -0.82684200 & -0.40251500 \\ \mathrm{C} & -3.20848400 & -0.56524100 & 0.22266500 \\ \mathrm{O} & -1.72050500 & -1.88285200 & -1.00674500 \\ \mathrm{C} & -3.43122700 & 0.23585000 & 1.26962500 \\ \mathrm{C} & 0.99247800 & -1.56462400 & 0.06831100 \\ \mathrm{C} & 2.31546500 & -1.88028600 & 0.28245900 \\ \mathrm{C} & 3.30043200 & -0.86916700 & 0.35709800 \\ \mathrm{C} & 2.93807500 & 0.44925100 & 0.21801600 \\ \mathrm{H} & -0.38157800 & 3.56903400 & -0.46576000 \\ \mathrm{H} & -2.13134100 & 1.81833600 & -0.55705700 \\ \mathrm{H} & 1.98456400 & 2.93303200 & -0.09769300 \\ \mathrm{H} & -4.00113900 & -1.19262800 & -0.17767200 \\ \mathrm{H} & -4.41359500 & 0.30226900 & 1.72717400 \\ \mathrm{H} & -2.64010500 & 0.83110000 & 1.71314800 \\ \mathrm{H} & 0.25141700 & -2.34787100 & -0.00920800 \\ \mathrm{H} & 2.60658700 & -2.92053600 & 0.39103500 \\ \mathrm{H} & 4.33922700 & -1.13687500 & 0.52355900 \\ \mathrm{H} & 3.68332600 & 1.23771200 & 0.27468800\end{array}$


<smiles>C=CC(=O)c1ccccc1Cl</smiles>

Enone-14

$\begin{array}{lrrr}\mathrm{C} & -2.18733800 & 0.86126400 & -0.09964700 \\ \mathrm{C} & -0.80582800 & 0.72399700 & 0.03264700 \\ \mathrm{C} & -0.19463500 & -0.53785000 & 0.03873700 \\ \mathrm{C} & -1.02645500 & -1.66515800 & -0.05897000 \\ \mathrm{C} & -2.40251700 & -1.54291400 & -0.20539200 \\ \mathrm{C} & -2.98310600 & -0.27385900 & -0.23184000 \\ \mathrm{C} & 1.28088200 & -0.81175900 & 0.16048500 \\ \mathrm{C} & 2.24068400 & 0.03595200 & -0.59878500 \\ \mathrm{O} & 1.65362500 & -1.78054000 & 0.80951200 \\ \mathrm{C} & 3.55754100 & -0.13996400 & -0.45832900 \\ \mathrm{Cl} & 0.12361500 & 2.19923600 & 0.28573900 \\ \mathrm{H} & -2.62727500 & 1.85158800 & -0.08545100 \\ \mathrm{H} & -0.55238900 & -2.63950600 & -0.01874400 \\ \mathrm{H} & -3.02051600 & -2.43022000 & -0.29369600 \\ \mathrm{H} & -4.05705600 & -0.16259600 & -0.34239100 \\ \mathrm{H} & 1.84060800 & 0.77786000 & -1.28025900 \\ \mathrm{H} & 4.27535500 & 0.44753200 & -1.02079300 \\ \mathrm{H} & 3.93546200 & -0.88560500 & 0.23423100\end{array}$<smiles>C=CC(=O)c1ccc2ccccc2c1</smiles>

Enone-15

$\begin{array}{lrrr}\mathrm{C} & 1.17985600 & -0.55255500 & -0.02021500 \\ \mathrm{C} & -0.22752400 & -0.72002500 & -0.02649400 \\ \mathrm{C} & -1.08054400 & 0.36843600 & 0.00587800 \\ \mathrm{C} & -0.53152900 & 1.68201600 & 0.04078300 \\ \mathrm{C} & 0.82476200 & 1.87509600 & 0.05588100 \\ \mathrm{C} & 1.72158100 & 0.77173200 & 0.02654900 \\ \mathrm{C} & -2.57038900 & 0.24921700 & -0.02006900 \\ \mathrm{C} & -3.20002100 & -1.09555500 & 0.13434400 \\ \mathrm{O} & -3.26529400 & 1.25134500 & -0.15883700 \\ \mathrm{C} & -4.52323100 & -1.23885600 & 0.02421800 \\ \mathrm{C} & 3.12960100 & 0.93487700 & 0.03869600 \\ \mathrm{C} & 3.96517000 & -0.15912000 & 0.00397000 \\ \mathrm{C} & 3.43026300 & -1.46881200 & -0.04476900 \\ \mathrm{C} & 2.06784100 & -1.65979000 & -0.05691500 \\ \mathrm{H} & -0.61262200 & -1.73260400 & -0.07469900\end{array}$




$$
\begin{array}{rrrc}
\mathrm{H} & -1.22466000 & 2.51482600 & 0.05465600 \\
\mathrm{H} & 1.23630700 & 2.88003100 & 0.08837000 \\
\mathrm{H} & -2.57298300 & -1.95228000 & 0.35451600 \\
\mathrm{H} & -5.00754500 & -2.20257200 & 0.14142000 \\
\mathrm{H} & -5.14390800 & -0.37345900 & -0.18676000 \\
\mathrm{H} & 3.53878400 & 1.94046400 & 0.07510400 \\
\mathrm{H} & 5.04185200 & -0.02063500 & 0.01315800 \\
\mathrm{H} & 4.10078600 & -2.32188200 & -0.07236500 \\
\mathrm{H} & 1.65133300 & -2.66261700 & -0.09384900
\end{array}
$$<smiles>C=CC(=O)c1ccc(Br)cc1</smiles>

Enone-16

$\begin{array}{lrrr}\mathrm{C} & -0.74053200 & 1.25382100 & 0.06165700 \\ \mathrm{C} & 0.64608300 & 1.32429500 & 0.05514100 \\ \mathrm{C} & 1.42987900 & 0.16139300 & -0.00487500 \\ \mathrm{C} & 0.78806000 & -1.08331100 & -0.07093000 \\ \mathrm{C} & -0.60223800 & -1.16975000 & -0.07303300 \\ \mathrm{C} & -1.35277900 & 0.00120300 & -0.00212600 \\ \mathrm{C} & 2.91737300 & 0.33173300 & -0.01331300 \\ \mathrm{C} & 3.78980700 & -0.87143800 & 0.11543300 \\ \mathrm{O} & 3.40582500 & 1.45221700 & -0.11624900 \\ \mathrm{C} & 5.11735900 & -0.75600800 & 0.02344200 \\ \mathrm{Br} & -3.25576400 & -0.10958800 & 0.00486900 \\ \mathrm{H} & -1.34472200 & 2.15174700 & 0.11395400 \\ \mathrm{H} & 1.15519300 & 2.28049800 & 0.09624200 \\ \mathrm{H} & 1.35985100 & -2.00111100 & -0.13896100 \\ \mathrm{H} & -1.09831900 & -2.13121200 & -0.12964400 \\ \mathrm{H} & 3.33599000 & -1.83859500 & 0.30097500 \\ \mathrm{H} & 5.77543200 & -1.61276600 & 0.12243200 \\ \mathrm{H} & 5.56365400 & 0.21766800 & -0.15378600\end{array}$<smiles>C=CC(=O)c1ccc(Cl)cc1</smiles>

Enone-17

$\begin{array}{lrrr}\mathrm{C} & -1.29399700 & -1.21557200 & -0.07299000 \\ \mathrm{C} & 0.09448300 & -1.10616400 & -0.07201500 \\ \mathrm{C} & 0.71625300 & 0.14843100 & -0.00440700 \\ \mathrm{C} & -0.08638900 & 1.29819000 & 0.05787300 \\ \mathrm{C} & -1.47144000 & 1.20571300 & 0.06553900\end{array}$




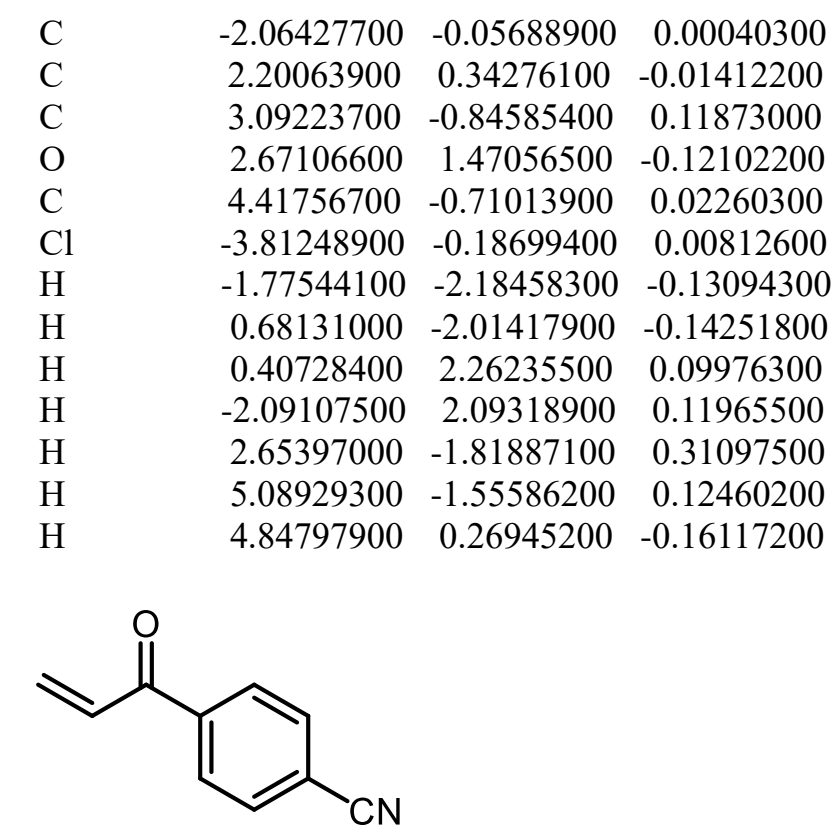

Enone-18

$\begin{array}{rrrr}\mathrm{C} & -1.42349600 & -1.22015300 & -0.09547700 \\ \mathrm{C} & -0.03732000 & -1.11067300 & -0.09553000 \\ \mathrm{C} & 0.57873500 & 0.14561700 & -0.00711500 \\ \mathrm{C} & -0.2211100 & 1.29587600 & 0.07576200 \\ \mathrm{C} & -1.60398900 & 1.19942300 & 0.08618100 \\ \mathrm{C} & -2.21315600 & -0.06478000 & 0.00116500 \\ \mathrm{C} & 2.06780600 & 0.33972800 & -0.02072900 \\ \mathrm{C} & 2.95629600 & -0.84404900 & 0.15035300 \\ \mathrm{O} & 2.53211300 & 1.46518500 & -0.16081400 \\ \mathrm{C} & 4.28145200 & -0.71570000 & 0.03839200 \\ \mathrm{C} & -3.64152900 & -0.17492000 & 0.01021400 \\ \mathrm{~N} & -4.80097000 & -0.26494300 & 0.01819900 \\ \mathrm{H} & -1.90075300 & -2.19059000 & -0.17033300 \\ \mathrm{H} & 0.55522500 & -2.01306400 & -0.18504500 \\ \mathrm{H} & 0.27422300 & 2.25826900 & 0.13037200 \\ \mathrm{H} & -2.22193200 & 2.08736600 & 0.15753200 \\ \mathrm{H} & 2.51566600 & -1.80644600 & 0.38608900 \\ \mathrm{H} & 4.95151800 & -1.55866600 & 0.16927800 \\ \mathrm{H} & 4.71381000 & 0.25404400 & -0.18807700\end{array}$<smiles>C=CC(=O)/C=C/c1ccccc1</smiles>

Enone-19 


$\begin{array}{rrrr}\mathrm{C} & 1.17096900 & 0.32077500 & 0.00016400 \\ \mathrm{C} & 2.55510600 & -0.20369300 & -0.00007300 \\ \mathrm{C} & 3.64519100 & 0.81583200 & 0.00031800 \\ \mathrm{O} & 2.80150100 & -1.40788500 & -0.00058000 \\ \mathrm{C} & 4.93053600 & 0.45402200 & 0.00040300 \\ \mathrm{C} & 0.12025700 & -0.52222700 & -0.00034700 \\ \mathrm{C} & -1.29936700 & -0.18726800 & -0.00021900 \\ \mathrm{C} & -2.23803000 & -1.23424000 & 0.00024500 \\ \mathrm{C} & -3.60545700 & -0.97241900 & 0.00049800 \\ \mathrm{C} & -4.06189500 & 0.34548200 & 0.00026500 \\ \mathrm{C} & -3.14177000 & 1.39863600 & -0.00025400 \\ \mathrm{C} & -1.77734500 & 1.13716700 & -0.00050000 \\ \mathrm{H} & 1.04132500 & 1.39944300 & 0.00080000 \\ \mathrm{H} & 3.35656400 & 1.86451300 & 0.00054500 \\ \mathrm{H} & 5.73521800 & 1.18176200 & 0.00069300 \\ \mathrm{H} & 5.19038800 & -0.60036800 & 0.00017100 \\ \mathrm{H} & 0.36566100 & -1.58323600 & -0.00084600 \\ \mathrm{H} & -1.88110100 & -2.26011400 & 0.00042700 \\ \mathrm{H} & -4.31362500 & -1.79506800 & 0.00086600 \\ \mathrm{H} & -5.12720600 & 0.55404900 & 0.00047000 \\ \mathrm{H} & -3.49346300 & 2.42571300 & -0.00049300 \\ \mathrm{H} & -1.07494200 & 1.96398100 & -0.00099400\end{array}$




\section{Cartesian Coordinates - Nucleophiles}<smiles>CCOC(=O)CC(=O)OCC</smiles>

Nucleophile-1

$\begin{array}{lrrr}\mathrm{O} & -1.92932100 & -0.26025200 & -0.80355600 \\ \mathrm{C} & -1.35185300 & -0.95276800 & 0.19562700 \\ \mathrm{C} & 0.04354600 & -1.38258900 & -0.20463400 \\ \mathrm{O} & -1.86513500 & -1.18879100 & 1.26579400 \\ \mathrm{C} & 0.99759000 & -0.20039600 & -0.14324600 \\ \mathrm{O} & 2.20251300 & -0.55041700 & -0.63167600 \\ \mathrm{O} & 0.72051000 & 0.89525400 & 0.29215400 \\ \mathrm{C} & -3.19393200 & 0.37329600 & -0.48989700 \\ \mathrm{C} & -2.96413700 & 1.68739800 & 0.23715600 \\ \mathrm{C} & 3.22971100 & 0.47329400 & -0.59043200 \\ \mathrm{C} & 3.88136100 & 0.54115100 & 0.78000300 \\ \mathrm{H} & 0.05291900 & -1.79288800 & -1.21802700 \\ \mathrm{H} & 0.39172100 & -2.15498100 & 0.48514600 \\ \mathrm{H} & -3.79387200 & -0.31598400 & 0.10861400 \\ \mathrm{H} & -3.67049300 & 0.52279300 & -1.46085800 \\ \mathrm{H} & -3.92072900 & 2.19232600 & 0.40586900 \\ \mathrm{H} & -2.48883900 & 1.50915400 & 1.20381300 \\ \mathrm{H} & -2.31843900 & 2.34466100 & -0.35069900 \\ \mathrm{H} & 2.77979000 & 1.43039900 & -0.86338400 \\ \mathrm{H} & 3.94008200 & 0.17065800 & -1.36224300 \\ \mathrm{H} & 3.15616000 & 0.85871100 & 1.53235600 \\ \mathrm{H} & 4.28777200 & -0.43329400 & 1.06502500 \\ \mathrm{H} & 4.70168000 & 1.26577900 & 0.76520600\end{array}$<smiles>CC(=O)CC1CC1(C#N)C#N</smiles>

Nucleophile-2

$\begin{array}{lrrr}\mathrm{C} & 3.21238200 & 0.19201400 & -0.39520000 \\ \mathrm{C} & 1.91507100 & -0.54592500 & -0.16334400 \\ \mathrm{C} & 1.14037100 & -0.17377100 & 1.10307200 \\ \mathrm{O} & 1.48239300 & -1.38020600 & -0.93306200 \\ \mathrm{C} & -0.29933900 & -0.61821100 & 1.02965400 \\ \mathrm{C} & -1.38350500 & 0.13743100 & 1.71045300 \\ \mathrm{C} & -1.27323200 & 0.22618900 & 0.17635500 \\ \mathrm{C} & -0.74987700 & 1.44342300 & -0.39781200 \\ \mathrm{C} & -2.25808800 & -0.47143100 & -0.61532400 \\ \mathrm{~N} & -3.05981500 & -1.05077800 & -1.22307000 \\ \mathrm{~N} & -0.26595000 & 2.41017100 & -0.82237200 \\ \mathrm{H} & 2.97621400 & 1.22033000 & -0.69524200 \\ \mathrm{H} & 3.80945300 & 0.25066300 & 0.52093400\end{array}$




$$
\begin{array}{rrrr}
\mathrm{H} & 3.78116500 & -0.29148800 & -1.18974700 \\
\mathrm{H} & 1.21936200 & 0.90399500 & 1.27990700 \\
\mathrm{H} & 1.63316500 & -0.65833100 & 1.95720300 \\
\mathrm{H} & -0.44365400 & -1.68364900 & 0.88828900 \\
\mathrm{H} & -2.24840300 & -0.39808800 & 2.08507400 \\
\mathrm{H} & -1.12879700 & 1.04415900 & 2.24905300
\end{array}
$$<smiles>O=[PH](Oc1ccccc1)c1ccccc1</smiles>

Nucleophile-3

$\begin{array}{lrrr}\mathrm{P} & -1.91604600 & 1.39860900 & -0.12693800 \\ \mathrm{O} & -2.40774400 & 1.15158300 & 1.24662900 \\ \mathrm{O} & -1.73792100 & 0.09517600 & -1.08308900 \\ \mathrm{O} & -0.50898200 & 2.20711100 & -0.26175300 \\ \mathrm{H} & -2.69946000 & 2.19038000 & -0.96746600 \\ \mathrm{C} & -1.03967400 & -1.04458100 & -0.65003500 \\ \mathrm{C} & 0.70995400 & 1.59726100 & 0.06090000 \\ \mathrm{C} & 0.08872400 & -1.41819800 & -1.37283200 \\ \mathrm{C} & 0.80686000 & -2.54184200 & -0.96861300 \\ \mathrm{C} & 0.39268500 & -3.27975300 & 0.14176800 \\ \mathrm{C} & -0.74829400 & -2.89350600 & 0.84595600 \\ \mathrm{C} & -1.47909500 & -1.77161800 & 0.45322400 \\ \mathrm{C} & 1.73640500 & 1.70737200 & -0.87215200 \\ \mathrm{C} & 2.95877500 & 1.09052200 & -0.60789000 \\ \mathrm{C} & 3.14234400 & 0.36899000 & 0.57265500 \\ \mathrm{C} & 2.10450800 & 0.28279800 & 1.50150800 \\ \mathrm{C} & 0.88151400 & 0.90746600 & 1.26004900 \\ \mathrm{H} & 0.40342300 & -0.81414800 & -2.21527900 \\ \mathrm{H} & 1.69556700 & -2.83397600 & -1.51869900 \\ \mathrm{H} & 0.95715700 & -4.15156100 & 0.45650000 \\ \mathrm{H} & -1.07516000 & -3.46466800 & 1.70917900 \\ \mathrm{H} & -2.35407500 & -1.44245600 & 0.99941900 \\ \mathrm{H} & 1.56096800 & 2.25747400 & -1.78989100 \\ \mathrm{H} & 3.76410500 & 1.16765300 & -1.33164000 \\ \mathrm{H} & 4.09086100 & -0.11994600 & 0.76950600 \\ \mathrm{H} & 2.24218400 & -0.27428700 & 2.42253000 \\ \mathrm{H} & 0.06406600 & 0.84597200 & 1.96837600 \\ & & & \end{array}$<smiles>O=C(CC(=O)c1ccccc1)c1ccccc1</smiles>

Nucleophile-4
$\begin{array}{llll}\text { C } & 3.55183100 & 1.42417400 & -0.41442300\end{array}$
$\begin{array}{llll}\mathrm{C} & 2.40980400 & 0.97269600 & 0.24497200\end{array}$
$\begin{array}{llll}\mathrm{C} & 2.08005500 & -0.39120400 & 0.21746800\end{array}$
$\begin{array}{lllll}\mathrm{C} & 2.90660500 & -1.28991900 & -0.47659900\end{array}$
$\begin{array}{llll}\text { C } & 4.04768600 & -0.83563800 & -1.12562200\end{array}$
$\begin{array}{llll}\mathrm{C} & 4.37235500 & 0.52404900 & -1.09452600\end{array}$ 


\begin{tabular}{|c|c|c|c|}
\hline $\mathrm{C}$ & 0.87245800 & -0.94074600 & 0.89361400 \\
\hline $\mathrm{C}$ & 0.00000100 & -0.00000400 & 1.73423500 \\
\hline $\mathrm{O}$ & 0.56912700 & -2.12142400 & 0.79473900 \\
\hline $\mathrm{C}$ & -0.87243300 & 0.94073400 & 0.89357900 \\
\hline $\mathrm{C}$ & -2.08005600 & 0.39119900 & 0.21746500 \\
\hline $\mathrm{O}$ & -0.56907300 & 2.12139900 & 0.79464600 \\
\hline $\mathrm{C}$ & -2.90664100 & 1.28993200 & -0.47653600 \\
\hline $\mathrm{C}$ & -4.04773900 & 0.83566600 & -1.12554000 \\
\hline $\mathrm{C}$ & -4.37238200 & -0.52402900 & -1.09450600 \\
\hline $\mathrm{C}$ & -3.55181800 & -1.42417500 & -0.41447900 \\
\hline $\mathrm{C}$ & -2.40978100 & -0.97271000 & 0.24490800 \\
\hline $\mathrm{H}$ & 3.79945400 & 2.48073100 & -0.39744700 \\
\hline $\mathrm{H}$ & 1.76732100 & 1.68453600 & 0.74988600 \\
\hline $\mathrm{H}$ & 2.62838200 & -2.33770000 & -0.48841400 \\
\hline $\mathrm{H}$ & 4.68520400 & -1.53524100 & -1.65710800 \\
\hline $\mathrm{H}$ & 5.26351600 & 0.88023800 & -1.60258000 \\
\hline $\mathrm{H}$ & 0.62368300 & 0.63433300 & 2.36606300 \\
\hline $\mathrm{H}$ & -0.62368600 & -0.63433400 & 2.36606300 \\
\hline $\mathrm{H}$ & -2.62843100 & 2.33771800 & -0.48831700 \\
\hline $\mathrm{H}$ & -4.68529000 & 1.53528600 & -1.65696300 \\
\hline $\mathrm{H}$ & -5.26355200 & -0.88020800 & -1.60254900 \\
\hline $\mathrm{H}$ & -3.79941700 & -2.48073900 & -0.39755800 \\
\hline $\mathrm{H}$ & -1.76727700 & -1.68456800 & 0.74976900 \\
\hline
\end{tabular}

Nucleophile-5

$\begin{array}{lrrr}\mathrm{C} & 1.96109900 & -0.99542100 & -0.04145000 \\ \mathrm{C} & 1.24573000 & 0.30083900 & 0.22907400 \\ \mathrm{C} & -0.05024700 & 0.17438300 & 0.97212000 \\ \mathrm{C} & 1.75635800 & 1.45159400 & -0.23000700 \\ \mathrm{C} & 1.11287900 & 2.70420000 & -0.00694100 \\ \mathrm{C} & -1.21224800 & -0.09962200 & 0.01714400 \\ \mathrm{O} & -2.18593000 & -0.76917000 & 0.65444700 \\ \mathrm{O} & -1.25266900 & 0.26822900 & -1.13397500 \\ \mathrm{C} & -3.39370400 & -1.02188500 & -0.11330300 \\ \mathrm{C} & -4.30622500 & 0.19191700 & -0.11151200 \\ \mathrm{~N} & 0.59131300 & 3.72516000 & 0.18966600 \\ \mathrm{~F} & 2.34047700 & -1.57494600 & 1.12089100 \\ \mathrm{~F} & 1.13912300 & -1.86595400 & -0.66680800 \\ \mathrm{~F} & 3.06067700 & -0.84369100 & -0.80008700 \\ \mathrm{H} & -0.27936300 & 1.11185400 & 1.48948500 \\ \mathrm{H} & -0.00034800 & -0.61604600 & 1.72354900 \\ \mathrm{H} & 2.68318100 & 1.46466300 & -0.79258700 \\ \mathrm{H} & -3.85210000 & -1.87661100 & 0.38729700 \\ \mathrm{H} & -3.10600200 & -1.30325300 & -1.12850400 \\ \mathrm{H} & -4.54979200 & 0.49331900 & 0.91104800 \\ \mathrm{H} & -3.83111300 & 1.02953100 & -0.62677900 \\ \mathrm{H} & -5.23919900 & -0.04676300 & -0.63166100\end{array}$


<smiles>CCC(=O)CC1CC1(C#N)C#N</smiles>

Nucleophile-6

$\begin{array}{lrrr}\mathrm{C} & -2.76951900 & 0.42554100 & -0.00545400 \\ \mathrm{C} & -1.52739100 & -0.33075200 & -0.42742200 \\ \mathrm{C} & -0.54127100 & 0.42683900 & -1.32102600 \\ \mathrm{O} & -1.29494400 & -1.47255400 & -0.08117500 \\ \mathrm{C} & 0.85212200 & -0.14466300 & -1.22204100 \\ \mathrm{C} & 2.05702600 & 0.71012100 & -1.38381700 \\ \mathrm{C} & 1.69078800 & 0.21239700 & 0.02760600 \\ \mathrm{C} & 1.11048000 & 1.15622000 & 0.95366800 \\ \mathrm{C} & 2.50751000 & -0.82332900 & 0.61367600 \\ \mathrm{~N} & 0.58563400 & 1.92143700 & 1.65235300 \\ \mathrm{~N} & 3.17733500 & -1.66312300 & 1.05404200 \\ \mathrm{C} & -3.80410800 & -0.44638000 & 0.69790500 \\ \mathrm{H} & -3.18872800 & 0.92859800 & -0.88797100 \\ \mathrm{H} & -2.42215700 & 1.23699800 & 0.65104300 \\ \mathrm{H} & -0.55560100 & 1.49328000 & -1.07514400 \\ \mathrm{H} & -0.89587900 & 0.34594500 & -2.35764800 \\ \mathrm{H} & 0.93770800 & -1.19127800 & -1.49246800 \\ \mathrm{H} & 1.92301400 & 1.77356200 & -1.55191200 \\ \mathrm{H} & 2.95801400 & 0.28240700 & -1.80870300 \\ \mathrm{H} & -3.36649000 & -0.92888200 & 1.57451400 \\ \mathrm{H} & -4.65975500 & 0.15310700 & 1.01918700 \\ \mathrm{H} & -4.16517900 & -1.23746500 & 0.03516900\end{array}$<smiles>CCCC(=O)CC1CC1(C#N)C#N</smiles>

Nucleophile-7

$\begin{array}{lrrr}\mathrm{C} & 2.65837200 & 0.08444400 & 0.23168600 \\ \mathrm{C} & 1.32460400 & -0.64233600 & 0.12807100 \\ \mathrm{C} & 0.33051600 & -0.41797100 & 1.26739700 \\ \mathrm{O} & 1.03657500 & -1.35318500 & -0.81588400 \\ \mathrm{C} & -1.06408300 & -0.85421500 & 0.89706100 \\ \mathrm{C} & -2.26605500 & -0.30517000 & 1.57624200 \\ \mathrm{C} & -1.97125900 & 0.13564500 & 0.12919100 \\ \mathrm{C} & -1.47203500 & 1.47451100 & -0.07630000 \\ \mathrm{C} & -2.80555300 & -0.39127100 & -0.92441500 \\ \mathrm{~N} & -1.02424500 & 2.53914100 & -0.19947800 \\ \mathrm{~N} & -3.48693900 & -0.83876800 & -1.75102500 \\ \mathrm{C} & 2.50408300 & 1.45573300 & -0.46197300 \\ \mathrm{C} & 3.78699000 & -0.73762900 & -0.39470700 \\ \mathrm{H} & 2.86840300 & 0.26050000 & 1.29551500 \\ \mathrm{H} & 0.35831600 & 0.63022700 & 1.58297900\end{array}$




$\begin{array}{rrrr}\mathrm{H} & 0.68147200 & -1.00084300 & 2.13077300 \\ \mathrm{H} & -1.13035800 & -1.86198500 & 0.50154900 \\ \mathrm{H} & -3.13812100 & -0.93645000 & 1.70366700 \\ \mathrm{H} & -2.13412800 & 0.46355000 & 2.33048200 \\ \mathrm{H} & 3.42907200 & 2.03025000 & -0.35891500 \\ \mathrm{H} & 2.30378900 & 1.31382400 & -1.52804100 \\ \mathrm{H} & 1.68478600 & 2.04719400 & -0.04435900 \\ \mathrm{H} & 3.55004300 & -0.96709500 & -1.43621200 \\ \mathrm{H} & 4.72786700 & -0.18093700 & -0.36231300 \\ \mathrm{H} & 3.93106700 & -1.68581100 & 0.13194700\end{array}$<smiles>CC(C)(C)C(=O)CC1CC1(C#N)C#N</smiles>

Nucleophile-8

\begin{tabular}{|c|c|c|c|}
\hline $\mathrm{C}$ & -2.42875300 & -0.02341900 & 0.12578400 \\
\hline $\mathrm{C}$ & -1.06098400 & -0.67958100 & -0.10136100 \\
\hline $\mathrm{C}$ & -0.17135800 & -0.13471100 & -1.22405100 \\
\hline $\mathrm{O}$ & -0.65803200 & -1.59502300 & 0.59126600 \\
\hline $\mathrm{C}$ & 1.25582900 & -0.59767700 & -1.07249000 \\
\hline $\mathrm{C}$ & 2.39100100 & 0.15584900 & -1.66500800 \\
\hline $\mathrm{C}$ & 2.19796300 & 0.19365300 & -0.13621500 \\
\hline $\mathrm{C}$ & 1.69128100 & 1.41353500 & 0.44632200 \\
\hline $\mathrm{C}$ & 3.12024900 & -0.55585200 & 0.68315400 \\
\hline $\mathrm{N}$ & 1.23868000 & 2.39294100 & 0.87643100 \\
\hline $\mathrm{N}$ & 3.87106400 & -1.17588000 & 1.31537900 \\
\hline $\mathrm{C}$ & -3.31110800 & -0.96924600 & 0.95172100 \\
\hline $\mathrm{C}$ & -3.11643300 & 0.31063000 & -1.21128800 \\
\hline C & -2.17447600 & 1.27889000 & 0.92210700 \\
\hline $\mathrm{H}$ & -0.23521600 & 0.95637400 & -1.26773400 \\
\hline $\mathrm{H}$ & -0.56989000 & -0.49835000 & -2.18029400 \\
\hline $\mathrm{H}$ & 1.37310100 & -1.66984300 & -0.96036200 \\
\hline $\mathrm{H}$ & 3.26354200 & -0.38557100 & -2.01257600 \\
\hline $\mathrm{H}$ & 2.18687100 & 1.08850900 & -2.18050300 \\
\hline $\mathrm{H}$ & -4.26770600 & -0.48683400 & 1.17397800 \\
\hline $\mathrm{H}$ & -3.51190500 & -1.89672600 & 0.40715000 \\
\hline $\mathrm{H}$ & -2.82341200 & -1.23577300 & 1.89078700 \\
\hline $\mathrm{H}$ & -3.26943600 & -0.58729200 & -1.81945400 \\
\hline $\mathrm{H}$ & -4.09935200 & 0.74973200 & -1.01591400 \\
\hline $\mathrm{H}$ & -2.54844500 & 1.03474500 & -1.80238100 \\
\hline $\mathrm{H}$ & -1.69083700 & 1.06388900 & 1.87868900 \\
\hline $\mathrm{H}$ & -1.53949100 & 1.98417400 & 0.37970300 \\
\hline $\mathrm{H}$ & -3.13103200 & 1.77128400 & 1.12406300 \\
\hline
\end{tabular}

Nucleophile-9 


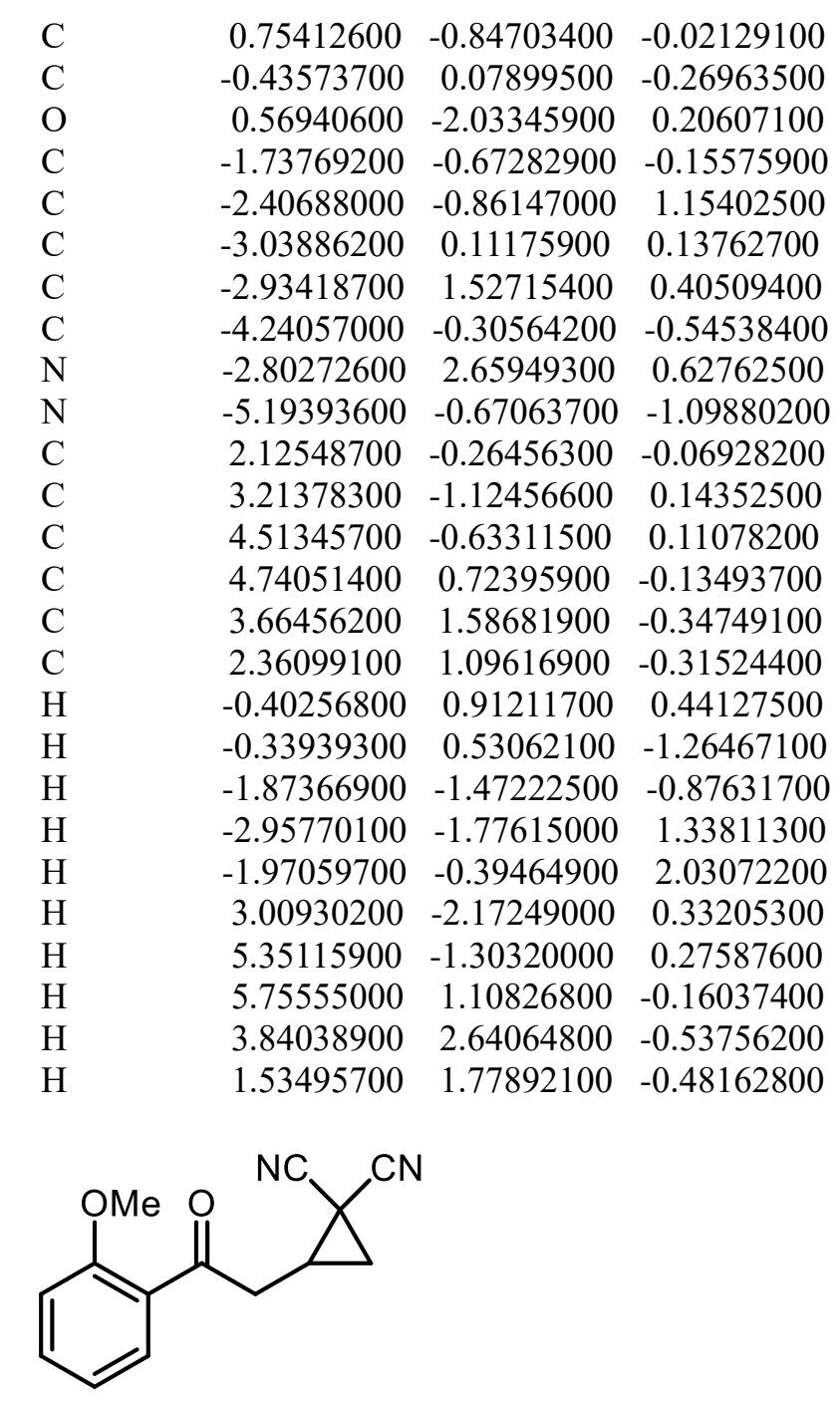

Nucleophile-10

$\begin{array}{lrrr}\mathrm{C} & -0.45586300 & -1.18667900 & 0.04005800 \\ \mathrm{C} & 0.68623300 & -0.19145800 & 0.19299600 \\ \mathrm{O} & -0.19811800 & -2.37844100 & -0.07744600 \\ \mathrm{C} & 2.02390200 & -0.88400500 & 0.12981800 \\ \mathrm{C} & 2.73191200 & -1.06619000 & -1.16002700 \\ \mathrm{C} & 3.27821900 & -0.02381300 & -0.16227200 \\ \mathrm{C} & 3.08227600 & 1.37502700 & -0.46516900 \\ \mathrm{C} & 4.49257100 & -0.33954400 & 0.55150300 \\ \mathrm{~N} & 2.87679200 & 2.49169700 & -0.71038000 \\ \mathrm{~N} & 5.45901000 & -0.62191400 & 1.13027100 \\ \mathrm{C} & -1.87720600 & -0.71986600 & 0.04300200 \\ \mathrm{C} & -2.30378200 & 0.62887600 & 0.12115000 \\ \mathrm{C} & -3.67167400 & 0.93110100 & 0.10679000 \\ \mathrm{C} & -4.61359300 & -0.09057500 & 0.01785900 \\ \mathrm{C} & -4.21182900 & -1.42457900 & -0.06014700 \\ \mathrm{C} & -2.85628200 & -1.72187200 & -0.04782700\end{array}$




$\begin{array}{lrrr}\mathrm{O} & -1.34321400 & 1.58669100 & 0.20596800 \\ \mathrm{C} & -1.72138200 & 2.95819000 & 0.23867100 \\ \mathrm{H} & 0.56614700 & 0.34894500 & 1.13843700 \\ \mathrm{H} & 0.60045800 & 0.58016100 & -0.57648900 \\ \mathrm{H} & 2.19248100 & -1.65042100 & 0.87859600 \\ \mathrm{H} & 3.34891000 & -1.94494500 & -1.30606700 \\ \mathrm{H} & 2.28378700 & -0.65534300 & -2.05856700 \\ \mathrm{H} & -4.00455100 & 1.95906100 & 0.16619300 \\ \mathrm{H} & -5.66909400 & 0.16404100 & 0.00980900 \\ \mathrm{H} & -4.94718900 & -2.21868900 & -0.12953700 \\ \mathrm{H} & -2.50369600 & -2.74513700 & -0.10916400 \\ \mathrm{H} & -0.78634400 & 3.51719100 & 0.27170500 \\ \mathrm{H} & -2.28327500 & 3.23723900 & -0.65971000 \\ \mathrm{H} & -2.31858400 & 3.18573200 & 1.12894800\end{array}$<smiles>COc1cccc(C(=O)CC2CC2(C#N)C#N)c1</smiles>

Nucleophile-11

$\begin{array}{lrrr}\mathrm{C} & 0.12589900 & -0.53585600 & -0.11660800 \\ \mathrm{C} & -1.18445100 & 0.23085700 & -0.28814300 \\ \mathrm{O} & 0.10908200 & -1.74818600 & 0.04096200 \\ \mathrm{C} & -2.36880800 & -0.69752700 & -0.19475700 \\ \mathrm{C} & -2.97225700 & -1.04752100 & 1.11383100 \\ \mathrm{C} & -3.75681700 & -0.11658700 & 0.16651900 \\ \mathrm{C} & -3.84053300 & 1.28288900 & 0.51329400 \\ \mathrm{C} & -4.90726100 & -0.65828200 & -0.51755000 \\ \mathrm{~N} & -3.86098000 & 2.40870100 & 0.79806300 \\ \mathrm{~N} & -5.81544200 & -1.12138400 & -1.07357600 \\ \mathrm{C} & 1.40387200 & 0.23085500 & -0.15223900 \\ \mathrm{C} & 2.59411000 & -0.50092000 & -0.00859200 \\ \mathrm{C} & 3.82088700 & 0.15874700 & -0.03230800 \\ \mathrm{C} & 3.85884400 & 1.55344700 & -0.19969000 \\ \mathrm{C} & 2.67899200 & 2.27140400 & -0.34092700 \\ \mathrm{C} & 1.44393100 & 1.61920300 & -0.31870600 \\ \mathrm{O} & 5.02943200 & -0.45192500 & 0.09731500 \\ \mathrm{C} & 5.04341400 & -1.86286500 & 0.26880000 \\ \mathrm{H} & -1.17566000 & 0.74586200 & -1.25647200 \\ \mathrm{H} & -1.24554100 & 1.01971600 & 0.46959900 \\ \mathrm{H} & -2.41323300 & -1.46752700 & -0.95754400 \\ \mathrm{H} & -3.38868000 & -2.03775200 & 1.25518200 \\ \mathrm{H} & -2.58119400 & -0.57336800 & 2.00777200 \\ \mathrm{H} & 2.51236000 & -1.57223000 & 0.11783500 \\ \mathrm{H} & 4.82569700 & 2.04466800 & -0.21465200 \\ \mathrm{H} & 2.71969700 & 3.34818900 & -0.46895100 \\ \mathrm{H} & 0.53340700 & 2.19604600 & -0.42991100 \\ \mathrm{H} & 4.59455100 & -2.37558400 & -0.59045900 \\ \mathrm{H} & 6.09332500 & -2.14439000 & 0.35111600\end{array}$


<smiles>N#CC1(C#N)CC1CC(=O)c1ccc(Cl)cc1</smiles>

Nucleophile-12

$\begin{array}{lrrr}\mathrm{C} & -0.14329400 & -1.01079200 & -0.01474100 \\ \mathrm{C} & -1.25094500 & 0.01137400 & -0.26022700 \\ \mathrm{O} & -0.42127500 & -2.18207300 & 0.19410300 \\ \mathrm{C} & -2.61147700 & -0.63195000 & -0.16636400 \\ \mathrm{C} & -3.30611400 & -0.77994600 & 1.13576200 \\ \mathrm{C} & -3.84499500 & 0.25463900 & 0.12675600 \\ \mathrm{C} & -3.62305300 & 1.65287100 & 0.41277300 \\ \mathrm{C} & -5.07091900 & -0.05204900 & -0.57185600 \\ \mathrm{~N} & -3.39514700 & 2.76668700 & 0.65030100 \\ \mathrm{~N} & -6.04604100 & -0.32908000 & -1.13813200 \\ \mathrm{C} & 1.27072400 & -0.54071700 & -0.04244900 \\ \mathrm{C} & 2.28424400 & -1.48841900 & 0.16574400 \\ \mathrm{C} & 3.62105000 & -1.11433300 & 0.15323600 \\ \mathrm{C} & 3.94619500 & 0.22557100 & -0.07031100 \\ \mathrm{C} & 2.95863500 & 1.18770800 & -0.27966200 \\ \mathrm{C} & 1.62250500 & 0.79833800 & -0.26468000 \\ \mathrm{Cl} & 5.62876400 & 0.70767600 & -0.08784400 \\ \mathrm{H} & -1.10911000 & 0.46545100 & -1.24885800 \\ \mathrm{H} & -1.15635500 & 0.83046500 & 0.46151600 \\ \mathrm{H} & -2.80633700 & -1.40831000 & -0.89849600 \\ \mathrm{H} & -3.93262600 & -1.64789700 & 1.30325700 \\ \mathrm{H} & -2.84086900 & -0.36193500 & 2.02214900 \\ \mathrm{H} & 1.99643300 & -2.51961100 & 0.33648600 \\ \mathrm{H} & 4.40710600 & -1.84288800 & 0.31323400 \\ \mathrm{H} & 3.23523400 & 2.22126900 & -0.45018600 \\ \mathrm{H} & 0.86072000 & 1.55253700 & -0.42763900\end{array}$<smiles>N#CC1(C#N)CC1CC(=O)c1ccccc1Cl</smiles>

Nucleophile-13
C
$0.05170800-0.05058900$
0.93148100
$\mathrm{C}$ $\begin{array}{lll}-0.68455600 & 1.17855300 & 0.40391300\end{array}$
$\mathrm{O}$ $-0.52891100-0.81225300$ 1.68814700
C $\begin{array}{lll}-2.17767800 & 1.01395300 & 0.56253700\end{array}$
$\begin{array}{llll}\text { C } & -3.12312000 & 1.58558500 & -0.43202300\end{array}$
$\begin{array}{lllll}\text { C } & -2.91328300 & 0.05955300 & -0.40383500\end{array}$ 


$\begin{array}{lrrr}\mathrm{C} & -2.13112700 & -0.54275600 & -1.45755300 \\ \mathrm{C} & -3.97147000 & -0.76024400 & 0.13640900 \\ \mathrm{~N} & -1.45394700 & -0.99652300 & -2.28475500 \\ \mathrm{~N} & -4.83522500 & -1.39244000 & 0.58603500 \\ \mathrm{C} & 1.43472300 & -0.39155000 & 0.45613300 \\ \mathrm{C} & 2.42445300 & 0.48869000 & -0.01029000 \\ \mathrm{C} & 3.66175100 & 0.02107400 & -0.45154800 \\ \mathrm{C} & 3.92942000 & -1.34493100 & -0.44259400 \\ \mathrm{C} & 2.96892200 & -2.24208100 & 0.02594500 \\ \mathrm{C} & 1.74631200 & -1.76340000 & 0.47466500 \\ \mathrm{Cl} & 2.21279500 & 2.23889700 & -0.01031000 \\ \mathrm{H} & -0.40976000 & 1.36253600 & -0.63791400 \\ \mathrm{H} & -0.35790000 & 2.06177200 & 0.96293800 \\ \mathrm{H} & -2.52620900 & 0.91629700 & 1.58439300 \\ \mathrm{H} & -2.71978300 & 2.09458000 & -1.30112600 \\ \mathrm{H} & -4.09694500 & 1.92905800 & -0.10217400 \\ \mathrm{H} & 4.40543400 & 0.73122400 & -0.79307600 \\ \mathrm{H} & 4.89069900 & -1.70224400 & -0.79756600 \\ \mathrm{H} & 3.17213600 & -3.30741200 & 0.03905200 \\ \mathrm{H} & 0.98398200 & -2.43744000 & 0.84715900\end{array}$<smiles>CC(=O)S</smiles>

Nucleophile-14

$$
\begin{array}{lrrr}
\mathrm{C} & 1.48313800 & 0.81393000 & 0.00000000 \\
\mathrm{C} & 0.00000000 & 0.51814200 & 0.00000000 \\
\mathrm{~S} & -0.36153000 & -1.27191800 & 0.00000000 \\
\mathrm{O} & -0.86939700 & 1.35175600 & 0.00000000 \\
\mathrm{H} & 1.62651300 & 1.89485500 & 0.00000000 \\
\mathrm{H} & 1.95576800 & 0.37452900 & 0.88364100 \\
\mathrm{H} & 1.95576800 & 0.37452900 & -0.88364100 \\
\mathrm{H} & -1.69722800 & -1.09971000 & 0.00000000 \\
\end{array}
$$

Nucleophile-15 


\begin{tabular}{|c|c|c|c|}
\hline $\mathrm{C}$ & -2.56056200 & 0.44674600 & 0.00017600 \\
\hline $\mathrm{O}$ & 2.36852700 & -0.89575900 & 0.00048200 \\
\hline $\mathrm{O}$ & 3.66483100 & 0.97857500 & -0.00001900 \\
\hline $\mathrm{C}$ & 3.49508000 & -1.83245900 & -0.00005900 \\
\hline $\mathrm{C}$ & 4.32870500 & -1.65150200 & 1.27053400 \\
\hline $\mathrm{C}$ & 4.32807100 & -1.65119500 & -1.27102000 \\
\hline $\mathrm{C}$ & 2.79638500 & -3.19204100 & -0.00011000 \\
\hline $\mathrm{O}$ & -2.36852900 & -0.89574900 & 0.00046000 \\
\hline $\mathrm{O}$ & -3.66486800 & 0.97856900 & -0.00003200 \\
\hline $\mathrm{C}$ & -3.49505500 & -1.83248500 & -0.00005700 \\
\hline $\mathrm{C}$ & -4.32812900 & -1.65118400 & -1.27095800 \\
\hline $\mathrm{C}$ & -4.32861000 & -1.65163900 & 1.27059900 \\
\hline $\mathrm{C}$ & -2.79631200 & -3.19204100 & -0.00022900 \\
\hline $\mathrm{H}$ & -0.00000400 & 4.14778000 & -0.00014900 \\
\hline $\mathrm{H}$ & -0.00002600 & -0.37637700 & 0.86446400 \\
\hline $\mathrm{H}$ & -0.00002800 & -0.37698300 & -0.86300300 \\
\hline $\mathrm{H}$ & 3.04381400 & 3.24712300 & -0.87090100 \\
\hline $\mathrm{H}$ & 2.08383700 & 4.46970500 & -0.00045100 \\
\hline $\mathrm{H}$ & 3.04383400 & 3.24754800 & 0.87056500 \\
\hline $\mathrm{H}$ & -3.04381100 & 3.24720200 & -0.87090200 \\
\hline $\mathrm{H}$ & -3.04386100 & 3.24756700 & 0.87056400 \\
\hline $\mathrm{H}$ & -2.08382400 & 4.46973500 & -0.00039500 \\
\hline $\mathrm{H}$ & 3.68913200 & -1.73467700 & 2.15445300 \\
\hline $\mathrm{H}$ & 5.08917800 & -2.43688200 & 1.32421800 \\
\hline $\mathrm{H}$ & 4.82036800 & -0.67937900 & 1.27854300 \\
\hline $\mathrm{H}$ & 3.68800500 & -1.73382400 & -2.15463800 \\
\hline $\mathrm{H}$ & 4.82009000 & -0.67926300 & -1.27897100 \\
\hline $\mathrm{H}$ & 5.08819300 & -2.43686700 & -1.32538900 \\
\hline $\mathrm{H}$ & 2.16493800 & -3.29778900 & -0.88651400 \\
\hline $\mathrm{H}$ & 3.53711800 & -3.99681200 & -0.00030700 \\
\hline $\mathrm{H}$ & 2.16520600 & -3.29799000 & 0.88646100 \\
\hline $\mathrm{H}$ & -3.68811000 & -1.73372700 & -2.15461900 \\
\hline $\mathrm{H}$ & -5.08821500 & -2.43689100 & -1.32533200 \\
\hline $\mathrm{H}$ & -4.82019600 & -0.67927600 & -1.27882100 \\
\hline $\mathrm{H}$ & -3.68897900 & -1.73482800 & 2.15447400 \\
\hline $\mathrm{H}$ & -4.82032500 & -0.67954400 & 1.27868800 \\
\hline $\mathrm{H}$ & -5.08903800 & -2.43706200 & 1.32428900 \\
\hline $\mathrm{H}$ & -2.16507300 & -3.29801600 & 0.88629700 \\
\hline $\mathrm{H}$ & -3.53701600 & -3.99683800 & -0.00042300 \\
\hline $\mathrm{H}$ & -2.16491600 & -3.29771800 & -0.88667800 \\
\hline
\end{tabular}

Nucleophile-16

$\begin{array}{lrrr}\mathrm{N} & -0.00000200 & 2.23117400 & -0.00033700 \\ \mathrm{C} & -1.22479000 & 1.58685500 & -0.00011300 \\ \mathrm{C} & -1.26417800 & 0.22813900 & -0.00008400 \\ \mathrm{C} & 0.00001200 & -0.61354500 & -0.00043500\end{array}$




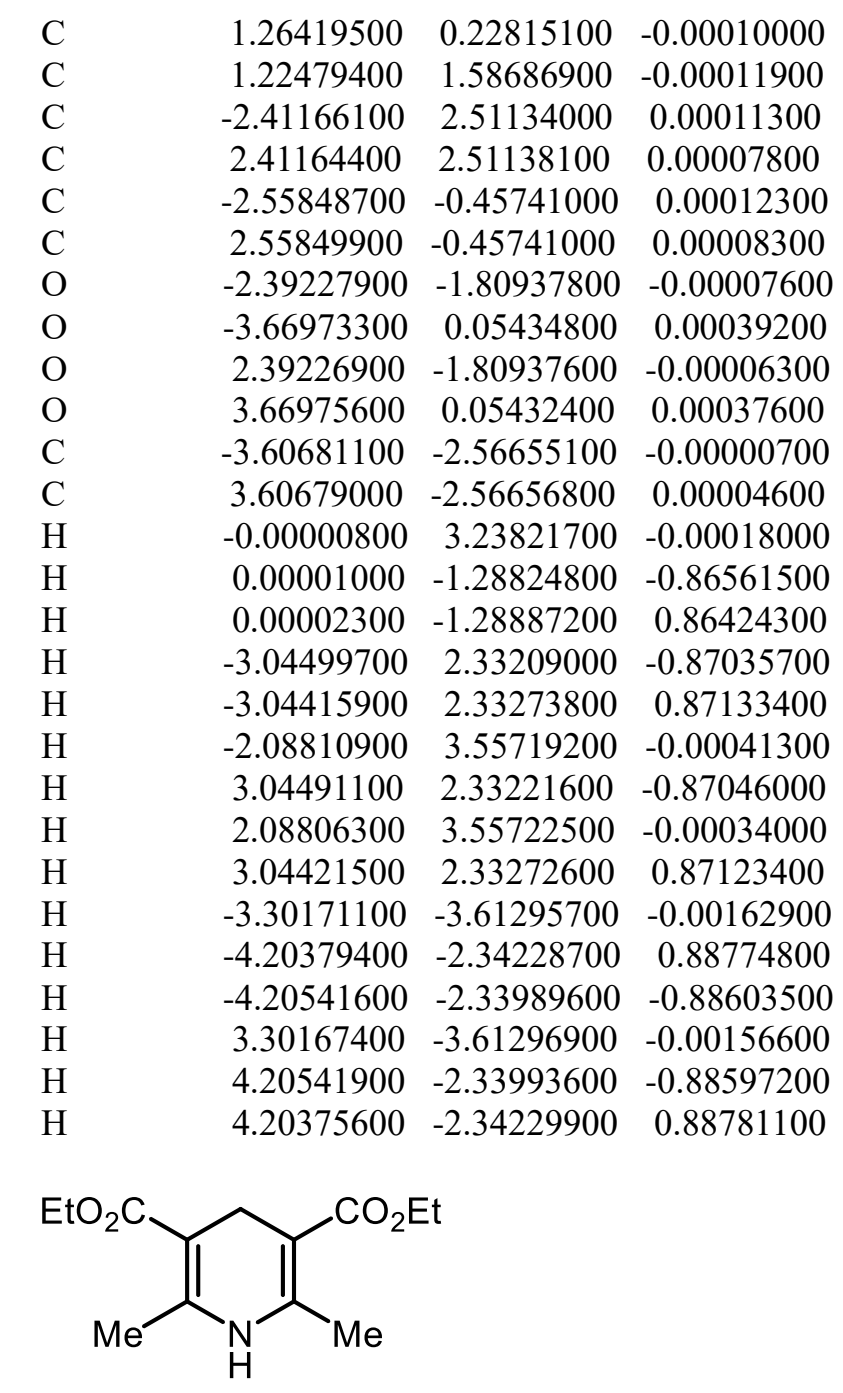

Nucleophile-17

$\begin{array}{lrrr}\mathrm{N} & 0.00000500 & -2.73287400 & 0.00038700 \\ \mathrm{C} & -1.22480500 & -2.08830600 & 0.00016500 \\ \mathrm{C} & -1.26413600 & -0.72973200 & 0.00000200 \\ \mathrm{C} & 0.00000800 & 0.11159600 & 0.00011800 \\ \mathrm{C} & 1.26415100 & -0.72973300 & -0.00000800 \\ \mathrm{C} & 1.22481700 & -2.08830900 & 0.00012600 \\ \mathrm{C} & -2.41207400 & -3.01232900 & 0.00016500 \\ \mathrm{C} & 2.41207600 & -3.01234500 & 0.00012400 \\ \mathrm{C} & -2.55785200 & -0.04175400 & -0.00023100 \\ \mathrm{C} & 2.55786200 & -0.04174400 & -0.00019800 \\ \mathrm{O} & -2.38753800 & 1.30816000 & -0.00046000 \\ \mathrm{O} & -3.66947600 & -0.55368200 & -0.00023600 \\ \mathrm{O} & 2.38753200 & 1.30816700 & -0.00055300 \\ \mathrm{O} & 3.66949300 & -0.55365600 & -0.00004000 \\ \mathrm{C} & -3.59669300 & 2.08870500 & -0.00053900 \\ \mathrm{C} & 3.59667800 & 2.08872700 & -0.00056300 \\ \mathrm{C} & -3.18882900 & 3.54854900 & 0.00078700\end{array}$




\begin{tabular}{|c|c|c|c|}
\hline $\mathrm{C}$ & 3.18879600 & 3.54856600 & 0.00068700 \\
\hline $\mathrm{H}$ & 0.00000400 & -3.73990900 & 0.00034600 \\
\hline $\mathrm{H}$ & 0.00001100 & 0.78712300 & 0.86474300 \\
\hline $\mathrm{H}$ & 0.00000600 & 0.78732700 & -0.86432900 \\
\hline $\mathrm{H}$ & -3.04481100 & -2.83322900 & 0.87110300 \\
\hline $\mathrm{H}$ & -3.04505400 & -2.83290400 & -0.87052200 \\
\hline $\mathrm{H}$ & -2.08898800 & -4.05836600 & -0.00006600 \\
\hline $\mathrm{H}$ & 3.04448200 & -2.83367900 & 0.87140100 \\
\hline $\mathrm{H}$ & 2.08897700 & -4.05837800 & -0.00071500 \\
\hline $\mathrm{H}$ & 3.04539000 & -2.83250200 & -0.87022400 \\
\hline $\mathrm{H}$ & -4.19171800 & 1.82619900 & 0.88002300 \\
\hline $\mathrm{H}$ & -4.19069300 & 1.82757600 & -0.88222000 \\
\hline $\mathrm{H}$ & 4.19074700 & 1.82757900 & -0.88219200 \\
\hline $\mathrm{H}$ & 4.19163900 & 1.82625300 & 0.88005100 \\
\hline $\mathrm{H}$ & -4.07813100 & 4.18614000 & 0.00069700 \\
\hline $\mathrm{H}$ & -2.59387700 & 3.78470100 & 0.88750600 \\
\hline $\mathrm{H}$ & -2.59276000 & 3.78601800 & -0.88482900 \\
\hline $\mathrm{H}$ & 4.07809100 & 4.18616700 & 0.00064300 \\
\hline $\mathrm{H}$ & 2.59279100 & 3.78600100 & -0.88498100 \\
\hline $\mathrm{H}$ & 2.59377600 & 3.78473800 & 0.88735500 \\
\hline
\end{tabular}

Nucleophile-18

$\begin{array}{lrrr}\mathrm{N} & -1.86967300 & -3.00997200 & 0.02496400 \\ \mathrm{C} & -2.60299600 & -1.83520800 & 0.02403300 \\ \mathrm{C} & -1.95162200 & -0.64355300 & 0.05974500 \\ \mathrm{C} & -0.43871800 & -0.55836000 & 0.12723000 \\ \mathrm{C} & 0.23099800 & -1.91615400 & 0.03366200 \\ \mathrm{C} & -0.48607700 & -3.06940200 & -0.00168800 \\ \mathrm{C} & -4.09383800 & -2.02863000 & -0.02646400 \\ \mathrm{C} & 0.07568200 & -4.46210600 & -0.08397100 \\ \mathrm{C} & -2.70925100 & 0.61055200 & 0.04040800 \\ \mathrm{C} & 1.69539600 & -1.95531900 & -0.01555600 \\ \mathrm{O} & -1.86167400 & 1.67276000 & 0.08487500 \\ \mathrm{O} & -3.92357500 & 0.74814900 & -0.01041800 \\ \mathrm{O} & 2.20378600 & -0.70060100 & 0.11646700 \\ \mathrm{O} & 2.41189800 & -2.93658500 & -0.15568600 \\ \mathrm{C} & -2.46078900 & 2.97897400 & 0.05556100 \\ \mathrm{C} & 3.63237800 & -0.57521000 & 0.04160500 \\ \mathrm{C} & -1.33111600 & 4.00369500 & -0.00070600 \\ \mathrm{C} & 3.98673400 & 0.88435200 & 0.30354100 \\ \mathrm{C} & 3.30806700 & 1.81923700 & -0.70400100 \\ \mathrm{C} & 5.51040000 & 1.05175200 & 0.29055400 \\ \mathrm{C} & -0.50947500 & 3.85955800 & -1.28800900 \\ \mathrm{C} & -0.43750200 & 3.92246600 & 1.24345300 \\ \mathrm{H} & -2.37679300 & -3.87917000 & -0.01640400 \\ \mathrm{H} & -0.12956400 & -0.04762600 & 1.04910300\end{array}$




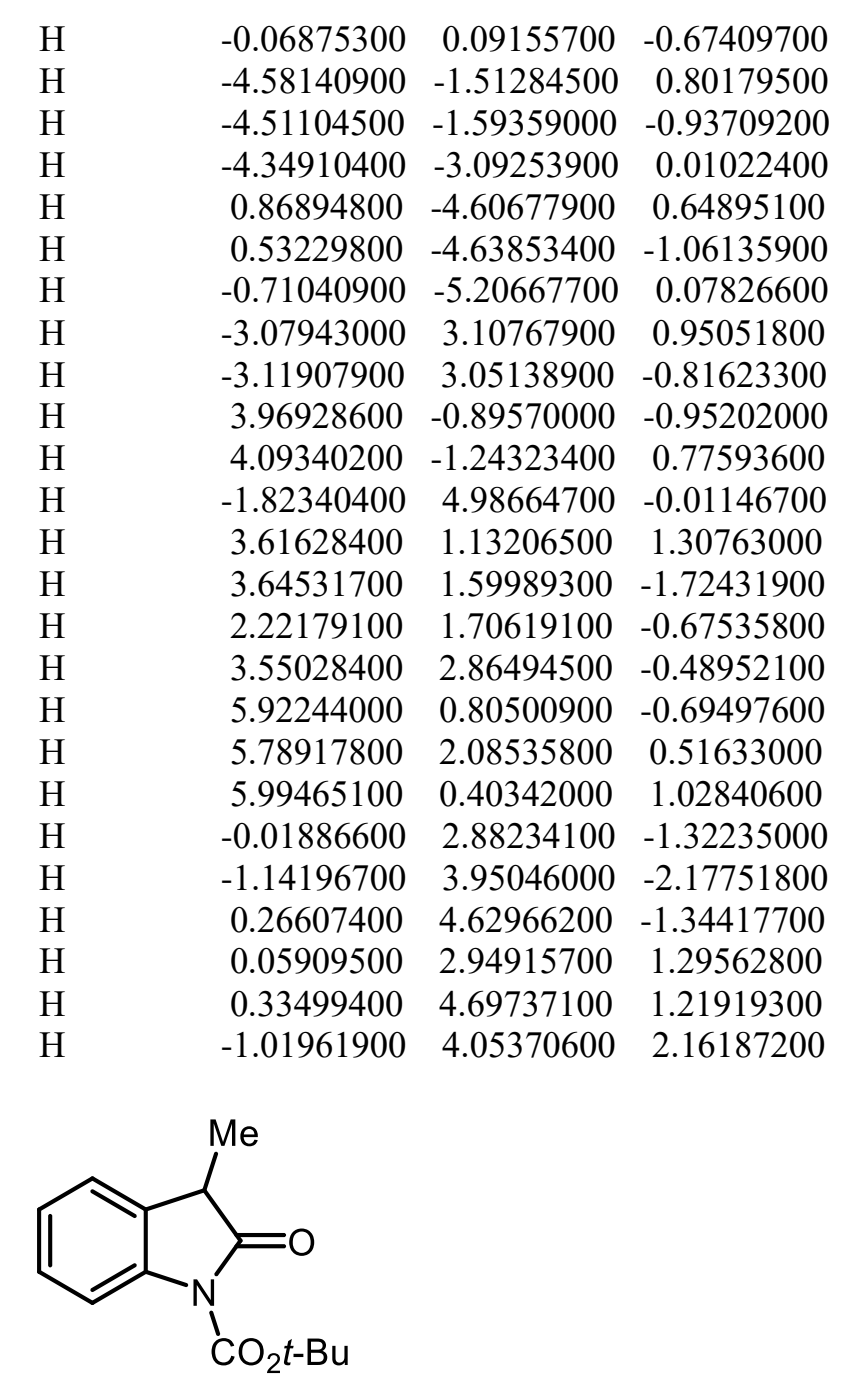

Nucleophile-19

$\begin{array}{lrrr}\mathrm{C} & -1.77917600 & -1.94938100 & 0.08490300 \\ \mathrm{C} & -1.51864100 & -0.58891100 & -0.05682000 \\ \mathrm{C} & -2.55589500 & 0.34129500 & -0.20837800 \\ \mathrm{C} & -3.87777900 & -0.07970600 & -0.21674100 \\ \mathrm{C} & -4.15956800 & -1.44202800 & -0.07465900 \\ \mathrm{C} & -3.11732600 & -2.35762100 & 0.07266200 \\ \mathrm{C} & -1.98600200 & 1.72424900 & -0.33922200 \\ \mathrm{C} & -0.47464500 & 1.50068800 & -0.21382700 \\ \mathrm{~N} & -0.26308300 & 0.09348400 & -0.08594000 \\ \mathrm{C} & 0.97459400 & -0.56384200 & 0.05010200 \\ \mathrm{O} & 1.05546400 & -1.74853300 & 0.31453100 \\ \mathrm{O} & 1.97934900 & 0.27681900 & -0.16296200 \\ \mathrm{C} & 3.38144400 & -0.15840300 & -0.00898300 \\ \mathrm{C} & 3.70197800 & -1.24357900 & -1.03655400 \\ \mathrm{C} & 3.62312400 & -0.62072900 & 1.42775900 \\ \mathrm{C} & 4.14647600 & 1.12883400 & -0.30815100 \\ \mathrm{O} & 0.36677400 & 2.36571100 & -0.20040100 \\ \mathrm{C} & -2.47607200 & 2.74834000 & 0.69311600\end{array}$




$\begin{array}{rrrr}\mathrm{H} & -0.97280200 & -2.65601000 & 0.20738800 \\ \mathrm{H} & -4.68105800 & 0.64195100 & -0.33247200 \\ \mathrm{H} & -5.18880400 & -1.78603100 & -0.07850800 \\ \mathrm{H} & -3.34146400 & -3.41399400 & 0.18361100 \\ \mathrm{H} & -2.15738500 & 2.12777900 & -1.34678700 \\ \mathrm{H} & 3.14655300 & -2.15814300 & -0.83018000 \\ \mathrm{H} & 3.45510200 & -0.89452700 & -2.04342100 \\ \mathrm{H} & 4.77317400 & -1.46481900 & -1.00579200 \\ \mathrm{H} & 4.68980300 & -0.81977600 & 1.56793300 \\ \mathrm{H} & 3.32709600 & 0.16308100 & 2.13094300 \\ \mathrm{H} & 3.06434900 & -1.52980600 & 1.64963200 \\ \mathrm{H} & 3.85396300 & 1.91660200 & 0.39012600 \\ \mathrm{H} & 5.22193000 & 0.95308800 & -0.21615600 \\ \mathrm{H} & 3.93201500 & 1.47452500 & -1.32250900 \\ \mathrm{H} & -2.34256300 & 2.36459000 & 1.70840300 \\ \mathrm{H} & -1.90845200 & 3.67631300 & 0.59260700 \\ \mathrm{H} & -3.53764800 & 2.96358100 & 0.54618600\end{array}$<smiles>CCC1C(=O)N(C(=O)OC(C)(C)C)c2ccccc21</smiles>

Nucleophile-20

$\begin{array}{lrrr}\mathrm{C} & -1.53065800 & -2.16430300 & 0.18362900 \\ \mathrm{C} & -1.34122700 & -0.82314400 & -0.13916500 \\ \mathrm{C} & -2.42153900 & 0.01234900 & -0.45284100 \\ \mathrm{C} & -3.71665700 & -0.48527000 & -0.44260200 \\ \mathrm{C} & -3.92760300 & -1.82928400 & -0.11893900 \\ \mathrm{C} & -2.84249200 & -2.65076400 & 0.18772800 \\ \mathrm{C} & -1.92611500 & 1.39688300 & -0.75552600 \\ \mathrm{C} & -0.40975300 & 1.28198100 & -0.56555100 \\ \mathrm{~N} & -0.12529200 & -0.07663400 & -0.22644100 \\ \mathrm{C} & 1.13938600 & -0.62938900 & 0.05097900 \\ \mathrm{O} & 1.27430000 & -1.75578300 & 0.49041000 \\ \mathrm{O} & 2.10432100 & 0.23264200 & -0.24513700 \\ \mathrm{C} & 3.51961300 & -0.08628500 & 0.02652000 \\ \mathrm{C} & 4.22401600 & 1.18907700 & -0.43080100 \\ \mathrm{C} & 3.95017200 & -1.28766100 & -0.81466700 \\ \mathrm{C} & 3.71769500 & -0.31713900 & 1.52448600 \\ \mathrm{O} & 0.38248600 & 2.18818300 & -0.65647600 \\ \mathrm{C} & -2.50519700 & 2.52845200 & 0.11764700 \\ \mathrm{C} & -2.31066200 & 2.30033100 & 1.61714600 \\ \mathrm{H} & -0.69156500 & -2.79685800 & 0.42931100 \\ \mathrm{H} & -4.55355200 & 0.16412600 & -0.68227100 \\ \mathrm{H} & -4.93513500 & -2.23224200 & -0.10598000 \\ \mathrm{H} & -3.01162200 & -3.69319300 & 0.43913900 \\ \mathrm{H} & -2.09217800 & 1.65433000 & -1.81028000 \\ \mathrm{H} & 5.30197600 & 1.09713200 & -0.27122700 \\ \mathrm{H} & 4.03953800 & 1.36823000 & -1.49299300\end{array}$




$\begin{array}{rrrr}\mathrm{H} & 3.85369300 & 2.05046600 & 0.13009300 \\ \mathrm{H} & 3.43836600 & -2.19549000 & -0.49625600 \\ \mathrm{H} & 3.73238200 & -1.10591400 & -1.87111300 \\ \mathrm{H} & 5.02951800 & -1.43376200 & -0.70960900 \\ \mathrm{H} & 3.20101900 & -1.21716300 & 1.85748400 \\ \mathrm{H} & 4.78590700 & -0.42608200 & 1.73457400 \\ \mathrm{H} & 3.34458600 & 0.54108900 & 2.09081200 \\ \mathrm{H} & -3.57164100 & 2.62866600 & -0.11391800 \\ \mathrm{H} & -2.01901400 & 3.46061600 & -0.18720600 \\ \mathrm{H} & -2.75025000 & 3.11934100 & 2.19347500 \\ \mathrm{H} & -2.78070400 & 1.36709200 & 1.94085400 \\ \mathrm{H} & -1.24702100 & 2.25072200 & 1.87156700\end{array}$<smiles>C=CCC1C(=O)N(C(=O)OC(C)(C)C)c2ccccc21</smiles>

Nucleophile-21

$\begin{array}{lrrr}\mathrm{C} & -1.48566700 & -2.25851600 & 0.37238100 \\ \mathrm{C} & -1.30331700 & -0.97897500 & -0.14587900 \\ \mathrm{C} & -2.38996200 & -0.20541600 & -0.57562200 \\ \mathrm{C} & -3.68248400 & -0.70193300 & -0.48816200 \\ \mathrm{C} & -3.88552100 & -1.98434000 & 0.03122700 \\ \mathrm{C} & -2.79495100 & -2.74515600 & 0.45333400 \\ \mathrm{C} & -1.90260300 & 1.12628200 & -1.06621800 \\ \mathrm{C} & -0.38130300 & 1.03188000 & -0.90916400 \\ \mathrm{~N} & -0.09166300 & -0.24885300 & -0.34868000 \\ \mathrm{C} & 1.17225300 & -0.73406300 & 0.03643700 \\ \mathrm{O} & 1.31180900 & -1.79389100 & 0.61742700 \\ \mathrm{O} & 2.13112100 & 0.10825500 & -0.32754500 \\ \mathrm{C} & 3.54202200 & -0.14973800 & 0.01974800 \\ \mathrm{C} & 4.01640200 & -1.43383600 & -0.66018300 \\ \mathrm{C} & 3.69825500 & -0.19763400 & 1.53957400 \\ \mathrm{C} & 4.24046800 & 1.07530900 & -0.56626300 \\ \mathrm{O} & 0.40755200 & 1.90205700 & -1.18577700 \\ \mathrm{C} & -2.42473700 & 2.35554900 & -0.28027000 \\ \mathrm{C} & -2.18472700 & 2.22722100 & 1.19883100 \\ \mathrm{C} & -1.24266600 & 2.89662400 & 1.86178600 \\ \mathrm{H} & -0.64267600 & -2.84435100 & 0.70481600 \\ \mathrm{H} & -4.52348300 & -0.09936900 & -0.81891700 \\ \mathrm{H} & -4.89064100 & -2.38661000 & 0.10563200 \\ \mathrm{H} & -2.95762000 & -3.73982400 & 0.85662800 \\ \mathrm{H} & -2.11707400 & 1.27630400 & -2.13168800 \\ \mathrm{H} & 3.50804300 & -2.30692600 & -0.25182500 \\ \mathrm{H} & 3.82961400 & -1.38179800 & -1.73672500 \\ \mathrm{H} & 5.09386000 & -1.54698700 & -0.50639600 \\ \mathrm{H} & 3.18760000 & -1.06276400 & 1.96216100 \\ & & & \end{array}$




$\begin{array}{lrrr}\mathrm{H} & 4.76132500 & -0.25768900 & 1.79143900 \\ \mathrm{H} & 3.29193200 & 0.71311700 & 1.98893600 \\ \mathrm{H} & 5.31507800 & 1.02070800 & -0.37083200 \\ \mathrm{H} & 4.08059800 & 1.12650100 & -1.64609900 \\ \mathrm{H} & 3.84426000 & 1.99001700 & -0.11888100 \\ \mathrm{H} & -3.49590000 & 2.46539700 & -0.48755000 \\ \mathrm{H} & -1.91411700 & 3.23971200 & -0.67308300 \\ \mathrm{H} & -2.80421500 & 1.50400000 & 1.72619100 \\ \mathrm{H} & -1.08356200 & 2.75284700 & 2.92611300 \\ \mathrm{H} & -0.59401300 & 3.60877000 & 1.35864600\end{array}$<smiles>CC(C)(C)ON1C(=O)C(Cc2ccc(Br)cc2)c2ccccc21</smiles>

Nucleophile-22

$\begin{array}{lrrr}\mathrm{C} & -2.56942200 & 0.32948700 & -2.09565400 \\ \mathrm{C} & -2.41180400 & 0.46689300 & -0.72038600 \\ \mathrm{C} & -3.23907700 & -0.21020100 & 0.18520500 \\ \mathrm{C} & -4.24565000 & -1.04529100 & -0.27776100 \\ \mathrm{C} & -4.42017500 & -1.19884200 & -1.65746000 \\ \mathrm{C} & -3.58655200 & -0.51871100 & -2.54695200 \\ \mathrm{C} & -2.78724200 & 0.07440900 & 1.58782400 \\ \mathrm{C} & -1.71570700 & 1.15505500 & 1.39860300 \\ \mathrm{~N} & -1.45022200 & 1.22819500 & 0.00350600 \\ \mathrm{C} & -0.33070100 & 1.82970600 & -0.60719400 \\ \mathrm{O} & -0.31056400 & 2.10582400 & -1.79051500 \\ \mathrm{O} & 0.64732800 & 1.98280500 & 0.27443000 \\ \mathrm{C} & 1.91700100 & 2.63842200 & -0.10239700 \\ \mathrm{C} & 1.63434800 & 4.08150400 & -0.51803600 \\ \mathrm{C} & 2.61698900 & 1.83769800 & -1.20008800 \\ \mathrm{C} & 2.70011700 & 2.58412600 & 1.20682100 \\ \mathrm{O} & -1.18204700 & 1.79978200 & 2.26887500 \\ \mathrm{C} & -2.11581700 & -1.15475400 & 2.28111600 \\ \mathrm{C} & -0.80500400 & -1.51300300 & 1.62093400 \\ \mathrm{C} & 0.39677300 & -1.03688800 & 2.15822400 \\ \mathrm{C} & 1.60711200 & -1.22580200 & 1.49639500 \\ \mathrm{C} & 1.60800300 & -1.88757100 & 0.27299000 \\ \mathrm{C} & 0.43336800 & -2.39072400 & -0.27979300 \\ \mathrm{C} & -0.76717500 & -2.20627100 & 0.40389500 \\ \mathrm{Br} & 3.25692900 & -2.08201000 & -0.67320900 \\ \mathrm{H} & -1.92605200 & 0.86265000 & -2.77988400 \\ \mathrm{H} & -4.88432800 & -1.57553500 & 0.42295800 \\ \mathrm{H} & -5.20565000 & -1.84490300 & -2.03573100 \\ & & & \end{array}$




$\begin{array}{rrrr}\mathrm{H} & -3.72814700 & -0.64238300 & -3.61606900 \\ \mathrm{H} & -3.58364200 & 0.45659800 & 2.23338500 \\ \mathrm{H} & 1.08897800 & 4.60303700 & 0.27370500 \\ \mathrm{H} & 2.58178600 & 4.60298200 & -0.68346100 \\ \mathrm{H} & 1.05088300 & 4.11814600 & -1.43836400 \\ \mathrm{H} & 3.61431800 & 2.25765100 & -1.36418600 \\ \mathrm{H} & 2.73558100 & 0.79135000 & -0.90852500 \\ \mathrm{H} & 2.05966900 & 1.88199800 & -2.13528000 \\ \mathrm{H} & 2.86976300 & 1.54665600 & 1.50432200 \\ \mathrm{H} & 3.66903400 & 3.07526100 & 1.08245900 \\ \mathrm{H} & 2.14541700 & 3.08601900 & 2.00334500 \\ \mathrm{H} & -2.82162500 & -1.99064900 & 2.24873500 \\ \mathrm{H} & -1.94525400 & -0.89883200 & 3.33014600 \\ \mathrm{H} & 0.38187300 & -0.47862500 & 3.08834000 \\ \mathrm{H} & 2.53358100 & -0.85155900 & 1.91484500 \\ \mathrm{H} & 0.45606900 & -2.90807700 & -1.23148400 \\ \mathrm{H} & -1.68728600 & -2.58154200 & -0.03154200\end{array}$<smiles>CCC(C)OC(=O)N1C(=O)C(/C=C/c2ccccc2)c2ccccc21</smiles>

Nucleophile-23

$\begin{array}{lrrr}\mathrm{C} & 1.33110800 & 2.86792000 & -0.75596600 \\ \mathrm{C} & 0.88288100 & 1.84457700 & 0.07546500 \\ \mathrm{C} & -0.31010200 & 1.96488700 & 0.80010700 \\ \mathrm{C} & -1.07422000 & 3.11983300 & 0.70742300 \\ \mathrm{C} & -0.64101400 & 4.15715200 & -0.12403400 \\ \mathrm{C} & 0.54633200 & 4.02268000 & -0.84420200 \\ \mathrm{C} & -0.54566500 & 0.72085400 & 1.60424200 \\ \mathrm{C} & 0.70677100 & -0.13208700 & 1.31625500 \\ \mathrm{~N} & 1.48223000 & 0.57725500 & 0.35163200 \\ \mathrm{C} & 2.67989700 & 0.14223500 & -0.24975500 \\ \mathrm{O} & 3.34604000 & 0.86541600 & -0.96580200 \\ \mathrm{O} & 2.93332100 & -1.12036200 & 0.06846900 \\ \mathrm{C} & 4.16189700 & -1.79225800 & -0.40029800 \\ \mathrm{C} & 4.02411500 & -3.17668500 & 0.22900500 \\ \mathrm{C} & 5.38892900 & -1.06017000 & 0.14239500 \\ \mathrm{C} & 4.15454000 & -1.87549200 & -1.92642000 \\ \mathrm{O} & 0.96103800 & -1.19848100 & 1.81782600 \\ \mathrm{C} & -1.77134900 & -0.09920500 & 1.27019400 \\ \mathrm{C} & -2.64472500 & 0.17985800 & 0.29510800 \\ \mathrm{C} & -3.82531000 & -0.60932200 & -0.07845700 \\ \mathrm{C} & -4.13537000 & -1.85518600 & 0.49552100\end{array}$




$\begin{array}{lrrr}\mathrm{C} & -5.27086200 & -2.55595900 & 0.10453800 \\ \mathrm{C} & -6.12426500 & -2.03342300 & -0.87123900 \\ \mathrm{C} & -5.82798100 & -0.80199700 & -1.45396600 \\ \mathrm{C} & -4.69023700 & -0.10050200 & -1.06105800 \\ \mathrm{H} & 2.25414700 & 2.76735200 & -1.30572900 \\ \mathrm{H} & -1.99727400 & 3.20767800 & 1.27193200 \\ \mathrm{H} & -1.22762500 & 5.06627900 & -0.20702500 \\ \mathrm{H} & 0.87882600 & 4.83095600 & -1.48807700 \\ \mathrm{H} & -0.54004500 & 0.92545600 & 2.68423500 \\ \mathrm{H} & 4.88959300 & -3.79209100 & -0.03201400 \\ \mathrm{H} & 3.96069000 & -3.09619500 & 1.31678700 \\ \mathrm{H} & 3.11864300 & -3.67185800 & -0.13046000 \\ \mathrm{H} & 5.48005600 & -0.06503000 & -0.29262700 \\ \mathrm{H} & 5.32465700 & -0.97148500 & 1.23071500 \\ \mathrm{H} & 6.28794800 & -1.63450100 & -0.10049300 \\ \mathrm{H} & 4.23926500 & -0.88630300 & -2.37573800 \\ \mathrm{H} & 4.99786200 & -2.48862400 & -2.25834300 \\ \mathrm{H} & 3.23163500 & -2.34864500 & -2.27405700 \\ \mathrm{H} & -1.87370700 & -0.99373100 & 1.87856200 \\ \mathrm{H} & -2.49218800 & 1.08317300 & -0.29091100 \\ \mathrm{H} & -3.47983100 & -2.28220300 & 1.24729400 \\ \mathrm{H} & -5.49111900 & -3.51707100 & 0.55936500 \\ \mathrm{H} & -7.00885800 & -2.58450600 & -1.17491000 \\ \mathrm{H} & -6.48155300 & -0.38709800 & -2.21531500 \\ \mathrm{H} & -4.46214500 & 0.85882100 & -1.51772400\end{array}$<smiles>CCOC(=O)CC(C)=O</smiles>

Nucleophile-24

$\begin{array}{lrrr}\mathrm{C} & -3.28273800 & 0.38461300 & 0.24761600 \\ \mathrm{C} & -1.80198700 & 0.32997900 & -0.06437100 \\ \mathrm{C} & -1.10270300 & -0.99545800 & 0.25963600 \\ \mathrm{O} & -1.20099400 & 1.26874600 & -0.54396200 \\ \mathrm{C} & 0.36678500 & -0.92694600 & -0.08929800 \\ \mathrm{O} & 1.04627500 & -0.27964000 & 0.87610900 \\ \mathrm{O} & 0.85399300 & -1.36852400 & -1.10565400 \\ \mathrm{C} & 2.42890800 & 0.03357800 & 0.57817400 \\ \mathrm{C} & 2.51841000 & 1.29380100 & -0.26574500 \\ \mathrm{H} & -3.45064500 & 0.21704600 & 1.31749500 \\ \mathrm{H} & -3.68326700 & 1.35702700 & -0.03989500 \\ \mathrm{H} & -3.81500200 & -0.40926500 & -0.28806100 \\ \mathrm{H} & -1.57085800 & -1.79892100 & -0.31679700 \\ \mathrm{H} & -1.24064900 & -1.21495200 & 1.32400200 \\ \mathrm{H} & 2.88151800 & -0.82093700 & 0.07007100 \\ \mathrm{H} & 2.89522800 & 0.16459700 & 1.55686500 \\ \mathrm{H} & 2.02105300 & 2.12864200 & 0.23445000 \\ \mathrm{H} & 3.56794100 & 1.55764000 & -0.43143100 \\ \mathrm{H} & 2.04044100 & 1.13707100 & -1.23472000\end{array}$


<smiles>CC(=O)CC(=O)OC(c1ccccc1)c1ccccc1</smiles>

Nucleophile-25

\begin{tabular}{lrrr}
$\mathrm{C}$ & 1.40391400 & -1.32194000 & 1.21917300 \\
$\mathrm{C}$ & 2.34649300 & -2.28862600 & 0.54247800 \\
$\mathrm{O}$ & 1.62506600 & -0.73201800 & 2.25414600 \\
$\mathrm{C}$ & 2.98063800 & -1.67943600 & -0.72299100 \\
$\mathrm{O}$ & 3.14958600 & -2.36229200 & -1.71056500 \\
$\mathrm{O}$ & 0.29665100 & -1.15327900 & 0.46306500 \\
$\mathrm{C}$ & -0.59541200 & -0.06759500 & 0.84836500 \\
$\mathrm{C}$ & -1.98114200 & -0.39397700 & 0.33987800 \\
$\mathrm{C}$ & -0.02664300 & 1.22155400 & 0.28731700 \\
$\mathrm{C}$ & 3.38253900 & -0.22341100 & -0.64977600 \\
$\mathrm{C}$ & 0.69546700 & 2.08838000 & 1.11267600 \\
$\mathrm{C}$ & 1.28587900 & 3.23582800 & 0.58169200 \\
$\mathrm{C}$ & 1.16506700 & 3.51858600 & -0.77864000 \\
$\mathrm{C}$ & 0.44652500 & 2.65368700 & -1.60778200 \\
$\mathrm{C}$ & -0.14973000 & 1.51223700 & -1.07670700 \\
$\mathrm{C}$ & -3.04550800 & 0.41408300 & 0.75722100 \\
$\mathrm{C}$ & -4.33709500 & 0.17367200 & 0.29755900 \\
$\mathrm{C}$ & -4.57978300 & -0.87831400 & -0.58890800 \\
$\mathrm{C}$ & -3.52281500 & -1.68353600 & -1.00838500 \\
$\mathrm{C}$ & -2.22634000 & -1.44383900 & -0.54738700 \\
$\mathrm{H}$ & 1.83105200 & -3.20370600 & 0.24612800 \\
$\mathrm{H}$ & 3.14523700 & -2.52870000 & 1.25056700 \\
$\mathrm{H}$ & -0.59727600 & -0.01464300 & 1.93966800 \\
$\mathrm{H}$ & 4.06448100 & 0.01066000 & -1.46759300 \\
$\mathrm{H}$ & 2.48593900 & 0.40061100 & -0.74570100 \\
$\mathrm{H}$ & 3.83570400 & 0.01789100 & 0.31621400 \\
$\mathrm{H}$ & 0.81295700 & 1.84585900 & 2.16336400 \\
$\mathrm{H}$ & 1.84429000 & 3.90420600 & 1.22978800 \\
$\mathrm{H}$ & 1.62722100 & 4.40945800 & -1.19272500 \\
$\mathrm{H}$ & 0.34849900 & 2.8711900 & -2.66687500 \\
$\mathrm{H}$ & -0.71604900 & 0.84211600 & -1.71546900 \\
$\mathrm{H}$ & -2.85557800 & 1.23848700 & 1.43920500 \\
$\mathrm{H}$ & -5.15456200 & 0.80565700 & 0.63073100 \\
$\mathrm{H}$ & -5.58666900 & -1.06722500 & -0.94800500 \\
$\mathrm{H}$ & -3.70300900 & -2.50320900 & -1.69726600 \\
$\mathrm{H}$ & -1.40499900 & -2.07198500 & -0.86989200 \\
& & & \\
\hline
\end{tabular}<smiles>CCCCOC(=O)CC(C)=O</smiles>

Nucleophile-26
C
$\begin{array}{lll}-0.21883300 & 0.94738200 & -0.09847500\end{array}$
$\begin{array}{lllll}\text { C } & -1.63052400 & 0.82699900 & -0.63538100\end{array}$ 


$\begin{array}{lrrr}\mathrm{O} & 0.12946800 & 1.79719300 & 0.69272500 \\ \mathrm{C} & -2.40181700 & -0.28558900 & 0.08279500 \\ \mathrm{O} & -1.88752600 & -0.97559600 & 0.93877700 \\ \mathrm{O} & 0.54245700 & -0.03021700 & -0.60900100 \\ \mathrm{C} & 1.89350500 & -0.29762200 & -0.07978400 \\ \mathrm{C} & 1.80117700 & -0.59753200 & 1.41710200 \\ \mathrm{C} & 2.81426300 & 0.88304500 & -0.38883100 \\ \mathrm{C} & 2.31364000 & -1.54146000 & -0.86011600 \\ \mathrm{C} & -3.84583800 & -0.45987800 & -0.33995500 \\ \mathrm{H} & -1.61901400 & 0.60385100 & -1.70810000 \\ \mathrm{H} & -2.15133700 & 1.77842200 & -0.49252400 \\ \mathrm{H} & 1.02966800 & -1.35029300 & 1.59805000 \\ \mathrm{H} & 2.76223000 & -0.98384300 & 1.77017600 \\ \mathrm{H} & 1.55168400 & 0.29904700 & 1.98408200 \\ \mathrm{H} & 2.81423400 & 1.09438900 & -1.46246700 \\ \mathrm{H} & 2.49648500 & 1.77554800 & 0.14961300 \\ \mathrm{H} & 3.83755800 & 0.63443100 & -0.09054400 \\ \mathrm{H} & 2.31165800 & -1.34031700 & -1.93508200 \\ \mathrm{H} & 3.32201300 & -1.84497600 & -0.56508100 \\ \mathrm{H} & 1.62715900 & -2.36837600 & -0.66003800 \\ \mathrm{H} & -4.40841800 & 0.46146100 & -0.15162100 \\ \mathrm{H} & -3.90793800 & -0.65841200 & -1.41571300 \\ \mathrm{H} & -4.29460200 & -1.28405200 & 0.21509800\end{array}$<smiles>CCOC(=O)CC(=O)c1ccccc1</smiles>

Nucleophile-27

$\begin{array}{lrrr}\mathrm{C} & 1.89596500 & 0.37006600 & -0.64217400 \\ \mathrm{C} & 1.13805400 & 1.67416400 & -0.80532900 \\ \mathrm{O} & 2.24542800 & -0.35478100 & -1.54919100 \\ \mathrm{C} & -0.11913500 & 1.73102600 & 0.07090900 \\ \mathrm{O} & -0.26483700 & 2.64143200 & 0.86791600 \\ \mathrm{C} & -1.14131500 & 0.65191100 & -0.07610300 \\ \mathrm{C} & -2.18890600 & 0.61198200 & 0.85640300 \\ \mathrm{C} & -3.16319600 & -0.37601500 & 0.77711200 \\ \mathrm{C} & -3.10611200 & -1.33233600 & -0.24093800 \\ \mathrm{C} & -2.07301400 & -1.29482600 & -1.17760100 \\ \mathrm{C} & -1.09172100 & -0.30775900 & -1.09832100 \\ \mathrm{O} & 2.10533400 & 0.11697600 & 0.66378800 \\ \mathrm{C} & 2.67580400 & -1.17571500 & 0.98734900 \\ \mathrm{C} & 1.58741800 & -2.23497500 & 1.03417400 \\ \mathrm{H} & 0.88946200 & 1.78863000 & -1.86303500 \\ \mathrm{H} & 1.77028200 & 2.50857500 & -0.49384500 \\ \mathrm{H} & -2.20991700 & 1.36744000 & 1.63383400 \\ \mathrm{H} & -3.96767400 & -0.40462500 & 1.50539500 \\ \mathrm{H} & -3.86696500 & -2.10442700 & -0.30363700 \\ \mathrm{H} & -2.02977500 & -2.03375900 & -1.97137600 \\ \mathrm{H} & -0.29193000 & -0.29876200 & -1.82949900 \\ \mathrm{H} & 3.14287300 & -1.02934200 & 1.96294900 \\ \mathrm{H} & 3.44115800 & -1.41829600 & 0.24733300\end{array}$




$$
\begin{array}{llll}
\mathrm{H} & 2.01172200 & -3.19248000 & 1.35224500 \\
\mathrm{H} & 0.80210000 & -1.94985400 & 1.73932600 \\
\mathrm{H} & 1.13821700 & -2.36725400 & 0.04732500
\end{array}
$$<smiles>CCCOC(=O)CC(=O)c1ccccc1</smiles>

Nucleophile-28

$\begin{array}{lrrr}\mathrm{C} & -1.44402900 & 0.75028000 & -0.97384100 \\ \mathrm{C} & -0.55931400 & 1.97003100 & -0.78851000 \\ \mathrm{O} & -1.70663700 & 0.23459800 & -2.03995900 \\ \mathrm{C} & 0.52944900 & 1.74237900 & 0.26609200 \\ \mathrm{O} & 0.61271000 & 2.48719000 & 1.22729900 \\ \mathrm{C} & 1.46740300 & 0.59378300 & 0.08614900 \\ \mathrm{C} & 1.50959100 & -0.17590500 & -1.08601300 \\ \mathrm{C} & 2.40266300 & -1.24129300 & -1.19016700 \\ \mathrm{C} & 3.25609100 & -1.54604000 & -0.12959200 \\ \mathrm{C} & 3.22169200 & -0.77978000 & 1.03912200 \\ \mathrm{C} & 2.33537200 & 0.28562500 & 1.14445800 \\ \mathrm{O} & -1.87553900 & 0.30890000 & 0.22038900 \\ \mathrm{C} & -2.61822800 & -0.94837400 & 0.23892000 \\ \mathrm{C} & -3.46897000 & -0.90852500 & 1.49637400 \\ \mathrm{C} & -1.62175400 & -2.09971100 & 0.20884200 \\ \mathrm{H} & -1.16143700 & 2.81047600 & -0.43613600 \\ \mathrm{H} & -0.12597100 & 2.22265300 & -1.75911200 \\ \mathrm{H} & 0.84767200 & 0.04122900 & -1.91616600 \\ \mathrm{H} & 2.43102300 & -1.83251400 & -2.09990600 \\ \mathrm{H} & 3.94823300 & -2.37850400 & -0.21267000 \\ \mathrm{H} & 3.88650300 & -1.01635400 & 1.86403600 \\ \mathrm{H} & 2.28892200 & 0.89794000 & 2.03804800 \\ \mathrm{H} & -3.24373600 & -0.97045400 & -0.65732300 \\ \mathrm{H} & -4.07111900 & -1.81896400 & 1.56834600 \\ \mathrm{H} & -2.83676700 & -0.84029300 & 2.38670600 \\ \mathrm{H} & -4.14203700 & -0.04739700 & 1.48334600 \\ \mathrm{H} & -0.94429700 & -2.03862000 & 1.06579000 \\ \mathrm{H} & -2.15184200 & -3.05614300 & 0.25010500 \\ \mathrm{H} & -1.02921800 & -2.07338100 & -0.70791300\end{array}$

\begin{tabular}{|c|c|c|c|}
\hline $\mathrm{C}$ & 0.24820600 & -1.38202900 & 1.88709900 \\
\hline $\mathrm{C}$ & 1.13059100 & -2.54652600 & 1.47831800 \\
\hline $\mathrm{O}$ & 0.34486100 & -0.74650100 & 2.91347400 \\
\hline $\mathrm{C}$ & 1.57002200 & -2.43188800 & 0.01398800 \\
\hline $\mathrm{O}$ & 1.26081400 & -3.30214500 & -0.78286200 \\
\hline $\mathrm{C}$ & 2.35771600 & -1.23434900 & -0.39991500 \\
\hline
\end{tabular}<smiles>O=C(CC(=O)c1ccccc1)OC(c1ccccc1)c1ccccc1</smiles>

Nucleophile-29 


$\begin{array}{lrrr}\mathrm{C} & 2.65667200 & -1.08623900 & -1.76255900 \\ \mathrm{C} & 3.37827900 & 0.01490900 & -2.20746400 \\ \mathrm{C} & 3.80985800 & 0.98077200 & -1.29428900 \\ \mathrm{C} & 3.51616200 & 0.84196500 & 0.06171000 \\ \mathrm{C} & 2.79330300 & -0.26023800 & 0.51077100 \\ \mathrm{O} & -0.65283100 & -1.13966300 & 0.91596900 \\ \mathrm{C} & -1.42512000 & 0.08813100 & 1.01758700 \\ \mathrm{C} & -2.66527900 & -0.08542200 & 0.16955600 \\ \mathrm{C} & -0.55056300 & 1.24437900 & 0.57246500 \\ \mathrm{C} & -0.05093300 & 2.15169800 & 1.50879400 \\ \mathrm{C} & 0.77299400 & 3.20171300 & 1.09996200 \\ \mathrm{C} & 1.11139800 & 3.33898500 & -0.24513400 \\ \mathrm{C} & 0.62703400 & 2.42305300 & -1.18208600 \\ \mathrm{C} & -0.20256500 & 1.38290900 & -0.77615200 \\ \mathrm{C} & -3.69004500 & 0.86007000 & 0.28918800 \\ \mathrm{C} & -4.83522700 & 0.76268300 & -0.49623000 \\ \mathrm{C} & -4.96717500 & -0.28217800 & -1.41379000 \\ \mathrm{C} & -3.94758400 & -1.22417600 & -1.53714500 \\ \mathrm{C} & -2.79821000 & -1.12895800 & -0.74967600 \\ \mathrm{H} & 0.56703900 & -3.47865400 & 1.56422900 \\ \mathrm{H} & 1.98361700 & -2.58164200 & 2.15947700 \\ \mathrm{H} & 2.30826800 & -1.85051600 & -2.44814800 \\ \mathrm{H} & 3.60484300 & 0.12482500 & -3.26350000 \\ \mathrm{H} & 4.36897300 & 1.84440800 & -1.64141700 \\ \mathrm{H} & 3.83541700 & 1.59922300 & 0.76935600 \\ \mathrm{H} & 2.56276500 & -0.34243000 & 1.56622400 \\ \mathrm{H} & -1.69747000 & 0.22520000 & 2.06701400 \\ \mathrm{H} & -0.29637100 & 2.02287400 & 2.55796600 \\ \mathrm{H} & 1.15216600 & 3.90713200 & 1.83315200 \\ \mathrm{H} & 1.75518700 & 4.15318000 & -0.56407800 \\ \mathrm{H} & 0.90092900 & 2.51756200 & -2.22781200 \\ \mathrm{H} & -0.58701200 & 0.67409900 & -1.50206100 \\ \mathrm{H} & -3.58286500 & 1.67867200 & 0.99581200 \\ \mathrm{H} & -5.62501500 & 1.50040000 & -0.39243600 \\ \mathrm{H} & -5.85997300 & -0.35982300 & -2.02654400 \\ \mathrm{H} & -4.04283200 & -2.03962000 & -2.24770600 \\ \mathrm{H} & -2.00762700 & -1.86400300 & -0.84216700 \\ & & & \\ & & & \end{array}$<smiles>CCOC(=O)CC(=O)[18OH]</smiles>

Nucleophile-30

$\begin{array}{lrrr}\mathrm{C} & 0.15142600 & 1.18080200 & -0.08155500 \\ \mathrm{C} & -1.20174400 & 1.79055600 & -0.13169800 \\ \mathrm{C} & -2.25455100 & 1.16693000 & -1.06085400 \\ \mathrm{O} & -1.49419600 & 2.76212000 & 0.54659400 \\ \mathrm{C} & -2.48987100 & -0.29942100 & -0.75604300 \\ \mathrm{O} & -2.78414500 & -0.46179700 & 0.54807200 \\ \mathrm{O} & -2.40059200 & -1.20548500 & -1.55803200 \\ \mathrm{C} & -2.85816800 & -1.82740500 & 1.02621100 \\ \mathrm{C} & -1.46667700 & -2.34982100 & 1.34313800\end{array}$




$\begin{array}{lrrr}\mathrm{C} & 1.05589300 & 1.65190600 & 0.88927900 \\ \mathrm{C} & 2.32489700 & 1.11722600 & 0.99660900 \\ \mathrm{C} & 2.73086800 & 0.09260100 & 0.12321000 \\ \mathrm{C} & 1.84801000 & -0.38145000 & -0.85524800 \\ \mathrm{C} & 0.56910800 & 0.16420600 & -0.95012600 \\ \mathrm{O} & 3.99323900 & -0.36984300 & 0.30826900 \\ \mathrm{C} & 4.46074300 & -1.40797700 & -0.54251900 \\ \mathrm{H} & -1.95806800 & 1.24966400 & -2.10900600 \\ \mathrm{H} & -3.17666800 & 1.72972900 & -0.90140200 \\ \mathrm{H} & -3.35476100 & -2.43989100 & 0.27082400 \\ \mathrm{H} & -3.48314700 & -1.76909800 & 1.91920000 \\ \mathrm{H} & -0.86137500 & -2.40261100 & 0.43530100 \\ \mathrm{H} & -0.96368000 & -1.69492000 & 2.05963900 \\ \mathrm{H} & -1.53321500 & -3.35381700 & 1.77406300 \\ \mathrm{H} & 0.72561000 & 2.44337700 & 1.55259500 \\ \mathrm{H} & 3.02878900 & 1.46654200 & 1.74396400 \\ \mathrm{H} & 2.14266700 & -1.16538500 & -1.54102500 \\ \mathrm{H} & -0.09921600 & -0.22483500 & -1.70920700 \\ \mathrm{H} & 4.46973100 & -1.09198500 & -1.59252500 \\ \mathrm{H} & 5.47989600 & -1.62270800 & -0.22076700 \\ \mathrm{H} & 3.84937600 & -2.31293400 & -0.44329800\end{array}$<smiles>CCOC(=O)CC(=O)c1cccc(C(F)(F)F)c1</smiles>

Nucleophile-31

$\begin{array}{lrrr}\mathrm{C} & 2.24572500 & -0.96727500 & -0.70534000 \\ \mathrm{C} & 2.46030500 & 0.36114200 & -1.40995300 \\ \mathrm{O} & 1.80722000 & -1.96802900 & -1.22951000 \\ \mathrm{C} & 1.91558000 & 1.55408600 & -0.62142000 \\ \mathrm{O} & 2.62977300 & 2.51028200 & -0.37902500 \\ \mathrm{C} & 0.49398200 & 1.51454500 & -0.15366100 \\ \mathrm{C} & 0.06243100 & 2.51784500 & 0.72692600 \\ \mathrm{C} & -1.23600700 & 2.50889900 & 1.22235500 \\ \mathrm{C} & -2.12095700 & 1.49546500 & 0.84836800 \\ \mathrm{C} & -1.69874900 & 0.50328200 & -0.03586200 \\ \mathrm{C} & -0.40009100 & 0.51320000 & -0.54499200 \\ \mathrm{O} & 2.59115300 & -0.86731600 & 0.59146200 \\ \mathrm{C} & 2.29576900 & -2.01440700 & 1.43105800 \\ \mathrm{C} & 0.86093200 & -1.95382200 & 1.92699700 \\ \mathrm{C} & -2.60748100 & -0.63798100 & -0.39813800 \\ \mathrm{~F} & -2.31668700 & -1.74549900 & 0.33039600 \\ \mathrm{~F} & -3.90570300 & -0.34740000 & -0.17206000 \\ \mathrm{~F} & -2.48256400 & -0.98352000 & -1.69673400 \\ \mathrm{H} & 1.99034700 & 0.29534000 & -2.39479100 \\ \mathrm{H} & 3.53013900 & 0.53825000 & -1.54338500 \\ \mathrm{H} & 0.77116300 & 3.28818100 & 1.00918500 \\ \mathrm{H} & -1.56392200 & 3.28629600 & 1.90447200 \\ \mathrm{H} & -3.13350200 & 1.47525600 & 1.23434400\end{array}$




$$
\begin{array}{lrrr}
\mathrm{H} & -0.10361000 & -0.27031300 & -1.23148000 \\
\mathrm{H} & 3.01186500 & -1.93906900 & 2.25130400 \\
\mathrm{H} & 2.48666700 & -2.92492100 & 0.85997300 \\
\mathrm{H} & 0.67085400 & -1.00785500 & 2.44163500 \\
\mathrm{H} & 0.67884500 & -2.77260400 & 2.63025700 \\
\mathrm{H} & 0.15194500 & -2.05015900 & 1.10262800
\end{array}
$$<smiles>COc1ccc(C(=O)CC(=O)c2ccc(Cl)cc2)cc1</smiles>

Nucleophile-32

$\begin{array}{lrrr}\mathrm{C} & 1.99146700 & 0.89438100 & 0.37134200 \\ \mathrm{C} & 0.73331300 & 1.44043500 & 0.92377700 \\ \mathrm{C} & 0.01501300 & 0.66467500 & 2.03984000 \\ \mathrm{O} & 0.25119800 & 2.49054800 & 0.51624500 \\ \mathrm{C} & -0.71545700 & -0.58253900 & 1.53716300 \\ \mathrm{O} & -0.25259200 & -1.69330800 & 1.75781800 \\ \mathrm{C} & 2.67368000 & 1.65086800 & -0.60285200 \\ \mathrm{C} & 3.85146600 & 1.19335900 & -1.15844700 \\ \mathrm{C} & 4.38055500 & -0.04703800 & -0.75740400 \\ \mathrm{C} & 3.71104000 & -0.81548500 & 0.20389100 \\ \mathrm{C} & 2.52745400 & -0.34032100 & 0.76310200 \\ \mathrm{C} & -1.98958300 & -0.41568100 & 0.78395800 \\ \mathrm{C} & -2.68787300 & -1.57573700 & 0.41289200 \\ \mathrm{C} & -3.88258000 & -1.49282500 & -0.28823300 \\ \mathrm{C} & -4.37817000 & -0.23165200 & -0.62774900 \\ \mathrm{C} & -3.69959800 & 0.93490100 & -0.28001200 \\ \mathrm{C} & -2.50580100 & 0.84022800 & 0.43069300 \\ \mathrm{O} & 5.53932600 & -0.41075900 & -1.36008600 \\ \mathrm{Cl} & -5.88632400 & -0.11585600 & -1.51410300 \\ \mathrm{C} & 6.12205800 & -1.65805000 & -1.00397600 \\ \mathrm{H} & -0.68911500 & 1.36089000 & 2.49850800 \\ \mathrm{H} & 0.72848800 & 0.32872500 & 2.79379300 \\ \mathrm{H} & 2.24818100 & 2.60164500 & -0.90330800 \\ \mathrm{H} & 4.38912300 & 1.76575800 & -1.90621100 \\ \mathrm{H} & 4.09772800 & -1.77634400 & 0.51825100 \\ \mathrm{H} & 2.01022300 & -0.95938800 & 1.48688700 \\ \mathrm{H} & -2.27059900 & -2.53751900 & 0.68881300 \\ \mathrm{H} & -4.42838100 & -2.38465800 & -0.57302600 \\ \mathrm{H} & -4.10001600 & 1.90090500 & -0.56376000 \\ \mathrm{H} & -1.96683900 & 1.74784900 & 0.67690000 \\ \mathrm{H} & 6.38177700 & -1.68864200 & 0.06081300 \\ \mathrm{H} & 7.03003400 & -1.74499100 & -1.60080800 \\ \mathrm{H} & 5.45154000 & -2.49364500 & -1.23683300\end{array}$


<smiles>COc1ccc(C(=O)C(F)C(=O)c2ccc(Cl)cc2)cc1</smiles>

Nucleophile-33

$\begin{array}{lrrr}\mathrm{C} & 2.05529700 & 0.79580400 & -0.30503900 \\ \mathrm{C} & 0.78955400 & 1.54911800 & -0.22534600 \\ \mathrm{C} & 0.03797300 & 1.61874600 & 1.12707300 \\ \mathrm{O} & 0.30056400 & 2.12801200 & -1.18259700 \\ \mathrm{C} & -0.68545700 & 0.29828300 & 1.44559300 \\ \mathrm{O} & -0.15089500 & -0.48025700 & 2.22309400 \\ \mathrm{C} & 2.73784400 & 0.78907300 & -1.53880800 \\ \mathrm{C} & 3.93045400 & 0.11056800 & -1.68455600 \\ \mathrm{C} & 4.47609300 & -0.59090200 & -0.59362200 \\ \mathrm{C} & 3.80715000 & -0.59992700 & 0.63773800 \\ \mathrm{C} & 2.60800300 & 0.09348300 & 0.77562100 \\ \mathrm{C} & -1.98025300 & -0.00632000 & 0.78872700 \\ \mathrm{C} & -2.65773800 & -1.16274800 & 1.20966400 \\ \mathrm{C} & -3.85849300 & -1.53557400 & 0.62377700 \\ \mathrm{C} & -4.38009500 & -0.74435400 & -0.40280800 \\ \mathrm{C} & -3.72312100 & 0.40316900 & -0.84385100 \\ \mathrm{C} & -2.52501400 & 0.77696700 & -0.24239400 \\ \mathrm{O} & 5.64821200 & -1.22566900 & -0.83439700 \\ \mathrm{Cl} & -5.89398600 & -1.20859900 & -1.15298400 \\ \mathrm{C} & 6.24926900 & -1.96037500 & 0.22519400 \\ \mathrm{~F} & -0.86336400 & 2.66603900 & 1.07036600 \\ \mathrm{H} & 0.73488900 & 1.81004000 & 1.94738300 \\ \mathrm{H} & 2.30099700 & 1.33205200 & -2.36925500 \\ \mathrm{H} & 4.46792800 & 0.09893700 & -2.62618700 \\ \mathrm{H} & 4.20668000 & -1.14105700 & 1.48565200 \\ \mathrm{H} & 2.09225800 & 0.04972900 & 1.72746500 \\ \mathrm{H} & -2.21782900 & -1.75957700 & 2.00060100 \\ \mathrm{H} & -4.38799900 & -2.42400400 & 0.94705200 \\ \mathrm{H} & -4.14672700 & 0.99615700 & -1.64561600 \\ \mathrm{H} & -2.01285000 & 1.66637800 & -0.58479700 \\ \mathrm{H} & 7.16627500 & -2.38163300 & -0.18660100 \\ \mathrm{H} & 6.49543400 & -1.30997700 & 1.07271600 \\ \mathrm{H} & 5.59713400 & -2.77196800 & 0.56844700\end{array}$<smiles>O=C(CC(=O)c1ccc(Cl)cc1)c1ccc(Cl)cc1</smiles>

Nucleophile-34
$\mathrm{C}$
$\mathrm{C}$
$-2.02463700-0.61692900$
0.62566100
$\begin{array}{lll}-0.76056700 & -1.02789000 & 1.29410900\end{array}$ 


$\begin{array}{lrrr}\mathrm{C} & 0.00001600 & 0.00202000 & 2.13769600 \\ \mathrm{O} & -0.32294300 & -2.16438400 & 1.18248400 \\ \mathrm{C} & 0.76057800 & 1.03036600 & 1.29218200 \\ \mathrm{O} & 0.32304400 & 2.16669400 & 1.17857000 \\ \mathrm{C} & -2.74954300 & -1.60098000 & -0.06575900 \\ \mathrm{C} & -3.93670300 & -1.28647900 & -0.71152300 \\ \mathrm{C} & -4.39804800 & 0.03163900 & -0.67012400 \\ \mathrm{C} & -3.69233800 & 1.02894500 & 0.00092000 \\ \mathrm{C} & -2.50655200 & 0.70065700 & 0.65249300 \\ \mathrm{C} & 2.02464300 & 0.61813600 & 0.62450500 \\ \mathrm{C} & 2.74970900 & 1.60092600 & -0.06853400 \\ \mathrm{C} & 3.93686100 & 1.28518100 & -0.71370800 \\ \mathrm{C} & 4.39802300 & -0.03292900 & -0.67007400 \\ \mathrm{C} & 3.69214600 & -1.02901000 & 0.00261500 \\ \mathrm{C} & 2.50637800 & -0.69946700 & 0.65358600 \\ \mathrm{Cl} & -5.89554100 & 0.43923200 & -1.48292100 \\ \mathrm{Cl} & 5.89550400 & -0.44208600 & -1.48210800 \\ \mathrm{H} & 0.68956700 & -0.55892300 & 2.77040500 \\ \mathrm{H} & -0.68950800 & 0.56413400 & 2.76939800 \\ \mathrm{H} & -2.35917200 & -2.61219100 & -0.08174700 \\ \mathrm{H} & -4.50282000 & -2.04263800 & -1.24257500 \\ \mathrm{H} & -4.06630800 & 2.04579500 & 0.01027200 \\ \mathrm{H} & -1.94786700 & 1.48378000 & 1.15160100 \\ \mathrm{H} & 2.35947500 & 2.61216100 & -0.08625100 \\ \mathrm{H} & 4.50311100 & 2.04037000 & -1.24599700 \\ \mathrm{H} & 4.06597600 & -2.04589400 & 0.01368800 \\ \mathrm{H} & 1.94756300 & -1.48167300 & 1.15398900\end{array}$<smiles>CCOC(=O)CC(=O)c1ccc(Cl)cc1</smiles>

Nucleophile-35

$\begin{array}{lrrr}\mathrm{C} & -2.55510400 & -0.15379100 & 0.69361500 \\ \mathrm{C} & -2.25783300 & 1.32104500 & 0.89352100 \\ \mathrm{O} & -2.58487600 & -0.98607300 & 1.57505400 \\ \mathrm{C} & -1.12446000 & 1.81176700 & -0.01385200 \\ \mathrm{O} & -1.31200500 & 2.74097400 & -0.77947400 \\ \mathrm{C} & 0.19921500 & 1.12456600 & 0.06195500 \\ \mathrm{C} & 1.16964400 & 1.46786300 & -0.89120400 \\ \mathrm{C} & 2.41786600 & 0.86023800 & -0.88742800 \\ \mathrm{C} & 2.69894500 & -0.09834700 & 0.08874100 \\ \mathrm{C} & 1.75645600 & -0.45117600 & 1.05293300 \\ \mathrm{C} & 0.50663900 & 0.16310200 & 1.03579700 \\ \mathrm{O} & -2.75263700 & -0.41331000 & -0.61192500 \\ \mathrm{C} & -2.89169300 & -1.80969200 & -0.97905000 \\ \mathrm{C} & -1.52423900 & -2.45460700 & -1.12934700 \\ \mathrm{Cl} & 4.27037800 & -0.87414500 & 0.10069700 \\ \mathrm{H} & -2.01586700 & 1.47589600 & 1.94765900 \\ \mathrm{H} & -3.13869800 & 1.91322700 & 0.63654200\end{array}$




$\begin{array}{rrrr}\mathrm{H} & 0.91743000 & 2.21766800 & -1.63254300 \\ \mathrm{H} & 3.16825600 & 1.11820900 & -1.62553200 \\ \mathrm{H} & 1.99880200 & -1.19437300 & 1.80322100 \\ \mathrm{H} & -0.22252700 & -0.12539200 & 1.78354100 \\ \mathrm{H} & -3.43694300 & -1.78736200 & -1.92419800 \\ \mathrm{H} & -3.49323700 & -2.31461800 & -0.22055400 \\ \mathrm{H} & -1.63437500 & -3.48493600 & -1.48157400 \\ \mathrm{H} & -0.91507700 & -1.90358100 & -1.85090700 \\ \mathrm{H} & -1.00066200 & -2.47281900 & -0.17083600\end{array}$<smiles>CCOC(=O)CC(=O)c1ccc(F)cc1</smiles>

Nucleophile-36

$\begin{array}{cccc}\mathrm{C} & -2.22643500 & 0.06757800 & -0.69459200 \\ \mathrm{C} & -1.72640500 & 1.48762500 & -0.88270400 \\ \mathrm{O} & -2.35503300 & -0.75126100 & -1.58007700 \\ \mathrm{C} & -0.53678000 & 1.80996500 & 0.03009400 \\ \mathrm{O} & -0.60362600 & 2.74415300 & 0.81040500 \\ \mathrm{C} & 0.68407200 & 0.95871400 & -0.06119500 \\ \mathrm{C} & 1.69975600 & 1.16947700 & 0.88563500 \\ \mathrm{C} & 2.86048200 & 0.40883500 & 0.86570000 \\ \mathrm{C} & 2.99809200 & -0.56522000 & -0.12034100 \\ \mathrm{C} & 2.01741900 & -0.79690300 & -1.07782200 \\ \mathrm{C} & 0.85663300 & -0.02862800 & -1.04441300 \\ \mathrm{O} & -2.48104900 & -0.16618600 & 0.60610700 \\ \mathrm{C} & -2.81769800 & -1.53098100 & 0.96221600 \\ \mathrm{C} & -1.55513000 & -2.35925200 & 1.13088000 \\ \mathrm{~F} & 4.11944700 & -1.30775800 & -0.14623300 \\ \mathrm{H} & -2.51662600 & 2.19422700 & -0.62048800 \\ \mathrm{H} & -1.46356700 & 1.61524800 & -1.93538300 \\ \mathrm{H} & 1.54949400 & 1.94067300 & 1.63249400 \\ \mathrm{H} & 3.65390800 & 0.55243200 & 1.59023100 \\ \mathrm{H} & 2.17122100 & -1.56366100 & -1.82816100 \\ \mathrm{H} & 0.08850700 & -0.21553400 & -1.78523200 \\ \mathrm{H} & -3.46993400 & -1.94540400 & 0.19083400 \\ \mathrm{H} & -3.37082400 & -1.43754200 & 1.89843300 \\ \mathrm{H} & -0.88976300 & -1.90204100 & 1.86819100 \\ \mathrm{H} & -1.81246200 & -3.36701800 & 1.47152000 \\ \mathrm{H} & -1.02134900 & -2.44246700 & 0.18144400\end{array}$<smiles>CCOC(=O)CC(=O)c1ccc([N+](=O)[O-])cc1</smiles>

Nucleophile-37 


\begin{tabular}{|c|c|c|c|}
\hline $\mathrm{C}$ & -2.77268900 & -0.27273900 & -0.65260800 \\
\hline $\mathrm{C}$ & -2.56093500 & 1.20269500 & -0.94509600 \\
\hline $\mathrm{O}$ & -2.79431300 & -1.15212100 & -1.48634100 \\
\hline $\mathrm{C}$ & -1.44586800 & 1.80688000 & -0.09022900 \\
\hline $\mathrm{O}$ & -1.65924600 & 2.77938300 & 0.60945800 \\
\hline $\mathrm{C}$ & -0.08664200 & 1.17226100 & -0.13034000 \\
\hline $\mathrm{C}$ & 0.86250000 & 1.61111900 & 0.80526700 \\
\hline $\mathrm{C}$ & 2.13539400 & 1.05863400 & 0.83230600 \\
\hline $\mathrm{C}$ & 2.44691700 & 0.06629600 & -0.09572000 \\
\hline $\mathrm{C}$ & 1.53233100 & -0.38342300 & -1.04255200 \\
\hline $\mathrm{C}$ & 0.25778500 & 0.17578400 & -1.05618200 \\
\hline $\mathrm{O}$ & -2.90319800 & -0.46548600 & 0.67221400 \\
\hline $\mathrm{C}$ & -2.96877300 & -1.84404000 & 1.12284000 \\
\hline $\mathrm{C}$ & -1.57100100 & -2.42471900 & 1.25209500 \\
\hline $\mathrm{H}$ & -3.47033400 & 1.76034800 & -0.71087200 \\
\hline $\mathrm{H}$ & -2.34218500 & 1.30719400 & -2.01061000 \\
\hline $\mathrm{H}$ & 0.57378300 & 2.38724800 & 1.50431800 \\
\hline $\mathrm{H}$ & 2.88425600 & 1.37373400 & 1.54703300 \\
\hline $\mathrm{H}$ & 1.82524500 & -1.15365400 & -1.74368700 \\
\hline $\mathrm{H}$ & -0.46040600 & -0.18056000 & -1.78455900 \\
\hline $\mathrm{H}$ & -3.47728600 & -1.78811900 & 2.08672300 \\
\hline $\mathrm{H}$ & -3.57805400 & -2.41431300 & 0.41897100 \\
\hline $\mathrm{H}$ & -0.95719700 & -1.80976400 & 1.91588700 \\
\hline $\mathrm{H}$ & -1.62474300 & -3.43590600 & 1.66690300 \\
\hline $\mathrm{H}$ & -1.08573100 & -2.48021200 & 0.27500600 \\
\hline $\mathrm{N}$ & 3.79465000 & -0.52992300 & -0.07286400 \\
\hline $\mathrm{O}$ & 4.58439300 & -0.11652300 & 0.77416400 \\
\hline $\mathrm{O}$ & 4.04436300 & -1.40388000 & -0.90121400 \\
\hline & & & \\
\hline
\end{tabular}

Nucleophile-38

$\begin{array}{lrrr}\mathrm{C} & 2.04924800 & -0.41453900 & 0.17302400 \\ \mathrm{C} & 0.82490200 & -0.68792300 & 0.96464400 \\ \mathrm{C} & -0.02034600 & 0.48623900 & 1.49971100 \\ \mathrm{O} & 0.43613700 & -1.82371800 & 1.19812800 \\ \mathrm{C} & -0.92258800 & 1.09757900 & 0.40330900 \\ \mathrm{O} & -0.60692100 & 2.17538500 & -0.06978600 \\ \mathrm{C} & 2.84192800 & -1.51915800 & -0.18326900 \\ \mathrm{C} & 3.98451500 & -1.34299900 & -0.95230100 \\ \mathrm{C} & 4.34305100 & -0.06141500 & -1.38173800 \\ \mathrm{C} & 3.55717800 & 1.03865900 & -1.03746900 \\ \mathrm{C} & 2.41562400 & 0.87223600 & -0.25637300 \\ \mathrm{C} & -2.13947700 & 0.36261700 & -0.02830300 \\ \mathrm{C} & -2.95637100 & 0.97867800 & -0.99104500 \\ \mathrm{C} & -4.11496300 & 0.35234200 & -1.43177100 \\ \mathrm{C} & -4.46837800 & -0.89950700 & -0.91902700\end{array}$




$\begin{array}{cccc}\mathrm{C} & -3.65893300 & -1.52045200 & 0.03238800 \\ \mathrm{C} & -2.49845500 & -0.89439400 & 0.48324800 \\ \mathrm{~F} & 0.79230600 & 1.48573600 & 2.00092700 \\ \mathrm{H} & -0.62755600 & 0.09363100 & 2.32001700 \\ \mathrm{H} & 2.53459100 & -2.50288800 & 0.15322000 \\ \mathrm{H} & 4.59547100 & -2.19873700 & -1.22167500 \\ \mathrm{H} & 5.23479400 & 0.07746300 & -1.98564400 \\ \mathrm{H} & 3.83456900 & 2.03205200 & -1.37505700 \\ \mathrm{H} & 1.81182800 & 1.72995100 & 0.00787200 \\ \mathrm{H} & -2.65741800 & 1.94807000 & -1.37346000 \\ \mathrm{H} & -4.74400900 & 0.83413900 & -2.17355400 \\ \mathrm{H} & -5.37365300 & -1.39025500 & -1.26343300 \\ \mathrm{H} & -3.92938600 & -2.49549600 & 0.42453900 \\ \mathrm{H} & -1.86532600 & -1.40065900 & 1.20192600\end{array}$

$\mathrm{NO}_{2}$
1
$\mathrm{Me}$

Nucleophile-39

$\begin{array}{lrrr}\mathrm{C} & -1.32424700 & -0.02724400 & -0.00204200 \\ \mathrm{~N} & 0.17375900 & 0.00013800 & -0.00827700 \\ \mathrm{H} & -1.64288600 & 0.23070200 & 1.00912500 \\ \mathrm{H} & -1.67114900 & 0.73101000 & -0.70218700 \\ \mathrm{H} & -1.64863800 & -1.02841600 & -0.27369300 \\ \mathrm{O} & 0.70333600 & 1.10670800 & 0.00220500 \\ \mathrm{O} & 0.75814400 & -1.07805700 & 0.00241300\end{array}$

$\underset{\mathrm{NO}}{\mathrm{NO}_{2}}$

Nucleophile-40

$\begin{array}{lrrr}\mathrm{C} & -0.62604700 & -0.76018700 & 0.00023500 \\ \mathrm{C} & -1.87416000 & 0.09721700 & -0.00018600 \\ \mathrm{H} & -0.54281900 & -1.39966600 & 0.88202800 \\ \mathrm{H} & -0.54263900 & -1.40031100 & -0.88107000 \\ \mathrm{H} & -2.75107100 & -0.55679800 & -0.00008900 \\ \mathrm{H} & -1.91497400 & 0.73620500 & 0.88369400 \\ \mathrm{H} & -1.91472600 & 0.73559900 & -0.88451300 \\ \mathrm{~N} & 0.65234600 & 0.05280700 & 0.00010100 \\ \mathrm{O} & 0.56503900 & 1.27606000 & 0.00008800 \\ \mathrm{O} & 1.69759200 & -0.58941800 & -0.00022000\end{array}$<smiles>N#CC(Cc1ccccc1)c1nc2ccccc2s1</smiles>

Nucleophile-41
C
C
$\begin{array}{lll}3.05347500 & -0.56234100 & -1.44270800\end{array}$
$\begin{array}{llll}2.05024200 & 0.11226100 & -0.74413000\end{array}$ 


$\begin{array}{lrrr}\mathrm{C} & 2.18276200 & 0.40667700 & 0.63567600 \\ \mathrm{C} & 3.33864300 & 0.01251000 & 1.32237400 \\ \mathrm{C} & 4.33352400 & -0.66219400 & 0.62733400 \\ \mathrm{C} & 4.19230000 & -0.94669300 & -0.74204700 \\ \mathrm{~N} & 1.10687700 & 1.08043700 & 1.19574300 \\ \mathrm{C} & 0.18913200 & 1.30374500 & 0.31136300 \\ \mathrm{~S} & 0.51438900 & 0.71916100 & -1.32528800 \\ \mathrm{C} & -1.13758600 & 1.91569100 & 0.69731700 \\ \mathrm{C} & -1.96291100 & 0.90882000 & 1.56871000 \\ \mathrm{C} & -1.91092500 & 2.35864400 & -0.46652300 \\ \mathrm{C} & -2.17367500 & -0.41595600 & 0.88024000 \\ \mathrm{C} & -1.26856600 & -1.46540500 & 1.08187200 \\ \mathrm{C} & -1.41339600 & -2.66895600 & 0.39433300 \\ \mathrm{C} & -2.46780700 & -2.83825700 & -0.50366800 \\ \mathrm{C} & -3.37649300 & -1.79955800 & -0.70799800 \\ \mathrm{C} & -3.23040300 & -0.59582100 & -0.01962400 \\ \mathrm{~N} & -2.55335100 & 2.69660600 & -1.37170100 \\ \mathrm{H} & 2.94863200 & -0.78380100 & -2.49922800 \\ \mathrm{H} & 3.43297600 & 0.24096000 & 2.37824600 \\ \mathrm{H} & 5.23370700 & -0.97460900 & 1.14661200 \\ \mathrm{H} & 4.98406800 & -1.47502900 & -1.26339700 \\ \mathrm{H} & -0.92332500 & 2.79810300 & 1.31135200 \\ \mathrm{H} & -2.91675800 & 1.38239200 & 1.81933000 \\ \mathrm{H} & -1.39559000 & 0.77494800 & 2.49254100 \\ \mathrm{H} & -0.44032300 & -1.32859500 & 1.77061900 \\ \mathrm{H} & -0.70242400 & -3.47273300 & 0.55913100 \\ \mathrm{H} & -2.58187200 & -3.77518100 & -1.04014200 \\ \mathrm{H} & -4.20002400 & -1.92484300 & -1.40411700 \\ \mathrm{H} & -3.93388000 & 0.21351000 & -0.18974900 \\ & & & \end{array}$<smiles>N#CC(Cc1ccccc1)C(Cc1ccccc1)c1nc2ccccc2s1</smiles>

Nucleophile-42

$\begin{array}{lrrr}\mathrm{C} & -2.97659700 & 0.16403400 & -1.30083100 \\ \mathrm{C} & -1.82730700 & -0.43834300 & -0.78656500 \\ \mathrm{C} & -1.80722500 & -1.00016200 & 0.51393300 \\ \mathrm{C} & -2.96032600 & -0.95318300 & 1.30792400 \\ \mathrm{C} & -4.10271600 & -0.35095400 & 0.79658100 \\ \mathrm{C} & -4.11077400 & 0.20216600 & -0.49571000 \\ \mathrm{~N} & -0.58924200 & -1.53772900 & 0.89821200 \\ \mathrm{C} & 0.29834700 & -1.38941700 & -0.03167500 \\ \mathrm{~S} & -0.24893200 & -0.60972000 & -1.51949700 \\ \mathrm{C} & 1.72773500 & -1.85595500 & 0.17527400 \\ \mathrm{C} & 2.34180700 & -1.27682000 & 1.48420700 \\ \mathrm{C} & 2.58322600 & -1.58171300 & -0.98393300 \\ \mathrm{C} & 2.85583400 & 0.16996900 & 1.38085100 \\ \mathrm{~N} & 3.28682400 & -1.35182900 & -1.87755000 \\ \mathrm{C} & 1.85548500 & 1.16396200 & 0.83087400 \\ \mathrm{C} & 0.63088600 & 1.38881700 & 1.47426200\end{array}$




$\begin{array}{lrrr}\mathrm{C} & -0.31132700 & 2.25777700 & 0.93079200 \\ \mathrm{C} & -0.03376300 & 2.93752800 & -0.25750400 \\ \mathrm{C} & 1.18960400 & 2.73940800 & -0.89443900 \\ \mathrm{C} & 2.12377600 & 1.85382400 & -0.35546400 \\ \mathrm{H} & -2.98480700 & 0.59688000 & -2.29525300 \\ \mathrm{H} & -2.93734500 & -1.38228400 & 2.30364700 \\ \mathrm{H} & -5.00228500 & -0.30497500 & 1.40187700 \\ \mathrm{H} & -5.01526000 & 0.66885800 & -0.87265700 \\ \mathrm{H} & 1.68242700 & -2.94662900 & 0.28939900 \\ \mathrm{H} & 3.17062700 & -1.92473400 & 1.78645600 \\ \mathrm{H} & 1.56089100 & -1.36629800 & 2.24286100 \\ \mathrm{H} & 3.75238700 & 0.18484000 & 0.75324900 \\ \mathrm{H} & 3.17534900 & 0.47372000 & 2.38554600 \\ \mathrm{H} & 0.39900100 & 0.85281700 & 2.38967400 \\ \mathrm{H} & -1.26622000 & 2.39638800 & 1.42808300 \\ \mathrm{H} & -0.77012700 & 3.61215100 & -0.68332800 \\ \mathrm{H} & 1.41377400 & 3.26023100 & -1.82034900 \\ \mathrm{H} & 3.06144100 & 1.67584300 & -0.87333200\end{array}$<smiles>COc1cc(CC(C#N)c2nc3ccccc3s2)cc(OC)c1</smiles>

Nucleophile-43

$\begin{array}{lrrr}\mathrm{C} & -2.96123200 & 0.27828800 & -1.86611600 \\ \mathrm{C} & -2.16291500 & -0.50031200 & -1.02545900 \\ \mathrm{C} & -2.59265200 & -0.85504900 & 0.27811500 \\ \mathrm{C} & -3.84197000 & -0.42008200 & 0.74065800 \\ \mathrm{C} & -4.63166800 & 0.35966800 & -0.09464300 \\ \mathrm{C} & -4.19485900 & 0.70604100 & -1.38536800 \\ \mathrm{~N} & -1.68209200 & -1.60192700 & 1.01065600 \\ \mathrm{C} & -0.60553300 & -1.81870100 & 0.32563900 \\ \mathrm{~S} & -0.56721600 & -1.15704200 & -1.31327100 \\ \mathrm{C} & 0.62007800 & -2.42204300 & 0.97090300 \\ \mathrm{C} & 1.24864200 & -1.37734400 & 1.96282800 \\ \mathrm{C} & 1.61140300 & -2.86875400 & -0.01238300 \\ \mathrm{C} & 1.49786900 & -0.05279800 & 1.28546800 \\ \mathrm{C} & 2.65566800 & 0.12689300 & 0.52594900 \\ \mathrm{C} & 2.80421400 & 1.29767500 & -0.23038000 \\ \mathrm{C} & 1.80973400 & 2.27651500 & -0.21920500 \\ \mathrm{C} & 0.65834700 & 2.08802100 & 0.54622000 \\ \mathrm{C} & 0.50209800 & 0.92768700 & 1.31517500 \\ \mathrm{~N} & 2.42526500 & -3.20135000 & -0.76973900 \\ \mathrm{O} & 3.88282700 & 1.55910500 & -1.02055100 \\ \mathrm{O} & -0.27059700 & 3.08299900 & 0.47141700 \\ \mathrm{C} & 4.86184500 & 0.53894900 & -1.16189000\end{array}$




$\begin{array}{rrrr}\mathrm{C} & -1.53250500 & 2.86069000 & 1.08569400 \\ \mathrm{H} & -2.62646700 & 0.55159100 & -2.86072600 \\ \mathrm{H} & -4.16286900 & -0.69372200 & 1.73974100 \\ \mathrm{H} & -5.59894700 & 0.70824000 & 0.25239100 \\ \mathrm{H} & -4.82817300 & 1.31925800 & -2.01824800 \\ \mathrm{H} & 0.29790300 & -3.29535400 & 1.54903700 \\ \mathrm{H} & 0.53165900 & -1.26585900 & 2.77900400 \\ \mathrm{H} & 2.17026100 & -1.80412600 & 2.36896400 \\ \mathrm{H} & 3.40634600 & -0.65146000 & 0.50541200 \\ \mathrm{H} & 1.92336600 & 3.17197300 & -0.81776800 \\ \mathrm{H} & -0.39626100 & 0.75379800 & 1.89170900 \\ \mathrm{H} & 4.42198900 & -0.38989400 & -1.54427800 \\ \mathrm{H} & 5.36760800 & 0.33092800 & -0.21060100 \\ \mathrm{H} & 5.59015100 & 0.91997400 & -1.87852600 \\ \mathrm{H} & -2.13413100 & 3.74162600 & 0.85886600 \\ \mathrm{H} & -2.02560600 & 1.96925800 & 0.68225900 \\ \mathrm{H} & -1.44080300 & 2.76048800 & 2.17451600\end{array}$<smiles>N#CC(Cc1ccc([N+](=O)[O-])cc1)c1nc2ccccc2s1</smiles>

Nucleophile-44

$\begin{array}{lrrr}\mathrm{C} & 2.79621700 & -1.83378300 & -1.38153300 \\ \mathrm{C} & 2.34878800 & -0.65157500 & -0.78872000 \\ \mathrm{C} & 2.77095000 & -0.27066500 & 0.50914400 \\ \mathrm{C} & 3.65289000 & -1.09301300 & 1.22263700 \\ \mathrm{C} & 4.09366000 & -2.27060000 & 0.63382000 \\ \mathrm{C} & 3.66935000 & -2.63726900 & -0.65530800 \\ \mathrm{~N} & 2.23950000 & 0.92627900 & 0.96867600 \\ \mathrm{C} & 1.45549300 & 1.45417900 & 0.08512500 \\ \mathrm{~S} & 1.25553600 & 0.55952300 & -1.42615100 \\ \mathrm{C} & 0.65223600 & 2.69648900 & 0.39747900 \\ \mathrm{C} & -0.42062000 & 2.37958200 & 1.49399200 \\ \mathrm{C} & 0.02332900 & 3.27407800 & -0.79388000 \\ \mathrm{C} & -1.31911200 & 1.23639100 & 1.09663900 \\ \mathrm{~N} & -0.51243000 & 3.73223100 & -1.71537000 \\ \mathrm{C} & -0.99248400 & -0.07467900 & 1.47139700 \\ \mathrm{C} & -1.76379300 & -1.15014500 & 1.04669300 \\ \mathrm{C} & -2.87187100 & -0.89681500 & 0.24277200 \\ \mathrm{C} & -3.22781900 & 0.39267500 & -0.14228200 \\ \mathrm{C} & -2.44398900 & 1.45704900 & 0.29074700 \\ \mathrm{H} & 2.47097300 & -2.12108000 & -2.37535000 \\ \mathrm{H} & 3.97212300 & -0.79541100 & 2.21531200 \\ \mathrm{H} & 4.77545600 & -2.91863100 & 1.17468900 \\ \mathrm{H} & 4.02735300 & -3.56335500 & -1.09317900\end{array}$




$\begin{array}{lrrr}\mathrm{H} & 1.34234400 & 3.44206200 & 0.80870200 \\ \mathrm{H} & -0.99783000 & 3.28921600 & 1.68191600 \\ \mathrm{H} & 0.13476500 & 2.13089300 & 2.40057100 \\ \mathrm{H} & -0.11573900 & -0.25122400 & 2.08525400 \\ \mathrm{H} & -1.52496500 & -2.16939300 & 1.32019900 \\ \mathrm{H} & -4.09816100 & 0.54110900 & -0.76776800 \\ \mathrm{H} & -2.70000000 & 2.46679000 & -0.01200400 \\ \mathrm{~N} & -3.69186600 & -2.02805500 & -0.21402100 \\ \mathrm{O} & -4.67166400 & -1.77755900 & -0.91498100 \\ \mathrm{O} & -3.34842000 & -3.15843100 & 0.13157800\end{array}$<smiles>N#CC(Cc1ccc2c(c1)OCO2)c1nc2ccccc2s1</smiles>

Nucleophile-45

$\begin{array}{lrrr}\mathrm{C} & -3.00263600 & -1.63728400 & 1.37216200 \\ \mathrm{C} & -2.41697300 & -0.50324600 & 0.80619400 \\ \mathrm{C} & -2.88683900 & 0.03679100 & -0.41677900 \\ \mathrm{C} & -3.95747800 & -0.57585200 & -1.08098200 \\ \mathrm{C} & -4.53580700 & -1.70648700 & -0.51936100 \\ \mathrm{C} & -4.06293300 & -2.23204000 & 0.69559000 \\ \mathrm{~N} & -2.20830500 & 1.16411400 & -0.85753700 \\ \mathrm{C} & -1.26717800 & 1.49038900 & -0.03207600 \\ \mathrm{~S} & -1.08216700 & 0.46374800 & 1.39628000 \\ \mathrm{C} & -0.29630600 & 2.60307900 & -0.35027600 \\ \mathrm{C} & 0.66570800 & 2.15907300 & -1.50646400 \\ \mathrm{C} & 0.46139700 & 3.04011400 & 0.82579700 \\ \mathrm{C} & 1.37624300 & 0.86428100 & -1.20524300 \\ \mathrm{~N} & 1.09253500 & 3.38730600 & 1.73552200 \\ \mathrm{C} & 0.83259800 & -0.34470000 & -1.64905100 \\ \mathrm{C} & 1.40375800 & -1.58431700 & -1.31424200 \\ \mathrm{C} & 2.53145100 & -1.55413600 & -0.52077200 \\ \mathrm{C} & 3.08424200 & -0.35347800 & -0.07786900 \\ \mathrm{C} & 2.53679000 & 0.86938500 & -0.40103200 \\ \mathrm{O} & 4.21520100 & -0.61099400 & 0.65890200 \\ \mathrm{C} & 4.24629800 & -2.03240800 & 0.83039700 \\ \mathrm{O} & 3.29437000 & -2.60655400 & -0.07467900 \\ \mathrm{H} & -2.64115800 & -2.04603800 & 2.30947100 \\ \mathrm{H} & -4.31144400 & -0.15712800 & -2.01658400 \\ \mathrm{H} & -5.36482900 & -2.19229700 & -1.02363900 \\ \mathrm{H} & -4.53157200 & -3.11668800 & 1.11465600 \\ \mathrm{H} & -0.88365400 & 3.45717700 & -0.70642600 \\ \mathrm{H} & 1.37414400 & 2.97298300 & -1.68813900 \\ \mathrm{H} & 0.03853800 & 2.05034000 & -2.39391300 \\ \mathrm{H} & -0.07019500 & -0.32609400 & -2.25028000 \\ \mathrm{H} & 0.98030400 & -2.51972600 & -1.65980200 \\ & & & \end{array}$




$$
\begin{array}{rrrr}
\mathrm{H} & 2.97233900 & 1.79238800 & -0.03721500 \\
\mathrm{H} & 3.96643600 & -2.28314900 & 1.86337500 \\
\mathrm{H} & 5.24557500 & -2.40629300 & 0.59237000
\end{array}
$$<smiles>N#CC(Cc1ccc(Br)cc1)c1nc2ccccc2s1</smiles>

Nucleophile-46

$\begin{array}{lrrr}\mathrm{C} & 2.66041900 & -2.25266000 & -1.31436900 \\ \mathrm{C} & 2.46111200 & -0.98641900 & -0.76090300 \\ \mathrm{C} & 2.99150400 & -0.64398700 & 0.50772700 \\ \mathrm{C} & 3.72913700 & -1.59036800 & 1.23112400 \\ \mathrm{C} & 3.92294000 & -2.85061600 & 0.68140900 \\ \mathrm{C} & 3.39363800 & -3.17844600 & -0.57898000 \\ \mathrm{~N} & 2.70983400 & 0.64766200 & 0.92983100 \\ \mathrm{C} & 2.00757300 & 1.28056000 & 0.04635700 \\ \mathrm{~S} & 1.59204300 & 0.38360100 & -1.41940900 \\ \mathrm{C} & 1.45962100 & 2.66113400 & 0.32570300 \\ \mathrm{C} & 0.35745800 & 2.58512700 & 1.43703500 \\ \mathrm{C} & 0.93961000 & 3.31341100 & -0.87954500 \\ \mathrm{C} & -0.74313000 & 1.61645100 & 1.08743000 \\ \mathrm{C} & -0.66855600 & 0.28371800 & 1.51024500 \\ \mathrm{C} & -1.63152400 & -0.64644000 & 1.12654300 \\ \mathrm{C} & -2.68107200 & -0.23244000 & 0.31031100 \\ \mathrm{C} & -2.78436000 & 1.08760000 & -0.12031200 \\ \mathrm{C} & -1.81207200 & 2.00558500 & 0.27249500 \\ \mathrm{~N} & 0.49391300 & 3.84040400 & -1.81222800 \\ \mathrm{Br} & -4.00485500 & -1.50052300 & -0.22791800 \\ \mathrm{H} & 2.25339000 & -2.51001400 & -2.28608300 \\ \mathrm{H} & 4.13200000 & -1.32138600 & 2.20132700 \\ \mathrm{H} & 4.49093200 & -3.59432400 & 1.23074800 \\ \mathrm{H} & 3.55837900 & -4.17044400 & -0.98704800 \\ \mathrm{H} & 2.28540800 & 3.27185300 & 0.70840600 \\ \mathrm{H} & -0.03539900 & 3.59352800 & 1.59617200 \\ \mathrm{H} & 0.86851500 & 2.26985500 & 2.34929400 \\ \mathrm{H} & 0.16231800 & -0.03431500 & 2.13213300 \\ \mathrm{H} & -1.56744500 & -1.67773900 & 1.45241000 \\ \mathrm{H} & -3.60898700 & 1.39166400 & -0.75380500 \\ \mathrm{H} & -1.88188600 & 3.03226800 & -0.07273400\end{array}$


<smiles>N#CC(Cc1ccco1)c1nc2ccccc2s1</smiles>

Nucleophile-47

$\begin{array}{lrrr}\mathrm{C} & 2.94113000 & -0.71271800 & -1.32663100 \\ \mathrm{C} & 1.90600800 & -0.02065700 & -0.69488700 \\ \mathrm{C} & 2.07387500 & 0.54044300 & 0.59547500 \\ \mathrm{C} & 3.29882500 & 0.39898100 & 1.26041800 \\ \mathrm{C} & 4.32594400 & -0.29331400 & 0.63300400 \\ \mathrm{C} & 4.14877100 & -0.84359300 & -0.64834100 \\ \mathrm{~N} & 0.96272500 & 1.20464600 & 1.09446200 \\ \mathrm{C} & -0.01560200 & 1.16728100 & 0.24960800 \\ \mathrm{~S} & 0.28736900 & 0.31555100 & -1.27193000 \\ \mathrm{C} & -1.37886800 & 1.71375000 & 0.59896600 \\ \mathrm{C} & -2.18733200 & 0.67732200 & 1.45361500 \\ \mathrm{C} & -2.15157000 & 2.10259400 & -0.58472300 \\ \mathrm{C} & -2.35921000 & -0.62996300 & 0.76623400 \\ \mathrm{~N} & -2.80014700 & 2.38390100 & -1.50470200 \\ \mathrm{O} & -1.43343800 & -1.59756900 & 1.06369200 \\ \mathrm{C} & -1.70193500 & -2.67186200 & 0.26644700 \\ \mathrm{C} & -2.77388700 & -2.41329000 & -0.53105000 \\ \mathrm{C} & -3.20139300 & -1.08314400 & -0.20711800 \\ \mathrm{H} & 2.80919800 & -1.13783300 & -2.31568400 \\ \mathrm{H} & 3.41937800 & 0.83006300 & 2.24806500 \\ \mathrm{H} & 5.27965900 & -0.41197400 & 1.13690100 \\ \mathrm{H} & 4.96709100 & -1.37949800 & -1.11852100 \\ \mathrm{H} & -1.21547800 & 2.60701100 & 1.21168100 \\ \mathrm{H} & -3.15984500 & 1.11644200 & 1.69255000 \\ \mathrm{H} & -1.63450100 & 0.53192800 & 2.38446600 \\ \mathrm{H} & -1.05590900 & -3.52533400 & 0.39578500 \\ \mathrm{H} & -3.21185400 & -3.08168900 & -1.25745300 \\ \mathrm{H} & -4.01471200 & -0.52819700 & -0.65085800\end{array}$<smiles>CC(C)CC(C#N)c1nc2ccccc2s1</smiles>

Nucleophile-48
C
3.66366000
$-0.75205100$
0.76319900
C
$\begin{array}{lll}2.37051200 & -0.48316300 & 0.31164700\end{array}$
C
$\begin{array}{llll}2.07090600 & 0.69912400 & -0.40904800\end{array}$
C
3.08688000
$1.62609000-0.67465800$
$\mathrm{C}$
4.37234400
$1.36026100-0.22118000$
C
4.65789800
0.18267900
0.49061900 


$\begin{array}{lrrr}\mathrm{N} & 0.74557200 & 0.82831800 & -0.80122300 \\ \mathrm{C} & 0.04540200 & -0.18802800 & -0.41757500 \\ \mathrm{~S} & 0.91646900 & -1.44778600 & 0.48009800 \\ \mathrm{C} & -1.44876300 & -0.25081400 & -0.62573600 \\ \mathrm{C} & -2.18950600 & 0.50191800 & 0.51451800 \\ \mathrm{C} & -1.93462800 & -1.63044800 & -0.74447700 \\ \mathrm{C} & -3.72059100 & 0.51570200 & 0.39090400 \\ \mathrm{~N} & -2.34452600 & -2.71403200 & -0.81118500 \\ \mathrm{C} & -4.32245100 & 1.24254300 & 1.59968700 \\ \mathrm{C} & -4.19210400 & 1.15353400 & -0.92150600 \\ \mathrm{H} & 3.88875500 & -1.66041700 & 1.31131400 \\ \mathrm{H} & 2.85031500 & 2.52892500 & -1.22667500 \\ \mathrm{H} & 5.16889800 & 2.07007700 & -0.41910200 \\ \mathrm{H} & 5.67048700 & -0.00333600 & 0.83394000 \\ \mathrm{H} & -1.64472400 & 0.25888000 & -1.57449700 \\ \mathrm{H} & -1.79967500 & 1.52582800 & 0.51224700 \\ \mathrm{H} & -1.89867100 & 0.04675700 & 1.46747600 \\ \mathrm{H} & -4.06885500 & -0.52544300 & 0.41104800 \\ \mathrm{H} & -4.00892400 & 0.78015400 & 2.54125500 \\ \mathrm{H} & -4.00839100 & 2.29275300 & 1.62033100 \\ \mathrm{H} & -5.41588700 & 1.22254200 & 1.56233500 \\ \mathrm{H} & -3.80782500 & 2.17587700 & -1.01978400 \\ \mathrm{H} & -3.86907500 & 0.58430400 & -1.79854900 \\ \mathrm{H} & -5.28459900 & 1.20359400 & -0.95443000\end{array}$<smiles>N#CC(Cc1cccs1)c1nc2ccccc2s1</smiles>

Nucleophile-49

$\begin{array}{lrrr}\mathrm{C} & 3.05007700 & -0.26510000 & -1.48122100 \\ \mathrm{C} & 2.00855400 & 0.28164500 & -0.72912900 \\ \mathrm{C} & 2.09913000 & 0.39889800 & 0.68011200 \\ \mathrm{C} & 3.25125200 & -0.04521700 & 1.34179900 \\ \mathrm{C} & 4.28430200 & -0.59300800 & 0.59314900 \\ \mathrm{C} & 4.18471500 & -0.70161600 & -0.80484800 \\ \mathrm{~N} & 0.98887400 & 0.96249400 & 1.29225200 \\ \mathrm{C} & 0.08596500 & 1.27091200 & 0.41869900 \\ \mathrm{~S} & 0.46725100 & 0.91217500 & -1.26976600 \\ \mathrm{C} & -1.27064600 & 1.77995600 & 0.84487400 \\ \mathrm{C} & -2.08205000 & 0.63039000 & 1.53540500 \\ \mathrm{C} & -2.03519000 & 2.35073600 & -0.26736100 \\ \mathrm{C} & -2.23720500 & -0.57484800 & 0.66115100 \\ \mathrm{~N} & -2.67470300 & 2.78054200 & -1.13478900 \\ \mathrm{~S} & -1.11387200 & -1.90763800 & 0.76333900 \\ \mathrm{C} & -1.87956000 & -2.72269100 & -0.56044100 \\ \mathrm{C} & -2.93248100 & -2.00257200 & -1.05340600 \\ \mathrm{C} & -3.13516600 & -0.77393000 & -0.35591100\end{array}$




$\begin{array}{rrrr}\mathrm{H} & 2.97738000 & -0.35089100 & -2.55993700 \\ \mathrm{H} & 3.31236800 & 0.04585000 & 2.42057600 \\ \mathrm{H} & 5.18212300 & -0.94275700 & 1.09230400 \\ \mathrm{H} & 5.00590500 & -1.13336800 & -1.36799300 \\ \mathrm{H} & -1.10718300 & 2.57158500 & 1.58530800 \\ \mathrm{H} & -1.54445300 & 0.38410500 & 2.45378400 \\ \mathrm{H} & -3.06364000 & 1.02456400 & 1.81468100 \\ \mathrm{H} & -1.51230200 & -3.68457600 & -0.88846100 \\ \mathrm{H} & -3.54645900 & -2.33442900 & -1.88209400 \\ \mathrm{H} & -3.90719300 & -0.05527300 & -0.60481700\end{array}$<smiles>N#CC(CBr)c1nc2ccccc2o1</smiles>

Nucleophile-50

$\begin{array}{lrrr}\mathrm{C} & 2.47799500 & 0.66511900 & 1.52468300 \\ \mathrm{C} & 1.75011900 & -0.15923800 & 0.68251400 \\ \mathrm{C} & 2.20233400 & -0.59495800 & -0.57162300 \\ \mathrm{C} & 3.45620600 & -0.19529100 & -1.03718600 \\ \mathrm{C} & 4.20711900 & 0.63759000 & -0.20892000 \\ \mathrm{C} & 3.72902000 & 1.05955700 & 1.04526400 \\ \mathrm{~N} & 1.21305000 & -1.40417100 & -1.14539000 \\ \mathrm{C} & 0.26626700 & -1.41900300 & -0.26383100 \\ \mathrm{O} & 0.49946800 & -0.69871200 & 0.87806700 \\ \mathrm{C} & -1.09302500 & -2.02552100 & -0.44355100 \\ \mathrm{C} & -1.97974600 & -1.09553600 & -1.34397300 \\ \mathrm{C} & -1.75575800 & -2.30322700 & 0.83474500 \\ \mathrm{C} & -2.06468200 & 0.31225100 & -0.81086500 \\ \mathrm{~N} & -2.33430600 & -2.52652400 & 1.81464800 \\ \mathrm{C} & -1.13426500 & 1.27524700 & -1.22068700 \\ \mathrm{C} & -1.15588200 & 2.55854500 & -0.67751900 \\ \mathrm{C} & -2.11349100 & 2.89535000 & 0.27978300 \\ \mathrm{C} & -3.04762700 & 1.94354000 & 0.68981700 \\ \mathrm{C} & -3.02271000 & 0.65906900 & 0.14830500 \\ \mathrm{H} & 2.09671000 & 0.98359200 & 2.48745200 \\ \mathrm{H} & 3.82139400 & -0.52318600 & -2.00383400 \\ \mathrm{H} & 5.18630300 & 0.97095800 & -0.53703700 \\ \mathrm{H} & 4.34720500 & 1.70893400 & 1.65642700 \\ \mathrm{H} & -0.95782500 & -2.97722300 & -0.96867000 \\ \mathrm{H} & -2.97022800 & -1.55253600 & -1.42390200 \\ \mathrm{H} & -1.52098000 & -1.10348300 & -2.33587000 \\ \mathrm{H} & -0.38321600 & 1.01112700 & -1.95967200 \\ \mathrm{H} & -0.42652800 & 3.29400600 & -1.00271000 \\ \mathrm{H} & -2.13311600 & 3.89506100 & 0.70267100 \\ \mathrm{H} & -3.79565400 & 2.19946300 & 1.43372500 \\ \mathrm{H} & -3.74226300 & -0.08311300 & 0.48035100\end{array}$


<smiles>Cc1csc(C(C#N)CCc2ccccc2)n1</smiles>

Nucleophile-51

$\begin{array}{lrrr}\mathrm{C} & -2.45739500 & -0.60219600 & 1.24072200 \\ \mathrm{C} & -2.80909500 & -0.62123100 & -0.08167800 \\ \mathrm{C} & -3.91259200 & -1.42759400 & -0.69416400 \\ \mathrm{~N} & -2.04562300 & 0.20249700 & -0.88877500 \\ \mathrm{C} & -1.14058200 & 0.83744200 & -0.20150500 \\ \mathrm{~S} & -1.12896100 & 0.47736200 & 1.50890900 \\ \mathrm{C} & -0.11217800 & 1.71620600 & -0.87381000 \\ \mathrm{C} & 0.87243600 & 0.85405600 & -1.73287600 \\ \mathrm{C} & 0.62108500 & 2.54894200 & 0.08444200 \\ \mathrm{C} & 1.58472100 & -0.19859400 & -0.92217500 \\ \mathrm{~N} & 1.23054400 & 3.20129900 & 0.82559800 \\ \mathrm{C} & 1.03971500 & -1.48274600 & -0.80008100 \\ \mathrm{C} & 1.65823300 & -2.44329800 & -0.00189900 \\ \mathrm{C} & 2.83148400 & -2.13065600 & 0.68562400 \\ \mathrm{C} & 3.38242400 & -0.85426800 & 0.56914900 \\ \mathrm{C} & 2.76283800 & 0.10539800 & -0.23048200 \\ \mathrm{H} & -2.90785500 & -1.14562900 & 2.05844400 \\ \mathrm{H} & -4.42230400 & -2.03332700 & 0.05833200 \\ \mathrm{H} & -4.64952900 & -0.77374700 & -1.17165800 \\ \mathrm{H} & -3.51989600 & -2.09403000 & -1.46904600 \\ \mathrm{H} & -0.65025800 & 2.38800900 & -1.55300800 \\ \mathrm{H} & 1.58486000 & 1.53140800 & -2.21326400 \\ \mathrm{H} & 0.26376800 & 0.39135400 & -2.51322700 \\ \mathrm{H} & 0.11976000 & -1.72082100 & -1.32549400 \\ \mathrm{H} & 1.22395200 & -3.43476700 & 0.08338800 \\ \mathrm{H} & 3.31462400 & -2.87790800 & 1.30761500 \\ \mathrm{H} & 4.29526300 & -0.60403800 & 1.10074800 \\ \mathrm{H} & 3.18997700 & 1.10036300 & -0.31074300\end{array}$<smiles>COC(=O)CC(=O)OC</smiles>

Nucleophile-52

$\begin{array}{lrrr}\mathrm{C} & 1.12594800 & -0.10260000 & 0.10560400 \\ \mathrm{C} & 0.05746700 & -0.99555700 & -0.50173200 \\ \mathrm{C} & -1.32324900 & -0.58595900 & -0.03311800 \\ \mathrm{O} & -1.68838100 & 0.57667800 & -0.60676600 \\ \mathrm{O} & -2.00901200 & -1.20999100 & 0.74280800 \\ \mathrm{O} & 2.30219100 & -0.28320300 & -0.52687200 \\ \mathrm{O} & 0.96316600 & 0.64941500 & 1.03889400 \\ \mathrm{C} & 3.39981900 & 0.48371800 & 0.00045200 \\ \mathrm{C} & -2.95274300 & 1.09995200 & -0.16673700 \\ \mathrm{H} & 0.24067600 & -2.02669700 & -0.18961200\end{array}$




$$
\begin{array}{lrrr}
\mathrm{H} & 0.12751800 & -0.94750500 & -1.59157700 \\
\mathrm{H} & 4.26155800 & 0.22076300 & -0.61157400 \\
\mathrm{H} & 3.18689200 & 1.55261100 & -0.07097900 \\
\mathrm{H} & 3.57690900 & 0.22768000 & 1.04746900 \\
\mathrm{H} & -3.09550800 & 2.02747400 & -0.71968000 \\
\mathrm{H} & -3.75688800 & 0.39302200 & -0.38353400 \\
\mathrm{H} & -2.92832600 & 1.29213800 & 0.90815700
\end{array}
$$

Nucleophile-53

$\begin{array}{lrrr}\mathrm{C} & -1.69730100 & -1.38706000 & 0.22000000 \\ \mathrm{C} & -0.45275600 & -0.81399000 & 0.02534300 \\ \mathrm{C} & -0.26716400 & 0.54608600 & -0.22239900 \\ \mathrm{C} & -1.37137000 & 1.38588900 & -0.27691600 \\ \mathrm{C} & -2.64542500 & 0.83742900 & -0.08326200 \\ \mathrm{C} & -2.80246200 & -0.52943300 & 0.16042000 \\ \mathrm{C} & 1.20913600 & 0.78787000 & -0.37439800 \\ \mathrm{C} & 1.78458400 & -0.61842400 & -0.15165800 \\ \mathrm{O} & 0.74390600 & -1.51378500 & 0.05984600 \\ \mathrm{O} & 2.93167500 & -0.96493300 & -0.13410800 \\ \mathrm{C} & 1.83248700 & 1.80113800 & 0.59608100 \\ \mathrm{H} & -1.80146400 & -2.44865700 & 0.41097100 \\ \mathrm{H} & -1.25141500 & 2.44839900 & -0.46599800 \\ \mathrm{H} & -3.51888500 & 1.47984500 & -0.12315100 \\ \mathrm{H} & -3.79718200 & -0.93763800 & 0.30798100 \\ \mathrm{H} & 1.46536600 & 1.07555500 & -1.40276300 \\ \mathrm{H} & 1.58344800 & 1.54839700 & 1.63016400 \\ \mathrm{H} & 2.91967700 & 1.80000200 & 0.48822800 \\ \mathrm{H} & 1.45743400 & 2.80680900 & 0.38940000\end{array}$<smiles>O=C1Oc2ccccc2C1c1ccccc1</smiles>

Nucleophile-54 


$\begin{array}{lrrr}\mathrm{O} & -0.49487000 & 2.85808200 & 0.14877700 \\ \mathrm{C} & -1.38493800 & -0.04169800 & -0.31321400 \\ \mathrm{C} & -2.59036100 & 0.47963100 & -0.79561000 \\ \mathrm{C} & -3.80719400 & -0.05396400 & -0.37745700 \\ \mathrm{C} & -3.83201400 & -1.11496500 & 0.52933600 \\ \mathrm{C} & -2.63346700 & -1.63394300 & 1.01726400 \\ \mathrm{C} & -1.41424100 & -1.09831200 & 0.60089500 \\ \mathrm{H} & 3.90452800 & 0.81215300 & 1.30823400 \\ \mathrm{H} & 0.96877000 & -2.06556100 & -1.54667500 \\ \mathrm{H} & 3.22391700 & -2.86897000 & -0.84962200 \\ \mathrm{H} & 4.67499700 & -1.45181900 & 0.56282700 \\ \mathrm{H} & -0.17120100 & 0.88449100 & -1.81163400 \\ \mathrm{H} & -2.57109100 & 1.31263400 & -1.49145800 \\ \mathrm{H} & -4.73576200 & 0.35794000 & -0.76038200 \\ \mathrm{H} & -4.77992400 & -1.53374100 & 0.85268500 \\ \mathrm{H} & -2.64403200 & -2.45701600 & 1.72524700 \\ \mathrm{H} & -0.48416200 & -1.50484400 & 0.98315800\end{array}$<smiles>CCc1ccc2c(c1)C(c1ccccc1)C(=O)O2</smiles>

Nucleophile-55

$\begin{array}{lrrr}\mathrm{C} & 2.38169400 & 1.76283300 & 0.76639700 \\ \mathrm{C} & 1.07653200 & 1.65741200 & 0.32068700 \\ \mathrm{C} & 0.63058300 & 0.59507100 & -0.46252100 \\ \mathrm{C} & 1.51464900 & -0.41329100 & -0.81760400 \\ \mathrm{C} & 2.84962100 & -0.35071900 & -0.38499300 \\ \mathrm{C} & 3.25930700 & 0.73664900 & 0.39902200 \\ \mathrm{C} & -0.82630100 & 0.81752500 & -0.75674500 \\ \mathrm{C} & -1.09457700 & 2.15217200 & -0.02262700 \\ \mathrm{O} & 0.07529800 & 2.57955100 & 0.58766000 \\ \mathrm{O} & -2.11377000 & 2.77585500 & 0.04809200 \\ \mathrm{C} & -1.80558300 & -0.25670300 & -0.31175000 \\ \mathrm{C} & -3.11000500 & -0.23877300 & -0.81778500 \\ \mathrm{C} & -4.04062600 & -1.18865000 & -0.40328000 \\ \mathrm{C} & -3.67733100 & -2.16741800 & 0.52343900 \\ \mathrm{C} & -2.38046100 & -2.18576100 & 1.03484500 \\ \mathrm{C} & -1.44832600 & -1.23297400 & 0.62215100 \\ \mathrm{C} & 3.80899100 & -1.47247900 & -0.71136900 \\ \mathrm{C} & 3.70241600 & -2.63766600 & 0.28537100 \\ \mathrm{H} & 2.70280000 & 2.60336000 & 1.37036900 \\ \mathrm{H} & 1.17656400 & -1.25187600 & -1.42017200 \\ \mathrm{H} & 4.29235600 & 0.78437000 & 0.73182000 \\ \mathrm{H} & -0.99160600 & 1.02054500 & -1.82280700 \\ \mathrm{H} & -3.39583700 & 0.53023600 & -1.52859900 \\ \mathrm{H} & -5.04912600 & -1.16516000 & -0.80446600 \\ \mathrm{H} & -4.40116200 & -2.91025400 & 0.84400500 \\ \mathrm{H} & -2.09063800 & -2.94147800 & 1.75857500 \\ \mathrm{H} & -0.44056300 & -1.25051900 & 1.02271700 \\ & & & \end{array}$




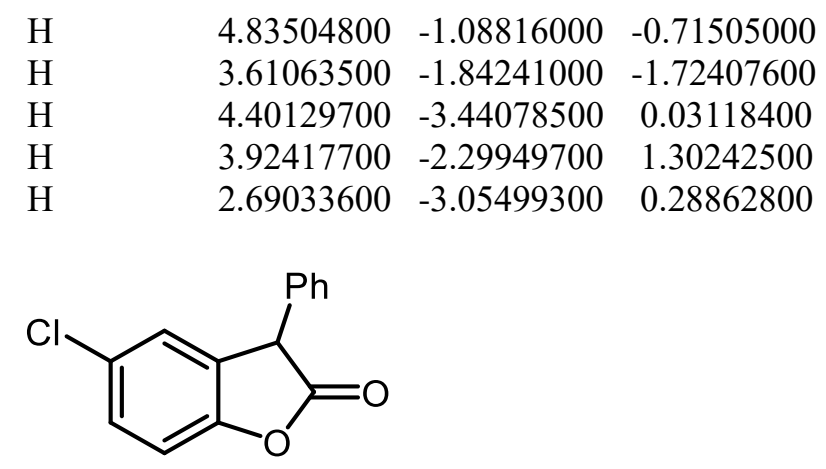

Nucleophile-56

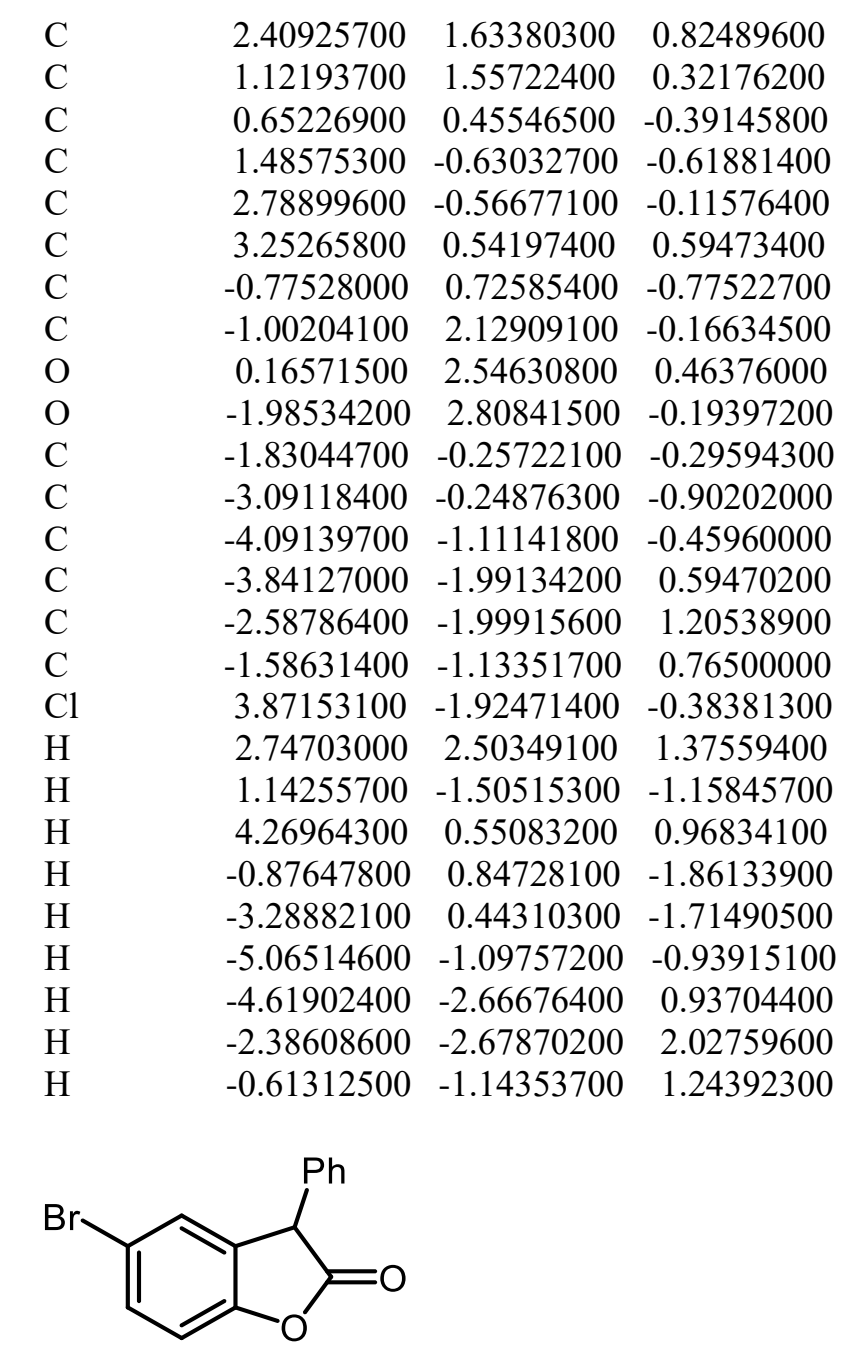

Nucleophile-57
$\begin{array}{llll}\text { C } & 1.50632600 & 2.23982800 & 0.85455500\end{array}$
$\begin{array}{llll}\mathrm{C} & 0.26725700 & 1.91763000 & 0.32771000\end{array}$
$\begin{array}{llll}\mathrm{C} & 0.02150900 & 0.72917800 & -0.35857400\end{array}$
$\begin{array}{llll}\mathrm{C} & 1.04263200 & -0.19434100 & -0.53231400\end{array}$ 


$\begin{array}{lrrr}\mathrm{C} & 2.29885900 & 0.11792300 & -0.00476700 \\ \mathrm{C} & 2.53766300 & 1.31137200 & 0.67784900 \\ \mathrm{C} & -1.42084500 & 0.72672200 & -0.78021900 \\ \mathrm{C} & -1.91166600 & 2.08257600 & -0.22186200 \\ \mathrm{O} & -0.85428200 & 2.72155300 & 0.41758800 \\ \mathrm{O} & -2.99989800 & 2.57252900 & -0.29116400 \\ \mathrm{C} & -2.29462500 & -0.41683700 & -0.29188600 \\ \mathrm{C} & -3.52378500 & -0.64695400 & -0.91894900 \\ \mathrm{C} & -4.36411200 & -1.66329800 & -0.47045000 \\ \mathrm{C} & -3.98409700 & -2.46003800 & 0.61086100 \\ \mathrm{C} & -2.76237300 & -2.23055600 & 1.24225600 \\ \mathrm{C} & -1.92128000 & -1.21088500 & 0.79581000 \\ \mathrm{Br} & 3.72960400 & -1.12941700 & -0.22116000 \\ \mathrm{H} & 1.66777000 & 3.17170600 & 1.38311700 \\ \mathrm{H} & 0.87638800 & -1.13159100 & -1.04987300 \\ \mathrm{H} & 3.52573800 & 1.51526200 & 1.07231700 \\ \mathrm{H} & -1.51363000 & 0.79760000 & -1.87157700 \\ \mathrm{H} & -3.82391700 & -0.02022900 & -1.75299000 \\ \mathrm{H} & -5.31453700 & -1.83403800 & -0.96632800 \\ \mathrm{H} & -4.63668800 & -3.25496800 & 0.95800200 \\ \mathrm{H} & -2.46050600 & -2.84415000 & 2.08540600 \\ \mathrm{H} & -0.97211200 & -1.03657700 & 1.29099600\end{array}$<smiles>O=C1Oc2ccccc2C1c1ccc(Cl)cc1</smiles>

Nucleophile-58

$\begin{array}{lrrr}\mathrm{C} & -3.91997900 & -0.30352600 & -0.85973700 \\ \mathrm{C} & -2.78542400 & 0.33996900 & -0.39663300 \\ \mathrm{C} & -1.90283600 & -0.23272100 & 0.51789900 \\ \mathrm{C} & -2.15219700 & -1.51086100 & 1.00124500 \\ \mathrm{C} & -3.29116700 & -2.18920000 & 0.55125200 \\ \mathrm{C} & -4.16002400 & -1.59108800 & -0.36581100 \\ \mathrm{C} & -0.80457300 & 0.75873500 & 0.78198800 \\ \mathrm{C} & -1.22746800 & 1.94344100 & -0.11818400 \\ \mathrm{O} & -2.40052500 & 1.61529800 & -0.78024700 \\ \mathrm{O} & -0.68458800 & 2.99943000 & -0.26706700 \\ \mathrm{C} & 0.62133400 & 0.33096600 & 0.47847400 \\ \mathrm{C} & 1.68124000 & 1.11589800 & 0.94602000 \\ \mathrm{C} & 3.00076300 & 0.77740600 & 0.66118100 \\ \mathrm{C} & 3.25829300 & -0.36039600 & -0.10218300 \\ \mathrm{C} & 2.22014000 & -1.15253800 & -0.58440400 \\ \mathrm{C} & 0.90312300 & -0.79874600 & -0.29315800 \\ \mathrm{Cl} & 4.92063300 & -0.80247200 & -0.45867200 \\ \mathrm{H} & -4.58348800 & 0.17406700 & -1.57090200\end{array}$




$\begin{array}{lrrr}\mathrm{H} & -1.47224300 & -1.97555900 & 1.70826000 \\ \mathrm{H} & -3.50135400 & -3.18842800 & 0.91772300 \\ \mathrm{H} & -5.03811400 & -2.13170100 & -0.70409400 \\ \mathrm{H} & -0.84505500 & 1.13265000 & 1.81332500 \\ \mathrm{H} & 1.47193200 & 2.00801100 & 1.52718100 \\ \mathrm{H} & 3.82083400 & 1.38513300 & 1.02514600 \\ \mathrm{H} & 2.43825500 & -2.03141300 & -1.17980800 \\ \mathrm{H} & 0.09202800 & -1.41258800 & -0.66859900\end{array}$<smiles>c1ccc2[nH]ccc2c1</smiles>

Nucleophile-59

$\begin{array}{lrrr}\mathrm{C} & -0.93479700 & -1.41825900 & 0.00000300 \\ \mathrm{C} & 0.24724200 & -0.67258900 & 0.00000300 \\ \mathrm{C} & 0.24923100 & 0.75170600 & 0.00000100 \\ \mathrm{C} & -0.98176800 & 1.42857200 & -0.00000200 \\ \mathrm{C} & -2.15821400 & 0.69146400 & -0.00000200 \\ \mathrm{C} & -2.13507700 & -0.71791500 & 0.00000000 \\ \mathrm{C} & 1.62533500 & 1.16675300 & 0.00000200 \\ \mathrm{C} & 2.39026600 & 0.03056700 & 0.00000000 \\ \mathrm{~N} & 1.56585000 & -1.08128900 & -0.00000200 \\ \mathrm{H} & -0.91770100 & -2.50424200 & 0.00000200 \\ \mathrm{H} & -1.00973400 & 2.51429000 & -0.00000300 \\ \mathrm{H} & -3.11428400 & 1.20577000 & -0.00000500 \\ \mathrm{H} & -3.07146100 & -1.26711300 & 0.00000000 \\ \mathrm{H} & 1.99600200 & 2.18135100 & 0.00000700 \\ \mathrm{H} & 3.46419000 & -0.08532400 & -0.00000300 \\ \mathrm{H} & 1.87873000 & -2.03750200 & -0.00001700\end{array}$<smiles>Cc1cc2ccccc2[nH]1</smiles>

Nucleophile-60

$\begin{array}{lrrr}\mathrm{C} & 0.87966500 & 1.77608800 & 0.00000000 \\ \mathrm{C} & 0.00000000 & 0.69207500 & 0.00000000 \\ \mathrm{C} & 0.45363400 & -0.65776400 & 0.00000000 \\ \mathrm{C} & 1.83553900 & -0.90461700 & 0.00000000 \\ \mathrm{C} & 2.71514500 & 0.17105700 & 0.00000000 \\ \mathrm{C} & 2.24265300 & 1.49770400 & 0.00000000 \\ \mathrm{C} & -0.71900600 & -1.48825600 & 0.00000000 \\ \mathrm{C} & -1.81672100 & -0.66440200 & 0.00000000 \\ \mathrm{~N} & -1.38096400 & 0.65420400 & 0.00000000 \\ \mathrm{C} & -3.27263400 & -0.99032000 & 0.00000000 \\ \mathrm{H} & 0.51537100 & 2.79937200 & 0.00000000 \\ \mathrm{H} & 2.20989900 & -1.92420700 & 0.00000000\end{array}$




$$
\begin{array}{rrrr}
\mathrm{H} & 3.78550200 & -0.01075300 & 0.00000000 \\
\mathrm{H} & 2.95334800 & 2.31830000 & 0.00000000 \\
\mathrm{H} & -0.74885500 & -2.56853100 & 0.00000000 \\
\mathrm{H} & -1.98497800 & 1.45953000 & 0.00000000 \\
\mathrm{H} & -3.41363400 & -2.07286500 & 0.00000000 \\
\mathrm{H} & -3.77977700 & -0.58483300 & 0.88426100 \\
\mathrm{H} & -3.77977700 & -0.58483300 & -0.88426100 \\
& & &
\end{array}
$$

Nucleophile-61

$$
\begin{array}{lrrr}
\mathrm{C} & -0.29468400 & 1.71413400 & 0.00000400 \\
\mathrm{C} & -1.23900200 & 0.68492900 & 0.00000300 \\
\mathrm{C} & -0.86260700 & -0.68853200 & 0.00000100 \\
\mathrm{C} & 0.50103900 & -1.02398700 & -0.00000600 \\
\mathrm{C} & 1.42543400 & 0.00785800 & -0.00000500 \\
\mathrm{C} & 1.04911400 & 1.36433900 & 0.00000100 \\
\mathrm{C} & -2.07740500 & -1.45502200 & 0.00002600 \\
\mathrm{C} & -3.11603800 & -0.56154600 & -0.00002600 \\
\mathrm{~N} & -2.61790300 & 0.72900300 & -0.00000500 \\
\mathrm{Cl} & 3.14579000 & -0.38156600 & -0.00000100 \\
\mathrm{H} & -0.59219300 & 2.75826500 & 0.00000500 \\
\mathrm{H} & 0.82789800 & -2.05746400 & -0.00000300 \\
\mathrm{H} & 1.81607900 & 2.12937400 & 0.00000400 \\
\mathrm{H} & -2.16452200 & -2.53140200 & 0.00004200 \\
\mathrm{H} & -4.18187700 & -0.73605300 & -0.00004800 \\
\mathrm{H} & -3.17359100 & 1.56785000 & 0.00006800 \\
& & & \\
\mathrm{MeO} & & &
\end{array}
$$

Nucleophile-62

$\begin{array}{lrrr}\mathrm{C} & 0.50370100 & 1.79050400 & -0.00000500 \\ \mathrm{C} & 1.31777700 & 0.65316000 & -0.00000300 \\ \mathrm{C} & 0.77206100 & -0.65761600 & 0.00000300 \\ \mathrm{C} & -0.62662500 & -0.82693200 & 0.00000900 \\ \mathrm{C} & -1.43124000 & 0.30654700 & 0.00000800 \\ \mathrm{C} & -0.86827400 & 1.60480400 & 0.00000000 \\ \mathrm{C} & 1.88116300 & -1.56958100 & -0.00001500 \\ \mathrm{C} & 3.02701600 & -0.81470400 & 0.00001800 \\ \mathrm{~N} & 2.69496600 & 0.52584200 & -0.00000700 \\ \mathrm{O} & -2.80314200 & 0.28401500 & 0.00001200 \\ \mathrm{C} & -3.43531600 & -0.98117000 & -0.00001100 \\ \mathrm{H} & 0.92619800 & 2.79089300 & -0.00001000 \\ \mathrm{H} & -1.04963800 & -1.82365600 & 0.00001000\end{array}$




$$
\begin{array}{rrrr}
\mathrm{H} & -1.54688900 & 2.45019500 & -0.00000300 \\
\mathrm{H} & 1.83312300 & -2.64876100 & -0.00002400 \\
\mathrm{H} & 4.06225400 & -1.12307700 & 0.00003000 \\
\mathrm{H} & 3.35059300 & 1.28872500 & -0.00002300 \\
\mathrm{H} & -4.50873500 & -0.78620700 & -0.00002500 \\
\mathrm{H} & -3.17403600 & -1.56559200 & -0.89243700 \\
\mathrm{H} & -3.17406300 & -1.56560900 & 0.89241300
\end{array}
$$<smiles>CCOP(=O)(OCC)C(C)[N+](=O)[O-]</smiles>

Nucleophile-63

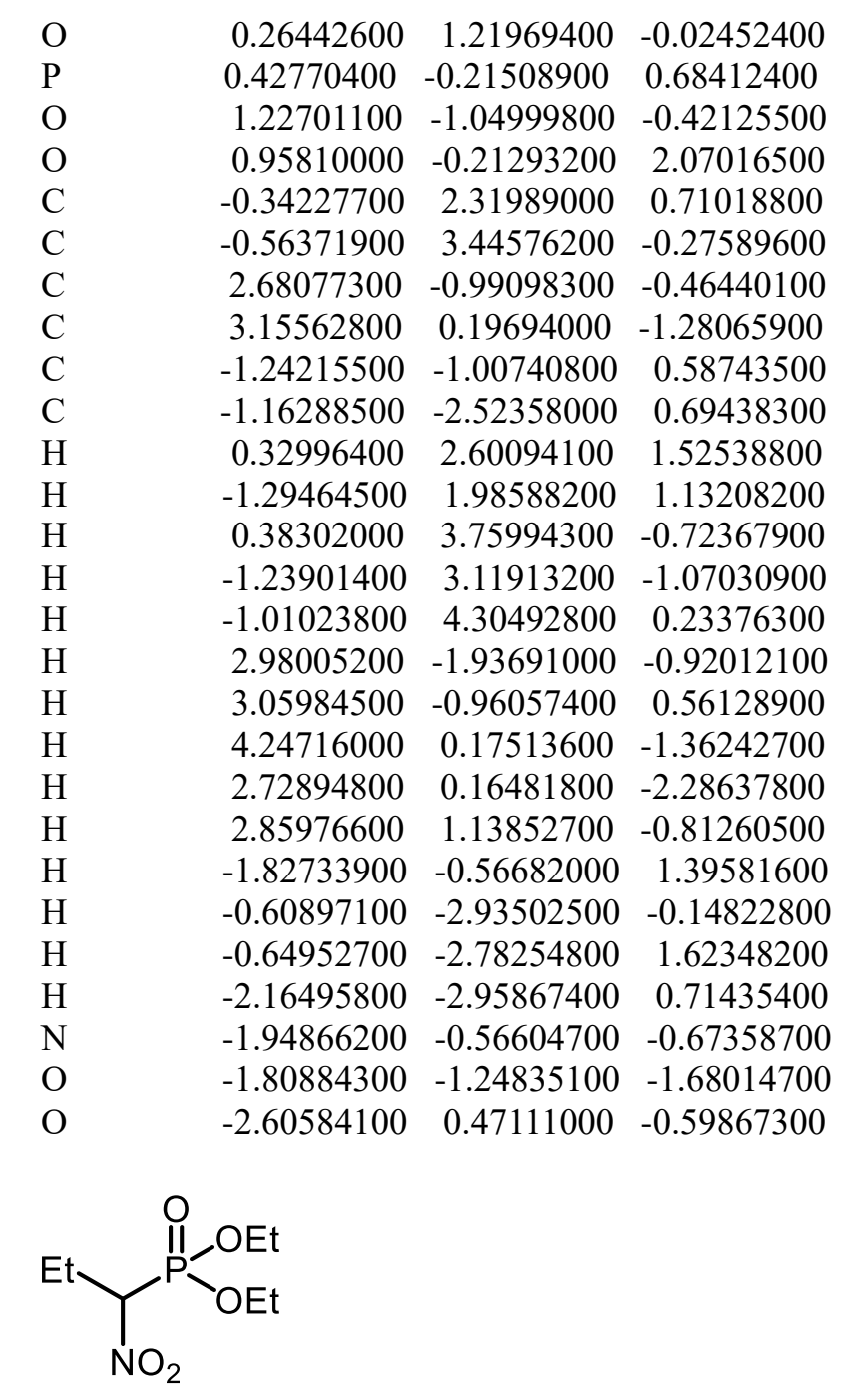

Nucleophile-64 


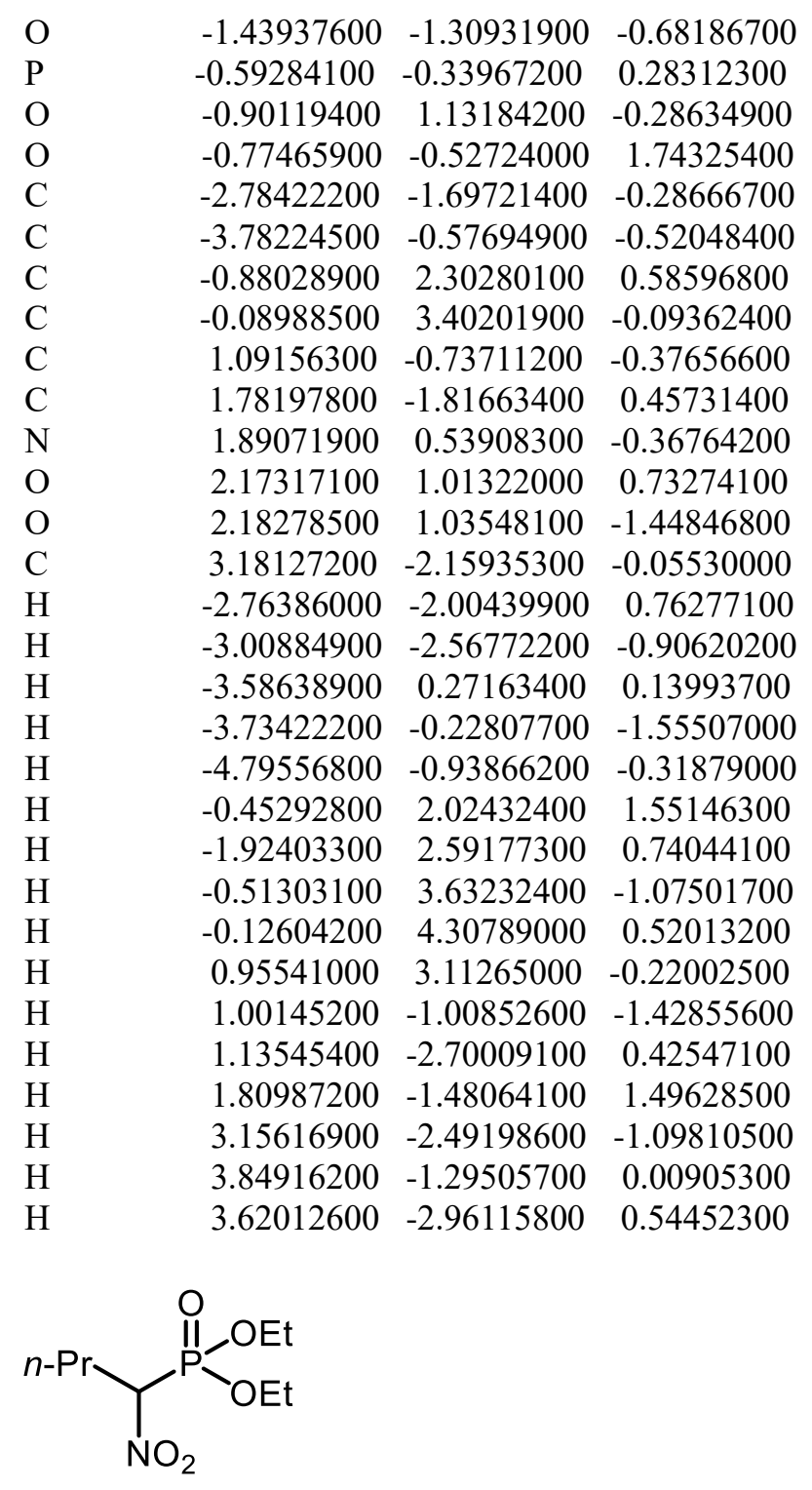

Nucleophile-65

$\begin{array}{lrrr}\mathrm{O} & -1.19313800 & -1.62034700 & -0.58504500 \\ \mathrm{P} & -0.75736400 & -0.37641500 & 0.33833300 \\ \mathrm{O} & -1.40934300 & 0.89517300 & -0.39970800 \\ \mathrm{O} & -1.02803900 & -0.50015200 & 1.79140900 \\ \mathrm{C} & -2.40181000 & -2.35609100 & -0.25040500 \\ \mathrm{C} & -3.65105400 & -1.60033300 & -0.66757900 \\ \mathrm{C} & -1.81914500 & 2.07954800 & 0.34885700 \\ \mathrm{C} & -1.31107200 & 3.31033300 & -0.37369900 \\ \mathrm{C} & 1.02333600 & -0.31311000 & -0.15874000 \\ \mathrm{C} & 1.90853000 & -1.10411200 & 0.80744200 \\ \mathrm{~N} & 1.43797500 & 1.13261600 & -0.19337600\end{array}$




$$
\begin{aligned}
& \begin{array}{llll}
\mathrm{O} & 1.76683600 & 1.60623500 & -1.27482500
\end{array} \\
& \begin{array}{llll}
\mathrm{O} & 1.40450500 & 1.74908500 & 0.87131700
\end{array} \\
& \begin{array}{lllll}
\mathrm{C} & 3.40889800 & -0.99593100 & 0.50420500
\end{array} \\
& \begin{array}{llll}
\mathrm{C} & 3.79683100 & -1.42185800 & -0.91432400
\end{array} \\
& \mathrm{H} \quad-2.30558700 \quad-3.29859500 \quad-0.79314000 \\
& \begin{array}{llll}
\mathrm{H} & -2.39768400 & -2.56298400 & 0.82348900
\end{array} \\
& \mathrm{H} \quad \begin{array}{llll}
-3.60366200 & -1.33247900 & -1.72608500
\end{array} \\
& \mathrm{H} \quad \begin{array}{llll}
-3.77216000 & -0.68403500 & -0.08413900
\end{array} \\
& \mathrm{H} \quad \begin{array}{llll}
-4.53313300 & -2.22750900 & -0.50346400
\end{array} \\
& \mathrm{H} \quad \begin{array}{llll}
\mathrm{H} & -1.43367100 & 2.01178500 & 1.36816900
\end{array} \\
& \begin{array}{llll}
\mathrm{H} & -2.91218100 & 2.05756800 & 0.38982700
\end{array} \\
& \mathrm{H} \quad-1.67007900 \quad 4.20796300 \quad 0.14006500 \\
& \mathrm{H} \quad-0.21936300 \quad 3.33414000 \quad-0.38588900 \\
& \mathrm{H} \quad-1.67641700 \quad 3.33247000 \quad-1.40391300 \\
& \mathrm{H} \quad 1.10541200-0.65811700 \quad-1.18824600 \\
& \mathrm{H} \quad \begin{array}{llll}
1.58335800 & -2.14856100 & 0.73630300
\end{array} \\
& \mathrm{H} \quad \begin{array}{llll}
1.69212600 & -0.76907900 & 1.82446300
\end{array} \\
& \mathrm{H} \quad 3.93951100 \quad-1.61517000 \quad 1.23614000 \\
& \mathrm{H} \quad 3.73858500 \quad 0.03418100 \quad 0.68540200 \\
& \mathrm{H} \quad 4.88392300 \quad-1.42709400 \quad-1.03454200 \\
& \mathrm{H} \quad 3.43125700 \quad-2.43004200 \quad-1.14046200 \\
& \mathrm{H} \quad 3.39073200 \quad-0.73714800 \quad-1.66507800
\end{aligned}
$$

Nucleophile-66

$\begin{array}{lrrr}\mathrm{O} & -1.56716300 & -0.68553600 & -0.49936600 \\ \mathrm{P} & -0.26342900 & -0.31386600 & 0.35934000 \\ \mathrm{O} & 0.29263200 & 0.99514200 & -0.38634700 \\ \mathrm{O} & -0.40283200 & -0.20516200 & 1.83342300 \\ \mathrm{C} & -2.76820700 & 0.14755000 & -0.36295600 \\ \mathrm{C} & -3.60171100 & -0.36769100 & 0.80002400 \\ \mathrm{C} & 1.09696300 & 1.99032200 & 0.34473000 \\ \mathrm{C} & 0.16061200 & 3.08095500 & 0.83556000 \\ \mathrm{C} & 0.83231000 & -1.70267800 & -0.21211000 \\ \mathrm{C} & 0.92791000 & -2.80378800 & 0.83526100 \\ \mathrm{~N} & 2.18619400 & -1.10167200 & -0.49301400 \\ \mathrm{O} & 2.86007700 & -0.75908100 & 0.47759600 \\ \mathrm{O} & 2.50855200 & -0.95811800 & -1.66619200 \\ \mathrm{C} & 2.16201100 & 2.46664100 & -0.62637500 \\ \mathrm{C} & -3.47288700 & 0.08579600 & -1.70606500 \\ \mathrm{H} & -2.43987700 & 1.17508100 & -0.16777300 \\ \mathrm{H} & -4.50590700 & 0.23914900 & 0.91056900 \\ \mathrm{H} & -3.90068700 & -1.40453500 & 0.61829500 \\ \mathrm{H} & -3.02890100 & -0.32315900 & 1.72841400 \\ \mathrm{H} & 1.55986000 & 1.48623400 & 1.19701100 \\ \mathrm{H} & 0.72739800 & 3.85719900 & 1.35895600\end{array}$




$\begin{array}{rrrr}\mathrm{H} & -0.36135200 & 3.54306000 & -0.00836900 \\ \mathrm{H} & -0.57401800 & 2.66425800 & 1.52896200 \\ \mathrm{H} & 0.47190600 & -2.05305100 & -1.17828000 \\ \mathrm{H} & 1.60338000 & -3.59539200 & 0.50065600 \\ \mathrm{H} & 1.27842600 & -2.39407500 & 1.78209100 \\ \mathrm{H} & -0.06450300 & -3.23405600 & 0.98866400 \\ \mathrm{H} & 2.79945100 & 3.21132000 & -0.14027500 \\ \mathrm{H} & 2.78789400 & 1.63332400 & -0.95243200 \\ \mathrm{H} & 1.69980600 & 2.92570000 & -1.50550500 \\ \mathrm{H} & -4.37841500 & 0.69939500 & -1.68327900 \\ \mathrm{H} & -2.81959600 & 0.45339200 & -2.50094800 \\ \mathrm{H} & -3.75890800 & -0.94474700 & -1.93709200\end{array}$<smiles>CCOP(=O)(OCC)C(C1CC1)[N+](=O)[O-]</smiles>

Nucleophile-67

$\begin{array}{lrrr}\mathrm{O} & -0.94189500 & -1.57283200 & -0.69544900 \\ \mathrm{P} & -0.53797100 & -0.35513000 & 0.27629800 \\ \mathrm{O} & -1.36890100 & 0.89089700 & -0.31070900 \\ \mathrm{O} & -0.66111100 & -0.59394700 & 1.73482600 \\ \mathrm{C} & -2.02347000 & -2.45898000 & -0.29664100 \\ \mathrm{C} & -3.38226300 & -1.81133500 & -0.49675100 \\ \mathrm{C} & -1.81044800 & 1.98214100 & 0.55253300 \\ \mathrm{C} & -1.46565000 & 3.29959000 & -0.11131800 \\ \mathrm{C} & 1.18108300 & -0.08844000 & -0.35844100 \\ \mathrm{C} & 2.18350000 & -0.84094100 & 0.47272100 \\ \mathrm{~N} & 1.45967800 & 1.39335600 & -0.33509200 \\ \mathrm{O} & 1.59140500 & 1.96446100 & -1.41054000 \\ \mathrm{O} & 1.50799300 & 1.93174700 & 0.77002500 \\ \mathrm{C} & 2.48270000 & -2.26846900 & 0.08652700 \\ \mathrm{C} & 3.52009900 & -1.19748400 & -0.12846700 \\ \mathrm{H} & -1.86796800 & -2.75069500 & 0.74576000 \\ \mathrm{H} & -1.90266300 & -3.33823400 & -0.93258500 \\ \mathrm{H} & -3.52039000 & -0.96600100 & 0.18231600 \\ \mathrm{H} & -3.49077400 & -1.45249900 & -1.52333900 \\ \mathrm{H} & -4.17209800 & -2.54193600 & -0.29490700 \\ \mathrm{H} & -1.33947100 & 1.87865600 & 1.53227400 \\ \mathrm{H} & -2.89102800 & 1.86192500 & 0.67409300 \\ \mathrm{H} & -0.38438800 & 3.42096000 & -0.20386100 \\ \mathrm{H} & -1.91379300 & 3.35990600 & -1.10670600 \\ \mathrm{H} & -1.85513200 & 4.12448700 & 0.49406400 \\ \mathrm{H} & 1.21207900 & -0.37097000 & -1.41075000 \\ \mathrm{H} & 2.13899800 & -0.60646700 & 1.53011400 \\ \mathrm{H} & 1.97397500 & -2.67312100 & -0.78269000 \\ \mathrm{H} & 2.62598600 & -2.98039800 & 0.89183300 \\ \mathrm{H} & 4.38483400 & -1.17391300 & 0.52587600 \\ \mathrm{H} & 3.72041900 & -0.87736100 & -1.14652400 \\ & & & \end{array}$


<smiles>CCOP(=O)(OCC)C(CCc1ccccc1)[N+](=O)[O-]</smiles>

Nucleophile-68

$\begin{array}{lrrr}\mathrm{O} & 2.56542000 & -0.81676500 & -0.08229000 \\ \mathrm{P} & 1.36131800 & 0.20281300 & -0.34462400 \\ \mathrm{O} & 1.91923000 & 1.56861300 & 0.29429300 \\ \mathrm{O} & 0.83115700 & 0.33650000 & -1.72862700 \\ \mathrm{C} & 3.84056800 & -0.63724200 & -0.75879800 \\ \mathrm{C} & 1.37346400 & 2.85070700 & -0.12554700 \\ \mathrm{C} & 0.11787900 & -0.46465400 & 0.84300000 \\ \mathrm{~N} & 0.66548000 & -0.38351700 & 2.24998600 \\ \mathrm{O} & 0.48975200 & 0.67246400 & 2.85241800 \\ \mathrm{O} & 1.24797500 & -1.36639500 & 2.69309800 \\ \mathrm{C} & -0.04012100 & 3.06750800 & 0.38605200 \\ \mathrm{C} & 3.80775100 & -1.23271900 & -2.15459900 \\ \mathrm{C} & -0.34622900 & -1.88493400 & 0.50787200 \\ \mathrm{C} & -1.21888500 & -1.93699200 & -0.76438600 \\ \mathrm{C} & -2.41645000 & -1.01582400 & -0.68371500 \\ \mathrm{C} & -3.51744400 & -1.34753800 & 0.11576400 \\ \mathrm{C} & -4.59851700 & -0.47597400 & 0.24112200 \\ \mathrm{C} & -4.59366500 & 0.74600600 & -0.43341500 \\ \mathrm{C} & -3.50354000 & 1.08569800 & -1.23484300 \\ \mathrm{C} & -2.42202000 & 0.21177000 & -1.36007200 \\ \mathrm{H} & 4.08487000 & 0.42947700 & -0.77366700 \\ \mathrm{H} & 4.56402400 & -1.14387500 & -0.11721800 \\ \mathrm{H} & 2.06538100 & 3.58186900 & 0.29643500 \\ \mathrm{H} & 1.41723400 & 2.90487800 & -1.21655200 \\ \mathrm{H} & -0.71853600 & 0.23466600 & 0.83263600 \\ \mathrm{H} & -0.09215700 & 2.87227900 & 1.45936000 \\ \mathrm{H} & -0.75329300 & 2.41556600 & -0.12733100 \\ \mathrm{H} & -0.34312200 & 4.10219300 & 0.19702000 \\ \mathrm{H} & 3.54747700 & -2.29372500 & -2.11015600 \\ \mathrm{H} & 4.79393500 & -1.13752600 & -2.62057200 \\ \mathrm{H} & 3.07065200 & -0.71974300 & -2.77626500 \\ \mathrm{H} & -0.92774600 & -2.24667500 & 1.36196000 \\ \mathrm{H} & 0.52085700 & -2.54079500 & 0.40886800 \\ \mathrm{H} & -1.55059900 & -2.97355900 & -0.88957100 \\ \mathrm{H} & -0.61395400 & -1.67811800 & -1.63560300 \\ \mathrm{H} & -3.52836700 & -2.29913700 & 0.64190300 \\ \mathrm{H} & -5.44521400 & -0.75118800 & 0.86288700 \\ \mathrm{H} & -5.43475900 & 1.42566100 & -0.33678000 \\ \mathrm{H} & -3.49388800 & 2.03222900 & -1.76745200 \\ \mathrm{H} & -1.56594700 & 0.47774100 & -1.97217800\end{array}$


<smiles>CCOC(=O)CCCC([N+](=O)[O-])P(=O)(OCC)OCC</smiles>

Nucleophile-69

$\begin{array}{crcc}\mathrm{O} & 0.45578200 & 1.47852700 & 0.18006700 \\ \mathrm{P} & 1.31957400 & 0.15209800 & 0.48472800 \\ \mathrm{O} & 2.25592600 & 0.05468300 & -0.82319300 \\ \mathrm{O} & 2.02327800 & 0.05783800 & 1.78566500 \\ \mathrm{C} & 1.12863500 & 2.75462500 & 0.00378900 \\ \mathrm{C} & 3.57354400 & -0.56371500 & -0.73071300 \\ \mathrm{C} & -0.05794000 & -1.04617600 & 0.25300700 \\ \mathrm{~N} & 0.38008200 & -2.41514000 & 0.69081700 \\ \mathrm{O} & 1.45181600 & -2.82886900 & 0.24996600 \\ \mathrm{O} & -0.38547300 & -3.05067900 & 1.40596800 \\ \mathrm{C} & 4.61381900 & 0.46368800 & -0.32984600 \\ \mathrm{C} & 1.16831800 & 3.11840900 & -1.46742000 \\ \mathrm{C} & -0.56035100 & -1.12318200 & -1.19585400 \\ \mathrm{C} & -1.80227900 & -2.00442200 & -1.38854400 \\ \mathrm{C} & -2.98902700 & -1.62711100 & -0.47640200 \\ \mathrm{C} & -3.24291600 & -0.13682100 & -0.55012700 \\ \mathrm{O} & -2.84850800 & 0.48354300 & 0.58528400 \\ \mathrm{O} & -3.66868100 & 0.44973100 & -1.52191600 \\ \mathrm{C} & -2.89141900 & 1.93081500 & 0.56799000 \\ \mathrm{C} & -2.33081000 & 2.41304800 & 1.88938500 \\ \mathrm{H} & 2.13205800 & 2.70522900 & 0.43745900 \\ \mathrm{H} & 0.54605800 & 3.47240900 & 0.58602200 \\ \mathrm{H} & 3.75775600 & -0.96282500 & -1.73049700 \\ \mathrm{H} & 3.53277700 & -1.39479300 & -0.02538100 \\ \mathrm{H} & -0.85490600 & -0.74998400 & 0.93367700 \\ \mathrm{H} & 5.61047700 & 0.01081900 & -0.34840900 \\ \mathrm{H} & 4.60886300 & 1.31135900 & -1.02175000 \\ \mathrm{H} & 4.41735000 & 0.82384300 & 0.68279700 \\ \mathrm{H} & 1.62640700 & 4.10415300 & -1.59790200 \\ \mathrm{H} & 0.15659000 & 3.15072600 & -1.88173800 \\ \mathrm{H} & 1.75033300 & 2.38097300 & -2.02411000 \\ \mathrm{H} & 0.25948400 & -1.47248100 & -1.82835200 \\ \mathrm{H} & -0.78434800 & -0.09784900 & -1.50351600 \\ \mathrm{H} & -1.55482200 & -3.05730500 & -1.21936700 \\ \mathrm{H} & -2.11422900 & -1.91039300 & -2.43303000 \\ \mathrm{H} & -2.79218400 & -1.92580200 & 0.55475700 \\ \mathrm{H} & -3.88664300 & -2.14690900 & -0.82145400 \\ \mathrm{H} & -3.92625200 & 2.24801800 & 0.41045200 \\ \mathrm{H} & -2.29938600 & 2.28188600 & -0.28172700 \\ \mathrm{H} & -2.35407100 & 3.50674900 & 1.92413300 \\ \mathrm{H} & -2.91982200 & 2.03129100 & 2.72769600 \\ \mathrm{H} & -1.29623200 & 2.08225200 & 2.00728700\end{array}$


<smiles></smiles>

Nucleophile-70

$\begin{array}{lrrr}\mathrm{C} & 0.19187300 & 3.07562500 & -1.80228200 \\ \mathrm{C} & -0.84567100 & 2.00694200 & -2.14817900 \\ \mathrm{C} & -1.94440900 & 1.86830500 & -1.08857100 \\ \mathrm{C} & -3.00192000 & 0.82254200 & -1.46694600 \\ \mathrm{C} & -3.97723900 & 0.45658500 & -0.33501400 \\ \mathrm{C} & -3.33933400 & -0.34100000 & 0.81524100 \\ \mathrm{C} & -2.91973900 & -1.76126800 & 0.40485700 \\ \mathrm{C} & -1.94439500 & -2.45869700 & 1.36527800 \\ \mathrm{C} & -0.64321700 & -1.68215500 & 1.63590400 \\ \mathrm{C} & -0.01306300 & -1.08077700 & 0.37996500 \\ \mathrm{P} & 1.49188600 & -0.09279400 & 0.78191700 \\ \mathrm{O} & 0.69754800 & 1.19628500 & 1.33560600 \\ \mathrm{O} & 2.49540600 & -0.70977400 & 1.68178500 \\ \mathrm{O} & 2.04573800 & 0.39427900 & -0.64840200 \\ \mathrm{C} & 3.37222500 & 0.01371600 & -1.11818900 \\ \mathrm{C} & 3.31696200 & -1.26331600 & -1.93316600 \\ \mathrm{C} & 1.45122400 & 2.30256800 & 1.89763700 \\ \mathrm{C} & 0.45534000 & 3.36108200 & 2.32106200 \\ \mathrm{H} & 0.95105000 & 3.16462400 & -2.58586600 \\ \mathrm{H} & -0.27633400 & 4.05895900 & -1.67703800 \\ \mathrm{H} & 0.70686300 & 2.82163600 & -0.87217600 \\ \mathrm{H} & -0.34173300 & 1.03945200 & -2.26831900 \\ \mathrm{H} & -1.30619600 & 2.23566200 & -3.11858900 \\ \mathrm{H} & -2.43439000 & 2.83958200 & -0.93146500 \\ \mathrm{H} & -1.47402200 & 1.61767600 & -0.12985900 \\ \mathrm{H} & -2.49529900 & -0.08108100 & -1.82943000 \\ \mathrm{H} & -3.57402800 & 1.19764400 & -2.32469600 \\ \mathrm{H} & -4.80941900 & -0.12595100 & -0.75134100 \\ \mathrm{H} & -4.41919500 & 1.37767700 & 0.06648600 \\ \mathrm{H} & -4.04254000 & -0.40356100 & 1.65496000 \\ \mathrm{H} & -2.47755500 & 0.21805700 & 1.19724500 \\ \mathrm{H} & -2.46852300 & -1.74017000 & -0.59286200 \\ \mathrm{H} & -3.81713800 & -2.38275800 & 0.30057600 \\ \mathrm{H} & -1.68939800 & -3.44235800 & 0.95519400 \\ \mathrm{H} & -2.42940200 & -2.64398800 & 2.33109300 \\ \mathrm{H} & 0.09342100 & -2.32300300 & 2.12544700 \\ \mathrm{H} & -0.83165000 & -0.84062600 & 2.30885500 \\ \mathrm{H} & -0.68718400 & -0.41019400 & -0.14662500 \\ \mathrm{H} & 4.03306400 & -0.08988800 & -0.25433700 \\ \mathrm{H} & 3.69956600 & 0.86358600 & -1.72119000 \\ \mathrm{H} & 4.30218000 & -1.46260000 & -2.36711200 \\ \mathrm{H} & 3.04251300 & -2.11797900 & -1.30964300 \\ \mathrm{H} & 2.58868800 & -1.17551400 & -2.74279500 \\ \mathrm{H} & 2.13505200 & 2.68192400 & 1.13051400\end{array}$




$\begin{array}{rrrr}\mathrm{H} & 2.04069700 & 1.93021100 & 2.74041800 \\ \mathrm{H} & 0.98196400 & 4.21463400 & 2.75833700 \\ \mathrm{H} & -0.12271900 & 3.71030800 & 1.46169600 \\ \mathrm{H} & -0.23630000 & 2.96051200 & 3.06699900 \\ \mathrm{~N} & 0.34189500 & -2.13259600 & -0.63336000 \\ \mathrm{O} & 0.03183100 & -1.91103000 & -1.80194300 \\ \mathrm{O} & 0.93380200 & -3.13142100 & -0.23395800\end{array}$<smiles>O=C1c2ccccc2CCC1C(=S)Nc1ccccc1</smiles>

Nucleophile-71

$\begin{array}{lrrr}\mathrm{C} & 4.25189500 & 0.21678900 & -1.09748200 \\ \mathrm{C} & 3.08835400 & 0.41925100 & -0.34888500 \\ \mathrm{C} & 2.46114500 & -0.70333300 & 0.22864900 \\ \mathrm{C} & 2.99802300 & -1.99009200 & 0.05097100 \\ \mathrm{C} & 4.15384900 & -2.17098400 & -0.69329300 \\ \mathrm{C} & 4.78061800 & -1.06004200 & -1.26941000 \\ \mathrm{C} & 2.50175900 & 1.79601200 & -0.17608800 \\ \mathrm{C} & 1.71760800 & 1.92201900 & 1.13092000 \\ \mathrm{C} & 0.65159700 & 0.83426400 & 1.27855600 \\ \mathrm{C} & 1.21657500 & -0.56783500 & 1.01165800 \\ \mathrm{O} & 0.61133100 & -1.55316700 & 1.43692800 \\ \mathrm{C} & -0.65251700 & 1.05570500 & 0.45983800 \\ \mathrm{~N} & -1.50222300 & 0.02270100 & 0.62690600 \\ \mathrm{~S} & -0.91152000 & 2.43862100 & -0.43079200 \\ \mathrm{C} & -2.78443900 & -0.27140200 & 0.11715600 \\ \mathrm{C} & -3.22063600 & -1.59301900 & 0.31374500 \\ \mathrm{C} & -4.47515800 & -1.99322800 & -0.12828900 \\ \mathrm{C} & -5.31547700 & -1.08228500 & -0.77216700 \\ \mathrm{C} & -4.88216200 & 0.22845100 & -0.96222500 \\ \mathrm{C} & -3.62560400 & 0.64694000 & -0.52418100 \\ \mathrm{H} & 4.74760600 & 1.07328200 & -1.54535000 \\ \mathrm{H} & 2.48145700 & -2.82636800 & 0.50852400 \\ \mathrm{H} & 4.56881200 & -3.16441900 & -0.82863500 \\ \mathrm{H} & 5.68821500 & -1.19164300 & -1.85100400 \\ \mathrm{H} & 1.82973100 & 2.01543300 & -1.01399900 \\ \mathrm{H} & 3.29998700 & 2.54442900 & -0.21168200 \\ \mathrm{H} & 2.41018800 & 1.82476800 & 1.97526600 \\ \mathrm{H} & 1.24469000 & 2.90349500 & 1.19427000 \\ \mathrm{H} & 0.31944600 & 0.80183900 & 2.32408100 \\ \mathrm{H} & -1.09706200 & -0.74998000 & 1.15845200 \\ \mathrm{H} & -2.56338300 & -2.30343900 & 0.80831800 \\ \mathrm{H} & -4.79518200 & -3.01845000 & 0.02887900 \\ \mathrm{H} & -6.29553200 & -1.39261000 & -1.11987700 \\ \mathrm{H} & -5.52793500 & 0.94622100 & -1.45868100 \\ \mathrm{H} & -3.29439900 & 1.66267300 & -0.67649500 \\ & & & \\ & & & \\ & & & \end{array}$


<smiles>COc1ccc2c(c1)CCC(C(=S)Nc1ccccc1)C2=O</smiles>

Nucleophile-72

$\begin{array}{rrrr}\mathrm{C} & 3.69227700 & 0.39323400 & -0.46408800 \\ \mathrm{C} & 2.44511800 & 0.58260900 & 0.13516700 \\ \mathrm{C} & 1.78847000 & -0.51695800 & 0.72161900 \\ \mathrm{C} & 2.39718800 & -1.78898100 & 0.69466300 \\ \mathrm{C} & 3.62842400 & -1.97317000 & 0.10463700 \\ \mathrm{C} & 4.28475000 & -0.87418500 & -0.48306800 \\ \mathrm{C} & 1.79589800 & 1.94237200 & 0.13177900 \\ \mathrm{C} & 0.85912700 & 2.12934000 & 1.32604900 \\ \mathrm{C} & -0.17681700 & 1.00879200 & 1.43378100 \\ \mathrm{C} & 0.46576000 & -0.38411600 & 1.34098100 \\ \mathrm{O} & -0.15524700 & -1.36120900 & 1.77063800 \\ \mathrm{C} & -1.38191500 & 1.11222800 & 0.45811400 \\ \mathrm{~N} & -2.20505600 & 0.05502700 & 0.60407600 \\ \mathrm{~S} & -1.58688500 & 2.41416700 & -0.56140900 \\ \mathrm{C} & -3.40477800 & -0.33364400 & -0.02746800 \\ \mathrm{C} & -3.79779000 & -1.66473100 & 0.19441100 \\ \mathrm{C} & -4.97446600 & -2.15273700 & -0.35956800 \\ \mathrm{C} & -5.77887600 & -1.32155200 & -1.14224200 \\ \mathrm{C} & -5.38912700 & -0.00091300 & -1.35704500 \\ \mathrm{C} & -4.21087300 & 0.50508500 & -0.80791500 \\ \mathrm{O} & 5.49025600 & -1.14577800 & -1.03765900 \\ \mathrm{C} & 6.20505100 & -0.08470700 & -1.65948100 \\ \mathrm{H} & 4.19091200 & 1.24500800 & -0.91031900 \\ \mathrm{H} & 1.86595600 & -2.61745000 & 1.14946000 \\ \mathrm{H} & 4.11279100 & -2.94254200 & 0.07575200 \\ \mathrm{H} & 2.56578000 & 2.72092100 & 0.12876600 \\ \mathrm{H} & 1.21897900 & 2.06778300 & -0.79203100 \\ \mathrm{H} & 0.34833900 & 3.09127600 & 1.25643400 \\ \mathrm{H} & 1.45017100 & 2.12522800 & 2.24936300 \\ \mathrm{H} & -0.62670400 & 1.03697100 & 2.43441000 \\ \mathrm{H} & -1.83059100 & -0.66086200 & 1.23106600 \\ \mathrm{H} & -3.16715800 & -2.31280500 & 0.79727900 \\ \mathrm{H} & -5.26160600 & -3.18421600 & -0.18105200 \\ \mathrm{H} & -6.69785700 & -1.70069200 & -1.57756700 \\ \mathrm{H} & -6.00816500 & 0.65550300 & -1.96092800 \\ \mathrm{H} & -3.91231800 & 1.52782400 & -0.98003300 \\ \mathrm{H} & 7.12634600 & -0.52730300 & -2.03803600 \\ \mathrm{H} & 5.63691000 & 0.34462700 & -2.49288500 \\ \mathrm{H} & 6.44916100 & 0.70697700 & -0.94145900\end{array}$


<smiles>Cc1ccc(NC(=S)C2CCc3ccccc3C2=O)cc1</smiles>

Nucleophile-73

$\begin{array}{lrrr}\mathrm{C} & 4.56314900 & -0.03221400 & -1.22735600 \\ \mathrm{C} & 3.44808600 & 0.27833600 & -0.44280400 \\ \mathrm{C} & 2.78404800 & -0.77302500 & 0.22106900 \\ \mathrm{C} & 3.23752000 & -2.09704900 & 0.09178500 \\ \mathrm{C} & 4.34632000 & -2.38544200 & -0.68925900 \\ \mathrm{C} & 5.00931400 & -1.34560800 & -1.35115600 \\ \mathrm{C} & 2.95005700 & 1.69483900 & -0.32090900 \\ \mathrm{C} & 2.23340100 & 1.93523300 & 1.00864100 \\ \mathrm{C} & 1.11408900 & 0.92232400 & 1.25898300 \\ \mathrm{C} & 1.58570000 & -0.52261700 & 1.04714600 \\ \mathrm{O} & 0.94544700 & -1.44679400 & 1.55117300 \\ \mathrm{C} & -0.20917100 & 1.17786700 & 0.48151700 \\ \mathrm{~N} & -1.10783600 & 0.20676400 & 0.73451400 \\ \mathrm{~S} & -0.42829700 & 2.52832400 & -0.46914600 \\ \mathrm{C} & -2.42529300 & -0.03421500 & 0.29071000 \\ \mathrm{C} & -2.93332400 & -1.31409200 & 0.56986700 \\ \mathrm{C} & -4.22601300 & -1.65589700 & 0.19993200 \\ \mathrm{C} & -5.05637200 & -0.73912200 & -0.45954100 \\ \mathrm{C} & -4.53859800 & 0.53190200 & -0.72261800 \\ \mathrm{C} & -3.24286000 & 0.89705200 & -0.35966000 \\ \mathrm{C} & -6.44952900 & -1.12401400 & -0.88890000 \\ \mathrm{H} & 5.08662700 & 0.76856100 & -1.74179900 \\ \mathrm{H} & 2.69478200 & -2.87580300 & 0.61575200 \\ \mathrm{H} & 4.69696700 & -3.40764600 & -0.78724400 \\ \mathrm{H} & 5.88072000 & -1.56144800 & -1.96221300 \\ \mathrm{H} & 3.78766900 & 2.39128100 & -0.43036700 \\ \mathrm{H} & 2.25479500 & 1.91038500 & -1.14069300 \\ \mathrm{H} & 1.82071700 & 2.94508800 & 1.03751700 \\ \mathrm{H} & 2.95606600 & 1.84092200 & 1.82776500 \\ \mathrm{H} & 0.82623100 & 0.96515800 & 2.31716500 \\ \mathrm{H} & -0.72711200 & -0.56072100 & 1.29084200 \\ \mathrm{H} & -2.30291100 & -2.04044500 & 1.07634400 \\ \mathrm{H} & -4.59748600 & -2.65160300 & 0.42606200 \\ \mathrm{H} & -5.16303500 & 1.26580900 & -1.22480300 \\ \mathrm{H} & -2.86666100 & 1.88541000 & -0.57512100 \\ \mathrm{H} & -6.43287800 & -1.68980300 & -1.82857400 \\ \mathrm{H} & -6.93870400 & -1.75524300 & -0.14051600 \\ \mathrm{H} & -7.07489500 & -0.24161600 & -1.04944000 \\ & & & \\ & & & \\ & & & \end{array}$


<smiles>O=C1c2ccccc2CCC1C(=S)Nc1ccc(Cl)cc1</smiles>

Nucleophile-74

$\begin{array}{lrrr}\mathrm{C} & 4.87498300 & -0.16084800 & -1.28046200 \\ \mathrm{C} & 3.78172500 & 0.20046700 & -0.48730400 \\ \mathrm{C} & 3.09678400 & -0.81554100 & 0.20998200 \\ \mathrm{C} & 3.50859600 & -2.15544000 & 0.10446400 \\ \mathrm{C} & 4.59662700 & -2.49409500 & -0.68535600 \\ \mathrm{C} & 5.28007100 & -1.48956400 & -1.38025500 \\ \mathrm{C} & 3.32760800 & 1.63366000 & -0.39061400 \\ \mathrm{C} & 2.64511900 & 1.92539700 & 0.94661700 \\ \mathrm{C} & 1.50025000 & 0.95383100 & 1.24077100 \\ \mathrm{C} & 1.91884100 & -0.51083700 & 1.04505800 \\ \mathrm{O} & 1.25169900 & -1.40313600 & 1.57184700 \\ \mathrm{C} & 0.16826000 & 1.23877900 & 0.49171200 \\ \mathrm{~N} & -0.75649100 & 0.30228100 & 0.78959300 \\ \mathrm{~S} & -0.03128200 & 2.57294100 & -0.48284600 \\ \mathrm{C} & -2.08939300 & 0.09792200 & 0.38377700 \\ \mathrm{C} & -2.63483000 & -1.15627900 & 0.70716100 \\ \mathrm{C} & -3.94628700 & -1.46952100 & 0.37795000 \\ \mathrm{C} & -4.72560700 & -0.51964100 & -0.28184700 \\ \mathrm{C} & -4.20224200 & 0.72808800 & -0.60642000 \\ \mathrm{C} & -2.88558800 & 1.04348600 & -0.27571000 \\ \mathrm{Cl} & -6.38574400 & -0.90627300 & -0.70544300 \\ \mathrm{H} & 5.41416200 & 0.61191900 & -1.82076300 \\ \mathrm{H} & 2.95114800 & -2.90598600 & 0.65350800 \\ \mathrm{H} & 4.91550800 & -3.52817000 & -0.76489800 \\ \mathrm{H} & 6.13543800 & -1.74511000 & -1.99852800 \\ \mathrm{H} & 4.18282300 & 2.30223200 & -0.53202900 \\ \mathrm{H} & 2.62390700 & 1.85063300 & -1.20281800 \\ \mathrm{H} & 3.38059600 & 1.82526400 & 1.75337800 \\ \mathrm{H} & 2.26567800 & 2.94847900 & 0.96232700 \\ \mathrm{H} & 1.23931000 & 1.02391100 & 2.30458000 \\ \mathrm{H} & -0.38287200 & -0.46790200 & 1.34822700 \\ \mathrm{H} & -2.01911400 & -1.89367000 & 1.21468000 \\ \mathrm{H} & -4.36065000 & -2.43926100 & 0.62676300 \\ \mathrm{H} & -4.82001200 & 1.45795400 & -1.11653400 \\ \mathrm{H} & -2.47542600 & 2.00922500 & -0.52888600 \\ & & & \\ & & & \\ & & & \end{array}$


<smiles>O=C1c2ccccc2CCC1C(=S)Nc1cccc(Cl)c1</smiles>

Nucleophile-75

$\begin{array}{lrrr}\mathrm{C} & -4.72033800 & -0.59722100 & -1.26739500 \\ \mathrm{C} & -3.60906500 & -0.61536400 & -0.41909100 \\ \mathrm{C} & -3.12228700 & 0.61345500 & 0.07148700 \\ \mathrm{C} & -3.74405300 & 1.82177800 & -0.28830200 \\ \mathrm{C} & -4.84659000 & 1.81948700 & -1.12894300 \\ \mathrm{C} & -5.33368300 & 0.60246900 & -1.61957000 \\ \mathrm{C} & -2.93032700 & -1.90872700 & -0.05017000 \\ \mathrm{C} & -2.25123500 & -1.82536900 & 1.31765000 \\ \mathrm{C} & -1.29061700 & -0.63880300 & 1.41995100 \\ \mathrm{C} & -1.93763700 & 0.67224900 & 0.94949200 \\ \mathrm{O} & -1.44305000 & 1.74620800 & 1.29692100 \\ \mathrm{C} & 0.09203200 & -0.83361200 & 0.73695800 \\ \mathrm{~N} & 0.84136500 & 0.28416200 & 0.85060000 \\ \mathrm{~S} & 0.53711600 & -2.27343200 & 0.03410500 \\ \mathrm{C} & 2.13341800 & 0.63063800 & 0.41192900 \\ \mathrm{C} & 3.08475700 & -0.28250600 & -0.05971600 \\ \mathrm{C} & 4.33388000 & 0.20109800 & -0.44108300 \\ \mathrm{C} & 4.67047500 & 1.54955300 & -0.36955800 \\ \mathrm{C} & 3.71090100 & 2.44404900 & 0.10702700 \\ \mathrm{C} & 2.45541200 & 1.99644100 & 0.49538400 \\ \mathrm{Cl} & 5.52856600 & -0.94518300 & -1.03254000 \\ \mathrm{H} & -5.10805600 & -1.53677900 & -1.65041400 \\ \mathrm{H} & -3.33486300 & 2.74450900 & 0.10748900 \\ \mathrm{H} & -5.32747600 & 2.75220500 & -1.40483800 \\ \mathrm{H} & -6.19874000 & 0.59079700 & -2.27589100 \\ \mathrm{H} & -3.66009300 & -2.72462600 & -0.06041000 \\ \mathrm{H} & -2.17591300 & -2.15511800 & -0.80651300 \\ \mathrm{H} & -1.71016900 & -2.74939300 & 1.52829200 \\ \mathrm{H} & -3.01785000 & -1.69893000 & 2.09103200 \\ \mathrm{H} & -1.05377200 & -0.46305800 & 2.47717800 \\ \mathrm{H} & 0.32974500 & 1.07228400 & 1.25286500 \\ \mathrm{H} & 2.85061400 & -1.33308500 & -0.12962200 \\ \mathrm{H} & 5.65259500 & 1.88684800 & -0.67762900 \\ \mathrm{H} & 3.94757700 & 3.50096500 & 0.17233500 \\ \mathrm{H} & 1.71149800 & 2.69989700 & 0.85777000\end{array}$


<smiles>O=C1c2ccccc2CCC1C(=S)Nc1ccccc1Cl</smiles>

Nucleophile-76

$\begin{array}{lrrr}\mathrm{C} & 2.88806800 & -1.23700200 & -1.42041200 \\ \mathrm{C} & 2.32315800 & -0.07752700 & -0.87923800 \\ \mathrm{C} & 2.31004800 & 0.06976400 & 0.52368500 \\ \mathrm{C} & 2.85447200 & -0.92890900 & 1.34809000 \\ \mathrm{C} & 3.40035700 & -2.07680000 & 0.79398800 \\ \mathrm{C} & 3.41579000 & -2.22831500 & -0.59763500 \\ \mathrm{C} & 1.72198900 & 0.97694600 & -1.78036000 \\ \mathrm{C} & 1.62625100 & 2.34103700 & -1.09697900 \\ \mathrm{C} & 0.92131000 & 2.22478700 & 0.27036100 \\ \mathrm{C} & 1.69541100 & 1.26248100 & 1.17332800 \\ \mathrm{O} & 1.77542300 & 1.45942600 & 2.37580200 \\ \mathrm{C} & -0.56059500 & 1.85514300 & 0.08068600 \\ \mathrm{~N} & -0.84278800 & 0.54382500 & 0.28407400 \\ \mathrm{~S} & -1.61807500 & 3.03770000 & -0.41059400 \\ \mathrm{C} & -2.04468200 & -0.18226900 & 0.17773200 \\ \mathrm{C} & -1.96079400 & -1.57395300 & -0.02633500 \\ \mathrm{C} & -3.09833500 & -2.36803000 & -0.10577500 \\ \mathrm{C} & -4.35643100 & -1.78162200 & 0.01729900 \\ \mathrm{C} & -4.45763700 & -0.40796000 & 0.22877900 \\ \mathrm{C} & -3.31820000 & 0.38721500 & 0.31278700 \\ \mathrm{Cl} & -0.38366600 & -2.34244000 & -0.18618300 \\ \mathrm{H} & 2.91004700 & -1.36079500 & -2.49941200 \\ \mathrm{H} & 2.82319900 & -0.77278100 & 2.42083300 \\ \mathrm{H} & 3.81229700 & -2.85149500 & 1.43241400 \\ \mathrm{H} & 3.84551500 & -3.12171200 & -1.04061800 \\ \mathrm{H} & 2.30965300 & 1.05052400 & -2.70110200 \\ \mathrm{H} & 0.71851100 & 0.65083800 & -2.08714500 \\ \mathrm{H} & 1.08219200 & 3.05548800 & -1.71906500 \\ \mathrm{H} & 2.63269300 & 2.74178900 & -0.92928700 \\ \mathrm{H} & 0.92380600 & 3.19272900 & 0.77264600 \\ \mathrm{H} & -0.04711700 & -0.05474800 & 0.47902000 \\ \mathrm{H} & -2.98919600 & -3.43416700 & -0.26608400 \\ \mathrm{H} & -5.24615100 & -2.39855000 & -0.05022000 \\ \mathrm{H} & -5.43195100 & 0.05733900 & 0.33441100 \\ \mathrm{H} & -3.39691400 & 1.45173400 & 0.47328300\end{array}$


<smiles>COc1cccc(NC(=S)C2CCc3ccccc3C2=O)c1</smiles>

Nucleophile-77

$\begin{array}{lrrr}\mathrm{C} & -4.64823500 & -0.71535000 & -1.23644800 \\ \mathrm{C} & -3.54471900 & -0.66214300 & -0.37935500 \\ \mathrm{C} & -3.08739900 & 0.60220900 & 0.04410000 \\ \mathrm{C} & -3.72986600 & 1.77477700 & -0.38933700 \\ \mathrm{C} & -4.82395700 & 1.70200900 & -1.23800000 \\ \mathrm{C} & -5.28205400 & 0.44933100 & -1.66213400 \\ \mathrm{C} & -2.84427600 & -1.91828100 & 0.06915100 \\ \mathrm{C} & -2.17323800 & -1.74156700 & 1.43204500 \\ \mathrm{C} & -1.23629800 & -0.53228400 & 1.46775300 \\ \mathrm{C} & -1.91303100 & 0.73598100 & 0.92927300 \\ \mathrm{O} & -1.45186300 & 1.83859600 & 1.22766900 \\ \mathrm{C} & 0.15037900 & -0.73458100 & 0.79182400 \\ \mathrm{~N} & 0.87093200 & 0.40260300 & 0.82863900 \\ \mathrm{~S} & 0.62391500 & -2.21561600 & 0.18816600 \\ \mathrm{C} & 2.16056300 & 0.75642200 & 0.37872700 \\ \mathrm{C} & 3.09956300 & -0.16156500 & -0.10747400 \\ \mathrm{C} & 4.35323000 & 0.30338200 & -0.51512000 \\ \mathrm{C} & 4.67612100 & 1.66404900 & -0.43826200 \\ \mathrm{C} & 3.73148500 & 2.55943700 & 0.05021400 \\ \mathrm{C} & 2.47558700 & 2.12054900 & 0.46047500 \\ \mathrm{O} & 5.32852200 & -0.51244500 & -1.00403100 \\ \mathrm{C} & 5.04036800 & -1.90006000 & -1.11030900 \\ \mathrm{H} & -5.01367600 & -1.68312100 & -1.56779600 \\ \mathrm{H} & -3.34271000 & 2.72658500 & -0.04333000 \\ \mathrm{H} & -5.32048400 & 2.60750200 & -1.57109500 \\ \mathrm{H} & -6.14044900 & 0.38244000 & -2.32393300 \\ \mathrm{H} & -3.55897800 & -2.74686300 & 0.10431700 \\ \mathrm{H} & -2.08108200 & -2.19496900 & -0.66766900 \\ \mathrm{H} & -1.61514300 & -2.64085100 & 1.69789200 \\ \mathrm{H} & -2.94557400 & -1.58495400 & 2.19428200 \\ \mathrm{H} & -1.00165800 & -0.29550400 & 2.51338400 \\ \mathrm{H} & 0.34762700 & 1.19962800 & 1.19549100 \\ \mathrm{H} & 2.83251300 & -1.20357000 & -0.16177800 \\ \mathrm{H} & 5.65769100 & 1.98904700 & -0.76357200 \\ \mathrm{H} & 3.97435300 & 3.61549000 & 0.11133200 \\ \mathrm{H} & 1.73886100 & 2.82525800 & 0.83424000 \\ \mathrm{H} & 4.18852100 & -2.08432000 & -1.77574800 \\ \mathrm{H} & 5.93589100 & -2.36040500 & -1.52896100 \\ \mathrm{H} & 4.82653000 & -2.34286100 & -0.13002300\end{array}$


<smiles>COc1ccccc1NC(=S)C1CCc2ccccc2C1=O</smiles>

Nucleophile-79

$\begin{array}{lrrr}\mathrm{C} & -4.59703500 & -0.18953300 & -1.00609600 \\ \mathrm{C} & -3.43906900 & -0.36227500 & -0.24074900 \\ \mathrm{C} & -2.65365000 & 0.77124300 & 0.04855700 \\ \mathrm{C} & -3.03234300 & 2.03936000 & -0.42364900 \\ \mathrm{C} & -4.18381700 & 2.19156800 & -1.18114700 \\ \mathrm{C} & -4.96740800 & 1.06906600 & -1.47248400 \\ \mathrm{C} & -3.02699200 & -1.72627400 & 0.24848300 \\ \mathrm{C} & -2.18581200 & -1.65134200 & 1.52377900 \\ \mathrm{C} & -0.98881700 & -0.70783800 & 1.38196600 \\ \mathrm{C} & -1.40636400 & 0.66447600 & 0.83677000 \\ \mathrm{O} & -0.69013500 & 1.64121000 & 1.04672000 \\ \mathrm{C} & 0.21591700 & -1.26719600 & 0.57547000 \\ \mathrm{~N} & 1.19660100 & -0.34964900 & 0.47156600 \\ \mathrm{~S} & 0.24156300 & -2.83635100 & 0.01157200 \\ \mathrm{C} & 2.47352400 & -0.37109400 & -0.11998700 \\ \mathrm{C} & 3.21012300 & 0.83417600 & 0.01810200 \\ \mathrm{C} & 4.48139600 & 0.95302200 & -0.53252900 \\ \mathrm{C} & 5.04065000 & -0.12688600 & -1.22335400 \\ \mathrm{C} & 4.32804800 & -1.31202000 & -1.35809600 \\ \mathrm{C} & 3.04868700 & -1.43956700 & -0.81088300 \\ \mathrm{O} & 2.57090300 & 1.81794000 & 0.71708600 \\ \mathrm{C} & 3.18233600 & 3.09361000 & 0.81523700 \\ \mathrm{H} & -5.21408100 & -1.05440400 & -1.23272500 \\ \mathrm{H} & -2.39720000 & 2.88364100 & -0.17984900 \\ \mathrm{H} & -4.47522000 & 3.17146200 & -1.54521000 \\ \mathrm{H} & -5.87317100 & 1.17871700 & -2.06153900 \\ \mathrm{H} & -3.91704900 & -2.34076900 & 0.41940900 \\ \mathrm{H} & -2.44323300 & -2.23141000 & -0.52968600 \\ \mathrm{H} & -1.83328500 & -2.64634500 & 1.80101300 \\ \mathrm{H} & -2.80901300 & -1.27311300 & 2.34293200 \\ \mathrm{H} & -0.58259900 & -0.49553600 & 2.37894100 \\ \mathrm{H} & 0.96975700 & 0.56691600 & 0.86483900 \\ \mathrm{H} & 5.03863700 & 1.87523400 & -0.42535200 \\ \mathrm{H} & 6.03385700 & -0.02926100 & -1.64981900 \\ \mathrm{H} & 4.75891000 & -2.15212800 & -1.89244100 \\ \mathrm{H} & 2.48917000 & -2.35663600 & -0.91366000 \\ \mathrm{H} & 3.36694500 & 3.52253100 & -0.17688900 \\ \mathrm{H} & 2.47601400 & 3.72089900 & 1.35876200 \\ \mathrm{H} & 4.12795000 & 3.04120500 & 1.36838800\end{array}$


<smiles>O=C1c2ccccc2CCC1C(=S)Nc1ccc2ccccc2c1</smiles>

Nucleophile-79

$\begin{array}{lrrr}\mathrm{C} & -5.13030000 & -0.23563900 & -1.43925300 \\ \mathrm{C} & -4.06820800 & -0.41267800 & -0.54716300 \\ \mathrm{C} & -3.50351800 & 0.72988000 & 0.05522500 \\ \mathrm{C} & -4.00115900 & 2.01095200 & -0.23940900 \\ \mathrm{C} & -5.05638800 & 2.16656100 & -1.12530100 \\ \mathrm{C} & -5.62061200 & 1.03570400 & -1.72679600 \\ \mathrm{C} & -3.52196700 & -1.78424800 & -0.24714400 \\ \mathrm{C} & -2.90246500 & -1.85835800 & 1.14915200 \\ \mathrm{C} & -1.84931400 & -0.77197500 & 1.37714000 \\ \mathrm{C} & -2.36383000 & 0.62065800 & 0.98766200 \\ \mathrm{O} & -1.80360600 & 1.61957800 & 1.44207900 \\ \mathrm{C} & -0.46109100 & -1.03636000 & 0.72756100 \\ \mathrm{~N} & 0.37270800 & 0.00179400 & 0.93916700 \\ \mathrm{~S} & -0.10815100 & -2.46382100 & -0.05350400 \\ \mathrm{C} & 1.70881100 & 0.26070200 & 0.57549500 \\ \mathrm{C} & 2.58574100 & -0.65636900 & 0.02886100 \\ \mathrm{C} & 3.91559200 & -0.27164900 & -0.28122800 \\ \mathrm{C} & 4.35322900 & 1.06647900 & -0.02678800 \\ \mathrm{C} & 3.42486000 & 1.98160600 & 0.53738000 \\ \mathrm{C} & 2.14428600 & 1.59473500 & 0.83016900 \\ \mathrm{C} & 4.83706800 & -1.19444700 & -0.84398100 \\ \mathrm{C} & 6.12538800 & -0.81164900 & -1.14000400 \\ \mathrm{C} & 6.55521700 & 0.51315900 & -0.88793600 \\ \mathrm{C} & 5.68581800 & 1.43115000 & -0.34287800 \\ \mathrm{H} & -5.57735700 & -1.10753600 & -1.90811400 \\ \mathrm{H} & -3.53478200 & 2.86296900 & 0.24236600 \\ \mathrm{H} & -5.44125800 & 3.15565000 & -1.3511100 \\ \mathrm{H} & -6.44963700 & 1.14761500 & -2.41924500 \\ \mathrm{H} & -4.31834500 & -2.52873800 & -0.34744000 \\ \mathrm{H} & -2.75654000 & -2.04370300 & -0.98792500 \\ \mathrm{H} & -2.45218400 & -2.83917100 & 1.31099600 \\ \mathrm{H} & -3.69025800 & -1.71965100 & 1.89891100 \\ \mathrm{H} & -1.64227300 & -0.69762100 & 2.45240600 \\ \mathrm{H} & -0.08222100 & 0.79808700 & 1.38909000 \\ \mathrm{H} & 2.26166900 & -1.66755200 & -0.16683200 \\ \mathrm{H} & 3.74314300 & 3.00121300 & 0.73407500 \\ \mathrm{H} & 1.44091800 & 2.30655300 & 1.25403100 \\ \mathrm{H} & 4.50377300 & -2.20994000 & -1.03686300 \\ \mathrm{H} & 6.81911800 & -1.52746000 & -1.57011900 \\ \mathrm{H} & 7.57400500 & 0.80225500 & -1.12653400 \\ \mathrm{H} & 6.00958400 & 2.44971800 & -0.14701500\end{array}$


<smiles>O=C(CC(=S)Nc1ccccc1)c1ccccc1</smiles>

Nucleophile-80

$\begin{array}{lrrr}\mathrm{C} & -4.53847600 & -1.45454300 & -0.82965700 \\ \mathrm{C} & -3.42209000 & -1.53341300 & -0.00679100 \\ \mathrm{C} & -2.66259500 & -0.38384800 & 0.26556600 \\ \mathrm{C} & -3.02411400 & 0.84177100 & -0.31531300 \\ \mathrm{C} & -4.13379300 & 0.91140000 & -1.15478600 \\ \mathrm{C} & -4.89529100 & -0.23084600 & -1.40481400 \\ \mathrm{C} & -0.76577900 & 0.72853700 & 1.65606400 \\ \mathrm{C} & -1.46787200 & -0.52194700 & 1.13160200 \\ \mathrm{O} & -0.99214000 & -1.62185500 & 1.40580800 \\ \mathrm{C} & 0.47493700 & 1.04418800 & 0.81341400 \\ \mathrm{~N} & 1.31648200 & -0.01007000 & 0.77108000 \\ \mathrm{~S} & 0.63250800 & 2.53298100 & 0.08777900 \\ \mathrm{C} & 2.55230800 & -0.23274600 & 0.12814000 \\ \mathrm{C} & 3.00733200 & -1.56176500 & 0.12855000 \\ \mathrm{C} & 4.22087900 & -1.88876200 & -0.46329600 \\ \mathrm{C} & 4.99904400 & -0.89594700 & -1.06247900 \\ \mathrm{C} & 4.54613700 & 0.42209600 & -1.05757400 \\ \mathrm{C} & 3.33041400 & 0.76773700 & -0.46770900 \\ \mathrm{H} & -5.13001800 & -2.34236500 & -1.02935100 \\ \mathrm{H} & -3.11491400 & -2.47226600 & 0.44021900 \\ \mathrm{H} & -2.42344300 & 1.72908700 & -0.14696300 \\ \mathrm{H} & -4.40293600 & 1.85705100 & -1.61388400 \\ \mathrm{H} & -5.76529200 & -0.17015800 & -2.05155600 \\ \mathrm{H} & -0.44842300 & 0.50026300 & 2.67913400 \\ \mathrm{H} & -1.40612800 & 1.60445400 & 1.67726700 \\ \mathrm{H} & 0.93801700 & -0.84652200 & 1.21324300 \\ \mathrm{H} & 2.39901300 & -2.33558100 & 0.58982300 \\ \mathrm{H} & 4.55739600 & -2.92065200 & -0.45728600 \\ \mathrm{H} & 5.94683200 & -1.14906000 & -1.52639100 \\ \mathrm{H} & 5.14385300 & 1.20248300 & -1.51831800 \\ \mathrm{H} & 2.98141700 & 1.78941900 & -0.46993700\end{array}$<smiles>COCC(=CCC(COC)COC)C(=O)OC</smiles>

Nucleophile-81

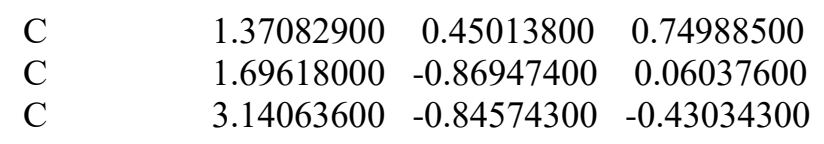




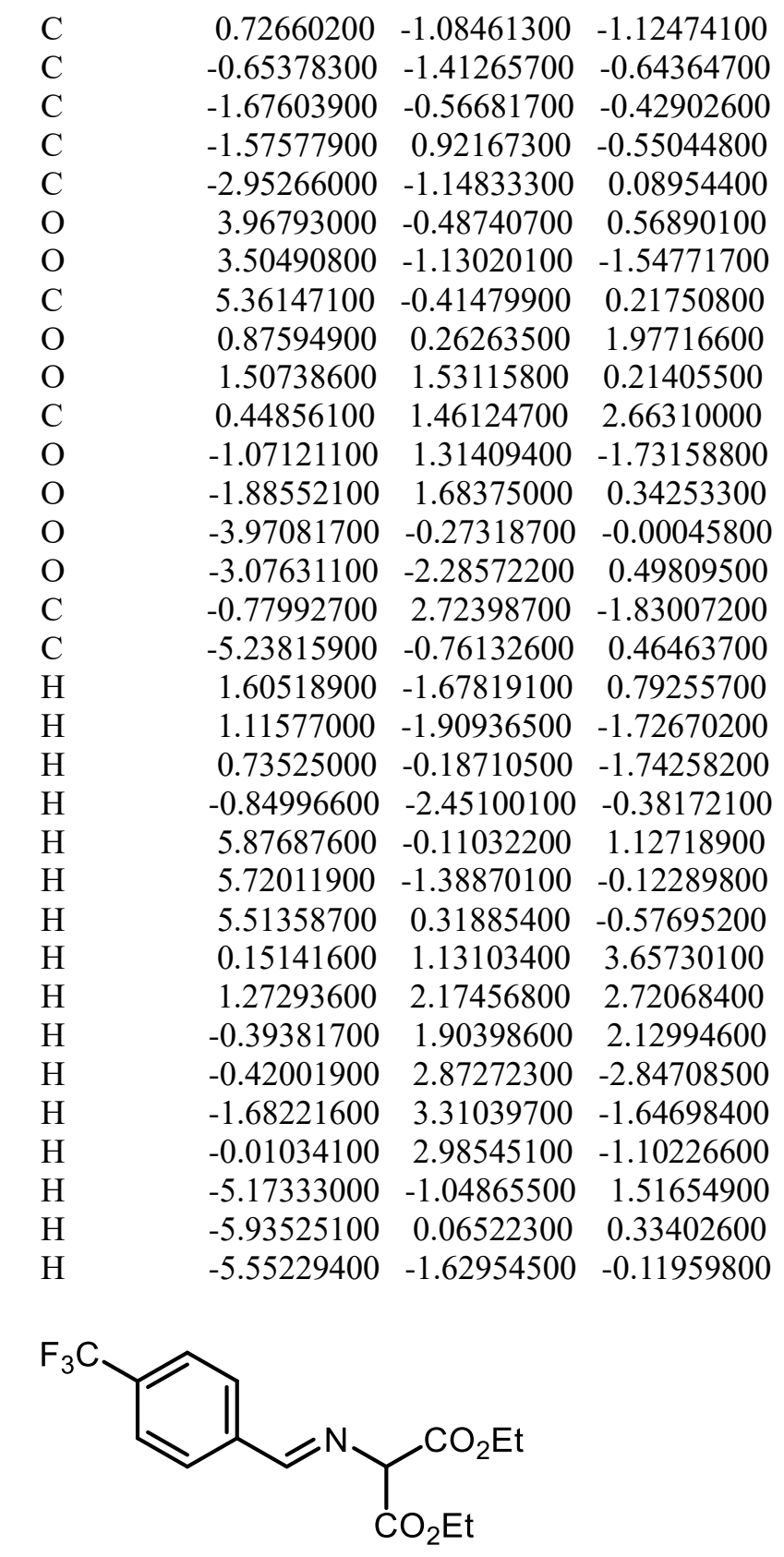

Nucleophile-82

$\begin{array}{lrrr}\mathrm{C} & -1.46626500 & -0.08602700 & -0.01623100 \\ \mathrm{C} & 0.00402300 & -0.12272200 & -0.03483400 \\ \mathrm{~N} & 0.65503500 & 0.15759700 & -1.09998700 \\ \mathrm{C} & 2.09824000 & 0.11075300 & -1.04889000 \\ \mathrm{C} & 2.67625900 & -1.23922600 & -0.57851800 \\ \mathrm{C} & 2.69666200 & 1.16625800 & -0.10301700 \\ \mathrm{O} & 3.66909600 & 1.85611200 & -0.71430500 \\ \mathrm{O} & 2.34591700 & 1.32229300 & 1.04616500 \\ \mathrm{C} & 4.41965500 & 2.77534000 & 0.12142000\end{array}$




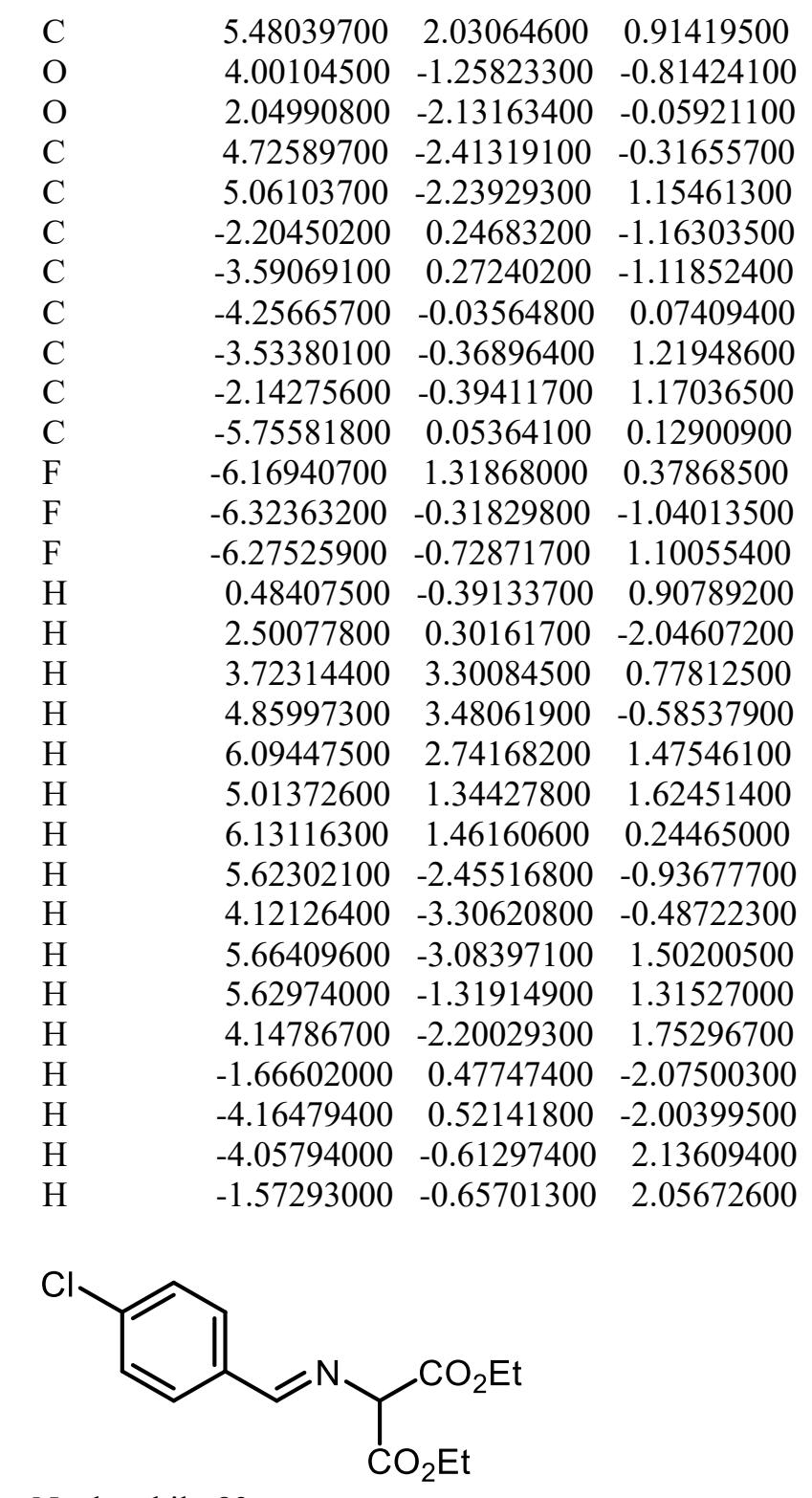

Nucleophile-83

$\begin{array}{lrrr}\mathrm{C} & 2.11545500 & -0.05099300 & 0.01554900 \\ \mathrm{C} & 0.64823100 & -0.10088400 & -0.01752800 \\ \mathrm{~N} & 0.00147200 & 0.18031000 & -1.08593500 \\ \mathrm{C} & -1.44137700 & 0.11843700 & -1.04456000 \\ \mathrm{C} & -2.05773700 & 1.16222200 & -0.09778700 \\ \mathrm{C} & -2.01040000 & -1.23944000 & -0.58678300 \\ \mathrm{O} & -3.33508200 & -1.26644200 & -0.82629400 \\ \mathrm{O} & -1.37979600 & -2.13225600 & -0.07343700 \\ \mathrm{C} & -4.05236400 & -2.42990400 & -0.33880900 \\ \mathrm{C} & -4.39133600 & -2.26986700 & 1.13311700 \\ \mathrm{O} & -3.02640200 & 1.85295100 & -0.71532100 \\ \mathrm{O} & -1.72308000 & 1.31096900 & 1.05724300 \\ \mathrm{C} & -3.79065000 & 2.76153900 & 0.11893100\end{array}$




$\begin{array}{lrrr}\mathrm{C} & -4.85694900 & 2.00542700 & 0.89338600 \\ \mathrm{C} & 2.78658100 & -0.35760100 & 1.20577800 \\ \mathrm{C} & 4.17713200 & -0.32021900 & 1.27334400 \\ \mathrm{C} & 4.89470400 & 0.02839100 & 0.13149300 \\ \mathrm{C} & 4.24966700 & 0.33813200 & -1.06894700 \\ \mathrm{C} & 2.86305100 & 0.29677700 & -1.12049900 \\ \mathrm{Cl} & 6.64868400 & 0.08000400 & 0.20033400 \\ \mathrm{H} & 0.16247000 & -0.38038100 & 0.91939300 \\ \mathrm{H} & -1.83926100 & 0.31086000 & -2.04345900 \\ \mathrm{H} & -3.44133900 & -3.31748900 & -0.51516900 \\ \mathrm{H} & -4.94837400 & -2.47373600 & -0.96061000 \\ \mathrm{H} & -4.98866300 & -3.12164900 & 1.47305900 \\ \mathrm{H} & -3.47949600 & -2.22836700 & 1.73328600 \\ \mathrm{H} & -4.96719700 & -1.35521200 & 1.29966400 \\ \mathrm{H} & -4.22630200 & 3.47085900 & -0.58679800 \\ \mathrm{H} & -3.10448500 & 3.28479300 & 0.78823400 \\ \mathrm{H} & -5.48094300 & 2.70871300 & 1.45351900 \\ \mathrm{H} & -5.49711100 & 1.43863200 & 0.21182500 \\ \mathrm{H} & -4.39483300 & 1.31539300 & 1.60314300 \\ \mathrm{H} & 2.21330100 & -0.62831100 & 2.08781800 \\ \mathrm{H} & 4.69929900 & -0.55677400 & 2.19287800 \\ \mathrm{H} & 4.83347900 & 0.60548700 & -1.94211700 \\ \mathrm{H} & 2.33235900 & 0.53107000 & -2.03642800\end{array}$<smiles>CCOC(=O)C(/N=C/c1ccccc1)C(=O)OCC</smiles>

Nucleophile-84

$\begin{array}{lrrr}\mathrm{C} & 2.60158600 & -0.29758700 & 0.05113200 \\ \mathrm{C} & 1.13416200 & -0.27330900 & 0.01462500 \\ \mathrm{~N} & 0.49666400 & -0.00366200 & -1.06311300 \\ \mathrm{C} & -0.95763600 & -0.01963800 & -1.00150000 \\ \mathrm{C} & -1.48075300 & 1.05078700 & -0.03783400 \\ \mathrm{C} & -1.54692900 & -1.36225500 & -0.54465900 \\ \mathrm{O} & -2.87570200 & -1.35254100 & -0.76308100 \\ \mathrm{O} & -0.93109300 & -2.27647000 & -0.05191100 \\ \mathrm{C} & -3.61574600 & -2.49129400 & -0.25254700 \\ \mathrm{C} & -3.89712000 & -2.32377100 & 1.23067500 \\ \mathrm{O} & -1.93857800 & 2.12046700 & -0.70346000 \\ \mathrm{O} & -1.44244000 & 0.94726900 & 1.17121100 \\ \mathrm{C} & -2.37664300 & 3.23907600 & 0.11161200 \\ \mathrm{C} & -1.19418600 & 4.08510100 & 0.55086200 \\ \mathrm{C} & 3.24874600 & -0.58512900 & 1.26014500 \\ \mathrm{C} & 4.64054700 & -0.61321500 & 1.32699600 \\ \mathrm{C} & 5.39549800 & -0.35368300 & 0.18320500 \\ \mathrm{C} & 4.75601500 & -0.06639500 & -1.02782600 \\ \mathrm{C} & 3.36853200 & -0.03817800 & -1.09605600 \\ \mathrm{H} & 0.63196800 & -0.48465300 & 0.96311300 \\ \mathrm{H} & -1.35338900 & 0.19833900 & -1.99580900\end{array}$




$\begin{array}{rrrr}\mathrm{H} & -3.04193300 & -3.39890200 & -0.45165600 \\ \mathrm{H} & -4.53357300 & -2.50305200 & -0.84339300 \\ \mathrm{H} & -2.96354200 & -2.31177900 & 1.79720100 \\ \mathrm{H} & -4.43384100 & -1.39003900 & 1.41894300 \\ \mathrm{H} & -4.51029800 & -3.15634300 & 1.58982100 \\ \mathrm{H} & -2.92910300 & 2.84981600 & 0.96967600 \\ \mathrm{H} & -3.05452000 & 3.79407400 & -0.53942900 \\ \mathrm{H} & -0.62075200 & 4.42755900 & -0.31478100 \\ \mathrm{H} & -1.54971100 & 4.96262100 & 1.10013900 \\ \mathrm{H} & -0.53709100 & 3.51085900 & 1.20747000 \\ \mathrm{H} & 2.65368900 & -0.78804900 & 2.14642800 \\ \mathrm{H} & 5.13440800 & -0.83738000 & 2.26734500 \\ \mathrm{H} & 6.48004100 & -0.37536100 & 0.23218400 \\ \mathrm{H} & 5.34593400 & 0.13426300 & -1.91704300 \\ \mathrm{H} & 2.85115000 & 0.18080700 & -2.02347500\end{array}$<smiles>CCOC(=O)C(/N=C/c1ccc(C)cc1)C(=O)OCC</smiles>

Nucleophile-85

$\begin{array}{lrrr}\mathrm{C} & -2.21059500 & -0.23984000 & 0.01703500 \\ \mathrm{C} & -0.74494300 & -0.23837700 & -0.00842100 \\ \mathrm{~N} & -0.09336500 & 0.01658300 & -1.08216300 \\ \mathrm{C} & 1.36008300 & -0.02339200 & -1.00613000 \\ \mathrm{C} & 1.92316300 & -1.37467200 & -0.54296300 \\ \mathrm{C} & 1.88939400 & 1.03954400 & -0.03826500 \\ \mathrm{O} & 2.34235500 & 2.11458400 & -0.69930000 \\ \mathrm{O} & 1.85690100 & 0.92908700 & 1.17045700 \\ \mathrm{C} & 2.77747700 & 3.23025700 & 0.12081200 \\ \mathrm{C} & 1.59225600 & 4.06843900 & 0.56779200 \\ \mathrm{O} & 3.25439200 & -1.38634900 & -0.74819900 \\ \mathrm{O} & 1.28849400 & -2.27898900 & -0.05585700 \\ \mathrm{C} & 3.97117900 & -2.53426700 & -0.22597800 \\ \mathrm{C} & 4.23939200 & -2.36525400 & 1.25957800 \\ \mathrm{C} & -2.96973200 & 0.02697900 & -1.13484500 \\ \mathrm{C} & -4.35479400 & 0.02100800 & -1.07526400 \\ \mathrm{C} & -5.02990100 & -0.25098400 & 0.12746100 \\ \mathrm{C} & -4.26909000 & -0.51506500 & 1.27033700 \\ \mathrm{C} & -2.87669700 & -0.51052500 & 1.21762700 \\ \mathrm{C} & -6.53674500 & -0.26485700 & 0.17075000 \\ \mathrm{H} & -0.25355200 & -0.45374300 & 0.94508600 \\ \mathrm{H} & 1.76898000 & 0.18766700 & -1.99671600 \\ \mathrm{H} & 3.45123900 & 3.79218100 & -0.52860400 \\ \mathrm{H} & 3.33381200 & 2.83886700 & 0.97541600 \\ \mathrm{H} & 1.01508900 & 4.41300300 & -0.29453000 \\ \mathrm{H} & 0.93940200 & 3.48728400 & 1.22255600 \\ \mathrm{H} & 1.94494700 & 4.94446700 & 1.12129200 \\ \mathrm{H} & 4.89500000 & -2.56268000 & -0.80689800\end{array}$




$\begin{array}{rrrr}\mathrm{H} & 3.38558700 & -3.43377400 & -0.42764400 \\ \mathrm{H} & 4.78844300 & -1.43915700 & 1.44993100 \\ \mathrm{H} & 3.30002700 & -2.33601900 & 1.81580200 \\ \mathrm{H} & 4.83548300 & -3.20586000 & 1.62869500 \\ \mathrm{H} & -2.44605100 & 0.23571400 & -2.06119700 \\ \mathrm{H} & -4.93267500 & 0.22947100 & -1.97206800 \\ \mathrm{H} & -4.77233100 & -0.72656500 & 2.20960700 \\ \mathrm{H} & -2.29760900 & -0.71878500 & 2.11330300 \\ \mathrm{H} & -6.95403800 & 0.66499000 & -0.23068200 \\ \mathrm{H} & -6.94119500 & -1.08505000 & -0.43377600 \\ \mathrm{H} & -6.90682700 & -0.38871300 & 1.19161000\end{array}$<smiles>O=C(C/C=C/c1ccccc1)c1ccccc1</smiles>

Nucleophile-86

$\begin{array}{lrrr}\mathrm{C} & -4.89029000 & -1.41589200 & -0.20377400 \\ \mathrm{C} & -3.53561000 & -1.11475200 & -0.33220600 \\ \mathrm{C} & -3.06348900 & 0.16818500 & -0.02050600 \\ \mathrm{C} & -3.97024300 & 1.14276100 & 0.42210200 \\ \mathrm{C} & -5.32092500 & 0.84073200 & 0.55323200 \\ \mathrm{C} & -5.78362200 & -0.44017200 & 0.23988600 \\ \mathrm{C} & -1.62272400 & 0.55540300 & -0.14201200 \\ \mathrm{C} & -0.62005100 & -0.50815300 & -0.59021500 \\ \mathrm{O} & -1.26063200 & 1.69794700 & 0.09210700 \\ \mathrm{C} & 0.79727900 & -0.02758600 & -0.55410600 \\ \mathrm{C} & 1.80698200 & -0.73380300 & -0.03065700 \\ \mathrm{C} & 3.22164700 & -0.34435800 & 0.03242200 \\ \mathrm{C} & 4.16326800 & -1.27934300 & 0.49233900 \\ \mathrm{C} & 5.51832200 & -0.96360500 & 0.56527300 \\ \mathrm{C} & 5.96204600 & 0.30110700 & 0.18144100 \\ \mathrm{C} & 5.03679100 & 1.24534000 & -0.27210100 \\ \mathrm{C} & 3.68472700 & 0.92896800 & -0.34482900 \\ \mathrm{H} & -5.24832700 & -2.41082000 & -0.44905500 \\ \mathrm{H} & -2.85296300 & -1.88245900 & -0.67924000 \\ \mathrm{H} & -3.58464000 & 2.12879500 & 0.65584800 \\ \mathrm{H} & -6.01560300 & 1.60016900 & 0.89840600 \\ \mathrm{H} & -6.83840700 & -0.67692800 & 0.34135500 \\ \mathrm{H} & -0.90631900 & -0.79906000 & -1.61423600 \\ \mathrm{H} & -0.74344900 & -1.41043600 & 0.01970200 \\ \mathrm{H} & 0.96825000 & 0.95544200 & -0.98237300 \\ \mathrm{H} & 1.58437900 & -1.71307600 & 0.39423000 \\ \mathrm{H} & 3.82232100 & -2.26660900 & 0.79279300 \\ \mathrm{H} & 6.22647400 & -1.70512800 & 0.92281000 \\ \mathrm{H} & 7.01654500 & 0.55263500 & 0.23838100 \\ \mathrm{H} & 5.37220500 & 2.23547100 & -0.56608000 \\ \mathrm{H} & 2.97994300 & 1.67943900 & -0.68711700\end{array}$


<smiles>O=C(C/C=C/c1ccccc1)c1cccc(Br)c1</smiles>

Nucleophile-87

$\begin{array}{lrrr}\mathrm{C} & -3.68593600 & 2.38684200 & 0.55387800 \\ \mathrm{C} & -2.30778000 & 2.40672700 & 0.37061100 \\ \mathrm{C} & -1.62163100 & 1.23000500 & 0.03966100 \\ \mathrm{C} & -2.33338800 & 0.03227300 & -0.10825900 \\ \mathrm{C} & -3.71219800 & 0.03251900 & 0.07592900 \\ \mathrm{C} & -4.40158000 & 1.19652500 & 0.40727100 \\ \mathrm{C} & -0.13511100 & 1.30828800 & -0.14734900 \\ \mathrm{C} & 0.62390300 & 0.02444800 & -0.47534500 \\ \mathrm{O} & 0.44294100 & 2.37889500 & -0.05694200 \\ \mathrm{C} & 2.10762500 & 0.21835800 & -0.52437300 \\ \mathrm{C} & 2.98088900 & -0.59745000 & 0.07895600 \\ \mathrm{C} & 4.44555500 & -0.49126000 & 0.07051700 \\ \mathrm{C} & 5.20315100 & -1.51800100 & 0.65709000 \\ \mathrm{C} & 6.59550800 & -1.47015000 & 0.67081100 \\ \mathrm{C} & 7.26231200 & -0.38781000 & 0.09848000 \\ \mathrm{C} & 6.52268600 & 0.64525100 & -0.48388100 \\ \mathrm{C} & 5.13317100 & 0.59622700 & -0.49739000 \\ \mathrm{Br} & -4.67961200 & -1.60225000 & -0.13016900 \\ \mathrm{H} & -4.21511800 & 3.29855400 & 0.81202400 \\ \mathrm{H} & -1.73387900 & 3.31985600 & 0.47723100 \\ \mathrm{H} & -1.83402900 & -0.89278000 & -0.36589700 \\ \mathrm{H} & -5.47582500 & 1.16996200 & 0.54716800 \\ \mathrm{H} & 0.24382400 & -0.32647400 & -1.44888600 \\ \mathrm{H} & 0.35456500 & -0.75547300 & 0.24640400 \\ \mathrm{H} & 2.44846600 & 1.08233000 & -1.08681300 \\ \mathrm{H} & 2.58886400 & -1.44746200 & 0.63820700 \\ \mathrm{H} & 4.68784100 & -2.36378300 & 1.10457600 \\ \mathrm{H} & 7.15850100 & -2.27758800 & 1.12922200 \\ \mathrm{H} & 8.34697800 & -0.34503000 & 0.10826400 \\ \mathrm{H} & 7.03351100 & 1.49542400 & -0.92600300 \\ \mathrm{H} & 4.57612800 & 1.41331000 & -0.94369000\end{array}$<smiles>O=C(C/C=C/c1ccccc1)c1cccc(Cl)c1</smiles>

Nucleophile-88
C
$-4.48432600$
1.78517900
0.56021800
C $\quad-3.11142200$
1.91988500
0.38714900
C
$-2.33280900$
0.81114800
0.02791500 


$\begin{array}{lrrr}\mathrm{C} & -2.94579800 & -0.43467900 & -0.15875800 \\ \mathrm{C} & -4.32175800 & -0.54974400 & 0.01575900 \\ \mathrm{C} & -5.10267900 & 0.54695100 & 0.37531200 \\ \mathrm{C} & -0.85631700 & 1.01325800 & -0.14598900 \\ \mathrm{C} & 0.00554600 & -0.19587800 & -0.50265500 \\ \mathrm{O} & -0.36660200 & 2.12386700 & -0.02252900 \\ \mathrm{C} & 1.46911200 & 0.11797000 & -0.53353400 \\ \mathrm{C} & 2.40153800 & -0.64120700 & 0.05498400 \\ \mathrm{C} & 3.85272700 & -0.41624300 & 0.06253000 \\ \mathrm{C} & 4.68828900 & -1.39780900 & 0.61981600 \\ \mathrm{C} & 6.07201300 & -1.23742700 & 0.64656100 \\ \mathrm{C} & 6.65140700 & -0.08510600 & 0.11727700 \\ \mathrm{C} & 5.83316900 & 0.90421100 & -0.43509800 \\ \mathrm{C} & 4.45236000 & 0.74290100 & -0.46165400 \\ \mathrm{Cl} & -5.08823200 & -2.11303900 & -0.22234600 \\ \mathrm{H} & -5.08530600 & 2.64430400 & 0.84035300 \\ \mathrm{H} & -2.61250700 & 2.87221200 & 0.52346100 \\ \mathrm{H} & -2.37397800 & -1.30998700 & -0.43941800 \\ \mathrm{H} & -6.17213200 & 0.42978500 & 0.50670200 \\ \mathrm{H} & -0.33878200 & -0.54847300 & -1.48882400 \\ \mathrm{H} & -0.20454500 & -1.01485700 & 0.19511500 \\ \mathrm{H} & 1.74274000 & 1.02184400 & -1.06932000 \\ \mathrm{H} & 2.07618300 & -1.53582300 & 0.58675000 \\ \mathrm{H} & 4.24126100 & -2.29781800 & 1.03368200 \\ \mathrm{H} & 6.69649900 & -2.01192000 & 1.08156700 \\ \mathrm{H} & 7.72889600 & 0.04538500 & 0.13753600 \\ \mathrm{H} & 6.27532600 & 1.80819600 & -0.84315200 \\ \mathrm{H} & 3.83280900 & 1.52740500 & -0.88334200 \\ & & & \end{array}$<smiles>O=C(C/C=C/c1ccc(F)cc1)c1ccccc1</smiles>

Nucleophile-89

$\begin{array}{lrrr}\mathrm{C} & -4.59702100 & -1.44511400 & -0.90524400 \\ \mathrm{C} & -4.10024500 & -0.14807000 & -0.85032600 \\ \mathrm{C} & -3.01041300 & 0.16178900 & -0.02225000 \\ \mathrm{C} & -2.42363600 & -0.85357700 & 0.74631400 \\ \mathrm{C} & -2.91978100 & -2.15429900 & 0.68723900 \\ \mathrm{C} & -4.00686900 & -2.45141300 & -0.13552400 \\ \mathrm{C} & -2.52859700 & 1.57607600 & -0.00362700 \\ \mathrm{C} & -1.37094300 & 1.96606400 & 0.92283700 \\ \mathrm{O} & -3.02375900 & 2.41845900 & -0.73671600 \\ \mathrm{C} & -0.04961700 & 1.55620800 & 0.33139600 \\ \mathrm{C} & 0.85899400 & 0.79782700 & 0.95992900 \\ \mathrm{C} & 2.15795800 & 0.35339700 & 0.44265900 \\ \mathrm{C} & 3.04915400 & -0.29872700 & 1.31122400 \\ \mathrm{C} & 4.29788800 & -0.73926400 & 0.88034100\end{array}$




$\begin{array}{lrrr}\mathrm{C} & 4.65549900 & -0.52481400 & -0.44372100 \\ \mathrm{C} & 3.80126000 & 0.11001100 & -1.34034200 \\ \mathrm{C} & 2.55872000 & 0.54229400 & -0.89289300 \\ \mathrm{~F} & 5.86109400 & -0.94774000 & -0.87670800 \\ \mathrm{H} & -5.44235500 & -1.67603100 & -1.54600700 \\ \mathrm{H} & -4.53728300 & 0.65123700 & -1.43827600 \\ \mathrm{H} & -1.56920200 & -0.63794600 & 1.37535000 \\ \mathrm{H} & -2.45694400 & -2.93612700 & 1.28118000 \\ \mathrm{H} & -4.39292500 & -3.46536800 & -0.17869400 \\ \mathrm{H} & -1.49628200 & 1.52952600 & 1.91864600 \\ \mathrm{H} & -1.42442600 & 3.05614400 & 1.01262100 \\ \mathrm{H} & 0.13918000 & 1.92272700 & -0.67562300 \\ \mathrm{H} & 0.63720500 & 0.47240100 & 1.97676000 \\ \mathrm{H} & 2.75794100 & -0.45902300 & 2.34537400 \\ \mathrm{H} & 4.98820200 & -1.24062200 & 1.54910400 \\ \mathrm{H} & 4.11491600 & 0.25031300 & -2.36859700 \\ \mathrm{H} & 1.88808700 & 1.02443900 & -1.59582900\end{array}$<smiles>COc1ccc(/C=C/CC(=O)c2ccccc2)cc1</smiles>

Nucleophile-90

$\begin{array}{lrrr}\mathrm{C} & 6.24539700 & 0.82924200 & -0.67152100 \\ \mathrm{C} & 4.89732300 & 1.13212100 & -0.51715100 \\ \mathrm{C} & 4.00595500 & 0.17295700 & -0.01395900 \\ \mathrm{C} & 4.49110100 & -1.09549000 & 0.33466100 \\ \mathrm{C} & 5.84323400 & -1.39749000 & 0.18291200 \\ \mathrm{C} & 6.72111400 & -0.43718400 & -0.32107600 \\ \mathrm{C} & 2.56656200 & 0.56063200 & 0.12934100 \\ \mathrm{C} & 1.58006600 & -0.48712700 & 0.64439800 \\ \mathrm{O} & 2.19593500 & 1.69264700 & -0.13980200 \\ \mathrm{C} & 0.15921000 & -0.01575100 & 0.62642400 \\ \mathrm{C} & -0.85797000 & -0.74652200 & 0.15192200 \\ \mathrm{C} & -2.27575700 & -0.37561500 & 0.10717000 \\ \mathrm{C} & -3.21098200 & -1.29673000 & -0.38093000 \\ \mathrm{C} & -4.57416400 & -1.00640000 & -0.44717800 \\ \mathrm{C} & -5.03080700 & 0.24194400 & -0.01632400 \\ \mathrm{C} & -4.11086300 & 1.18243600 & 0.47398100 \\ \mathrm{C} & -2.76260300 & 0.87706700 & 0.53290200 \\ \mathrm{O} & -6.33512300 & 0.64156600 & -0.03205100 \\ \mathrm{C} & -7.30088700 & -0.27236600 & -0.52388100 \\ \mathrm{H} & 6.92797000 & 1.57682800 & -1.06362100 \\ \mathrm{H} & 4.50178700 & 2.10721000 & -0.77863300 \\ \mathrm{H} & 3.82049700 & -1.85098500 & 0.72874100 \\ \mathrm{H} & 6.21133000 & -2.38116100 & 0.45714800 \\ \mathrm{H} & 7.77387700 & -0.67462400 & -0.44057700 \\ \mathrm{H} & 1.89667200 & -0.73777000 & 1.67041300 \\ & & & \end{array}$




$\begin{array}{lrrc}\mathrm{H} & 1.69266000 & -1.41191500 & 0.06662200 \\ \mathrm{H} & -0.00732000 & 0.98193200 & 1.02141600 \\ \mathrm{H} & -0.63473200 & -1.73569600 & -0.24978600 \\ \mathrm{H} & -2.86553900 & -2.27022100 & -0.71885500 \\ \mathrm{H} & -5.25951500 & -1.75135400 & -0.83182600 \\ \mathrm{H} & -4.48440200 & 2.14728400 & 0.79962200 \\ \mathrm{H} & -2.07264600 & 1.62428800 & 0.91047500 \\ \mathrm{H} & -8.26289700 & 0.23638700 & -0.45246000 \\ \mathrm{H} & -7.10863000 & -0.53810000 & -1.57117500 \\ \mathrm{H} & -7.33117900 & -1.19014400 & 0.07716500\end{array}$




\section{Cartesian Coordinates for Solvents}

$\begin{array}{lrrr}\text { Acetonitrile } & & & \\ & & & \\ \mathrm{C} & 0.00000000 & 0.27959700 & 0.00000000 \\ \mathrm{~N} & 0.00013700 & 1.43985900 & 0.00000000 \\ \mathrm{C} & -0.00009400 & -1.18032600 & 0.00000000 \\ \mathrm{H} & 1.02590800 & -1.55827400 & 0.00000000 \\ \mathrm{H} & -0.51315200 & -1.55818500 & 0.88855800 \\ \mathrm{H} & -0.51315200 & -1.55818500 & -0.88855800\end{array}$

\section{Benzene}

$\begin{array}{lrrr}\mathrm{C} & -0.51346300 & -1.29789400 & -0.00000100 \\ \mathrm{C} & -1.38074300 & -0.20427600 & 0.00000100 \\ \mathrm{C} & -0.86727800 & 1.09361700 & -0.00000200 \\ \mathrm{C} & 0.51346300 & 1.29789500 & 0.00000100 \\ \mathrm{C} & 1.38074300 & 0.20427500 & -0.00000100 \\ \mathrm{C} & 0.86727800 & -1.09361800 & 0.00000200 \\ \mathrm{H} & -0.91295200 & -2.30770200 & -0.00000200 \\ \mathrm{H} & -2.45500700 & -0.36320600 & 0.00000200 \\ \mathrm{H} & -1.54205700 & 1.94448500 & -0.00000100 \\ \mathrm{H} & 0.91295200 & 2.30770300 & 0.00000200 \\ \mathrm{H} & 2.45500700 & 0.36320600 & -0.00000200 \\ \mathrm{H} & 1.54205700 & -1.94448500 & 0.00000100\end{array}$

\section{Chloroform}

$\begin{array}{lrrr}\mathrm{Cl} & -0.61774800 & 1.58727500 & -0.08369200 \\ \mathrm{C} & 0.00004200 & 0.00000600 & 0.45471000 \\ \mathrm{Cl} & 1.68351200 & -0.25866700 & -0.08369600 \\ \mathrm{Cl} & -1.06577900 & -1.32861000 & -0.08369200 \\ \mathrm{H} & -0.00000200 & -0.00001400 & 1.54010300\end{array}$

\section{Dichloromethane (DCM)}

\begin{tabular}{|c|c|c|c|}
\hline $\mathrm{Cl}$ & -1.49470700 & -0.21644500 & -0.00000100 \\
\hline $\mathrm{C}$ & 0.00004000 & 0.76705600 & -0.00000100 \\
\hline $\mathrm{Cl}$ & 1.49468500 & -0.21646300 & -0.00000200 \\
\hline $\mathrm{H}$ & 0.00007900 & 1.37851600 & 0.89889000 \\
\hline 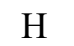 & 0.00006500 & 1.37859700 & -0.89884100 \\
\hline
\end{tabular}

\section{Diethyl Ether}

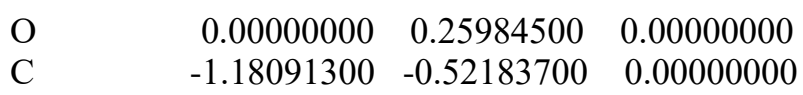




$\begin{array}{lrrr}\mathrm{C} & -2.37595300 & 0.41512800 & 0.00000000 \\ \mathrm{C} & 1.18091300 & -0.52182600 & 0.00000000 \\ \mathrm{C} & 2.37595400 & 0.41514600 & 0.00000000 \\ \mathrm{H} & -1.20325700 & -1.17864900 & 0.88652500 \\ \mathrm{H} & -1.20325700 & -1.17864900 & -0.88652500 \\ \mathrm{H} & -3.31084000 & -0.15371000 & 0.00000000 \\ \mathrm{H} & -2.35736400 & 1.05587800 & 0.88608300 \\ \mathrm{H} & -2.35736400 & 1.05587800 & -0.88608300 \\ \mathrm{H} & 1.20325800 & -1.17863900 & -0.88652500 \\ \mathrm{H} & 1.20325800 & -1.17863900 & 0.88652500 \\ \mathrm{H} & 3.31084000 & -0.15369200 & 0.00000000 \\ \mathrm{H} & 2.35736100 & 1.05589500 & -0.88608400 \\ \mathrm{H} & 2.35736100 & 1.05589500 & 0.88608400\end{array}$

\section{Ethyl Acetate}

$\begin{array}{lrrr}\mathrm{O} & -0.17829600 & -0.44649000 & 0.00022600 \\ \mathrm{C} & 1.03730400 & 0.14627800 & 0.00040800 \\ \mathrm{C} & 2.13710500 & -0.88731400 & -0.00016200 \\ \mathrm{O} & 1.19739400 & 1.34717800 & -0.00016100 \\ \mathrm{C} & -1.31045900 & 0.45235100 & 0.00003000 \\ \mathrm{C} & -2.56399900 & -0.39853700 & -0.00013900 \\ \mathrm{H} & 2.04648200 & -1.52935600 & -0.88097400 \\ \mathrm{H} & 3.10473800 & -0.38747000 & -0.00078400 \\ \mathrm{H} & 2.04750900 & -1.52912700 & 0.88092000 \\ \mathrm{H} & -1.24926200 & 1.09769400 & -0.88166700 \\ \mathrm{H} & -1.24952200 & 1.09773300 & 0.88171500 \\ \mathrm{H} & -3.44988300 & 0.24362900 & -0.00028500 \\ \mathrm{H} & -2.60114300 & -1.03766100 & -0.88668500 \\ \mathrm{H} & -2.60140800 & -1.03762000 & 0.88642500\end{array}$

\section{p-Xylene}

$\begin{array}{lrrr}\mathrm{C} & 0.69666300 & 1.19827700 & -0.00816800 \\ \mathrm{C} & -0.69666300 & 1.19827700 & 0.00816800 \\ \mathrm{C} & -1.41939700 & -0.00003400 & 0.01421100 \\ \mathrm{C} & -0.69668200 & -1.19831200 & 0.00816400 \\ \mathrm{C} & 0.69668200 & -1.19831300 & -0.00816400 \\ \mathrm{C} & 1.41939700 & -0.00003500 & -0.01421100 \\ \mathrm{C} & -2.92811300 & 0.00003600 & -0.00512800 \\ \mathrm{C} & 2.92811300 & 0.00003600 & 0.00512800 \\ \mathrm{H} & 1.23239500 & 2.14422300 & -0.01366900 \\ \mathrm{H} & -1.23239500 & 2.14422300 & 0.01366900 \\ \mathrm{H} & -1.23241100 & -2.14425700 & 0.01365100 \\ \mathrm{H} & 1.23241000 & -2.14425700 & -0.01365100 \\ \mathrm{H} & -3.33404800 & -0.88628900 & 0.49132200 \\ \mathrm{H} & -3.33402400 & 0.88440800 & 0.49484000 \\ \mathrm{H} & 3.33404700 & -0.88630800 & -0.49128900 \\ \mathrm{H} & 3.33402500 & 0.88438900 & -0.49487200 \\ \mathrm{H} & 3.31265900 & 0.00215900 & 1.03263500\end{array}$




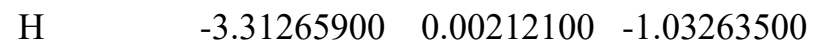

$m$-Xylene

$\begin{array}{lrrr}\mathrm{C} & 1.22321400 & -0.27062400 & -0.01075100 \\ \mathrm{C} & 0.00000600 & -0.94938500 & -0.01981100 \\ \mathrm{C} & -1.22319400 & -0.27065400 & -0.01072800 \\ \mathrm{C} & -1.20890400 & 1.12876000 & -0.00061800 \\ \mathrm{C} & -0.00001600 & 1.82250100 & 0.00490600 \\ \mathrm{C} & 1.20889600 & 1.12876900 & -0.00063900 \\ \mathrm{C} & -2.52673500 & -1.03093300 & 0.01078800 \\ \mathrm{C} & 2.52673800 & -1.03092500 & 0.01080300 \\ \mathrm{H} & 0.00001900 & -2.03724900 & -0.03771100 \\ \mathrm{H} & -2.14856400 & 1.67473000 & -0.00321600 \\ \mathrm{H} & -0.00001000 & 2.90868100 & 0.00498200 \\ \mathrm{H} & 2.14853900 & 1.67476900 & -0.00326700 \\ \mathrm{H} & -3.31535100 & -0.48718500 & -0.51769600 \\ \mathrm{H} & -2.42299400 & -2.01582700 & -0.45354400 \\ \mathrm{H} & 3.31551400 & -0.48696900 & -0.51722400 \\ \mathrm{H} & 2.87436300 & -1.19041900 & 1.03899700 \\ \mathrm{H} & 2.42311900 & -2.01562200 & -0.45397100 \\ \mathrm{H} & -2.87466500 & -1.18996700 & 1.03895000\end{array}$

Methyl, $t$-Bu-Ether (MTBE)

$\begin{array}{lrrr}\mathrm{O} & 0.44961600 & -1.03291800 & 0.00000000 \\ \mathrm{C} & 0.14128000 & 0.37380900 & 0.00000000 \\ \mathrm{C} & -0.63571400 & 0.76697200 & 1.26445700 \\ \mathrm{C} & -0.63571400 & -1.93611600 & 0.00000000 \\ \mathrm{C} & -0.63571400 & 0.76697200 & -1.26445700 \\ \mathrm{C} & 1.52006400 & 1.03708400 & 0.00000000 \\ \mathrm{H} & -0.10485100 & 0.42089300 & 2.15609700 \\ \mathrm{H} & -0.73806700 & 1.85473700 & 1.32321800 \\ \mathrm{H} & -1.64374400 & 0.34244300 & 1.27449900 \\ \mathrm{H} & -0.20020400 & -2.93831400 & 0.00000000 \\ \mathrm{H} & -1.27205600 & -1.83822400 & 0.89053200 \\ \mathrm{H} & -1.27205600 & -1.83822400 & -0.89053200 \\ \mathrm{H} & -0.10485100 & 0.42089300 & -2.15609700 \\ \mathrm{H} & -1.64374400 & 0.34244300 & -1.27449900 \\ \mathrm{H} & -0.73806700 & 1.85473700 & -1.32321800 \\ \mathrm{H} & 2.08350800 & 0.73129200 & -0.88572800 \\ \mathrm{H} & 1.42848700 & 2.12704900 & 0.00000000 \\ \mathrm{H} & 2.08350800 & 0.73129200 & 0.88572800\end{array}$

\section{Tetrahydrofuran (THF)}

\begin{tabular}{|c|c|c|c|}
\hline $\mathrm{C}$ & -0.73210600 & 0.99497800 & -0.22985900 \\
\hline $\mathrm{C}$ & -1.16429900 & -0.42939200 & 0.13305100 \\
\hline $\mathrm{O}$ & 0.00025800 & -1.25149000 & 0.00034200 \\
\hline $\mathrm{C}$ & 1.16427800 & -0.42907100 & -0.13347800 \\
\hline
\end{tabular}




$\begin{array}{rrrr}\mathrm{C} & 0.73190200 & 0.99505700 & 0.23005500 \\ \mathrm{H} & -1.34398100 & 1.76058300 & 0.25437900 \\ \mathrm{H} & -0.78913100 & 1.14762300 & -1.31328200 \\ \mathrm{H} & -1.95021000 & -0.82126900 & -0.52203000 \\ \mathrm{H} & -1.53104400 & -0.47630900 & 1.16963700 \\ \mathrm{H} & 1.95102700 & -0.82076600 & 0.52072900 \\ \mathrm{H} & 1.53013700 & -0.47555100 & -1.17044200 \\ \mathrm{H} & 1.34357800 & 1.76099400 & -0.25389700 \\ \mathrm{H} & 0.78891800 & 1.14718600 & 1.31354900\end{array}$

Toluene

$\begin{array}{lrrr}\mathrm{C} & 1.19965700 & -1.20294000 & 0.00000000 \\ \mathrm{C} & 1.20121900 & 0.19052400 & 0.00000000 \\ \mathrm{C} & 0.00000000 & 0.91237300 & 0.00000000 \\ \mathrm{C} & -1.20315000 & 0.19929600 & 0.00000000 \\ \mathrm{C} & -1.21053400 & -1.19667200 & 0.00000000 \\ \mathrm{C} & -0.00857400 & -1.90280500 & 0.00000000 \\ \mathrm{C} & 0.01421500 & 2.42236600 & 0.00000000 \\ \mathrm{H} & 2.14160200 & -1.74419200 & 0.00000000 \\ \mathrm{H} & 2.14606200 & 0.72875800 & 0.00000000 \\ \mathrm{H} & -2.14379700 & 0.74400700 & 0.00000000 \\ \mathrm{H} & -2.15607100 & -1.73150100 & 0.00000000 \\ \mathrm{H} & -0.01158900 & -2.98880400 & 0.00000000 \\ \mathrm{H} & -1.00030800 & 2.82931900 & 0.00000000 \\ \mathrm{H} & 0.53355300 & 2.81477800 & 0.88161700 \\ \mathrm{H} & 0.53355300 & 2.81477800 & -0.88161700\end{array}$

\section{Trifluorotoluene $\left(\mathrm{PhCF}_{3}\right)$}

$\begin{array}{lrrr}\mathrm{C} & -0.03488500 & 2.83356000 & 0.00000000 \\ \mathrm{C} & -0.03605400 & 2.13641100 & 1.20901200 \\ \mathrm{C} & -0.03605400 & 0.74297600 & 1.21183000 \\ \mathrm{C} & -0.03677200 & 0.04910800 & 0.00000000 \\ \mathrm{C} & -0.03605400 & 0.74297600 & -1.21183000 \\ \mathrm{C} & -0.03605400 & 2.13641100 & -1.20901200 \\ \mathrm{C} & 0.02374600 & -1.45240500 & 0.00000000 \\ \mathrm{~F} & -0.57534000 & -1.98227600 & -1.09031300 \\ \mathrm{~F} & 1.30152000 & -1.90141800 & 0.00000000 \\ \mathrm{~F} & -0.57534000 & -1.98227600 & 1.09031300 \\ \mathrm{H} & -0.03601200 & 3.91907400 & 0.00000000 \\ \mathrm{H} & -0.03992800 & 2.67712700 & 2.15003200 \\ \mathrm{H} & -0.04446100 & 0.19309400 & 2.14599800 \\ \mathrm{H} & -0.04446100 & 0.19309400 & -2.14599800 \\ \mathrm{H} & -0.03992800 & 2.67712700 & -2.15003200\end{array}$

\section{Cyclopentyl, Methyl-Ether (CPME)}

C

$\begin{array}{lll}-1.96977400 & -0.33953400 & -0.34301800\end{array}$ 


$\begin{array}{lrrr}\mathrm{C} & -0.85357000 & -1.26284700 & 0.22044500 \\ \mathrm{C} & 0.33147200 & -0.34385600 & 0.58333300 \\ \mathrm{C} & -0.29190000 & 1.05933000 & 0.67748600 \\ \mathrm{C} & -1.31965500 & 1.05352800 & -0.46163800 \\ \mathrm{O} & 1.26800200 & -0.43140400 & -0.49306300 \\ \mathrm{C} & 2.51891900 & 0.15679900 & -0.21220900 \\ \mathrm{H} & -2.81148700 & -0.29656800 & 0.35721500 \\ \mathrm{H} & -2.36631900 & -0.69832000 & -1.29665900 \\ \mathrm{H} & -0.50767800 & -1.99895400 & -0.50898600 \\ \mathrm{H} & -1.19830400 & -1.81100100 & 1.10206500 \\ \mathrm{H} & 0.83051500 & -0.64684700 & 1.51657200 \\ \mathrm{H} & 0.44674600 & 1.86283200 & 0.60653200 \\ \mathrm{H} & -0.79733300 & 1.16228600 & 1.64652800 \\ \mathrm{H} & -0.79143900 & 1.14132800 & -1.41662600 \\ \mathrm{H} & -2.04307400 & 1.87164100 & -0.39976700 \\ \mathrm{H} & 3.16475800 & -0.03510300 & -1.07242100 \\ \mathrm{H} & 2.45528400 & 1.24435400 & -0.06123500 \\ \mathrm{H} & 2.98137200 & -0.28493800 & 0.68489800\end{array}$

\section{Mesitylene}

$\begin{array}{lrrr}\mathrm{C} & 1.36910200 & 0.22941000 & -0.00020300 \\ \mathrm{C} & 0.49777200 & 1.31713400 & -0.00004000 \\ \mathrm{C} & -0.88325400 & 1.07098000 & 0.00019700 \\ \mathrm{C} & -1.38957100 & -0.22747200 & 0.00023900 \\ \mathrm{C} & -0.48587600 & -1.30039100 & 0.00016300 \\ \mathrm{C} & 0.89178000 & -1.08966900 & -0.00006900 \\ \mathrm{C} & -2.87664100 & -0.48549100 & -0.00018800 \\ \mathrm{C} & 1.01789400 & 2.73397500 & -0.00000200 \\ \mathrm{C} & 1.85878400 & -2.24848400 & 0.00002000 \\ \mathrm{H} & 2.44237000 & 0.40664600 & -0.00036600 \\ \mathrm{H} & -1.57336600 & 1.91185100 & 0.00034200 \\ \mathrm{H} & -0.86904400 & -2.31847700 & 0.00030500 \\ \mathrm{H} & -3.17969400 & -1.06747000 & 0.87753500 \\ \mathrm{H} & -3.44384500 & 0.44897100 & 0.00560000 \\ \mathrm{H} & 0.67104500 & 3.28499900 & 0.88150900 \\ \mathrm{H} & 2.11077300 & 2.75789400 & -0.00100200 \\ \mathrm{H} & 2.51081900 & -2.22250300 & -0.88040900 \\ \mathrm{H} & 2.50939700 & -2.22353900 & 0.88153200 \\ \mathrm{H} & 1.33313000 & -3.20694500 & -0.00094600 \\ \mathrm{H} & 0.66938300 & 3.28567100 & -0.88042700 \\ \mathrm{H} & -3.18090300 & -1.05705000 & -0.88437400\end{array}$




\section{Collected Parameters}

The parameters calculated are reported in separate tables encompassed in the accompanying excel spreadsheets. Sterimol parameters L, B1 and B5, respectively, represent the length, minimum and maximum widths of the considered substituent and are calculated using the Bondi radii. ${ }^{16-17}$ IR stretching frequencies/intensities, NMR values and NBO charges have been found to be useful descriptors of electronic and steric properties. ${ }^{18}$ Solvent topological and connectivity descriptors were acquired from DFT optimized geometries and calculated with the molecular descriptors application available in Maestro using default settings. 


\section{Catalyst Parameter Table}

NBO, Sterimol, and IR vibration/intensities $(\mathrm{N}-\mathrm{H})$ were collected for various atoms, using the catalyst model below as a guide. Molecular surface area (MSA) was calculated for the full catalyst structure using Macromodel. The NBO/NMR $(\mathrm{C}=\mathrm{X})$ values of the HBD carbon adjacent to the two nitrogen atoms was reported as an average for squaramide catalysts. One catalyst in the training set featured multiple points of chirality and was treated as shown below. HOMO, LUMO and polarizability were also collected as descriptors.

Model Catalyst

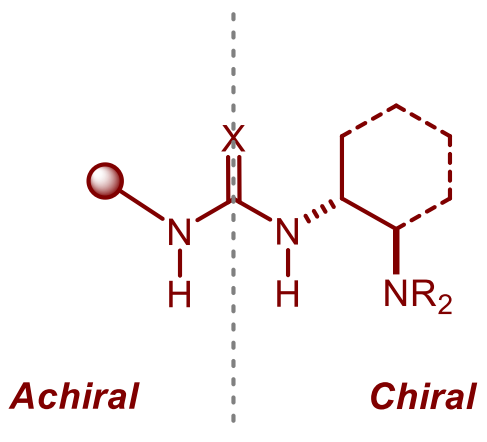

\section{Thiourea-9}

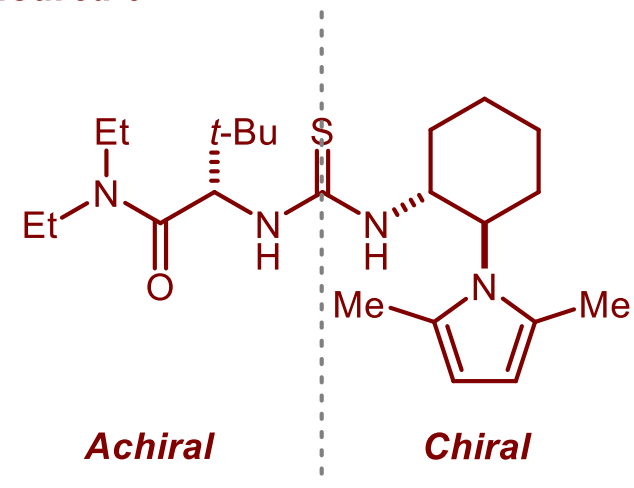




\section{Nucleophile Parameter Table}

NBO charges from the common $\mathrm{H}-\mathrm{X}$ subunit (where $\mathrm{X}$ is $\mathrm{N}, \mathrm{S}, \mathrm{P}$ or $\mathrm{C}$ ) were calculated and collected. Bond lengths of the $\mathrm{H}-\mathrm{X}$ bond was collected in addition to Sterimol values representing the general steric environment of the nucleophile. In many cases, two protons were present for possible activation, requiring average Sterimol values be calculated. Sterimol sets were classified as large and small based on the relative magnitude of the L term. HOMO, LUMO, dipole and polarizability descriptors were also collected.

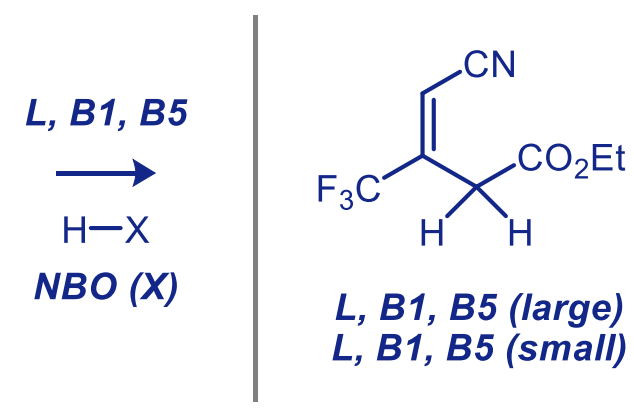




\section{Nitroalkene Parameter Table}

NBO charges were calculated and collected for common atoms of all nitroalkenes as shown below. In addition to global properties (HOMO, LUMO, polarizability), Sterimol values of the substituent $\left(\mathrm{R}^{1}\right)$ were collected as descriptors.
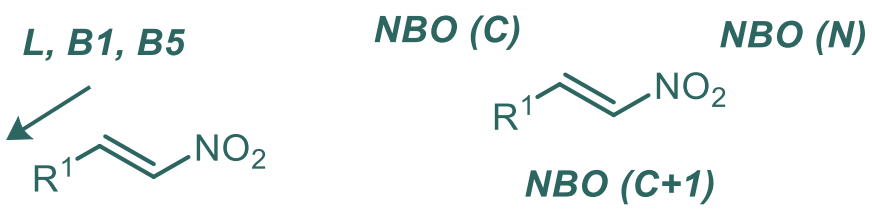


\section{Amine/Imine Parameter Table}

NBO charges were calculated and collected for common atoms of all amines and imines as shown below. In addition to global properties (HOMO, LUMO, polarizability), Sterimol values of the substituent $\left(\mathrm{R}^{1}\right)$ and along the $\mathrm{C}-\mathrm{N}$ bond were collected as descriptors.
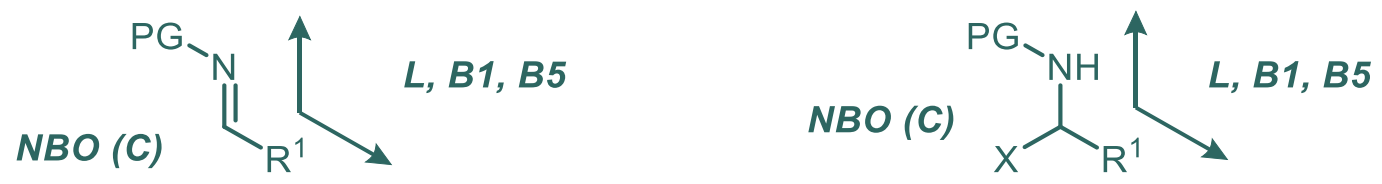


\section{Enone Parameter Table}

NBO charges were calculated and collected for common atoms of enones as shown below. In addition to global properties (HOMO, LUMO, polarizability), Sterimol values along the $\mathrm{C}-\mathrm{C}+1$ bond were collected.

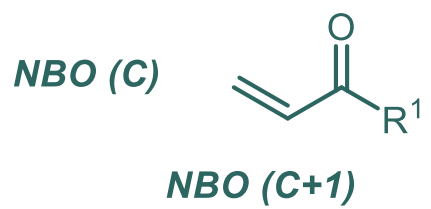




\section{Data Curation}

Relevant data were manually extracted from the following literature reports:

(1) Martin, N. J. A.; Ozores, L.; List, B. J. Am. Chem. Soc. 2007, 129, 8976-8977.

(2) Blom, J.; Vidal-Alabat, A.; Jørgensen, J.; Barløse, C. L.; Jessen, K. S.; Iversen, M. V.; Jørgensen, K. A. Angew. Chem. Int. Ed. 2017, 56, 11831-11835.

(3) Zhu, Y.; Malerich, J. P.; Rawal, V. H. Angew. Chem. Int. Ed. 2010, 49, 153-156.

(4) He, X.; Yang, L.; Ji, Y.; Zhao, Q.; Yang, M.; Huang, W.; Peng, C.; Han, B. Chem. Eur. J. 2018, 24, 1947-1955.

(5) Kimmel, K. L.; Robak, M. T.; Ellman, J. A. J. Am. Chem. Soc. 2009, 131, 8754-8755.

(6) Bui, T.; Syed, S.; Barbas III, C. F. J. Am. Chem. Soc. 2009, 131, 8758-8759.

(7) Yang, C.; Wang, J.; Liu, Y.; Ni, X.; Li, X.; Cheng, J. Chem. Eur. J. 2017, 23, 5488-5497.

\section{Out of Sample Predictions}

(1) Martinelli, E.; Vicini, A. C.; Mancinelli, M.; Mazanti, A.; Zani, P.; Bernardi, L.; Fochi, M. Chem. Commun. 2015, 51, 658.

(2) Li, X.; Xue, X.; Liu, C.; Wang, B.; Tan, B.; Jin, J.; Zhang, Y.; Dong, N.; Cheng, J. Org. Biomol. Chem. 2012, 10, 413.

(3) Herrera, R. P.; Sgarzani, V.; Bernardi, L.; Ricci, A. Angew. Chem. Int. Ed. 2005, 44, 6576-6579.

(4) Wasa, M.; Liu, R. Y.; Roche, S. P.; Jacobsen, E. N. J. Am. Chem. Soc. 2014, 136, 1287212875.

(5) Okino, T.; Nakamura, S.; Furukawa, T.; Takemoto, Y. Org. Lett. 2004, 6, 625-627.

(6) Wang, K.; Chen, C.; Liu, X.; Li, D.; Peng, T.; Liu, X.; Yang, D.; Wang, L. Org. Lett. 2018, 20, 5260-5263

\section{Cascade Reaction Predictions}

(1) Enders, D.; Göddertz, D. P.; Beceño, C.; Raabe, G. Adv. Synth. Catal. 2010, 352, 28632868.

(2) Zeng, X.; Meng, C.; Bao, J.; Xu, D.; Xie, J.; Zhu, W. J. Org. Chem. 2015, 80, 1152111528.

(3) Xie, J.; Yoshida, K.; Takasu, K.; Takemoto, Y. Tetrahedron Lett. 2008, 49, 6910-6913. 
(4) Akula, P. V.; Hong, B.; Lee, G. Org. Lett. 2018, 20, 7835-7839.

Nitrophosphonate Addition to Enones

(1) Bera, K.; Namboothiri, I. N. N. J. Org. Chem. 2015, 80, 1402-1413. 


\section{Transfer Hydrogenation of Nitroolefins (List)}<smiles>[R]OC(=O)C1=C(C)NC(C)=C(C([R])O[R])C1</smiles><smiles>[R]C([R])=C[N+](=O)[O-]</smiles>

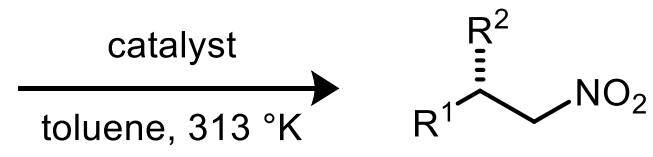

\begin{tabular}{|c|c|c|c|c|c|c|c|c|}
\hline entry & $\mathbf{R}^{1}$ & $\mathbf{R}^{2}$ & $\mathbf{R}^{3}$ & catalyst & ee (\%) & \multicolumn{2}{|c|}{ er } & $\Delta \Delta \mathrm{G}^{\ddagger}(\mathrm{kcal} / \mathrm{mol})$ \\
\hline 1 & $\mathrm{Ph}$ & $\mathrm{Me}$ & Et & Thiourea-1 & 4 & 52 & 48 & 0.05 \\
\hline 2 & 2-napthyl & $\mathrm{Me}$ & $t-\mathrm{Bu}$ & Thiourea-9 & 92 & 96 & 4 & 1.98 \\
\hline 3 & 3-Cl Ph & $\mathrm{Me}$ & $t-\mathrm{Bu}$ & Thiourea-9 & 90 & 95 & 5 & 1.83 \\
\hline 4 & 4-Cl Ph & $\mathrm{Me}$ & $t$-Bu & Thiourea-9 & 94 & 97 & 3 & 2.16 \\
\hline 5 & 4-CN Ph & $\mathrm{Me}$ & $t-\mathrm{Bu}$ & Thiourea-9 & 90 & 95 & 5 & 1.83 \\
\hline 6 & 4-F Ph & $\mathrm{Me}$ & $t-\mathrm{Bu}$ & Thiourea-9 & 90 & 95 & 5 & 1.83 \\
\hline 7 & 4-Me Ph & $\mathrm{Me}$ & $t-\mathrm{Bu}$ & Thiourea-9 & 94 & 97 & 3 & 2.16 \\
\hline 8 & Et & $\mathrm{Me}$ & $t$-Bu & Thiourea-9 & 64 & 82 & 18 & 0.94 \\
\hline 9 & 2 -furyl & $\mathrm{Me}$ & $t-\mathrm{Bu}$ & Thiourea-9 & 92 & 96 & 4 & 1.98 \\
\hline 10 & $\mathrm{Ph}$ & Et & $t-\mathrm{Bu}$ & Thiourea-9 & 94 & 97 & 3 & 2.16 \\
\hline 11 & $\mathrm{Ph}$ & $\mathrm{Me}$ & $t-\mathrm{Bu}$ & Thiourea-9 & 94 & 97 & 3 & 2.16 \\
\hline 12 & $\mathrm{Ph}$ & $n-\mathrm{Pr}$ & $t-\mathrm{Bu}$ & Thiourea-9 & 96 & 98 & 2 & 2.42 \\
\hline 13 & $t-B u$ & $\mathrm{Me}$ & $t-\mathrm{Bu}$ & Thiourea-9 & 92 & 96 & 4 & 1.98 \\
\hline
\end{tabular}




\section{1,3-Dipolar Cycloaddition}

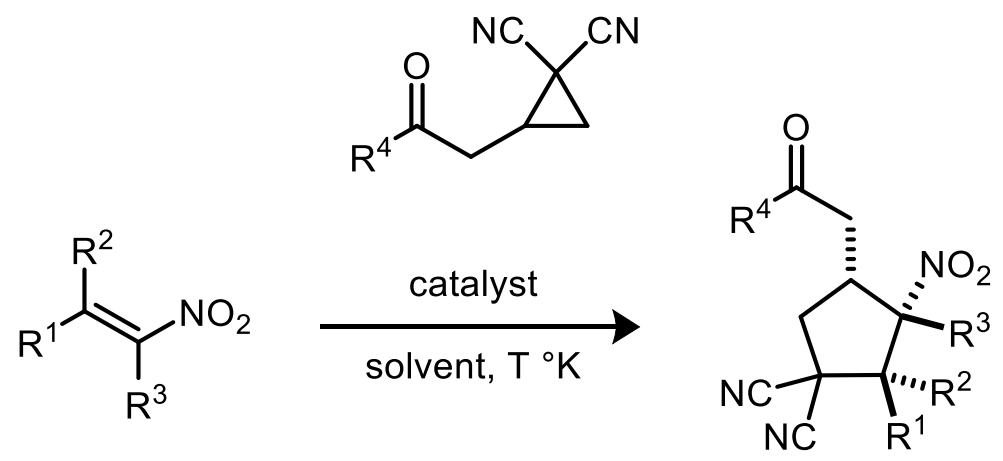

\begin{tabular}{|c|c|c|c|c|c|c|c|c|c|c|c|}
\hline entry & $\mathbf{R}^{1}$ & $\mathbf{R}^{2}$ & $\mathbf{R}^{3}$ & $\mathbf{R}^{4}$ & Catalyst & Solvent & Temp (K) & ee (\%) & \multicolumn{2}{|c|}{ er } & $\Delta \Delta \mathrm{G}^{\ddagger}(\mathrm{kcal} / \mathrm{mol})$ \\
\hline 1 & $\mathrm{Ph}$ & $\mathrm{H}$ & $\mathrm{H}$ & $\mathrm{Me}$ & Thiourea-7 & Benzene & 296 & 5 & 52.5 & 47.5 & 0.06 \\
\hline 2 & $\mathrm{Ph}$ & $\mathrm{H}$ & $\mathrm{H}$ & $\mathrm{Me}$ & Thiourea-6 & Benzene & 296 & 13 & 56.5 & 43.5 & 0.15 \\
\hline 3 & $\mathrm{Ph}$ & $\mathrm{H}$ & $\mathrm{H}$ & $\mathrm{Me}$ & Thiourea-1 & Benzene & 296 & 14 & 57 & 43 & 0.17 \\
\hline 4 & $\mathrm{Ph}$ & $\mathrm{H}$ & $\mathrm{H}$ & $\mathrm{Me}$ & Thiourea-1 & DCM & 296 & 52 & 76 & 24 & 0.68 \\
\hline 5 & $\mathrm{Ph}$ & $\mathrm{H}$ & $\mathrm{H}$ & $\mathrm{Me}$ & Thiourea-1 & DCM & 248 & 62 & 81 & 19 & 0.71 \\
\hline 6 & $\mathrm{Ph}$ & $\mathrm{H}$ & $\mathrm{H}$ & $\mathrm{Me}$ & Thiourea-3 & DCM & 248 & 65 & 82.5 & 17.5 & 0.76 \\
\hline 7 & $\mathrm{Ph}$ & $\mathrm{H}$ & $\mathrm{H}$ & $\mathrm{Me}$ & Thiourea-2 & $\mathrm{DCM}$ & 248 & 69 & 84.5 & 15.5 & 0.84 \\
\hline 8 & $\mathrm{Ph}$ & $\mathrm{H}$ & $\mathrm{H}$ & $\mathrm{Me}$ & Thiourea-5 & DCM & 248 & 79 & 89.5 & 10.5 & 1.06 \\
\hline 9 & $\mathrm{Ph}$ & $\mathrm{H}$ & $\mathrm{H}$ & $\mathrm{Me}$ & Thiourea-1 & EtOAc & 296 & 26 & 63 & 37 & 0.31 \\
\hline 11 & $\mathrm{Ph}$ & $\mathrm{H}$ & $\mathrm{H}$ & $\mathrm{Me}$ & Thiourea-1 & Toluene & 296 & 27 & 63.5 & 36.5 & 0.33 \\
\hline 12 & $\mathrm{Ph}$ & $\mathrm{H}$ & $\mathrm{H}$ & $\mathrm{Me}$ & Thiourea-1 & $\mathrm{Et}_{2} \mathrm{O}$ & 296 & 37 & 68.5 & 31.5 & 0.46 \\
\hline 13 & $\mathrm{Ph}$ & $\mathrm{H}$ & $\mathrm{H}$ & $\mathrm{Me}$ & Thiourea-5 & DCM & 248 & 80 & 90 & 10 & 1.08 \\
\hline 14 & $\mathrm{Ph}$ & $\mathrm{H}$ & $\mathrm{H}$ & Et & Thiourea-5 & DCM & 248 & 76 & 88 & 12 & 0.98 \\
\hline 15 & $\mathrm{Ph}$ & $\mathrm{H}$ & $\mathrm{H}$ & $i-\operatorname{Pr}$ & Thiourea-5 & DCM & 248 & 72 & 86 & 14 & 0.89 \\
\hline 16 & $\mathrm{Ph}$ & $\mathrm{H}$ & $\mathrm{H}$ & $t-\mathrm{Bu}$ & Thiourea-5 & DCM & 248 & 84 & 92 & 8 & 1.20 \\
\hline 17 & $\mathrm{Ph}$ & $\mathrm{H}$ & $\mathrm{H}$ & $\mathrm{Ph}$ & Thiourea-5 & DCM & 248 & 74 & 87 & 13 & 0.94 \\
\hline 18 & $\mathrm{Ph}$ & $\mathrm{H}$ & $\mathrm{H}$ & 2-OMe Ph & Thiourea-5 & DCM & 248 & 74 & 87 & 13 & 0.94 \\
\hline 19 & $\mathrm{Ph}$ & $\mathrm{H}$ & $\mathrm{H}$ & 3-OMe Ph & Thiourea-5 & DCM & 248 & 75 & 87.5 & 12.5 & 0.96 \\
\hline 21 & $\mathrm{Ph}$ & $\mathrm{H}$ & $\mathrm{H}$ & $2-\mathrm{Cl} \mathrm{Ph}$ & Thiourea-5 & DCM & 248 & 74 & 87 & 13 & 0.94 \\
\hline 22 & 4-OMe Ph & $\mathrm{H}$ & $\mathrm{H}$ & $\mathrm{Me}$ & Thiourea-5 & DCM & 248 & 78 & 89 & 11 & 1.03 \\
\hline 23 & 4-Me Ph & $\mathrm{H}$ & $\mathrm{H}$ & $\mathrm{Me}$ & Thiourea-5 & $\mathrm{DCM}$ & 248 & 82 & 91 & 9 & 1.14 \\
\hline 24 & $\mathrm{Ph}$ & $\mathrm{H}$ & $\mathrm{Me}$ & $\mathrm{Me}$ & Thiourea-5 & DCM & 248 & 81 & 90.5 & 9.5 & 1.11 \\
\hline 25 & 4-F Ph & $\mathrm{H}$ & $\mathrm{Me}$ & $\mathrm{Me}$ & Thiourea-5 & DCM & 248 & 75 & 87.5 & 12.5 & 0.96 \\
\hline 26 & 4-Br Ph & $\mathrm{H}$ & $\mathrm{Me}$ & $\mathrm{Me}$ & Thiourea-5 & DCM & 248 & 76 & 88 & 12 & 0.98 \\
\hline 27 & Cyclohexyl & $\mathrm{H}$ & $\mathrm{H}$ & $\mathrm{Me}$ & Thiourea-5 & DCM & 248 & 82 & 91 & 9 & 1.14 \\
\hline
\end{tabular}




\begin{tabular}{|c|c|c|c|c|c|c|c|c|c|c|c|}
\hline 28 & Cyclohexyl & $\mathrm{H}$ & $\mathrm{H}$ & $\mathrm{Ph}$ & Thiourea-5 & DCM & 248 & 78 & 89 & 11 & 1.03 \\
\hline 29 & Butyl & $\mathrm{H}$ & H & $\mathrm{Ph}$ & Thiourea-5 & DCM & 248 & 78 & 89 & 11 & 1.03 \\
\hline 30 & $\left(\mathrm{CH}_{2} \mathrm{OCH}_{2}\right)$ & N/A & $\mathrm{Me}$ & $\mathrm{Me}$ & Thiourea-5 & DCM & 248 & 66 & 83 & 17 & 0.78 \\
\hline 31 & $\left(\mathrm{CH}_{2}\right)_{4}$ & $\mathrm{H}$ & N/A & $\mathrm{Ph}$ & Thiourea-5 & DCM & 248 & 89 & 94.5 & 5.5 & 1.40 \\
\hline 32 & $\left(\mathrm{CH}_{2}\right)_{4}$ & $\mathrm{H}$ & N/A & 4-Cl Ph & Thiourea-5 & DCM & 248 & 91 & 95.5 & 4.5 & 1.51 \\
\hline 33 & $\left(\mathrm{CH}_{2}\right)_{4}$ & $\mathrm{H}$ & N/A & 2-Cl Ph & Thiourea-5 & DCM & 248 & 88 & 94 & 6 & 1.36 \\
\hline 34 & $\left(\mathrm{CH}_{2}\right)_{4}$ & $\mathrm{H}$ & N/A & 3-OMe Ph & Thiourea-5 & DCM & 248 & 86 & 93 & 7 & 1.27 \\
\hline 35 & $\left(\mathrm{CH}_{2}\right)_{4}$ & $\mathrm{H}$ & N/A & 2-OMe Ph & Thiourea-5 & DCM & 248 & 89 & 94.5 & 5.5 & 1.40 \\
\hline
\end{tabular}




\section{Michael Addition of Diphenyl Phosphite to Nitroalkenes}

\begin{tabular}{|c|c|c|c|c|c|c|c|c|}
\hline entry & $\mathbf{R}^{1}$ & Catalyst & Solvent & Temp (K) & ee (\%) & \multicolumn{2}{|c|}{ er } & $\Delta \Delta \mathbf{G}^{\ddagger}(\mathrm{kcal} / \mathrm{mol})$ \\
\hline 1 & $\mathrm{Ph}$ & Squaramide-1 & DCM & 296 & 81 & 90.5 & 9.5 & 1.33 \\
\hline 2 & $\mathrm{Ph}$ & Squaramide-2 & DCM & 296 & 88 & 94 & 6 & 1.62 \\
\hline 3 & $\mathrm{Ph}$ & Squaramide-3 & DCM & 296 & 95 & 97.5 & 2.5 & 2.15 \\
\hline 4 & $\mathrm{Ph}$ & Squaramide-4 & DCM & 296 & 96 & 98 & 2 & 2.29 \\
\hline 5 & $\mathrm{Ph}$ & Squaramide-4 & DCM & 273 & 97 & 98.5 & 1.5 & 2.27 \\
\hline 6 & $\mathrm{Ph}$ & Squaramide-4 & DCM & 263 & 98 & 99 & 1 & 2.40 \\
\hline 7 & $\mathrm{Ph}$ & Squaramide-4 & DCM & 253 & 98.4 & 99.2 & 0.8 & 2.42 \\
\hline 8 & $\mathrm{Ph}$ & Squaramide-4 & Toluene & 296 & 94 & 97 & 3 & 2.04 \\
\hline 9 & $\mathrm{Ph}$ & Squaramide-4 & $\mathrm{Et}_{2} \mathrm{O}$ & 296 & 93 & 96.5 & 3.5 & 1.95 \\
\hline 10 & $\mathrm{Ph}$ & Squaramide-4 & MTBE & 296 & 94 & 97 & 3 & 2.04 \\
\hline 11 & $\mathrm{Ph}$ & Squaramide-4 & THF & 296 & 93 & 96.5 & 3.5 & 1.95 \\
\hline 12 & $\mathrm{Ph}$ & Squaramide-4 & $\mathrm{MeCN}$ & 296 & 90 & 95 & 5 & 1.73 \\
\hline 13 & $2-\mathrm{FPh}$ & Squaramide-4 & DCM & 273 & 99 & 99.5 & 0.5 & 2.87 \\
\hline 14 & 2-napthyl & Squaramide-4 & DCM & 273 & 99 & 99.5 & 0.5 & 2.87 \\
\hline 15 & 1-napthyl & Squaramide-4 & DCM & 273 & 97 & 98.5 & 1.5 & 2.27 \\
\hline 16 & 2-furyl & Squaramide-4 & DCM & 273 & 97 & 98.5 & 1.5 & 2.27 \\
\hline 17 & 2-thienyl & Squaramide-4 & DCM & 273 & 97 & 98.5 & 1.5 & 2.27 \\
\hline 18 & 4-OMe Ph & Squaramide-4 & DCM & 273 & 99 & 99.5 & 0.5 & 2.87 \\
\hline 19 & $2,4-\mathrm{Cl} \mathrm{Ph}$ & Squaramide-4 & DCM & 273 & 97 & 98.5 & 1.5 & 2.27 \\
\hline 20 & $i-\operatorname{Pr}$ & Squaramide-4 & DCM & 273 & 95 & 97.5 & 2.5 & 1.99 \\
\hline 21 & Cyclohexyl & Squaramide-4 & DCM & 273 & 97 & 98.5 & 1.5 & 2.27 \\
\hline 22 & 3-indole & Squaramide-4 & DCM & 273 & 98 & 99 & 1 & 2.49 \\
\hline 23 & $i$-Bu & Squaramide-4 & DCM & 273 & 96 & 98 & 2 & 2.11 \\
\hline 24 & Pentyl & Squaramide-4 & DCM & 273 & 96 & 98 & 2 & 2.11 \\
\hline 25 & Mesityl & Squaramide-4 & DCM & 273 & 96 & 98 & 2 & 2.11 \\
\hline 26 & $2-\mathrm{NO}_{2} \mathrm{Ph}$ & Squaramide-4 & DCM & 273 & 97 & 98.5 & 1.5 & 2.27 \\
\hline 27 & $3,4-\left(\mathrm{OCH}_{2} \mathrm{O}\right) \mathrm{Ph}$ & Squaramide-4 & DCM & 273 & 98 & 99 & 1 & 2.49 \\
\hline 28 & $2-\mathrm{Br} \mathrm{Ph}$ & Squaramide-4 & DCM & 273 & 97 & 98.5 & 1.5 & 2.27 \\
\hline 29 & 4-Br Ph & Squaramide-4 & DCM & 273 & 97 & 98.5 & 1.5 & 2.27 \\
\hline
\end{tabular}




\section{Cross Rauhut-Currier-Type Reaction of Tri-substituted Alkenes}

\begin{tabular}{|c|c|c|c|c|c|c|c|c|}
\hline entry & $R^{1}$ & Catalyst & Solvent & Temp (K) & ee (\%) & \multicolumn{2}{|c|}{ er } & $\Delta \Delta \mathrm{G}^{\ddagger}(\mathrm{kcal} / \mathrm{mol})$ \\
\hline 1 & $\mathrm{Ph}$ & Thiourea-1 & DCM & 296 & 44 & 72 & 28 & 0.56 \\
\hline 2 & $\mathrm{Ph}$ & Thiourea-6 & DCM & 296 & 41 & 70.5 & 29.5 & 0.51 \\
\hline 3 & $\mathrm{Ph}$ & Squaramide- 5 & DCM & 296 & 83 & 91.5 & 8.5 & 1.40 \\
\hline 4 & $\mathrm{Ph}$ & Squaramide-5 & Toluene & 296 & 67 & 83.5 & 16.5 & 0.95 \\
\hline 5 & $\mathrm{Ph}$ & Squaramide-5 & THF & 296 & 55 & 77.5 & 22.5 & 0.73 \\
\hline 6 & $\mathrm{Ph}$ & Squaramide- 5 & $\mathrm{MeCN}$ & 296 & 64 & 82 & 18 & 0.89 \\
\hline 7 & $\mathrm{Ph}$ & Squaramide-5 & $\mathrm{CHCl}_{3}$ & 296 & 79 & 89.5 & 10.5 & 1.26 \\
\hline 8 & $\mathrm{Ph}$ & Squaramide-5 & DCM & 273 & 81 & 90.5 & 9.5 & 1.22 \\
\hline 9 & $\mathrm{Ph}$ & Squaramide-5 & DCM & 253 & 80 & 90 & 10 & 1.10 \\
\hline 10 & 2-thienyl & Squaramide- 5 & DCM & 296 & 77 & 88.5 & 11.5 & 1.20 \\
\hline 11 & 4-i-Pr Ph & Squaramide- 5 & DCM & 296 & 83 & 91.5 & 8.5 & 1.40 \\
\hline 12 & 3,4-OMe Ph & Squaramide-5 & DCM & 296 & 91 & 95.5 & 4.5 & 1.80 \\
\hline 13 & $2-\mathrm{FPh}$ & Squaramide-5 & DCM & 296 & 72 & 86 & 14 & 1.07 \\
\hline 14 & 3-Cl Ph & Squaramide- 5 & DCM & 296 & 82 & 91 & 9 & 1.36 \\
\hline 15 & 4-Br Ph & Squaramide-5 & DCM & 296 & 82 & 91 & 9 & 1.36 \\
\hline 16 & $3,4-\mathrm{Cl} \mathrm{Ph}$ & Squaramide- 5 & DCM & 296 & 83 & 91.5 & 8.5 & 1.40 \\
\hline 17 & 2-Me Ph & Squaramide- 5 & DCM & 296 & 75 & 87.5 & 12.5 & 1.14 \\
\hline 18 & 3-OMe Ph & Squaramide-5 & DCM & 296 & 87 & 93.5 & 6.5 & 1.57 \\
\hline 19 & 2-napthyl & Squaramide-5 & DCM & 296 & 56 & 78 & 22 & 0.74 \\
\hline 20 & 4-OMe Ph & Squaramide-5 & DCM & 296 & 88 & 94 & 6 & 1.62 \\
\hline
\end{tabular}




\section{Enantioselective Addition of Thioacetic Acid to Nitroalkenes}

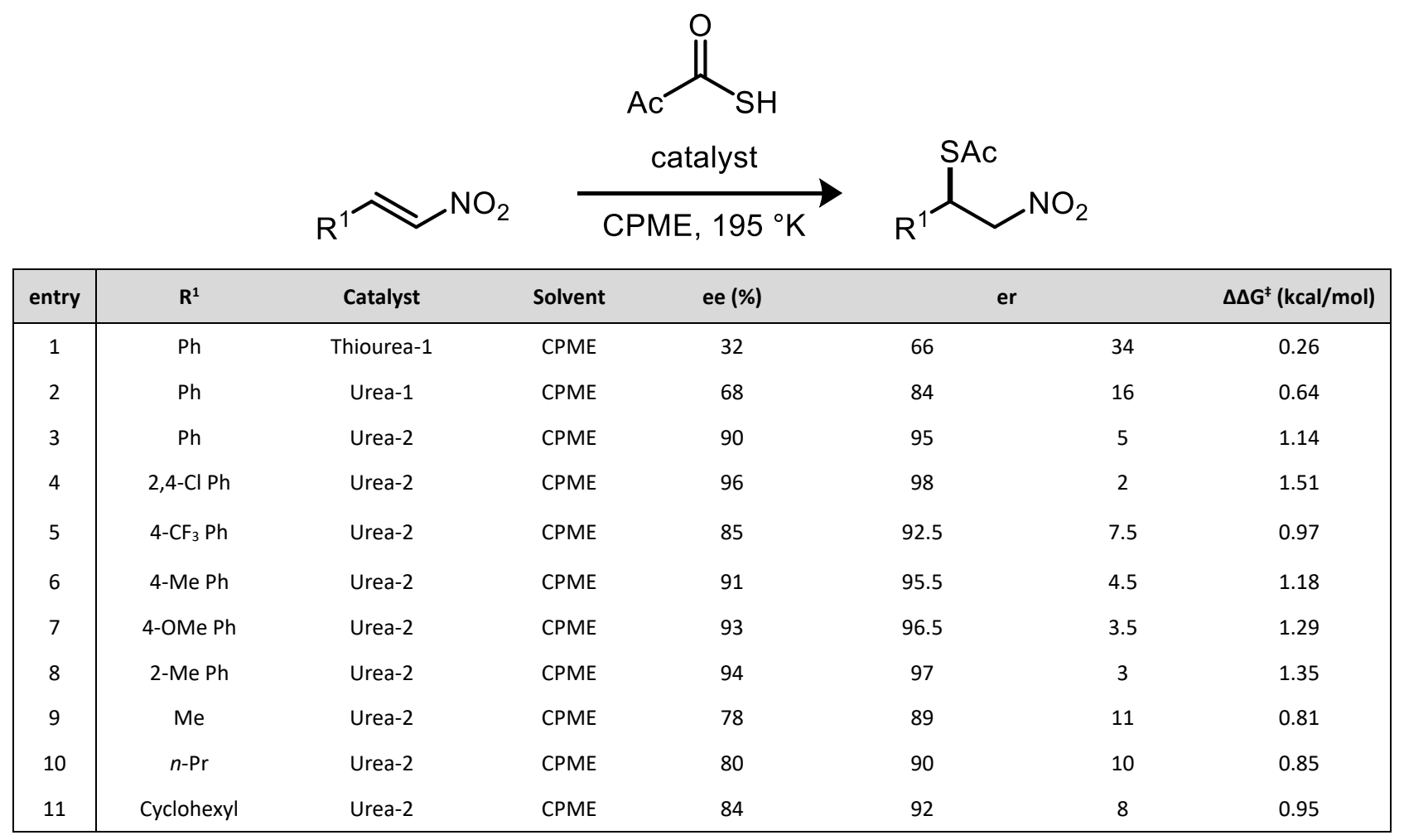




\section{Addition of Oxindoles to Nitroolefins}

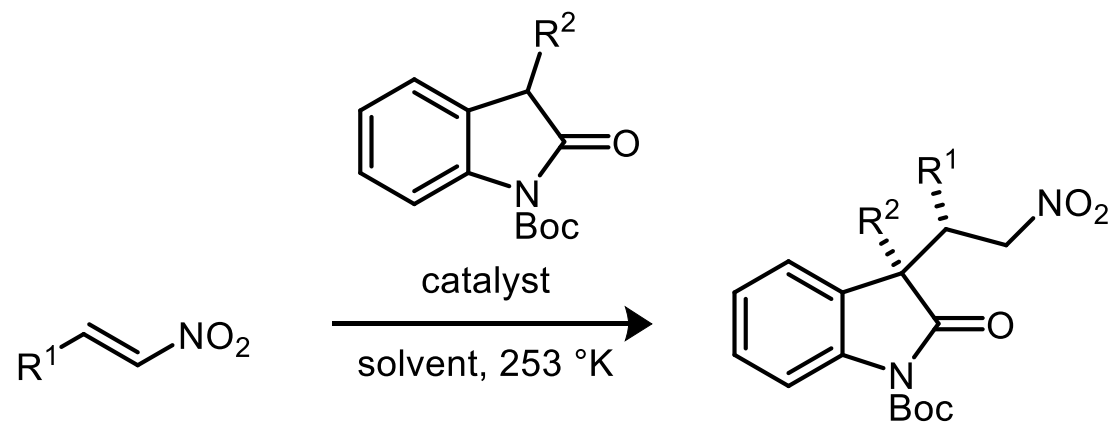

\begin{tabular}{|c|c|c|c|c|c|c|c|c|}
\hline entry & $\mathbf{R}^{1}$ & $\mathbf{R}^{2}$ & Catalyst & Solvent & ee (\%) & \multicolumn{2}{|c|}{ er } & $\Delta \Delta \mathrm{G}^{\ddagger}(\mathrm{kcal} / \mathrm{mol})$ \\
\hline 1 & $\mathrm{Ph}$ & Me & Thiourea-1 & THF & 78 & 89 & 11 & 1.05 \\
\hline 2 & $\mathrm{Ph}$ & $\mathrm{Me}$ & Thiourea-1 & xylene & 89 & 94.5 & 5.5 & 1.43 \\
\hline 3 & $\mathrm{Ph}$ & Me & Thiourea-1 & Toluene & 87 & 93.5 & 6.5 & 1.34 \\
\hline 4 & $\mathrm{Ph}$ & Me & Thiourea-1 & DCM & 93 & 96.5 & 3.5 & 1.67 \\
\hline 5 & $\mathrm{Ph}$ & $\mathrm{Me}$ & Thiourea-1 & $\mathrm{CHCl}_{3}$ & 95 & 97.5 & 2.5 & 1.84 \\
\hline 6 & $\mathrm{Ph}$ & $\mathrm{Me}$ & Thiourea-10 & $\mathrm{CHCl}_{3}$ & 99 & 99.5 & 0.5 & 2.66 \\
\hline 7 & $\mathrm{Ph}$ & $\mathrm{Me}$ & Thiourea-10 & DCM & 92 & 96 & 4 & 1.60 \\
\hline 8 & $\mathrm{Ph}$ & Et & Thiourea-10 & $\mathrm{CHCl}_{3}$ & 96 & 98 & 2 & 1.96 \\
\hline 9 & $\mathrm{Ph}$ & Allyl & Thiourea-10 & $\mathrm{CHCl}_{3}$ & 94 & 97 & 3 & 1.75 \\
\hline 10 & $\mathrm{Ph}$ & Cinnamyl & Thiourea-10 & $\mathrm{CHCl}_{3}$ & 92 & 96 & 4 & 1.60 \\
\hline 11 & $\mathrm{Ph}$ & $4-\mathrm{Br} B n$ & Thiourea-10 & $\mathrm{CHCl}_{3}$ & 88 & 94 & 6 & 1.38 \\
\hline 12 & 4-Br Ph & Allyl & Thiourea-10 & $\mathrm{CHCl}_{3}$ & 91 & 95.5 & 4.5 & 1.54 \\
\hline 13 & $3-\mathrm{Br} \mathrm{Ph}$ & Allyl & Thiourea-10 & $\mathrm{CHCl}_{3}$ & 95 & 97.5 & 2.5 & 1.84 \\
\hline 14 & 4-OMe Ph & Allyl & Thiourea-10 & $\mathrm{CHCl}_{3}$ & 90 & 95 & 5 & 1.48 \\
\hline 15 & 4-OH Ph & Allyl & Thiourea-10 & $\mathrm{CHCl}_{3}$ & 92 & 96 & 4 & 1.60 \\
\hline 16 & $3,4-\mathrm{Cl} \mathrm{Ph}$ & Allyl & Thiourea-10 & $\mathrm{CHCl}_{3}$ & 89 & 94.5 & 5.5 & 1.43 \\
\hline 17 & 2 -furyl & Allyl & Thiourea-10 & $\mathrm{CHCl}_{3}$ & 99 & 99.5 & 0.5 & 2.66 \\
\hline 18 & Heptyl & Allyl & Thiourea-10 & $\mathrm{CHCl}_{3}$ & 94 & 97 & 3 & 1.75 \\
\hline 19 & $(\mathrm{OMe})_{2} \mathrm{CH}$ & Allyl & Thiourea-10 & $\mathrm{CHCl}_{3}$ & 93 & 96.5 & 3.5 & 1.67 \\
\hline
\end{tabular}


Michael Addition of Malonates to Nitroalkenes

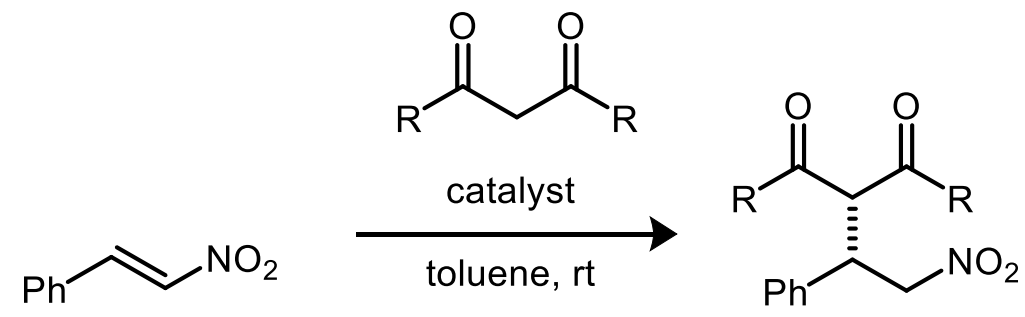

\begin{tabular}{|c|ccc|}
\hline entry & R & Catalyst & $\Delta \mathbf{G}^{\mathbf{*}}(\mathbf{k c a l} / \mathbf{m o l})$ \\
\hline 1 & OEt & Thiourea-11 & 1.25 \\
2 & OEt & Thiourea-12 & 1.07 \\
3 & OEt & Thiourea-13 & 1.02 \\
4 & OEt & Thiourea-14 & 0.93 \\
5 & OEt & Thiourea-15 & 1.22 \\
6 & OEt & Thiourea-16 & 0.84 \\
7 & OEt & Thiourea-17 & 1.04 \\
8 & OEt & Thiourea-18 & 0.57 \\
9 & OEt & Thiourea-19 & 0.42 \\
10 & OEt & Thiourea-20 & 0.96 \\
11 & OEt & Thiourea-21 & 1.13 \\
12 & OEt & Thiourea-22 & 1.08 \\
13 & OEt & Thiourea-23 & 1.05 \\
14 & OEt & Thiourea-24 & 1.21 \\
15 & OEt & Thiourea-25 & 1.08 \\
16 & OEt & Thiourea-26 & 1.34 \\
17 & OEt & Thiourea-27 & 0.59 \\
18 & Ph & Squaramide-7 & 1.48 \\
19 & Ph & Squaramide-8 & 1.40 \\
20 & Ph & Squaramide-9 & 1.24 \\
21 & Ph & Squaramide-10 & 1.30 \\
22 & Ph & Squaramide-11 & 0.74 \\
23 & Ph & Squaramide-12 & 0.54 \\
\hline
\end{tabular}




\section{Transfer Hydrogenation of Trifluoromethyl Nitroalkenes}<smiles>[R]OC([R])=C1CC(C([R])=O)=C(C)NC1[R]</smiles><smiles>[R]/C(=C/[N+](=O)[O-])C(F)(F)F</smiles><smiles>CC(C)(C)C(NC(=S)Nc1cc(C(F)(F)F)cc(C(F)(F)F)c1)C(=O)N(Cc1ccccc1)C(C)(C)C</smiles>

\begin{tabular}{|c|c|c|c|c|c|c|c|c|}
\hline entry & $\mathbf{R}^{1}$ & $\mathbf{R}^{2}$ & Solvent & Temp (K) & ee (\%) & \multicolumn{2}{|c|}{ er } & $\Delta \Delta \mathrm{G}^{\ddagger}(\mathrm{kcal} / \mathrm{mol})$ \\
\hline 1 & $\mathrm{Ph}$ & Et & Toluene & 313 & 77 & 88.5 & 11.5 & 1.27 \\
\hline 2 & $\mathrm{Ph}$ & $\mathrm{Me}$ & Toluene & 313 & 69 & 84.5 & 15.5 & 1.05 \\
\hline 3 & $\mathrm{Ph}$ & $i-\mathrm{Bu}$ & Toluene & 313 & 70 & 85 & 15 & 1.08 \\
\hline 4 & $\mathrm{Ph}$ & $t-\mathrm{Bu}$ & Toluene & 313 & 89 & 94.5 & 5.5 & 1.77 \\
\hline 5 & $\mathrm{Ph}$ & $t$-Bu & Toluene & 253 & 94 & 97 & 3 & 1.75 \\
\hline 6 & $\mathrm{Ph}$ & $t$-Bu & $\mathrm{PhCF}_{3}$ & 253 & 95 & 97.5 & 2.5 & 1.84 \\
\hline 7 & 4-Me Ph & $t-\mathrm{Bu}$ & $\mathrm{PhCF}_{3}$ & 253 & 94 & 97 & 3 & 1.75 \\
\hline 8 & 3-Me Ph & $t-\mathrm{Bu}$ & $\mathrm{PhCF}_{3}$ & 253 & 97 & 98.5 & 1.5 & 2.10 \\
\hline 9 & 2-Me Ph & $t-\mathrm{Bu}$ & $\mathrm{PhCF}_{3}$ & 253 & 96 & 98 & 2 & 1.96 \\
\hline 10 & $4-\mathrm{FPh}$ & $t$-Bu & $\mathrm{PhCF}_{3}$ & 253 & 93 & 96.5 & 3.5 & 1.67 \\
\hline 11 & 4-Br Ph & $t-\mathrm{Bu}$ & $\mathrm{PhCF}_{3}$ & 253 & 96 & 98 & 2 & 1.96 \\
\hline 12 & 4-Br Ph & $t-\mathrm{Bu}$ & Toluene & 253 & 95 & 97.5 & 2.5 & 1.84 \\
\hline 13 & 4-OMe Ph & $t$-Bu & $\mathrm{PhCF}_{3}$ & 253 & 98 & 99 & 1 & 2.31 \\
\hline 14 & 4-OMe Ph & $t-\mathrm{Bu}$ & Toluene & 253 & 94 & 97 & 3 & 1.75 \\
\hline 15 & $4-\mathrm{CF}_{3} \mathrm{Ph}$ & $t-\mathrm{Bu}$ & $\mathrm{PhCF}_{3}$ & 253 & 94 & 97 & 3 & 1.75 \\
\hline 16 & 2-napthyl & $t$-Bu & $\mathrm{PhCF}_{3}$ & 253 & 97 & 98.5 & 1.5 & 2.10 \\
\hline 17 & 2-napthyl & $t-\mathrm{Bu}$ & Toluene & 253 & 95 & 97.5 & 2.5 & 1.84 \\
\hline 18 & 3-thienyl & $t-\mathrm{Bu}$ & $\mathrm{PhCF}_{3}$ & 253 & 95 & 97.5 & 2.5 & 1.84 \\
\hline 19 & $3-N$-Ts-Indolyl & $t$-Bu & $\mathrm{PhCF}_{3}$ & 253 & 93 & 96.5 & 3.5 & 1.67 \\
\hline 20 & $\mathrm{Bn}$ & $t-\mathrm{Bu}$ & $\mathrm{PhCF}_{3}$ & 253 & 90 & 95 & 5 & 1.48 \\
\hline 21 & $\mathrm{Bn}$ & $t-\mathrm{Bu}$ & Toluene & 253 & 85 & 92.5 & 7.5 & 1.26 \\
\hline 22 & Nonyl & $t$-Bu & $\mathrm{PhCF}_{3}$ & 253 & 91 & 95.5 & 4.5 & 1.54 \\
\hline
\end{tabular}




\section{Addition of 3-substituted Benzofuran-2(3H)-ones to Nitroolefins}<smiles>[R]C1C(=O)Oc2ccccc21</smiles><smiles>[R]C=C[N+](=O)[O-]</smiles><smiles>CN(C)C(c1ccccc1)[C@H](NC(=S)Nc1cc(C(F)(F)F)cc(C(F)(F)F)c1)c1ccccc1</smiles>

\begin{tabular}{|c|c|c|c|c|c|c|c|c|}
\hline \multirow{2}{*}{$\frac{\text { entry }}{1}$} & \multirow{2}{*}{$\frac{\mathbf{R}^{1}}{\mathrm{Ph}}$} & \multirow{2}{*}{$\frac{\mathbf{R}^{2}}{\mathrm{Me}}$} & \multirow{2}{*}{$\frac{\mathbf{R}^{3}}{\mathrm{H}}$} & \multirow{2}{*}{$\begin{array}{c}\text { Temp (K) } \\
293\end{array}$} & \multirow{2}{*}{$\begin{array}{c}\text { ee (\%) } \\
57\end{array}$} & \multicolumn{2}{|c|}{ er } & \multirow{2}{*}{$\frac{\Delta \Delta \mathbf{G}^{\ddagger}(\mathbf{k c a l} / \mathrm{mol})}{0.75}$} \\
\hline & & & & & & 78.5 & 21.5 & \\
\hline 2 & $\mathrm{Ph}$ & Me & $\mathrm{H}$ & 253 & 62 & 81 & 19 & 0.73 \\
\hline 3 & $\mathrm{Ph}$ & Me & $\mathrm{H}$ & 213 & 66 & 83 & 17 & 0.67 \\
\hline 4 & 4-OMe Ph & Me & $\mathrm{H}$ & 213 & 65 & 82.5 & 17.5 & 0.66 \\
\hline 5 & 4-F Ph & Me & $\mathrm{H}$ & 213 & 64 & 82 & 18 & 0.64 \\
\hline 6 & 4-Ph Ph & Me & $\mathrm{H}$ & 213 & 58 & 79 & 21 & 0.56 \\
\hline 7 & 4-Cl Ph & Me & $\mathrm{H}$ & 213 & 52 & 76 & 24 & 0.49 \\
\hline 8 & 3- $\mathrm{NO}_{2} \mathrm{Ph}$ & Me & $\mathrm{H}$ & 213 & 75 & 87.5 & 12.5 & 0.82 \\
\hline 9 & 2-Cl Ph & Me & $\mathrm{H}$ & 213 & 86 & 93 & 7 & 1.09 \\
\hline 10 & 2-Br Ph & Me & $\mathrm{H}$ & 213 & 80 & 90 & 10 & 0.93 \\
\hline 11 & 2-F,6-Cl Ph & $\mathrm{Me}$ & $\mathrm{H}$ & 213 & 82 & 91 & 9 & 0.98 \\
\hline 12 & 2,6-Cl Ph & Me & $\mathrm{H}$ & 213 & 91 & 95.5 & 4.5 & 1.29 \\
\hline 13 & 2-napthyl & Me & $\mathrm{H}$ & 213 & 77 & 88.5 & 11.5 & 0.86 \\
\hline 14 & $\mathrm{CH}_{2} \mathrm{CH}_{2} \mathrm{Ph}$ & Me & $\mathrm{H}$ & 213 & 85 & 92.5 & 7.5 & 1.06 \\
\hline 15 & $i$-Bu & Me & $\mathrm{H}$ & 213 & 82 & 91 & 9 & 0.98 \\
\hline 16 & $\mathrm{Ph}$ & $\mathrm{Ph}$ & $\mathrm{H}$ & 213 & 55 & 77.5 & 22.5 & 0.52 \\
\hline 17 & $\mathrm{Ph}$ & $\mathrm{Ph}$ & Et & 213 & 58 & 79 & 21 & 0.56 \\
\hline 18 & $\mathrm{Ph}$ & $\mathrm{Ph}$ & $\mathrm{Cl}$ & 213 & 73 & 86.5 & 13.5 & 0.79 \\
\hline 19 & $\mathrm{Ph}$ & $\mathrm{Ph}$ & $\mathrm{Br}$ & 213 & 77 & 88.5 & 11.5 & 0.86 \\
\hline 20 & $\mathrm{Ph}$ & 4-Cl Ph & $\mathrm{H}$ & 213 & 53 & 76.5 & 23.5 & 0.50 \\
\hline
\end{tabular}




\section{Alkylation of Indoles to Nitroalkenes}<smiles>[R]c1ccc2[nH]c([R])cc2c1</smiles>

$\mathrm{R}^{1} \searrow \mathrm{NO}_{2}$

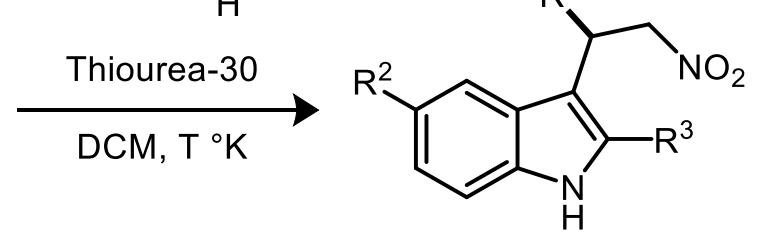<smiles>OC1Cc2ccccc2[C@H]1NC(=S)Nc1cc(C(F)(F)F)cc(C(F)(F)F)c1</smiles>

$\Delta \Delta \mathrm{G}^{\ddagger}(\mathrm{kcal} / \mathrm{mol})$

\begin{tabular}{ccl}
74 & 26 & 0.61 \\
92.5 & 7.5 & 1.24 \\
87 & 13 & 0.86 \\
94.5 & 5.5 & 1.29 \\
85.5 & 14.5 & 0.88 \\
86.5 & 13.5 & 0.92 \\
86.5 & 13.5 & 0.92 \\
91.5 & 8.5 & 1.18 \\
90.5 & 9.5 & 1.12 \\
\hline
\end{tabular}




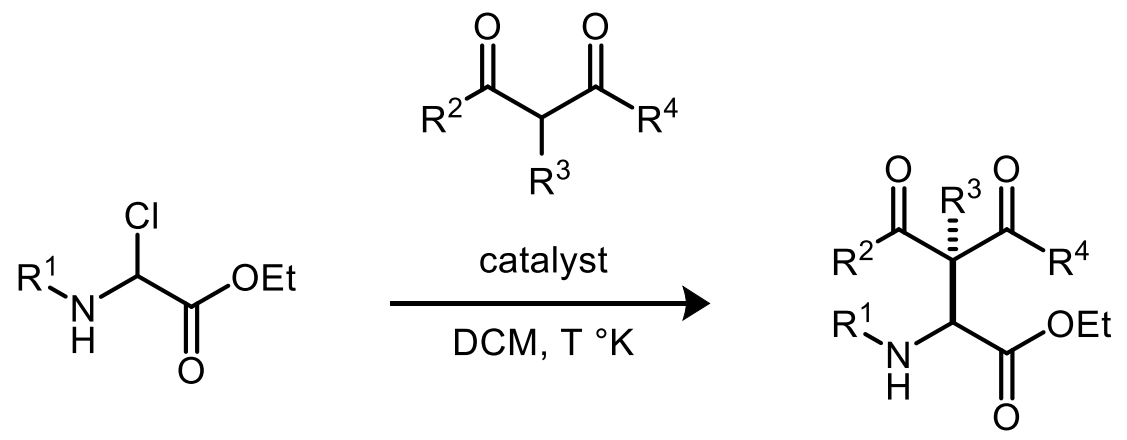

\begin{tabular}{|c|c|c|c|c|c|c|c|c|c|c|}
\hline entry & $\mathbf{R}^{1}$ & $\mathbf{R}^{2}$ & $\mathbf{R}^{3}$ & $\mathbf{R}^{4}$ & Catalyst & Temp (K) & ee (\%) & \multicolumn{2}{|c|}{ er } & $\Delta \Delta \mathrm{G}^{\ddagger}(\mathrm{kcal} / \mathrm{mol})$ \\
\hline 1 & $\mathrm{Bn}$ & $\mathrm{Ph}$ & $\mathrm{H}$ & $\mathrm{Ph}$ & Thiourea-1 & 243 & 43 & 71.5 & 28.5 & 0.44 \\
\hline 2 & $\mathrm{Cbz}$ & $\mathrm{Ph}$ & $\mathrm{H}$ & $\mathrm{Ph}$ & Thiourea-1 & 243 & 93 & 96.5 & 3.5 & 1.60 \\
\hline 3 & $\mathrm{CO}_{2} \mathrm{Me}$ & $\mathrm{Ph}$ & $\mathrm{H}$ & $\mathrm{Ph}$ & Thiourea-1 & 243 & 82 & 91 & 9 & 1.12 \\
\hline 4 & $\mathrm{CO}_{2} \mathrm{Ph}$ & $\mathrm{Ph}$ & $\mathrm{H}$ & $\mathrm{Ph}$ & Thiourea-1 & 243 & 44 & 72 & 28 & 0.46 \\
\hline 5 & Fmoc & $\mathrm{Ph}$ & $\mathrm{H}$ & $\mathrm{Ph}$ & Thiourea-1 & 243 & 66 & 83 & 17 & 0.77 \\
\hline 6 & TFA & $\mathrm{Ph}$ & $\mathrm{H}$ & $\mathrm{Ph}$ & Thiourea-1 & 243 & 25 & 62.5 & 37.5 & 0.25 \\
\hline 7 & Troc & $\mathrm{Ph}$ & $\mathrm{H}$ & $\mathrm{Ph}$ & Thiourea-1 & 243 & 58 & 79 & 21 & 0.64 \\
\hline 8 & $A c$ & $\mathrm{Ph}$ & $\mathrm{H}$ & $\mathrm{Ph}$ & Thiourea-1 & 244 & 76 & 88 & 12 & 0.97 \\
\hline 9 & $\mathrm{Cbz}$ & $\mathrm{Ph}$ & $\mathrm{H}$ & $\mathrm{Ph}$ & Thiourea-1 & 296 & 44 & 72 & 28 & 0.56 \\
\hline 10 & $\mathrm{Cbz}$ & $\mathrm{Ph}$ & $\mathrm{H}$ & $\mathrm{Ph}$ & Thiourea-1 & 273 & 88 & 94 & 6 & 1.49 \\
\hline 11 & $\mathrm{Cbz}$ & $\mathrm{Ph}$ & $\mathrm{H}$ & $\mathrm{Ph}$ & Thiourea-1 & 195 & 90 & 95 & 5 & 1.14 \\
\hline 12 & $\mathrm{Cbz}$ & $\mathrm{Ph}$ & $\mathrm{H}$ & $\mathrm{Ph}$ & Squaramide-1 & 243 & 77 & 88.5 & 11.5 & 0.99 \\
\hline 13 & $\mathrm{Cbz}$ & $\mathrm{Ph}$ & $\mathrm{H}$ & $\mathrm{Ph}$ & Urea-1 & 243 & 90 & 95 & 5 & 1.42 \\
\hline 14 & $\mathrm{Cbz}$ & Me & $\mathrm{H}$ & OEt & Thiourea-1 & 243 & 58 & 79 & 21 & 0.64 \\
\hline 15 & $\mathrm{Cbz}$ & $\mathrm{Me}$ & $\mathrm{H}$ & $\mathrm{OPh}_{2}$ & Thiourea-1 & 243 & 95 & 97.5 & 2.5 & 1.77 \\
\hline 16 & $\mathrm{Cbz}$ & Me & $\mathrm{H}$ & $\mathrm{O} t-\mathrm{Bu}$ & Thiourea-1 & 243 & 51 & 75.5 & 24.5 & 0.54 \\
\hline 17 & $\mathrm{Cbz}$ & $\mathrm{Ph}$ & $\mathrm{H}$ & OEt & Thiourea-1 & 243 & 89 & 94.5 & 5.5 & 1.37 \\
\hline 18 & $\mathrm{Cbz}$ & $\mathrm{Ph}$ & $\mathrm{H}$ & $\mathrm{O} i-\mathrm{Pr}$ & Thiourea-1 & 243 & 91 & 95.5 & 4.5 & 1.48 \\
\hline 19 & $\mathrm{Cbz}$ & $\mathrm{Ph}$ & $\mathrm{H}$ & $\mathrm{OPh}_{2}$ & Thiourea-1 & 243 & 96 & 98 & 2 & 1.88 \\
\hline 20 & $\mathrm{Cbz}$ & 4-OMe Ph & $\mathrm{H}$ & OEt & Thiourea-1 & 243 & 73 & 86.5 & 13.5 & 0.90 \\
\hline 21 & $\mathrm{Cbz}$ & $3-\mathrm{CF}_{3} \mathrm{Ph}$ & $\mathrm{H}$ & OEt & Thiourea-1 & 243 & 89 & 94.5 & 5.5 & 1.37 \\
\hline 22 & $\mathrm{Cbz}$ & 4-Cl Ph & $\mathrm{H}$ & 4-OMe Ph & Thiourea-1 & 243 & 97 & 98.5 & 1.5 & 2.02 \\
\hline 23 & $\mathrm{Cbz}$ & $4-\mathrm{Cl} \mathrm{Ph}$ & $\mathrm{F}$ & 4-OMe Ph & Thiourea-1 & 243 & 95 & 97.5 & 2.5 & 1.77 \\
\hline 24 & $\mathrm{Cbz}$ & $4-\mathrm{Cl} \mathrm{Ph}$ & $\mathrm{H}$ & 4-Cl Ph & Thiourea-1 & 243 & 93 & 96.5 & 3.5 & 1.60 \\
\hline 25 & $\mathrm{Cbz}$ & 4- $\mathrm{Cl} \mathrm{Ph}$ & $\mathrm{H}$ & OEt & Thiourea-1 & 243 & 90 & 95 & 5 & 1.42 \\
\hline 26 & $\mathrm{Cbz}$ & 4-F Ph & $\mathrm{H}$ & OEt & Thiourea-1 & 243 & 88 & 94 & 6 & 1.33 \\
\hline 27 & $\mathrm{Cbz}$ & $4-\mathrm{NO}_{2} \mathrm{Ph}$ & $\mathrm{H}$ & OEt & Thiourea-1 & 243 & 92 & 96 & 4 & 1.53 \\
\hline
\end{tabular}




\begin{tabular}{|l|llllllllll|}
28 & $\mathrm{Cbz}$ & $\mathrm{Ph}$ & $\mathrm{F}$ & $\mathrm{Ph}$ & Thiourea-1 & 243 & 94 & 97 & 3 & 1.68 \\
29 & $\mathrm{Cbz}$ & $\mathrm{Me}$ & $\mathrm{H}$ & $\mathrm{Me}$ & Thiourea-1 & 243 & 86 & 93 & 7 & 1.25 \\
\hline
\end{tabular}




\section{Aza-Henry}

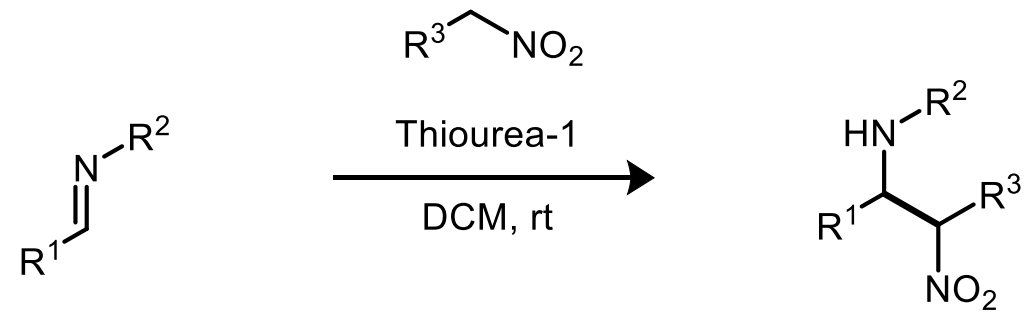

\begin{tabular}{|c|c|c|c|c|c|c|c|}
\hline entry & $\mathbf{R}^{1}$ & $\mathbf{R}^{2}$ & $\mathbf{R}^{\mathbf{3}}$ & ee (\%) & \multicolumn{2}{|c|}{ er } & $\Delta \Delta \mathrm{G}^{\ddagger}(\mathrm{kcal} / \mathrm{mol})$ \\
\hline 1 & $\mathrm{Ph}$ & Ts & $\mathrm{H}$ & 4 & 52 & 48 & 0.05 \\
\hline 2 & $\mathrm{Ph}$ & $\mathrm{P}(\mathrm{O}) \mathrm{Ph}_{2}$ & $\mathrm{H}$ & 67 & 83.5 & 16.5 & 0.95 \\
\hline 3 & 2-furyl & $\mathrm{P}(\mathrm{O}) \mathrm{Ph}_{2}$ & $\mathrm{H}$ & 76 & 88 & 12 & 1.17 \\
\hline 5 & 2-pyridyl & $\mathrm{P}(\mathrm{O}) \mathrm{Ph}_{2}$ & $\mathrm{H}$ & 68 & 84 & 16 & 0.98 \\
\hline 6 & 2-thienyl & $\mathrm{P}(\mathrm{O}) \mathrm{Ph}_{2}$ & $\mathrm{H}$ & 64 & 82 & 18 & 0.89 \\
\hline 7 & 4-Cl Ph & $\mathrm{P}(\mathrm{O}) \mathrm{Ph}_{2}$ & $\mathrm{H}$ & 67 & 83.5 & 16.5 & 0.95 \\
\hline 8 & 4-Me Ph & $\mathrm{P}(\mathrm{O}) \mathrm{Ph}_{2}$ & $\mathrm{H}$ & 63 & 81.5 & 18.5 & 0.87 \\
\hline 9 & Cinnamyl & $\mathrm{P}(\mathrm{O}) \mathrm{Ph}_{2}$ & $\mathrm{H}$ & 65 & 82.5 & 17.5 & 0.91 \\
\hline 10 & $\mathrm{Ph}$ & $\mathrm{P}(\mathrm{O}) \mathrm{Ph}_{2}$ & $\mathrm{Me}$ & 67 & 83.5 & 16.5 & 0.95 \\
\hline
\end{tabular}




\section{Addition of 2-(Cyanomethyl)azaarenes to $\mathrm{N}$-Boc-amine Sulfones}

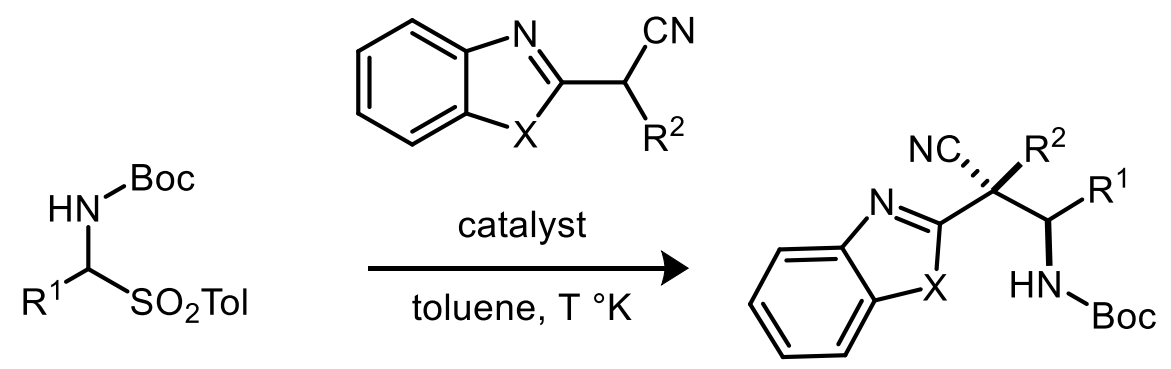

\begin{tabular}{|c|c|c|c|c|c|c|c|c|c|}
\hline entry & $\mathbf{R}^{1}$ & $\mathbf{R}^{2}$ & $x$ & Catalyst & Temp (K) & ee (\%) & \multicolumn{2}{|c|}{ er } & $\Delta \Delta \mathrm{G}^{\ddagger}(\mathrm{kcal} / \mathrm{mol})$ \\
\hline 1 & $\mathrm{Ph}$ & $\mathrm{Bn}$ & $\mathrm{S}$ & Thiourea-2 & 296 & 38 & 69 & 31 & 0.47 \\
\hline 2 & $\mathrm{Ph}$ & $\mathrm{Bn}$ & $\mathrm{S}$ & Thiourea-6 & 296 & 68 & 84 & 16 & 0.98 \\
\hline 3 & $\mathrm{Ph}$ & $\mathrm{Bn}$ & $\mathrm{S}$ & Squaramide-2 & 296 & 94 & 97 & 3 & 2.04 \\
\hline 4 & $\mathrm{Ph}$ & $\mathrm{Bn}$ & $\mathrm{S}$ & Squaramide-2 & 303 & 79 & 89.5 & 10.5 & 1.29 \\
\hline 5 & 2-furyl & $\mathrm{Bn}$ & $S$ & Squaramide-2 & 296 & 80 & 90 & 10 & 1.29 \\
\hline 6 & $3,4-\left(\mathrm{OCH}_{2} \mathrm{O}\right)$ & $\mathrm{Bn}$ & $\mathrm{S}$ & Squaramide-2 & 296 & 85 & 92.5 & 7.5 & 1.48 \\
\hline 7 & 2,3,4-OMe Ph & $\mathrm{Bn}$ & $\mathrm{S}$ & Squaramide-2 & 296 & 78 & 89 & 11 & 1.23 \\
\hline 8 & 2,3-OMe Ph & $\mathrm{Bn}$ & $\mathrm{S}$ & Squaramide-2 & 296 & 93 & 96.5 & 3.5 & 1.95 \\
\hline 9 & $2-\mathrm{FPh}$ & $\mathrm{Bn}$ & $\mathrm{S}$ & Squaramide-2 & 296 & 94 & 97 & 3 & 2.04 \\
\hline 10 & 3,5-OMe Ph & $\mathrm{Bn}$ & $\mathrm{S}$ & Squaramide-2 & 296 & 88 & 94 & 6 & 1.62 \\
\hline 11 & 4-Br Ph & $\mathrm{Bn}$ & $\mathrm{S}$ & Squaramide-2 & 296 & 78 & 89 & 11 & 1.23 \\
\hline 12 & 4-Me Ph & $\mathrm{Bn}$ & $\mathrm{S}$ & Squaramide-2 & 296 & 82 & 91 & 9 & 1.36 \\
\hline 13 & $4-\mathrm{NO}_{2} \mathrm{Ph}$ & $\mathrm{Bn}$ & $\mathrm{S}$ & Squaramide-2 & 296 & 92 & 96 & 4 & 1.87 \\
\hline 14 & 4-Br,2-thienyl & $\mathrm{Bn}$ & $S$ & Squaramide-2 & 296 & 80 & 90 & 10 & 1.29 \\
\hline 15 & 3-benzothiophene & $\mathrm{Bn}$ & $\mathrm{S}$ & Squaramide-2 & 296 & 83 & 91.5 & 8.5 & 1.40 \\
\hline 16 & 2-thienyl & $\mathrm{Bn}$ & $\mathrm{S}$ & Squaramide-2 & 296 & 77 & 88.5 & 11.5 & 1.20 \\
\hline 17 & $\mathrm{Ph}$ & $\mathrm{CH}_{2} \mathrm{CH}_{2} \mathrm{Ph}$ & $\mathrm{S}$ & Squaramide-2 & 296 & 86 & 93 & 7 & 1.52 \\
\hline 18 & $\mathrm{Ph}$ & 3,5-OMe Bn & $\mathrm{S}$ & Squaramide-2 & 296 & 89 & 94.5 & 5.5 & 1.67 \\
\hline 20 & $\mathrm{Ph}$ & $3,4-\left(\mathrm{OCH}_{2} \mathrm{O}\right) \mathrm{Bn}$ & $\mathrm{S}$ & Squaramide-2 & 296 & 93 & 96.5 & 3.5 & 1.95 \\
\hline 21 & $\mathrm{Ph}$ & 4-Br Bn & $\mathrm{S}$ & Squaramide-2 & 296 & 89 & 94.5 & 5.5 & 1.67 \\
\hline 22 & $\mathrm{Ph}$ & $\mathrm{CH}_{2}$-2-furyl & $\mathrm{S}$ & Squaramide-2 & 296 & 92 & 96 & 4 & 1.87 \\
\hline 23 & $\mathrm{Ph}$ & $\mathrm{CH}_{2}-\mathrm{i}-\mathrm{Pr}$ & $\mathrm{S}$ & Squaramide-2 & 296 & 92 & 96 & 4 & 1.87 \\
\hline 24 & $\mathrm{Ph}$ & $\mathrm{CH}_{2}$-2-thienyl & $\mathrm{S}$ & Squaramide-2 & 296 & 90 & 95 & 5 & 1.73 \\
\hline 25 & $\mathrm{Ph}$ & $\mathrm{Bn}$ & $\mathrm{O}$ & Squaramide-2 & 296 & 92 & 96 & 4 & 1.87 \\
\hline 26 & $\mathrm{Ph}$ & $\mathrm{Bn}$ & $\mathrm{S}$ & Squaramide-2 & 296 & 86 & 93 & 7 & 1.52 \\
\hline
\end{tabular}




\section{Nitrophosphonate Addition to Enones}

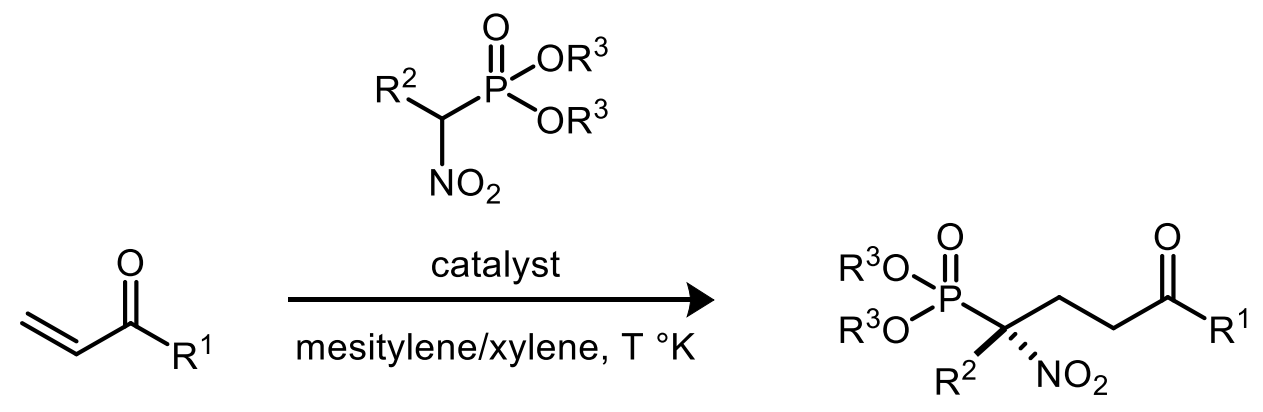

\begin{tabular}{|c|c|c|c|c|c|c|c|c|c|}
\hline entry & $\mathbf{R}^{1}$ & $\mathbf{R}^{2}$ & $\mathbf{R}^{3}$ & Catalyst & Temp (K) & ee (\%) & \multicolumn{2}{|c|}{ er } & $\Delta \Delta \mathrm{G}^{\ddagger}(\mathrm{kcal} / \mathrm{mol})$ \\
\hline 1 & $\mathrm{Ph}$ & $\mathrm{Me}$ & Et & Thiourea-31 & 208 & 45 & 72.5 & 27.5 & 0.40 \\
\hline 2 & 2-thienyl & $\mathrm{Me}$ & Et & Thiourea-31 & 208 & 43 & 71.5 & 28.5 & 0.38 \\
\hline 3 & 4-Me Ph & $\mathrm{Me}$ & Et & Thiourea-31 & 208 & 74 & 87 & 13 & 0.79 \\
\hline 4 & 2-furyl & $\mathrm{Me}$ & Et & Thiourea-31 & 208 & 42 & 71 & 29 & 0.37 \\
\hline 5 & Cyclohexyl & $\mathrm{Me}$ & Et & Thiourea-31 & 208 & 44 & 72 & 28 & 0.39 \\
\hline 6 & 3,4,5-OMe Ph & $\mathrm{Me}$ & Et & Thiourea-31 & 208 & 78 & 89 & 11 & 0.86 \\
\hline 7 & 3,4-OMe Ph & $\mathrm{Me}$ & Et & Thiourea-31 & 208 & 87 & 93.5 & 6.5 & 1.10 \\
\hline 8 & 4-OMe Ph & $\mathrm{Me}$ & Et & Thiourea-31 & 208 & 72 & 86 & 14 & 0.75 \\
\hline 9 & $4-\mathrm{NO}_{2} \mathrm{Ph}$ & $\mathrm{Me}$ & Et & Thiourea-31 & 208 & 35 & 67.5 & 32.5 & 0.30 \\
\hline 11 & $4-\mathrm{CF}_{3} \mathrm{Ph}$ & Me & Et & Thiourea-31 & 208 & 69 & 84.5 & 15.5 & 0.70 \\
\hline 12 & $\mathrm{Ph}$ & $\mathrm{Me}$ & Et & Squaramide- 6 & 208 & 92 & 96 & 4 & 1.31 \\
\hline 13 & 2-thienyl & $\mathrm{Me}$ & Et & Squaramide- 6 & 208 & 93 & 96.5 & 3.5 & 1.37 \\
\hline 14 & 4-Me Ph & $\mathrm{Me}$ & Et & Squaramide-6 & 208 & 97 & 98.5 & 1.5 & 1.73 \\
\hline 15 & 2-furyl & $\mathrm{Me}$ & Et & Squaramide-6 & 208 & 85 & 92.5 & 7.5 & 1.04 \\
\hline 16 & Cyclohexyl & $\mathrm{Me}$ & Et & Squaramide-6 & 208 & 51 & 75.5 & 24.5 & 0.47 \\
\hline 17 & 3,4-OMe Ph & $\mathrm{Me}$ & Et & Squaramide-6 & 208 & 94 & 97 & 3 & 1.44 \\
\hline 18 & 4-OMe Ph & Me & Et & Squaramide-6 & 208 & 93 & 96.5 & 3.5 & 1.37 \\
\hline 19 & $4-\mathrm{NO}_{2} \mathrm{Ph}$ & $\mathrm{Me}$ & Et & Squaramide- 6 & 208 & 92 & 96 & 4 & 1.31 \\
\hline 21 & $\alpha-M e$ Cinnamyl & $\mathrm{Me}$ & Et & Squaramide- 6 & 208 & 90 & 95 & 5 & 1.22 \\
\hline 22 & 1-napthyl & $\mathrm{Me}$ & Et & Squaramide- 6 & 208 & 15 & 57.5 & 42.5 & 0.12 \\
\hline 23 & $2-\mathrm{Cl} \mathrm{Ph}$ & $\mathrm{Me}$ & Et & Squaramide- 6 & 208 & 74 & 87 & 13 & 0.79 \\
\hline 24 & 2-napthyl & $\mathrm{Me}$ & Et & Squaramide- 6 & 208 & 96 & 98 & 2 & 1.61 \\
\hline 25 & 4-Br Ph & $\mathrm{Me}$ & Et & Squaramide-6 & 208 & 90 & 95 & 5 & 1.22 \\
\hline 26 & 4-Cl Ph & $\mathrm{Me}$ & Et & Squaramide-6 & 208 & 92 & 96 & 4 & 1.31 \\
\hline 27 & 4-CN Ph & $\mathrm{Me}$ & Et & Squaramide- 6 & 208 & 88 & 94 & 6 & 1.14 \\
\hline 28 & Cinnamyl & $\mathrm{Me}$ & Et & Squaramide- 6 & 208 & 95 & 97.5 & 2.5 & 1.51 \\
\hline
\end{tabular}




\begin{tabular}{|c|c|c|c|c|c|c|c|c|c|}
\hline 29 & 3-Br Ph & $\left(\mathrm{CH}_{2}\right)_{3} \mathrm{CO}_{2} \mathrm{Me}$ & Et & Squaramide-6 & 208 & 91 & 95.5 & 4.5 & 1.26 \\
\hline 30 & $3-\mathrm{Br} \mathrm{Ph}$ & $\mathrm{CH}_{2} \mathrm{CH}_{2} \mathrm{Ph}$ & Et & Squaramide-6 & 208 & 98 & 99 & 1 & 1.90 \\
\hline 31 & 3-Br Ph & Cyclopropyl & Et & Squaramide-6 & 233 & 92 & 96 & 4 & 1.47 \\
\hline 32 & 3-Br Ph & Et & Et & Squaramide- 6 & 208 & 92 & 96 & 4 & 1.31 \\
\hline 33 & $3-\mathrm{Br} \mathrm{Ph}$ & $n-\operatorname{Pr}$ & Et & Squaramide-6 & 208 & 96 & 98 & 2 & 1.61 \\
\hline 34 & 4-NO2 Ph & $\mathrm{Me}$ & $i-\operatorname{Pr}$ & Squaramide-6 & 208 & 74 & 87 & 13 & 0.79 \\
\hline 35 & 3-Br Ph & Nonyl & Et & Squaramide-6 & 208 & 95 & 97.5 & 2.5 & 1.51 \\
\hline
\end{tabular}




\section{Mannich/Aza-Michael}

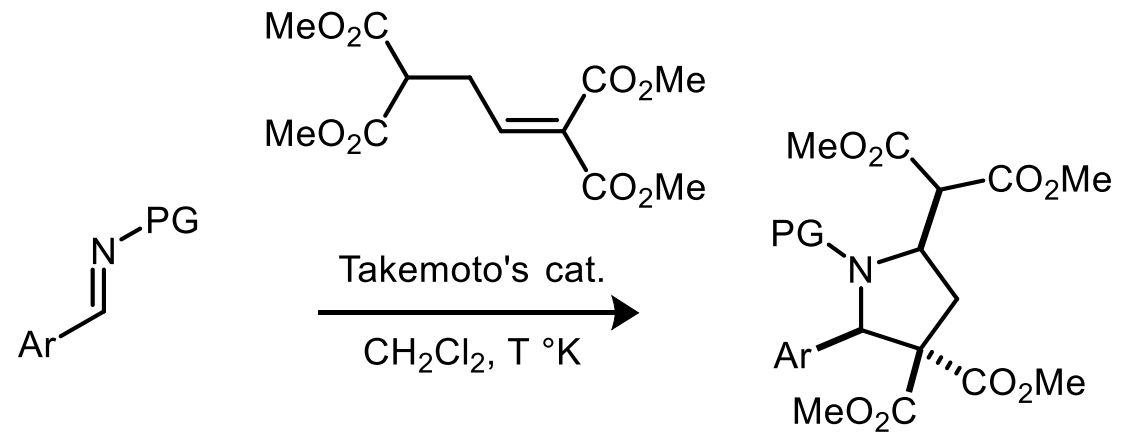

\begin{tabular}{|c|c|c|c|c|c|c|c|c|}
\hline entry & Ar & PG & Catalyst & Temp (K) & ee & \multicolumn{2}{|c|}{ er } & $\Delta \Delta \mathbf{G}^{\ddagger}(\mathrm{kcal} / \mathrm{mol})$ \\
\hline 1 & $\mathrm{Ph}$ & Boc & Thiourea-1 & 296 & 84 & 92 & 8 & 1.44 \\
\hline 2 & $\mathrm{Ph}$ & Boc & Thiourea-1 & 274 & 85 & 92.5 & 7.5 & 1.37 \\
\hline 3 & $\mathrm{Ph}$ & Boc & Thiourea-1 & 247 & 91 & 95.5 & 4.5 & 1.50 \\
\hline 4 & $\mathrm{Ph}$ & $\mathrm{Cbz}$ & Thiourea-1 & 296 & 70 & 85 & 15 & 1.02 \\
\hline 5 & $\mathrm{Ph}$ & Etoc & Thiourea-1 & 296 & 60 & 80 & 20 & 0.82 \\
\hline 6 & $\mathrm{Ph}$ & Boc & Thiourea-6 & 296 & 78 & 89 & 11 & 1.23 \\
\hline 7 & 2-Me Ph & Boc & Thiourea-1 & 296 & 75 & 87.5 & 12.5 & 1.14 \\
\hline 8 & 4-F Ph & Boc & Thiourea-1 & 296 & 83 & 91.5 & 8.5 & 1.40 \\
\hline 9 & 4-OMe Ph & Boc & Thiourea-1 & 296 & 78 & 89 & 11 & 1.23 \\
\hline 11 & 2-furyl & Boc & Thiourea-1 & 296 & 94 & 97 & 3 & 2.04 \\
\hline 12 & 2-thienyl & Boc & Thiourea-1 & 296 & 83 & 91.5 & 8.5 & 1.40 \\
\hline
\end{tabular}




\section{Michael/Spirocyclization}<smiles></smiles><smiles>[R]C=C[N+](=O)[O-]</smiles><smiles>[R]c1ccc2c(c1)CC[C@@]1(C(=O)/C(=N\[Al])S/C1=N\O)C2[R1]</smiles>

\begin{tabular}{|c|c|c|c|c|c|c|c|c|c|}
\hline entry & $\mathrm{Ar}$ & $\mathbf{R}^{1}$ & $\mathbf{R}^{2}$ & Catalyst & Temp. (K) & ee & & & $\Delta \Delta \mathrm{G}^{\ddagger}(\mathrm{kcal} / \mathrm{mol})$ \\
\hline 1 & $\mathrm{Ph}$ & 4-Me Ph & $\mathrm{H}$ & Thiourea- 6 & 298 & 0 & 50 & 50 & 0.00 \\
\hline 2 & $\mathrm{Ph}$ & 4-Me Ph & $\mathrm{H}$ & Thiourea-7 & 298 & 25 & 62.5 & 37.5 & 0.30 \\
\hline 3 & $\mathrm{Ph}$ & 4-Me Ph & $\mathrm{H}$ & Thiourea-1 & 298 & 46 & 73 & 27 & 0.59 \\
\hline 4 & $\mathrm{Ph}$ & 4-Me Ph & $\mathrm{H}$ & Thiourea-1 & 268 & 74 & 87 & 13 & 1.01 \\
\hline 5 & $\mathrm{Ph}$ & 4-Me Ph & $\mathrm{H}$ & Thiourea-1 & 243 & 83 & 91.5 & 8.5 & 1.15 \\
\hline 6 & $\mathrm{Ph}$ & 4-Me Ph & $\mathrm{H}$ & Thiourea-1 & 223 & 87 & 93.5 & 6.5 & 1.18 \\
\hline 7 & $\mathrm{Ph}$ & 4-Me Ph & $\mathrm{H}$ & Thiourea-1 & 213 & 91 & 95.5 & 4.5 & 1.29 \\
\hline 8 & $\mathrm{Ph}$ & 4-Me Ph & $\mathrm{H}$ & Thiourea-1 & 203 & 90 & 95 & 5 & 1.19 \\
\hline 9 & $\mathrm{Ph}$ & 4-OMe Ph & $\mathrm{H}$ & Thiourea-1 & 213 & 80 & 90 & 10 & 0.93 \\
\hline 10 & $\mathrm{Ph}$ & 2-Me Ph & $\mathrm{H}$ & Thiourea-1 & 213 & 80 & 90 & 10 & 0.93 \\
\hline 11 & $\mathrm{Ph}$ & 4-Cl Ph & $\mathrm{H}$ & Thiourea-1 & 213 & 93 & 96.5 & 3.5 & 1.40 \\
\hline 12 & $\mathrm{Ph}$ & 3-Cl Ph & $\mathrm{H}$ & Thiourea-1 & 213 & 91 & 95.5 & 4.5 & 1.29 \\
\hline 13 & $\mathrm{Ph}$ & 2-Cl Ph & $\mathrm{H}$ & Thiourea-1 & 213 & 88 & 94 & 6 & 1.16 \\
\hline 14 & $\mathrm{Ph}$ & $4-B r$ & $\mathrm{H}$ & Thiourea-1 & 213 & 92 & 96 & 4 & 1.35 \\
\hline 15 & $\mathrm{Ph}$ & $\mathrm{Ph}$ & $\mathrm{H}$ & Thiourea-1 & 213 & 93 & 96.5 & 3.5 & 1.40 \\
\hline 16 & $\mathrm{Ph}$ & 2-Napthyl & $\mathrm{H}$ & Thiourea-1 & 213 & 94 & 97 & 3 & 1.47 \\
\hline 17 & $\mathrm{Ph}$ & 2-furyl & $\mathrm{H}$ & Thiourea-1 & 213 & 79 & 89.5 & 10.5 & 0.91 \\
\hline 18 & $\mathrm{Ph}$ & 4-Me Ph & 6-OMe & Thiourea-1 & 213 & 92 & 96 & 4 & 1.35 \\
\hline 19 & 4-Me Ph & 4-Me Ph & $\mathrm{H}$ & Thiourea-1 & 213 & 90 & 95 & 5 & 1.25 \\
\hline 20 & 4-Cl Ph & 4-Me Ph & $\mathrm{H}$ & Thiourea-1 & 213 & 84 & 92 & 8 & 1.03 \\
\hline 21 & 3-Cl Ph & 4-Me Ph & $\mathrm{H}$ & Thiourea-1 & 213 & 92 & 96 & 4 & 1.35 \\
\hline 22 & 2-Cl Ph & 4-Me Ph & $\mathrm{H}$ & Thiourea-1 & 213 & 90 & 95 & 5 & 1.25 \\
\hline 23 & 3-OMe & 4-Me Ph & $\mathrm{H}$ & Thiourea-1 & 213 & 88 & 94 & 6 & 1.16 \\
\hline 24 & 2-OMe & 4-Me Ph & $\mathrm{H}$ & Thiourea-1 & 213 & 92 & 96 & 4 & 1.35 \\
\hline 25 & 2-Napthyl & 4-Me Ph & $\mathrm{H}$ & Thiourea-1 & 213 & 82 & 91 & 9 & 0.98 \\
\hline 26 & $\mathrm{Ph}$ & 4-Me Ph & $\mathrm{H}$ & Thiourea-1 & 213 & 33 & 66.5 & 33.5 & 0.29 \\
\hline 27 & $\mathrm{Ph}$ & 3-Me Ph & $\mathrm{H}$ & Thiourea-1 & 213 & 88 & 94 & 6 & 1.16 \\
\hline 28 & $\mathrm{Ph}$ & $4-\mathrm{NO}_{2} \mathrm{Ph}$ & $\mathrm{H}$ & Thiourea-1 & 213 & 92 & 96 & 4 & 1.35 \\
\hline
\end{tabular}




\section{Michael/Aza-Henry}

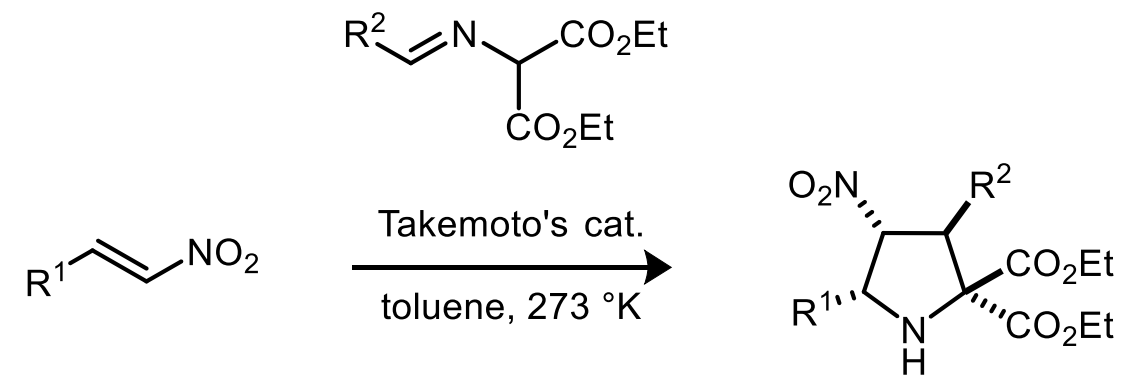

\begin{tabular}{|c|c|c|c|c|c|c|c|c|}
\hline entry & $\mathbf{R}^{1}$ & $\mathbf{R}^{2}$ & Catalyst & Temp. (K) & ee & \multicolumn{2}{|c|}{ er } & $\Delta \Delta \mathrm{G}^{\ddagger}(\mathrm{kcal} / \mathrm{mol})$ \\
\hline 1 & $\mathrm{Ph}$ & $4-\mathrm{CF}_{3} \mathrm{Ph}$ & Thiourea-1 & 273 & 92 & 96 & 4 & 1.72 \\
\hline 2 & 4-OMe Ph & $4-\mathrm{CF}_{3} \mathrm{Ph}$ & Thiourea-1 & 273 & 91 & 95.5 & 4.5 & 1.66 \\
\hline 3 & 4-Cl Ph & $4-\mathrm{CF}_{3} \mathrm{Ph}$ & Thiourea-1 & 273 & 90 & 95 & 5 & 1.60 \\
\hline 4 & 3-Cl Ph & $4-\mathrm{CF}_{3} \mathrm{Ph}$ & Thiourea-1 & 273 & 90 & 95 & 5 & 1.60 \\
\hline 5 & 2-Cl Ph & $4-\mathrm{CF}_{3} \mathrm{Ph}$ & Thiourea-1 & 273 & 92 & 96 & 4 & 1.72 \\
\hline 6 & 2-thienyl & $4-\mathrm{CF}_{3} \mathrm{Ph}$ & Thiourea-1 & 273 & 84 & 92 & 8 & 1.32 \\
\hline 7 & 1-Napthyl & $4-\mathrm{CF}_{3} \mathrm{Ph}$ & Thiourea-1 & 273 & 88 & 94 & 6 & 1.49 \\
\hline 8 & $\mathrm{Ph}$ & 4-Cl Ph & Thiourea-1 & 273 & 91 & 95.5 & 4.5 & 1.66 \\
\hline 9 & $\mathrm{Ph}$ & $\mathrm{Ph}$ & Thiourea-1 & 273 & 80 & 90 & 10 & 1.19 \\
\hline
\end{tabular}




\section{[2+2] Annulation}

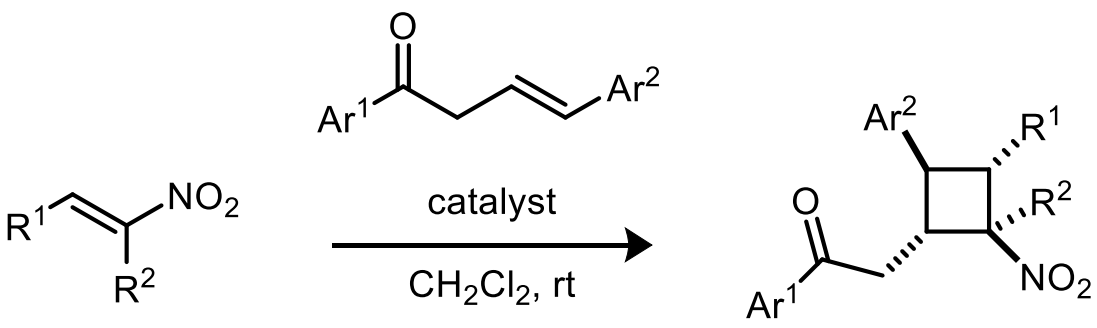

\begin{tabular}{|c|c|c|c|c|c|c|c|c|c|c|}
\hline entry & $\mathrm{Ar}^{1}$ & $A r^{2}$ & $\mathbf{R}^{1}$ & $\mathbf{R}^{2}$ & Catalyst & Temp. (K) & ee & \multicolumn{2}{|c|}{ er } & $\Delta \Delta \mathrm{G}^{\ddagger}(\mathrm{kcal} / \mathrm{mol})$ \\
\hline 1 & $\mathrm{Ph}$ & $\mathrm{Ph}$ & $\mathrm{Ph}$ & $\mathrm{Me}$ & Thiourea-1 & 296 & 87 & 93.5 & 6.5 & 1.57 \\
\hline 2 & $\mathrm{Ph}$ & $\mathrm{Ph}$ & $\mathrm{Ph}$ & $\mathrm{Me}$ & Thiourea- 6 & 296 & 91 & 95.5 & 4.5 & 1.80 \\
\hline 3 & $\mathrm{Ph}$ & $\mathrm{Ph}$ & $\mathrm{Ph}$ & $\mathrm{Me}$ & Squaramide-1 & 296 & 86 & 93 & 7 & 1.52 \\
\hline 4 & $\mathrm{Ph}$ & $\mathrm{Ph}$ & $\mathrm{Ph}$ & $\mathrm{Me}$ & Squaramide-3 & 296 & 98 & 99 & 1 & 2.70 \\
\hline 5 & $\mathrm{Ph}$ & $\mathrm{Ph}$ & $\mathrm{Ph}$ & $\mathrm{Me}$ & Squaramide-5 & 296 & 98 & 99 & 1 & 2.70 \\
\hline 6 & $\mathrm{Ph}$ & $\mathrm{Ph}$ & 4-Br Ph & $\mathrm{Me}$ & Squaramide-5 & 296 & 98 & 99 & 1 & 2.70 \\
\hline 7 & $\mathrm{Ph}$ & $\mathrm{Ph}$ & 4-F Ph & $\mathrm{Me}$ & Squaramide-5 & 296 & 98 & 99 & 1 & 2.70 \\
\hline 8 & $\mathrm{Ph}$ & $\mathrm{Ph}$ & 3-Br Ph & $\mathrm{Me}$ & Squaramide-5 & 296 & 98 & 99 & 1 & 2.70 \\
\hline 9 & $\mathrm{Ph}$ & $\mathrm{Ph}$ & 4-Cl Ph & Me & Squaramide-5 & 296 & 98 & 99 & 1 & 2.70 \\
\hline 11 & $\mathrm{Ph}$ & $\mathrm{Ph}$ & $2-\mathrm{Cl} \mathrm{Ph}$ & $\mathrm{Me}$ & Squaramide-5 & 296 & 98 & 99 & 1 & 2.70 \\
\hline 12 & $\mathrm{Ph}$ & $\mathrm{Ph}$ & $2-\mathrm{Br} \mathrm{Ph}$ & $\mathrm{Me}$ & Squaramide-5 & 296 & 96 & 98 & 2 & 2.29 \\
\hline 13 & $\mathrm{Ph}$ & $\mathrm{Ph}$ & $4-\mathrm{NO}_{2} \mathrm{Ph}$ & $\mathrm{Me}$ & Squaramide-5 & 296 & 98 & 99 & 1 & 2.70 \\
\hline 14 & 3-Br Ph & $\mathrm{Ph}$ & $\mathrm{Ph}$ & $\mathrm{Me}$ & Squaramide-5 & 296 & 93 & 96.5 & 3.5 & 1.95 \\
\hline 15 & 3-Cl Ph & $\mathrm{Ph}$ & $4-\mathrm{NO}_{2} \mathrm{Ph}$ & $\mathrm{Me}$ & Squaramide-5 & 296 & 98 & 99 & 1 & 2.70 \\
\hline 16 & $\mathrm{Ph}$ & $\mathrm{Ph}$ & $\mathrm{CH}_{2} \mathrm{CH}_{2} \mathrm{Ph}$ & $\mathrm{Me}$ & Squaramide-5 & 296 & 98 & 99 & 1 & 2.70 \\
\hline 17 & $\mathrm{Ph}$ & $\mathrm{Ph}$ & $n-\mathrm{Bu}$ & $\mathrm{Me}$ & Squaramide-5 & 296 & 99 & 99.5 & 0.5 & 3.11 \\
\hline 18 & $\mathrm{Ph}$ & 4-OMe Ph & $\mathrm{Ph}$ & $\mathrm{Me}$ & Squaramide-5 & 296 & 98 & 99 & 1 & 2.70 \\
\hline 19 & $\mathrm{Ph}$ & 4-F Ph & $\mathrm{Ph}$ & $\mathrm{Me}$ & Squaramide-5 & 296 & 98 & 99 & 1 & 2.70 \\
\hline
\end{tabular}




\section{Model Development}

Measured $\Delta \Delta \mathrm{G}^{\ddagger}$ values were calculated using the formula $\Delta \Delta \mathrm{G}^{\ddagger}=-\mathrm{RT} \ln \mid$ er $\mid$ where $\mathrm{R}$ is the gas constant, $\mathrm{T}$ is temperature, and er is the enantiomeric ratio. Absolute values of enantiomeric ratio were used for modeling. Linear regression models were developed using an in-house script implemented in MATLAB ${ }^{R}$ (versionR2019b), to obtain the predicted $\Delta \Delta \mathrm{G}^{\ddagger}$. Forward stepwise linear regression was employed for model development with previously reported algorithms. ${ }^{19}$ Parameters that measure similar features i.e. collinear parameters were automatically removed, defined by 0.3 collinear criteria. As the model search process can produce a large pool of model candidates, we truncated the models on the recorded statistics as well as the number of included parameters and presence of cross-terms. The predictions provided by the models would then be fitted to the measured results to visualize and further evaluate the model performances. Normalized parameters were employed so that the coefficients would reflect the importance of each descriptor. A good linear correlation $\left(\mathrm{R}^{2}\right.$ close to 1.0 and intercept close to 0.0$)$ between the predicted $\Delta \Delta \mathrm{G}^{\ddagger}$ and the measured $\Delta \Delta \mathrm{G}^{\ddagger}$ indicates that the obtained model adequately approximates the system under study.

Models were validated using external and cross-validation techniques. Cross-validation generally refers to the statistical method of excluding part of the dataset as validations, predicting their values using the remaining set of data. Such analysis is generally employed to test the stability of a model and overall robustness. Additionally, these methods are used to identify over-fitting of data. Leave-one-out (LOO) cross-validation is where only one data point would be removed each time and predicted by the remaining set. All validation predictions would then be combined and fitted to the measured values, the $\mathrm{R}^{2}$ value of the resulting fit is called $\mathrm{Q}^{2}$. Another method to test model robustness is k-fold cross-validation. In this validation method, the dataset is divided randomly into $\mathrm{k}$ subsets with same/similar sizes, each set would then be predicted out by the other $\mathrm{k}^{-1}$ subsets, and the goodness of fit would be tested based on these predictions. However, it is important to note that the k-fold statistics is dependent on the partitioning of the dataset, and that there's a necessity of executing the process multiple times for average results. The 150 reactions were split into 50:50 TS:VS sets for external validation. The split was partitioned based on the response values using the MATLAB "equidistant" function. This process can be described as pseudorandom as it is generated using a deterministic algorithm. 
Nitroalkene Model (50:50 TS:VS)

Training Set

\begin{tabular}{|c|c|c|}
\hline Predicted $\Delta \Delta \mathbf{G}^{\ddagger}$ & Measured $\Delta \Delta \mathrm{G}^{\ddagger}$ & LOO \\
\hline 0.69 & 0.56 & 0.70 \\
\hline 0.51 & 0.51 & 0.51 \\
\hline 1.24 & 1.40 & 1.23 \\
\hline 1.24 & 0.95 & 1.27 \\
\hline 1.04 & 0.89 & 1.09 \\
\hline 1.24 & 1.26 & 1.24 \\
\hline 1.51 & 1.80 & 1.47 \\
\hline 1.24 & 1.07 & 1.26 \\
\hline 1.40 & 1.36 & 1.41 \\
\hline 1.43 & 1.40 & 1.43 \\
\hline 1.98 & 1.33 & 2.05 \\
\hline 1.97 & 1.62 & 2.00 \\
\hline 1.99 & 2.15 & 1.97 \\
\hline 2.23 & 2.29 & 2.23 \\
\hline 2.10 & 1.95 & 2.12 \\
\hline 2.10 & 2.04 & 2.11 \\
\hline 2.23 & 2.87 & 2.18 \\
\hline 2.54 & 2.27 & 2.59 \\
\hline 2.22 & 2.27 & 2.21 \\
\hline 2.39 & 2.87 & 2.34 \\
\hline 2.00 & 1.99 & 2.00 \\
\hline 2.19 & 2.27 & 2.18 \\
\hline 2.44 & 2.49 & 2.44 \\
\hline 2.14 & 2.11 & 2.15 \\
\hline 2.35 & 2.27 & 2.36 \\
\hline 0.33 & 0.06 & 0.38 \\
\hline 0.54 & 0.17 & 0.58 \\
\hline 0.54 & 0.71 & 0.53 \\
\hline 0.53 & 0.76 & 0.51 \\
\hline 0.18 & 0.31 & 0.01 \\
\hline 0.54 & 0.57 & 0.54 \\
\hline 0.92 & 1.08 & 0.91 \\
\hline 1.00 & 1.20 & 0.98 \\
\hline 1.30 & 0.94 & 1.32 \\
\hline 1.54 & 0.98 & 1.61 \\
\hline 1.02 & 1.14 & 1.01 \\
\hline
\end{tabular}




\begin{tabular}{|c|c|c|}
\hline 0.98 & 1.11 & 0.97 \\
\hline 0.99 & 0.96 & 0.99 \\
\hline 1.22 & 1.03 & 1.23 \\
\hline 1.06 & 1.40 & 1.03 \\
\hline 1.34 & 1.51 & 1.31 \\
\hline 1.05 & 1.36 & 1.02 \\
\hline 2.01 & 1.83 & 2.04 \\
\hline 1.98 & 2.16 & 1.95 \\
\hline 1.60 & 0.94 & 1.81 \\
\hline 1.80 & 1.98 & 1.77 \\
\hline 1.88 & 2.16 & 1.84 \\
\hline 2.03 & 2.42 & 1.96 \\
\hline 0.44 & 0.64 & 0.39 \\
\hline 1.11 & 1.14 & 1.10 \\
\hline 1.19 & 0.97 & 1.25 \\
\hline 1.21 & 1.18 & 1.22 \\
\hline 1.26 & 1.29 & 1.26 \\
\hline 0.72 & 0.81 & 0.67 \\
\hline 1.17 & 1.02 & 1.18 \\
\hline 1.00 & 0.93 & 1.00 \\
\hline 1.22 & 1.22 & 1.22 \\
\hline 1.02 & 0.84 & 1.03 \\
\hline 1.11 & 1.04 & 1.12 \\
\hline 0.59 & 0.42 & 0.62 \\
\hline 1.02 & 1.08 & 1.02 \\
\hline 1.40 & 1.48 & 1.39 \\
\hline 0.97 & 1.24 & 0.96 \\
\hline 1.19 & 0.74 & 1.22 \\
\hline 1.09 & 1.05 & 1.10 \\
\hline 1.23 & 1.43 & 1.19 \\
\hline 1.23 & 1.34 & 1.21 \\
\hline 1.23 & 1.84 & 1.13 \\
\hline 1.88 & 2.66 & 1.78 \\
\hline 1.88 & 1.60 & 1.91 \\
\hline 1.87 & 1.96 & 1.86 \\
\hline 1.84 & 1.75 & 1.86 \\
\hline 2.01 & 1.54 & 2.09 \\
\hline 1.90 & 1.60 & 1.94 \\
\hline 1.65 & 1.67 & 1.65 \\
\hline
\end{tabular}


Validation Set

\begin{tabular}{|c|c|}
\hline Predicted $\Delta \Delta \mathbf{G}^{\ddagger}$ & Measured $\Delta \Delta \mathbf{G}^{\ddagger}$ \\
\hline 1.10 & 0.73 \\
\hline 1.24 & 1.22 \\
\hline 1.24 & 1.10 \\
\hline 1.22 & 1.20 \\
\hline 1.50 & 1.40 \\
\hline 1.32 & 1.36 \\
\hline 1.32 & 1.14 \\
\hline 1.35 & 1.57 \\
\hline 1.60 & 0.74 \\
\hline 1.40 & 1.62 \\
\hline 2.23 & 2.27 \\
\hline 2.23 & 2.40 \\
\hline 2.23 & 2.42 \\
\hline 2.23 & 2.04 \\
\hline 2.10 & 1.95 \\
\hline 2.04 & 1.73 \\
\hline 2.60 & 2.87 \\
\hline 2.14 & 2.27 \\
\hline 2.41 & 2.27 \\
\hline 2.07 & 2.11 \\
\hline 2.49 & 2.11 \\
\hline 2.31 & 2.27 \\
\hline 2.38 & 2.49 \\
\hline 2.40 & 2.27 \\
\hline 0.36 & 0.15 \\
\hline 0.54 & 0.68 \\
\hline 0.53 & 0.84 \\
\hline 0.92 & 1.06 \\
\hline 0.54 & 0.33 \\
\hline 0.41 & 0.46 \\
\hline 0.99 & 0.98 \\
\hline 1.00 & 0.89 \\
\hline 1.27 & 0.94 \\
\hline 1.55 & 0.96 \\
\hline 1.26 & 0.94 \\
\hline 1.08 & 1.03 \\
\hline 1.14 & 0.98 \\
\hline 0.87 & 1.14 \\
\hline
\end{tabular}




\begin{tabular}{|c|c|}
\hline 1.11 & 1.03 \\
\hline 0.68 & 0.78 \\
\hline 1.34 & 1.27 \\
\hline 1.09 & 1.40 \\
\hline 0.50 & 0.05 \\
\hline 2.22 & 1.98 \\
\hline 1.97 & 1.83 \\
\hline 1.98 & 2.16 \\
\hline 1.89 & 1.83 \\
\hline 1.95 & 2.16 \\
\hline 1.74 & 1.98 \\
\hline 0.39 & 0.26 \\
\hline 1.29 & 1.51 \\
\hline 1.18 & 1.35 \\
\hline 0.87 & 0.85 \\
\hline 1.06 & 0.95 \\
\hline 1.36 & 1.25 \\
\hline 1.21 & 1.07 \\
\hline 0.57 & 0.57 \\
\hline 1.24 & 0.96 \\
\hline 1.08 & 1.13 \\
\hline 0.85 & 1.05 \\
\hline 0.67 & 1.21 \\
\hline 0.83 & 1.08 \\
\hline 0.95 & 1.34 \\
\hline 0.41 & 0.59 \\
\hline 1.19 & 1.40 \\
\hline 1.14 & 1.30 \\
\hline 0.59 & 0.54 \\
\hline 1.23 & 1.67 \\
\hline 1.82 & 1.60 \\
\hline 1.68 & 1.38 \\
\hline 1.97 & 1.84 \\
\hline 2.00 & 1.48 \\
\hline 2.03 & 1.43 \\
\hline 1.75 & 2.66 \\
\hline 1.91 & 1.75 \\
\hline
\end{tabular}




\section{Leave one reaction out (LORO) analysis}

In this separate validation test, the catalyst, nitroalkene and nucleophile structures are varied by removing each distinct reaction (as determined by individual publications) and using the left-out data as a validation set. The analysis was evaluated by external validations' goodness of fit, pred $R^{2}$. List of predicted $R^{2}$ when each individual reaction was left out:

(1) Martin, N. J. A.; Ozores, L.; List, B. J. Am. Chem. Soc. 2007, 129, 8976-8977. predR ${ }^{2}=$ 0.74

(2) Blom, J.; Vidal-Alabat, A.; Jørgensen, J.; Barløse, C. L.; Jessen, K. S.; Iversen, M. V.; Jørgensen, K. A. Angew. Chem. Int. Ed. 2017, 56, 11831-11835. predR ${ }^{2}=0.87$

(3) Zhu, Y.; Malerich, J. P.; Rawal, V. H. Angew. Chem. Int. Ed. 2010, 49, 153-156. predR ${ }^{2}=$ 0.84

(4) He, X.; Yang, L.; Ji, Y.; Zhao, Q.; Yang, M.; Huang, W.; Peng, C.; Han, B. Chem. Eur. J. 2018, 24, 1947-1955. predR $^{2}=0.64$

(5) Kimmel, K. L.; Robak, M. T.; Ellman, J. A. J. Am. Chem. Soc. 2009, 131, 8754-8755. $\operatorname{predR}^{2}=0.91$

(6) Bui, T.; Syed, S.; Barbas III, C. F. J. Am. Chem. Soc. 2009, 131, 8758-8759. predR ${ }^{2}=$ 0.24

(7) Yang, C.; Wang, J.; Liu, Y.; Ni, X.; Li, X.; Cheng, J. Chem. Eur. J. 2017, 23, 5488-5497. $\operatorname{predR}^{2}=0.80$ 


\section{Cinchona Alkaloid Validation}

To further test the model's recognition of varied catalyst types, all data points consisting of cinchona alkaloid-based catalysts were excluded from the training set and used as the validation set (28 reactions).

\section{Training Set}

\begin{tabular}{|c|c|}
\hline Predicted $\Delta \Delta \mathbf{G}^{\ddagger}$ & Measured $\Delta \Delta \mathbf{G}^{\ddagger}$ \\
\hline 0.72 & 0.56 \\
\hline 2.02 & 1.33 \\
\hline 2.00 & 1.62 \\
\hline 2.03 & 2.15 \\
\hline 2.24 & 2.29 \\
\hline 2.24 & 2.27 \\
\hline 2.24 & 2.40 \\
\hline 2.24 & 2.42 \\
\hline 2.24 & 2.04 \\
\hline 2.07 & 1.95 \\
\hline 2.07 & 2.04 \\
\hline 2.07 & 1.95 \\
\hline 2.00 & 1.73 \\
\hline 2.24 & 2.87 \\
\hline 2.47 & 2.87 \\
\hline 2.43 & 2.27 \\
\hline 2.18 & 2.27 \\
\hline 2.23 & 2.27 \\
\hline 2.34 & 2.87 \\
\hline 2.35 & 2.27 \\
\hline 2.09 & 1.99 \\
\hline 2.21 & 2.27 \\
\hline 2.37 & 2.49 \\
\hline 2.14 & 2.11 \\
\hline 2.19 & 2.11 \\
\hline 2.40 & 2.11 \\
\hline 2.29 & 2.27 \\
\hline 2.33 & 2.49 \\
\hline 2.32 & 2.27 \\
\hline
\end{tabular}




\begin{tabular}{|c|c|}
\hline 2.34 & 2.27 \\
\hline 0.57 & 0.17 \\
\hline 0.57 & 0.68 \\
\hline 0.57 & 0.71 \\
\hline 0.56 & 0.76 \\
\hline 0.56 & 0.84 \\
\hline 0.90 & 1.06 \\
\hline 0.12 & 0.31 \\
\hline 0.57 & 0.57 \\
\hline 0.57 & 0.33 \\
\hline 0.40 & 0.46 \\
\hline 0.90 & 1.08 \\
\hline 0.97 & 0.98 \\
\hline 0.97 & 0.89 \\
\hline 0.97 & 1.20 \\
\hline 1.23 & 0.94 \\
\hline 1.26 & 0.94 \\
\hline 1.50 & 0.96 \\
\hline 1.49 & 0.98 \\
\hline 1.22 & 0.94 \\
\hline 1.00 & 1.03 \\
\hline 0.97 & 1.14 \\
\hline 0.94 & 1.11 \\
\hline 0.94 & 0.96 \\
\hline 1.04 & 0.98 \\
\hline 0.87 & 1.14 \\
\hline 1.21 & 1.03 \\
\hline 1.13 & 1.03 \\
\hline 0.75 & 0.78 \\
\hline 1.10 & 1.40 \\
\hline 1.36 & 1.51 \\
\hline 1.09 & 1.36 \\
\hline 1.37 & 1.27 \\
\hline 1.13 & 1.40 \\
\hline 0.52 & 0.05 \\
\hline 2.12 & 1.98 \\
\hline 1.97 & 1.83 \\
\hline 1.98 & 2.16 \\
\hline 1.99 & 1.83 \\
\hline 1.92 & 1.83 \\
\hline 1.98 & 2.16 \\
\hline
\end{tabular}




\begin{tabular}{|c|c|}
\hline 1.74 & 0.94 \\
\hline 1.86 & 1.98 \\
\hline 1.96 & 2.16 \\
\hline 1.92 & 2.16 \\
\hline 2.01 & 2.42 \\
\hline 1.83 & 1.98 \\
\hline 0.40 & 0.26 \\
\hline 0.45 & 0.64 \\
\hline 1.12 & 1.14 \\
\hline 1.23 & 1.51 \\
\hline 1.17 & 0.97 \\
\hline 1.18 & 1.18 \\
\hline 1.22 & 1.29 \\
\hline 1.17 & 1.35 \\
\hline 0.88 & 0.81 \\
\hline 0.97 & 0.85 \\
\hline 1.09 & 0.95 \\
\hline 1.30 & 1.25 \\
\hline 1.19 & 1.07 \\
\hline 1.16 & 1.02 \\
\hline 1.00 & 0.93 \\
\hline 1.19 & 1.22 \\
\hline 1.03 & 0.84 \\
\hline 1.09 & 1.04 \\
\hline 0.63 & 0.57 \\
\hline 0.66 & 0.42 \\
\hline 1.24 & 0.96 \\
\hline 1.35 & 1.48 \\
\hline 1.15 & 1.40 \\
\hline 0.96 & 1.24 \\
\hline 1.11 & 1.30 \\
\hline 1.16 & 0.74 \\
\hline 0.63 & 0.54 \\
\hline 1.05 & 1.05 \\
\hline 1.23 & 1.43 \\
\hline 1.23 & 1.34 \\
\hline 1.23 & 1.67 \\
\hline 1.23 & 1.84 \\
\hline 1.80 & 2.66 \\
\hline 1.80 & 1.60 \\
\hline 1.80 & 1.96 \\
\hline
\end{tabular}




\begin{tabular}{|ll|}
\hline 1.77 & 1.75 \\
1.75 & 1.60 \\
1.62 & 1.38 \\
1.88 & 1.54 \\
1.85 & 1.84 \\
1.87 & 1.48 \\
1.81 & 1.60 \\
1.89 & 1.43 \\
1.71 & 2.66 \\
1.81 & 1.75 \\
1.65 & 1.67 \\
\hline
\end{tabular}


Validation Set

\begin{tabular}{|c|c|}
\hline Predicted $\Delta \Delta \mathbf{G}^{\ddagger}$ & Measured $\Delta \Delta \mathbf{G}^{\ddagger}$ \\
\hline 0.53 & 0.51 \\
\hline 1.29 & 1.40 \\
\hline 1.29 & 0.95 \\
\hline 1.12 & 0.73 \\
\hline 1.05 & 0.89 \\
\hline 1.29 & 1.26 \\
\hline 1.29 & 1.22 \\
\hline 1.29 & 1.10 \\
\hline 1.28 & 1.20 \\
\hline 1.46 & 1.40 \\
\hline 1.47 & 1.80 \\
\hline 1.29 & 1.07 \\
\hline 1.34 & 1.36 \\
\hline 1.40 & 1.36 \\
\hline 1.41 & 1.40 \\
\hline 1.34 & 1.14 \\
\hline 1.37 & 1.57 \\
\hline 1.52 & 0.74 \\
\hline 1.39 & 1.62 \\
\hline 0.35 & 0.06 \\
\hline 0.38 & 0.15 \\
\hline 1.04 & 1.13 \\
\hline 0.99 & 1.08 \\
\hline 0.85 & 1.05 \\
\hline 0.65 & 1.21 \\
\hline 0.82 & 1.08 \\
\hline 0.92 & 1.34 \\
\hline 0.46 & 0.59 \\
\hline
\end{tabular}




\section{Out of Sample Predictions}

The out of sample test cases were treated as validations sets to obtain predicted $\Delta \Delta \mathrm{G}^{\ddagger}$ values. The model's ability to predict was evaluated by the average absolute $\Delta \Delta \mathrm{G}^{\ddagger}$ error $\left(\Delta \Delta \mathrm{G}^{\ddagger}\right.$ error $=$ absolute(model predicted - experimentally measured/ no. of examples) and associated standard deviation.

\section{Transfer Hydrogenation of Trifluoromethyl Nitroalkenes}

\begin{tabular}{|cc|}
\hline Predicted $\Delta \mathbf{\Delta} \mathbf{G}^{\ddagger}$ & Measured $\Delta \Delta \mathbf{G}^{\ddagger}$ \\
\hline 1.21 & 1.27 \\
1.22 & 1.05 \\
1.37 & 1.08 \\
1.40 & 1.77 \\
1.40 & 1.75 \\
1.40 & 1.84 \\
1.46 & 1.75 \\
1.45 & 2.10 \\
1.44 & 1.96 \\
1.40 & 1.67 \\
1.49 & 1.96 \\
1.49 & 1.84 \\
1.49 & 2.31 \\
1.49 & 1.75 \\
1.45 & 1.75 \\
1.59 & 2.10 \\
1.59 & 1.84 \\
1.40 & 1.84 \\
1.90 & 1.67 \\
1.43 & 1.48 \\
1.43 & 1.26 \\
1.56 & 1.54 \\
\hline
\end{tabular}


Addition of 3-substituted Benzofuran-2(3H)-ones to Nitroolefins

\begin{tabular}{|c|c|}
\hline Predicted $\Delta \Delta \mathrm{G}^{\ddagger}$ & Measured $\Delta \Delta \mathbf{G}^{\ddagger}$ \\
\hline 0.54 & 0.75 \\
\hline 0.54 & 0.73 \\
\hline 0.54 & 0.67 \\
\hline 0.64 & 0.66 \\
\hline 0.55 & 0.64 \\
\hline 0.85 & 0.56 \\
\hline 0.61 & 0.49 \\
\hline 0.60 & 0.82 \\
\hline 0.59 & 1.09 \\
\hline 0.61 & 0.93 \\
\hline 0.59 & 0.98 \\
\hline 0.60 & 1.29 \\
\hline 0.76 & 0.86 \\
\hline 0.58 & 1.06 \\
\hline 0.45 & 0.98 \\
\hline 0.59 & 0.52 \\
\hline 0.83 & 0.56 \\
\hline 0.74 & 0.79 \\
\hline 0.78 & 0.86 \\
\hline 0.83 & 0.50 \\
\hline
\end{tabular}


Alkylation of Indoles to Nitroalkenes

\begin{tabular}{|cc|}
\hline Predicted $\Delta \mathbf{\Delta} \mathbf{G}^{\ddagger}$ & Measured $\boldsymbol{\Delta} \boldsymbol{\Delta} \mathbf{G}^{\ddagger}$ \\
\hline 1.10 & 0.61 \\
1.10 & 1.24 \\
1.10 & 0.86 \\
1.13 & 1.29 \\
1.18 & 0.88 \\
1.05 & 0.92 \\
1.09 & 0.92 \\
1.05 & 1.18 \\
0.96 & 1.12 \\
\hline
\end{tabular}


Asymmetric Mannich Synthesis of $\alpha$-Amino Esters by Anion-Binding Catalysis

\begin{tabular}{|c|c|}
\hline Predicted $\Delta \Delta \mathbf{G}^{\ddagger}$ & Measured $\Delta \Delta \mathbf{G}^{\ddagger}$ \\
\hline 0.96 & 0.44 \\
\hline 1.03 & 1.60 \\
\hline 0.79 & 1.12 \\
\hline 0.99 & 0.46 \\
\hline 1.32 & 0.77 \\
\hline 0.78 & 0.25 \\
\hline 0.97 & 0.64 \\
\hline 0.78 & 0.97 \\
\hline 1.03 & 0.56 \\
\hline 1.03 & 1.49 \\
\hline 1.03 & 1.14 \\
\hline 0.91 & 0.99 \\
\hline 1.09 & 1.42 \\
\hline 0.73 & 0.64 \\
\hline 1.23 & 1.77 \\
\hline 0.86 & 0.54 \\
\hline 0.92 & 1.37 \\
\hline 1.01 & 1.48 \\
\hline 1.30 & 1.88 \\
\hline 1.10 & 0.90 \\
\hline 0.95 & 1.37 \\
\hline 1.31 & 2.02 \\
\hline 1.68 & 1.77 \\
\hline 1.27 & 1.60 \\
\hline 1.04 & 1.42 \\
\hline 0.98 & 1.33 \\
\hline 1.07 & 1.53 \\
\hline 1.37 & 1.68 \\
\hline 0.70 & 1.25 \\
\hline
\end{tabular}




\section{Aza-Henry}

\begin{tabular}{|cc|}
\hline Predicted $\boldsymbol{\Delta} \boldsymbol{\Delta} \mathbf{G}^{\ddagger}$ & Measured $\boldsymbol{\Delta} \boldsymbol{\Delta} \mathbf{G}^{\ddagger}$ \\
\hline 0.57 & 0.05 \\
0.76 & 0.95 \\
0.69 & 1.17 \\
0.98 & 1.02 \\
0.73 & 0.98 \\
0.74 & 0.89 \\
0.82 & 0.95 \\
0.82 & 0.87 \\
0.93 & 0.91 \\
0.86 & 0.95 \\
\hline
\end{tabular}




\section{Addition of 2-(Cyanomethyl)azaarenes to $\mathrm{N}$-Boc-amine Sulfones}

\begin{tabular}{|cc|}
\hline Predicted $\Delta \Delta \mathbf{G}^{\ddagger}$ & Measured $\Delta \Delta \mathbf{G}^{\ddagger}$ \\
\hline 1.61 & 0.47 \\
1.45 & 0.98 \\
1.49 & 2.04 \\
1.49 & 1.29 \\
1.42 & 1.29 \\
1.56 & 1.48 \\
1.69 & 1.23 \\
1.62 & 1.95 \\
1.49 & 2.04 \\
1.62 & 1.62 \\
1.58 & 1.23 \\
1.55 & 1.36 \\
1.57 & 1.87 \\
1.55 & 1.29 \\
1.67 & 1.40 \\
1.47 & 1.20 \\
1.47 & 1.52 \\
1.48 & 1.67 \\
1.49 & 1.52 \\
1.49 & 1.95 \\
1.49 & 1.67 \\
1.50 & 1.87 \\
1.49 & 1.87 \\
1.49 & 1.73 \\
1.44 & 1.87 \\
1.26 & \\
& \\
\hline
\end{tabular}


Mannich/Aza-Michael

\begin{tabular}{|cc|}
\hline Predicted $\Delta \mathbf{\Delta} \mathbf{G}^{\ddagger}$ & Measured $\Delta \boldsymbol{\Delta} \mathbf{G}^{\ddagger}$ \\
\hline 1.41 & 1.44 \\
1.41 & 1.37 \\
1.41 & 1.50 \\
1.51 & 1.02 \\
1.32 & 0.82 \\
1.21 & 1.23 \\
1.46 & 1.14 \\
1.42 & 1.40 \\
1.50 & 1.23 \\
1.60 & 1.40 \\
1.35 & 2.04 \\
1.40 & 1.40 \\
\hline
\end{tabular}




\section{Michael/Spirocyclization}

\begin{tabular}{|c|c|}
\hline Predicted $\Delta \Delta \mathbf{G}^{\ddagger}$ & Measured $\Delta \Delta \mathbf{G}^{\ddagger}$ \\
\hline 1.14 & 0.00 \\
\hline 1.11 & 0.30 \\
\hline 1.32 & 0.59 \\
\hline 1.32 & 1.01 \\
\hline 1.32 & 1.15 \\
\hline 1.32 & 1.18 \\
\hline 1.32 & 1.29 \\
\hline 1.32 & 1.19 \\
\hline 1.35 & 0.93 \\
\hline 1.30 & 0.93 \\
\hline 1.32 & 1.40 \\
\hline 1.30 & 1.29 \\
\hline 1.30 & 1.16 \\
\hline 1.35 & 1.35 \\
\hline 1.26 & 1.40 \\
\hline 1.47 & 1.47 \\
\hline 1.20 & 0.91 \\
\hline 1.32 & 1.35 \\
\hline 1.52 & 1.25 \\
\hline 1.57 & 1.03 \\
\hline 1.35 & 1.35 \\
\hline 1.08 & 1.25 \\
\hline 1.34 & 1.16 \\
\hline 1.32 & 1.35 \\
\hline 1.73 & 0.98 \\
\hline 1.05 & 0.29 \\
\hline 1.31 & 1.16 \\
\hline 1.33 & 1.35 \\
\hline
\end{tabular}


Michael/Aza-Henry

\begin{tabular}{|cc|}
\hline Predicted $\boldsymbol{\Delta} \mathbf{\Delta} \mathbf{G}^{\mathbf{}}$ & Measured $\boldsymbol{\Delta} \mathbf{\Delta} \mathbf{G}^{\mathbf{}}$ \\
\hline 1.41 & 1.72 \\
1.51 & 1.66 \\
1.48 & 1.60 \\
1.46 & 1.60 \\
1.46 & 1.72 \\
1.40 & 1.32 \\
1.60 & 1.49 \\
1.33 & 1.66 \\
1.09 & 1.19 \\
1.29 & 0.66 \\
\hline
\end{tabular}


$[2+2]$ Annulation

\begin{tabular}{|cc|}
\hline Predicted $\Delta \mathbf{\Delta} \mathbf{G}^{\ddagger}$ & Measured $\boldsymbol{\Delta} \mathbf{\Delta \mathbf { G } ^ { \ddagger }}$ \\
\hline 1.15 & 1.57 \\
0.98 & 1.80 \\
1.03 & 1.52 \\
1.04 & 2.70 \\
1.67 & 2.70 \\
1.77 & 2.70 \\
1.67 & 2.70 \\
1.75 & 2.70 \\
1.73 & 2.70 \\
1.76 & 2.70 \\
1.71 & 2.70 \\
1.74 & 2.29 \\
1.74 & 2.70 \\
1.67 & 1.95 \\
1.74 & 2.70 \\
1.71 & 2.70 \\
1.58 & 3.11 \\
2.07 & 2.70 \\
1.78 & 2.70 \\
1.72 & 2.70 \\
\hline
\end{tabular}


Nitrophosphonate Addition to Enones

\begin{tabular}{|c|c|c|}
\hline Predicted $\Delta \Delta \mathbf{G}^{\ddagger}$ & Measured $\Delta \Delta \mathbf{G}^{\ddagger}$ & LOO \\
\hline 0.46 & 0.40 & 0.46 \\
\hline 0.57 & 0.38 & 0.59 \\
\hline 0.54 & 0.79 & 0.51 \\
\hline 0.61 & 0.37 & 0.63 \\
\hline 0.19 & 0.39 & 0.16 \\
\hline 0.93 & 0.86 & 0.95 \\
\hline 0.90 & 1.10 & 0.85 \\
\hline 0.70 & 0.75 & 0.69 \\
\hline 0.53 & 0.30 & 0.59 \\
\hline 0.86 & 0.72 & 0.88 \\
\hline 0.48 & 0.70 & 0.44 \\
\hline 1.12 & 1.31 & 1.11 \\
\hline 1.23 & 1.37 & 1.23 \\
\hline 1.21 & 1.73 & 1.18 \\
\hline 1.27 & 1.04 & 1.29 \\
\hline 0.86 & 0.47 & 0.91 \\
\hline 1.60 & 1.44 & 1.62 \\
\hline 1.37 & 1.37 & 1.37 \\
\hline 1.19 & 1.31 & 1.17 \\
\hline 1.53 & 1.61 & 1.52 \\
\hline 1.32 & 1.22 & 1.33 \\
\hline 0.11 & 0.12 & 0.06 \\
\hline 1.14 & 0.79 & 1.16 \\
\hline 1.66 & 1.61 & 1.66 \\
\hline 1.43 & 1.22 & 1.44 \\
\hline 1.35 & 1.31 & 1.35 \\
\hline 1.31 & 1.14 & 1.33 \\
\hline 1.32 & 1.51 & 1.30 \\
\hline 1.43 & 1.26 & 1.44 \\
\hline 1.71 & 1.90 & 1.54 \\
\hline 1.38 & 1.47 & 1.35 \\
\hline 1.38 & 1.31 & 1.39 \\
\hline 1.38 & 1.61 & 1.33 \\
\hline 1.06 & 0.79 & 1.13 \\
\hline 1.08 & 1.51 & 0.99 \\
\hline
\end{tabular}


Mechanistic Plots

LUMO vs. p $K_{\mathbf{a}}$

\begin{tabular}{|ccc|}
\hline catalyst & pKa & LUMO \\
\hline Thiourea-1 & 13.65 & -0.02450 \\
Thiourea-6 & 13.20 & -0.03130 \\
Squaramide-2 & 13.30 & -0.03659 \\
Squaramide-5 & 10.54 & -0.04606 \\
Thiourea-9 & 19.60 & 0.02684 \\
Thiourea-16 & 20.50 & 0.03646 \\
Thiourea-17 & 17.00 & 0.00357 \\
Thiourea-26 & 15.80 & -0.02373 \\
Squaramide-1 & 11.83 & -0.03690 \\
Thiourea-12 & 20.50 & 0.03589 \\
Thiourea-13 & 21.00 & 0.03418 \\
Thiourea-14 & 21.60 & 0.03527 \\
Thiourea-15 & 20.20 & 0.01488 \\
Thiourea-18 & 21.40 & 0.03422 \\
Thiourea-19 & 21.20 & 0.03274 \\
\hline
\end{tabular}




\section{LUMO Linear Regression Data}

\begin{tabular}{|c|c|c|}
\hline Predicted LUMO & Computed LUMO & LOO \\
\hline-0.004 & -0.025 & -0.003 \\
\hline-0.009 & -0.024 & -0.009 \\
\hline-0.012 & -0.024 & -0.011 \\
\hline-0.009 & -0.025 & -0.009 \\
\hline-0.021 & -0.022 & -0.020 \\
\hline-0.030 & -0.037 & -0.030 \\
\hline-0.032 & -0.036 & -0.032 \\
\hline-0.023 & -0.028 & -0.023 \\
\hline-0.034 & -0.046 & -0.028 \\
\hline-0.046 & -0.031 & -0.048 \\
\hline-0.060 & -0.045 & -0.062 \\
\hline-0.041 & -0.035 & -0.042 \\
\hline-0.042 & -0.034 & -0.043 \\
\hline 0.023 & 0.027 & 0.020 \\
\hline-0.009 & -0.008 & -0.009 \\
\hline-0.033 & -0.020 & -0.034 \\
\hline 0.038 & 0.030 & 0.039 \\
\hline 0.035 & 0.036 & 0.035 \\
\hline 0.030 & 0.034 & 0.030 \\
\hline 0.026 & 0.035 & 0.025 \\
\hline 0.016 & 0.015 & 0.016 \\
\hline 0.017 & 0.036 & 0.016 \\
\hline 0.016 & 0.004 & 0.017 \\
\hline 0.023 & 0.034 & 0.023 \\
\hline 0.010 & 0.033 & 0.009 \\
\hline 0.004 & 0.006 & 0.004 \\
\hline-0.007 & -0.021 & -0.006 \\
\hline-0.012 & -0.021 & -0.012 \\
\hline-0.017 & -0.021 & -0.016 \\
\hline-0.024 & -0.025 & -0.024 \\
\hline-0.022 & -0.019 & -0.023 \\
\hline-0.027 & -0.024 & -0.027 \\
\hline-0.019 & -0.021 & -0.019 \\
\hline 0.010 & 0.010 & 0.010 \\
\hline 0.005 & 0.008 & 0.005 \\
\hline 0.000 & 0.008 & 0.000 \\
\hline-0.009 & 0.000 & -0.010 \\
\hline
\end{tabular}




\begin{tabular}{|lll|}
\hline-0.006 & -0.014 & -0.005 \\
-0.002 & 0.007 & -0.002 \\
-0.024 & -0.037 & -0.023 \\
\hline
\end{tabular}




\section{Polarizability Linear Regression Data}

\begin{tabular}{|c|c|c|}
\hline Predicted Pol. & Computed Pol. & LOO \\
\hline 122.91 & 117.43 & 123.07 \\
\hline 123.05 & 114.40 & 123.42 \\
\hline 151.33 & 160.54 & 150.49 \\
\hline 174.19 & 162.47 & 175.66 \\
\hline 124.21 & 130.77 & 124.03 \\
\hline 141.37 & 148.11 & 141.16 \\
\hline 140.86 & 136.21 & 141.44 \\
\hline 165.21 & 177.05 & 164.42 \\
\hline 122.67 & 117.28 & 122.78 \\
\hline 151.81 & 167.78 & 148.14 \\
\hline 122.54 & 101.31 & 123.74 \\
\hline 140.39 & 146.94 & 140.19 \\
\hline 73.47 & 78.27 & 72.92 \\
\hline 100.16 & 109.77 & 99.48 \\
\hline 158.91 & 151.44 & 160.32 \\
\hline 91.80 & 91.13 & 91.87 \\
\hline 115.09 & 102.68 & 116.34 \\
\hline 149.38 & 159.27 & 148.96 \\
\hline 115.43 & 129.77 & 114.51 \\
\hline 151.65 & 141.60 & 152.27 \\
\hline 128.85 & 136.65 & 128.48 \\
\hline 141.47 & 144.15 & 141.30 \\
\hline 139.65 & 134.41 & 139.89 \\
\hline 126.85 & 127.15 & 126.84 \\
\hline 133.31 & 128.16 & 133.50 \\
\hline 144.86 & 153.45 & 144.33 \\
\hline 103.04 & 90.27 & 104.07 \\
\hline 76.55 & 78.34 & 76.28 \\
\hline 91.37 & 83.46 & 92.48 \\
\hline 122.88 & 125.20 & 122.81 \\
\hline 166.95 & 180.21 & 166.00 \\
\hline 126.15 & 139.22 & 125.72 \\
\hline 137.70 & 141.72 & 137.46 \\
\hline 134.43 & 145.84 & 133.06 \\
\hline 129.33 & 125.99 & 129.47 \\
\hline 139.62 & 141.46 & 139.52 \\
\hline 82.28 & 78.25 & 82.61 \\
\hline
\end{tabular}




\begin{tabular}{|ccc|}
\hline 124.82 & 111.34 & 125.65 \\
122.24 & 136.25 & 121.79 \\
122.70 & 148.93 & 121.83 \\
126.87 & 131.38 & 126.53 \\
158.03 & 143.17 & 159.41 \\
131.63 & 130.07 & 131.70 \\
53.71 & 54.25 & 53.58 \\
84.94 & 78.20 & 85.57 \\
126.91 & 138.78 & 126.47 \\
141.27 & 127.21 & 141.96 \\
122.54 & 101.31 & 123.74 \\
144.97 & 127.45 & 149.46 \\
80.31 & 85.26 & 79.66 \\
174.54 & 165.46 & 181.68 \\
\hline
\end{tabular}




\section{References}

1 Becke, A. D. D. Phys. Rev. A. 1988, 38, 3098-3100.

2 Lee, C., Yang, W.; Parr, R. G. Phys. Rev. B. 1988, 37, 785-789.

3 Krishnan, R., Binkley, J. S., Seeger, R. \& Pople, J. A. J. Chem. Phys. 1980, 72, 650-654.

4 Gill, P. M. W., Johnson, B. G., Pople, J. A. \& Frisch, M. J. Chem. Phys. Lett. 1992, 197, 499-505.

5 Gaussian 16, Revision A.03, Frisch, M. J.; Trucks, G. W.; Schlegel, H. B.; Scuseria, G. E.; Robb, M. A.; Cheeseman, J. R.; Scalmani, G.; Barone, V.; Petersson, G. A.; Nakatsuji, H.; Li, X.; Caricato, M.; Marenich, A. V.; Bloino, J.; Janesko, B. G.; Gomperts, R.; Mennucci, B.; Hratchian, H. P.; Ortiz, J. V.; Izmaylov, A. F.; Sonnenberg, J. L.; Williams-Young, D.; Ding, F.; Lipparini, F.; Egidi, F.; Goings, J.; Peng, B.; Petrone, A.; Henderson, T.; Ranasinghe, D.; Zakrzewski, V. G.; Gao, J.; Rega, N.; Zheng, G.; Liang, W.; Hada, M.; Ehara, M.; Toyota, K.; Fukuda, R.; Hasegawa, J.; Ishida, M.; Nakajima, T.; Honda, Y.; Kitao, O.; Nakai, H.; Vreven, T.; Throssell, K.; Montgomery, J. A., Jr.; Peralta, J. E.; Ogliaro, F.; Bearpark, M. J.; Heyd, J. J.; Brothers, E. N.; Kudin, K. N.; Staroverov, V. N.; Keith, T. A.; Kobayashi, R.; Normand, J.; Raghavachari, K.; Rendell, A. P.; Burant, J. C.; Iyengar, S. S.; Tomasi, J.; Cossi, M.; Millam, J. M.; Klene, M.; Adamo, C.; Cammi, R.; Ochterski, J. W.; Martin, R. L.; Morokuma, K.; Farkas, O.; Foresman, J. B.; Fox, D. J. Gaussian, Inc., Wallingford CT, 2016.

6 Zhao, Y.; Truhlar, D. G. Theor. Chem. Acc. 2008, 120, 215-24.

7 Weigend, F.; Ahlrichs, R. Phys. Chem. Chem. Phys. 2005, 7, 3297-3305.

8 NBO Version 3.1, E. D. Glendening, A. E. Reed, J. E. Carpenter, and F. Weinhold.

9 Cheeseman, J. R.; Trucks, G. W.; Keith, T. A.; Frisch, M. J. J. Chem. Phys. 1996, 104, 54975509.

10 Piou, T.; Michailidis, F.; Michaelides, M.; Jackson, K. E.; Semakul, N.; Taggart, T. D.; Newell, B. S.; Rithner, C. D.; Patono, R. S.; Rovis, T. J. Am. Chem. Soc. 2017, 139, 1296-1310.

11 MacroModel, version 11.8, Schrödinger, LLC, New York, NY, 2017. S627.

12 Harder, E.; Damm, W.; Maple, J.; Wu, C.; Reboul, M.; Xiang, J. Y.; Wang, L.; Lupyan, D.; Dahlgren, M. K.; Knight, J. L.; Kaus, J. W.; Cerutti, D. S.; Krilov, G.; Jorgensen, W. L; Abel, R.; Friesner, R. A. J. Chem. Theory Comput. 2016, 12, 281-296.

13 Wittkopp, A.; Schreiner, P. R. Chem. Eur. J. 2003, 9, 407-414. 
14 Supady, A.; Hecht, S.; Baldauf, C. Org. Lett. 2017, 19, 4199-4202.

15 MATLAB and Statistics Toolbox Release R2019b, The MathWorks, Inc., Natick, Massachusetts, United States

16 Verloop, A.; Tipker, J. Pharmacochem. Libr. 1977, 2, 63-81.

17 Verloop, A.; Tipker, J. Pharmacochem. Libr. 1987, 10, 97-102.

18 Santiago, C. B.; Guo, J.-Y.; Sigman, M. S. Chem. Sci. 2018, 9, 2398-2412.

19 Guo, J.-Y.; Minko, Y.; Santiago, C. B.; Sigman, M. S. ACS Catal. 2017, 7, 4144-415. 